Universidade de São Paulo

INSTITUTO DE FÍSICA

\title{
Seções de choque de bremsstrahlung de elétrons, estudo experimental no Acelerador Microtron de São Paulo
}

\author{
Juan Alejandro García Alvarez \\ Orientadora: Prof ${ }^{\mathrm{a}}$. Dr ${ }^{\mathrm{a}}$. Nora Lía Maidana \\ Co-orientador: Prof. Dr. José María Fernández Varea
}

Banca Examinadora:

Prof $^{a}$. Dra ${ }^{\mathrm{a}}$. Nora Lía Maidana (IFUSP)

Prof. Dr. Manfredo Harri Tabacniks (IFUSP)

Prof $^{a}$. Dra ${ }^{\mathrm{a}}$. Márcia de Almeida Rizzutto (IFUSP)

Prof. Dr. Johnny Ferraz Dias (UFRGS)

Prof. Dr. Josep Sempau (UPC-Barcelona)

Tese de doutorado apresentada ao

Instituto de Física para a obtenção

do título de Doutor em Ciências

São Paulo 


\section{FICHA CATALOGRÁFICA \\ Preparada pelo Serviço de Biblioteca e Informação do Instituto de Física da Universidade de São Paulo}

García Alvarez, Juan Alejandro

Seções de choque de bremsstrahlung de elétrons, estudo experimental no Acelerador Microtron de São Paulo. São Paulo, 2016.

Tese (Doutorado) - Universidade de São Paulo. Instituto de Física. Depto. Física Experimental.

Orientador: Profa. Dra. Nora Lía Maidana

Co-orientador: Prof. Dr. José María Fernández Varea

Área de Concentração: Física

Unitermos: 1. Física experimental; 2. Elétrons; 3. Acelerador de partículas; 4. Espectroscopia. 
"La verdadera ciencia enseña, sobre todo, a dudar y a ser ignorante" Miguel de Unamuno 


\section{Resumo}

\section{Seções de choque de bremsstrahlung de elétrons, estudo experimental no Acelerador Microtron de São Paulo}

Foram determinadas de maneira absoluta seções de choque duplamente diferenciais de produção de bremsstrahlung, para elétrons com energia cinética de 20, 50, 75 e $100 \mathrm{keV}$, nos elementos C, Al, Ag, Te, Ta e Au, e nos ângulos de emissão dos fótons $35^{\circ}, 90^{\circ}$ e $131^{\circ}$. A linha de feixe de baixa energia do Acelerador Microtron do Instituto de Física da Universidade de São Paulo foi utilizada para realizar as medidas, em um arranjo que contou com três detetores de Ge hiperpuro: dois planares e um coaxial. Os alvos finos foram confeccionados pela técnica de deposição física de vapores, enquanto suas espessuras foram caracterizadas a partir da espectrometria de retroespalhamento de Rutherford. Foi desenvolvido um modelo analítico para descrever a função reposta de detetores de Ge, que permitiu a deconvolução dos espectros de bremsstrahlung entre os $12 \mathrm{keV}$ e o ponto de máxima transferência de energia, com uma resolução de $1 \mathrm{keV}$. As seções de choque obtidas apresentam uma incerteza relativa entre $3 \%$ e $5 \%$, e foram comparadas com os cálculos teóricos que constituem o estado da arte na temática, e com os poucos dados experimentais encontrados na literatura. 


\section{Abstract}

\section{Electron bremsstrahlung cross sections, experimental study in the São}

Paulo Microtron Accelerator

Bremsstrahlung doubly differential cross sections were absolutely determined for electrons with kinetic energies of 20,50, 75 and $100 \mathrm{keV}$, in the elements $\mathrm{C}, \mathrm{Al}, \mathrm{Ag}, \mathrm{Te}$, Ta e Au, and the photon emission angles of $35^{\circ}, 90^{\circ}$ and $131^{\circ}$. The measurements were

performed at the low energy beam line of the São Paulo Microtron Accelerator, using three high purity Ge detectors: two planar and one coaxial. Thin targets were obtained by the Physical Vapor Deposition technique, and their thicknesses were characterized through Rutherford Backscattering Spectroscopy. An analytical model was developed to describe the response function of Ge detectors, which allowed to unfold the bremsstrahlung spectra between $12 \mathrm{keV}$ and the maximum energy transfer point, with an energetic resolution of $1 \mathrm{keV}$. The obtained cross sections present relative uncertainties between $3 \%$ and $5 \%$. These results were compared with the higher accurate theoretical calculations and also with the experimental reports found in literature. 


\section{Agradecimentos}

Agradeço em primeiro lugar aos meus pais. Não caberia em apenas umas linhas todo o amor e os ensinamentos de uma vida. Por serem meu exemplo, meu fôlego e a minha inspiração.

Às minhas avós, pelo carinho infinito. Por me dedicar parte de suas vidas e pelos anos de ausência sem desfrutar de vocês.

A meu irmão, por me acompanhar sempre.

A meu tio Juan César, por me fazer sentir como um filho, e pelo apoio incondicional.

A meu tio Jorge Luis, minhas primas Any e Angy, e a Consuelo.

A Alla e al Leovy, desejando que tivessem chegado até aqui....

À professora Nora, por ter me aceito para este trabalho, por me abrir as portas do Microtron, pela confiança e compreensão, e pela orientação entusiasta e precisa na parte experimental. Também pela sua ajuda no plano pessoal.

Ao professor José María, por ter me animado em todo momento a continuar, pelos múltiplos ensinamentos, por acreditar em mim, e pela rigorosa orientação teórica.

Ao professor Vito, pelas inúmeras dúvidas respondidas, pelas dicas e ideias para a realização deste trabalho, e pelo excelente curso de Estatística.

A Yadira, por estar ao meu lado o tempo todo.

A Malvy e sua familia.

Aos meus amigos de toda a vida, Yaima, Ana Bárbara, Carlete e Mercy.

A Camilo, que iniciou este caminho comigo e esteve durante muitos anos.

A Neivy, que me recomendou vir para a USP. Também a Fátima.

A Vicky (la vieja), pelo sorriso, pelas caronas e pelo apoio profissional e pessoal.

Aos amigos cubanos que me ajudaram a esquecer a saudade, por compartilhar a experiência no Brasil e pelos bons momentos. Principalmente a Israel e Martica. Também a Eudar, Ahmed, José Luis, Made, Ivón, Maria Adelfa, Mantecón, Yansel, Yoelvis, Eduardo, e todos os outros, que agora são muitos.

Aos meus amigos e companheiros de grupo Suelen e Osvaldo, pela disposição, os intercâmbios, os sábios conselhos, pela ajuda com o português, pelos bolos e também pelos cafés. Vocês são excelentes. 
A Iván, pela amizade, as sugestões e a leitura do texto.

À equipe do LAL, em especial a Alexandre, pelas muitas horas de medidas e repetições de experimentos. Aos professores Marcos Martins e Alessio Mangiarotti, pelos seminários, conferências e ensinamentos, e pelo trabalho conjunto. Ao professor Otaviano Helene, pelas interessantes conversas, os dados curiosos de diversos temas, e os esclarecimentos estatísticos. Ao professor João Arruda-Neto, pelos almoços, a ajuda e a afinidade com os cubanos. Também a Edu, Adriano, Bianca, Eliana, Roberto, Alfredo, Marcelo, Kaka, Sergio, Renato, e os funcionários da oficina mecânica.

A Wanda Engel, por me ensinar pacientemente o processo de confecção de filmes finos. Por todos os alvos realizados especialmente para este trabalho. Também ao professor Antonio Domingues pela preparação de muitos alvos.

Ao pessoal do LAMFI, pelas medidas de RBS. Ao professor Manfredo Harri Tabacniks pelo tempo dedicado ao grupo e pelas monitorias, a Thiago Silva, Marcos Moro e Cleber Lima Rodrigues pelas dicas para o processamento dos espectros. A Marquinhos pelas longas medidas.

Aos professores de Cuba, Katia, Oscar Díaz, Doris, César, Rubayo, Maikel, Guzmán, Amaya, Yasiel, Arturo, Yuri e Bárbara Garea, pela confiança e apoio.

Aos funcionários da CPG, pelo permanente e gentil atendimento.

Ao CNPq pelo fundamental e imprescindível apoio financeiro. À FAPESP e ao CAPES.

Minha gratidão também a todos aqueles que não mencionei mas contribuíram à culminação deste trabalho. 



\section{Sumário}

Resumo iv

Agradecimentos vi

Sumário viii

Lista de Figuras $\quad$ xiii

Lista de Tabelas $\quad$ xix

$\begin{array}{ll}\text { Abreviaturas } & \text { xxi }\end{array}$

1 Introdução 1

1.1 Motivação . . . . . . . . . . . . . . . . . . . . . . 1

1.2 Estrutura do trabalho . . . . . . . . . . . . . . . 3

2 Bremsstrahlung de elétrons: estado da arte teórico e experimental 5

2.1 Conceitos teóricos da emissão de bremsstrahlung de elétrons . . . . . . . . 5

2.2 Aproximação de Born. Fórmula de Bethe-Heitler . . . . . . . . . . . . . . 8

2.2.1 Efeitos da blindagem atômica . . . . . . . . . . . . . . . 10

2.3 Aproximação de Sommerfeld-Maue . . . . . . . . . . . . . . . . . . . . . . . . . . . . . .

2.3 .1 Limite não relativístico $\ldots \ldots \ldots \ldots$

2.3.2 Efeitos da blindagem atômica no formalismo de Sommerfeld-Maue 15

2.4 Expansão relativística em ondas parciais . . . . . . . . . . . . . . . . 16

2.5 Resultados de cálculos e tabulações publicadas para as seções de choque de emissão de bremsstrahlung . . . . . . . . . . . . . . . . . . . . . 19

2.5.1 Seção de choque diferencial em energia . . . . . . . . . . . . . . . . 21

2.5.2 Distribuição angular dos fótons . . . . . . . . . . . . . . . 25

2.6 Experimentos para a determinação de seções de choque de bremsstrahlung 27

3 Descrição dos experimentos $\quad 37$

3.1 Acelerador Microtron: linha de feixe de baixa energia . . . . . . . . . . . 37

3.2 Alvos finos para medidas de bremsstrahlung de elétrons . . . . . . . . . . . 40

3.2 .1 Confecção dos alvos . . . . . . . . . . . . . . . . 40

3.3 Detetores, sistema de aquisição e arranjo experimental para medidas de bremsstrahlung ......................... 42 
3.4 Desenho dos experimentos: justificativa da escolha de elementos, energias e ângulos de medida . . . . . . . . . . . . . . . . . . . . 4 45

4 Modelagem da função resposta de detetores de Ge $\quad 47$

4.1 Metodologia para a deconvolução dos espectros de bremsstrahlung . . . . 47

4.2 Função reposta de detetores semicondutores: estado da arte . . . . . . . . . . . . . . . . . . . 50 50

4.3 Modelo analítico de função resposta para detetores planares . . . . . . . . 52

4.3.1 Função reposta intrínseca . . . . . . . . . . . . . . . . . . . 52

4.3.2 Escape dos raios-x K do Ge . . . . . . . . . . . . . . 53

4.3.3 Escape de fótons após um espalhamento Compton . . . . . . . . 56

4.3.3.1 Seção de choque duplamente diferencial na AIR . . . . . 59

4.3.4 Escape de fotoelétrons do volume ativo . . . . . . . . . . . 60

4.3.5 Absorção total da energia do fóton . . . . . . . . . . . . . 65

4.3.6 Contribuições das interações externas à função resposta . . . . . . 65

4.3.6.1 Espectro produzido pelo espalhamento inelástico de fótons na janela de $\mathrm{Be} \ldots \ldots \ldots 6$

4.3.6.2 Contribuição do espalhamento elástico de fótons na janela de Be . . . . . . . . . . . . . 69

4.3.6.3 Espectro produzido pelos fotoelétrons originados na camada morta de Ge . . . . . . . . . . . . . . . 69

4.4 Simulações Monte Carlo para testar o modelo analítico de função resposta de detetores planares . . . . . . . . . . . . . . . . . 71

4.4.1 Espectro de escape Compton . . . . . . . . . . . . . . 73

4.4 .2 Função resposta total . . . . . . . . . . . . . . . . . . . . 74

4.5 Modelo analítico de função resposta para detetores coaxiais . . . . . . . . 79

4.5.1 Teste do modelo, comparação com simulações Monte Carlo . . . . 83

4.6 Teste do procedimento de deconvolução de espectros contínuos . . . . . 85

5 Influência da geometria na função resposta: teste experimental $\quad 89$

5.1 Função resposta: experimento de teste . . . . . . . . . . . . . . . . . 89

5.1.1 Comparação do modelo de FR com o experimento . . . . . . . . . 92

5.2 Influência do colimador na função resposta . . . . . . . . . . . . . . . 95

5.2 .1 Transparência do colimador . . . . . . . . . . . . . . 98

5.2.2 Contribuição do espalhamento Compton no colimador . . . . . . . 99

5.3 Influência da fonte na função resposta . . . . . . . . . . . . . . . . . 102

5.4 Quantificação dos efeitos da geometria . . . . . . . . . . . . . . . . . 104

6 Caracterização do arranjo experimental no Microtron e dos alvos $\quad 107$

6.1 Otimização do sistema de aquisição . . . . . . . . . . . . . . . . 107

6.1.1 Conformação de pulsos . . . . . . . . . . . . . . . . . . 108

6.1.2 Estudo do tempo morto do sistema de aquisição . . . . . . . . . . 110

6.2 Caracterização experimental dos detetores . . . . . . . . . . . . . . . 114

6.2.1 Calibração da resolução dos detetores: determinação dos parâmetros de dispersão Gaussiana da resposta . . . . . . . . . . . . . . . 115

6.2.2 Calibração de eficiência: determinação do ângulo sólido . . . . . . 117

6.3 Determinação da espessura dos alvo . . . . . . . . . . . . . . . . . . . . . . . . . . 123

6.4 Alguns critérios quantitativos em relação aos alvos . . . . . . . . . . . . 129 
7 Medidas das SCDDs de bremsstrahlung: tratamento de dados 133

7.1 Correções aos espectros de bremsstrahlung coletados . . . . . . . . . . . 133

7.2 Determinação da energia do feixe de elétrons e da intensidade do espectro de bremsstrahlung no ponto de máxima transferência de energia . . . . . 141

7.3 Deconvolução dos espectros de bremsstrahlung . . . . . . . . . . . . . . . 148

7.4 Verificação cruzada: Ionização da camada K da Ag por impacto de elétrons 150

8 Resultados das SCDDs de emissão de bremsstrahlung de elétrons $\mathbf{1 5 5}$

8.1 Estrutura de apresentação dos resultados . . . . . . . . . . . . . . . . 155

8.2 Resultados da SCDD de emissão de bremsstrahlung no ponto de máxima transferência de energia e da SCIK da Ag, discussão . . . . . . . . . . . 156

8.3 Resultados da SCDD de emissão de bremsstrahlung para fótons de diferentes energias, discussão . . . . . . . . . . . . . . . . . . . 164

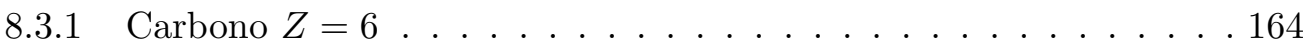

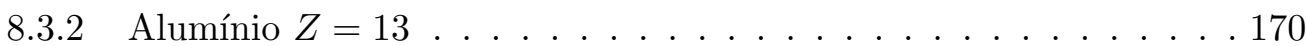

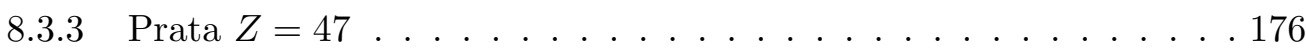

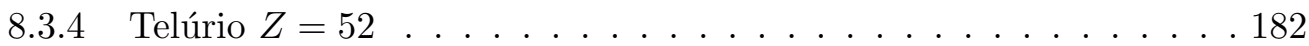

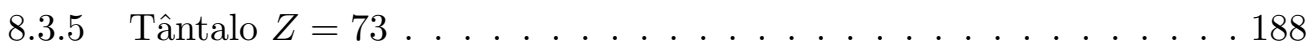

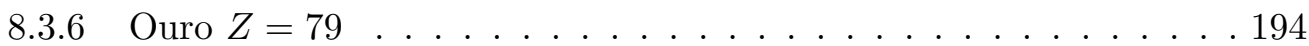

8.3.7 Comparação com os resultados publicados na literatura . . . . . . 200

8.4 Discussão das tendências gerais observadas nas SCDDs experimentais . . 202

9 Conclusões $\quad 205$

$\begin{array}{ll}\text { A Perfis de Compton do átomo de Ge } & 207\end{array}$

B Coeficientes $B_{n}$ para o cálculo da distribuição angular de bremsstrahlung 



\section{Lista de Figuras}

2.1 Esquema do arranjo experimental tipicamente utilizado nas medidas de SCDDs de emissão de bremsstrahlung. . . . . . . . . . . . . . . . . 27

3.1 Esquema do Acelerador Microtron do IFUSP. . . . . . . . . . . . . . . . 38

3.2 Esquema da câmara de irradiação correspondente à linha de $100 \mathrm{keV}$. . . 39

3.3 Fotografias da câmara de irradiação correspondente à linha de $100 \mathrm{keV}$. . 39

3.4 Torre de alvos da câmara de $100 \mathrm{keV}$. . . . . . . . . . . . . . . . . . . 40

3.5 Substratos colocados sobre as molduras de carbono. . . . . . . . . . . . 41

3.6 Processo de evaporação de Ag por aquecimento Joule para a confecção de um alvo típico. . . . . . . . . . . . . . . . . . . . 42

3.7 Fotografia de um alvo típico de Au produzido pelo processo de sputtering. 42

3.8 Fotografias tomadas durante o alinhamento do sistema detetor-colimador com o ponto central da câmara de irradiação. . . . . . . . . . . . . . . 4 44 . . 4

3.9 Montagem final dos detetores na câmara de irradiação. . . . . . . . . . . . 45

4.1 Representação geométrica de um detetor planar de Ge típico. . . . . . . . 52

4.2 Representação geométrica do processo de escape de raios- $\mathrm{x} K$ característicos no detetor . . . . . . . . . . . . . . . . . . . . 54

4.3 Probabilidade de escape do detetor dos raios-x K $\alpha$ e K $\beta$ do Ge. . . . . . . 55

4.4 SCDD de espalhamento Compton em Ge para fótons de $60 \mathrm{keV}$, e ângulos $\theta=60^{\circ}, \theta=90^{\circ}$ e $\theta=180^{\circ} \ldots \ldots \ldots \ldots \ldots$

4.5 Comparação entre a SCD de espalhamento Compton em Ge fornecida pela AIR, e as aproximações de WH e KN, para fótons de 60 e $100 \mathrm{keV}$. . 61

4.6 Representação geométrica do processo de escape dos fotoelétrons pelas superfícies do detetor. . . . . . . . . . . . . . . . . . . 62

4.7 Distribuição angular de fotoelétrons da camada K do Ge segundo a SCD de Sauter. . . . . . . . . . . . . . . . . . 6 63

4.8 Coeficientes de atenuação mássica para espalhamento incoerente, coerente e efeito fotoelétrico no Be. . . . . . . . . . . . . . . . . . 66

4.9 Representação geométrica do processo de espalhamento inelástico na janela do detetor. . . . . . . . . . . . . . . . . . . 6 67

4.10 Comparação entre a SCD de espalhamento Compton no Be fornecida pela AIR e a aproximação de KN, para fótons de 60 e $100 \mathrm{keV}$. . . . . . . . . 68

4.11 Representação geométrica do processo de migração de fotoelétrons da camada morta de Ge ao volume ativo. . . . . . . . . . . . . . . 70

4.12 Simulação Penelope de espectros de energia deposita no cristal de Ge para um fóton incidente de $60 \mathrm{keV}$. . . . . . . . . . . . . . . 72

4.13 Convolução Gaussiana do espectro de altura de pulso simulado com o código PENELOPE para $E=20 \mathrm{keV}$. 
4.14 Contínuo de Compton produzido pelo escape de fótons para energias incidentes $E=40,60,80 \mathrm{e} 100 \mathrm{keV} \ldots \ldots \ldots \ldots \ldots$

4.15 Componentes da FR calculadas (modelo) e simuladas (sim. MC) para

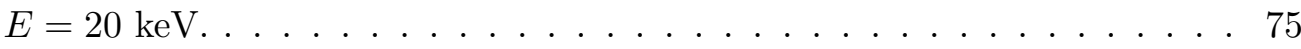

4.16 Componentes da FR calculadas (modelo) e simuladas (sim. MC) para

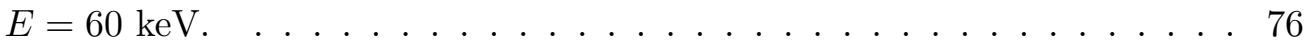

4.17 Componentes da FR calculadas (modelo) e simuladas (sim. MC) para

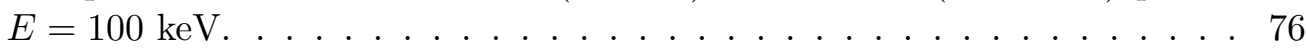

4.18 Função resposta total acompanhada pela sua respectiva função de acumulação de probabilidade para $E=20,60$ e $100 \mathrm{keV}$. . . . . . . . . 78

4.19 Área do espectro que representa cada mecanismo de deposição parcial de energia. . . . . . . . . . . . . . . . . . . 79

4.20 Representação geométrica simplificada do cristal de um detetor coaxial de Ge. . . . . . . . . . . . . . . . . . . . . 80

4.21 Comparação dos espectros de escape Compton nas configurações planar e coaxial para energias dos fótons incidentes entre 100 e $50 \mathrm{keV} . \quad$. . . . . 84

4.22 Comparação entre a área que representa o contínuo de Compton em relação à área total observada no EAP, nas configurações coaxial e planar. 85

4.23 Comparação entre o modelo e a simulação PENELOPE dos espectros de escape Compton na geometria coaxial. . . . . . . . . . . . . . 85

4.24 Espectro de fótons incidentes e distribuição de altura de pulso simulados com parâmetros de entrada $W_{\min }=32 \mathrm{keV}$ e $W_{\max }=95 \mathrm{keV} \ldots \ldots$. . . 87

4.25 Comparação entre o EAP e o espectro deconvoluído. . . . . . . . . . . . . 87

4.26 Comparação entre o espectro deconvoluído com a matriz $\mathbf{R}$ calculada segundo o modelo analítico e o espectro verdadeiro utilizado na simulação MC . . . . . . . . . . . . . . . . . 88

4.27 Comparação entre o espectro deconvoluído com a matriz $\mathbf{R}$ calculada segundo o modelo analítico e o espectro verdadeiro utilizado na simulação

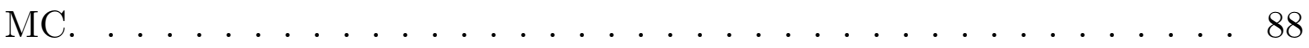

5.1 Esquema bidimensional do arranjo experimental e fotografia da fonte de ${ }^{241}$ Am utilizada nas medidas de teste da FR. . . . . . . . . . . . . . 90

5.2 Espectro de ${ }^{241} \mathrm{Am}$ coletado durante $t_{\mathrm{v}}=446400 \mathrm{~s}$ e do fundo radioativo normalizado ao mesmo tempo. . . . . . . . . . . . . . . . 91

5.3 Espectros netos de ${ }^{241} \mathrm{Am}$ coletados na primeira experiencia (Exp. 1) e na segunda (Exp. 2) incluindo as lâminas atenuadoras entre a fonte e o colimador. . . . . . . . . . . . . . . . . . . 92

5.4 Espectros da fonte de ${ }^{241} \mathrm{Am}$ medido e calculado segundo o modelo de FR na primeira experiência. . . . . . . . . . . . . . . . 94

5.5 Espectros da fonte de ${ }^{241} \mathrm{Am}$ medido e calculado segundo o modelo de FR na segunda experiência. . . . . . . . . . . . . . . . 95

5.6 Esquema bidimensional da geometria do colimador, onde se representam as possíveis trajetórias dos fótons. . . . . . . . . . . . . . 96

5.7 Iluminação das paredes do colimador quando o parâmetro $d_{\mathrm{f}}=3 \mathrm{ou}$

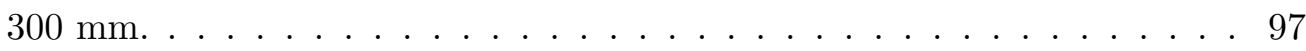

5.8 Relação $\Omega_{\mathrm{ef}} / \Omega_{\mathrm{g}}$ em porcento, para parâmetros $d_{\mathrm{f}}=3$ ou $300 \mathrm{~mm}$. . . . . . 98

5.9 Esquema tridimensional da geometria do espalhamento incoerente de fótons no colimador. . . . . . . . . . . . . . . . . . 99

5.10 Esquema do espalhamento Compton no material que compõe a fonte. . . . 103 
5.11 Comparação entre o espectro experimental e os modelos analíticos da contribuição dos elementos do arranjo na função resposta. . . . . . . . . . 105

5.12 Função de acumulação de probabilidade de cada uma das contribuições

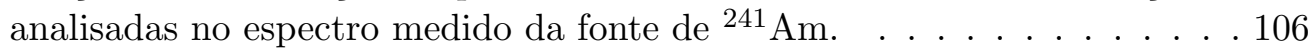

6.1 Forma dos pulsos amplificados no DSPEC para diferentes valores de $t_{\mathrm{s}} \mathrm{e}$

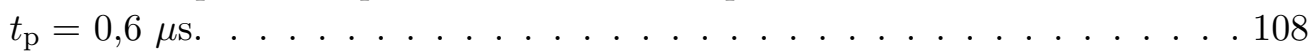

6.2 Variação da resolução da linha $\mathrm{L} \alpha$ do Au em função do $t_{\mathrm{s}} \operatorname{com} t_{\mathrm{p}}=0,6 \mu \mathrm{s} .109$

6.3 Resolução da linha $\mathrm{L} \alpha$ do $\mathrm{Au}$, no detetor colocado a $\theta=35^{\circ}$, em função

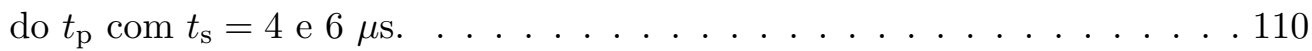

6.4 Exemplo dos EAP coletados para diferentes correntes $I$ do feixe de elétrons, que equivalem às taxas $m$ de contagens, no detetor colocado a $\theta=35^{\circ} \ldots 111$

6.5 Ajuste do tempo morto segundo o MP e o MNP. . . . . . . . . . . . . . 113

6.6 Resíduos reduzidos do ajuste segundo o MP considerando as incertezas

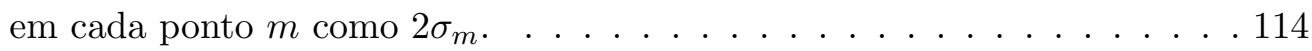

6.7 Processo de preparação e formato final das fontes radioativas utilizadas nas calibrações em resolução e eficiência dos detetores. . . . . . . . . . . 115

6.8 Calibração de resolução dos três detetores na faixa de energia 20-160 keV. 116

6.9 Minimização da função de mérito $Q(\Omega, d)$ considerando os modelos de ajuste M-1 e M-2, nos três detetores. . . . . . . . . . . . . . 120

6.10 Eficiências experimentais e curvas ajustadas para os três detetores. . . . . 121

6.11 Representação do retroespalhamento das partículas no alvo. . . . . . . . . 123

6.12 Fator de Andersen para a blindagem da carga nuclear em C, Ag e Au quando incidem partículas $\alpha$ de $2200 \mathrm{keV}$. . . . . . . . . . . . 126

6.13 Comparação entre as SCD Rutherford e non-Rutherford para a reação

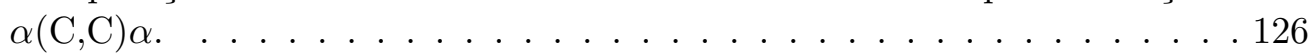

6.14 Espectros RBS experimentais e simulados com o programa MultiSIMNRA para o ajuste dos parâmetros dos alvos de Te e Au. . . . . . . . . . . . 128

6.15 Relação $\langle\Delta d\rangle / d$ em função da energia do elétron incidente para cada um dos alvos utilizados nas medidas de bremsstrahlung. . . . . . . . . . . 130

6.16 Perdas de energia total $\left(\Delta E_{0}\right)$ e relativa $\left(\Delta E_{0} / E_{0}\right)$ experimentadas pelos elétrons ao atravessar os filmes utilizados nas medidas de emissão de bremsstrahlung. . . . . . . . . . . . . . . . . . . . . . . 131

7.1 Espectro coletado para o alvo de Te, com $E_{0}=51,19 \mathrm{keV}$ a $90^{\circ}$, e correções pela radiação de fundo (Fun.) e por empilhamento de pulsos (EP) . . . . . . . . . . . . . . . . . . . . 135

7.2 Faixa do espectro de Te em que se observam as contagens por EP. . . . . 136

7.3 Correção do espectro de bremsstrahlung do alvo de Te, coletado a $90^{\circ}$, para elétrons de $51,19 \mathrm{keV}$, pela radiação gerada no substrato de C. . . . 137

7.4 Espectros de bremsstrahlung corrigidos por fundo radioativo, empilhamento de pulsos e radiação gerada no substrato, para $E_{0}$ em torno de $20 \mathrm{keV}$, nos três ângulos de coleção e os elementos Al, Ag e Au. . . . . . 138

7.5 Espectros de bremsstrahlung corrigidos por fundo radioativo, empilhamento de pulsos e radiação gerada no substrato, para $E_{0}$ em torno de $50 \mathrm{keV}$, nos três ângulos de coleção e os elementos Al, Ag e Au. . . . . . . 139

7.6 Espectros de bremsstrahlung corrigidos por fundo radioativo, empilhamento de pulsos e radiação gerada no substrato, para $E_{0}$ em torno de $100 \mathrm{keV}$, nos três ângulos de coleção e os elementos Al, Ag e Au. . . . . . 140 
7.7 Espectro de bremsstrahlung do alvo Al, coletado para a energia incidente $E_{0}=50,96 \mathrm{keV}$ no ângulo $\theta=35^{\circ}$ na faixa de $h 6-14 \mathrm{keV} . \ldots . . . .141$

7.8 Funções densidade de probabilidade para os espectros de fótons gerado no alvo $(B)$ e medido no detector $(F)$ quando $E_{0}=100 \mathrm{keV}$, com larguras

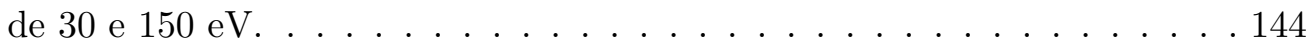

7.9 Curvas de nível de $\chi^{2}$ do ajuste dos parâmetros $E_{0}$ e $\sigma_{0}$, para diferentes energias do feixe de elétrons e o alvo de Ag. . . . . . . . . . . . 145

7.10 Ajuste do modelo proposto às contagens coletadas no tip, para o alvo de $\mathrm{Ag}$ e os três detetores. Os parâmetros obtidos são $E_{0}=26,97 \mathrm{keV}$ e $\sigma_{0}=0,11 \mathrm{keV}$ (coluna esquerda) e $E_{0}=51,09 \mathrm{keV}$ e $\sigma_{0}=0,09 \mathrm{keV}$ (coluna direita). . . . . . . . . . . . . . . . . 146

7.11 Ajuste do modelo proposto às contagens coletadas no tip, para o alvo de $\mathrm{Ag}$ e os três detetores. Os parâmetros obtidos são $E_{0}=70,63 \mathrm{keV}$ e $\sigma_{0}=0,08 \mathrm{keV}$ (coluna esquerda) e $E_{0}=100,44 \mathrm{keV}$ e $\sigma_{0}=0,10 \mathrm{keV}$ (coluna direita). . . . . . . . . . . . . . . . . . . . . . 147

7.12 Representação da matriz resposta $\mathbf{R}$ do detetor colocado a $35^{\circ}$. . . . . . 149

7.13 Representação da inversa da matriz resposta $\mathbf{R}^{-1}$ do detetor colocado a $35^{\circ} \ldots \ldots \ldots \ldots \ldots \ldots \ldots \ldots$

7.14 Espectro medido $N(h)$ e deconvoluído $B(W)$, para o alvo de Ta a $35^{\circ}$ e as energias incidentes $E_{0}=51,19$ e $100,80 \mathrm{keV} \ldots \ldots \ldots$. . . . . 150

7.15 Comparação entre os espectros deconvoluído com a matriz resposta $\mathbf{R}$ $(B(W))$ e calculado com a eficiência intrínseca $\varepsilon_{\mathrm{i}}(W)\left(B^{\prime}(W)\right)$, para o mesmo alvo de Ta da Fig. 7.14. . . . . . . . . . . . . . . . . . 151

8.1 Comparação entre a SCDD experimental e teórica no ponto $\kappa=1$, para os elementos C e Al. . . . . . . . . . . . . . . . . . . . . 160

8.2 Comparação entre a SCDD experimental e teórica no ponto $\kappa=1$, para os elementos Te e Ta. . . . . . . . . . . . . . . . . . . 161

8.3 Comparação entre a SCDD experimental e teórica no ponto $\kappa=1$, para os elementos Ag e Au. . . . . . . . . . . . . . . . . . . 162

8.4 Comparação entre as SCIKs em Ag obtidas no presente experimento, os resultados de Vanin e colaboradores e o cálculo segundo a ABOD. . . . . 163

8.5 Comparação entre as SCIKs em Ag obtidas no presente experimento, os dados experimentais encontrados na literatura e o cálculo segundo a ABOD.164

8.6 Resultados das SCDDs de emissão de bremsstrahlung em C para elétrons incidentes de $22,39 \mathrm{keV}$. . . . . . . . . . . . . . . . . 165

8.7 Resultados das SCDDs de emissão de bremsstrahlung em C para elétrons incidentes de $51,01 \mathrm{keV} \ldots \ldots \ldots$. . . . . . . . . . . . 166

8.8 Resultados das SCDDs de emissão de bremsstrahlung em $\mathrm{C}$ para elétrons incidentes de $75,46 \mathrm{keV}$. . . . . . . . . . . . . . . . 167

8.9 Resultados das SCDDs de emissão de bremsstrahlung em C para elétrons incidentes de 100,57 keV. . . . . . . . . . . . . . . . . . 168

8.10 Razão experimento/teoria entre as SCDDs obtidas para o C, considerando todas energias e ângulos medidos. . . . . . . . . . . . . . . . . 169

8.11 Resultados das SCDDs de emissão de bremsstrahlung em Al para elétrons incidentes de $20,36 \mathrm{keV}$. . . . . . . . . . . . . . . . . . 171

8.12 Resultados das SCDDs de emissão de bremsstrahlung em Al para elétrons incidentes de $50,96 \mathrm{keV}$. . . . . . . . . . . . . . . . . . . 172 
8.13 Resultados das SCDDs de emissão de bremsstrahlung em Al para elétrons

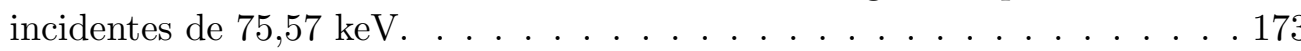

8.14 Resultados das SCDDs de emissão de bremsstrahlung em Al para elétrons incidentes de 100,48 keV. . . . . . . . . . . . . . . . . . . 174

8.15 Razão experimento/teoria entre as SCDDs obtidas para o Al, considerando todas energias e ângulos medidos. . . . . . . . . . . . . 175

8.16 Resultados das SCDDs de emissão de bremsstrahlung em Ag para elétrons incidentes de 20,30 keV. . . . . . . . . . . . . . . . 177

8.17 Resultados das SCDDs de emissão de bremsstrahlung em Ag para elétrons

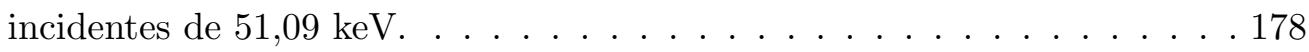

8.18 Resultados das SCDDs de emissão de bremsstrahlung em Ag para elétrons incidentes de $70,63 \mathrm{keV}$. . . . . . . . . . . . . . . . . . . . 179

8.19 Resultados das SCDDs de emissão de bremsstrahlung em Ag para elétrons incidentes de 100,44 keV. . . . . . . . . . . . . . . . . . . 180

8.20 Razão experimento/teoria entre as SCDDs obtidas para o Ag, considerando todas energias e ângulos medidos. . . . . . . . . . . . . . . . 181

8.21 Resultados das SCDDs de emissão de bremsstrahlung em Te para elétrons incidentes de $22,57 \mathrm{keV} . \ldots \ldots$. . . . . . . . . . . . . . 183

8.22 Resultados das SCDDs de emissão de bremsstrahlung em Te para elétrons

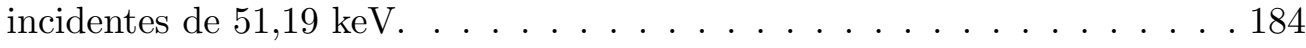

8.23 Resultados das SCDDs de emissão de bremsstrahlung em Te para elétrons incidentes de $75,71 \mathrm{keV}$. . . . . . . . . . . . . . . . . 185

8.24 Resultados das SCDDs de emissão de bremsstrahlung em Te para elétrons incidentes de 100,73 keV. . . . . . . . . . . . . . . . . 186

8.25 Razão experimento/teoria entre as SCDDs obtidas para o Te, considerando todas energias e ângulos medidos. . . . . . . . . . . . . . 187

8.26 Resultados das SCDDs de emissão de bremsstrahlung em Ta para elétrons incidentes de $22,58 \mathrm{keV} \ldots \ldots \ldots$. . . . . . . . . . . . . 189

8.27 Resultados das SCDDs de emissão de bremsstrahlung em Ta para elétrons

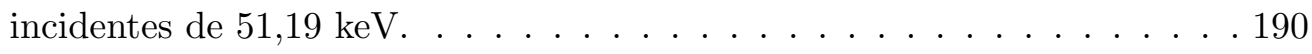

8.28 Resultados das SCDDs de emissão de bremsstrahlung em Ta para elétrons incidentes de $75,69 \mathrm{keV}$. . . . . . . . . . . . . . . . . . 191

8.29 Resultados das SCDDs de emissão de bremsstrahlung em Ta para elétrons

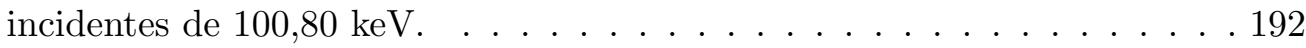

8.30 Razão experimento/teoria entre as SCDDs obtidas para o Ta, considerando todas energias e ângulos medidos. . . . . . . . . . . . . . 193

8.31 Resultados das SCDDs de emissão de bremsstrahlung em Au para elétrons incidentes de $20,38 \mathrm{keV}$. . . . . . . . . . . . . . . . . 195

8.32 Resultados das SCDDs de emissão de bremsstrahlung em Au para elétrons

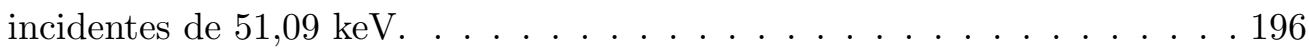

8.33 Resultados das SCDDs de emissão de bremsstrahlung em Au para elétrons incidentes de $75,68 \mathrm{keV}$. . . . . . . . . . . . . . . . . . 197

8.34 Resultados das SCDDs de emissão de bremsstrahlung em Au para elétrons incidentes de 100,48 keV. . . . . . . . . . . . . . . . . . . 198

8.35 Razão experimento/teoria entre as SCDDs obtidas para o $\mathrm{Au}$, considerando todas energias e ângulos medidos. . . . . . . . . . . . . . . . 199 
8.36 Comparação entre os resultados obtidos neste trabalho e os dados encontrados na literatura, para $E_{0} \sim 50 \mathrm{keV}, \theta=90^{\circ}$ e os elementos $\mathrm{Al}, \mathrm{Ag}$, Te e Au. . . . . . . . . . . . . . . . . . . . 201

A.1 Perfis de Compton para os orbitais 1s, 2s, 2p e 3s do átomo de Ge. . . . . 207

A.2 Perfis de Compton para os orbitais $3 \mathrm{p}, 3 \mathrm{~d}, 4 \mathrm{~s}, 4 \mathrm{p}$ e contribuição de todos os orbitais (total) do átomo de Ge. . . . . . . . . . . . . . 208 


\section{Lista de Tabelas}

2.1 Comparação entre os resultados publicados por Pratt e colaboradores $\left(^{\dagger}\right)$ em 1977, e Seltzer e Berger $\left(^{\star}\right)$ em 1986, para a SCD escalada. A unidade de $\chi\left(Z, E_{0}, \kappa\right)$ é mb. $\delta$ é a razão entre os cálculos de Pratt e colaboradores (sem incluir a componente do bremsstrahlung no campo dos elétrons atômicos) e as tabulações de Seltzer e Berger. . . . . . . . . . 25

2.2 Desvio dos dados de Quarles e Heroy em relação ao cálculo de Tseng, Pratt e Lee para $S\left(Z, E_{0}, \kappa, \theta\right)$, com $E_{0}=50 \mathrm{keV}$. Os valores $\pm 1, \pm 2, \pm 3$ indicam a quantidade de desvios padrões experimentais acima ou abaixo da curva teórica. . . . . . . . . . . . . . . . . . 31

3.1 Distâncias características do posicionamento dos detetores e colimadores em referência à câmara de irradiação. . . . . . . . . . . . . . . . . . . 44

6.1 Parâmetros $\vartheta$ e $\zeta$ ajustados segundo o MP e o MNP, e tempo morto $\tau$ determinado em cada caso. . . . . . . . . . . . . . . . . . . 113

6.2 Resultados do ajuste dos coeficientes da largura à meia altura $\left(a^{\prime}\right.$ e $\left.b^{\prime}\right)$ e dos parâmetros de dispersão Gaussiana $(a$ e $b) . \ldots . . .117$

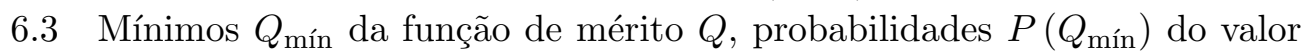
de $\chi_{\nu}^{2}=Q_{\text {mín }}$ exceder o valor crítico e resultados dos parâmetros $d$ e $\omega_{\mathrm{g}}$, ajustados segundo os modelos 1 (M-1) e 2 (M-2). Os graus de liberdade são $\nu=18,21$ e 16 para os detetores colocados a $\theta=35^{\circ}, 90^{\circ}$ e $131^{\circ}$ respectivamente. . . . . . . . . . . . . . . . . 122

6.4 Resultados do ajuste da densidade superficial de átomos $t$, equivalente à espessura $d$, das camadas do elemento e do substrato (C) que conformam cada alvo. . . . . . . . . . . . . . . . . . . . . . 129

8.1 Resultados das SCDDs no ponto de máxima transferência de energia ( $\kappa=$ $W / E_{0}=1$ ), para elétrons incidentes entre 20 e $100 \mathrm{keV}$, em alvos de C, $\mathrm{Al}$, Te e Ta. Os valores teóricos são interpolados das tabelas de Seltzer e Berger e Kissel e colaboradores. R é a razão experimento/teoria. . . . . . 158

8.2 Resultados das SCDDs no ponto de máxima transferência de energia $(\kappa=$ $W / E_{0}=1$ ), para elétrons incidentes entre 20 e $100 \mathrm{keV}$, em alvos de Ag e Au. Os valores teóricos são interpolados das tabelas de Seltzer e Berger e Kissel e colaboradores. R é a razão experimento/teoria. . . . . . . . . . 159

8.3 Resultados das SCIKs da Ag obtidos em cada ângulo $\theta$ de medida, valor médio experimental e estimativa teórica segundo a ABOD. $\mathrm{R}$ é a razão entre a média experimental e a SCIK teórica. . . . . . . . . . . . . 163

B.1 Coeficientes $B_{n}$ tabulados por Kissel e colaboradores para o cálculo da distribuição angular de bremsstrahlung (Parte 1). . . . . . . . . . . . 210 
B.2 Coeficientes $B_{n}$ tabulados por Kissel e colaboradores para o cálculo da distribuição angular de bremsstrahlung (Parte 2). . . . . . . . . . . . . 211

B.3 Coeficientes $B_{n}$ tabulados por Kissel e colaboradores para o cálculo da distribuição angular de bremsstrahlung (Parte 3) . . . . . . . . . . . . . 212 


\section{Abreviaturas}

AB Aproximação de Born

ABOD Aproximação de Born de Ondas Distorcidas

AIR Aproximação de Impulso Relativística

CF Copo de Faraday

CI1 Câmara de Irradiação 1

CI2 Câmara de Irradiação 2

EAP Espectro de Altura de Pulso

EP Empilhamento de Pulsos

Eq. Equação

Fig. Figura

FFB Função de Forma do Bremsstrahlung

FWHM Full Width at Half Maximum

FR Função Resposta

HFS Hartree-Fock-Slater

KN Klein-Nishina

MC Monte Carlo

MMQ Método dos Mínimos Quadrados

PC Perfil Compton

SCD Seção Choque Diferencial

SCDD Seção Choque Duplamante Diferencial

SCIK Seção Choque de Ionização da camada K

SCTD Seção Choque Triplamante Diferencial

SM Sommerfeld-Maue

TF Thomas-Fermi

TFM Thomas-Fermi-Modificado 
UN Unidades Naturais

WH Waller-Hartree 
Como en La Habana de 2010 y 2012, a mis abuelas, mis padres y mi hermano. 



\section{Capítulo 1}

\section{Introdução}

\subsection{Motivação}

A emissão de bremsstrahlung é um dos mecanismos mais importantes de perdas de energia dos elétrons ao interagir com a matéria. Por seu caráter fundamental constitui um processo amplamente estudado do ponto de vista teórico [1]. Porém, não existe um formalismo único capaz de descrever as distribuições angulares e de momentos para qualquer energia dos elétrons incidentes ou dos fótons emitidos, e ao mesmo tempo, para todos os números atômicos.

As seções de choque diferenciais na energia do fóton tem sido calculadas para elétrons entre $1 \mathrm{keV}$ e $10 \mathrm{GeV}$ em algumas espécies atômicas (He, O, Al, Ag, Au e U), aplicando diferentes aproximações de acordo com o intervalo energético [2,3]. A partir da interpolação matemática dos resultados obtidos nesses cálculos, Seltzer e Berger [3] estimaram essa grandeza para a maioria dos elementos da tabela periódica. Para energias cinéticas dos elétrons menores que $2 \mathrm{MeV}$, os dados dos autores apresentam a maior incerteza de toda a compilação: em torno de $10 \%$.

No entanto, as seções de choque diferenciais só na energia do fóton, não são quantidades diretamente mensuráveis. Logo, os esforços teóricos se centraram também no estudo da função de forma do bremsstrahlung que descreve a distribuição angular da emissão dos fótons $[4,5]$. Nesse tema, as tabulações publicadas por Kissel e colaboradores [5] constituem o que se considera o estado da arte, e os autores estimaram um $5 \%$ de precisão nos seus cálculos.

A combinação dos resultados teóricos da seção de choque de emissão de bremsstrahlung diferencial na energia do fóton, e da distribuição angular, permitiram calcular as seções de choque duplamente diferenciais em ângulo de emissão e energia do fóton, com a maior 
precisão disponível até hoje. Consequentemente, para energias cinéticas dos elétrons inferiores a $2 \mathrm{MeV}$, estima-se um $11 \%$ de margem de erro nesses dados.

A seção de choque duplamente diferencial de emissão de bremsstrahlung de elétrons tem sido medida por diferentes métodos, mas existem poucos experimentos onde essa grandeza seja determinada de maneira absoluta. Especificamente, para energias inferiores a $100 \mathrm{keV}$, os principais trabalhos experimentais datam das décadas do 60 e 70 do século passado, realizaram-se para $\mathrm{Al}$ e $\mathrm{Au}$, e utilizaram detetores de baixa resolução e sistemas eletrônicos analógicos para a aquisição de espectros $[6,7]$. As incertezas relativas associadas a essas medidas estiveram entre $10 \%$ e $15 \%$, com a maior contribuição relacionada com a determinação da espessura dos alvos, e com a deconvolução dos espectros de bremsstrahlung. Outras experiências onde foi estudada a forma do espectro de fótons de maneira relativa $[8,9]$, também apresentaram incertezas superiores a 20\%. Portanto, os resultados disponíveis na literatura não permitem estabelecer critérios conclusivos em relação à precisão das tabulações de Seltzer e Berger [3] e Kissel et al. [5], quando se consideram na comparação experimento-teoria as diferentes variáveis envolvidas no mecanismo de interação elétron-átomo: elemento alvo (ou número atômico); energia incidente; ângulos e energias de emissão dos fótons. Diante disso, a determinação absoluta de seções de choque duplamente diferenciais de emissão de bremsstrahlung de elétrons, parâmetros considerados fundamentais no campo da física atômica, continua sendo um tema de interesse nas pesquisas experimentais.

No Laboratório do Acelerador Microtron do Instituto de Física da Universidade de São Paulo, a linha de baixa energia para elétrons entre 10 e $100 \mathrm{keV}$ está operando há aproximadamente 2 anos. A instalação, desenhada em especial para experimentos de interação de elétrons com a matéria onde são emitidos fótons, possui uma câmara de irradiação com janelas espectroscópicas situadas em diferentes ângulos, que facilitam medidas de distribuição angular. O equipamento associado à câmara possibilita a coleção da totalidade da carga incidente para feixes contínuos de corrente da ordem dos nA. Além disso, o Laboratório dispõe de detetores de Ge e espectrômetros digitais de última geração, que permitem a aquisição de espectros com alta resolução. Adicionalmente, o Instituto conta com a infraestrutura necessária para confeccionar e caracterizar alvos de diferentes elementos, com técnicas como a espectrometria de retroespalhamento de Rutherford, que conferem uma elevada confiabilidade a esses resultados.

No contexto descrito, o objetivo principal deste trabalho se concentrou em:

- Determinar de maneira absoluta seções de choque de bremsstrahlung de elétrons para energias incidentes entre 20 e $100 \mathrm{keV}$, vários elementos atômicos e ângulos de emissão dos fótons. 
Outros objetivos específicos que complementam o propósito central são:

i) Desenvolver um modelo analítico de função resposta de detetores de Ge que permita a deconvolução dos espectros de bremsstrahlung, a partir da interpretação rigorosa dos espectros de altura de pulso medidos.

ii) Testar o modelo por intermédio de simulações Monte Carlo, e também de maneira experimental, utilizando fontes radioativas calibradas cujos espectros de emissão são conhecidos.

iii) Caracterizar a espessura dos alvos com alta precisão aplicando a espectrometria de retroespalhamento de Rutherford. Neste caso, a utilização da técnica representa uma vantagem qualitativa em relação a experimentos publicados, onde a incerteza relativa na espessura dos alvos alcançou até $20 \%$.

iv) Comparar os resultados experimentais de seções de choque de emissão de bremsstrahlung de elétrons com os cálculos teóricos disponíveis de maior precisão.

A interação de elétrons com a matéria é uma das linhas de pesquisa do Laboratório do Acelerador Linear do Instituto de Física da Universidade de São Paulo. O presente estudo forma parte desse tópico e dá continuidade a trabalhos anteriores desenvolvidos no grupo [10-13].

\subsection{Estrutura do trabalho}

Esta tese se divide em 9 Capítulos, sendo o primeiro deles a Introdução, mais 2 Apêndices.

No Capítulo 2 se abordam os conceitos teóricos de emissão de bremsstrahlung de elétrons e as aproximações desenvolvidas até hoje, discutindo as regiões de aplicação de cada uma delas. Além disso, são apresentados os principais resultados de tabulações e cálculos encontrados na literatura sobre este tema. Por outro lado, se faz uma revisão bibliográfica dos resultados experimentais obtidos e as técnicas utilizadas em cada caso.

No Capítulo 3 se descrevem os materiais utilizados na investigação. É apresentado de maneira geral o acelerador Microtron de São Paulo, com maior ênfase na linha de feixe de baixa energia, bem como a câmara de irradiação associada a mesma. Além disso, é detalhada a montagem experimental, mostrando as características dos detetores, a eletrônica de aquisição de espectros, blindagens, colimadores, etc. Também se particularizam as técnicas de confecção dos alvos finos usados nas medidas de bremsstrahlung.

No Capítulo 4 se expõe a metodologia seguida para calcular analiticamente a função resposta de detetores planares e coaxiais de Ge. Os modelos são validados com simulações 
Monte Carlo para as duas configurações do cristal de Ge, verificando cada um dos efeitos de detecção considerados nos mesmos. O objetivo desta modelagem consiste na construção de uma ferramenta que permita a deconvolução dos espectros de bremsstrahlung.

No Capítulo 5 é desenvolvido um experimento específico para testar a função resposta analítica, utilizando nesse caso um detetor planar de Ge. Consequentemente, investiga-se também a influência na função resposta de componentes típicos da montagem experimental: colimador e fonte.

Os capítulos 4 e 5 constituem uma unidade independente e paralela dentro do tema fundamental de pesquisa deste trabalho. Pela extensão desse fragmento dentro do texto, é importante que o leitor tenha ciência antecipadamente, que as expressões derivadas da modelagem proposta em ambos os capítulos, são utilizadas a posteriori para a caracterização do arranjo experimental do Microtron.

Sobre essa base, logo depois no Capítulo 6 se caracterizam experimentalmente os detetores na configuração do arranjo de irradiação no Microtron, e se obtêm grandezas necessárias para avaliar tanto a função resposta, quanto a seção de choque. Ao mesmo tempo, relata-se o procedimento de otimização dos parâmetros da eletrônica de aquisição para medidas de bremsstrahlung, e são expostos os métodos utilizados para a determinação da espessura dos alvos.

No Capítulo 7 se explicam as correções realizadas aos espectros coletados de bremsstrahlung, por efeitos como fundo radioativo, empilhamento de pulsos e radiação gerada no substrato dos alvos. Apresentam-se vários exemplos de espectros corrigidos, mostrando as diferenças de intensidades em função do ângulo de medida e da energia dos elétrons incidentes. Descreve-se a metodologia para a determinação da energia média e a dispersão do feixe de elétrons, bem como a intensidade do espectro de bremsstrahlung no ponto de máxima transferência de energia. São mostrados também exemplos de espectros deconvoluídos a partir da inversão da matriz resposta dos detetores.

Os resultados das medidas de SCDDs são apresentados no Capítulo 8, acompanhados por uma comparação com as estimativas teóricas derivadas das tabelas de Seltzer e Berger [3] e Kissel e colaboradores [5]. Em todos os casos se discutem as tendências observadas.

No Capítulo 9 se relatam as conclusões derivadas do presente trabalho.

No Apêndices A se apresentam os resultados da aproximação seguida para o cálculo de perfiles de Compton no átomo de Ge. No Apêndice B se tabulam os coeficientes utilizados para a interpolação e posterior cálculo da distribuição angular de bremsstrahlung. 


\section{Capítulo 2}

\section{Bremsstrahlung de elétrons: estado da arte teórico e experimental}

Neste capítulo são descritos os conceitos teóricos da emissão de bremsstrahlung de elétrons. Abordam-se as principais teorias utilizadas nos cálculos de seções de choque: a aproximação de Born; a aproximação de Sommerfeld-Maue e o formalismo de ondas parciais. São analisados os resultados dos cálculos e tabulações publicadas. Finalmente, detalham-se os experimentos fundamentais encontrados na literatura onde se determinaram seções de choque para elétrons com energia cinética entre 10 e 100 keV.

\subsection{Conceitos teóricos da emissão de bremsstrahlung de elétrons}

A emissão de bremsstrahlung é um dos mecanismos fundamentais de interação dos elétrons com a matéria. Quando uma dessas partículas é acelerada no campo eletrostático do átomo, existe uma probabilidade de que o elétron passe de um estado inicial com energia cinética $E_{0}$ e momento $\mathbf{p}_{1}$, a um estado com energia $E_{e}$ e momento $\mathbf{p}_{2}$, produzindo um fóton de energia $W$ e momento $\mathbf{k}$ [1]. Considerando que o estado do átomo alvo não varia durante a transição, o momento transferido é

$$
\mathbf{q}=\mathbf{p}_{1}-\mathbf{p}_{2}-\mathbf{k}
$$

Levando em conta a diferença entre as massas $m_{\mathrm{e}}$ do elétron e $M$ do núcleo, a energia de recuo deste último $E_{\mathrm{r}} \approx \mathbf{q}^{2} /(2 M)$ pode ser desprezada para valores de $E_{0} \ll M c^{2} / 2 \approx$ 
469A MeV (sendo $A$ o número de massa do núcleo). De acordo com a lei de conservação da energia

$$
W=E_{0}-E_{e}
$$

Este resultado indica que o espectro dos fótons varia desde $W=0$, onde a interação equivale a um espalhamento elástico, até a máxima transferência de energia $W=E_{0}$.

Os cálculos das seções de choque de bremsstrahlung partem da equação de onda relativística de Dirac independente do tempo [14], para um elétron interagindo com um campo eletromagnético representado pelo potencial escalar $\varphi$ e o potencial vetor $\mathbf{A}$

$$
H \psi(\mathbf{r})=\left\{-c \boldsymbol{\alpha} \cdot(\mathrm{i} \hbar \nabla+e \mathbf{A})+\boldsymbol{\beta} m_{\mathrm{e}} c^{2}+e \varphi\right\} \psi(\mathbf{r})=E \psi(\mathbf{r}),
$$

onde $e$ é a carga elementar, e $\boldsymbol{\alpha}$ e $\boldsymbol{\beta}$ são matrizes $4 \times 4$, que podem se expressar em função das matrizes $2 \times 2$ de Pauli $\sigma_{i}(i=1,2,3)$ e a matriz unidade $\mathbf{1}$

$$
\boldsymbol{\alpha}=\left(\begin{array}{cc}
0 & \boldsymbol{\sigma} \\
\boldsymbol{\sigma} & 0
\end{array}\right), \quad \boldsymbol{\beta}=\left(\begin{array}{cc}
\mathbf{1} & 0 \\
0 & -1
\end{array}\right)
$$

Para um dado valor do momento do elétron existem quatro autofunções diferentes que correspondem às duas direções do spin, e valores de energia positivos ou negativos [1].

A linearidade da Eq. (2.3) permite separar o Hamiltoniano $H$ em duas partes: 1) a interação do elétron com o campo elétrico do átomo $H_{\text {el }}$, e 2) a interação com o campo de radiação $H_{\text {int }}$ que causa a transição de estado do elétron

$$
H=H_{\mathrm{el}}+H_{\mathrm{int}},
$$

sendo

$$
H_{\mathrm{int}}=-e c \boldsymbol{\alpha} \cdot \mathbf{A}
$$

O potencial vetor pode ser escrito como uma série de ondas planas confinadas em um cubo de volume $L^{3}$ [1]. Logo, o elemento matricial de $H_{\text {int }}$ para a emissão de um fóton de energia $W$ e momento $\mathbf{k}$ na transição do elétron $1 \rightarrow 2$ resulta

$$
M_{1 \rightarrow 2}=-\frac{e \hbar c}{\sqrt{2 \epsilon_{0} W L^{3}}} \int \psi_{2}^{\dagger}(\mathbf{r})(\boldsymbol{\alpha} \cdot \hat{\mathbf{e}}) \mathrm{e}^{-\mathrm{i} \mathbf{k} \cdot \mathbf{r} / \hbar} \psi_{1}(\mathbf{r}) \mathrm{d}^{3} r .
$$

Na expressão (2.7) $\epsilon_{0}$ é a permissividade do vácuo, os subíndices 1 e 2 referem-se aos estados inicial e final do elétron, $\psi^{\dagger}$ indica a matriz adjunta de $\psi$, enquanto ê denota o vetor unitário de polarização que é sempre perpendicular a $\mathbf{k}[1]$. 
De acordo com a teoria de perturbação dependente do tempo [14], a taxa de transição de um sistema quântico do estado $|i\rangle$ a um grupo de estados finais do contínuo $|f\rangle$, está dada em primeira ordem pela Regra de Ouro de Fermi

$$
W_{i \rightarrow f}=\frac{2 \pi}{\hbar}|\langle f|V| i\rangle|^{2} \rho_{f},
$$

onde $V$ é a perturbação dos autoestados $|i\rangle$ e $|f\rangle$ de $H_{\text {el }}$, e $\rho_{f}$ a densidade de estados finais. Pode-se demonstrar a partir da relação relativística da energia e do momento que

$$
\rho_{f}=\frac{p_{2}\left(E_{2}+m_{\mathrm{e}} c^{2}\right) \mathrm{d} \Omega_{p_{2}} k^{2} \mathrm{~d} k \mathrm{~d} \Omega_{k}}{\left(2 \pi m_{\mathrm{e}}\right)^{6} c^{8}}
$$

na qual, $\mathrm{d} \Omega_{p_{2}}$ e $\mathrm{d} \Omega_{k}$ são os elementos diferenciais de ângulo sólido nas direções de $\mathbf{p}_{2}$ e $\mathbf{k}$ respectivamente [1].

A seção de choque de um processo é definida como o número de interações por unidade de tempo e unidade de fluxo de partículas incidentes. Portanto, inserindo as Eqs. (2.7) e (2.9) em (2.8) e dividindo $W_{i \rightarrow f}$ pela densidade de corrente de elétrons no cubo

$$
J_{1}=\frac{v_{1}}{L^{3}}=\frac{1}{L^{3}} \frac{p_{1} c^{2}}{E_{1}+m_{\mathrm{e}} c^{2}},
$$

obtemos a seção de choque diferencial para o bremsstrahlung,

$$
\frac{\mathrm{d}^{3} \sigma}{\mathrm{d} k \mathrm{~d} \Omega_{k} \mathrm{~d} \Omega_{p_{2}}}=\frac{\alpha}{(2 \pi)^{4}}\left(\frac{\hbar}{m_{\mathrm{e}} c}\right)^{2} \frac{p_{2}\left(E_{1}+m_{\mathrm{e}} c^{2}\right)\left(E_{2}+m_{\mathrm{e}} c^{2}\right) k^{2}}{m_{\mathrm{e}}^{4} c^{5} p_{1} W}|M|^{2} .
$$

Aqui $\alpha=e^{2} /\left(4 \pi \epsilon_{0} \hbar c\right) \approx 1 / 137$ é a constante de estrutura fina e

$$
M=\int \psi_{2}^{\dagger}(\mathbf{r})(\boldsymbol{\alpha} \cdot \hat{\mathbf{e}}) \mathrm{e}^{-\mathrm{ik} \cdot \mathbf{r} / \hbar} \psi_{1}(\mathbf{r}) \mathrm{d}^{3} r .
$$

As unidades naturais (UN) muitas vezes são escolhidas para realizar os cálculos de seções de choque. Nesse sistema as constantes $\hbar \equiv 1, m_{\mathrm{e}} \equiv 1$ e $c \equiv 1$, desse modo, as unidades de energia, momento e longitude são então $m_{\mathrm{e}} c^{2}, m_{\mathrm{e}} c$ e $\hbar / m_{\mathrm{e}} c$, respectivamente. Como consequência, as grandezas da física atômica tomam valores na ordem da unidade facilitando os procedimentos numéricos. Além disso, é frequente definir a energia total do elétron como $\epsilon=E / m_{\mathrm{e}} c^{2}+1$, o que reduz a relação relativística entre o momento e a energia a uma forma simples,

$$
\epsilon^{2}=p^{2}+1
$$

enquanto para o fóton os valores absolutos de energia e momento coincidem, por conseguinte

$$
\frac{W}{m_{\mathrm{e}} c^{2}}=|\mathbf{k}|=k
$$


Assim a Eq. (2.11) pode ser reescrita de maneira compacta como

$$
\frac{\mathrm{d}^{3} \sigma}{\mathrm{d} k \mathrm{~d} \Omega_{k} \mathrm{~d} \Omega_{p_{2}}}=\frac{\alpha}{(2 \pi)^{4}} \frac{\epsilon_{1} \epsilon_{2} p_{2} k}{p_{1}}|M|^{2} .
$$

Os princípios gerais para derivar teoricamente a seção de choque finalizam na expressão (2.15). Neste campo, o problema fundamental das pesquisas esteve centrado durante décadas nos métodos aplicados para calcular o elemento matricial (2.12). Atualmente não exite uma formulação única que forneça resultados precisos para qualquer energia do elétron incidente ou do fóton emitido, nem para qualquer número atômico $[1,3,15-$ 19]. São discutidas a seguir as principais teorias utilizadas: a aproximação de Born, a aproximação de Sommerfeld-Maue e a expansão relativística em ondas parciais, bem como as restrições de cada uma. No restante do capítulo são utilizadas as UN.

\subsection{Aproximação de Born. Fórmula de Bethe-Heitler}

A aproximação de Born ( $\mathrm{AB}$ ) é o método mais simples e difundido no cálculo do elemento matricial de bremsstrahlung (2.12). O potencial escalar que experimenta um elétron se movimentando no campo elétrico de um núcleo com número atômico $Z$ é $\varphi=-\alpha Z / r$, e a equação de Dirac toma a forma

$$
\left\{-\mathrm{i} \boldsymbol{\alpha} \cdot \nabla+\boldsymbol{\beta}-\epsilon-\frac{a}{r}\right\} \psi(\mathbf{r})=0
$$

onde $a=\alpha Z$ é o parâmetro de Coulomb [1]. Para resolver a equação anterior a função de onda $\psi(\mathbf{r})$ se expande em uma série de potências de $a$ :

$$
\psi(\mathbf{r})=\Phi_{0}(\mathbf{r})+a \Phi_{1}(\mathbf{r})+a^{2} \Phi_{2}(\mathbf{r})+\ldots
$$

$\Phi_{0}(\mathbf{r})$ é a onda plana incidente, solução da Eq. (2.16) de ordem zero $(a=0$, partícula livre)

$$
\Phi_{0}(\mathbf{r})=\mathrm{e}^{\mathrm{i} \mathbf{p} \cdot \mathbf{r}} u(\mathbf{p}),
$$

enquanto $u(\mathbf{p})$ é o espinor do elétron livre que pode ser representado como um vetor de quatro componentes. Para um dado valor do momento $\mathbf{p}, u(\mathbf{p})$ tem quatro autofunções correspondentes aos dois valores de spin e soluções com energia positiva e negativa [1]. Os outros termos da expansão (2.17) caracterizam a distorção introduzida pelo potencial. A condição básica da $\mathrm{AB}$ de primeira ordem indica que, se o produto $\alpha Z$ for pequeno comparado com a unidade, só o primeiro termo precisa ser considerado, o que fisicamente significa que as ondas incidentes e emergentes são pouco deformadas em relação à onda 
plana. Substituindo a série (2.17) até a potência linear de $a$ em (2.16) se obtém

$$
\{-\mathrm{i} \boldsymbol{\alpha} \cdot \nabla+\boldsymbol{\beta}-\epsilon\} \Phi_{1}(\mathbf{r})=\frac{1}{r} \Phi_{0}(\mathbf{r}) .
$$

$\Phi_{1}(\mathbf{r})$ se calcula introduzindo a função de Green e passando do espaço das coordenadas $\mathbf{r}$ ao espaço dos momentos $\mathbf{k}$, mediante a transformada de Fourier [1]. De fato, do ponto de vista analítico é mais conveniente trabalhar com $\Phi_{1}(\mathbf{k})$ levando em conta a forma funcional do elemento (2.12).

A fórmula de Bethe-Heitler se obtém quando o elemento (2.12) é calculado com o parâmetro de Coulomb de ordem 1. Em um sistema de coordenadas, onde a direção do eixo $z$ coincide com a direção $\hat{\mathbf{k}}$ do fóton emitido, e o elétron incidente se movimenta no plano $(x, z)$, os momentos $\mathbf{p}_{1}, \mathbf{p}_{2}$ e $\mathbf{k}$ em coordenadas polares adquirem a forma

$$
\begin{aligned}
\mathbf{p}_{1} & =p_{1}\left(\sin \theta_{1}, 0, \cos \theta_{1}\right), \\
\mathbf{p}_{2} & =p_{2}\left(\sin \theta_{2} \cos \phi, \sin \theta_{2} \sin \phi, \cos \theta_{2}\right), \\
\mathbf{k} & =k(0,0,1),
\end{aligned}
$$

enquanto a seção de choque triplamente diferencial (SCTD) [1] é dada por

$$
\begin{aligned}
\frac{\mathrm{d}^{3} \sigma}{\mathrm{d} k \mathrm{~d} \Omega_{k} \mathrm{~d} \Omega_{p_{2}}}= & \frac{\alpha Z^{2} r_{\mathrm{e}}^{2}}{4 \pi^{2}} \frac{p_{2}}{k p_{1} q^{4}}\left\{\left(4 \epsilon_{2}^{2}-q^{2}\right) \frac{p_{1}^{2} \sin ^{2} \theta_{1}}{\left(\epsilon_{1}-p_{1} \cos \theta_{1}\right)^{2}}\right. \\
& +\left(4 \epsilon_{1}^{2}-q^{2}\right) \frac{p_{1}^{2} \sin ^{2} \theta_{2}}{\left(\epsilon_{2}-p_{2} \cos \theta_{2}\right)^{2}} \\
& -\left(4 \epsilon_{1} \epsilon_{2}-q^{2}+2 k^{2}\right) \frac{2 p_{1} p_{2} \sin \theta_{1} \sin \theta_{2} \cos \phi}{\left(\epsilon_{1}-p_{1} \cos \theta_{1}\right)\left(\epsilon_{2}-p_{2} \cos \theta_{2}\right)} \\
& \left.+2 k^{2} \frac{p_{1}^{2} \sin ^{2} \theta_{1}+p_{2}^{2} \sin ^{2} \theta_{2}}{\left(\epsilon_{1}-p_{1} \cos \theta_{1}\right)\left(\epsilon_{2}-p_{2} \cos \theta_{2}\right)}\right\},
\end{aligned}
$$

com

$$
\begin{aligned}
q^{2}= & 2 k\left[\left(\epsilon_{1}-p_{1} \cos \theta_{1}\right)-\left(\epsilon_{2}-p_{2} \cos \theta_{2}\right)\right] \\
& +2\left[\epsilon_{1} \epsilon_{2}-p_{1} p_{2}\left(\cos \theta_{1} \cos \theta_{2}+\sin \theta_{1} \sin \theta_{2} \cos \phi\right)-1\right] .
\end{aligned}
$$

A SCTD (2.21) reflete a probabilidade de que um elétron com energia total $\epsilon_{1}$ seja espalhado na direção de $\hat{\mathbf{p}}_{2}$ emitindo um fóton de momento $\mathbf{k}$, e está expressada em função do raio clássico do elétron $r_{\mathrm{e}}=\alpha\left(\hbar / m_{\mathrm{e}} c\right)$, levando em conta as UN, para enfatizar as dimensões de área. A fórmula de Bethe-Heitler é uma média avaliada sobre os spins dos elétrons incidente e emergente, e as direções de polarização do fóton emitido [1]. A aproximação só fornece bons resultados se o comprimento de onda de de Broglie do elétron, $\hbar / m_{\mathrm{e}} v$, for longo comparado com o tamanho $d$ do campo Coulombiano. Uma 
estimativa deste parâmetro é dada pela condição energética

$$
\frac{1}{4 \pi \epsilon_{0}} \frac{Z e^{2}}{d} \approx m_{\mathrm{e}} v^{2}
$$

por consequência a validade da $\mathrm{AB}$ se restringe a

$$
\frac{\alpha Z}{\beta_{1}} \ll 1, \quad \frac{\alpha Z}{\beta_{2}} \ll 1,
$$

onde $\beta_{1}=p_{1} / \epsilon_{1}$ e $\beta_{2}=p_{2} / \epsilon_{2}$ são as velocidades dos elétrons em unidades de $c$. Considerando que $\alpha \approx 1 / 137$, as inequações descritas em (2.24) são satisfeitas para elementos com $Z$ baixos e energias relativísticas $\beta_{1,2} \approx 1$. Contudo, mesmo para $\epsilon_{1} \gg 1$ perto do limite de comprimentos de ondas menores, a segunda condição de (2.24) é violada já que $\beta_{2} \ll 1$. Além disso, nesta região em que o fóton recebe a energia cinética total do elétron $\left(p_{2}=0\right)$ a seção de Bethe-Heitler tende a zero. Então, pode se concluir que esta aproximação é válida só para altas energias dos elétrons incidentes, elementos leves e energias baixas e intermediárias do espectro de bremsstrahlung.

\subsubsection{Efeitos da blindagem atômica}

A SCTD (2.21) foi derivada supondo que a emissão de bremsstrahlung seja consequência de um potencial Coulombiano puro. O efeito de blindagem do campo elétrico do núcleo causado pelos elétrons das diferentes camadas atômicas pode ser considerado introduzindo o fator de forma $F(\mathbf{q})$. Neste caso o potencial atômico é dado por

$$
V(\mathbf{r})=-\frac{\alpha Z}{r}+\alpha \int \frac{\rho\left(\mathbf{r}^{\prime}\right)}{\left|\mathbf{r}-\mathbf{r}^{\prime}\right|} \mathrm{d}^{3} r^{\prime}
$$

onde $\rho(\mathbf{r})$ é a densidade de elétrons normalizada a $Z$. O elemento matricial de bremsstrahlung (2.12) é proporcional a

$$
V(\mathbf{q})=\int V(\mathbf{r}) \mathrm{e}^{\mathrm{i} \mathbf{q} \cdot \mathbf{r}} \mathrm{d}^{3} r=-\frac{4 \pi \alpha Z}{q^{2}}[1-F(\mathbf{q})],
$$

com

$$
F(\mathbf{q})=\frac{1}{Z} \int \rho(\mathbf{r}) \mathrm{e}^{\mathrm{i} \mathbf{q} \cdot \mathbf{r}} \mathrm{d}^{3} r
$$

Para um potencial de Coulomb puro o termo entre colchetes da Eq. (2.26) é igual a 1, portanto o efeito da blindagem é levado em conta multiplicando o elemento matricial pelo fator $1-F(\mathbf{q})$. Uma prática comum é considerar a densidade de carga esfericamente simétrica, $\rho(\mathbf{r})=\rho(r)$, deste modo o fator de forma é uma função de $q, F(\mathbf{q})=F(q)$. Salvat e colaboradores [20], por exemplo, desenvolveram uma aproximação analítica para a função de blindagem atômica a partir da superposição de três potenciais de Yukawa. 
O método permite obter o fator $1-F(q)$ para elementos na faixa $1 \leqslant Z \leqslant 92$ a partir da tabulação dos parâmetros $b_{i}$ e $\mu_{i}$ :

$$
1-F(q)=\sum_{i=1}^{3} b_{i} \frac{q^{2}}{q^{2}+\mu_{i}^{2}}, \quad \text { com } \quad \sum_{i=1}^{3} b_{i}=1
$$

Em geral a incorporação destes fatores na fórmula de Bethe-Heitler indica que a blindagem atômica é mais importante no limite de baixos comprimentos de onda, sendo praticamente desprezível no outro limite, onde $\epsilon_{2} \approx 1$. Aliás, levando em conta as bases da $\mathrm{AB}$ os melhores resultados da aproximação dos fatores de forma são obtidos para elementos de baixo $Z$ e altas energias dos elétrons $\epsilon_{1} \gg 1$.

Uma tentativa para melhorar estes resultados se baseia em tomar a série (2.17) até o termo quadrático. Porém, uma comparação da $\mathrm{AB}$ de segunda ordem com outras teorias (ver secção 2.4) demonstra que é limitada a concordância fornecida pelo método, indicando que a série de Born converge lentamente [1].

\subsection{Aproximação de Sommerfeld-Maue}

A aproximação de Sommerfeld-Maue (SM) propõe uma descrição da interação do elétron no campo atômico mais rigorosa do que a AB; utiliza melhores soluções da equação de Dirac com o Hamiltoniano não perturbado $\left(H_{\mathrm{el}}\right)$ para avaliação do elemento matricial de bremsstrahlung (2.12). No caso não relativístico, a equação de Schrödinger para um elétron no campo Coulombiano pode ser resolvida de forma fechada [14], porém isto não é possível no caso relativístico. A solução exata da equação de Dirac para $H_{\mathrm{el}}$ só pode ser expressa como uma expansão em polinômios de Legendre [1]. Dessa maneira, calcular a seção de choque exige um grande número de elementos matriciais a serem considerados. Nesse contexto, é mais simples usar uma solução aproximada da equação de Dirac com potencial de Coulomb, mesmo quando o método só é preciso para $\alpha Z \ll 1$ e todas as energias, ou para todo $\alpha Z$, valores de energias elevados dos elétrons incidente e emergente e ângulos pequenos.

Para calcular as funções de onda de SM o operador

$$
(-\mathrm{i} \boldsymbol{\alpha} \cdot \nabla+\boldsymbol{\beta}+\epsilon+a / r)
$$


é aplicado à equação de Dirac com o potencial Coulombiano (2.16), transformando-a em uma equação de segunda ordem

$$
\left(\nabla^{2}+p^{2}+2 \epsilon a / r\right) \psi(\mathbf{r})=\left[\mathrm{i} \boldsymbol{\alpha} \cdot \nabla(a / r)-a^{2} / r^{2}\right] \psi(\mathbf{r})
$$

O membro esquerdo tem a estrutura da equação de Schrödinger para um campo Coulombiano puro cuja solução é bem conhecida. O lado direito é da ordem de $a=\alpha Z$, portanto pode ser considerado pequeno para $Z$ baixos. Uma solução aproximada da equação anterior se obtém escrevendo $\psi(\mathbf{r})$ como uma série em termos do parâmetro $a$ :

$$
\psi(\mathbf{r})=\psi_{0}(\mathbf{r})+a \psi_{1}(\mathbf{r})+a^{2} \psi_{2}(\mathbf{r})+\ldots
$$

Em contraste com a AB, a expressão (2.31) não é estritamente uma expansão em termos do parâmetro de Coulomb, devido a que o potencial proporcional a a continua no membro esquerdo da Eq. (2.30). Logo, a função de menor ordem $\psi_{0}(\mathbf{r})$, solução da equação

$$
\left(\nabla^{2}+p^{2}+2 \epsilon a / r\right) \psi_{0}(\mathbf{r})=0
$$

representa uma onda distorcida, diferente da onda plana $\Phi_{0}(\mathbf{r})$, e vem dada por

$$
\psi_{0}(\mathbf{r})=N \mathrm{e}^{\mathrm{i} \mathbf{p} \cdot \mathbf{r}}{ }_{1} F_{1}(\mathrm{i} a \epsilon / p ; 1 ; \mathrm{i} p r-\mathrm{ip} \cdot \mathbf{r}) u(\mathbf{p})
$$

onde $N$ é um fator de normalização e ${ }_{1} F_{1}$ denota a função hipergeométrica confluente $[1,21]$. Assim, substituindo os dois primeiros termos da série (2.31) na Eq. (2.30) se obtém

$$
\left(\nabla^{2}+p^{2}+2 \epsilon a / r\right) \psi_{1}(\mathbf{r})=\mathrm{i} \boldsymbol{\alpha} \cdot \nabla(1 / r) \psi_{0}(\mathbf{r})
$$

Pode-se demonstrar [1] que

$$
\psi_{1}(\mathbf{r})=-N \mathrm{e}^{\mathrm{i} \mathbf{p} \cdot \mathbf{r}}\left(\frac{\mathrm{i}}{2 \epsilon a}\right) \boldsymbol{\alpha} \cdot \nabla_{1} F_{1}(\mathrm{i} a \epsilon / p ; 1 ; \mathrm{i} p r-\mathrm{ip} \cdot \mathbf{r}) u(\mathbf{p}) .
$$

Se for repetido o processo de iteração tomando mais um termo na série (2.31), a equação diferencial que surge não tem solução analítica [1]. Por consequência, a função de onda de SM se compõe por

$$
\psi_{\mathrm{SM}}=\psi_{0}+a \psi_{1} .
$$


A precisão desta aproximação pode ser comprovada inserindo $\psi_{\mathrm{SM}}$ na equação de Dirac para o campo Coulombiano

$$
\begin{aligned}
& (\mathrm{i} \boldsymbol{\alpha} \cdot \nabla-\boldsymbol{\beta}+\epsilon+a / r) \psi_{\mathrm{SM}}(\mathbf{r})= \\
& =\mathrm{i} N \mathrm{e}^{\mathrm{i} \mathbf{p} \cdot \mathbf{r}} \frac{a^{2}}{2 r}{ }_{1} F_{1}(1+\mathrm{i} a \epsilon / p ; 2 ; \mathrm{i} p r-\mathrm{i} \mathbf{p} \cdot \mathbf{r}) \boldsymbol{\alpha} \cdot(\hat{\mathbf{r}}-\hat{\mathbf{p}}) u(\mathbf{p}) \\
& =\mathrm{i} N \mathrm{e}^{\mathrm{i} \mathbf{p} \cdot \mathbf{r}}\left(\frac{a^{2}}{r} \sin \vartheta / 2\right){ }_{1} F_{1}(1+\mathrm{i} a \epsilon / p ; 2 ; \mathrm{i} p r-\mathrm{i} \mathbf{p} \cdot \mathbf{r}) \boldsymbol{\sigma}\left(\begin{array}{c}
\cos \vartheta / 2 \\
\sin \vartheta / 2
\end{array}\right) u(\mathbf{p}) .
\end{aligned}
$$

Logo, $\psi_{\mathrm{SM}}$ satisfaz a equação de onda $(2.30)$ para termos da ordem $\left(a^{2} / r\right) \sin \vartheta / 2$. Se o ângulo $\vartheta$ entre os versores $\hat{\mathbf{r}}$ e $\hat{\mathbf{p}}$ tende a zero, a Eq. (2.30) é resolvida exatamente. Além disso, a Eq. (2.30) é satisfeita até a primeira potência de $a=\alpha Z$ em todo o espaço, e na região de ângulos $\vartheta$ pequenos é válida com um alto grau de aproximação. Por outro lado, para altas energias, a contribuição dominante na seção de choque vem dos eventos em que tanto o elétron espalhado quanto o fóton produzido emergem dentro de um ângulo da ordem de $1 / \epsilon$ com respeito à direção do elétron incidente. Sob essa condição, o momento q transferido ao átomo é pequeno, implicando parâmetros de impacto $r$ grandes, da ordem de $\epsilon$. De acordo com esse argumento, o membro direito de (2.37) também é pequeno, e se espera que a função de onda de SM seja uma boa aproximação para altas energias, mesmo quando a possua valores elevados (altos $Z$ ).

A expressão analítica exata da SCTD obtida através do método de SM é extremamente complexa desde o ponto de vista algébrico (Haug e Nakel, fórmula 3.184 [1]). Porém, alguns casos individuais de interesse para este trabalho podem ser analisados: a aproximação não relativística e o limite para comprimentos de onda pequenos.

\subsubsection{Limite não relativístico}

No caso de energias não relativísticas se satisfazem os limites

$$
\epsilon_{1,2} \rightarrow 1, \quad p_{1,2} \rightarrow \beta_{1,2} \ll 1, \quad k \rightarrow \frac{1}{2}\left(\beta_{1}^{2}-\beta_{2}^{2}\right)^{1 / 2},
$$

onde $\beta_{1}$ e $\beta_{2}$ são as velocidades do elétron nos estados inicial e final respectivamente em UN. Se os termos da ordem $\beta^{2}$ não são considerados em comparação com a unidade [1], se obtém

$$
\begin{aligned}
\frac{\mathrm{d}^{3} \sigma_{\mathrm{nr}}}{\mathrm{d} k \mathrm{~d} \Omega_{k} \mathrm{~d} \Omega_{p_{2}}}= & \frac{2 \pi a / \beta_{1}}{\mathrm{e}^{2 \pi a / \beta_{1}}-1} \frac{2 \pi a / \beta_{2}}{1-\mathrm{e}^{-2 \pi a / \beta_{2}}} \frac{\alpha Z^{2} r_{\mathrm{e}}^{2}}{\pi^{2}} \frac{\beta_{2}}{\beta_{1} k q^{4}} \\
& \times\left|\frac{\hat{\mathbf{p}}_{1} \times \hat{\mathbf{k}}}{S_{1}}\left[\beta_{1} U-\mathrm{i} a(1-x) V\right]-\frac{\hat{\mathbf{p}}_{2} \times \hat{\mathbf{k}}}{S_{2}}\left[\beta_{2} U-\mathrm{i} a(1-x) V\right]\right|^{2},
\end{aligned}
$$


sendo

$$
S_{1}=1-\hat{\mathbf{k}} \cdot \mathbf{p}_{1}=1-\beta_{1} \cos \theta_{1}, \quad S_{2}=1-\hat{\mathbf{k}} \cdot \mathbf{p}_{2}=1-\beta_{2} \cos \theta_{2},
$$

enquanto

$$
\begin{aligned}
& U={ }_{2} F_{1}\left(\frac{\mathrm{i} a}{\beta_{1}}, \frac{\mathrm{i} a}{\beta_{2}} ; 1 ; x\right), \quad V={ }_{2} F_{1}\left(\frac{\mathrm{i} a}{\beta_{1}}+1, \frac{\mathrm{i} a}{\beta_{2}}+1 ; 2 ; x\right), \\
& x=1-\frac{\left(\mathbf{p}_{1}-\mathbf{p}_{2}\right)^{2}}{\left(\beta_{1}-\beta_{2}\right)^{2} S_{1} S_{2}} .
\end{aligned}
$$

${ }_{2} F_{1}$ denota a função hipergeométrica [21]. A SCTD (2.39) coincide com o resultado derivado da equação de Schrödinger para elétrons não relativísticos quando é levado em conta o efeito de retardo [1]. Observa-se que, como os fatores $S_{1}$ e $S_{2}$ contém as velocidades do elétron até a primeira ordem, a distribuição angular não é simétrica com repeito a $\theta_{1,2}=90^{\circ}$ mesmo para baixas energias. Além disso, quando $p_{2}=0$ a SCTD não relativística de SM possui um valor finito, a diferença da SCTD fornecida pela $\mathrm{AB}$ $(2.21)$.

Por outro lado, no caso em que a energia cinética total é transferida ao fóton, o momento do elétron emergente $p_{2}$ é zero, por conseguinte $k \rightarrow \epsilon_{1}-1$. O limite de comprimentos de onda curtos é conhecido como região do tip do espectro de bremsstrahlung. Considerando a condição anterior, a seção de choque da aproximação de SM não relativística para essa faixa energética [1] é dada por

$$
\begin{aligned}
& \frac{\mathrm{d}^{3} \sigma}{\mathrm{d} k \mathrm{~d} \Omega_{k} \mathrm{~d} \Omega_{p_{2}}}=\frac{2 \pi a_{1}}{\mathrm{e}^{2 \pi a_{1}}-1} \frac{\alpha^{2} Z^{3} r_{\mathrm{e}}^{2}}{\pi k p_{1} q^{4}}\left\{2\left(\frac{4}{q^{2}}+k-1\right) \frac{\left(\mathbf{p}_{1} \times \mathbf{k}\right)^{2}}{q^{2}}\left|U_{0}-\mathrm{i} a_{1} V_{0}\right|^{2}\right. \\
& +\left[2\left\{k^{2} / p_{1}+(1-k)\left(\hat{\mathbf{k}} \cdot \hat{\mathbf{p}}_{2}\right)\right\} \frac{\left(\mathbf{p}_{1} \times \mathbf{k}\right)^{2}}{q^{2}}\right. \\
& \left.\left(2 \frac{\epsilon_{1}^{2}+\epsilon_{1}+2}{q^{2}}-1\right)\left(\mathbf{p}_{1} \times \mathbf{k}\right) \cdot\left(\hat{\mathbf{p}}_{2} \times \mathbf{k}\right)\right] a\left[\operatorname{Im}\left(U_{0} V_{0}^{*}\right)-a_{1}\left|V_{0}\right|^{2}\right] \\
& +\left[\left(2 \epsilon_{1}^{2}-k\right)\left(\hat{\mathbf{p}}_{2} \times \hat{\mathbf{k}}\right)^{2}+\left(\hat{\mathbf{k}} \cdot \hat{\mathbf{p}}_{2}\right)\left(\mathbf{p}_{1} \times \mathbf{k}\right) \cdot\left(\hat{\mathbf{p}}_{2} \times \hat{\mathbf{k}}\right)\right. \\
& \left.\left.k^{3}\left\{1-\left(\hat{\mathbf{k}} \cdot \hat{\mathbf{p}}_{1}\right)\left(\hat{\mathbf{k}} \cdot \hat{\mathbf{p}}_{2}\right)\right\}\right] a^{2}\left|V_{0}\right|^{2}\right\} .
\end{aligned}
$$

Nesta expressão

$$
\begin{aligned}
& U_{0}={ }_{1} F_{1}\left(\mathrm{i} a_{1} ; 1 ; \mathrm{i} \xi\right), \quad V_{0}={ }_{1} F_{1}\left(1+\mathrm{i} a_{1} ; 2 ; \mathrm{i} \xi\right), \\
& a_{1}=\frac{\alpha Z}{\beta_{1}}, \quad \xi=a\left(2 \frac{\mathbf{q} \cdot \hat{\mathbf{p}}_{2}}{q^{2}}-\frac{\mathbf{k} \cdot \hat{\mathbf{p}}_{2}}{k}-\frac{p_{1}}{k}\right)
\end{aligned}
$$


O resultado (2.42) não apresenta os mesmos problemas no limite de comprimentos de ondas curtos do que a SCTD da AB (2.21), já que se deriva de uma aproximação em $\alpha Z$, e não em $\alpha Z / \beta$. Portanto, a fórmula (2.42) é precisa para números atômicos $Z$ baixos. Como $p_{2}=0$ na região analisada, a seção de choque duplamente diferencial (SCDD) pode ser calculada de maneira mais simples em relação às expressões (2.21) e (2.39). Integrando sobre todos os ângulos do elétron emergente, considerando que $a_{1} \ll 1$, e desprezando os termos da ordem $a_{1}^{2}[1]$ se obtém

$$
\frac{\mathrm{d}^{2} \sigma}{\mathrm{d} k \mathrm{~d} \Omega_{k}} \approx 8 \alpha^{2} Z^{3} r_{\mathrm{e}}^{2} \frac{2 \pi a_{1}}{\mathrm{e}^{2 \pi a_{1}}-1} \frac{\left(\mathbf{p}_{1} \times \mathbf{k}\right)^{2}}{k p_{1} q^{6}} \frac{4}{q^{2}+k-1} .
$$

A SCDD (2.44) é proporcional à terceira potência de $Z$, assim, pode se entender porque a intensidade do espectro de bremsstrahlung no tip se incrementa para os elementos pesados, ainda quando a formula não seja precisa nesta faixa de $Z$. Além disso, a distribuição angular dos fótons é dada pelo fator $\left(\mathbf{p}_{1} \times \mathbf{k}\right)^{2} / q^{6}$, característica da aproximação dipolar relativística.

\subsubsection{Efeitos da blindagem atômica no formalismo de Sommerfeld- Maue}

Sob a AB, a SCDD pode ser obtida em forma de expressões fechadas, em ambos os casos em que são o não considerados os efeitos da blindagem atômica [18]. De maneira similar, Haug apresenta uma fórmula analítica para a SCDD dentro da aproximação de SM [18]. Com a finalidade de incorporar nesse último caso a blindagem dos elétrons ao campo elétrico do núcleo, o autor propõe a seguinte correção

$$
\left(\frac{\mathrm{d}^{2} \sigma}{\mathrm{d} k \mathrm{~d} \Omega_{k}}\right)_{\mathrm{SM}}^{\mathrm{b}} \approx\left(\frac{\mathrm{d}^{2} \sigma}{\mathrm{d} k \mathrm{~d} \Omega_{k}}\right)_{\mathrm{SM}}^{\mathrm{nb}}+\left[\left(\frac{\mathrm{d}^{2} \sigma}{\mathrm{d} k \mathrm{~d} \Omega_{k}}\right)_{\mathrm{AB}}^{\mathrm{b}}-\left(\frac{\mathrm{d}^{2} \sigma}{\mathrm{d} k \mathrm{~d} \Omega_{k}}\right)_{\mathrm{AB}}^{\mathrm{nb}}\right],
$$

onde as siglas "b" e "nb" representam as situações em que é considerada a blindagem atômica o não, respectivamente.

A Eq. (2.45) espera-se forneça melhores resultados para elementos atômicos leves, porém, para energias cinéticas dos elétrons incidentes acima de $5 \mathrm{MeV}$, Haug sugere utiliza-la em elementos de $Z$ alto [18]. O esquema de trabalho de Haug pode ser adotado na faixa de energia onde não existem cálculos de SCDDs segundo formalismo de ondas parciais $\left(E_{0}>2 \mathrm{MeV}\right.$, ver próxima seção). 


\subsection{Expansão relativística em ondas parciais}

O método de maior precisão para calcular a seção de choque de bremsstrahlung utiliza a aproximação relativística de ondas parciais para descrever o movimento do elétron no campo estático do átomo alvo, supondo que o estado inicial e final deste coincidam [1]. O fóton é tratado como uma expansão em multipolos. Se o potencial numérico exato $V(\mathbf{r})$ for inserido no Hamiltoniano não perturbado, a interação do elétron leva em conta os efeitos da blindagem atômica. Assim, a equação de Dirac toma a forma:

$$
\{-\mathrm{i} \boldsymbol{\alpha} \cdot \nabla+\boldsymbol{\beta}-\epsilon+V(\mathbf{r})\} \Phi(\mathbf{r})=0
$$

Nesse caso é preciso utilizar a representação ampliada do espinor, de maneira que os operadores de Dirac $\boldsymbol{\alpha}$ e $\boldsymbol{\beta}$ são substituídos pelas matrizes de Pauli e as matrizes unidade $2 \times 2$ respectivamente, enquanto a função de onda $\Phi(\mathbf{r})$, é expressa em termos dos espinores $v$ e $w$,

$$
\Phi(\mathbf{r})=\left(\begin{array}{c}
v(\mathbf{r}) \\
w(\mathbf{r})
\end{array}\right)
$$

Quando a função (2.47) é substituída na Eq. (2.46) se obtém um sistema de duas equações acopladas que permitem eliminar $w[1]$ :

$$
\Phi(\mathbf{r})=\left(\begin{array}{c}
\mathbf{1} \\
-\mathrm{i}(\epsilon+1-V)^{-1} \boldsymbol{\sigma} \cdot \nabla
\end{array}\right) v(\mathbf{r}),
$$

onde $v(\mathbf{r})$ é determinada pela equação diferencial de segunda ordem

$$
\nabla^{2} v(\mathbf{r})+(\epsilon+1-V)\left[\boldsymbol{\sigma} \cdot \nabla(\epsilon+1-V)^{-1}\right] \boldsymbol{\sigma} \cdot \nabla v(\mathbf{r})+\left[(\epsilon-V)^{2}-1\right] v(\mathbf{r})=0 .
$$

A situação mais frequente é considerar $V$ como uma função só de $r$, de modo que $v(\mathbf{r})$ pode ser dividida em uma parte radial e outra angular

$$
v(\mathbf{r})=g(r) \chi(\hat{\mathbf{r}})
$$

O momento angular total $\mathbf{J}$ é composto pelo momento angular orbital $\mathbf{L}$ e o momento de spin:

$$
\mathbf{J}=\mathbf{L}+\frac{1}{2} \boldsymbol{\sigma}
$$

Elevando ao quadrado (2.51) se demonstra que

$$
\boldsymbol{\sigma} \cdot \mathbf{L}=\mathbf{J}^{2}-\mathbf{L}^{2}-\frac{3}{4}
$$


Expressando a parte angular dos operadores $\nabla$ e $\nabla^{2}$ em função de $\mathbf{L}^{2}$, empregando a relação (2.52) e substituindo $v(\mathbf{r})$ na equação (2.49) se obtém

$$
\chi_{j j_{z} l}(\hat{\mathbf{r}})=\left(\begin{array}{c}
\sqrt{\left(j+j_{z}\right) / 2 j} Y_{l, j_{z}-1 / 2} \\
\sqrt{\left(j-j_{z}\right) / 2 j} Y_{l, j_{z}+1 / 2}
\end{array}\right) \quad \text { para } \quad j=l+\frac{1}{2},
$$

e

$$
\chi_{j j_{z} l}(\hat{\mathbf{r}})=\left(\begin{array}{c}
-\sqrt{\left(j+1-j_{z}\right) /(2 j+2)} Y_{l, j_{z}-1 / 2} \\
\sqrt{\left(j+1+j_{z}\right) /(2 j+2)} Y_{l, j_{z}+1 / 2}
\end{array}\right) \quad \text { para } \quad j=l-\frac{1}{2}
$$

As soluções anteriores representam autoestados de $\mathbf{J}^{2}$ e $\mathbf{L}^{2}$, enquanto que $j, j_{z}$ e $l$ são os números quânticos correspondentes aos operadores $\mathbf{J}^{2}, \mathbf{J}_{z}$ e $\mathbf{L}^{2}$ respectivamente, e $Y_{l, j_{z} \pm 1 / 2}$ são os harmônicos esféricos normalizados [1]. Por outro lado, a função radial se deriva da equação de onda

$$
\begin{aligned}
\frac{\mathrm{d}^{2} g_{\kappa}}{\mathrm{d} r^{2}}+ & \frac{2}{r} \frac{\mathrm{d} g_{\kappa}}{\mathrm{d} r}+\left\{[\epsilon-V(r)]^{2}-1-\frac{l(l+1)}{r^{2}}\right\} g_{\kappa} \\
& +\frac{\mathrm{d} V}{\mathrm{~d} r}[\epsilon-V(r)+1]^{-1}\left(\frac{\mathrm{d}}{\mathrm{d} r}+\frac{\kappa(\kappa+1)}{r}\right) g_{\kappa}=0,
\end{aligned}
$$

definida em termos do número quântico relativístico do momento angular:

$$
\kappa=l(l+1)-j(j+1)-\frac{1}{4} .
$$

A introdução de $\kappa$ permite conhecer como o momento orbital $l$ e o spin do elétron estão acoplados no momento angular total $j$. Dado que

$$
\kappa=\left\{\begin{array}{cc}
-(l+1) \quad \text { para } & j=l+\frac{1}{2} \\
l & \text { para } j=l-\frac{1}{2}
\end{array},\right.
$$

só com esse número é possível caracterizar um estado, já que seu valor absoluto fornece tanto $j$ quanto $l$ :

$$
j=|\kappa|-\frac{1}{2}, \quad l=\left\{\begin{array}{cc}
\kappa & \text { para } \quad \kappa>0 \\
-(\kappa+1) & \text { para } \quad \kappa<0
\end{array} .\right.
$$

Logo, $g_{\kappa}$ depende unicamente de $\kappa$ e geralmente é chamada de componente maior da função de onda, enquanto $\chi_{j j_{z} l}(\hat{\mathbf{r}})=\chi_{\kappa j_{z}}(\hat{\mathbf{r}})$ e $\Phi(\mathbf{r})=\Phi_{\kappa, j_{z}}(\mathbf{r})$ [1]. Definindo $\bar{g}_{\kappa}=r g_{\kappa}$ 
a Eq. (2.55) se transforma em

$$
\begin{aligned}
\frac{\mathrm{d}^{2} \bar{g}_{\kappa}}{\mathrm{d} r^{2}} & +\left\{[\epsilon-V(r)]^{2}-1-\frac{\kappa(\kappa+1)}{r^{2}}\right\} \bar{g}_{\kappa} \\
& +\frac{\mathrm{d} V}{\mathrm{~d} r}[\epsilon-V(r)+1]^{-1}\left(\frac{\mathrm{d}}{\mathrm{d} r}+\frac{\kappa}{r}\right) \bar{g}_{\kappa}=0 .
\end{aligned}
$$

Métodos numéricos são utilizados para resolver a equação anterior, apenas no caso de um potencial Coulombiano $V=a / r$ é possível obter uma solução analítica em termos da função hipergeométrica confluente $[1,21]$.

O cálculo da seção de choque de bremsstrahlung requer funções de onda que assimptoticamente se comportem como a superposição de ondas planas mais uma componente de ondas esféricas incidentes ou emergentes, denotadas por $\psi^{+}(\mathbf{p}, \hat{\zeta}, \mathbf{r})$ e $\psi^{-}(\mathbf{p}, \hat{\zeta}, \mathbf{r})$ respectivamente, sendo $\hat{\zeta}=\mathbf{s} / s,(s=1 / 2)$ o vetor unitário que indica a direção do spin no sistema do elétron. Para valores grandes de $r$, a Eq. (2.59) se reduz a d ${ }^{2} \bar{g}_{\kappa} / \mathrm{d} r^{2}+p^{2} \bar{g}_{\kappa}=0$, assumindo que $V(r)$ tende a zero mais rápido do que o termo $1 / r$. Por conseguinte a forma assimptótica de qualquer solução $g_{\kappa}=\bar{g}_{\kappa} / r$ é

$$
g_{\kappa} \sim \frac{A}{p r} \sin \left(p r-l \frac{\pi}{2}+\eta_{\kappa}\right)
$$

onde $A$ é uma constante, enquanto a mudança de fase $\eta_{\kappa}$ depende de $p$ e do potencial $V(r)$ e em geral pode ser obtida numericamente [1].

A solução da equação de Dirac para um potencial $V(r)$ que satisfaça as condições de contorno da emissão de bremsstrahlung pode ser expressa como uma combinação linear de autoestados $\Phi_{\kappa, j_{z}}$ e os coeficientes de Clebsch-Gordan $C\left(j, j_{z} ; l, m, 1 / 2, s\right)[1,14]$ :

$$
\psi^{ \pm}(\mathbf{p}, \hat{\zeta}, \mathbf{r})=4 \pi \sqrt{\frac{\epsilon+1}{2 \epsilon}} \sum_{\kappa j_{z}} \mathrm{i}^{l} C\left(j, j_{z} ; l, m, 1 / 2, s\right) Y_{l m}^{*}(\hat{\mathbf{p}}) \mathrm{e}^{ \pm \mathrm{i} \eta_{\kappa}} \Phi_{\kappa j_{z}}(r)
$$

sendo os números quânticos $m$ e $s$ os autovalores dos operadores de momento angular orbital e de spin no eixo $z$.

Por outro lado o operador de interação do fóton no elemento matricial (2.12), $\boldsymbol{\alpha} \cdot \hat{\mathbf{e}}^{*} e^{-i \mathbf{k} \cdot \mathbf{r}}$, é expandido também em uma série multipolar em termos dos harmônicos esféricos $Y_{l m}$ e das funções esféricas de Bessel $j_{l}(k r)$ (expansão de Rayleigh) [1, 21]:

$$
\mathrm{e}^{\mathrm{i} \mathbf{k} \cdot \mathbf{r}}=4 \pi \sum_{l=0}^{\infty} \sum_{m=-l}^{m=l} \mathrm{i}^{l} j_{l}(k r) Y_{l m}^{*}(\hat{\mathbf{k}}) Y_{l m}(\hat{\mathbf{r}})
$$


Inserindo a função de onda do elétron $\psi^{ \pm}(\mathbf{p}, \vec{\zeta}, \mathbf{r})$ e a expansão (2.62) no elemento matricial (2.12), a integração angular pode se realizar analiticamente, logo, os elementos radiais são as principais integrais a serem obtidas numericamente [1, 22, 23]:

$$
\begin{aligned}
& R_{1}(\kappa)=\int_{0}^{\infty} r^{2} j_{l}(k r) g_{\kappa_{1}}(r) f_{\kappa_{2}}(r) \mathrm{d} r \\
& R_{2}(\kappa)=\int_{0}^{\infty} r^{2} j_{l}(k r) g_{\kappa_{2}}(r) f_{\kappa_{1}}(r) \mathrm{d} r
\end{aligned}
$$

onde $f_{\kappa}(r)$ é a chamada componente pequena da função de onda, definida a partir de $(2.48)$ e $g_{\kappa}(r)$ como

$$
f_{\kappa}(r)=\frac{1}{\epsilon+1-V}\left(\frac{\mathrm{d}}{\mathrm{d} r}+\frac{\kappa+1}{r}\right) g_{\kappa}(r)
$$

$R_{1}$ e $R_{2}$ dependem de funções de $p_{1}, p_{2}, k$ e $Z$, e convergem lentamente. Além disso, a sua avaliação requer somas sobre os números quânticos do fóton $l$ e $m$ e do elétron $\kappa_{1}, \kappa_{2}, j_{1 z}$ e $j_{2 z}$. As integrais angulares limitam o somatório sobre $l$ já que só são obtidos termos diferentes de zero se $\left|l_{2}-l_{1}\right| \leqslant l \leqslant\left|l_{2}+l_{1}\right|$, onde $l_{1,2}$ são os números quânticos correspondentes a $\kappa_{1,2}$ de acordo com (2.58). A maior dificuldade do cálculo é garantir a convergência dos somatórios infinitos sobre $\kappa_{1}$ e $\kappa_{2}$. A quantidade de elementos matriciais radiais a serem considerados cresce rapidamente com o incremento da energia do elétron, e por anos foi a maior limitante do método, especialmente devido à ausência de computadores com suficiente capacidade de armazenamento e precisão. $\mathrm{O}$ formalismo de ondas parciais no cálculo da SCTD é mais complexo do que a seção de choque integrada para todos os ângulos do elétron espalhado e o fóton emitido, em razão da ortogonalidade das funções angulares [1]. Como consequência, este tipo de cálculo surge primeiro do que aqueles para a SCTD (ver seção 2.5). Além disso, as integrais radiais $R_{1}$ e $R_{2}$ precisam se estender até raios numericamente grandes quando a energia diminui, portanto o método não tem sido aplicado para energias do elétron incidente menores do que $1 \mathrm{keV}$.

\subsection{Resultados de cálculos e tabulações publicadas para as seções de choque de emissão de bremsstrahlung}

O trabalho de Koch e Motz de 1959 [24] é a primeira revisão publicada de fórmulas de seções de choque de bremsstrahlung. Ainda que já na época era conhecida a solução em forma de série infinita da equação de Dirac para o elétron em um campo Coulombiano blindado, do ponto de vista computacional resultava impossível um cálculo desse tipo. 
Mesmo que, em geral, a AB é menos precisa a medida que: i) o número atômico aumenta, ii) a energia inicial do elétron diminui e iii) a energia do fóton se aproxima no limite de altas frequências, os autores fizeram maior ênfase nestas fórmulas analíticas incluindo vários fatores de correção teóricos e empíricos. Só no caso de energias altamente relativísticas $\left(\epsilon_{1}, \epsilon_{2}, k \gg 1\right)$, onde a função hipergeométrica tem um comportamento assimptótico analiticamente mais simples [1], são fornecidas um conjunto de fórmulas derivadas das funções de onda de SM.

Koch e Motz [24] dividem as seções de choque de acordo com os parâmetros diferenciais em três grupos:

A) Com respeito à energia do fóton e aos ângulos de emissão do fóton e do elétron (SCTD)

$$
\frac{\mathrm{d}^{3} \sigma}{\mathrm{d} k \mathrm{~d} \Omega_{k} \mathrm{~d} \Omega_{p_{2}}}
$$

B) Com respeito à energia e o ângulo de emissão do fóton (SCDD)

$$
\frac{\mathrm{d}^{2} \sigma}{\mathrm{d} k \mathrm{~d} \Omega_{k}}=\int \frac{\mathrm{d}^{3} \sigma}{\mathrm{d} k \mathrm{~d} \Omega_{k} \mathrm{~d} \Omega_{p_{2}}} \mathrm{~d} \Omega_{p_{2}}
$$

C) Com respeito à energia do fóton (SCD)

$$
\frac{\mathrm{d} \sigma}{\mathrm{d} k}=\iint \frac{\mathrm{d}^{3} \sigma}{\mathrm{d} k \mathrm{~d} \Omega_{k} \mathrm{~d} \Omega_{p_{2}}} \mathrm{~d} \Omega_{p_{2}} \mathrm{~d} \Omega_{k}
$$

Outras subdivisões são feitas nas fórmulas: $i$ ) para levar em conta (ou não) a blindagem do campo Coulombiano pelos elétrons das camadas atômicas; ii) para os casos nãorelativísticos e relativísticos; iii) se são usadas aproximações para pequenos ou grandes ângulos de espalhamento. A região de validade e a precisão de cada fórmula é discutida por Koch e Motz [24]. Os autores também plotaram as seções de choque mostrando as dependências com a energia do elétron incidente (acima de $1 \mathrm{MeV}$ ), com a energia e o ângulo do fóton emitido, e as correções por blindagem, tomando como referência um átomo alvo $\operatorname{com} Z=78$.

Dez anos depois, em 1969, Elwert e Haug [25] publicaram os primeiros cálculos de seções de choque utilizando as autofunções de SM. Além das expressões descritas na seção 2.3, nesse artigo também é deduzida a aproximação da SCTD no limite de comprimentos de ondas grandes $\left(p_{2} \simeq p_{1}, k \ll p_{2}\right)$ e a fórmula de Bethe-Heitler (2.21), como casos particulares da teoria. O fator multiplicativo de correção $F_{E}$, determinou-se para a 
SCTD da AB no limite de altas frequências:

$$
F_{E}=\frac{a_{2}\left(1-\mathrm{e}^{-2 \pi a_{1}}\right)}{a_{1}\left(1-\mathrm{e}^{-2 \pi a_{2}}\right)},
$$

com o parâmetro

$$
a_{2}=\frac{\alpha Z}{\beta_{2}}=\alpha Z \frac{\epsilon_{2}}{p_{2}}
$$

de maneira que se consegue um valor finito dessa grandeza quando $p_{2} \rightarrow 0$.

Elwert e Haug [25] também calcularam a SCDD integrando numericamente a SCTD para todos os ângulos do elétron espalhado. A avaliação foi realizada para átomos com $Z=8$ e $Z=13$ em que se satisfaz a condição $a \ll 1$, e energias $E_{0}=45 \mathrm{keV}$ e $W=$ $40 \mathrm{keV}$. Dessa forma foi estudada a distribuição angular dos fótons e comparada com SCDDs experimentais não publicadas, obtidas na Universidade de Würzburg para um alvo de $\mathrm{Al}_{2} \mathrm{O}_{3}$ [25]. Os resultados mostraram compatibilidade na faixa $30^{\circ} \leqslant \theta \leqslant 120^{\circ}$, porém, devido à pouca disponibilidade de dados experimentais, não foram estabelecidas conclusões gerais. Por outro lado, os autores recomendaram não utilizar a aproximação não relativística (2.39) ainda para $E_{0}=45 \mathrm{keV}$, já que fornece valores discrepantes da expressão exata nessa energia.

De maneira similar, Elwert e Haug [25] calcularam a SCD para átomos com $Z=13$ e 79 . No caso de baixas energias, por exemplo $E_{0}=45 \mathrm{keV}$, sugeriram a utilização dos resultados da teoria tanto para $Z$ baixos quanto altos, no entanto, para $E_{0}=500 \mathrm{keV}$, eles só obtiveram bons resultados para $Z$ baixos. As conclusões anteriores são derivadas de comparações com alguns experimentos disponíveis na época.

\subsubsection{Seção de choque diferencial em energia}

Em 1971, Tseng e Pratt [26] publicaram o primeiro cálculo de seções de choque utilizando a expansão relativística de ondas parciais. O estudo determina tanto SCDD quanto a $\mathrm{SCD}$, e se concentra em energias dos elétrons incidentes entre $5 \mathrm{keV}$ e $1 \mathrm{MeV}$, elementos com $Z=1,8,13$ e 79, empregando quatro potenciais centrais:

1) Coulombiano pontual

$$
V(r)=-\frac{a}{r}
$$

2) Thomas-Fermi (TF) [27]

$$
V(r)=-\frac{a}{r} V_{0}(x),
$$


onde $V_{0}(x)$ é a função universal de Thomas-Fermi em termos da variável adimensional $x$. Os valores de $V_{0}(x)$ são determinados numericamente e encontram-se tabulados (ver $p$. ex. a referência [27]).

3) Thomas-Fermi modificado (TFM) [28]

$$
V(r)=-\frac{a}{r}\left(0,711 \mathrm{e}^{-0,175 a_{0} r}+0,2889 \mathrm{e}^{-1,6625 a_{0} r}\right)^{2},
$$

sendo $a_{0}=2(3 \pi / 4)^{-2 / 3} a^{1 / 3}$.

4) Hartree-Fock-Slater (HFS) [29]

$$
r V(r)=-Z+\int_{0}^{r} n\left(r^{\prime}\right) \mathrm{d} r^{\prime}+r \int_{r}^{\infty} \frac{n\left(r^{\prime}\right)}{r^{\prime}} \mathrm{d} r^{\prime}-\frac{3}{2}\left(\frac{81}{32 \pi^{2}} r n(r)\right)^{1 / 3}
$$

com $n(r)=4 \pi r^{2} \rho(r)$ a densidade radial de carga dos elétrons.

As componentes maior $g_{\kappa}(r)$ e menor $f_{\kappa}(r)$ da função de onda foram obtidas numericamente mediante o método de Runge-Kutta de quarta ordem [21]. Segundo Tseng e Pratt as principais fontes de erro nesse cálculo foram: a largura finita do passo de integração em $R_{1}(\kappa)$ e $R_{2}(\kappa)$, e o truncamento da série (2.61) para uma quantidade limitada de números $\kappa_{1}$ e $\kappa_{2}$. Porém, eles lograram uma alta precisão, estimando erros numéricos para as SCDD e SCD entorno de $1 \%$ e $0,5 \%$, respectivamente [26].

O método usado por Tseng e Pratt [26] forneceu a maior exatidão até hoje, e permitiu testar teorias como a $\mathrm{AB}$ e as derivadas das autofunções de SM, considerando diferentes intervalos angulares e números atômicos. Para $Z=1,8$ e 13 os resultados de Elwert e Haug [25] mostram boa concordância na SCD para todas as energias, e na SCDD para ângulos de emissão dos fótons com respeito a direção do elétron incidente maiores que $30^{\circ}$. Como era de esperar, o acordo entre as duas teorias desaparece para $Z=79$, onde os cálculos de Elwert e Haug [25] tendem a subestimar as seções choque.

Além disso, a utilização dos potenciais anteriormente mencionados possibilitou estudar de maneira rigorosa os efeitos da blindagem atômica e a sensibilidade do método na escolha de $V(r)$. As diferenças nas seções de choque quando o cálculo é desenvolvido com um potencial tipo TF, TFM ou HFS para as energias analisadas mostrou discrepâncias de $1 \%[26]$.

Finalmente, com o aumento da velocidade e capacidade de armazenamento dos computadores, as primeiras tabulações de seções de choque foram publicadas em 1976 e 1977 [2, 30]. Assim, Lee, Kissel, Pratt e Tseng lograram aumentar o número de elementos e energias dos elétrons incidentes no cálculo da SCD [30]. A metodologia seguida 
foi similar ao trabalho anterior de Tseng e Pratt [26], descrevendo o bremsstrahlung como uma transição simples do elétron em um potencial auto-consistente do tipo HFS relativístico [29]. As funções de onda do elétron foram obtidas mediante a série (2.61), integrando numericamente a equação radial de Dirac (2.59) e somando posteriormente sobre os valores de $\kappa_{1,2}$.

Uma comparação entre os resultados da SCD calculada com um potencial Coulombiano pontual e um potencial HFS para $Z=2,8,13,47,79$ e 92 , e energias $E_{0}=5,10,25$, $50,75,100,200,300,400$ e $500 \mathrm{keV}$, permitiu as seguintes conclusões [30] ${ }^{1}$ :

I) Para um $Z$ e um $E_{0}$ dados, no caso do potencial Coulombiano pontual, a SCD começa em um valor finito no tip $\left(W / E_{0}=1\right)$ aumentando gradualmente na medida em que $W / E_{0}$ diminui, e finalmente diverge para $W / E_{0} \rightarrow 0$.

II) A intensidade do espectro de bremsstrahlung de um átomo neutro se encontra abaixo em relação ao caso de um potencial pontual. Quando a blindagem é considerada, a SCD é zero no tip, mas cresce rapidamente nos primeiros $5-50 \mathrm{eV}$, além de que no limite $W / E_{0} \rightarrow 0$ apresenta um valor finito.

III) Em ambos casos o espectro se torna mais plano com o aumento de $Z$ para um valor fixo de $E_{0}$, e para um valor fixo de $Z$ com o aumento de $E_{0}$.

IV) Para um átomo neutro a SCD tem um máximo com a diminuição de $W / E_{0}$ e depois diminui perto da região final do espectro. Este efeito se define primeiro para elementos de alto $Z$ : em torno de $10 \mathrm{keV}$ para $\mathrm{Au}(Z=79), 5 \mathrm{keV}$ para $\mathrm{Ag}$ $(Z=47)$ e $1 \mathrm{keV}$ para $\mathrm{Al}(Z=13)$.

V) Para um dado valor de $W / E_{0}$, a blindagem é mais importante para pequenos $E_{0}$, e para um valor fixo de $E_{0}$, a blindagem é mais importante para pequenos valores de $W / E_{0}$.

VI) O espectro de átomos parcialmente ionizados se encontra entre os dois casos analisados.

Posteriormente, Pratt e colaboradores [2] acrescentaram à tabulação da SCD valores para elétrons com energias entre $1 \mathrm{keV}$ e $2 \mathrm{MeV}$, e todos os átomos na faixa $2 \leqslant Z \leqslant 92$. Essas tabelas dependem de três parâmetros: $Z, E_{0}$ e a fração de energia radiada $\kappa \equiv W / E_{0}$ ou energia reduzida do fóton. A grandeza fornecida nesse trabalho se conhece como seção de choque escalada (ou reduzida):

$$
\chi\left(Z, E_{0}, \kappa\right)=\frac{\beta^{2}}{Z^{2}} W \frac{\mathrm{d} \sigma}{\mathrm{d} W},
$$

\footnotetext{
${ }^{1}$ A fim de permitir uma fácil comparação com dados experimentais, as energias são expressas em unidades medidas no laboratório, $E_{0}$ para os elétrons incidentes e $W$ para os fótons.
} 
onde $\beta=v_{0} / c$ é a velocidade do elétron incidente em unidades da velocidade da luz $c$, que generalmente se expressa em termos de $E_{0}$ e a energia em repouso do elétron $m_{\mathrm{e}} c^{2}$ como:

$$
\beta=\sqrt{1-\left(1+\frac{E_{0}}{m_{\mathrm{e}} c^{2}}\right)^{-2}} .
$$

Uma das vantagens de tabular $\chi\left(Z, E_{0}, \kappa\right)$ em lugar da SCD, reside no fato que, para um valor fixo de alguns dos parâmetros $Z, E_{0}$ ou $\kappa, \chi\left(Z, E_{0}, \kappa\right)$ varia suavemente com os outros dois, e portanto, um procedimento de interpolação resulta matematicamente mais simples. Pratt e colaboradores [2] calcularam esta função para os valores de referência:

$$
\begin{aligned}
& Z=2,8,13,47,79 \text { e } 92, \\
& E_{0}=1,5,10,50,100,500,1000 \text { e } 2000 \mathrm{keV}, \\
& \kappa=0,0,0,4,0,6 \text { e } 0,95
\end{aligned}
$$

e expandiram, mediante a interpolação, os resultados para todos os elementos da tabela periódica anteriormente citados. A interpolação se baseou em ajustar uma função tipo spline-cúbico, pelo método dos mínimos quadrados, aos pontos dados pela razão entre seus resultados e as predições analíticas da $\mathrm{AB}$ com correções de blindagem. A estimativa da incerteza da SCD fornecida pelos autores é de 10\% [2].

Mais tarde, as tabelas de Pratt e colaboradores [2], foram estendidas por Seltzer e Berger $[3,16]$ em três sentidos: a) as energias $E_{0}$ dos elétrons incidentes foram aumentadas até $10 \mathrm{GeV}$; b) adicionaram-se elementos, $1 \leqslant Z \leqslant 100$; c) foi incluída na SCD uma componente que leva em conta a produção de bremsstrahlung no campo elétrico do elétron atômico:

$$
\frac{\mathrm{d} \sigma}{\mathrm{d} W}=\frac{\mathrm{d} \sigma_{n}}{\mathrm{~d} W}+Z \frac{\mathrm{d} \sigma_{e}}{\mathrm{~d} W}
$$

onde $\mathrm{d} \sigma_{n} / \mathrm{d} W$ representa a radiação produzida no campo blindado do núcleo atômico, e $Z\left(\mathrm{~d} \sigma_{e} / \mathrm{d} W\right)$ o bremsstrahlung gerado no campo dos $Z$ elétrons do átomo. Assim, o primeiro termo da SCD é calculado combinando teorias analíticas para altas energias e os dados de ondas parciais de Pratt [2] abaixo de $E_{0}=2 \mathrm{MeV}$. Por outro lado, a SCD da interação elétron-elétron foi obtida da teoria de Haug [31]. Seltzer e Berger [3] também tabularam o parâmetro

$$
\eta=\frac{\mathrm{d} \sigma_{e}}{\mathrm{~d} W} / \frac{1}{Z^{2}} \frac{\mathrm{d} \sigma_{n}}{\mathrm{~d} W}
$$


a partir do qual podem se avaliar as duas componentes da SCD:

$$
\begin{aligned}
& \frac{\mathrm{d} \sigma_{n}}{\mathrm{~d} W}=\frac{Z}{Z+\eta} \frac{\mathrm{d} \sigma}{\mathrm{d} W}, \\
& \frac{\mathrm{d} \sigma_{e}}{\mathrm{~d} W}=\frac{\eta}{Z+\eta} \frac{1}{Z} \frac{\mathrm{d} \sigma}{\mathrm{d} W} .
\end{aligned}
$$

Na tabela 2.1 são comparados os valores de $\chi\left(Z, E_{0}, \kappa\right)$ fornecidos por Pratt et al. [2] e Seltzer e Berger [3] para $\mathrm{Al}$ e $\mathrm{Au}$, e varias energias $E_{0}$ e $\kappa$. A razão $\delta$ entre ambas grandezas é calculada para obter a contribuição relativa da componente eletrônica. Pode se concluir que na faixa analisada, a emissão de bremsstrahlung é uma consequência totalmente dominada pelo campo Coulombiano blindado do núcleo.

As tabelas de Seltzer e Berger [3] constituem os dados teóricos mais confiáveis de SCDs disponíveis até o momento. Segundo os autores, as incertezas das SCDs estão: i) entre $3 \%$ e $5 \%$ na região de altas energias, $E_{0} \geqslant 50 \mathrm{MeV} ;$ ii) entre $5 \%$ e $10 \%$ para energias intermediárias, $2 \mathrm{MeV} \leqslant E_{0} \leqslant 5 \mathrm{MeV}$; e iii) em torno de $10 \%$ para $E_{0}<2 \mathrm{MeV}$.

\subsubsection{Distribuição angular dos fótons}

Além do espectro energético de bremsstrahlung, a distribuição angular dos fótons também tem sido tabulada $[4,5]$. O objetivo desse tipo de cálculo é fornecer uma grandeza que

TABela 2.1: Comparação entre os resultados publicados por Pratt e colaboradores $\left(^{\dagger}\right)$ em 1977, e Seltzer e Berger $\left(^{\star}\right)$ em 1986, para a SCD escalada. A unidade de $\chi\left(Z, E_{0}, \kappa\right)$ é mb. $\delta$ é a razão entre os cálculos de Pratt e colaboradores (sem incluir a componente do bremsstrahlung no campo dos elétrons atômicos) e as tabulações de Seltzer e Berger.

\begin{tabular}{c||ccc||ccc}
\hline \hline \multirow{2}{*}{\multicolumn{1}{c||}{$E_{0}(\mathrm{keV}) \backslash \kappa$}} & \multicolumn{3}{c||}{$Z=13$} & \multicolumn{3}{c}{$Z=79$} \\
\cline { 2 - 7 }$\dagger$ & 0,1 & 0,5 & 1,0 & 0,1 & 0,5 & 1,0 \\
\hline${ }^{\dagger} 10$ & 7,926 & 6,660 & 5,510 & 3,663 & 4,067 & 4,449 \\
$\delta \%$ & 7,850 & 6,564 & 5,488 & 3,687 & 4,116 & 4,459 \\
$\delta \%$ & 100,97 & 101,46 & 100,41 & 99,34 & 98,80 & 99,77 \\
\hline$\dagger 50$ & 9,488 & 6,019 & 3,900 & 7,141 & 6,207 & 5,591 \\
$\star 50$ & 9,534 & 6,023 & 3,902 & 7,146 & 6,183 & 5,596 \\
$\delta \%$ & 99,51 & 99,91 & 99,94 & 99,93 & 100,38 & 99,91 \\
\hline$\dagger 100$ & 9,573 & 5,380 & 2,813 & 8,523 & 6,594 & 5,397 \\
$\star 100$ & 9,659 & 5,384 & 2,819 & 8,524 & 6,595 & 5,385 \\
$\delta \%$ & 99,11 & 99,93 & 99,78 & 99,98 & 99,98 & 100,22 \\
\hline$\dagger 500$ & 9,168 & 3,987 & 0,939 & 9,750 & 5,640 & 3,135 \\
$\star 500$ & 9,647 & 4,055 & 0,940 & 9,802 & 5,652 & 3,136 \\
$\delta \%$ & 95,03 & 98,32 & 99,89 & 99,47 & 99,79 & 99,97 \\
\hline$\dagger 1000$ & 9,562 & 4,000 & 0,666 & 9,770 & 5,160 & 2,322 \\
$\star 1000$ & 10,170 & 4,111 & 0,665 & 9,843 & 5,177 & 2,315 \\
$\delta \%$ & 94,91 & 97,30 & 100,15 & 99,26 & 99,67 & 100,30 \\
\hline \hline
\end{tabular}


permita a avaliação da SCDD. Em um trabalho que complementa o prévio [2], Tseng, Pratt e Lee [4] descrevem a metodologia aplicada para determinar a função de forma do bremsstrahlung (FFB), definida como a razão entre a SCDD e a SCD

$$
S\left(Z, E_{0}, \kappa, \theta\right)=\frac{\mathrm{d}^{2} \sigma}{\mathrm{d} W \mathrm{~d} \Omega_{k}} / \frac{\mathrm{d} \sigma}{\mathrm{d} W} .
$$

A FFB depende dos mesmos parâmetros do espectro energético e, além disso, do ângulo $\theta$ entre as direções dos momentos do elétron incidente e do fóton emitido. Da relação anterior se observa que $S$ é uma função densidade de probabilidade que satisfaz a condição:

$$
\int S \mathrm{~d} \Omega_{k}=1
$$

O cálculo da FFB foi realizado seguindo o procedimento descrito pelos autores para a SCD [2, 26], com os mesmos valores de referência enumerados em (2.69), considerando energias $E_{0}$ somente até $500 \mathrm{keV}[4,5]$. A partir do estudo do comportamento funcional da FFB com base nas teorias analíticas existentes (AB e SM), Tseng, Pratt e Lee [4] mostraram que para $Z, E_{0}$ e $\kappa$ fixos, $S$ depende apenas do $\cos \theta$ e sugeriram a sua parametrização segundo

$$
S=\frac{A}{4 \pi(1-\beta \cos \theta)^{m}} \sum_{n=0}^{N} B_{n} P_{n}(\cos \theta),
$$

onde $A$ é definido pela condição de normalização $(2.74), P_{n}(\cos \theta)$ são os polinômios de Legendre e $B_{0} \equiv 1$.

Kissel, Quarles e Pratt [5] ajustaram a fórmula anterior aos pontos calculados e determinaram que estatisticamente os melhores resultados são obtidos para $N=5$ e $m=4,5$. Com esses valores a distribuição teórica é reproduzida até o terceiro decimal. Logo, para cada ponto de referência $\left(Z, E_{0}, \kappa\right)$ foi obtido um conjunto de cinco parâmetros: $B_{1}\left(Z, E_{0}, \kappa\right)-B_{5}\left(Z, E_{0}, \kappa\right)$, que posteriormente se interpolaram em cada uma das suas variáveis. Por último, $A\left(Z, E_{0}, \kappa\right)$ foi calculada de modo que se satisfaça a Eq. (2.74) em cada caso.

Duas tabulações são apresentadas no artigo [5]: 1) os parâmetros de ajuste $B_{1}\left(Z, E_{0}, \kappa\right)$ $B_{5}\left(Z, E_{0}, \kappa\right)$ e $\left.A\left(Z, E_{0}, \kappa\right), 2\right)$ o resultado da interpolação e, portanto, de $S\left(Z, E_{0}, \kappa, \theta\right)$ para 23 elementos na faixa $2 \leqslant Z \leqslant 92$ e ângulos $0^{\circ} \leqslant \theta \leqslant 180^{\circ}$, com um passo $\Delta \theta=5^{\circ}$. A incerteza estimada para a FFB é de $5 \%$, sendo esperado que seja menor para valores de $Z$ e $E_{0}$ próximos às referências. 
Por meio das tabulações de $\chi\left(Z, E_{0}, \kappa\right)$ e $S\left(Z, E_{0}, \kappa, \theta\right)$ a SCDD pode ser avaliada como:

$$
\frac{\mathrm{d}^{2} \sigma}{\mathrm{d} W \mathrm{~d} \Omega_{k}}=\frac{Z^{2}}{\beta^{2}} \frac{1}{W} \chi\left(Z, E_{0}, \kappa\right) S\left(Z, E_{0}, \kappa, \theta\right) .
$$

Segundo os critérios expostos, a incerteza dos resultados fornecidos pela fórmula anterior está em torno de $11 \%$.

\subsection{Experimentos para a determinação de seções de cho- que de bremsstrahlung}

Nas medidas de SCDDs de emissão de bremsstrahlung, um feixe monoenergético de elétrons incide sobre um alvo do material a ser estudado, e a radiação de freamento gerada é coletada mediante sistemas espectrométricos. A Fig. 2.1 apresenta um esquema do arranjo tipicamente utilizado nesses experimentos.

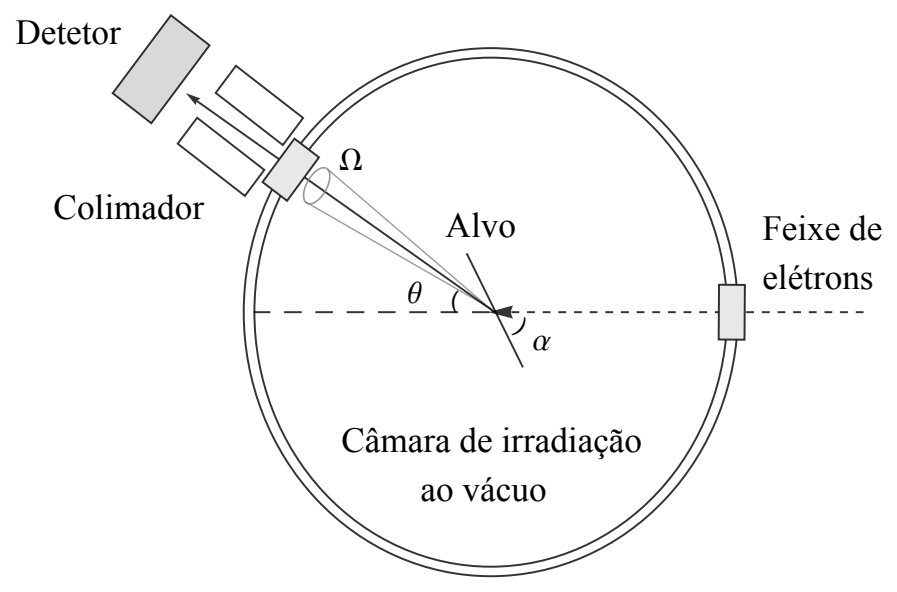

FigurA 2.1: Esquema do arranjo experimental tipicamente utilizado nas medidas de SCDDs de emissão de bremsstrahlung. Numa câmara de irradiação ao vácuo se produz a interação entre o feixe de elétrons e o alvo. Os fótons gerados são coletados com detetores posicionados em vários ângulos $\theta$.

A SCDD se determina segundo a relação

$$
\frac{\mathrm{d}^{2} \sigma}{\mathrm{d} W \mathrm{~d} \Omega}=\frac{B(W)}{N_{0} t \Delta \Omega},
$$

onde $B(W)$ é o número de fótons emitidos no elemento estudado, à energia $W$, na direção $\theta, N_{0}$ é o número de elétrons incidentes durante o tempo vivo de medida, $t$ é a 
densidade superficial de átomos do alvo, $\Delta$ é a largura do canal correspondente a $W$ no espectro de bremsstrahlung, e $\Omega$ é o ângulo sólido subtendido pelo detetor em relação ao alvo. O parâmetro $t$ se calcula como

$$
t=\mathcal{N} \frac{d}{\sin \alpha}
$$

sendo $\mathcal{N}$ o número de átomos por unidade de volume do elemento, $d$ a espessura do alvo, e $\alpha$ o ângulo de inclinação do alvo em relação à direção de incidência do feixe de elétrons, como se ilustra na Fig. 2.1.

A fórmula (2.77) é válida para alvos homogêneos e valores de $t$ suficientemente pequenos, que garantam, no máximo, uma interação radiativa por elétron incidente. Essas condições permitem obter o que se conhece como bremsstrahlung de alvo fino: um espectro caracterizado por um valor finito na região de máxima transferência de energia. Nesse caso, é possível realizar uma comparação direta entre as estimativas teóricas da SCDD e os resultados que fornece a Eq. (2.77). Do ponto de vista experimental o maior desafio radica na determinação absoluta de cada uma das grandezas envolvidas na relação (2.77).

Para energias incidentes dos elétrons na faixa $10 \mathrm{keV} \lesssim E \lesssim 100 \mathrm{keV}$, um grupo reduzido de experimentos foram desenvolvidos nos últimos 60 anos $[6-9,32-37]$. O primeiro destes trabalhos, publicado em 1958 por Motz e Placious [6], é uma das experiências mais completas considerando as limitações instrumentais da época. Eles obtiveram de maneira absoluta as SCDDs, realizando um estudo com elétrons incidentes de $50 \mathrm{keV}$ sobre alvos de $\mathrm{Al}$ e Au, em um amplo intervalo angular: 10, 20, 30, 40, 50, 60, 70, 90, 110 e 140 graus.

Na confecção dos alvos, substratos de collodion foram utilizados para a evaporação de $\mathrm{Al}$ e $\mathrm{Au}[6]$. A densidade $t$ foi determinada a partir de medidas numa microbalança da massa dos alvos. Os autores [6] prepararam dois filmes de cada material e obtiveram para $\mathrm{Al}$ as espessuras mássicas ${ }^{2} 17,3(8) \mu \mathrm{g} / \mathrm{cm}^{2}$ e $38,2(14) \mu \mathrm{g} / \mathrm{cm}^{2}$, e para Au 9,9(5) $\mu \mathrm{g} / \mathrm{cm}^{2}$ e $22,1(10) \mu \mathrm{g} / \mathrm{cm}^{2}$.

Motz e Placious [6] contabilizaram o número de elétrons $N_{0}$ por meio um integrador de corrente acoplado à câmara de alvos eletricamente isolada. O espectro de fótons $N(h)$ ( $h$ é a altura dos pulsos registrados no histograma multicanal) foi coletado com um detetor cintilador do tipo NaI ativado com $\mathrm{Tl}$ (12,7 mm de diâmetro, 63,5 mm de espessura do cristal), na faixa energética compreendida entre os $7 \mathrm{keV}$ e a região

\footnotetext{
${ }^{2}$ As espessura mássicas de $\mathrm{Al} 17,3$ e $38,2 \mu \mathrm{g} / \mathrm{cm}^{2}$ equivalem as densidades $t=386 \times 10^{15}$ e $732 \times 10^{15}$ átomos $/ \mathrm{cm}^{2}$, enquanto no caso do $\mathrm{Au}$, as espessuras 9,9 e $22,1 \mu \mathrm{g} / \mathrm{cm}^{2}$ equivalem a $t=30,3 \times 10^{15} \mathrm{e}$ $67,6 \times 10^{15}$ átomos $/ \mathrm{cm}^{2}$ respectivamente.
} 
do tip. Os autores deduziram o espectro de bremsstrahlung $B(W)$ a partir de $N(h)$ seguindo dois precedimentos: 1) dividindo $N(h)$ pela eficiência intrínseca de fotopico, e 2) multiplicando as contagens de cada canal por um fator de calibração que leva em conta a resposta do detetor fora do fotopico [6].

A incerteza relativa das SCDDs obtida por Motz e Placious é de 15\%, onde a maior contribuição foi associada à determinação do espectro $B(W)$ [6]. Devido à baixa resolução do detetor cintilador empregado, 25-30\% em $50 \mathrm{keV}$, na região entre $10-50 \mathrm{keV}$ os espectros sofrem grandes distorções. No caso do Au, por exemplo, foi impossível descontar a contribuição dos raios-x L, portanto a SCDD é superestimada nesta faixa energética $(\sim 9-14 \mathrm{keV})$.

Um experimento similar foi desenvolvido em 1970 por Rester, Edmonson e Peasley para elétrons de $50 \mathrm{keV}$ e os mesmos elementos [7]. Qualitativamente, representa um estudo superior considerando que o espectro $N(h)$ foi coletado com um detetor planar de $\mathrm{Ge}(\mathrm{Li})$ de $10 \mathrm{~mm}$ de diâmetro e $5 \mathrm{~mm}$ de espessura do cristal. Entre a saída da câmara de irradiação e o detetor foi colocado um colimador com abertura de $4 \mathrm{~mm}$ (não se especificou o material). Com esse dispositivo, Rester, Edmonson e Peasley procuraram evitar a interação dos fótons perto das bordas do cristal, onde os fotoelétrons podem escapar, e por consequência, a carga gerada no volume ativo não ser coletada completamente [7]. Dessa maneira foram maximizados os eventos de absorção total no detetor. Os autores comprovaram a eficácia do sistema colimação medindo a resposta do espectrômetro para linhas monocromáticas entre 10 e $50 \mathrm{keV}$, fornecidas por um sistema de difração de raios-x (cristal monocromador de LiF). Os resultados indicaram que a parte da resposta, fora do pico gaussiano de absorção total, representou uma pequena fração da área total do espectro (não é fornecido um número) e não foi considerada a sua contribuição [7]. A fórmula (2.77) sob as condições acima descritas se transforma em

$$
\frac{\mathrm{d}^{2} \sigma}{\mathrm{d} W \mathrm{~d} \Omega}=\frac{N(W) / \varepsilon_{\mathrm{int}}(W)}{N_{0} t \Delta \Omega}
$$

onde se assume que $N(h)=\varepsilon_{\text {int }}(W) B(W)$, com $h=W$, sendo $\varepsilon_{\text {int }}(W)$ a eficiência intrínseca de foto pico à energia $W$.

Os outros parâmetros do experimento foram análogos aos de Motz e Placious [6]: alvos de $\mathrm{Al}$ e $\mathrm{Au}$ de aproximadamente $20 \mu \mathrm{g} / \mathrm{cm}^{2}$ e faixa angular entre $10^{\circ}$ e $120^{\circ}$ [7]. A incerteza nas SCDDs foi estimada em $5 \%$ para energias dos fótons de $50 \mathrm{keV}$, enquanto na região do espectro perto de $10 \mathrm{keV}$ o desvio padrão aumentou para 10\% [7]. 
Os resultados dos dois experimentos anteriores [6, 7] foram comparados com a teoria de ondas parciais por Tseng e Pratt [26]. Eles calcularam a distribuição angular para $E_{0}=50 \mathrm{keV}$ e $W=40,30$ e $20 \mathrm{keV}$ no caso do Al, e $W=30$ e $20 \mathrm{keV}$ no Au, utilizando um potencial HFS. Para esses valores de $W$, longe da região dos raios-x L do Au, os dados de ambas experiências [6, 7] tem maior precisão. Da comparação pode-se concluir que:

I) Em geral os dados de Rester, Edmonson e Peasley tem maior concordância com a teoria do que os resultados de Motz e Placious.

II) Para $W=30$ e $40 \mathrm{keV}(\kappa=0,6$ e 0,8), tanto no Al quanto no $\mathrm{Au}$, experimentos e teoria são compatíveis dentro dos respectivos desvios padrão. O acordo maior se observa para ângulos $\theta \geqslant 90^{\circ}$.

III) Para $W=20 \mathrm{keV}(\kappa=0,4)$ os dados de Rester, Edmonson e Peasley estão sistematicamente abaixo da curva teórica.

IV) Para $W=20 \mathrm{keV}$ os dados de Motz e Placious estão sistematicamente abaixo da teoria no caso do Al, e sistematicamente acima no caso do Au.

A conclusão IV é um resultado esperado para o Au, devido à baixa resolução do detetor utilizado [6] e, portanto, para $W=20 \mathrm{keV}$ o espectro pode ter sido distorcido pelos raios-x L emitidos pelo alvo.

Outros quatro trabalhos foram publicados durante as décadas de 1980 e 1990 por um grupo da Universidade Cristiana de Texas [8, 9, 33, 34]. No primeiro destes, Quarles e Heroy mediram os espectros de bremsstrahlung produzidos em alvos de $\mathrm{Al}, \mathrm{Cu}, \mathrm{Ag}$ e $\mathrm{Au}$, por elétrons de $E_{0}=50,100$ e $140 \mathrm{keV}$, nos ângulos $\theta=30^{\circ}, 45^{\circ}, 60^{\circ}, 90^{\circ} \mathrm{e}$ $135^{\circ}$, utilizando um detetor planar de $\mathrm{Ge}(\mathrm{Li})$ [8]. A espessura dos alvos foi determinada de forma semelhante aos experimentos de Motz e Placious [6], e Rester, Edmonson e Peasley [7]. O procedimento forneceu incertezas relativas de $5 \%$ para a massa dos filmes de $\mathrm{Al}, \mathrm{Cu}$ e $\mathrm{Au}$, e de $15 \%$ para $\mathrm{Ag}$ [8].

Quarles e Heroy [8], determinaram a eficiência do detetor usando fontes radiativas calibradas, e ajustaram a função analítica proposta por Gallagher e Cipolla $[38]^{3}$ aos dados experimentais. No entanto, a expressão obtida apresentou grandes incertezas: até $22 \%$ para energias inferiores a $25 \mathrm{keV}$ e superiores a $100 \mathrm{keV}$. Esse resultado indica que o modelo não descreve corretamente o comportamento da eficiência nessas regiões energéticas.

\footnotetext{
${ }^{3} \mathrm{O}$ modelo de Gallagher e Cipolla [38], publicado em 1974, é baseado em um ajuste não linear de seis parâmetros de uma função semi-empírica para a eficiência. A aproximação utiliza a proporcionalidade da seção de choque de efeito fotoelétrico com uma potência do inverso da energia do fóton, mas não os coeficientes de atenuação que descrevem exatamente a interação.
} 
Para $E_{0}=50 \mathrm{keV}$ e alvos de $\mathrm{Al}$ e $\mathrm{Au}$, Quarles e Heroy [8] não apresentaram diretamente as SCDDs. Eles determinaram a FFB experimental dividindo seus dados, obtidos mediante a Eq. (2.79), pela SCD tabulada por Pratt e colaboradores [2] segundo a fórmula (2.73). Logo, os valores de $S$ foram comparados com os cálculos de Tseng, Pratt e Lee [4]. A Tabela 2.2 mostra os resultados desta comparação; pode se concluir que para as energias $W=20$ e $30 \mathrm{keV}$ ( $\kappa=0,4$ e 0,6 respectivamente), os resultados tendem a estar sistematicamente acima dos valores teóricos, sendo esse desvio menor no caso do $\mathrm{Au}$ e $\kappa=0,6$. Os autores sugeriram que a diferença observada para $\kappa=0,4$ no Al poderia ter relação com a produção de bremsstrahlung elétron-elétron, porém, de acordo com os dados da Tabela 2.1 essa hipótese parece não ser correta. Por outro lado, tanto no $\mathrm{Al}$ quanto no Au se observa que para $\theta=135^{\circ}$, e todos os valores de $\kappa$, a FFB experimental é também sistematicamente superior aos cálculos de Tseng, Pratt e Lee $[4]$.

Quarles e Heroy [8] estudaram além disso o espectro de energia de bremsstrahlung invertendo o procedimento descrito acima. Eles dividiram seus valores de SCDD pela FFB tabulada por Tseng, Pratt e Lee [4], e compararam os resultados com os cálculos de Pratt e colaboradores [2] para a SCD, normalizando pelo fator $W / Z^{2}$ :

$$
\frac{W}{Z^{2}} \frac{\mathrm{d} \sigma}{\mathrm{d} W}=\frac{W}{Z^{2}} \frac{\mathrm{d}^{2} \sigma}{\mathrm{d} W \mathrm{~d} \Omega} / S\left(Z, E_{0}, \kappa, \theta\right) .
$$

Desta maneira, os resultados deveriam ser independentes do ângulo de medida. Quarles e Heroy [8] apresentam os pontos experimentais obtidos com a relação anterior, para valores fixos de $E_{0}$ e $\kappa$, em função de $\theta$, só no caso do Al. Nota-se uma grande dispersão dos dados para $\kappa \leqslant 0,6$ e todas as energias $E_{0}$. Para o resto dos elementos foi calculada a média da SCD para todos os ângulos da medida. A comparação [8] mostrou que: $i$ ) para $Z=47$ e $E_{0}=50 \mathrm{keV}$, os valores experimentais oscilam em torno dos cálculos de Pratt et al. [2], enquanto para $E_{0}=100 \mathrm{keV}$ e todas energias dos fótons, o experimento está abaixo da curva teórica em mais de 3 desvios padrões, ii) para $Z=29$ os resultados estão sistematicamente abaixo da teoria para todos os valores de $E_{0}$ e $\kappa$, iii) para $Z=79$ e

TABela 2.2: Desvio dos dados de Quarles e Heroy em relação ao cálculo de Tseng, Pratt e Lee para $S\left(Z, E_{0}, \kappa, \theta\right)$, com $E_{0}=50 \mathrm{keV}$. Os valores $\pm 1, \pm 2, \pm 3$ indicam a quantidade de desvios padrões experimentais acima ou abaixo da curva teórica.

\begin{tabular}{c||ccccc||ccccc}
\hline \hline \multicolumn{1}{c||}{} & \multicolumn{5}{c||}{$Z=13$} & \multicolumn{5}{c}{$Z=79$} \\
\cline { 2 - 10 }$\kappa \backslash \theta$ & $30^{\circ}$ & $45^{\circ}$ & $60^{\circ}$ & $90^{\circ}$ & $135^{\circ}$ & $30^{\circ}$ & $45^{\circ 0}$ & $60^{\circ}$ & $90^{\circ}$ & $135^{\circ}$ \\
\hline 0,40 & +2 & +2 & +1 & +3 & +2 & +2 & +2 & +1 & +1 & +2 \\
0,60 & +2 & +2 & +1 & +3 & +2 & +1 & +1 & +1 & -1 & +2 \\
0,80 & +1 & -1 & -3 & +1 & +2 & -1 & -1 & -1 & -1 & +2 \\
0,95 & -2 & -2 & -2 & +1 & +1 & & & & & \\
\hline \hline
\end{tabular}


$\kappa \geqslant 0,6$ os pontos experimentais encontram-se abaixo da teoria, porém, quando $\kappa<0,6$ se observa o efeito contrário.

Pode se concluir que o experimento realizado por Quarles e Heroy [8] não melhorou a precisão dos dados obtidos por Rester, Edmonson e Peasley [7] nem por Motz e Placious [6], mas, foram publicados pela primeira vez resultados para $\mathrm{Cu}$ e $\mathrm{Ag}$ [8].

Em 1987 R. Ambrose, Altman e Quarles [9] mediram SCDDs para os elementos C, Al, $\mathrm{Cu}, \mathrm{Y}, \mathrm{Ag}, \mathrm{Te}, \mathrm{Tb}, \mathrm{Au}, \mathrm{Bi}$ e U, energias $E_{0}=50$, 75 e $100 \mathrm{keV}$, e ângulo de emissão $\theta=90^{\circ}$. Para a coleção dos espectros os autores utilizaram um detetor planar de Ge hiper puro (HPGe) com um colimador de diâmetro 15,8 mm [9]. Os alvos foram preparados utilizando o métodos de deposição de vapores sobre filmes de C, colocados posteriormente em suportes de $\mathrm{Al}$ com $1 \mathrm{~cm}^{2}$ de abertura. Nos casos de $\mathrm{Y}, \mathrm{Tb}$ e U os alvos foram construídos a partir de compostos de flúor: $\mathrm{YF}_{3}, \mathrm{TbF}_{3}$ e $\mathrm{UF}_{4}$. A densidade $t$ dos alvos se determinou a partir de medidas de massa numa balança, e o procedimento forneceu incertezas relativas entre $5 \%$ e 10\%. Além disso, R. Ambrose et al. [9] estimaram que a rugosidade ${ }^{4}$ dos alvos pode introduzir erros de até $20 \%$ na SCDD.

No experimento de R. Ambrose, Altman e Quarles [9] se assumem as condições antes mencionadas para a aplicação da Eq. (2.79). A eficiência do HPGe foi determinada usando os espectros de bremsstrahlung medidos para alvos de $\mathrm{C}, \mathrm{Cu}, \mathrm{Ag}, \mathrm{Au}$ e $\mathrm{UF}_{4}$, no caso de elétrons com $E_{0}=100 \mathrm{keV}[39]^{5}$. As distribuições de altura de pulso foram divididas pelos valores teóricos de $\operatorname{SCDD}[3,5]$ e obteve-se a função:

$$
N_{0} t \Omega \varepsilon_{\text {int }}(W)=\frac{N(W)}{\Delta} / \frac{\mathrm{d}^{2} \sigma}{\mathrm{d} W \mathrm{~d} \Omega}
$$

A dependência energética deste fator está dada pela eficiência sempre que: 1) as contagens $N(W)$ sejam estritamente devidas à absorção total dos fótons produzidos no alvo, nesse caso não é levada em conta a função resposta do detetor, e 2) a SCDD, que é a grandeza a ser estudada, seja correta. Desvios dessas condições conduzem a erros em $\varepsilon_{\text {int }}(W)$ no mesmo sentido. R. Ambrose et al. [9], ajustaram as curvas obtidas com cada alvo a cinco pontos de eficiência, calculados a partir de medidas com fontes radiativas calibradas, deixando só um parâmetro livre: o fator constante que multiplica a $\varepsilon_{\text {int }}(W)$ no membro esquerdo da Eq. (2.81). Finalmente, com as curvas normalizadas foi realizada uma média ponto a ponto na região de interesse.

A incerteza total das SCDDs medidas por R. Ambrose et al. [9] foram avaliadas em 22\%, onde $20 \%$ é associado à espessura dos alvos, $5 \%$ à eficiência do detetor, $5 \%$ ao ângulo

\footnotetext{
${ }^{4} \mathrm{~A}$ rugosidade do alvo é uma medida da não homogeneidade da superfície deste.

${ }^{5}$ A descrição do procedimento de calibração em eficiência do detetor encontra-se na referência [39], publicada de maneira independente.
} 
sólido e $3 \%$ produto da energia incidente, a coleção de carga, a resolução energética e a atenuação dos fótons na janela da câmara de irradiação e a janela do HPGe.

Os resultados de R. Ambrose et al. [9] são apresentados em forma de gráficos onde se compara o experimento com a SCDD teórica, calculada por meio das tabelas de Kissell, Quarles e Pratt [5]. Em geral, a forma funcional descrita pelos pontos experimentais é similar à SCDD teórica, porém, em valor absoluto, as medidas sempre ficam acima ou abaixo da curva teórica, com grandes desvios para todos os $Z$ e $\kappa$ [9]. A tendência observada foi a seguinte: a) para $E_{0}=50 \mathrm{keV}$ a SCDD experimental é menor que a teórica, b) para $E_{0}=75 \mathrm{keV}$ não existe um comportamento fixo, a SCDD experimental é superior que a teórica para alguns elementos e inferior para outros, c) para $E_{0}=100 \mathrm{keV}$ a SCDD experimental foi maior que a teórica.

É conhecido que a seção de choque de espalhamento elástico de elétrons é maior em varias ordens de grandeza para ângulos dianteiros do que para traseiros [40]. Por consequência, nas medidas de bremsstrahlung de alvos finos para pequenos ângulos de emissão dos fótons, o espectro coletado $N(h)$ é distorcido pela radiação produzida nas janelas da câmara de irradiação ou do detetor, pelos elétrons espalhados elasticamente nessas direções. Um método diferente para tentar eliminar esse efeito na determinação da SCDD foi publicado por R. Ambrose, Kahler, Lehtihet e Quarles em 1991, [33].

O experimento se baseou em medidas de dois tipos. Em uma primeira etapa, foi coletado o espectro $N_{\mathrm{t}}(h)$ produzido por um alvo fino do elemento a estudar, colocado sobre uma folha de $\mathrm{C}$ de espessura tal que o feixe é completamente detido [33]. Posteriormente, foi medido o bremsstrahlung de alvo grosso ${ }^{6} N_{\mathrm{b}}(h)$ gerado na folha de C. A contribuição neta do elemento pesquisado $N(h)$ pode se deduzir segundo

$$
N(h)=N_{\mathrm{t}}(h)-N_{\mathrm{b}}(h) \frac{N_{0}}{N_{0 \mathrm{~b}}}(1+r),
$$

onde $N_{0}$ e $N_{0 \mathrm{~b}}$ são a quantidade de elétrons que atingem os alvos durante as duas medidas respectivamente, enquanto $r$, é um parâmetro de correção introduzido pelos autores para levar em conta, no fator de normalização $N_{0} / N_{0 \mathrm{~b}}$, "pequenas flutuações aleatórias na eficiência de coleção dos elétrons de uma medição à outra". Logo, R. Ambrose e colaboradores [33], expressaram o espectro $B(W)$ como uma função de $r$ :

$$
B(W)=\frac{1}{\varepsilon_{\text {int }}(W)}\left[N_{\mathrm{t}}(W)-N_{\mathrm{b}}(W) \frac{N_{0}}{N_{0 \mathrm{~b}}}(1+r)\right] .
$$

\footnotetext{
${ }^{6}$ Bremsstrahlung de alvo grosso é a radiação produzida em um filme espesso, onde o elétron perde toda sua energia ou parte significativa desta [35].
} 
Nesse experimento [33] foram medidos alvos de $\mathrm{Cu}$ e $\mathrm{Au}$ para elétrons de $71 \mathrm{keV}$, nos ângulos $\theta=0^{\circ}, 30^{\circ}, 45^{\circ}$ e $90^{\circ}$. Dois detetores planares HPGe permitiram coletar os espectros simultaneamente nas posições: $0^{\circ}$ e $45^{\circ}$, e $30^{\circ}$ e $90^{\circ}$. A espessura mássica dos alvos esteve em torno a $50 \mu \mathrm{g} / \mathrm{cm}^{2}$ com incertezas estimadas de $20 \%$. Finalmente, os autores [33] determinaram o parâmetro constante $r$ que melhor ajustara a forma de seus dados

$$
\frac{\mathrm{d}^{2} \sigma}{\mathrm{d} W \mathrm{~d} \Omega}=\frac{N_{\mathrm{t}}(W)-N_{\mathrm{b}}(W) \frac{N_{0}}{N_{0 \mathrm{~b}}}(1+r)}{A(W) \varepsilon_{\text {int }}(W) N_{0} t \Delta \Omega},
$$

às curvas teóricas de Kissel [5], sendo $A(W)$ um fator de correção devido a absorção de fótons na folha de carbono. Os valores de $r$ para todos os espectros estiveram entre $1 \%$ e $5 \%$.

Os resultados de R. Ambrose et al. [33] mostraram que o método é mais sensível para elementos com $Z$ altos, observando-se menores flutuações nas curvas de Au do que nas de $\mathrm{Cu}$. Por outro lado, os espectros tiveram boa concordância para $\theta=30^{\circ}, 45^{\circ} \mathrm{e}$ $90^{\circ}$, porém, no caso de $\theta=0^{\circ}$, apreciaram-se grandes discrepâncias na forma da função teórica em relação à do experimento.

Em um trabalho posterior do mesmo grupo (1997), V. Ambrose, Quarles e R. Ambrose [34] encontraram que as diferenças com a teoria para $\theta=0^{\circ}$ no experimento anterior [33], deviam-se à radiação produzida em um colimador para elétrons colocado na frente do alvo. O bremsstrahlung gerado no dispositivo distorcia os espectros coletados nesta posição [34]. Seguindo o mesmo método do parâmetro $r$, esses autores conseguiram ajustar a forma dos dados experimentais à teoria, para elétrons de $50 \mathrm{keV}$ e alvos de $\mathrm{Ag}, \mathrm{Tb}$ e $\mathrm{U}$ a zero graus. Um outro artigo onde a forma do espectro experimental é comparada com diversas teorias por meio de parâmetros de ajuste foi publicado por Chapman e colaboradores [32].

Sob outra perspectiva, e com o uso de alvos grossos ou gasosos, Shanker [36] estudou a intensidade do espectro de bremsstrahlung para elétrons incidentes entre 2 e $30 \mathrm{keV}$. Trabalhos similares foram desenvolvidos por Williams e Quarles [37], Tian et al. [41] e Czarnecki et al. [42]. Em estes últimos casos os resultados experimentais foram comparados com espectros simulados com o código Monte Carlo Penelope [43]. O bremsstrahlung produzido por elétrons de espectro contínuo (fontes radioativas $\beta^{-}$) em alvos grossos também foi analisado por alguns pesquisadores [44-46]. Para a análise dos dados em esses experimentos são introduzidas várias aproximações, logo, torna-se mais complexa uma comparação entre os resultados obtidos e as estimativas teóricas. 
Em resumo, existem poucos experimentos em que as SCDDs foram obtidas de maneira absoluta, mediante a determinação de cada grandeza da fórmula (2.77). A grande limitante para atingir esse objetivo foi a elevada incerteza associada à espessura dos alvos finos. Neste apartado os dados de Rester, Edmonson e Peasley [7] continuam tendo a maior precisão. Uma interpretação mais rigorosa do espectro de altura de pulso $N(h)$, a partir do qual é obtido $B(W)$, especialmente para baixas energias ( $W<30 \mathrm{keV}$ ), também poderia melhorar os resultados experimentais atuais e permitiria estabelecer conclusões precisas em relação a teoria. 



\section{Capítulo 3}

\section{Descrição dos experimentos}

Neste capítulo é apresentado de forma geral o acelerador Microtron e a linha de baixa energia. São descritos os detetores e a eletrônica utilizados na aquisição dos espectros de fótons, bem como as características do arranjo experimental desenhado para as medidas de SCDDs. Detalham-se os procedimentos de confecção dos alvos finos usados nos experimentos.

\subsection{Acelerador Microtron: linha de feixe de baixa energia}

As medidas de bremsstrahlung de alvo fino do presente trabalho foram realizadas no Acelerador Microtron do Departamento de Física Experimental do IFUSP, para elétrons com energias entre 20 e $100 \mathrm{keV}$, na câmara de irradiação 1 (CI-1), que corresponde à linha de baixa energia.

A Fig. 3.1 apresenta um esquema das estruturas que compõem o acelerador. Os elétrons gerados no canhão são conduzidos em vácuo através de um tubo de aço e podem ser desviados por um dipolo seletor diretamente até CI-1. Os elétrons também podem passar pelo injetor para as cavidades ressonante aceleradoras lineares, e ser desviados por meio de dipolos seletores para a câmara de irradiação 2 (CI-2) com energias de 0,9 ou 1,9 MeV. Para atingir os $5 \mathrm{MeV}$ o feixe pode ser igualmente desviado por um dipolo magnético para o sistema Racetrack Microtron. Neste dispositivo os elétrons percorrem órbitas fechadas, confinados por eletroímãs, passando varias vezes pela mesma estrutura aceleradora.

O desenho e as fotografias das Figs. 3.2-3.3 mostram detalhes da CI-1, uma estrutura cilíndrica que conta com paredes laterias de aço e tampas superior e inferior de Al, possui um diâmetro externo de $500 \mathrm{~mm}$ e um peso de 13,2 kg. As medidas podem ser realizadas 


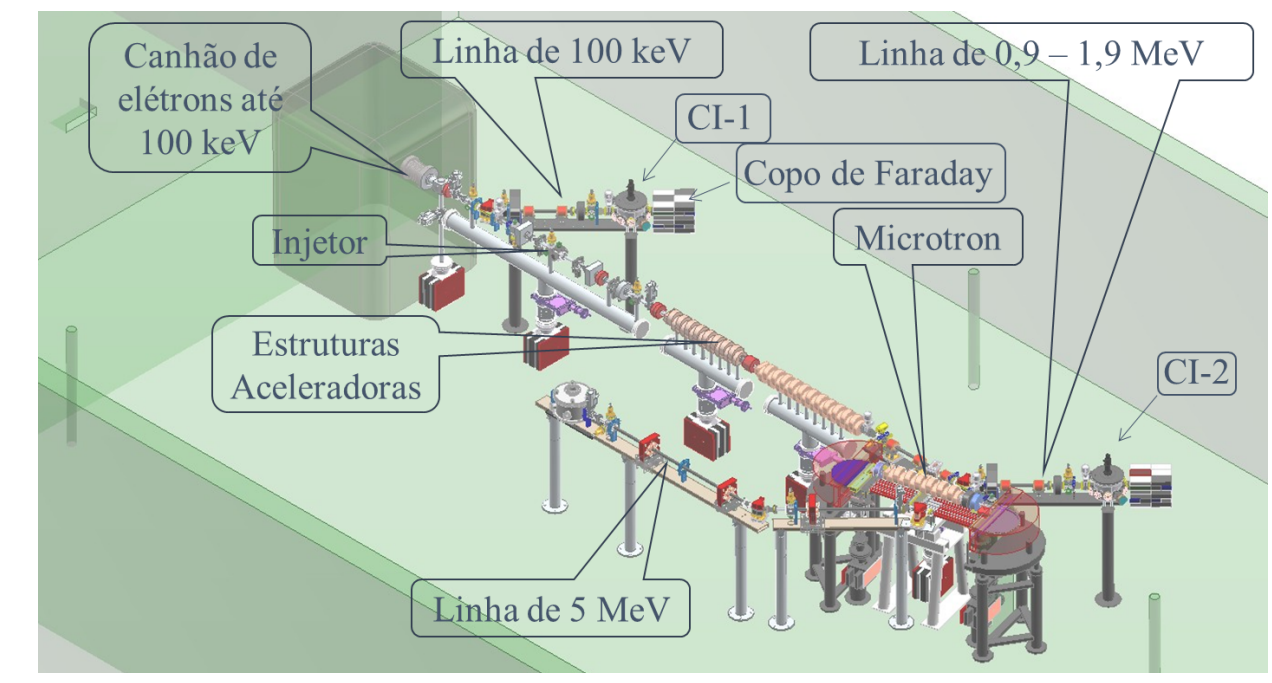

Figura 3.1: Esquema do Acelerador Microtron do IFUSP.

nos ângulos $31,1^{\circ}, 48,9^{\circ}, 69,9^{\circ}, 89,8^{\circ}, 125,0^{\circ}, 149,9^{\circ}$ em uma semicircunferência da câmara, e na outra, nas posições $34,8^{\circ}, 55,0^{\circ}, 90,2^{\circ}, 109,9^{\circ}, 131,1^{\circ}, 149,1^{\circ}$ e $166,0^{\circ}$. Alguns desses valores coincidem com os zeros e extremos dos polinômios de Legendre de ordem 2 e $4^{1}$. As janelas espectroscópicas da câmara são também de Al, com uma espessura de $50 \mu \mathrm{m}$.

$\mathrm{O}$ copo de Faraday, colocado a $0^{\circ}$ em relação à direção de incidência do feixe, e a própria câmara, que se encontra eletricamente isolada, permitem a coleção de toda a carga incidente durante os experimentos por meio de dois integradores de corrente. O copo foi desenhado com uma geometria cônica a fim de diminuir a probabilidade de saída dos elétrons uma vez que alcançam essa região. As paredes internas do copo estão cobertas com lâminas de C para minimizar a emissão de bremsstrahlung (ver Fig. 3.2).

No centro da CI-1 uma torre de fibra de C permite a colocação e medida de seis alvos sem a quebra do vácuo (Fig. 3.4). A parte superior dessa peça possui um monitor de óxido de Be que fluoresce quando os elétrons interagem, logo, com a ajuda de uma câmara de vídeo posicionada na janela mais próxima à direção de incidência, é possível observar em tempo real a forma geométrica do feixe. Os quadrupolos do sistema de aceleração conseguem focalizar os elétrons de maneira que o feixe se concentra numa área aproximadamente circular com diâmetro em torno de $2 \mathrm{~mm}$. Para a realização das medidas, a torre se inclinou um ângulo $\alpha=60^{\circ}$ (ver Fig. 2.1), a fim de evitar que os fótons gerados no alvo interagissem com a própria moldura de $\mathrm{C}$ dos alvos (ver seção 3.2.1) ou com a torre no caminho entre o ponto de emissão e os detetores.

\footnotetext{
${ }^{1}$ A câmara foi originalmente desenhada para o estudo da emissão de raios-x L, cuja distribuição angular depende de dos polinômios de Legendre de ordem 2 e 4.
} 


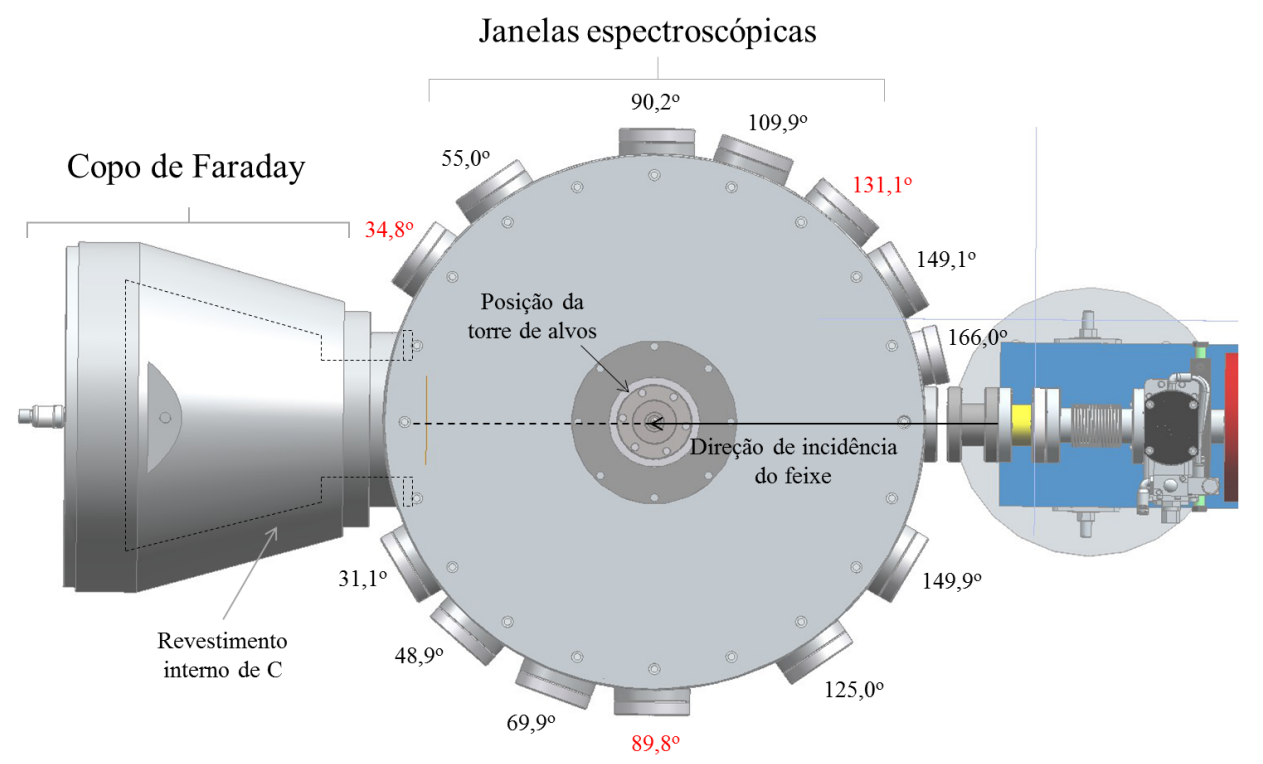

Figura 3.2: Esquema da câmara de irradiação correspondente à linha de $100 \mathrm{keV}$. São detalhados os ângulos onde é possível realizar medidas, sendo representadas em vermelho as posições utilizadas neste trabalho.

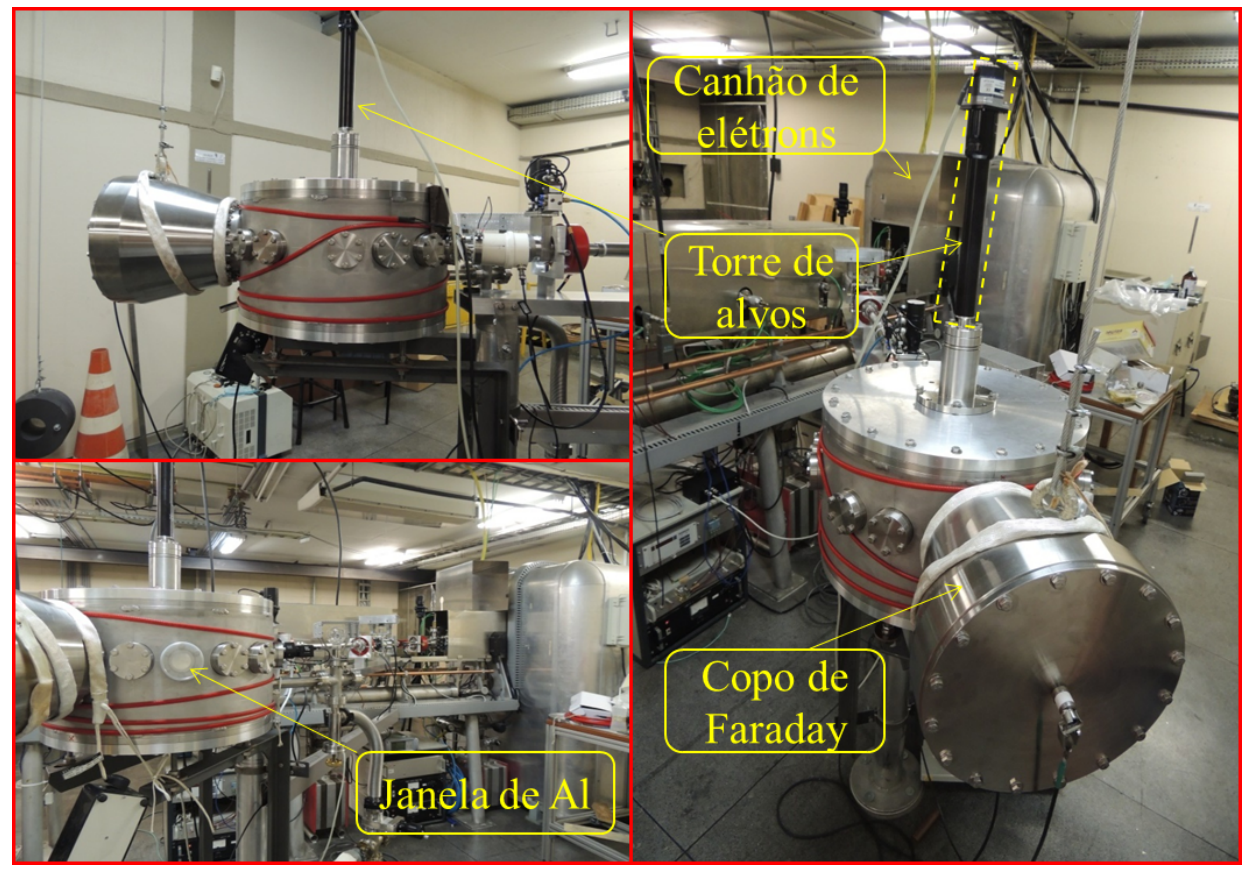

Figura 3.3: Fotografias da câmara de irradiação correspondente à linha de $100 \mathrm{keV}$. Observe-se a o canhão de elétrons, a torre de alvos, as janelas espectroscópicas da câmara e o copo de Faraday. 


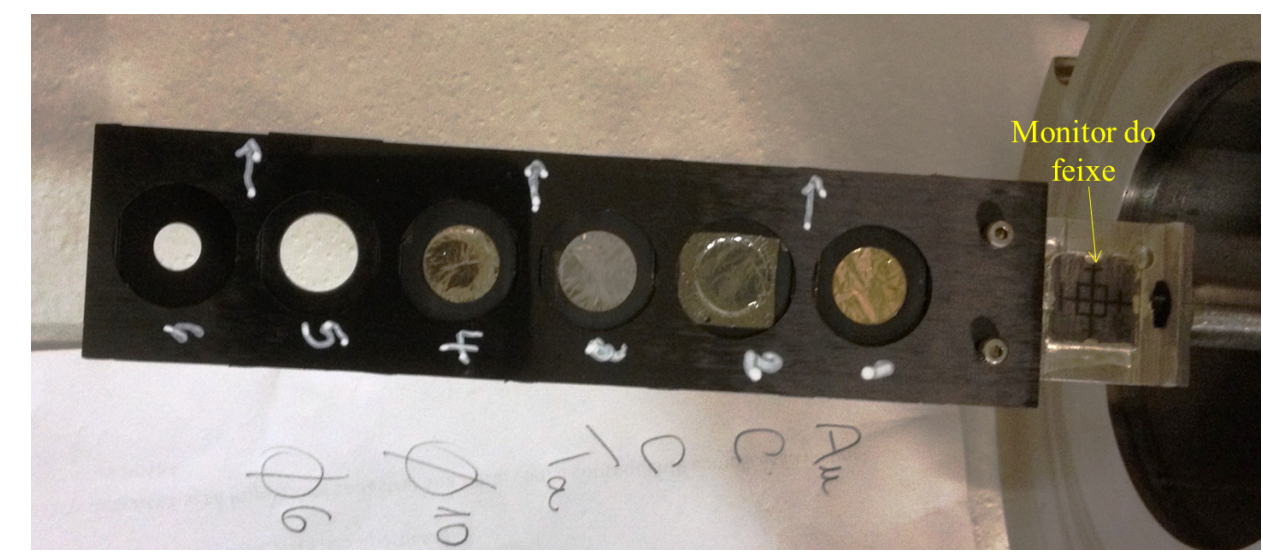

Figura 3.4: Torre de alvos da câmara de 100 keV.

\subsection{Alvos finos para medidas de bremsstrahlung de elétrons}

Dois mecanismos fundamentais produzem as perdas de energia dos elétrons ao interagirem com a matéria: colisões inelásticas com os elétrons atômicos do meio e emissão de bremsstrahlung. Os respectivos poderes de freamento $S_{\text {col }}\left(E_{0}\right)$ e $S_{\text {rad }}\left(E_{0}\right)$ caracterizam esses processos em função da energia $E_{0}$ do elétron. Na faixa dos keV é bem conhecido que $S_{\text {col }}\left(E_{0}\right) \gg S_{\text {rad }}\left(E_{0}\right)$, logo, as interações inelásticas vão determinar as perdas de energia do elétron [47]. Por outro lado, as partículas carregadas leves se espalham de maneira elástica (ou inelástica) com os núcleos e elétrons que compõem um material, e podem experimentar grandes deflexões em relação a sua trajetória original.

Com a fabricação de um alvo fino para a medição das SCDD de emissão de bremsstrahlung, espera-se que as variações da energia e da direção inicial de incidência do elétron sejam mínimas ao atravessar o filme. Desta forma é garantido que, se acontecer uma interação radiativa, esta ocorra sob as condições mais próximas às ideais.

\subsubsection{Confecção dos alvos}

Os alvos deste trabalho foram confeccionados pela técnica de deposição física de vapores $\left(\mathrm{PVD}^{2}\right)$ utilizando três métodos: (1) bombardeamento eletrônico com focalização eletromagnética, (2) efeito Joule e (3) pulverização catódica (conhecida comumente por sua denominação em inglês: magnetron sputtering). As evaporações foram realizadas no Laboratório de Alvos Nucleares (1 e 2) e no Laboratório de Materiais Magnéticos (3) do Instituto de Física da Universidade de São Paulo (IFUSP).

\footnotetext{
${ }^{2}$ PVD: siglas em inglês de "Physical Vapour Deposition".
} 
A maioria dos elementos da tabela periódica não são auto suportáveis em forma de filmes com espessuras necessárias para serem considerados finos. No entanto, o C que sim cumpre com essa propriedade, pode ser utilizado como substrato para que camadas finas de outros elementos sejam depositadas sobre ele. Esse procedimento foi adotado para a confecção dos alvos.

Os substratos foram construídos pelo método (1) em uma evaporadora que focaliza um feixe de elétrons, produzido em um filamento incandescente, sobre uma amostra de C [48]. O material evaporado depositou-se sobre suportes de vidro, que são previamente sometidos a um banho de ultra-som em álcool isopropílico como parte de um processo de polimento fino. Antes da evaporação, na superfície da lâmina onde foi depositado o C, colocou-se uma camada fina de detergente RBS [49] que permitiu a posterior descolagem do filme ao ser submergido em água destilada [48].

Na etapa seguinte os filmes de C são colocados sobre molduras também de fibras de C, com forma de paralelepípedo, e dimensões $30 \mathrm{~mm} \times 15 \mathrm{~mm} \times 0,3 \mathrm{~mm}$. Esses suportes possuem uma abertura circular de $10 \mathrm{~mm}$ de diâmetro que permite a passagem do feixe de elétrons nas medidas de bremsstrahlung (Fig. 3.5).

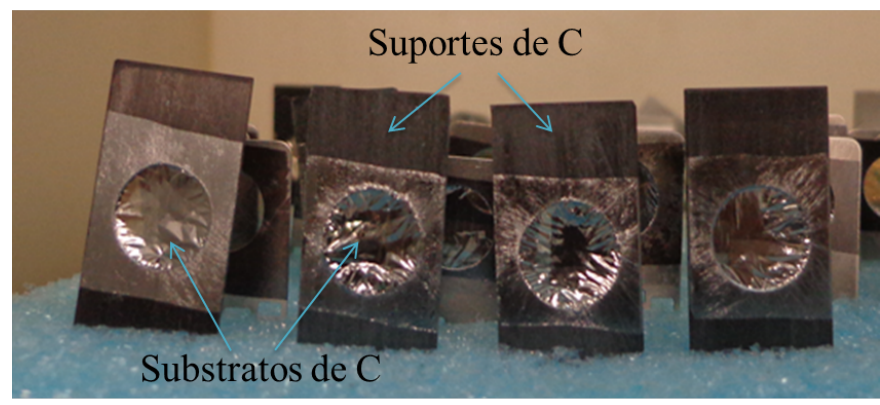

Figura 3.5: Substratos colocados sobre as molduras de C. O material a estudar é evaporado do lado contrário ao mostrado na imagem.

O método (2) foi utilizado para a confecção de alvos de Ag, Te e Ta. No processo, que se baseia no aquecimento por efeito Joule, a corrente elétrica passa através de um cadinho que contém o elemento a ser evaporado [48]. A Fig. 3.6 apresenta imagens do dispositivo de evaporação em vácuo no momento da confecção de um alvo típico de Ag.

Alvos de $\mathrm{Al}$ e Au foram preparados pelo método (3). Nesse caso íons de Ar formados por um campo elétrico são acelerados para um cátodo composto do material a ser evaporado. Os átomos desprendidos dessa superfície durante as colisões são depositados sobre o substrato. Um campo magnético perpendicular ao campo elétrico confina os elétrons secundários gerados numa região próxima ao cátodo para aumentar a ionização do gás, e por tanto, o rendimento do processo [50]. A Fig. 3.7 apresenta um alvo típico de $\mathrm{Au}$ obtido por sputtering. Tanto no método (2) quanto no (3), uma máscara circular de aço 


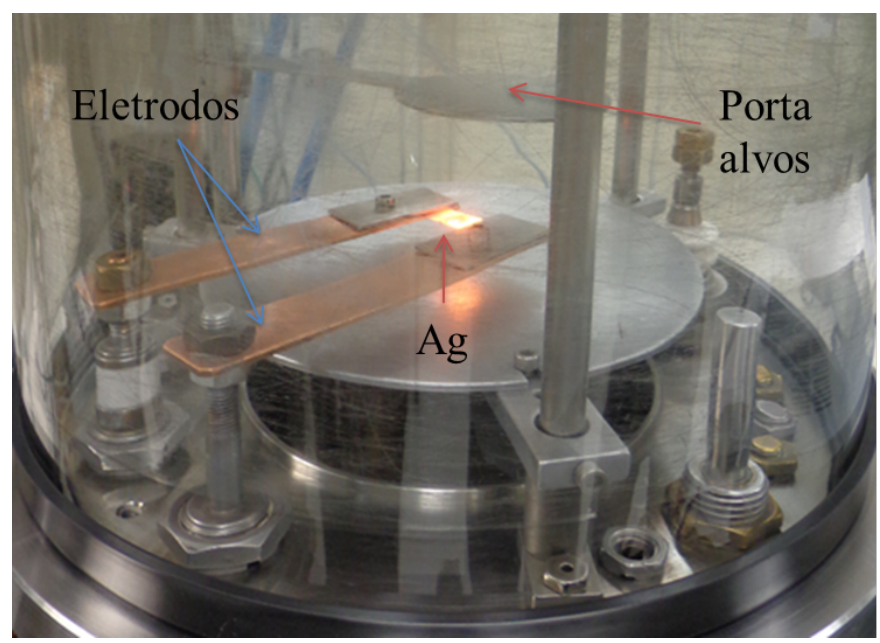

Figura 3.6: Imagem obtida durante o processo de evaporação de Ag por aquecimento Joule para a confecção de um alvo típico.

com $8 \mathrm{~mm}$ de diâmetro foi colocada sobre os suportes restringindo a área de evaporação à parte central do substrato.

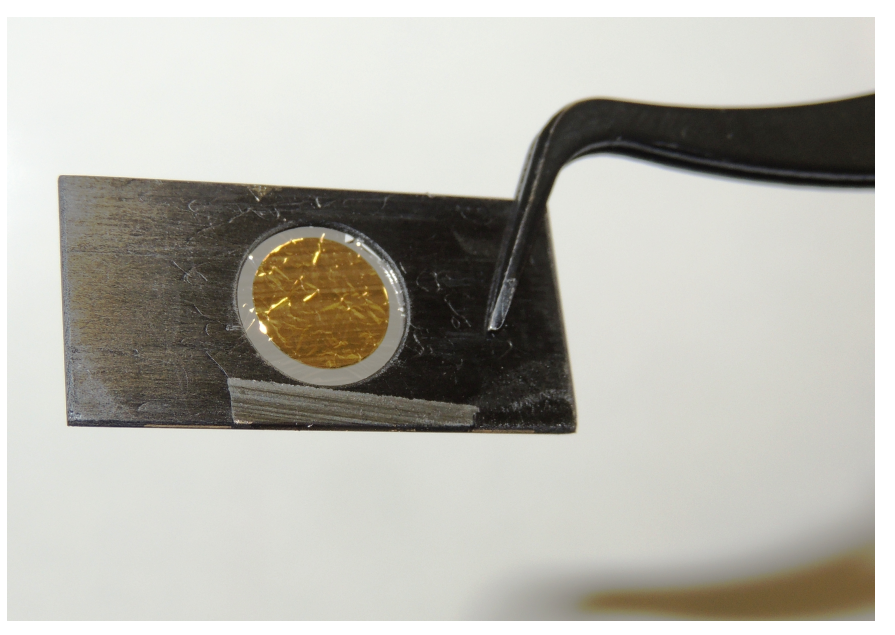

Figura 3.7: Fotografia de um alvo típico de Au produzido pelo processo de sputtering. Durante a evaporação foi utilizada uma máscara de aço que reduz a área deposita de Au a uma região de $8 \mathrm{~mm}$ de diâmetro.

\subsection{Detetores, sistema de aquisição e arranjo experimental para medidas de bremsstrahlung}

Nas medidas de bremsstrahlung se utilizaram três detetores HPGe da ORTEC posicionados a $35^{\circ}, 90^{\circ}$ e $131^{\circ}$. No primeiro ângulo foi colocado um detetor planar de $8 \mathrm{~cm}^{3} \mathrm{de}$

\footnotetext{
${ }^{3}$ Os valores exatos dos ângulo são $34,8^{\circ}, 89,8^{\circ}$ e $131,1^{\circ}$ mas pela quantidade de vezes em que serão mencionados a seguir são tomados números inteiros para sua referência.
} 
volume ativo, modelo GLP Series [51], com raio e espessura do cristal de Ge $r=16 \mathrm{~mm}$ e $l=10 \mathrm{~mm}$ respectivamente, e janela de $\mathrm{Be} l_{\mathrm{Be}}=0,254 \mathrm{~mm}$. Um espectrômetro digital DSPEC (também da ORTEC) acoplou-se ao detetor. O DSPEC integra e diferencia numericamente o pulso do pré-amplificador, digitalizado em alta frequência, com uma voltagem de trabalho $V_{\mathrm{T}}=-1000 \mathrm{~V}$.

Em $\theta=90^{\circ}$ foi posicionado um detetor planar de $5 \mathrm{~cm}^{3}$ modelo 1000 Series [51], $\left(r=12,5 \mathrm{~mm}, l=10 \mathrm{~mm}\right.$ e janela de Be $\left.l_{\mathrm{Be}}=0,25 \mathrm{~mm}\right)$. Neste caso se selecionou uma eletrônica analógica, também do fabricante ORTEC, composta por uma fonte de alto voltagem $A T$ (operada a $V_{\mathrm{T}}=-1000 \mathrm{~V}$ ), um amplificador 572 e um analisador multicanal ASPEC 92\%.

O último detetor, situado a $\theta=131^{\circ}$, é um coaxial modelo GMX30P4-56 70-CW-SMN acoplado a um espectrômetro digital DSPEC-Jr 2.0 [51] $\left(V_{\mathrm{T}}=-2800 \mathrm{~V}\right)$. O cristal coaxial possui um diâmetro de $58 \mathrm{~mm}$ e um comprimento de $66,3 \mathrm{~mm}$, com um orifício interno de diâmetro $10 \mathrm{~mm}$ e profundidade $58 \mathrm{~mm}$. A janela deste detetor é de $0,9 \mathrm{~mm}$ de C. O controle da aquisição dos espectros correspondentes aos três detetores foi realizado com o software MAESTRO [52].

Entre as janelas de Al correspondentes a cada posição da câmara e as superfícies frontais dos detetores foram colocados colimadores de $\mathrm{Cu}$. Os eixos de simetria dos sistemas detetor-colimador foram alinhados com o ponto central da câmara de irradiação, posição que ocupa o alvo durante as medidas, com ajuda de um dispositivo laser (ver Fig. 3.8). Posteriormente, uma blindagem de $\mathrm{Cu}$ e $\mathrm{Pb}$ foi colocada em torno à região dos detetores que contem os cristais de Ge, a fim de evitar a deteç̧ão de bremsstrahlung produzido no copo de Faraday ou nas paredes da câmara por elétrons espalhados, e diminuir a interferência nos espectros da radiação de fundo. Na Tabela 3.1 são resumidos os parâmetros geométricos que caracterizam o posicionamento dos detetores e colimadores, sendo: $d_{1}$ a separação entre a parede exterior da câmara e a superfície anterior do colimador, $d_{2}$ a distância entre a parede posterior do colimador e a parte frontal do detector, $d_{\mathrm{c}}$ o comprimento do colimador, $r_{\mathrm{c}}$ o raio da abertura do colimador, $\Omega_{\mathrm{g}} \mathrm{o}$ ângulo sólido geométrico subtendido pelo detetor em relação ao alvo, calculado segundo

$$
\Omega_{\mathrm{g}}=\frac{\pi r_{\mathrm{c}}^{2}}{\left(R+d_{1}+d_{\mathrm{c}}\right)^{2}},
$$

com $R=250 \mathrm{~mm}$ (raio da câmara), e $\omega_{\mathrm{g}}=\Omega_{\mathrm{g}} / 4 \pi$ a fração de ângulo sólido que representa a eficiência geométrica de detecção. A Fig. 3.9 mostra uma fotografia da montagem final do experimento. 


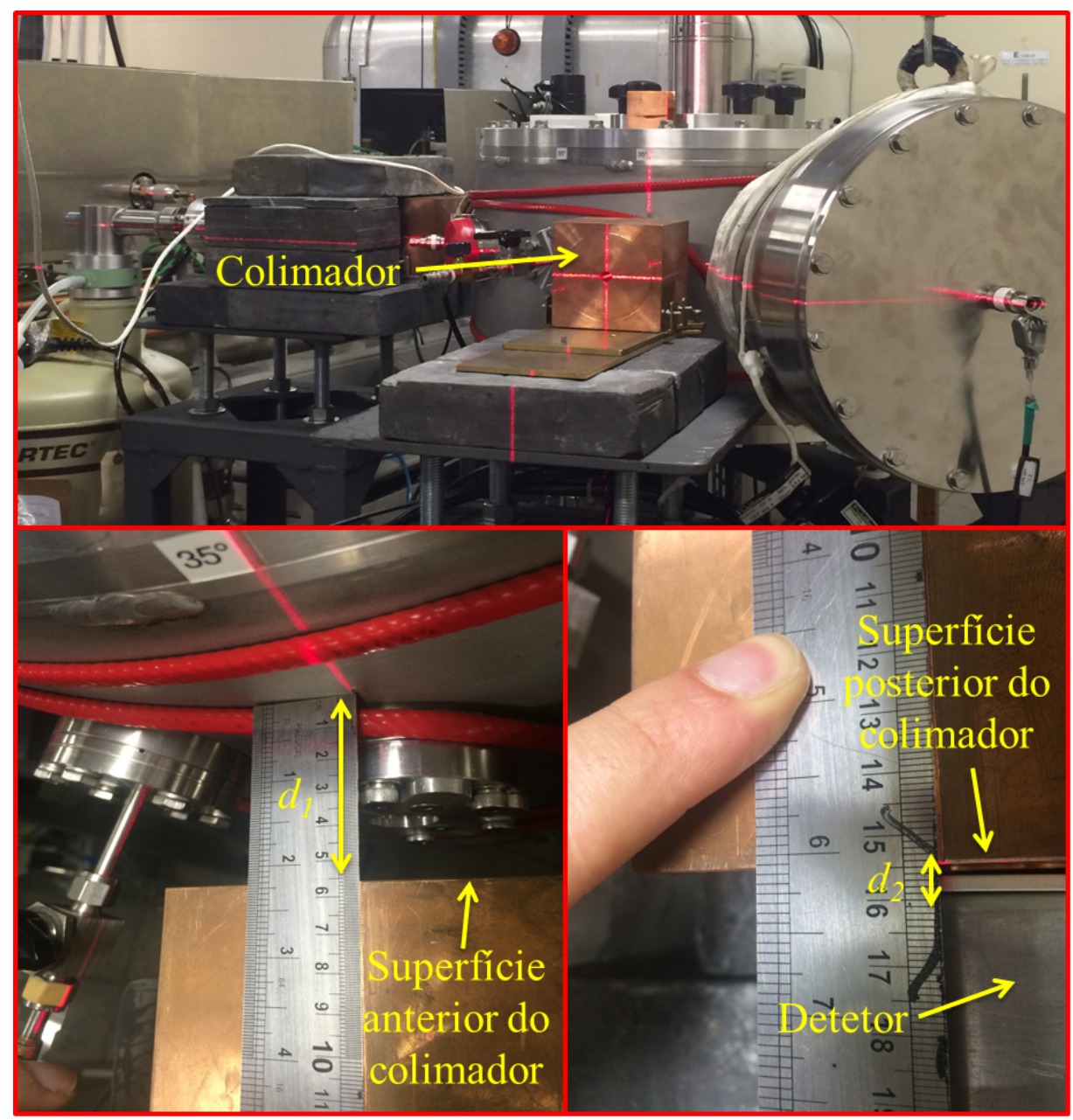

FiguRA 3.8: Fotografias tomadas durante o alinhamento do sistema detetor-colimador com o ponto central da câmara de irradiação.

TABELA 3.1: Distâncias características do posicionamento dos detetores e colimadores em referência à câmara de irradiação.

\begin{tabular}{c||cccccc}
\hline \hline Detetor & $d_{1}(\mathrm{~mm})$ & $d_{2}(\mathrm{~mm})$ & $d_{\mathrm{c}}(\mathrm{mm})$ & $r_{\mathrm{c}}(\mathrm{mm})$ & $\Omega_{\mathrm{g}} \times 10^{-4}(\mathrm{sr})$ & $\omega_{\mathrm{g}} \times 10^{-5}$ \\
\hline \hline & & & & & & \\
$\theta=35^{\circ}$ & $55,6(5)$ & $3,5(5)$ & $100,0(5)$ & $5,00(5)$ & $4,77(10)$ & $3,80(8)$ \\
$\theta=90^{\circ}$ & $77,5(5)$ & $10(1)$ & $70,0(5)$ & $5,00(5)$ & $4,97(10)$ & $3,96(8)$ \\
$\theta=131^{\circ}$ & $52,5(5)$ & $8,0(5)$ & $100,0(5)$ & $5,00(5)$ & $4,85(10)$ & $3,86(8)$ \\
\hline \hline
\end{tabular}




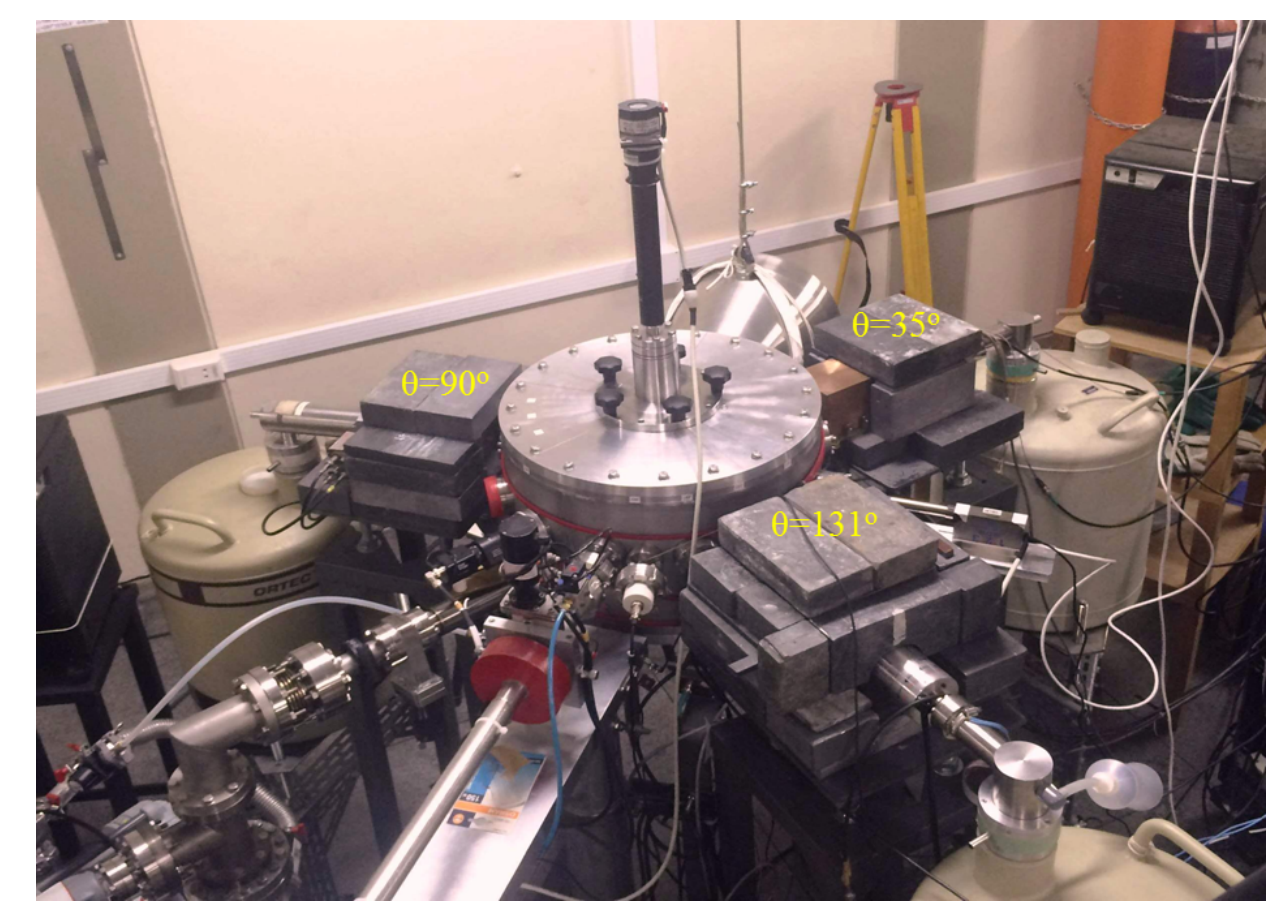

Figura 3.9: Montagem final dos detetores na câmara de irradiação.

\subsection{Desenho dos experimentos: justificativa da escolha de elementos, energias e ângulos de medida}

Os elementos selecionados neste trabalho para estudar a emissão de bremsstrahlung de elétrons são: $\mathrm{C}(Z=6), \mathrm{Al}(Z=13), \mathrm{Ag}(Z=47)$, Te $(Z=52)$, Ta $(Z=73)$ e $\mathrm{Au}(Z=$ 79). A escolha compreende dois tipos de átomos: $i$ ) aqueles tomados como referência para os cálculos da SCDD segundo a teoria de ondas parciais: Al, Ag e $\mathrm{Au}[3,5]$, e ii) aqueles cuja SCDD é interpolada em número atômico a partir dos resultados obtidos para outros elementos: C, Te e Ta.

Como descrito na secção 3.1, os detetores para a coleção dos espectros de bremsstrahlung foram colocados nos ângulos de emissão $\theta=35^{\circ}, 90^{\circ}$ e $131^{\circ}$, em relação à direção do feixe de elétrons. A escolha permite estudar a distribuição angular em três pontos cujas intensidades variam em função da energia incidente $E_{0}$. Particularmente, a posição $\theta=90^{\circ}$ resulta atrativa do ponto de vista comparativo, considerando que na maioria dos trabalhos experimentais onde é determinada a SCDD utilizou-se esse ângulo [6-9]. Por outro lado, espera-se que os cálculos teóricos de SCDDs sejam mais precisos também a $90^{\circ}$. Nesse caso o procedimento de interpolação dos coeficientes $B_{n}$, que multiplicam aos polinômios de Legendre $P_{n}(\cos \theta)$ na Eq. (2.75), e que são necessários para avaliar a distribuição angular de fótons, é reduzido somente aos termos pares, toda vez que $P_{n}(0)=0$ para $n=1,3$ e 5 . 
A seleção das energias $E_{0}$ dos elétrons também responde a critérios de comparação com outros dados experimentais e verificação dos cálculos teóricos. Assim os valores $E_{0}=50$ e $100 \mathrm{keV}$ foram analisados em todos os alvos devido a que formam parte da grade de interpolação (2.69), tanto da seção de choque reduzida $\chi\left(Z, E_{0}, \kappa\right)$ quanto da distribuição angular $S\left(Z, E_{0}, \kappa, \theta\right)$ [2-5]. Além disso, Motz e Placious [6], e Ambrose e colaboradores [9] realizaram medidas de SCDD para elétrons de 50 e $100 \mathrm{keV}$. As outras energias consideradas neste trabalho são $E_{0}=20 \mathrm{keV}$ e $75 \mathrm{keV}$, $(70 \mathrm{keV}$ no caso da $\mathrm{Ag}$ ), representam pontos intermediários de interesse na comprovação do referido procedimento de interpolação da SCDD teórica. Para os elementos Ag e Au, tomaram-se medidas com intervalos menores de $E_{0}$ entre 20 e $100 \mathrm{keV}$ a fim de determinar a SCDD no tip do espectro bremsstrahlung $(\kappa=1)$. 


\section{Capítulo 4}

\section{Modelagem da função resposta de detetores de $\mathrm{Ge}$}

Neste capítulo se descreve o formalismo matemático para obter o espectro de bremsstrahlung a partir da distribuição de altura de pulso medida. Desenvolve-se um modelo analítico para calcular a função resposta de detetores planares de Ge baseado em primeiros princípios. Tomando como referência um dos detetores utilizado nas medidas de bremsstrahlung, o modelo é validado mediante a comparação com simulações Monte Carlo. Posteriormente a metodologia proposta é estendida à configuração coaxial do cristal de Ge. No final do capítulo é testado o procedimento de deconvolução de espectros com o modelo analítico adotado e simulações Monte Carlo.

\subsection{Metodologia para a deconvolução dos espectros de brems- strahlung}

As contagens registradas em um canal correspondente à energia $h$ de um espectro de altura de pulso medido, são produto da convolução da função resposta (FR) do detetor $R(h, E)$, e o espectro real de energia $F(E)$ da radiação que atinge seu volume ativo. Em termos das duas grandezas pode se expressar a função densidade de probabilidade $N(h)$ que descreve o espectro coletado como:

$$
N(h)=\int_{0}^{\infty} R(h, E) F(E) \mathrm{d} E .
$$

Define-se $R(h, E)$ como a densidade de probabilidade de que um fóton de energia $E$ seja detetado à energia $h$. Em um espectro multicanal é observado a discretização de $N(h)$ 
nos diferentes canais

$$
N_{i}=\int_{h_{i}-\delta / 2}^{h_{i}+\delta / 2} \mathrm{~d} h \int_{0}^{\infty} R(h, E) F(E) \mathrm{d} E+\epsilon_{i},
$$

onde $N_{i}, h_{i}$ e $\delta$ são as contagens, energia e largura do $i$-ésimo canal respectivamente, enquanto $\epsilon_{i}$ é o erro associado a $N_{i}$. Mudando a ordem de integração e definindo a função

$$
r\left(h_{i}, E\right)=\int_{h_{i}-\delta / 2}^{h_{i}+\delta / 2} R(h, E) \mathrm{d} h
$$

é possível discretizar a integral $N_{i}$ ao longo dos $M$ canais do histograma

$$
N_{i}=\int_{0}^{\infty} r\left(h_{i}, E\right) F(E) \mathrm{d} E+\epsilon_{i} \cong \delta \sum_{j=1}^{M} r\left(h_{i}, E_{j}\right) F\left(E_{j}\right)+\epsilon_{i} .
$$

Logo, esse resultado pode ser generalizado para todo o espectro escrevendo a relação anterior em forma matricial

$$
\mathbf{N}=\mathbf{R} \mathbf{F}+\boldsymbol{\epsilon}
$$

sendo $\mathbf{N}, \boldsymbol{\epsilon}$ e $\mathbf{F}$ vetores coluna com componentes dadas por $N_{i}, \epsilon_{i}$ e o número de fótons incidentes no detetor com energias entre $E_{j}-\delta / 2$ e $E_{j}+\delta / 2$, respectivamente. Os elementos da matriz quadrada $\mathbf{R}(M \times M)$, também chamada de matriz resposta do detetor, são dados por:

$$
R_{i, j}=r\left(h_{i}, E_{j}\right)
$$

Em geral os vetores $\mathbf{F}$ e $\boldsymbol{\epsilon}$ são desconhecidos e suas componentes são estimadas a partir do Método dos Mínimos Quadrados (MMQ) [53]. Nesse caso assume-se que R e a matriz de covariância dos dados experimentais, $\mathbf{V}$, dada por

$$
V_{i, j}=\left\langle\epsilon_{i} \epsilon_{j}\right\rangle
$$

são conhecidas (a notação $\langle y\rangle$ representa o valor médio de $y$ ). Além disso, considera-se que os dados experimentais são não tendenciosos [53]. No caso de um espectro multicanal significa que o sinal $\mathbf{N}$ foi previamente corrigido por efeitos como o fundo radioativo e empilhamento de pulsos. Essa hipótese implica que $\left\langle\epsilon_{i}\right\rangle=0$, logo, $\langle\mathbf{N}\rangle=\mathbf{R} \mathbf{F}$.

A relação linear estabelecida pela Eq. (4.5) entre os elementos de $\mathbf{N}$ e $\mathbf{F}$ permite determinar o estimador de variância mínima de $\mathbf{F}$ por meio do MMQ [53, 54],

$$
\widetilde{\mathbf{F}}=\left(\mathbf{R}^{\mathrm{t}} \mathbf{V}^{-1} \mathbf{R}\right)^{-1} \mathbf{R}^{\mathrm{t}} \mathbf{V}^{-1} \mathbf{N}
$$

com matriz de covariância

$$
\mathbf{V}_{\widetilde{\mathbf{F}}}=\left(\mathbf{R}^{\mathrm{t}} \mathbf{V}^{-1} \mathbf{R}\right)^{-1}
$$


Como foi definido com anterioridade, $\mathbf{R}$ é uma matriz quadrada, o que reduz as Eqs. (4.8) e (4.9) a

$$
\widetilde{\mathbf{F}}=\mathbf{R}^{-1} \mathbf{N}
$$

e

$$
\mathbf{V}_{\widetilde{\mathbf{F}}}=\mathbf{R}^{-1} \mathbf{V}\left(\mathbf{R}^{\mathrm{t}}\right)^{-1}
$$

respectivamente. Observa-se que quando o espectro $\mathbf{N}$ é não tendencioso, o estimador $\widetilde{\mathbf{F}}$ é também não tendencioso

$$
\langle\widetilde{\mathbf{F}}\rangle=\mathbf{R}^{-1}\langle\mathbf{N}\rangle=\mathbf{F}
$$

ou seja, espera-se que em média $\widetilde{\mathbf{F}}$ seja igual ao valor verdadeiro $\mathbf{F}$.

Apontando a outras especifidades deste problema, e seguindo a definição de FR, pode se dizer que a matriz $\mathbf{R}$ é praticamente triangular. Sustenta essa afirmação o fato de que um fóton de energia $E$ só produz pulsos no intervalo compreendido entre $h=0$ e $h \approx E+3 \sigma(E)$, sendo $\sigma(E)$ a largura característica do detetor na coleção de carga (ver seção 4.3). Assim, dependendo da resolução energética com que é construída $\mathbf{R}$, os elementos à esquerda da diagonal $\left(R_{i, j}, i<j\right)$ irão ser iguais a zero, ou apenas alguns destes vão ser diferentes de zero. Por outro lado a matriz $\mathbf{V}$ é diagonal devido a que as contagens que conformam o vetor $\mathbf{N}$ não estão correlacionadas. Como a estatística de Poisson determina as flutuações de $N_{i}$, o limiar definido para as variâncias é $V_{i, i} \geqslant N_{i}$. Quanto menores forem as correções em $\mathbf{N}$ pelos efeitos acima mencionados, o desvio padrão associado a $N_{i}$ estará mais próximo de $\sqrt{N_{i}}$ e consequentemente $V_{i, i} \simeq N_{i}$.

A metodologia descrita até aqui é geral e válida para qualquer forma de espectro de fótons incidentes $\mathbf{F}$. Nas próximas seções será desenvolvido um modelo analítico para a FR dos detetores utilizados nas medidas deste trabalho. Para testa-lo serão comparadas no próximo capítulo as distribuições de altura de pulso produzidas por uma fonte radioativa com o espectro predito pela Eq. (4.5). Nesse caso, onde F é conhecido, manteremos a mesma notação. Enquanto, no procedimento de deconvolução de espectros de bremsstrahlung na Eq. (4.10) será utilizada a letra B em lugar de F, assim como $W$ para representar a energia desses fótons, em acordo com nomenclatura utilizada no Capítulo 2,

$$
\mathbf{B}=\mathbf{R}^{-1} \mathbf{N}
$$

A maioria dos resultados apresentados nas seções 4.2-4.4 foram publicados na referência [55]. 


\subsection{Função reposta de detetores semicondutores: estado da arte}

A FR de detetores de raios-x e $\gamma$ é uma grandeza que tem sido estudada durante os últimos 50 anos [56-75]. Essa propriedade reflete os complexos mecanismos de interação da radiação e depende da energia dos fótons incidentes, do material semicondutor de detecção e da geometria do arranjo experimental. Existem três métodos aplicados frequentemente para sua caracterização: parametrizações empíricas, simulações Monte Carlo e modelos analíticos ou semi-analíticos.

A primeira metodologia baseia-se na coleção de espectros de fontes radiativas com linhas de emissão na faixa de energia de interesse [57-62]. As estruturas observadas na distribuição de altura de pulso se associam com funções analíticas cujos parâmetros são ajustados aos dados experimentais obtidos. Para levar em conta a dependência energética, esses parâmetros são tomados como nodos em um posterior ajuste de um polinômio na energia do fóton incidente ( $p$. ex. a variância de uma distribuição Gaussiana). O tratamento tem sido aplicado a detetores de $\mathrm{Si}(\mathrm{Li})$ para fótons menores de $20 \mathrm{keV}$ [57-59] e também a espectrômetros de Ge desde dezenas de keV até $6 \mathrm{MeV}$ [60-62].

As técnicas de simulação Monte Carlo (MC) também são usadas para estudar a FR dos detetores [63-67]. Nesse contexto, por exemplo, Campbell e colaboradores [65] desenvolveram um código para simular interações de fótons na região 1-10 keV. Detetores de Si ou Ge podem ser modelados incluindo os principais materiais dos contatos elétricos com que são fabricados: $\mathrm{Al}, \mathrm{Cr}, \mathrm{Ni}, \mathrm{Pd}$ ou Au. O código só considera o fotoefeito como mecanismo de interação e transporta os produtos de relaxação atômica: raios-x característicos, elétrons Auger e Coster-Kronig, e fotoelétrons. Em um trabalho posterior, O'Meara e Campbell incluíram na simulação a contribuição ao foto pico dos eventos de espalhamento Rayleigh e Compton seguidos por absorção fotoelétrica [66].

Com a mesma finalidade, Fernández e Scot [67] publicaram MCSHAPE, um código MC para simular a difusão de fótons (polarizados ou não) que incorpora como possíveis interações o efeito fotoelétrico, e os espalhamentos Compton e Rayleigh. Ao contrário do trabalho de Campbell et al. [65], Fernández e Scot, assumem que os elétrons que surgem das interações anteriores são localmente absorbidos, e transportam os fótons primários e secundários. Os autores estudaram o espectro de escape em espectrômetros de Ge e CdTe para energias incidentes entre 30 e $200 \mathrm{keV}$ [67]. 
Os códigos MC de propósito geral como MCNP [76], Geant4 [77] e Penelope [43] também tem sido satisfatoriamente aplicados ao estudo da FR de vários tipos de detetores utilizados em diferentes aplicações [68-71]. A principal vantagem desses softwares é que permitem simular o transporte da radiação em materiais arbitrários e geometrias complexas. Porém, é preciso verificar a validade dos modelos físicos incorporados neles para a faixa de energia que se pretende estudar.

Por outro lado, Seltzer [56] publicou um modelo analítico para calcular a FR e a eficiência de fotopico de detetores planares de Ge. A sua aproximação, válida para energias de até $\sim 300 \mathrm{keV}$, considera o escape dos raios-x K do Ge, o contínuo de Compton produzido por uma ou mais interações inelásticas e o pico de absorção total. A fórmula de Klein-Nishina (KN) [78] é utilizada para descrever a distribuição relativa de energias finais na região do espectro que corresponde a um único espalhamento, sendo renormalizada pela seção de choque incoerente total de Waller-Hartree (WH) [79, 80], com o objetivo de levar em conta os efeitos de ligação dos elétrons no átomo de Ge. Seltzer simulou espectros correspondentes a diferentes energias de fótons incidentes e estimou a contribuição de espalhamentos inelásticos múltiplos dentro do volume ativo do detetor. Seguidamente, incorporou esses efeitos ao seu modelo recorrendo a uma distribuição triangular empírica, normalizada pela área observada no MC nessa faixa.

Outros modelos analíticos tem sido propostos por Goto [72], Lowe [73] e Sholze e Procop [75] para explicar o contínuo produzido pelo escape de elétrons gerados perto da superfície do volume ativo, nos contatos elétricos e nas camadas mortas de detetores, bem como a coleção incompleta de carga. Esses autores estudaram espectrômetros de Si e Ge para energias menores que $10 \mathrm{keV}$.

A continuação é apresentado um modelo para calcular a FR de detetores planares de Ge para fótons incidentes no intervalo $10 \mathrm{keV} \leqslant E \leqslant 100 \mathrm{keV}$. Basicamente o esquema de Seltzer [56] é expandido, incorporando novas contribuições à FR e melhorando os modelos físicos que descrevem os processos de interação de fótons.

Um enfoque deste tipo poderia ser questionado uma vez que as técnicas MC tem sido exitosamente utilizadas para a avaliação da FR. Porém, na deconvolução de espectros contínuos de radiação que são coletados por vários detetores, em diferentes configurações geométricas, a solução analítica converte-se numa ferramenta competitiva. Nesse caso a geração da matriz resposta para cada um dos espectrômetros via MC implicaria centenares de simulações, acrescentando o fato de que uma mudança no arranjo experimental conduz à repetição de todo o processo. 


\subsection{Modelo analítico de função resposta para detetores planares}

Considera-se um detetor cilíndrico de Ge com raio $r$ e espessura $l$, e fótons de energia $E$ que atingem seu volume ativo ao longo do eixo de simetria. Essa condição é garantida experimentalmente utilizando colimadores para o feixe (Fig. 4.1).

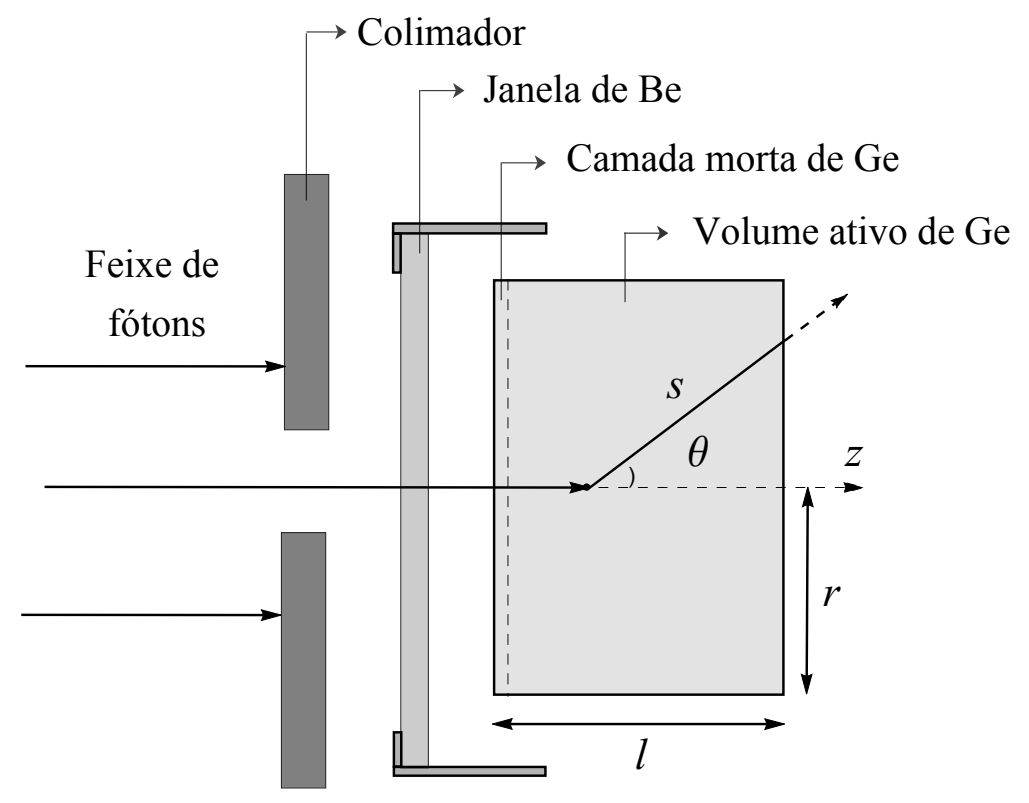

FigurA 4.1: Representação geométrica de um detetor planar de Ge típico. Assume-se simetria cilíndrica em torno ao eixo z. Esquema fora de escala.

Primeiro, são analisadas as contribuições à FR produzidas pela interação dos fótons dentro do volume ativo: absorção total de energia, escape de raios-x K do Ge, escape de fótons após um espalhamento Compton e escape de fotoelétrons. Esses fenômenos geram o que se define de FR intrínseca do cristal $R(h, E ; l, r)$.

Por outro lado, quando o feixe de fótons interage com alguns dos elementos do detetor antes de chegar ao volume ativo, o espectro de energia e partículas que atingem o cristal muda. Em particular são estudadas as modificações introduzidas pela janela de Be e a camada morta de Ge.

\subsubsection{Função reposta intrínseca}

A FR intrínseca de detetores semicondutores de radiação pode ser calculada como a convolução de um espectro de energia depositada $D(\epsilon, E ; l, r)$ e uma função de distribuição 
Gaussiana $\mathcal{G}(h, \epsilon ; \sigma)$

$$
R(h, E ; l, r)=T(E) \int_{0}^{\infty} \mathcal{G}(h, \epsilon ; \sigma) D(\epsilon, E ; l, r) \mathrm{d} \epsilon,
$$

com

$$
T(E)=\exp [-\boldsymbol{\mu}(E) \cdot \mathbf{d}]
$$

e

$$
\mathcal{G}(h, \epsilon ; \sigma)=\frac{1}{\sqrt{2 \pi} \sigma(\epsilon)} \exp \left[-\frac{1}{2} \frac{(h-\epsilon)^{2}}{\sigma^{2}(\epsilon)}\right] .
$$

As funções da expressão anterior tem o mesmo significado descrito no modelo de Seltzer [56]:

1. $R(h, E ; l, r)$ é a densidade de probabilidade de que um fóton de energia $E$ gere um pulso de altura $h$ no analisador multicanal.

2. $T(E)$ leva em conta a fração de fótons transmitidos através dos materiais na frente do volume ativo. Os vetores $\boldsymbol{\mu}(E)=\left(\mu_{1}, \mu_{2}, \ldots, \mu_{n}\right)$ e $\mathbf{d}=\left(d_{1}, d_{2}, \ldots, d_{n}\right)$ contêm os coeficientes de atenuação linear e espessuras dos diferentes elementos.

3. $D(\epsilon, E ; l, r)$ é a densidade de probabilidade de que um fóton de energia $E$ deposite a energia $\epsilon$ no cristal de Ge.

4. $\mathcal{G}(h, \epsilon ; \sigma)$ é a densidade de probabilidade de que a energia depositada $\epsilon$ dê origem a um pulso de altura $h$. A variância de $\mathcal{G}(h, \epsilon ; \sigma)$ está determinada pelo ruido eletrônico, pela energia necessária para criar um par elétron-buraco e pelo fator de Fano [81].

Todas as grandezas da integral (4.14) estão normalizadas por fóton incidente, enquanto $R(h, E ; l, r)$ e $D(\epsilon, E ; l, r)$ se referem a um detetor com parâmetros $l$ e $r$. O espectro de energia depositada pode ser calculado como

$$
D(\epsilon, E ; l, r)=\sum_{j} P_{j}(\epsilon, E ; l, r)
$$

onde $P_{j}(\epsilon, E ; l, r)$ denota a probabilidade de depositar total o parcialmente a energia $E$ através das componentes da FR intrínseca.

\subsubsection{Escape dos raios- $\mathrm{K}$ do Ge}

A emissão de raios-x é um mecanismo importante de relaxação em átomos de Ge com vacantes na camada $\mathrm{K}$, como indica seu valor de rendimento fluorescente $\omega_{\mathrm{K}}=$ 
0,553(13) [82]. Quando fótons característicos $\mathrm{K} \alpha$ ou $\mathrm{K} \beta$ escapam do detetor, a energia remanescente é $\epsilon=E-E_{\mathrm{K} i}$, com $i=\alpha$ ou $\beta$, e $E_{\mathrm{K} i}$ a energia média das transições $\mathrm{K} \alpha_{1,2}$ e $\mathrm{K} \beta_{1,3}{ }^{1}$ [83]. Como resultado aparecem no espectro os denominados picos de escape. A Fig. 4.2 apresenta um esquema desse processo.

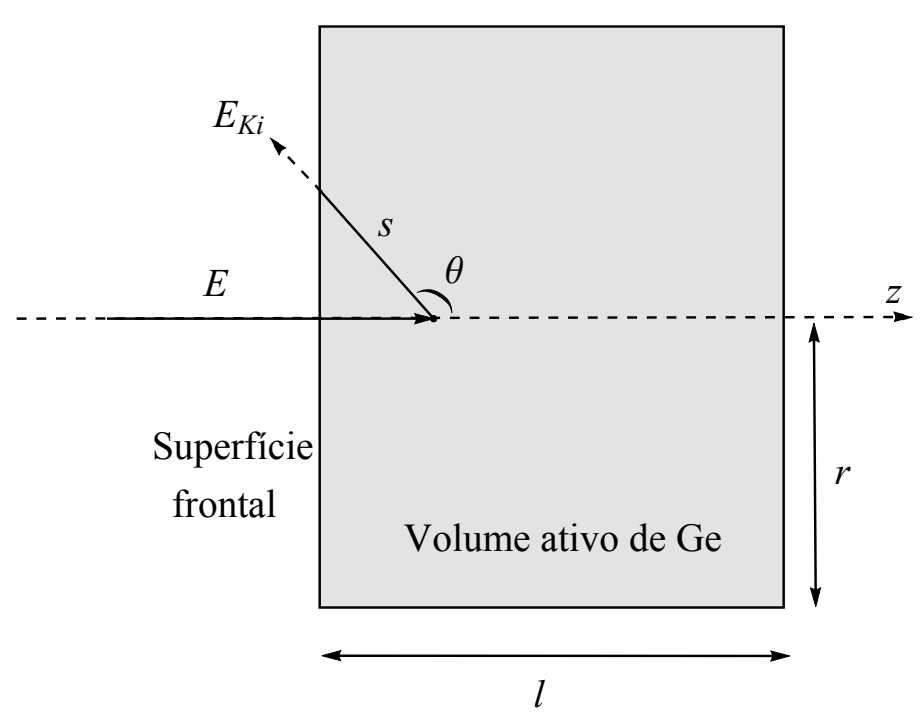

FigurA 4.2: Representação geométrica do processo de escape de raios-x K característicos pela superfície frontal do detetor.

O caminho livre médio dos raios-x $\mathrm{K}$ do Ge é $~ 0,05 \mathrm{~mm}$ [56], logo, o escape é somente possível quando o fóton é produzido perto de uma das superfícies do cristal. A probabilidade de ser absorbido perto da superfície frontal é maior quando o fóton tem menor energia. Sob essas condições, o escape pelas superfícies traseira e lateral é praticamente desprezível, portanto o detetor pode ser considerado como um plano semi-infinito $(l=r=\infty)$ para os fins desse cálculo [56].

A probabilidade diferencial de que um fóton de energia $E$ produza um raio-x K $i$ e este escape pela superfície frontal do detetor pode ser expressa como

$$
\begin{aligned}
\mathrm{d}^{3} P_{\mathrm{K} i}(E, \theta, \phi)= & \mu_{\mathrm{Ge}}^{\mathrm{pe}}(E) \exp \left[-\mu_{\mathrm{Ge}}(E) z\right] \mathrm{d} z \\
& \times \omega_{\mathrm{K}} p_{\mathrm{K} i} q_{\mathrm{K}}(E) \frac{1}{4 \pi} \mathrm{d}(\cos \theta) \mathrm{d} \phi \\
& \times \exp \left[-\mu_{\mathrm{Ge}}\left(E_{\mathrm{K} i}\right) s\right]
\end{aligned}
$$

\footnotetext{
${ }^{1}$ As energias das transições $\mathrm{KL}_{2}\left(\mathrm{~K} \alpha_{2}\right), \mathrm{KL}_{3}\left(\mathrm{~K} \alpha_{1}\right), \mathrm{KM}_{2}\left(\mathrm{~K} \beta_{3}\right), \mathrm{KM}_{3}\left(\mathrm{~K} \beta_{1}\right)$ no Ge são 9855,42(10), $9886,52(11), 10978,1(13)$ e 10982,19(29) eV respectivamente [83].
} 
onde o primeiro fator do membro direito representa a probabilidade de que exista fotoabsorção a uma profundidade entre $z$ e $z+\mathrm{d} z$ ao longo do eixo de simetria; o segundo fator é a probabilidade de que o fóton $\mathrm{K} i$ seja emitido no intervalo $(\theta, \theta+\mathrm{d} \theta)$, em relação à direção de incidência dos fótons, e $(\phi, \phi+\mathrm{d} \phi)$ na direção azimutal ${ }^{2}$; enquanto o último exponencial representa a probabilidade de escape do fóton $\mathrm{K} i$ pelo plano de entrada após viajar um caminho $s=z /|\cos \theta|$. Integrando $z$ entre 0 e $\infty, \cos \theta$ entre $0 \mathrm{e}-1$, e $\phi$ entre 0 e $2 \pi$ obtemos a probabilidade total escape

$$
\begin{aligned}
P_{\mathrm{K} i}(E)= & \frac{1}{2} \omega_{\mathrm{K}} p_{\mathrm{K} i} q_{\mathrm{K}}(E) \frac{\mu_{\mathrm{Ge}}^{\mathrm{pe}}(E)}{\mu_{\mathrm{Ge}}(E)} \\
& \times\left\{1-\frac{\mu_{\mathrm{Ge}}\left(E_{\mathrm{K} i}\right)}{\mu_{\mathrm{Ge}}(E)} \ln \left[1+\frac{\mu_{\mathrm{Ge}}(E)}{\mu_{\mathrm{Ge}}\left(E_{\mathrm{K} i}\right)}\right]\right\}
\end{aligned}
$$

sendo $\mu_{\mathrm{Ge}}^{\mathrm{pe}}(E)$ e $\mu_{\mathrm{Ge}}(E)$ os coeficientes lineares de absorção fotoelétrica e absorção total respectivamente, $q_{\mathrm{K}}(E)$ a probabilidade de ionizar a camada $\mathrm{K}$ com a energia $E$, e $p_{\mathrm{K} i}$ a probabilidade de emitir um fóton característico K $i$. Note-se que $P_{\mathrm{K} i}$ não depende dos parâmetros geométricos $l$ e $r$. Esse resultado é uma consequência direta da ordem de grandeza do caminho livre meio dos fótons $\mathrm{K} \alpha$ e $\mathrm{K} \beta$ no Ge comparado com os valores típicos de $l$ e $r$ (na faixa dos centímetros).

Na Fig. 4.3 são apresentadas as probabilidades de escape K $\alpha$ e K $\beta$ segundo a Eq. (4.19). Os valores de $p_{\mathrm{K} \alpha}, p_{\mathrm{K} \beta}$ e $q_{\mathrm{K}}$ foram obtidos do trabalho de Seltzer [56], $\omega_{\mathrm{K}}$ de Sampaio et al. [82], enquanto os coeficientes de atenuação do programa XCOM [84]. Observa-se que para energias $E \leqslant 25 \mathrm{keV} P_{\mathrm{K} \alpha}$ excede o $5 \%$ e alcança um $12 \%$ em $15 \mathrm{keV}$.

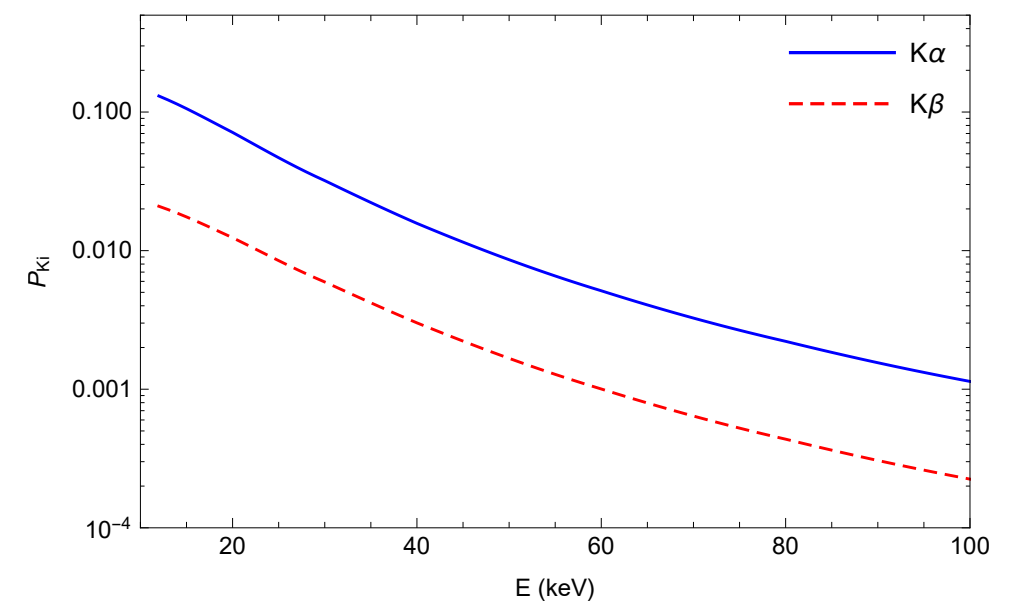

Figura 4.3: Probabilidade de escape do detetor dos raios-x $\mathrm{K} \alpha$ e $\mathrm{K} \beta$ do Ge.

Por outra parte, a probabilidade $P_{\mathrm{K} i}(\epsilon, E)$, de que a energia $\epsilon=E-E_{\mathrm{K} i}$ seja deposita no detetor após o escape, pode ser calculada a partir de $P_{\mathrm{K} i}(E)$ introduzindo a função

\footnotetext{
${ }^{2}$ Utiliza-se a condição de que a distribuição angular dos raios-x K é isotrópica.
} 
delta de Dirac

$$
P_{\mathrm{K} i}(\epsilon, E)=P_{\mathrm{K} i}(E) \delta\left(\epsilon-\left(E-E_{\mathrm{K} i}\right)\right)
$$

\subsubsection{Escape de fótons após um espalhamento Compton}

Os fótons que escapam do volume ativo do detetor após experimentar colisões inelásticas dão origem ao continuo de Compton observado na distribuição de altura de pulso. $\mathrm{O}$ espectro de energia produzido por esses eventos pode ser expresso como

$$
P_{\text {inc }}(\epsilon, E ; l, r)=\sum_{n} P_{\text {inc }, n}(\epsilon, E ; l, r),
$$

onde cada componente $n$ do somatório considera o número de interações inelásticas dos fótons antes de escapar. Neste trabalho é analisado apenas um espalhamento Compton para o cálculo do espectro

$$
P_{\mathrm{inc}}(\epsilon, E ; l, r) \approx P_{\mathrm{inc}, 1}(\epsilon, E ; l, r)
$$

Para energias incidentes $10 \mathrm{keV} \lesssim E \lesssim 100 \mathrm{keV}$ a seção de choque diferencial de KN [78] não descreve de maneira precisa o processo de colisão inelástica fóton-átomo. Neste intervalo de energia, o alargamento Doppler causado pelo movimento dos elétrons no átomo, bem como a energia de ligação do elétron ativo da $i$-ésima camada, não podem ser ignorados. A Aproximação de Impulso Relativística (AIR) [79] considera os dois efeitos, e consequentemente, esse formalismo é empregado para a descrição do espalhamento Compton dos fótons no cristal de Ge.

A probabilidade de que um fóton com energia $E$ escape do detetor com uma energia $E^{\prime}$, em uma direção definida pelo diferencial de ângulo sólido $\mathrm{d} \Omega^{\prime}$, depois de ser espalhado em um ponto do eixo de simetria entre $z$ e $z+\mathrm{d} z$ pode ser calculada como

$$
\begin{aligned}
\mathrm{d}^{2} P_{\text {inc }}^{\prime}\left(E, E^{\prime}, x, z ; l, r\right)= & \mu_{\mathrm{Ge}}^{\mathrm{inc}}(E) \exp \left[-\mu_{\mathrm{Ge}}(E) z\right] \mathrm{d} z \\
& \times \frac{2 \pi}{\sigma_{\mathrm{Ge}}^{\text {inc }}(E)} \frac{\mathrm{d}^{2} \sigma_{\mathrm{AIR}}\left(E, E^{\prime}, x\right)}{\mathrm{d} E^{\prime} \mathrm{d} \Omega^{\prime}} \mathrm{d} x \\
& \times \exp \left[-\mu_{\mathrm{Ge}}\left(E^{\prime}\right) s(z, x ; l, r)\right] .
\end{aligned}
$$

Os fatores da primeira linha do membro direito desta equação representam a probabilidade de que o fóton incidente seja espalhado inelasticamente no intervalo $(z, z+\mathrm{d} z)$, onde $\mu_{\mathrm{Ge}}^{\text {inc }}(E)$ é o coeficiente linear de atenuação incoerente. Os fatores da segunda linha fornecem a probabilidade de que o fóton seja emitido com energia $E^{\prime}$ sob o ângulo sólido 
$\mathrm{d} \Omega^{\prime}=2 \pi \mathrm{d} x$. Essa probabilidade é descrita pela SCDD $\mathrm{d}^{2} \sigma_{\mathrm{AIR}} / \mathrm{d} E^{\prime} \mathrm{d} \Omega^{\prime}$ calculada segundo a AIR. O coseno do ângulo de emissão $\theta$ (em relação à direção do fóton incidente) foi escrito como $x=\cos \theta$ utilizando a condição de simetria cilíndrica. O fator de normalização $\sigma_{\mathrm{Ge}}^{\text {inc }}(E)$ é a seção de choque total de espalhamento inelástico dada pela AIR, para a energia $E$. Finalmente, o último exponencial considera a probabilidade de escape do fóton depois de percorrer o caminho $s(z, x ; l, r)$ dentro do detetor (ver Fig. 2.1).

Em seu modelo, Seltzer [56] adota a seção de KN para descrever a distribuição angular dos fótons (ou a distribuição de energia de retrocesso dos elétrons), e os efeitos de ligação são incluídos utilizando $\mu_{\mathrm{Ge}}^{\mathrm{inc}}(E)$ calculados segundo a aproximação de $\mathrm{WH}[79,80]$ (ver Fig. 4.5 da próxima seção). Desta forma, a expressão (4.23) se transforma em uma equação diferencial de primeira ordem [56]:

$$
\begin{aligned}
\mathrm{d} P_{\mathrm{inc}}^{\prime}\left(E, E^{\prime}, z ; l, r\right)= & \mu_{\mathrm{Ge}}^{\mathrm{inc}}(E) \exp \left[-\mu_{\mathrm{Ge}}(E) z\right] \mathrm{d} z \\
& \times \frac{1}{\sigma_{\mathrm{KN}}(E)} \frac{\mathrm{d} \sigma_{\mathrm{KN}}\left(E, E^{\prime}\right)}{\mathrm{d} E^{\prime}} \\
& \times \exp \left[-\mu_{\mathrm{Ge}}\left(E^{\prime}\right) s(z, x ; l, r)\right],
\end{aligned}
$$

por meio da fórmula de Compton que relaciona diretamente a energia do fóton $E^{\prime}$ e o ângulo de espalhamento $\theta$

$$
E^{\prime}=E[1+\varepsilon(1-x)]^{-1}
$$

sendo $\varepsilon \equiv E / m_{\mathrm{e}} c^{2}$. A aproximação de Seltzer [56] é viável só se for necessário conhecer a área do continuo de Compton. Porém, para uma descrição precisa da forma do espectro, a equação duplamente diferencial (4.23) fornece resultados mais realistas.

Para obter as contribuições de todos os pontos $z$ ao longo do eixo de simetria, a Eq. (4.23) foi integrada entre 0 e $l$

$$
\mathrm{d} P_{\text {inc }}^{\prime}\left(E, E^{\prime}, x ; l, r\right)=\frac{2 \pi \mathcal{N}_{\mathrm{Ge}}}{\mu_{\mathrm{Ge}}(E)} \frac{\mathrm{d}^{2} \sigma_{\mathrm{AIR}}\left(E, E^{\prime}, x\right)}{\mathrm{d} E^{\prime} \mathrm{d} \Omega^{\prime}} \mathrm{d} x G\left(E, E^{\prime}, x ; l, r\right),
$$

sendo $\mathcal{N}_{\mathrm{Ge}}$ o número de átomos por unidade de volume do Ge. O fator adimensional $G\left(E, E^{\prime}, x ; l, r\right)$ contem todas as dependências geométricas do problema e é similar àquele descrito por Seltzer [56]

$$
G\left(E, E^{\prime}, x ; l, r\right)=\mu_{\mathrm{Ge}}(E) \int_{0}^{l} \exp \left[-\mu_{\mathrm{Ge}}(E) z\right] \exp \left[-\mu_{\mathrm{Ge}}\left(E^{\prime}\right) s(z, x ; l, r)\right] \mathrm{d} z .
$$

A diferença na aproximação escolhida neste trabalho se centra em que o coseno do ângulo de espalhamento $x$ não define a energia $E^{\prime}$. Além disso, os cálculos da função $G\left(E, E^{\prime}, x ; l, r\right)$ foram sempre realizados utilizando os coeficientes de atenuação totais. 
Seltzer [56] emprega para ângulos de retroespalhamento os coeficientes de absorção mássica. Assim, usando a notação reduzida: $\mu \equiv \mu_{\mathrm{Ge}}(E), \mu^{\prime} \equiv \mu_{\mathrm{Ge}}\left(E^{\prime}\right)$, denotando os limites de integração de $x$ em função dos parâmetros do detetor $l$ e $r$

$$
x_{\mathrm{g}}=\frac{l}{\sqrt{l^{2}+r^{2}}}
$$

e introduzindo a notação

$$
\xi \equiv \frac{\mu^{\prime}}{\mu x}-1
$$

obtemos a forma analítica do fator geométrico em função de $x$ e $E^{\prime}$, para cada uma das possíveis direções de escape do fóton

$$
G= \begin{cases}\mu l \exp (-\mu l) & \text { se } x=1, \\ \xi^{-1} \exp (-\mu l)[1-\exp (-\xi \mu l)] & \text { se } 1>x \geqslant x_{\mathrm{g}} \\ \exp \left[-\frac{(\xi+1) \mu r x}{\sqrt{1-x^{2}}}\right]-\exp (-\mu l) & \\ \times\left(1+\xi^{-1}\right) \exp \left[-\frac{\xi \mu r x}{\sqrt{1-x^{2}}}-\xi^{-1}\right] & \text { se } x_{\mathrm{g}}>x>0 \\ \exp \left(-\mu^{\prime} r\right)[1-\exp (-\mu l)] & \text { se } x=0 \\ \left(1+\xi^{-1}\right) \exp \left[-\frac{\xi \mu r x}{\sqrt{1-x^{2}}}\right]-\xi^{-1} & \text { se } 0>x>-x_{\mathrm{g}} \\ -\exp (-\mu l) \exp \left[-\frac{(\xi+1) \mu r x}{\sqrt{1-x^{2}}}\right] & \text { se }-x_{\mathrm{g}} \geqslant x \geqslant-1 . \\ \xi^{-1}[\exp (\xi \mu l)-1] & \end{cases}
$$

A probabilidade de que o fóton incidente de energia $E$ escape do detetor com energia $E^{\prime}$ pode ser agora determinada integrando a Eq. (4.26) no intervalo angular $-1 \leqslant x \leqslant 1$

$$
P_{\mathrm{inc}}^{\prime}\left(E, E^{\prime} ; l, r\right)=\frac{2 \pi \mathcal{N}_{\mathrm{Ge}}}{\mu_{\mathrm{Ge}}(E)} C_{\mathrm{AIR}}\left(E, E^{\prime} ; l, r\right)
$$

com

$$
C_{\mathrm{AIR}}\left(E, E^{\prime} ; l, r\right)=\int_{-1}^{1} \frac{\mathrm{d}^{2} \sigma_{\mathrm{AIR}}\left(E, E^{\prime}, x\right)}{\mathrm{d} E^{\prime} \mathrm{d} \Omega^{\prime}} G\left(E, E^{\prime}, x ; l, r\right) \mathrm{d} x
$$

Logo, a probabilidade de que a energia $\epsilon$ seja depositada no volume ativo de Ge pode ser escrita como

$$
P_{\text {inc }}(\epsilon, E ; l, r)=P_{\text {inc }}^{\prime}(E, E-\epsilon ; l, r) .
$$




\subsubsection{Seção de choque duplamente diferencial na AIR}

A seção de choque duplamente diferencial de espalhamento inelástico foi calculada por Ribberfors e Berggren [79] usando a AIR. Nesta aproximação a SCDD de Compton pode ser avaliada como

$$
\begin{aligned}
\frac{\mathrm{d}^{2} \sigma_{\mathrm{AIR}}}{\mathrm{d} E^{\prime} \mathrm{d} \Omega^{\prime}}= & \frac{r_{\mathrm{e}}^{2}}{2} \frac{m_{\mathrm{e}}}{\hbar K} \frac{E^{\prime}}{E}\left[1+x^{2}+\frac{E-E^{\prime}}{m_{\mathrm{e}} c^{2}}(1-x)\right] \\
& \times\left[\sum_{i} Z_{i} J_{i}\left(p_{z}\right) \Theta\left(E-E^{\prime}-U_{i}\right)\right],
\end{aligned}
$$

onde

$$
\hbar K=\frac{1}{c} \sqrt{E^{2}+E^{\prime 2}-2 E E^{\prime} x}
$$

é o modulo do vetor transferência de momento. Na Eq. (4.34), $Z_{i}$ é o número de elétrons na $i$-ésima camada atômica, $J_{i}\left(p_{z}\right)$ é o perfil de Compton do elétron da mesma camada e $p_{z}$ é a projeção do momento inicial do elétron alvo na direção do vetor de espalhamento,

$$
p_{z}=\frac{E E^{\prime}(1-x)-m_{\mathrm{e}} c^{2}\left(E-E^{\prime}\right)}{\hbar K} .
$$

A função degrau de Heaviside, $\Theta\left(E-E^{\prime}-U_{i}\right)$, leva em conta os efeitos energéticos de ligação permitindo espalhamento Compton somente se a energia transferida $E-E^{\prime}$ for maior do que a energia de ligação $U_{i}$ da camada ativa.

Os perfis de Compton (PC) tem sido calculados aplicando diferentes métodos [80, 85, 86]. Biggs, Mendelson e Mann [85], tabularam os PC orbitais e totais usando funções de onda Hartree-Fock para números atômicos $1 \leqslant Z \leqslant 36$, e funções de onda Dirac-HartreeFock para elementos $36 \leqslant Z \leqslant 102$. Por outro lado, Mendelson e Bloch [86] introduziram um enfoque simples para determinar a carga nuclear efetiva e utilizar funções de onda hidrogenoides não relativísticas, que são analíticas, nos cálculos dos PC. A aproximação consiste em impor o requerimento de que o PC hidrogenoide tenha o mesmo valor que o PC Hartree-Fock em $J_{i}(0)$ para cada orbital atômico [86]. Sob outra perspectiva, o código PENELOPE [43] simula os eventos de espalhamento Compton a partir do algoritmo proposto por Brusa e colaboradores [80]. Estes últimos autores, parametrizaram os PC de Biggs, Mendelson e Mann [85] para avaliar a fórmula de Ribberfors-Berggren (4.34):

$$
J_{i}\left(p_{z}\right)=J_{i}(0)\left(1+2 J_{i}(0)\left|p_{z}\right|\right) \exp \left[J_{i}(0)\left|p_{z}\right|\left(1+2 J_{i}(0)\left|p_{z}\right|\right)\right]
$$

O valor $J_{i}(0)$ se obteve das tabelas de Biggs, Mendelson e Mann [85]. Neste trabalho foi adotado o procedimento de Brusa et al. [80] para descrever o espalhamento Compton no cristal de Ge (ver Apêndice A). 


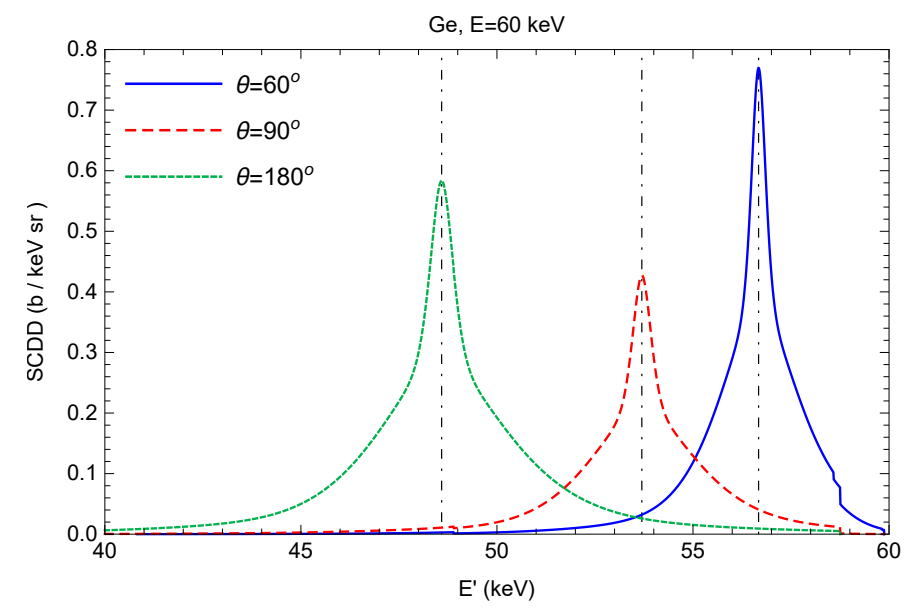

Figura 4.4: SCDD de espalhamento Compton em Ge para fótons de $60 \mathrm{keV}$, e ângulos $\theta=60^{\circ}, \theta=90^{\circ}$ e $\theta=180^{\circ}$. As linhas verticais representam as energias estabelecidas pela fórmula de Compton, Eq. (4.25).

A Fig. 4.4 mostra a SCDD de espalhamento inelástico para fótons de $60 \mathrm{keV}$ em Ge, obtida mediante a AIR [79], com PCs avaliados segundo a parametrização de Brusa e colaboradores [80]. Nota-se que o máximo da seção de choque coincide com a energia estabelecida pela fórmula de Compton (4.25) para o ângulo $\theta$, no entanto, a inclusão da distribuição de momentos dos elétrons nas camadas atômicas origina o alargamento observado em torno desse valor. No exemplo apresentado na Fig. 4.4, percebe-se que o fóton de $60 \mathrm{keV}$ pode ser inelasticamente espalhado com a mesma energia $E^{\prime}$ e $\theta=60^{\circ}$, $90^{\circ}$ ou $180^{\circ}$. Essa probabilidade implica que o escape pode acontecer tanto pela superfície frontal, quanto pelas laterais ou pela parte posterior do cristal, mantendo $E^{\prime}$ o mesmo valor. Em contrapartida, a Fig. 4.5 apresenta uma comparação entre as probabilidades de que um elétron do átomo de Ge seja emitido no intervalo energético $(\epsilon, \epsilon+\mathrm{d} \epsilon)$, depois de que um fóton de 60 ou $100 \mathrm{keV}$ experimente uma interação inelástica. Nos cálculos foram utilizadas as SCDs de KN, de WH e da AIR. A Eq. (4.31) foi integrada numericamente por meio do software Wolfram Mathematica 10.0.1 [87]. Constata-se que o efeito Doppler permite que os elétrons sejam emitidos acima do limiar estabelecido pela Eq. (4.25), suavizando a forma do espectro. Resulta evidente que KN e WH não modelam esse fenômeno, mas esta última é capaz de reproduzir razoavelmente a redução progressiva da SCD com a diminuição de $\epsilon$ a causa dos efeitos de ligação dos elétrons.

\subsubsection{Escape de fotoelétrons do volume ativo}

Quando os fótons incidentes experimentam uma interação perto das superfícies do volume ativo, além de raios-x característicos, também os fotoelétrons emitidos podem escapar. Como consequência, aparece uma região continua na distribuição de altura de pulso. Modelos analíticos que consideram esse fenômeno tem sido publicados por Goto 

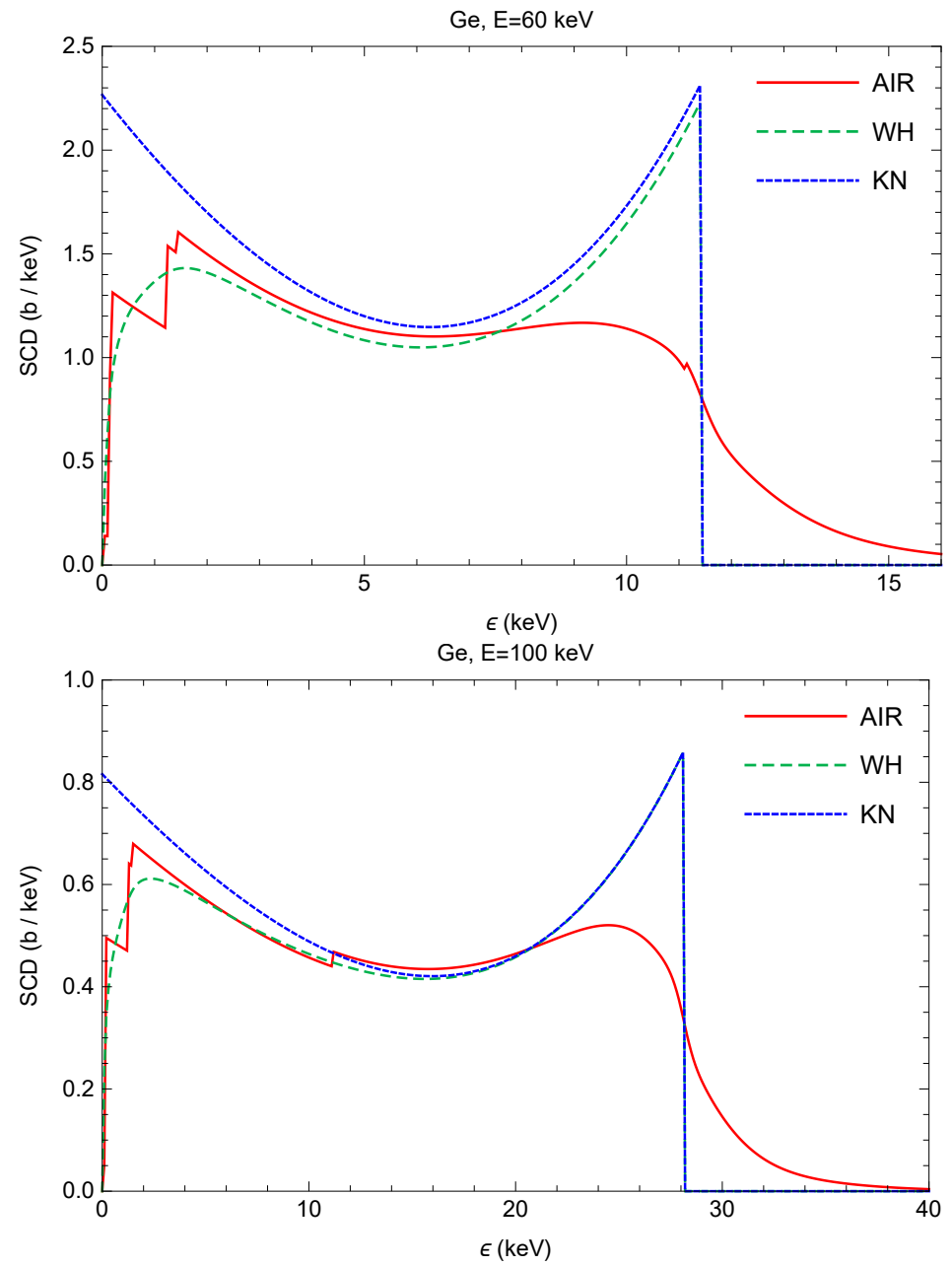

Figura 4.5: Comparação entre a SCD de espalhamento Compton em Ge fornecida pela AIR, e as aproximações de WH e KN, para fótons de 60 e $100 \mathrm{keV}$. Os gráficos apresentam a probabilidade de que o elétron seja emitido no intervalo energético $(\epsilon, \epsilon+$ $\mathrm{d} \epsilon)$.

[72], Lowe [73] e por Scholze e Procop [74, 75]. Nesses trabalhos foi calculado o espectro produzido por fotoelétrons e elétrons Auger que escapam do volume ativo, ou entram nele, provenientes do contato frontal do detetor. O estudo foi realizado em detetores planares de $\mathrm{Si}(\mathrm{Li}), \mathrm{SDD}^{3}$ e HPGe para energias $E \leqslant 10 \mathrm{keV}[72-75]$.

Nesta subseção é descrito o espectro produzido por fotoelétrons que escapam do volume ativo pelas superfícies anterior e posterior, para energias incidentes $10 \lesssim E \lesssim 100 \mathrm{keV}$. Quando o fotoelétron penetra no cristal proveniente da camada morta de Ge, o fóton original interatuou fora do volume ativo, portanto o processo não contribui à FR intrínseca. Esse tipo de eventos são analisados na subseção 4.3.6.3.

O modelo analítico desenvolvido neste trabalho postula que o fotoelétron atravessa um

\footnotetext{
${ }^{3}$ As siglas SDD significam Silicon Drift Detectors, detetores de silício de deriva.
} 
caminho reto $t$, definido pelo ângulo de emissão $\theta$, entre seu ponto de origem à profundidade z e a superfície do volume ativo (Fig. 4.6). Com a simplificação anterior, a energia $\epsilon$ depositada antes de escapar pode se estimar como

$$
\epsilon \simeq S\left(E_{0, i}\right) t
$$

onde $S\left(E_{0, i}\right)$ é o poder de freamento, calculado com a fórmula relativística de Bethe desconsiderando a contribuição radiativa [81], e $E_{0, i}=E-U_{i}$ equivale à energia inicial do fotoelétron.
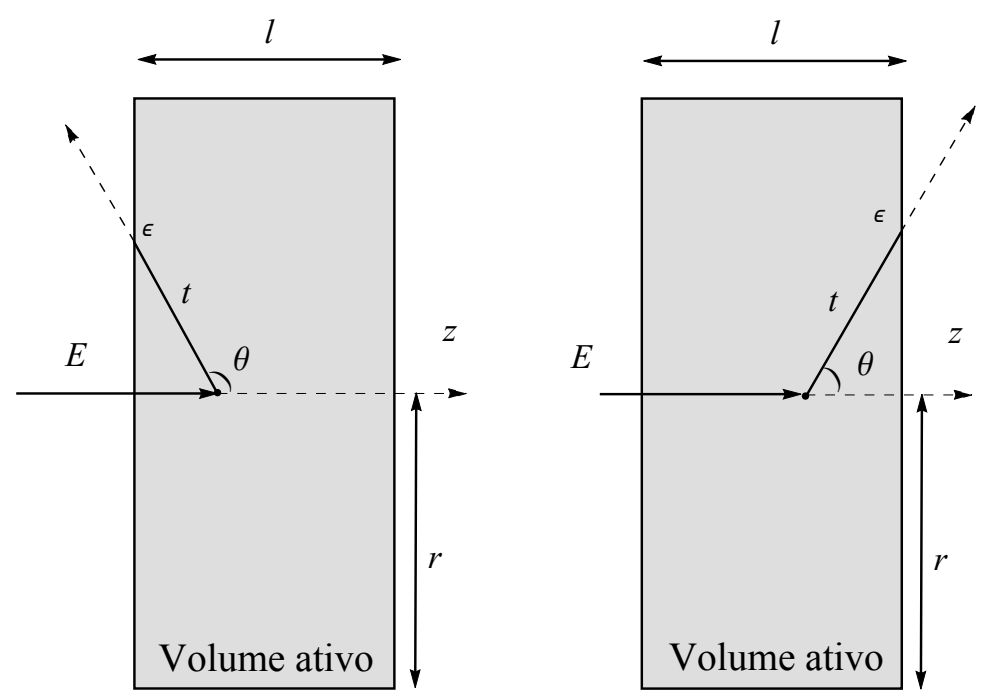

FiguRA 4.6: Representação geométrica do processo de escape dos fotoelétrons pelas superfícies frontal (esquerda) e posterior (direita) do detetor.

A probabilidade de que um fóton de energia $E$ gere um elétron em um ponto entre $z \mathrm{e}$ $z+\mathrm{d} z$ do eixo de simetria, e seja emitido no intervalo angular $(x, x+\mathrm{d} x)$ é dada por

$$
\mathrm{d} P_{\mathrm{ev}}^{\prime}(E, x, z)=\mu_{\mathrm{Ge}}^{\mathrm{pe}}(E) \exp \left[-\mu_{\mathrm{Ge}}(E) z\right] \mathrm{d} z p_{\mathrm{pe}}(E, x),
$$

onde $p_{\text {pe }}(E, x)$ é a distribuição angular dos fotoelétrons

$$
p_{\mathrm{pe}}(E, x)=\frac{2 \pi}{\sigma_{\mathrm{pe}}(E)} \frac{\mathrm{d} \sigma_{\mathrm{pe}}(E, x)}{\mathrm{d} \Omega_{\mathrm{e}}} .
$$

Nesta equação se supõe que o feixe incidente é não polarizado, portanto, o resultado independe do ângulo azimutal $\phi$, que é uniformemente distribuído no intervalo $[0,2 \pi]$. Alguns autores [73, 75] tem empregado a distribuição angular de um processo geral 
de absorção dipolar para determinar a emissão dos fotoelétrons na Eq. (4.40). Esse tratamento é válido para raios-x na faixa: 200-1500 eV [88, 89]. Neste trabalho foi utilizada a distribuição angular derivada no artigo de Sabbatucci e Salvat [90], a partir da SCD de Sauter para o fotoefeito na camada K [91]:

$$
\begin{aligned}
p_{\mathrm{pe}}(E, x)= & \frac{3}{4 \gamma^{4}}\left[1+\frac{3}{4} \frac{\gamma(\gamma-2)}{\gamma+1}\left(1-\frac{1}{2 \beta \gamma^{2}} \ln \frac{1+\beta}{1-\beta}\right)\right]^{-1} \\
& \times \frac{1-x^{2}}{(1-\beta x)^{4}}\left[1+\frac{1}{2} \gamma(\gamma-1)(\gamma-2)(1-\beta x)\right]
\end{aligned}
$$

com

$$
\gamma=1+\frac{E_{0, i}}{m_{\mathrm{e}} c^{2}}, \quad \beta=\sqrt{1-\gamma^{-2}}
$$

A equação de Sauter é adequada somente para a ionização da camada $\mathrm{K}$ por fótons de alta energia (da ordem dos MeV). Esses fatos fazem parecer limitada a Eq. (4.41) para a faixa analisada, porém, a expressão está implementada na maioria dos códigos de simulação MC de propósito geral, incluindo PENELOPE, para descrever os eventos de fotoionização. Sua utilização tem demostrado que não são apreciáveis os erros introduzidos nos resultados [43].

Na Fig. 4.7 é apresentada a distribuição angular para fotoelétrons ejetados da camada $\mathrm{K}$ do Ge por fótons de 20, 60 e $100 \mathrm{keV}$ segundo a Eq. (4.41). Nota-se que a emissão para ângulos dianteiros aumenta com o incremento da energia.

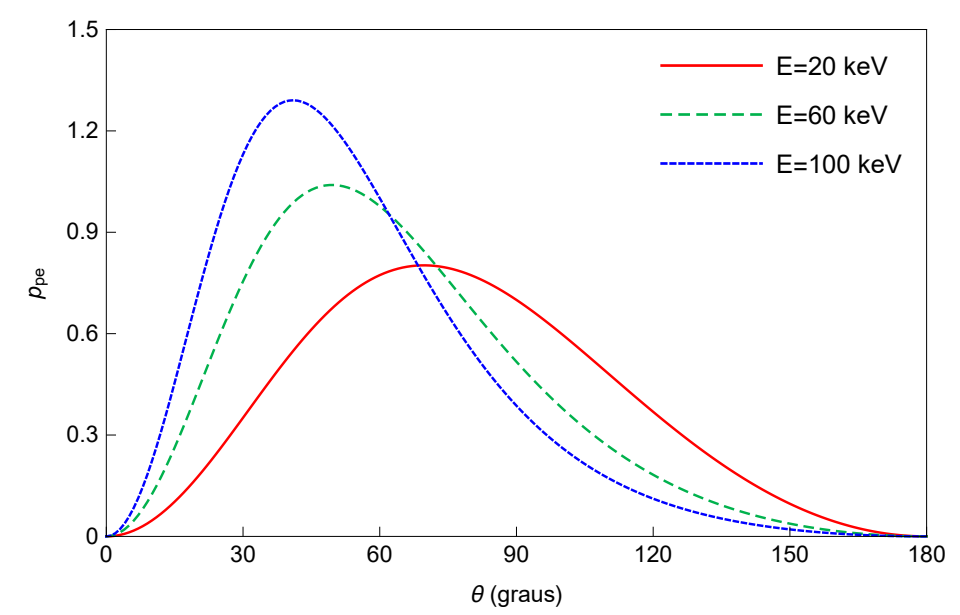

Figura 4.7: Distribuição angular de fotoelétrons da camada K do Ge segundo a SCD de Sauter, quando são absorvidos fótons de 20, 60 e $100 \mathrm{keV}$. 
De acordo com a Fig. 4.6 e devido à consideração de que o ângulo de emissão do fotoelétron define um caminho reto, a Eq. (4.41) pode se reescrever em termos de $t$ :

$$
x= \begin{cases}-z / t & \text { para escape pela superfície anterior } \\ (l-z) / t & \text { para escape pela superfície posterior }\end{cases}
$$

Logo, substituindo as relações anteriores na Eq. (4.41), é possível calcular a probabilidade de que o fóton seja absorbido à profundidade $z$, e o elétron emitido atravesse a espessura $t$ dentro do volume ativo antes de escapar, como

$$
\mathrm{d} P_{\mathrm{ev}}^{\mathrm{b}}(E, t, z ; l)=\mu_{\mathrm{Ge}}^{\mathrm{pe}}(E) \exp \left[-\mu_{\mathrm{Ge}}(E) z\right]\left(\frac{t^{2}}{l-z}\right) p_{\mathrm{pe}}\left(E, \frac{l-z}{t}\right) \mathrm{d} z
$$

se o elétron escapa pela superfície posterior do detetor, e

$$
\mathrm{d} P_{\mathrm{ev}}^{\mathrm{f}}(E, t, z ; l)=\mu_{\mathrm{Ge}}^{\mathrm{pe}}(E) \exp \left[-\mu_{\mathrm{Ge}}(E) z\right]\left(\frac{t^{2}}{z}\right) p_{\mathrm{pe}}\left(E, \frac{-z}{t}\right) \mathrm{d} z
$$

se o escape acontece pela superfície frontal do detetor. As expressões anteriores são diferentes de zero quando $t<R_{\mathrm{p}}\left(E_{0, i}\right)$, onde $R_{\mathrm{p}}\left(E_{0, i}\right)$ é o alcance projetado do elétron ${ }^{4}$. Para computar todas as profundidades que forneçam o mesmo caminho $t$, a Eq. (4.44) foi integrada numericamente sobre todos os pontos $z$ entre $l-t$ e $t$, considerando que $l \gg t$, por conseguinte $l-t$ é sempre uma grandeza positiva,

$$
P_{\mathrm{ev}}^{\mathrm{b}}(E, t ; l)=\int_{l-t}^{t} \mathrm{~d} P_{\mathrm{ev}}^{\mathrm{b}}(E, t, z ; l)
$$

Um procedimento similar se realizou com a Eq. (4.45) integrando de 0 a $t$,

$$
P_{\mathrm{ev}}^{\mathrm{f}}(E, t ; l)=\int_{0}^{t} \mathrm{~d} P_{\mathrm{ev}}^{\mathrm{f}}(E, t, z ; l)
$$

A partir das probabilidades (4.46) e (4.47) e a relação (4.38) pode ser calculado o espectro produzido pelo escape dos elétrons das diferentes camadas do átomo como

$$
P_{\mathrm{ev}}(\epsilon, E ; l)=\sum_{i} \frac{q_{i}(E)}{S\left(E_{0, i}\right)}\left[P_{\mathrm{ev}}^{\mathrm{b}}\left(E, t_{\epsilon i}\right)+P_{\mathrm{ev}}^{\mathrm{f}}\left(E, t_{\epsilon i}\right)\right] \Theta\left(E_{0, i}-\epsilon\right),
$$

onde

$$
t_{\epsilon i}=\frac{\epsilon}{S\left(E_{0, i}\right)} .
$$

\footnotetext{
${ }^{4} \mathrm{O}$ alcance projetado do elétron, $R_{\mathrm{p}}(E)$, é o caminho meio que este percorre em um material quando atravessa a espessura $t$ [92].
} 
A função degrau de Heaviside $\Theta\left(E_{0, i}-\epsilon\right)$ foi introduzida para restringir o espectro a energias inferiores a $E_{0, i}$, enquanto, o fator $q_{i}$ é a probabilidade de ionização da $i$-ésima camada.

\subsubsection{Absorção total da energia do fóton}

Se a energia $E$ for completamente absorbida no volume ativo, o evento se registra no pico de absorção total. Portanto, a probabilidade intrínseca $\varepsilon_{\text {int }}$ de que um fóton deposite totalmente a sua energia pode ser calculada subtraindo da probabilidade de experimentar qualquer tipo de interação no cristal, a probabilidade de depositar qualquer energia $\epsilon$ diferente de $E$. A partir das funções (4.20), (4.33) e (4.48) se obtém

$$
\begin{aligned}
\varepsilon_{\mathrm{int}}(E ; l, r)=1 & -\exp \left[-\mu_{\mathrm{Ge}}(E) l\right]-\int_{0}^{E}\left[P_{\mathrm{K} \alpha}(\epsilon, E)+P_{\mathrm{K} \beta}(\epsilon, E)\right] \mathrm{d} \epsilon \\
& -\int_{0}^{E}\left[P_{\mathrm{inc}}(\epsilon, E ; l, r)+P_{\mathrm{ev}}(\epsilon, E ; l)\right] \mathrm{d} \epsilon .
\end{aligned}
$$

Para incorporar este resultado à função de energia depositada $D(\epsilon, E ; l, r)$, (note-se que as unidades são de inverso de energia), é preciso escrever a Eq. (4.50) em termos da delta de Dirac (de igual forma que no caso da probabilidade de escape dos raios-x K) como

$$
P_{\mathrm{int}}(\epsilon, E ; l, r)=\varepsilon_{\mathrm{int}}(E ; l, r) \delta(\epsilon-E) \text {. }
$$

Por outro lado, como foi mencionado na subseção 4.3.3, o cálculo da área total produzida pelo escape Compton na Eq. (4.50) pode ser simplificado a uma integral unidimensional se for empregada a relação (4.24), já que os efeitos de ligação dos elétrons se levam em conta no coeficiente $\mu_{\mathrm{Ge}}^{\mathrm{inc}}(E)$. Finalmente, as componentes da FR intrínseca consideradas neste modelo analítico são

$$
\begin{aligned}
D(\epsilon, E ; l, r)= & P_{\mathrm{int}}(\epsilon, E ; l, r)+P_{\mathrm{K} \alpha}(\epsilon, E)+P_{\mathrm{K} \beta}(\epsilon, E) \\
& +P_{\mathrm{inc}}(\epsilon, E ; l, r)+P_{\mathrm{ev}}(\epsilon, E ; l) .
\end{aligned}
$$

\subsubsection{Contribuições das interações externas à função resposta}

Os detetores de radiação são compostos por vários elementos que garantem seu mecanismo de funcionamento. Particularmente, aqueles materiais na frente do volume ativo podem modificar o espectro incidente se os fótons interagem neles. Na folha de dados técnicos dos fabricantes de detetores HPGe, são fornecidos os valores de espessura da janela de Be (material tipicamente usado em espectrômetros de baixas energias) e da 
camada morta de Ge. A influência de ambos os componentes na FR será abordada a seguir.

\subsubsection{Espectro produzido pelo espalhamento inelástico de fótons na janela de Be}

O modelo proposto descreve o espectro produzido por espalhamento Compton de fótons na janela de Be do detetor (espessura $l_{\text {Be }}$ ). O gráfico da Fig. 4.8 apresenta os coeficientes de atenuação linear no Be, para o intervalo $10 \mathrm{keV} \leqslant E \leqslant 100 \mathrm{keV}$. Observa-se que os processos inelásticos são os mecanismos de interação dominantes acima de $E=15 \mathrm{keV}$. Neste caso, o interesse se centra em ângulos de espalhamento frontais: fótons com energia $E^{\prime}$ próxima a $E$ (Fig. 4.9).

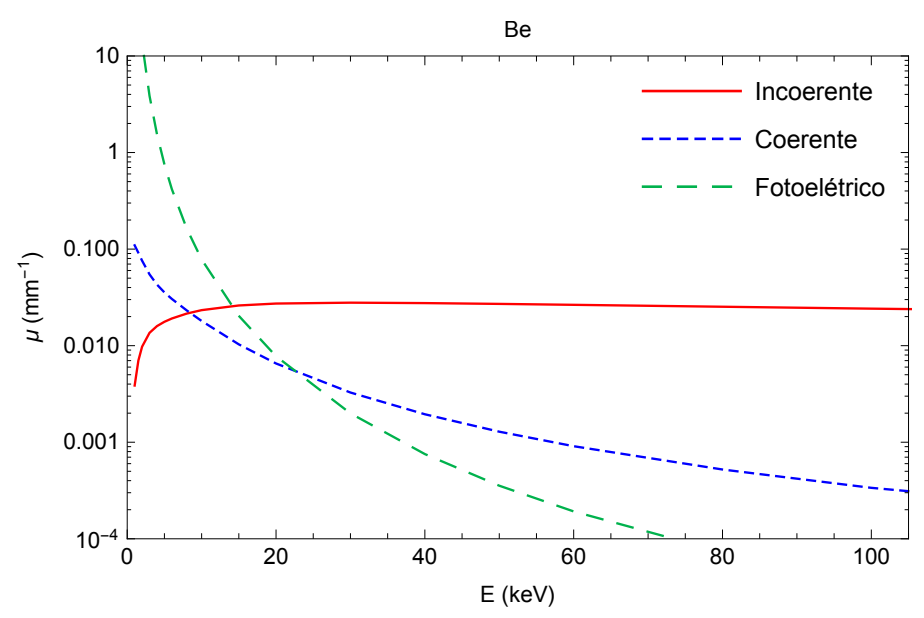

Figura 4.8: Coeficientes de atenuação mássica para espalhamento incoerente, coerente e efeito fotoelétrico no Be. Os dados foram extraídos do programa XCOM [84].

Como o intervalo energético $\left[E^{\prime}, E\right]$ encontra-se longe da região de máxima transferência de momento, onde o alargamento Doppler é mais importante, e levando em conta que o Be é um elemento leve $(Z=4)$, os efeitos de ligação podem ser desprezados, aliás, a energia $E^{\prime}$ é corretamente descrita pela fórmula de Compton (4.25). Por conseguinte, é também possível utilizar a distribuição angular de fótons fornecida pela SCD de KN [78]

$$
\begin{aligned}
p_{\mathrm{KN}}(E, x) & =\frac{2 \pi}{\sigma_{\mathrm{KN}}(E)} \frac{\mathrm{d} \sigma_{\mathrm{KN}}}{\mathrm{d} \Omega^{\prime}}(E, x) \\
& =N(E)[1+\varepsilon(1-x)]^{-2}\left\{1+x^{2}+\varepsilon(1-x)[1+\varepsilon(1-x)]^{-1}\right\},
\end{aligned}
$$

onde $N(E)$ é a constante de normalização. A Fig. 4.10 mostra uma comparação entre as SCDs para espalhamento inelástico de fótons de 60 e $100 \mathrm{keV}$ no Be, calculadas segundo a AIR e a teoria de KN. Observa-se a concordância que existe para energias $E^{\prime}$ próximas a $E$. 


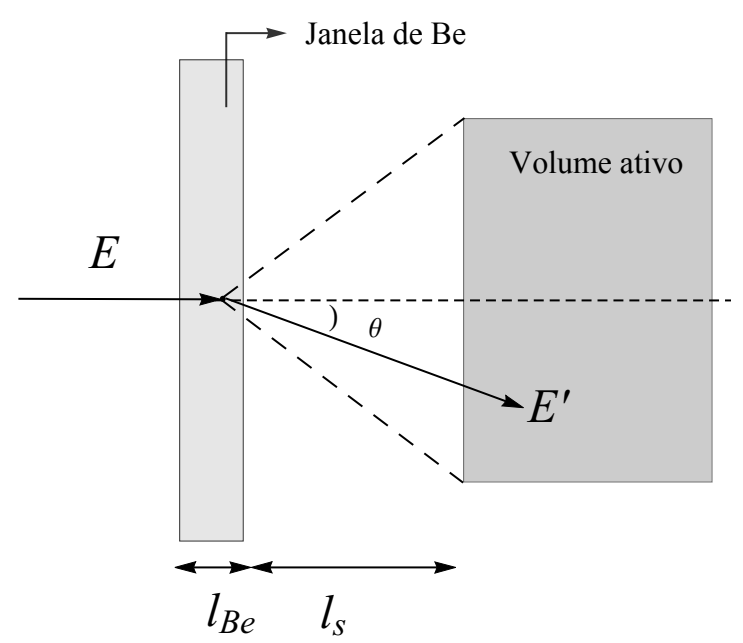

FiguRA 4.9: Representação geométrica do processo de espalhamento inelástico na janela do detetor. Esquema fora de escala.

A simplificação anterior representa uma vantagem devido a que existe uma relação direta entre o ângulo de espalhamento e a energia $E^{\prime}$. O modelo aqui desenvolvido supõe simetria cilíndrica e incidência normal ao longo do eixo da janela de Be (Fig. 4.9). A probabilidade de que um fóton seja espalhado a uma profundidade entre $z \mathrm{e} z+\mathrm{d} z$, com um ângulo descrito por $x$, e alcance a superfície frontal do detetor é dada por

$$
\begin{aligned}
\mathrm{d} P_{\mathrm{Be}}^{\prime}(E, x, z)= & \mu_{\mathrm{Be}}^{\mathrm{inc}}(E) \exp \left[-\mu_{\mathrm{Be}}(E) z\right] \mathrm{d} z \\
& \times p_{\mathrm{KN}}(E, x) \exp \left[-\mu_{\mathrm{Be}}\left(E^{\prime}\right)\left(\frac{l_{\mathrm{Be}}-z}{x}\right)\right],
\end{aligned}
$$

sendo $\mu_{\mathrm{Be}}^{\text {inc }}$ e $\mu_{\mathrm{Be}}$ os coeficientes de espalhamento incoerente e absorção total do Be. O último exponencial representa a probabilidade de que o fóton não seja absorbido uma vez espalhado. Se for considerado que só acontece uma interação dentro da janela, este fator pode ser tomado como 1 . Logo, integrando sobre todos os pontos $z$ entre 0 e $l_{\mathrm{Be}}$ se obtém

$$
P_{\mathrm{Be}}^{\prime}\left(E, x ; l_{\mathrm{Be}}\right)=\frac{\mu_{\mathrm{Be}}^{\mathrm{inc}}(E)}{\mu_{\mathrm{Be}}(E)}\left\{1-\exp \left[-\mu_{\mathrm{Be}}(E) l_{\mathrm{Be}}\right]\right\} p_{\mathrm{KN}}(E, x) .
$$

A expressão acima é válida para para todos os ângulos no intervalo $\left(x_{\min }, 1\right)$, sendo

$$
x_{\min }=\frac{l_{\mathrm{s}}}{\sqrt{l_{\mathrm{s}}^{2}+r^{2}}} .
$$

Para determinar esse limite, se considerou que nos detetores HPGe, a distância $l_{\mathrm{s}}$ é maior do que a espessura de janela $l_{\mathrm{Be}}$ em ao menos 20 vezes, então, o ponto real de interação dentro da janela apenas modifica o valor de $x_{\min }$. Assim, o espectro de energia 

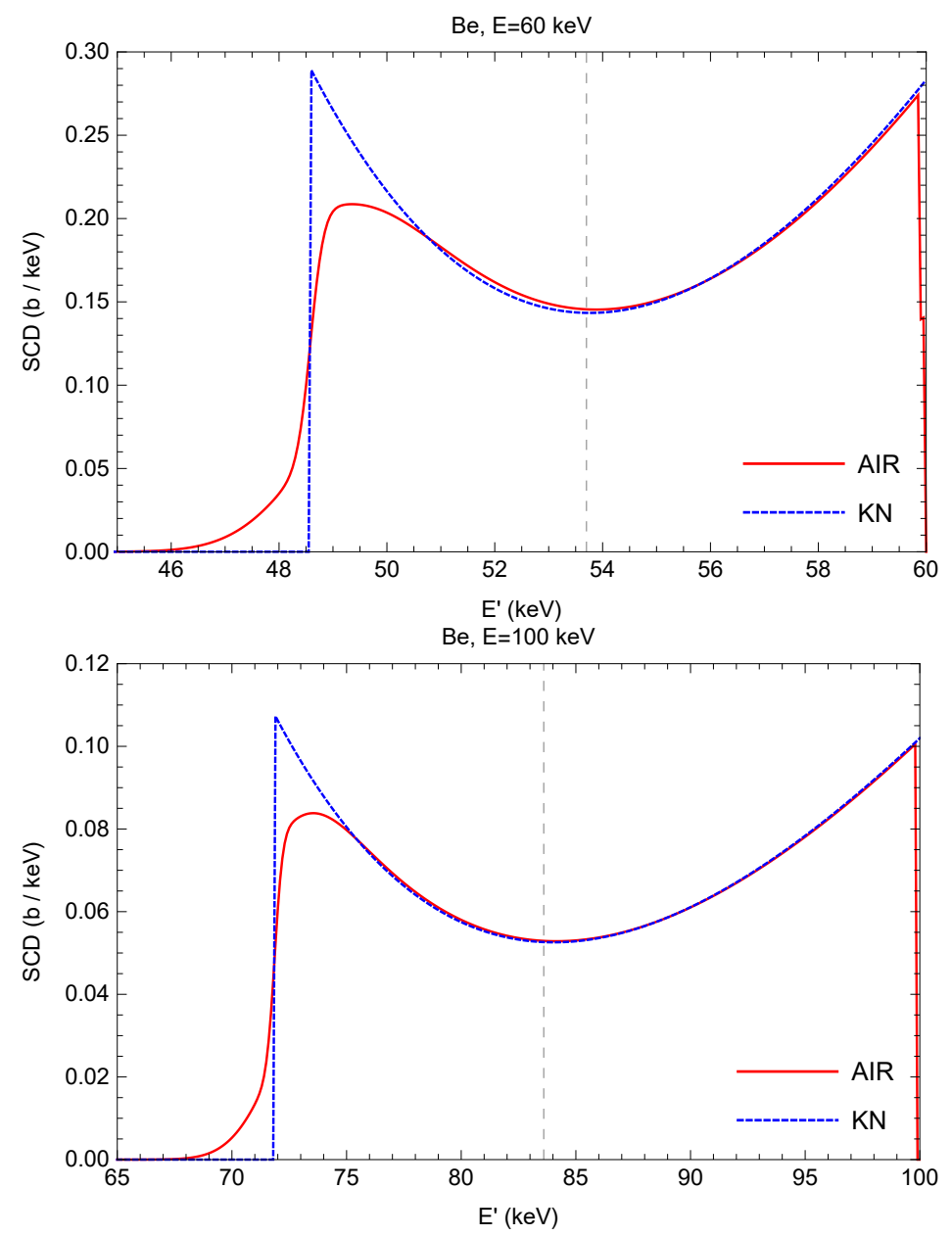

Figura 4.10: Comparação entre a SCD de espalhamento Compton no Be fornecida pela AIR e a aproximação de KN, para fótons de 60 e $100 \mathrm{keV}$. As linhas tracejadas verticais estão localizadas nas energias correspondentes ao ângulo de espalhamento $\theta=90^{\circ}$.

que atinge a superfície do detetor pode ser avaliado a partir das equações (4.25) e (4.55)

$$
P_{\mathrm{Be}}\left(E, E^{\prime} ; l_{\mathrm{Be}}\right)=\frac{m_{\mathrm{e}} c^{2}}{E^{\prime 2}} P_{\mathrm{Be}}^{\prime}\left(E, x_{\mathrm{c}} ; l_{\mathrm{Be}}\right) \Theta\left(E^{\prime}-E_{\min }^{\prime}\right)
$$

com

$$
x_{\mathrm{c}}=1-m_{\mathrm{e}} c^{2}\left(\frac{1}{E^{\prime}}-\frac{1}{E}\right), \quad \text { e } \quad E_{\min }^{\prime}=E\left[1+\varepsilon\left(1-x_{\min }\right)\right]^{-1} .
$$

O espalhamento na janela de Be irá produzir uma distribuição de altura de pulso dada por componentes similares às descritas para a FR intrínseca do cristal de Ge (não exatamente igual porque os fótons não atingem a superfície do detetor de maneira normal). Considerando as probabilidades relativas de cada um dos processos de interação, no modelo se adota que uma vez espalhado, o fóton irá ser totalmente absorbido no volume ativo de Ge, com uma probabilidade dada por $\varepsilon_{\text {int }}\left(E^{\prime} ; l, r\right)$. Esta hipótese é equivalente a 
supor que o espalhamento Compton só produz uma mudança na energia do fóton, mas não na sua direção original.

\subsubsection{Contribuição do espalhamento elástico de fótons na janela de Be}

A seção de choque de espalhamento elástico no Be, para energias entre 10 e $100 \mathrm{keV}$, é aproximadamente uma ordem de grandeza menor do que a seção inelástica (Fig. 4.8). No modelo analítico deste trabalho, não é avaliada a contribuição externa desse efeito à FR. Porém, abaixo de $10 \mathrm{keV}$, os processos de Rayleigh se tornam o mecanismo dominante de espalhamento. Ainda quando os detetores de Ge são utilizados acima deste valor, a fim de completar o modelo analítico, nesta subseção, é analisado o procedimento geral de cálculo do pico produzido pelas interações elásticas na janela de Be. A metodologia pode ser aplicada a detetores de $\mathrm{Si}(\mathrm{Li})$ e $\mathrm{SDD}$, usados principalmente em medidas abaixo de $\sim 10 \mathrm{keV}$.

Seguindo as mesmas aproximações da subseção 4.3.6.1, a probabilidade $P_{\mathrm{Be}}^{\prime c}\left(E, x ; l_{\mathrm{Be}}\right)$ de que um fóton de energia $E$ experimente um espalhamento elástico na janela de Be, e atinja a superfície do detetor sob um ângulo descrito por $x$, pode ser derivada da Eq. (4.55) substituindo o coeficiente de atenuação incoerente pelo coerente $\mu_{\mathrm{Be}}^{\mathrm{coe}}(E)$, e a distribuição angular de KN pela distribuição de Rayleigh $p_{\text {coe }}(E, x)$

$$
P_{\mathrm{Be}}^{\prime c}\left(E, x ; l_{\mathrm{Be}}\right)=\frac{\mu_{\mathrm{Be}}^{\mathrm{coe}}(E)}{\mu_{\mathrm{Be}}(E)}\left\{1-\exp \left[-\mu_{\mathrm{Be}}(E) l_{\mathrm{Be}}\right]\right\} p_{\mathrm{coe}}(E, x)
$$

com

$$
\begin{aligned}
p_{\mathrm{coe}}(E, x) & =\frac{2 \pi}{\sigma_{\mathrm{coe}}(E)} \frac{\mathrm{d} \sigma_{\mathrm{coe}}}{\mathrm{d} \Omega^{\prime}}(E, x) \\
& =N(E) r_{\mathrm{e}}^{2} \frac{1+x^{2}}{2}\left[F_{\mathrm{Be}}(E, x)\right]^{2},
\end{aligned}
$$

onde $N(E)$ é o fator de normalização e $F_{\mathrm{Be}}(E, x)$ é o fator de forma atômico do Be $[20,93]$.

\subsubsection{Espectro produzido pelos fotoelétrons originados na camada morta de Ge}

O efeito fotoelétrico é o principal mecanismo de interação no Ge para energias de fótons incidentes na faixa $10 \mathrm{keV} \leqslant E \leqslant 100 \mathrm{keV}$. Em um evento desse tipo, os átomos ionizados emitem fotoelétrons e raios-x característicos ou elétrons Auger. Essa radiação fluorescente, produzida na camada morta do detetor, pode atingir o volume ativo e adicionar contribuições à FR. Os detetores de Ge são utilizados tipicamente para medidas 
de fótons com energias acima da borda $\mathrm{K}\left(U_{\mathrm{K}}=11013,76(74) \mathrm{eV}\right.$ [83]). Considerando esse fato, no modelo descrito a seguir, só são analisadas aquelas partículas com energias superiores a $U_{\mathrm{K}}$ : os fotoelétrons.

As mesmas condições de simetria cilíndrica são consideradas para uma camada morta de espessura $d$ (Fig. 4.11). O foco, neste caso, são os elétrons que atingem o volume ativo com uma energia $\epsilon$. No modelo se supõe que: i) a energia $\epsilon$ é totalmente depositada no volume ativo uma vez que o elétron alcança o mesmo; ii) o ângulo de emissão $\theta$ define um caminho reto $t$, entre o ponto de origem $z$ e a superfície do volume ativo. Logo, $\epsilon$ pode ser aproximada a

$$
\epsilon \simeq E_{0, i}-S\left(E_{0, i}\right) t
$$

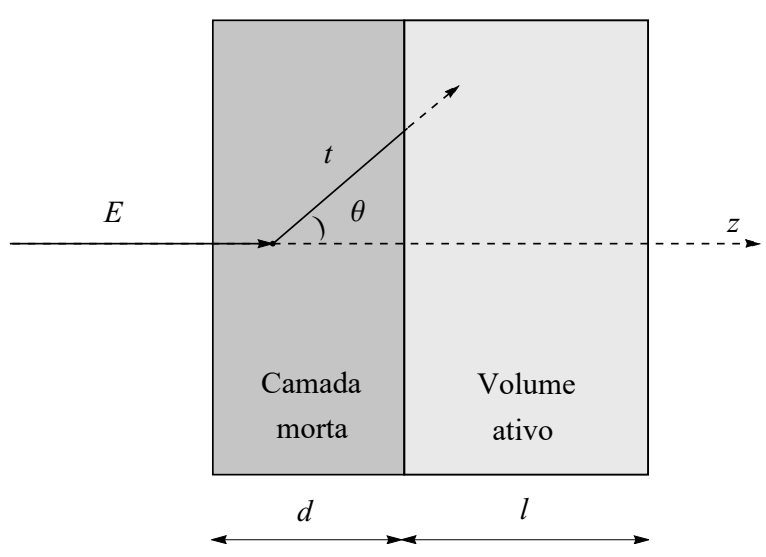

FigurA 4.11: Representação geométrica do processo de migração de fotoelétrons da camada morta de Ge ao volume ativo. Esquema fora de escala.

A probabilidade $P_{\text {ed }}^{\prime}(E, t ; d)$ de que um fotoelétron gerado na camada morta atinja o volume ativo depois de atravessar o caminho $t$ é descrita por uma relação igual à (4.46), substituindo a espessura do detetor $l$ pela espessura da camada morta $d$. Além disso, como os valores de $d$ geralmente são de apenas poucos $\mu \mathrm{m}^{5}$, trajetórias $t>d$ também serão possíveis, portanto é necessário somar um termo integrando a Eq. (4.44) entre 0 e $d$ para considerar esses caminhos.

A partir de $P_{\text {ed }}^{\prime}(E, t ; d)$ e a relação (4.61) pode ser calculado o espectro produzido pelos fotoelétrons referidos acima como

$$
P_{\text {ed }}(\epsilon, E ; d)=\sum_{i} \frac{q_{i}(E)}{S\left(E_{0, i}\right)} P_{\text {ed }}^{\prime}\left(E, t_{\epsilon i} ; d\right) \Theta\left(\epsilon-E_{0, i}\right)
$$

\footnotetext{
${ }^{5} \mathrm{~A}$ afirmação é válida para detetores de Ge de tipo n. Nos detetores de tipo p as camadas mortas podem alcançar os milímetros.
} 
com

$$
t_{\epsilon i}=\frac{E_{0, i}-\epsilon}{S\left(E_{0, i}\right)}
$$

\subsection{Simulações Monte Carlo para testar o modelo analítico de função resposta de detetores planares}

Com o objetivo de testar o modelo analítico de FR descrito na seção 4.3, realizaram-se simulações de espectros de altura de pulso (EAP) utilizando o código PENELOPE [43]. Este é um código MC de fonte aberta, escrito em Fortran, que permite a simulação do transporte de fótons, elétrons e pósitrons, no intervalo energético entre $50 \mathrm{eV}$ e $1 \mathrm{GeV}$. O programa principal penEasy [94] foi escolhido para avaliar o EAP de um detetor com parâmetros $l=10 \mathrm{~mm}$ e $r=16 \mathrm{~mm}$, que reproduzem as dimensões fornecidas pelo fabricante ORTEC na folha de dados do HPGe (GLP Series 32355 [51]) utilizado nas medidas de bremsstahlung deste trabalho $\left(\theta=35^{\circ}\right)$. Uma camada morta de espessura $d=1,50 \mu \mathrm{m}$ foi considerada na geometria da simulação. Esse dado foi determinado experimentalmente (ver subseção 6.2.2) pelo método sugerido por Maidana e colaboradores [95]. A janela de $\mathrm{Be}\left(t_{\mathrm{Be}}=0,254 \mathrm{~mm}\right)$ foi incluída à distância $l_{\mathrm{s}}=7 \mathrm{~mm}$ da superfície frontal do cristal de Ge, como se detalha no manual do detetor.

Para identificar cada uma das componentes da FR, as simulações foram feitas em diferentes etapas, de acordo com a hipótese original: incidência normal de um feixe puntual e monoenergético. Primeiro, foi obtido o EAP do cristal cilíndrico de Ge transportando unicamente fótons. Sob essas condições os elétrons secundários são forçados a depositar sua energia no ponto de origem. Logo, os eventos de absorção total, de escape Compton e de raios-x podem ser isolados, fornecendo um teste apropriado para as componentes $P_{\text {int }}, P_{\mathrm{K} \alpha}, P_{\mathrm{K} \beta}$ e $P_{\text {inc. }}$ Em uma segunda fase, o transporte de elétrons foi ativado para estudar o efeito de escape dessas partículas através das superfícies do cilindro $\left(P_{\mathrm{ev}}\right)$. Finalmente foram adicionadas a camada morta de Ge e a janela de Be, para checar as contribuições externas à FR: $P_{\text {ed }}$ e $P_{\mathrm{Be}}$.

Os EAP simulados se computaram desde os $5 \mathrm{keV}$ até a energia $E$ do fóton incidente, em histogramas com intervalos de classes $\delta=100 \mathrm{eV}$. A Fig. 4.12 apresenta um exemplo dos resultados obtidos para fótons de $60 \mathrm{keV}$ em cada uma das etapas de simulação. O espectro marcado pela letra A, corresponde ao transporte unicamente de fótons, observando-se as deltas dos picos de absorção total e de escape de raios-x K, bem como a estrutura característica do escape Compton. Quando se aciona o transporte de elétrons (espectro B), aparece um contínuo que une as componentes mencionadas, produto do escape próprio destas partículas ou de fótons gerados por elas. Ao ser adicionada a camada 
morta (espectro C), percebe-se um incremento do contínuo junto ao picos do absorção total e de escape simples de raios-x K. O efeito está associado a fotoelétrons K, L, etc, criados na camada morta que atingem o volume ativo de Ge. Finalmente, a introdução da janela de Be (espectro D), produz o efeito de degrau do lado esquerdo do pico de absorção total, causado pelo espalhamento inelástico dos fótons.

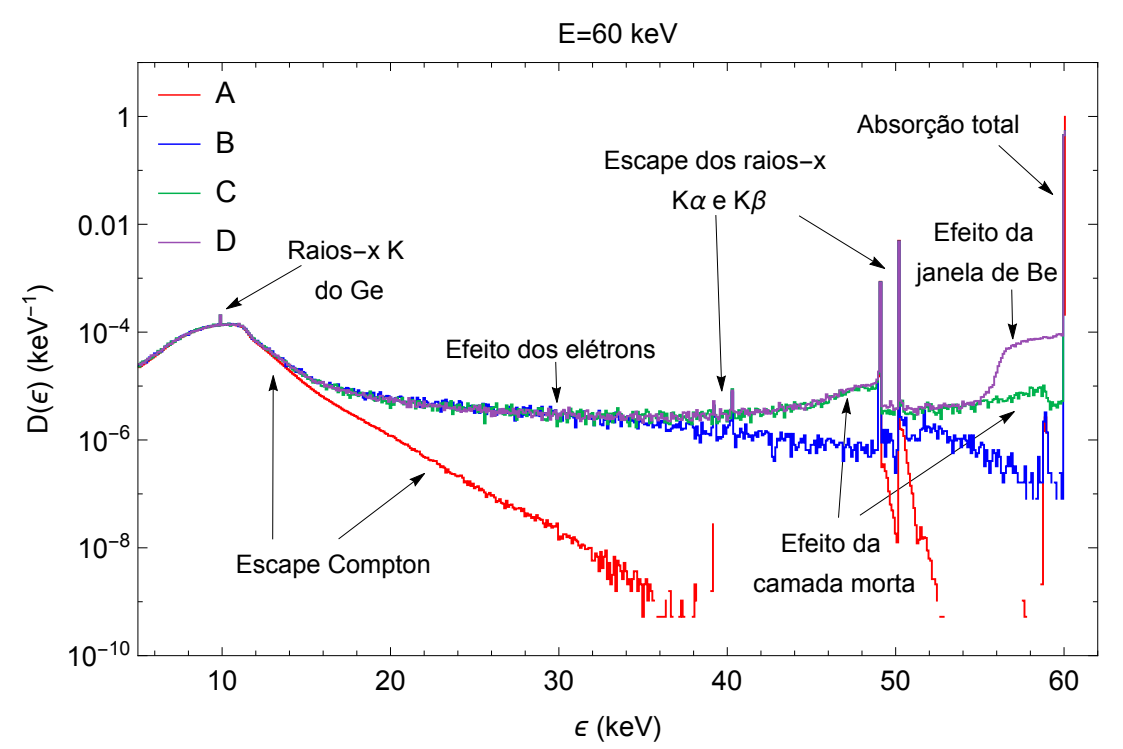

Figura 4.12: Simulação Penelope de espectros de energia deposita no cristal de Ge para um fóton incidente de $60 \mathrm{keV}$. No espectro marcado com a letra A foram transportados somente fótons no volume ativo, em B se transportaram fótons e elétrons, em $\mathrm{C}$ foi adicionada a camada morta e em D a janela de Be.

A fim de fazer uma comparação mais realística, tanto os espectros $D(\epsilon, E ; l, r)$ simulados quanto os analíticos foram convoluidos com uma função Gaussiana de acordo com a Eq. (4.14). A variância da distribuição foi

$$
\sigma^{2}(\epsilon)=a+b \epsilon
$$

O ruido eletrônico foi simulado adotando $a=(0,2 \mathrm{keV})^{2}$, que é um valor tipicamente obtido em medidas (ver subseção 6.2.1). Por outro lado, a constante $b=0,3 \mathrm{eV}$ foi derivada do trabalho de Gao e colaboradores [96], como o produto do fator de Fano em Ge $F_{\mathrm{Ge}}=0,11$ e a energia requerida para criar um par elétron-buraco $W_{\mathrm{Ge}}=2,64 \mathrm{eV}$.

A convolução Gaussiana de um espectro simulado para $E=20 \mathrm{keV}$, incluindo todos os elementos da geometria do detetor, é apresentada na Fig. 4.13. O EAP da saída do PENELOPE [43], simbolizado por $D(\epsilon)$, foi multiplicado pela largura da classe $\delta$ para obter a probabilidade absoluta de depositar uma energia $\epsilon$. O espectro convoluído é 
designado por $N(h)$. Nota-se como o efeito da janela de Be resulta imperceptível devido à resolução do pico de absorção total. Por outro lado, no espectro $D(\epsilon)$, os picos de escape de raios-x $\mathrm{K}$ se observam em $\epsilon=10,1 \mathrm{keV}$ e $\epsilon=9,0 \mathrm{keV}$, e além disso, aparecem picos em $\epsilon=9,9 \mathrm{keV}$ e $\epsilon=11 \mathrm{keV}$, de menor intensidade, correspondentes as energias dos raios-x $\mathrm{K} \alpha$ e $\mathrm{K} \beta$ do Ge respectivamente. Este é outro fenômeno introduzido no espectro pela camada morta, mas não é levado em conta no modelo analítico proposto neste trabalho devido a que esses fótons possuem energias abaixo da borda $\mathrm{K}$ do Ge, fora da faixa de interesse para a deconvolução dos espectros de bremsstrahlung.

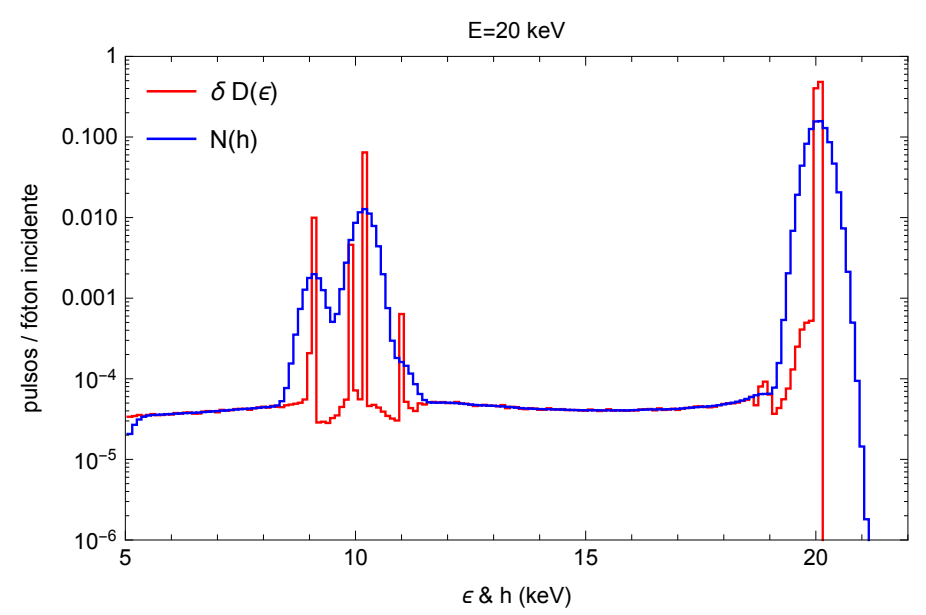

Figura 4.13: Convolução Gaussiana do espectro de altura de pulso simulado com o código PENELOPE para $E=20 \mathrm{keV}$.

\subsubsection{Espectro de escape Compton}

A Fig. 4.14 apresenta os espectros produzidos pelo escape de fótons via espalhamento Compton para radiação incidente de 40, 60, 80 e $100 \mathrm{keV}$, calculados utilizando a SCDD da AIR, a SCD de KN normalizada pela seção de choque incoerente de WH e a simulação MC. O EAP de PENELOPE foi obtido em simulações em que unicamente são transportados fótons, similar ao espectro A da Fig. 4.12. Na FR analítica se considera que a energia do elétron ejetado depois de uma colisão Compton é totalmente absorbida no volume ativo, portanto, essas simulações são coerentes com as hipóteses do modelo. Devido a que os cálculos de $P_{\text {inc }}(\epsilon, E ; l, r)$ incluem somente uma interação inelástica, as diferenças observadas nos espectros se devem ao espalhamento múltiplo dos fótons no cristal de Ge. Porém, nota-se uma excelente coincidência entre as formas dos espectros calculado com a AIR e o simulado. Como era de esperar a SCD de KN não é adequada para descrever corretamente o EAP no intervalo de energias de interesse neste trabalho.

A fórmula de Compton (4.25) estabelece que a energia máxima adquirida pelo elétron após uma colisão é

$$
\epsilon_{1 \mathrm{C}}(E)=E\left[1-(1+2 \varepsilon)^{-1}\right]
$$


Se duas colisões sucessivas acontecem $\operatorname{com} \theta=\pi$, os elétrons recebem uma energia máxima dada por

$$
\epsilon_{2 \mathrm{C}}(E)=E\left[1-(1+4 \varepsilon)^{-1}\right]
$$
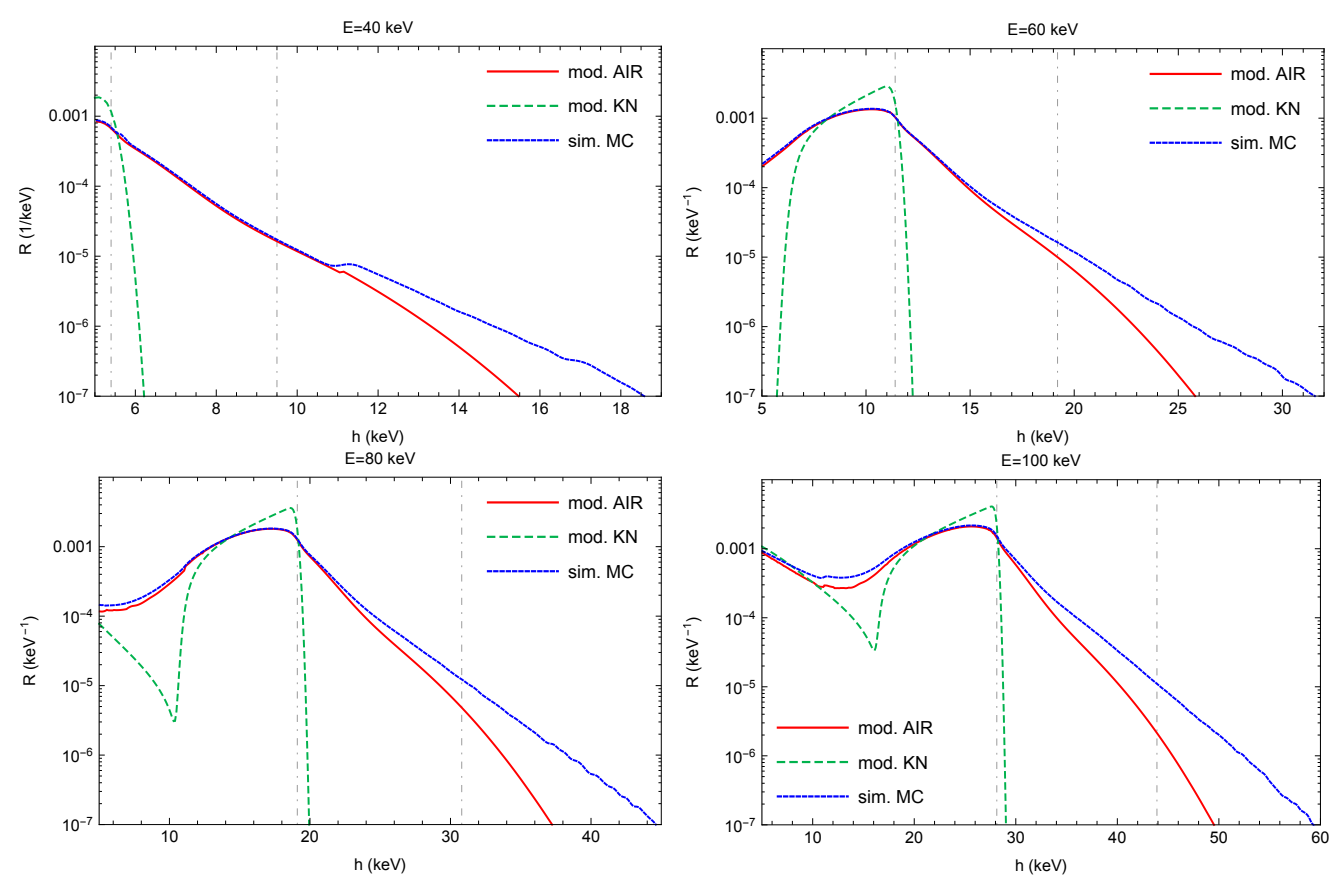

Figura 4.14: Contínuo de Compton produzido pelo escape de fótons para energias incidentes $E=40,60,80$ e $100 \mathrm{keV}$. Comparação entre o modelo analítico e a simulação PENELOPE. As linhas verticais estão situadas nos valores de $\epsilon_{1 \mathrm{C}}(E)$ Eq. (4.65) e $\epsilon_{2 \mathrm{C}}(E)$

Eq. (4.66).

As linhas descontinuas verticais nos gráficos da Fig. 4.14 marcam as posições $\epsilon_{1 \mathrm{C}}$ e $\epsilon_{2 \mathrm{C}}$. Observa-se que um único evento de interação é suficiente para explicar a maior parte da área acima de $\epsilon_{1 \mathrm{C}},\left[\epsilon_{1 \mathrm{C}}(40)=5,4 \mathrm{keV}, \epsilon_{1 \mathrm{C}}(60)=11,4 \mathrm{keV}, \epsilon_{1 \mathrm{C}}(80)=19,1 \mathrm{keV}\right.$, $\left.\epsilon_{1 \mathrm{C}}(100)=28,1 \mathrm{keV}\right]$ utilizando a AIR. De fato, para 40, 60 e $80 \mathrm{keV}$, o espectro analítico reproduz em mais de $95 \%$ a área da simulação. No caso de $100 \mathrm{keV}$, a fração é 90\%. Seltzer [56] introduz em $P_{\text {inc }}$ um termo empírico de correção por espalhamento inelástico múltiplo para energias depositadas superiores a $\epsilon_{1 \mathrm{C}}(E)$, onde a sua FR diminui abruptamente. A partir dos cálculos e simulações realizadas pode se concluir que a contribuição à FR por duplo ou múltiplo espalhamento é apreciável somente bem acima de $\epsilon_{1 \mathrm{C}}(E)$.

\subsubsection{Função resposta total}

Seguindo a ordem descrita na seção 4.4, e para fótons de 20, 60 e $100 \mathrm{keV}$, são apresentados uma sequência de espectros introduzindo sucessivamente as componentes do modelo de FR. 
Os espectros A das Figs. 4.15, 4.16 e 4.17 são produzidos por eventos de escape dos fótons do cilindro de $\mathrm{Ge}\left(P_{\mathrm{K} \alpha}, P_{\mathrm{K} \beta}\right.$ e $\left.P_{\text {inc }}\right)$ e de absorção total $\left(P_{\text {int }}\right)$. Um bom acordo é alcançado entre o modelo analítico e o simulado. Além das diferenças discutidas no contínuo de Compton, as principais discrepâncias são devidas ao escape de raios-x L do Ge (esquerda do fotopico) e escape de raios-x K após uma colisão inelástica (direita do pico de escape $\mathrm{K} \alpha$ ). Esses fenômenos tem maior visibilidade no espectro de $20 \mathrm{keV}$ pelo pequeno intervalo de energia analisado, e são evidentemente desprezíveis.
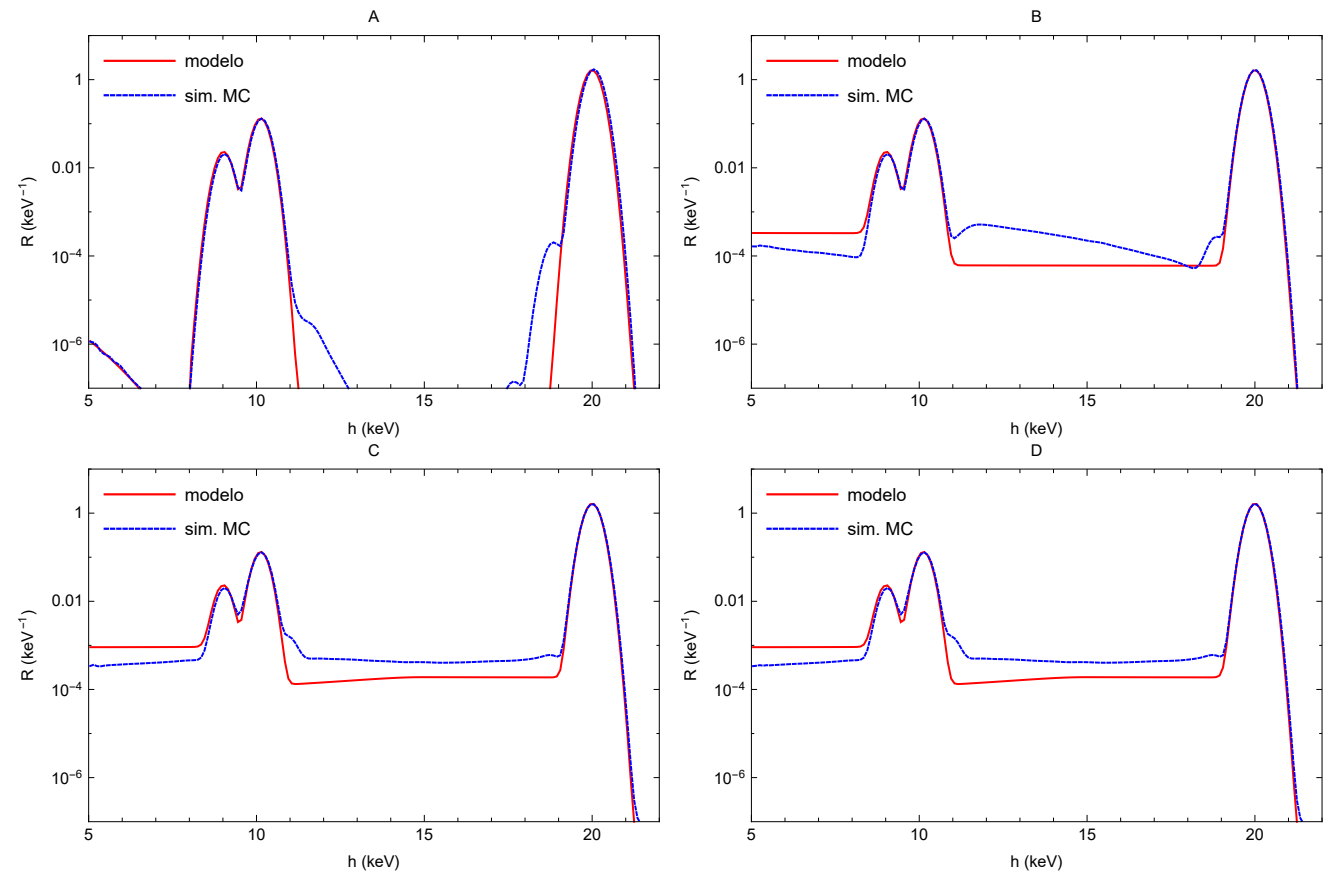

Figura 4.15: Componentes da FR calculadas (modelo) e simuladas (sim. MC) para $E=20 \mathrm{keV}$. Os gráficos incorporam aditivamente a sequência: (A) simulação somente do transporte de fótons no cilindro de $\mathrm{Ge},(\mathrm{B})$ simulação do transporte de fótons e elétrons no cilindro de Ge, (C) a camada morta de Ge foi adicionada, e (D) a janela de

Be foi incluída.

Quando o transporte de elétrons é acionado (espectros B), aparece o contínuo que une as estruturas observadas em A. O modelo analítico, por meio da Eq. (4.48), prevê um EAP praticamente constante para todas as energias. Esta é uma consequência direta da integração da distribuição angular de Sauter (4.41) sob os mesmo limites para todas as trajetórias retas $t$, e também, devido a que o fator exponencial nas Eqs. (4.44) e (4.45) é quase constante no intervalo de integração. No entanto, levando em conta os valores típicos de resolução dos detetores HPGe e as energias das bordas das diferentes camadas do átomo de Ge, para avaliar a expressão (4.48) se consideraram explicitamente os elétrons ejetados da camada K, e aqueles das restantes camadas foram tomados como um único grupo, utilizando a probabilidade efetiva de fotoionização $q_{\mathrm{ef}}=1-q_{\mathrm{K}}(E)$, e desprezando a suas energias de ligação. Dessa forma, o contínuo entre o pico de absorção total e os picos de escape, foi modelado por elétrons das camadas diferentes 

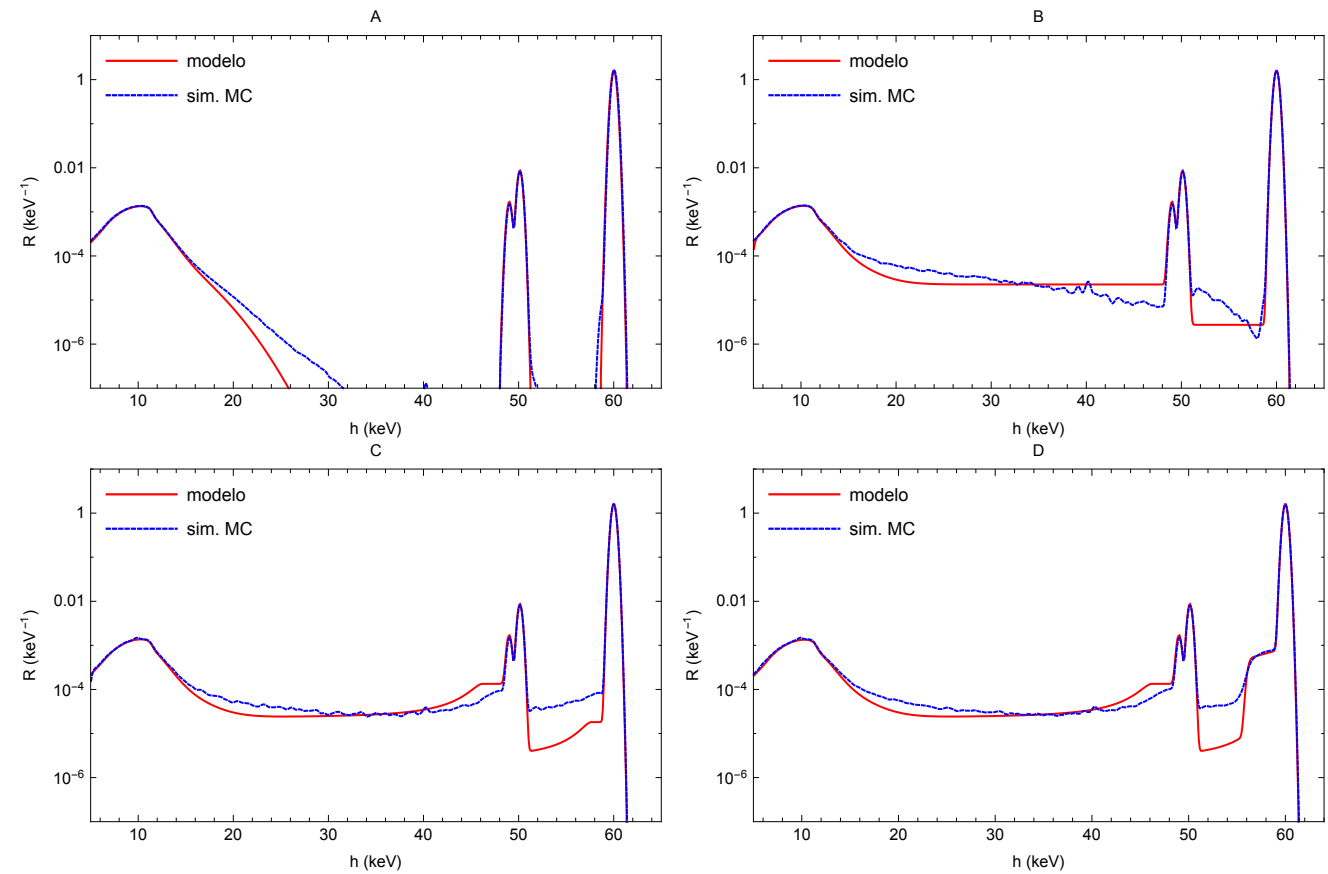

Figura 4.16: Componentes da FR calculadas (modelo) e simuladas (sim. MC) para $E=60 \mathrm{keV}$. A sequência (A)-(D) segue a mesma ordem da Fig. 4.15.
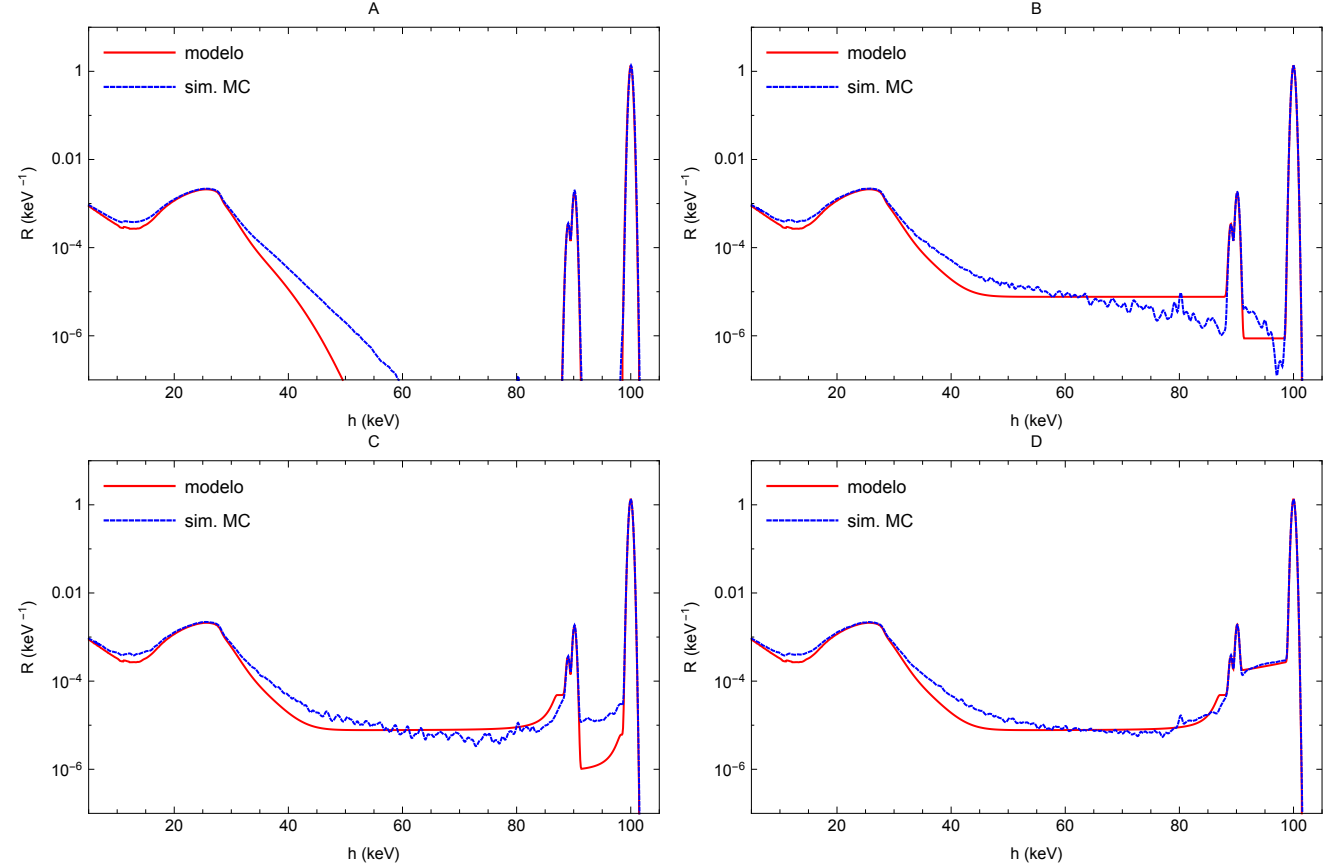

Figura 4.17: Componentes da FR calculadas (modelo) e simuladas (sim. MC) para $E=100 \mathrm{keV}$. A sequência (A)-(D) segue a mesma ordem da Fig. 4.15.

à $\mathrm{K}$, e para energias inferiores a $E-U_{\mathrm{K}}$ por todos os elétrons do Ge. O formalismo funciona melhor quanto maior for a energia do fóton incidente, sendo a aproximação de trajetórias retilíneas mais adequada. Para $E=60$ e $100 \mathrm{keV}$, pode se observar como o modelo analítico gera um espectro contínuo próximo ao valor médio do EAP simulado nessa região. 
Pequenas modificações são introduzidas no espectro pela camada morta de Ge (gráficos $\mathrm{C}$ das Figs. 4.15, 4.16 e 4.17). Nota-se o incremento das contagens à esquerda do pico de absorção total e dos picos de escape. O modelo analítico fornece uma distribuição praticamente constante para energias correspondentes a trajetórias entre 0 e $d$, de maneira similar ao caso anterior, mas para caminhos maiores, um rápido decrescimento é previsto. O comportamento pode ser entendido a partir das condições geométricas assumidas; no intervalo $t \in[0, d]$, são adicionadas contribuições de elétrons emitidos entre 0 e 90 graus, contudo, para caminhos maiores que $d$, o intervalo angular começa ser menor e portanto se reduz a intensidade do processo. Assim, as variações do espectro podem ser intuitivamente explicadas com esse argumento, ainda no caso das simulações, onde os elétrons não seguem uma linha reta ao longo da sua história. Para elétrons da camada K os cálculos analíticos superestimam este fenômeno quando comparado com EAP do PENELOPE, entretanto o efeito contrário se produz para elétrons das restantes camadas do átomo de Ge.

Finalmente, nos gráficos D das Figs. 4.16 e 4.17, observa-se claramente o efeito da janela de Be à esquerda do pico de absorção total. Uma excelente concordância entre a simulação e o modelo analítico é atingida nesta região do espectro.

Para quantificar a importância que tem no espectro os fenômenos não considerados no modelo de FR, calculou-se a função de acumulação de probabilidade de $R(h, E ; l, r)$

$$
\Gamma(h)=\int_{0}^{h} R\left(E, h^{\prime} ; l, r\right) \mathrm{d} h^{\prime} .
$$

A Fig. 4.18 apresenta o EAP analítico e da simulação, acompanhado da correspondente função $\Gamma(h)$ para $E=20,60$ e $100 \mathrm{keV}$. As variações em $\Gamma(h)$ se devem à aparição das diferentes contribuições à FR. Observa-se que ambos espectros estão igualmente distribuídos no intervalo de energias analisado. As diferenças na área computada na simulação e no modelo à esquerda da estrutura do foto pico são menores que $1 \%$.

Adicionalmente foi calculada a área que cada componente de $R(h, E ; l, r)$ em que a energia é depositada parcialmente (Fig. 4.19). O escape de raios-x K de Ge constitui a contribuição principal no intervalo $12 \mathrm{keV} \lesssim E \lesssim 55 \mathrm{keV}$. Além disso, os elétrons que entram ou escapam do volume ativo são importantes para $E \lesssim 35 \mathrm{keV}$, onde a área deste fenômeno oscila entre $0,5 \%$ e $1 \%$. Para energias acima de $55 \mathrm{keV}$, o escape Compton é dominante, alcançando valores de $3 \%$ da área total do EAP em $100 \mathrm{keV}$. Os processos de absorção total se maximizam na região $50 \mathrm{keV} \lesssim E \lesssim 70 \mathrm{keV}$, sendo os mecanismos de deposição incompleta menores que $2 \%$. Em concordância com os argumentos expostos, a forma da linha do gráfico que corresponde à soma de todos os processos representa uma imagem espelho das habituais curvas de eficiência de detetores de Ge (ver Fig. 6.10). 

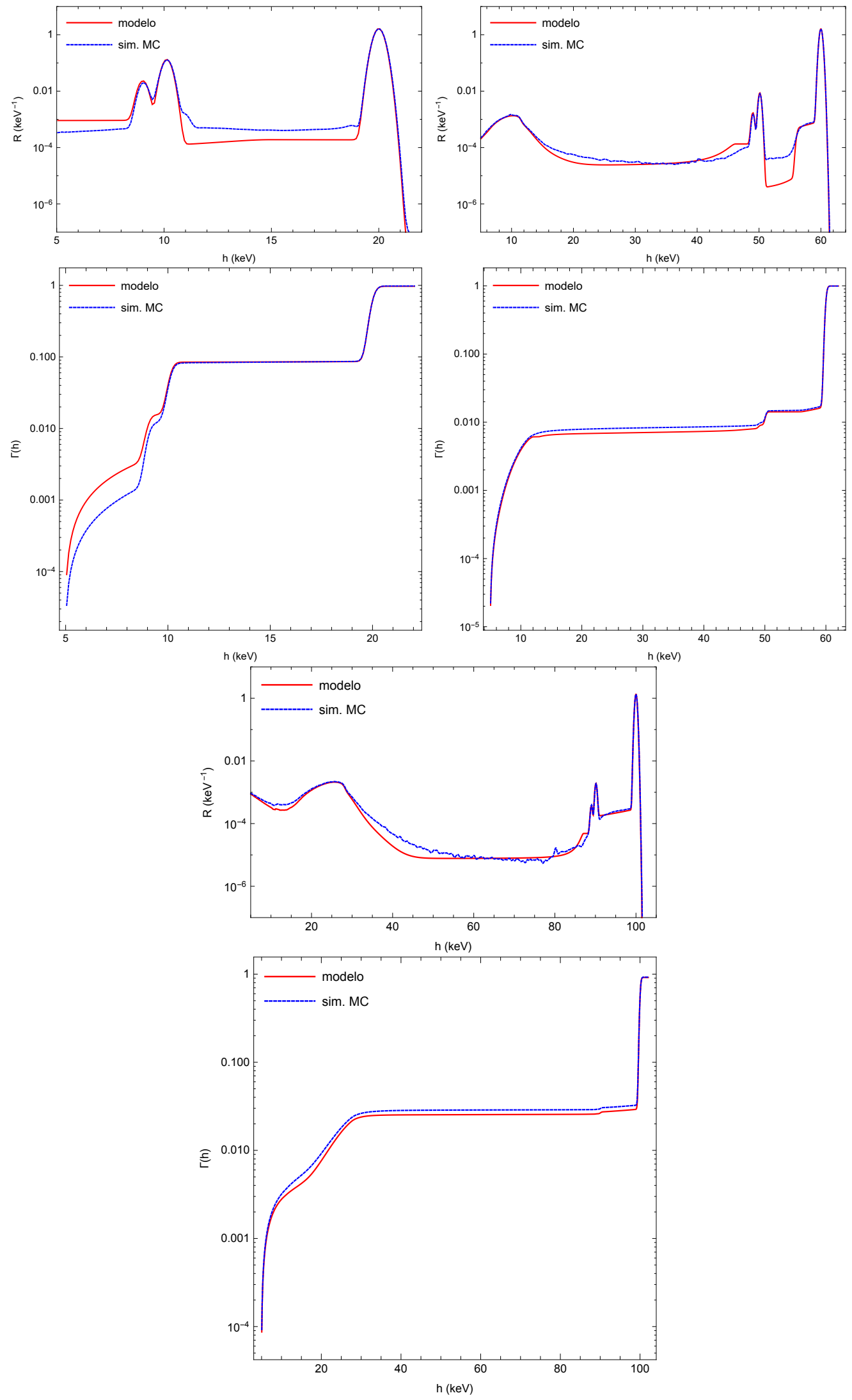

FIgURA 4.18: Função resposta total acompanhada pela sua respectiva função de acumulação de probabilidade para $E=20,60$ e $100 \mathrm{keV}$. 


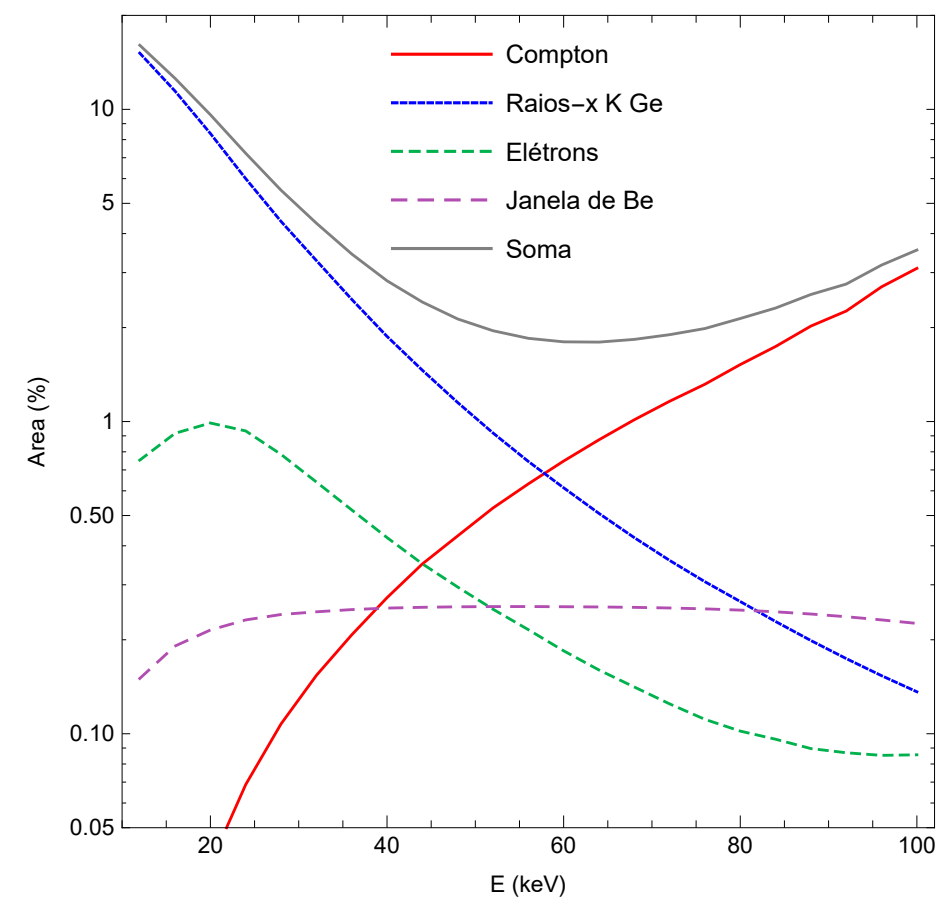

FIGURA 4.19: Área do espectro que representa cada mecanismo de deposição parcial de energia.

\subsection{Modelo analítico de função resposta para detetores co- axiais}

Os detetores coaxiais de Ge são desenhados para espectrometria de fótons na faixa energética de até os poucos $\mathrm{MeV}$. Em relação aos espectrômetros planares o volume de interação é aumentado, apresentando um cristal com uma configuração aproximadamente como a mostrada na Fig. 4.20. O campo elétrico para a coleção da carga é gerado na direção radial, e o volume ativo é refrigerado por meio do dedo frio introduzido no orifício central. Esse último dispositivo conduz o calor entre o depósito de nitrogênio líquido e o cristal Ge. Uma descrição detalhada do funcionamento dos detetores coaxiais pode se encontrar na referência [81].

O objetivo de esta seção é modelar a FR de detetores coaxiais na faixa de energia de interesse do presente trabalho. Assim, partindo da geometria simplificada do cristal que representa a Fig. 4.20 é possível generalizar o modelo desenvolvido para a configuração planar ao caso coaxial. Assume-se incidência de fótons ao longo do eixo de simetria z. Algumas componentes da FR podem ser derivadas diretamente. Como foi mostrado na seção 4.3.2, o escape de raios-x $\mathrm{K}$ de Ge pela superfície frontal do detetor independe dos parâmetros geométricos do cristal, logo, esta contribuição é calculada segundo a Eq. (4.20). De maneira similar, as equações desenvolvidas na seção 4.3 .4 que descrevem o escape de elétrons só dependem da espessura $l$ do detetor. Para a modelação desse 


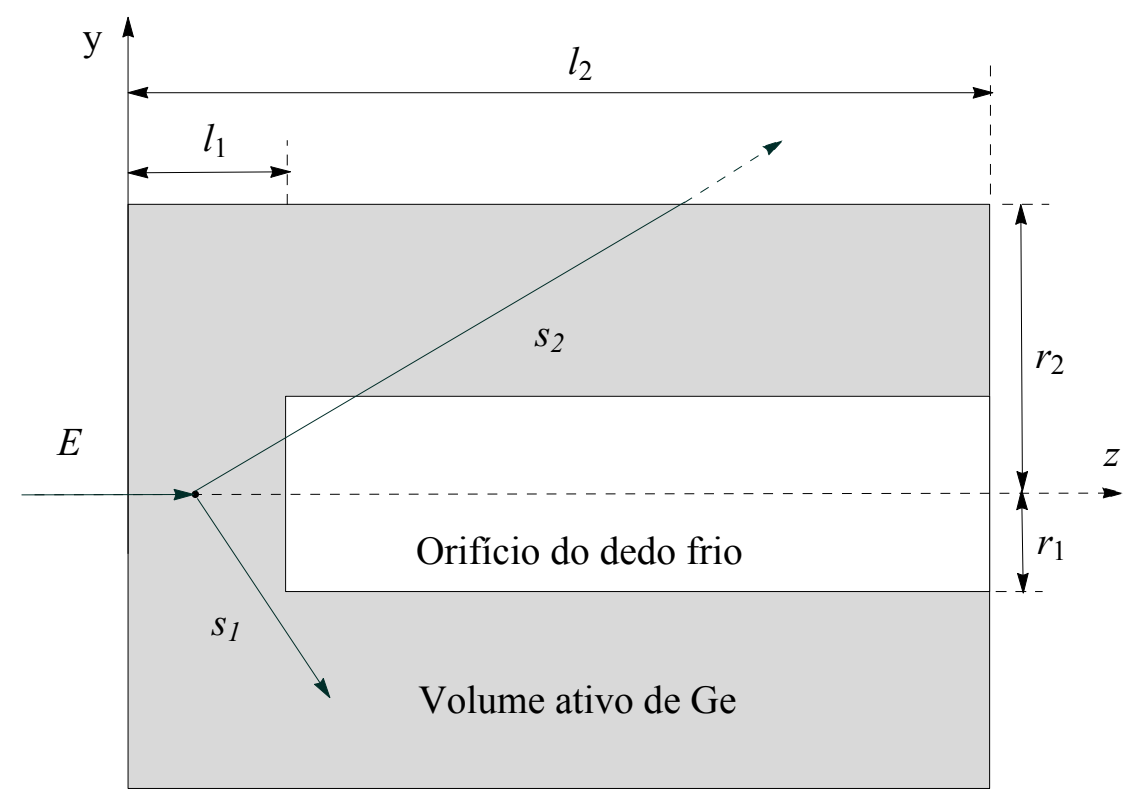

FiguRA 4.20: Representação geométrica simplificada do cristal de um detetor coaxial de Ge.

fenômeno no coaxial e considerando a sua ordem de grandeza, pode se admitir $l=l_{1}$ (Fig. 4.20) sem grandes riscos devido à hipótese da incidência do feixe.

Por outro lado, deve se esperar que a contribuição à FR intrínseca do escape Compton experimente modificações. Para esclarecer melhor esta ideia é construtivo fazer a comparação entre a geometria da Fig. 4.20 e um cilindro de raio $r_{2}$ e espessura $l_{1}$, que representa somente, a parte da frente do mencionado esquema. De maneira qualitativa se entende que os fótons espalhados no intervalo $0 \leqslant z \leqslant l_{1}$, na direção positiva do eixo de simetria, tenham uma probabilidade maior de serem posteriormente absorvidos na configuração coaxial em relação à planar (caminho $s_{1}$ na Fig. 4.20). Por consequência a probabilidade de escape será menor, e o continuo de Compton para baixas energias do EAP deve ser também menos intenso. Porém, se o fóton sofre um retroespalhamento, as probabilidades de escape em ambas geometrias tem que ser iguais. O planteamento anterior se traduz em formas do contínuo Compton iguais na região de máxima energia depositada no EAP. Como resultado adicional se prevê um aumento da componente de absorção total.

$\mathrm{Na}$ modelagem do espectro produzido pelo escape Compton em um detetor coaxial resulta fundamental a hipótese de simetria escolhida na incidência dos fótons. Sob essa condição pode se interpretar o volume ativo de Ge como um cilindro de raio $r_{2} e$ espessura $l_{2}$ que possui um coeficiente de atenuação $\mu$ dependente de $E$, e das variáveis 
de posição y e z (Fig. 4.20) segundo

$$
\mu(E ; y, z)=\mu_{\mathrm{Ge}}(E) \delta(y, z)
$$

com

$$
\delta(y, z)=\left\{\begin{array}{llll}
1 & 0 \leqslant z \leqslant l_{1} & \text { e } & 0 \leqslant y \leqslant r_{2} \\
0 & l_{1}<z \leqslant l_{2} & \text { e } & 0 \leqslant y<r_{1} \\
1 & l_{1} \leqslant z \leqslant l_{2} & \text { e } & r_{1} \leqslant y \leqslant r_{2}
\end{array}\right.
$$

A faixa $\delta(y, z)=0$ corresponde à região do orifício do dedo frio, onde se assume que os fótons não interagem durante seu passo. Por tanto o espectro de escape Compton vai estar definido pelos eventos de espalhamento que acontecem no intervalo $0 \leqslant z \leqslant l_{1}$. Assim, a probabilidade de que um fóton de energia $E$ escape do detetor com uma energia $E^{\prime}$, após experimentar uma colisão inelástica na direção definida pelo ângulo sólido $\mathrm{d} \Omega^{\prime}=2 \pi \mathrm{d} x$ pode ser escrita como (ver Eq. (4.26))

$$
\mathrm{d} P_{\mathrm{inc}}^{\prime}\left(E, E^{\prime}, x ; \mathbf{g}\right)=\frac{2 \pi \mathcal{N}_{\mathrm{Ge}}}{\mu_{\mathrm{Ge}}(E)} \frac{\mathrm{d}^{2} \sigma_{\mathrm{AIR}}\left(E, E^{\prime}, x\right)}{\mathrm{d} E^{\prime} \mathrm{d} \Omega^{\prime}} \mathrm{d} x G\left(E, E^{\prime}, x ; \mathbf{g}\right) .
$$

O fator adimensional $G\left(E, E^{\prime}, x ; \mathbf{g}\right)$ contem as dependências geométricas do problema no vetor dos parâmetros $\mathbf{g}=\left(l_{1}, l_{2}, r_{1}, r_{2}\right)$ e está dado por

$$
G\left(E, E^{\prime}, x ; \mathbf{g}\right)=\mu(E ; y, z) \int_{0}^{l_{1}} \exp [-\mu(E ; y, z) z] \exp \left[-\mu\left(E^{\prime}, y, z\right) s(z, x ; \mathbf{g})\right] \mathrm{d} z .
$$

Como a integração é realizada no intervalo $0 \leqslant z \leqslant l_{1}$, o coeficiente de atenuação no primeiro exponencial da expressão anterior pode ser tomado diretamente como $\mu(E ; y, z)=$ $\mu_{\mathrm{Ge}}(E)$. Por outro lado, a avaliação do caminho $s$ que o fóton percorre antes de sair do volume ativo tem dois casos:

A) $\mathrm{O}$ escape produto de espalhamentos nos ângulos $90^{\circ} \leqslant \theta \leqslant 180^{\circ}$, situação que é descrita pelas mesmas equações obtidas para um detetor planar de raio $r_{2}$ e espessura $l_{1}, \operatorname{com} \mu(E ; y, z)=\mu_{\mathrm{Ge}}(E)$.

B) O escape devido a espalhamentos frontais $\left(0^{\circ} \leqslant \theta<90^{\circ}\right)$ onde os fótons podem sair do volume ativo, atravessar o orifício do dedo frio e voltar entrar no cristal de Ge.

Na situação B), o argumento do segundo exponencial da Eq. (4.71) é diferente de zero na fração $s_{\mathrm{ef}}(z, x ; \mathbf{g})$ do caminho real $s(z, x ; \mathbf{g})$ em que é possível a interação do fóton, 
portanto pode se redefinir como

$$
\mu\left(E^{\prime} ; y, z\right) s(z, x ; \mathbf{g})=\mu_{\mathrm{Ge}}\left(E^{\prime}\right) s_{\mathrm{ef}}(z, x ; \mathbf{g})=\mu_{\mathrm{Ge}}\left(E^{\prime}\right) \int_{s} \delta(y, z) \mathrm{d} s .
$$

A integral de linha acima é resolvida dependendo da superfície de saída do fóton. Se o escape se produz pela superfície posterior $\left(z=l_{2}, y>r_{1}\right)$, se obtém

$$
s_{\mathrm{ef}}(z, x ; \mathbf{g})=\int_{s} \delta(y, z) \mathrm{d} s=\frac{l_{1}+l_{2}}{x}-\frac{r_{1}}{\sqrt{1-x^{2}}}-\frac{2 z}{x}=g_{1}(x)-\frac{2 z}{x} .
$$

Enquanto os escapes pelas laterais (caminho $s_{2}$ na Fig. 4.20) são descritos por

$$
s_{\mathrm{ef}}(z, x ; \mathbf{g})=\int_{s} \delta(y, z) \mathrm{d} s=\frac{r_{2}-r_{1}}{\sqrt{1-x^{2}}}+\frac{l_{1}-z}{x}=g_{2}(x)+\frac{l_{1}-z}{x} .
$$

Logo, o fator $G\left(E, E^{\prime}, x ; \mathbf{g}\right)$ pode se expressar em termos de $x$ e $E^{\prime}$ para cada uma das possíveis direções de escape do fóton ${ }^{6}$ :

1) Para $1 \geqslant x \geqslant x_{1}$, sendo $x_{1}=l_{2} / \sqrt{l_{2}^{2}+r_{1}^{2}}$,

$$
G=\xi_{1}^{-1} \mathrm{e}^{-\mu l_{1}}\left[1-\mathrm{e}^{-\xi_{1} \mu l_{1}}\right]
$$

$\operatorname{com} \xi_{1}=\left(\mu^{\prime} / \mu x\right)-1$. No limite $x=1\left(\theta=0^{\circ}\right), \xi_{1} \rightarrow 0$ e $G=\mu l_{1} \mathrm{e}^{-\mu l_{1}}$.

2) Para $x_{1}>x \geqslant x_{2}$, sendo $x_{2}=l_{2} / \sqrt{l_{2}^{2}+r_{2}^{2}}$,

$$
G=\xi_{2}^{-1} \mathrm{e}^{-\mu^{\prime} g_{1}}\left[\mathrm{e}^{\xi_{2} \mu l_{1}}-1\right]
$$

com $\xi_{2}=\left(2 \mu^{\prime} / \mu x\right)-1$. Neste caso se considerou que para o intervalo angular analisado é válida a relação $l_{2}-r_{1} x / \sqrt{1-x^{2}}>l_{1}$ e o escape sempre acontece para valores $y>r_{1}$.

3) Para $x_{2}>x \geqslant x_{3}$, sendo $x_{3}=l_{1} / \sqrt{l_{1}^{2}+r_{1}^{2}}$,

$$
\begin{aligned}
& G=\xi_{1}^{-1} \mathrm{e}^{-\mu^{\prime} g_{2}} \mathrm{e}^{-\left(\xi_{1}+1\right) \mu l_{1}}\left[\mathrm{e}^{\xi_{1} \mu z_{1}}-1\right]+\xi_{2}^{-1} \mathrm{e}^{-\mu^{\prime} g_{1}}\left[\mathrm{e}^{\xi_{2} \mu l_{1}}-\mathrm{e}^{\xi_{2} \mu z_{1}}\right], \\
& \operatorname{com} z_{1}=l_{2}-r_{2} x / \sqrt{1-x^{2}} .
\end{aligned}
$$

\footnotetext{
${ }^{6}$ É utilizada a notação reduzida: $\mu \equiv \mu_{\mathrm{Ge}}(E), \mu^{\prime} \equiv \mu_{\mathrm{Ge}}\left(E^{\prime}\right), g_{1}(x) \equiv g_{1}, g_{2}(x) \equiv g_{2}$.
} 
4) Para $x_{3}>x>0$,

$$
\begin{aligned}
G & =\mathrm{e}^{-\left(\xi_{1}+1\right) \mu r_{2} x / \sqrt{1-x^{2}}}\left[1-\mathrm{e}^{-\mu z_{2}}\right] \\
& +\xi_{1}^{-1} \mathrm{e}^{-\mu^{\prime} g_{2}} \mathrm{e}^{-\left(\xi_{1}+1\right) \mu l_{1}}\left[\mathrm{e}^{\xi_{1} \mu l_{1}}-\mathrm{e}^{\xi_{1} \mu z_{2}}\right],
\end{aligned}
$$

$\operatorname{com} z_{2}=l_{1}-r_{1} x / \sqrt{1-x^{2}}$.

5) Para $x=0$,

$$
G=\mathrm{e}^{-\mu^{\prime} r_{2}}\left[1-\mathrm{e}^{-\mu l_{1}}\right]
$$

6) Para $0>x>-x_{4}$, sendo $x_{4}=l_{1} / \sqrt{l_{1}^{2}+r_{2}^{2}}$,

$$
G=\left(1+\xi_{1}^{-1}\right) \mathrm{e}^{-\xi_{1} \mu r_{2} x / \sqrt{1-x^{2}}}-\xi_{1}^{-1}-\mathrm{e}^{-\mu l_{1}} \mathrm{e}^{-\left(\xi_{1}+1\right) \mu r_{2} x / \sqrt{1-x^{2}}} .
$$

7) Para $-x_{4} \geqslant x \geqslant-1$,

$$
G=\xi_{1}^{-1}\left[\mathrm{e}^{-\xi_{1} \mu l_{1}}-1\right]
$$

O espectro de energia depositada no detetor $P_{\text {inc }}(E, \epsilon ; \mathbf{g})$ se calcula integrando a Eq. (4.70) para todos os valores de $x$ entre -1 e 1 , e levando em conta a relação entre a energia do fóton espalhado $E^{\prime}$ e do elétron $\epsilon=E-E^{\prime}$.

\subsubsection{Teste do modelo, comparação com simulações Monte Carlo}

O formalismo anterior foi aplicado a um detetor com parâmetros: $l_{1}=8,3 \mathrm{~mm}, l_{2}=$ $66,3 \mathrm{~mm}, r_{1}=5,0 \mathrm{~mm}$ e $r_{2}=29 \mathrm{~mm}$. Os limites angulares nesse caso são $x_{1}=0,997$ $\left(\theta=4,31^{\circ}\right), x_{2}=0,916\left(\theta=23,62^{\circ}\right), x_{3}=0,857\left(\theta=31,07^{\circ}\right)$ e $x_{4}=0,275(\theta=$ $\left.74,03^{\circ}\right)$. Essas dimensões reproduzem aquelas fornecidas pelo fabricante ORTEC do detetor GMX30P [51], utilizado nas medidas de bremsstrahlung $\left(\theta=131^{\circ}\right)$. Retomando a comparação proposta no início da seção, os cálculos foram realizados também para um detetor planar de espessura $l_{1}=8,3 \mathrm{~mm}$ e raio $r_{2}=29 \mathrm{~mm}$, estudando a resposta Compton que ambas configurações fornecem a diferentes energias dos fótons incidentes.

A Fig. 4.21 apresenta os resultados da comparação. Segundo o esperado, as diferenças se observam para energias acima de $70 \mathrm{keV}$, na região do espectro correspondente a espalhamentos na faixa angular $0^{\circ} \leqslant \theta \leqslant 90^{\circ}$. Em eventos deste tipo, a probabilidade de escape no detetor coaxial (com os parâmetros utilizados) é muito menor, portanto diminui o contínuo gerado em relação ao detetor planar. Abaixo de $70 \mathrm{keV}$, ambos detetores mostram uma resposta análoga em concordância com o fato de que o caminho livre médio dos fótons é menor assim que a energia diminui, e portanto a interação dos fótons acontece nos primeiros milímetros do volume ativo. 

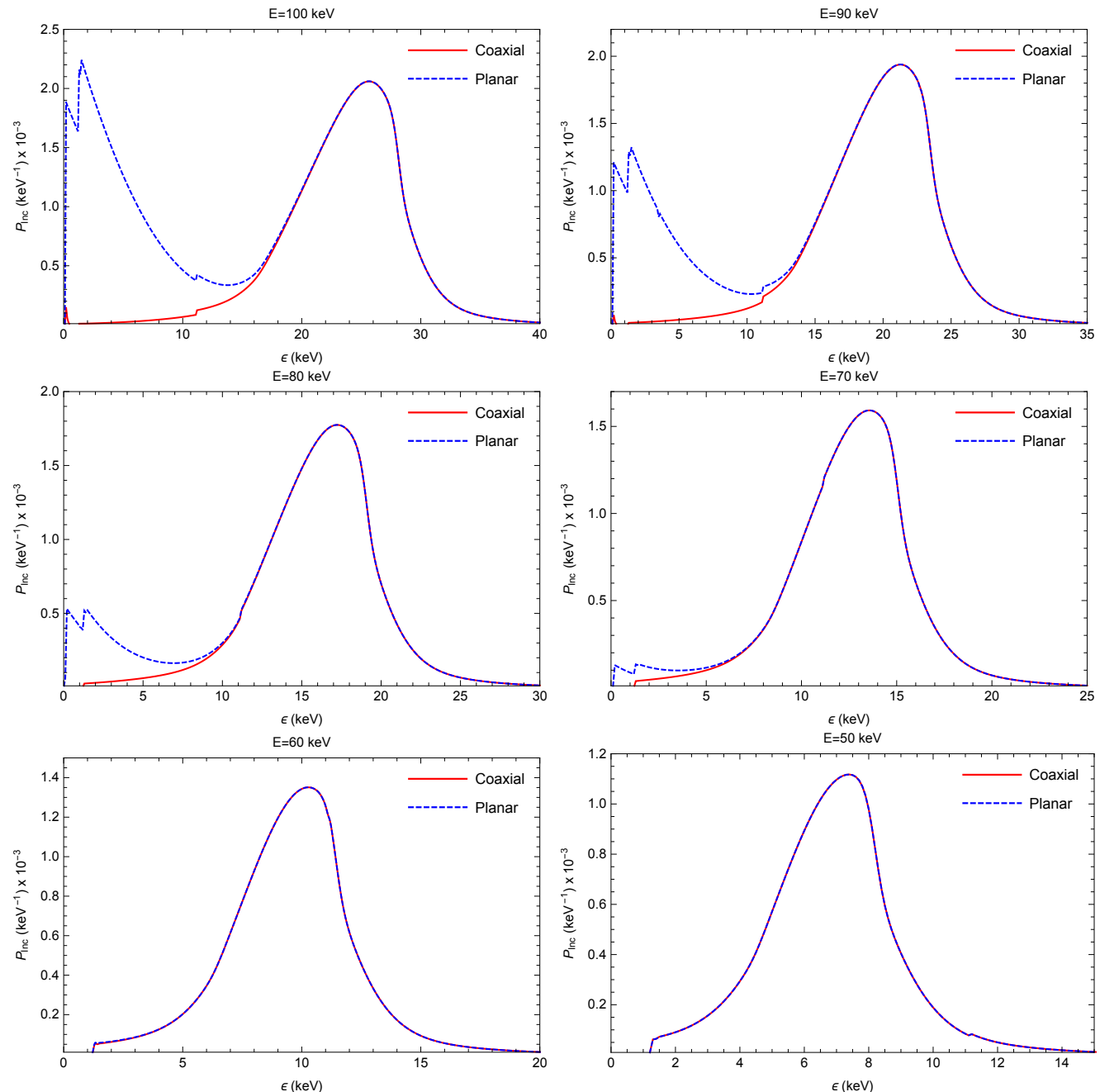

FIGURA 4.21: Comparação dos espectros de escape Compton nas configurações planar e coaxial para energias dos fótons incidentes entre 100 e $50 \mathrm{keV}$.

A Fig. 4.22 mostra a fração da área total do espectro que aporta o contínuo de Compton, em função da energia incidente. A discrepância observada entre as duas curvas acima de $\sim 65 \mathrm{keV}$ se traduz em um aumento do pico de absorção total na configuração coaxial na mesma porcentagem. Uma conclusão direta do estudo é que para $E \lesssim 65 \mathrm{keV}$ o detetor coaxial pode ser modelado simplesmente como um planar de parâmetros $l=l_{1}$ e $r=r_{2}$.

Por outro lado, foi realizada uma simulação PENELOPE em que só foram transportados fótons na geometria coaxial com as dimensões acima descritas. A Fig. 4.23 apresenta a comparação entre os resultados do cálculo analítico e do EAP PENELOPE na região do espectro que corresponde ao escape Compton, para fótons de 100, 90 e $80 \mathrm{keV}$. Observa-se uma boa concordância na forma dos espectros e como já foi discutido, as pequenas discrepâncias são devidas ao espalhamento múltiplo não considerado no modelo analítico. A análise da fração da área total do EAP que representa o Compton, fornecida pela simulação é 2,38\%, 1,92\% e 1,50\% para 100, 90 e $80 \mathrm{keV}$ respectivamente. O valores calculados analiticamente são $2,20 \%, 1,83 \%$ e $1,45 \%$ em cada caso, que constituem frações 


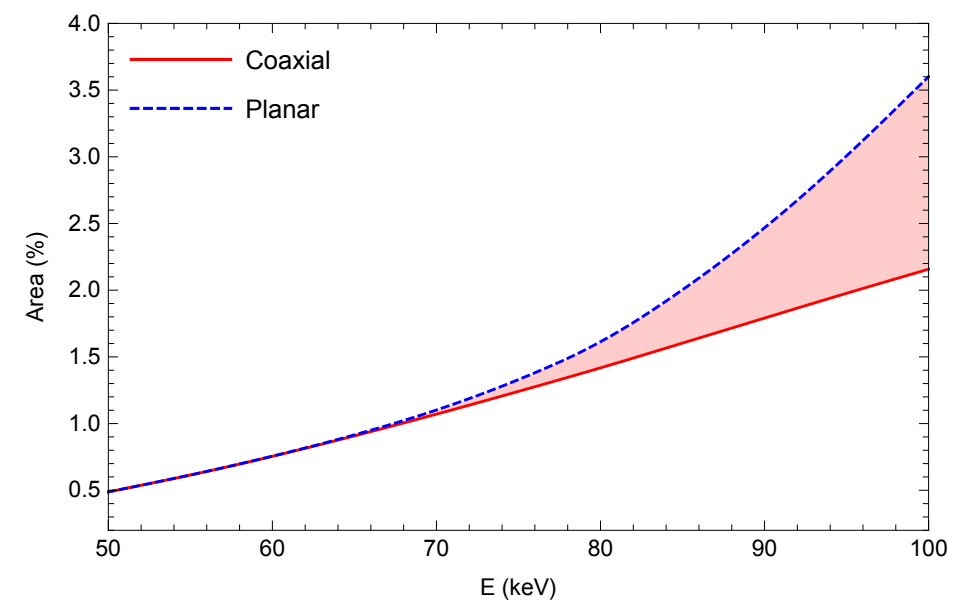

FigurA 4.22: Comparação entre a área que representa o contínuo de Compton em relação à área total observada no EAP, nas configurações coaxial e planar.

de $92,4 \%, 95,4 \%$ e $97,6 \%$ em relação os resultados MC.

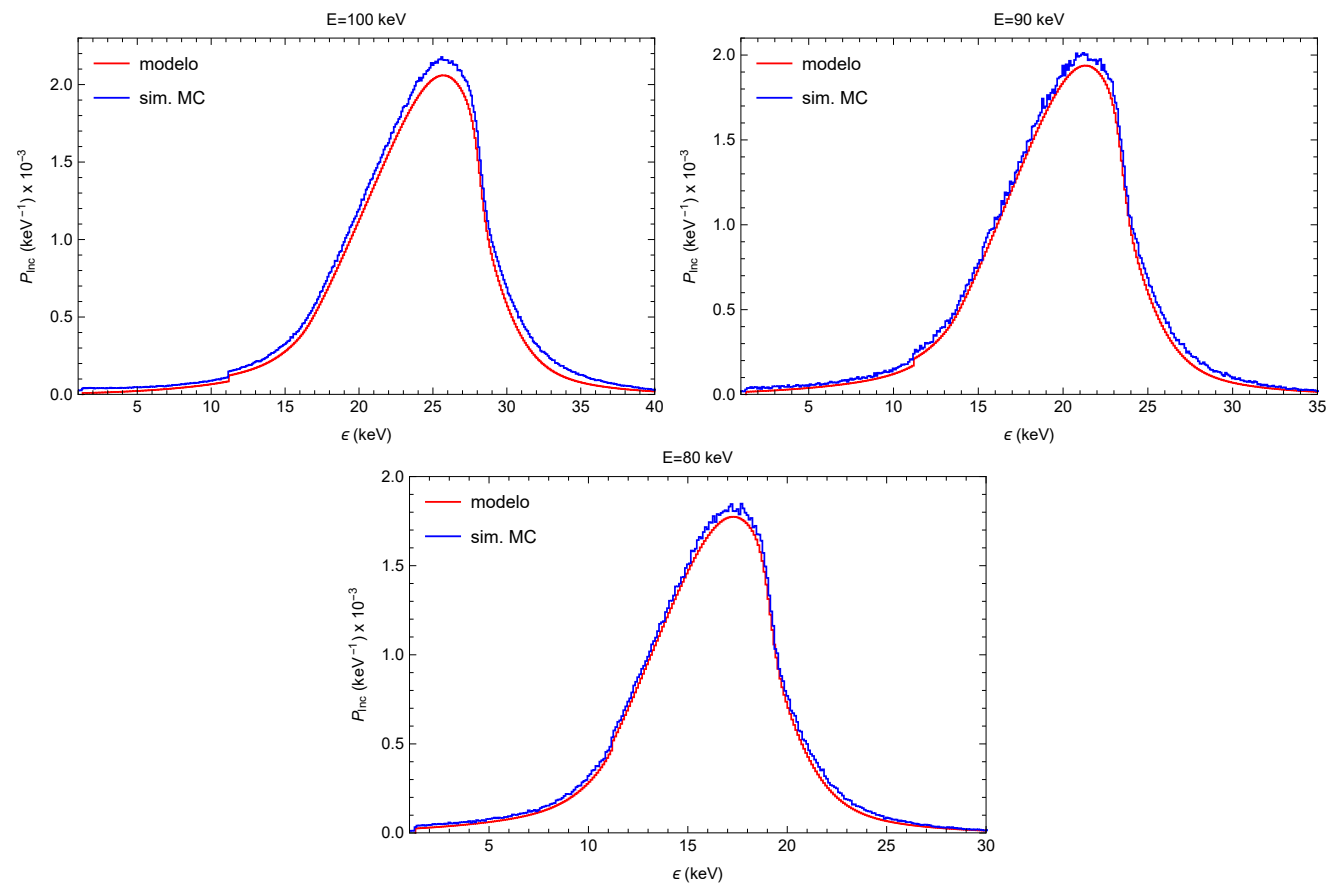

Figura 4.23: Comparação entre o modelo e a simulação PENELOPE dos espectros de escape Compton na geometria coaxial, para energias incidentes $E=100,90$ e $80 \mathrm{keV}$.

\subsection{Teste do procedimento de deconvolução de espectros contínuos}

Uma vez desenvolvido o modelo de FR resulta ilustrativo testa-lo na deconvolução de espectros contínuos de radiação. A prova utiliza a geometria do detetor planar da seção $4.4\left(l=10 \mathrm{~mm}, r=16 \mathrm{~mm}, d=1,50 \mu \mathrm{m}\right.$ e $\left.d_{\mathrm{Be}}=25,4 \mu \mathrm{m}\right)$ para a simulação 
PENELOPE do EAP gerado por um espectro de fótons incidentes da forma

$$
b(W)=\frac{C}{W} \Theta\left(W_{\max }-W\right) \Theta\left(W-W_{\min }\right) .
$$

A função densidade de probabilidade $b(W)$ representa uma aproximação da dependência energética do espectro de bremsstrahlung (ver Eq. (2.76)). Os parâmetros $W_{\max }$ e $W_{\min }$ estão associados respectivamente à energia do elétron incidente e a energia mínima de emissão dos fótons numa colisão radiativa. A constate de normalização $C$ pode ser calculada em função de $W_{\max }$ e $W_{\min }$ como

$$
C=\left[\ln \left(\frac{W_{\max }}{W_{\min }}\right)\right]^{-1}
$$

Teoricamente $W_{\min }=0$, mas com fins ilustrativos e para evitar a divergência de $b(W)$ em $W=0$ serão escolhidos valores diferentes a conveniência. Igualmente, os EAP simulados são convoluídos com uma função Gaussiana para levar em conta os efeitos de resolução. O espectro de entrada no PENELOPE é calculado segundo

$$
B_{i}=\left[\ln \left(\frac{W_{\max }}{W_{\min }}\right)\right]^{-1} \ln \left(\frac{W_{i+1}}{W_{i}}\right),
$$

com $i=1,2, \ldots, n$. As probabilidades de emissão $B_{i}$ compõem o que chamaremos vetor B. Logo, o EAP simulado $(\mathbf{N})$ e a matriz resposta calculada segundo a modelo analítico são substituídos na Eq. (4.13) para obter um novo vetor $\mathbf{B}_{\mathrm{d}}$, que representa a melhor estimativa possível de $\mathbf{B}$. O teste tem a vantagem da comparação direta de $\mathbf{B}_{\mathrm{d}}$ com o valor verdadeiro $\mathbf{B}$, que no caso real é sempre desconhecido. Também pretende determinar se os efeitos não considerados na FR tem um reflexo notável em $\mathbf{B}_{\mathrm{d}}$. A deconvolução dos espectros é realizada entre $h=12 \mathrm{keV}$ e $h=100 \mathrm{keV}$ com uma largura energética $\Delta=1 \mathrm{keV}$.

A Fig. 4.24 mostra os espectros $\mathbf{B}$ e $\mathbf{N}$ para parâmetros de entrada $W_{\min }=32 \mathrm{keV}$ e $W_{\max }=95 \mathrm{keV}$. Pode se observar que essa escolha produz uma distribuição de pulsos no intervalo $12 \mathrm{keV} \leqslant h \leqslant 32 \mathrm{keV}$ associados a fótons com $W \geqslant 32 \mathrm{keV}$. Na Fig. 4.25 se aprecia como o espectro deconvoluído nesta faixa devolve um sinal que é compatível com zero. No gráfico da direita são comparados os espectros $\mathbf{N}$ e $\mathbf{B}_{\mathrm{d}}$ na região energética de incidência dos fótons.

De maneira similar, na Fig. 4.26 são apresentados os espectros deconvoluído e real. Pode se verificar como as componentes de $\mathbf{B}_{\mathrm{d}}$ oscilam em torno do valor verdadeiro, salvo nos pontos extremos ( $W_{\min }$ e $\left.W_{\max }\right)$ onde muda bruscamente o espectro $\mathbf{B}$, passando de zero a $B_{i}\left(W_{\min }\right)$ ou de $B_{i}\left(W_{\max }\right)$ a zero. Porém nenhum dos dois casos representa um 


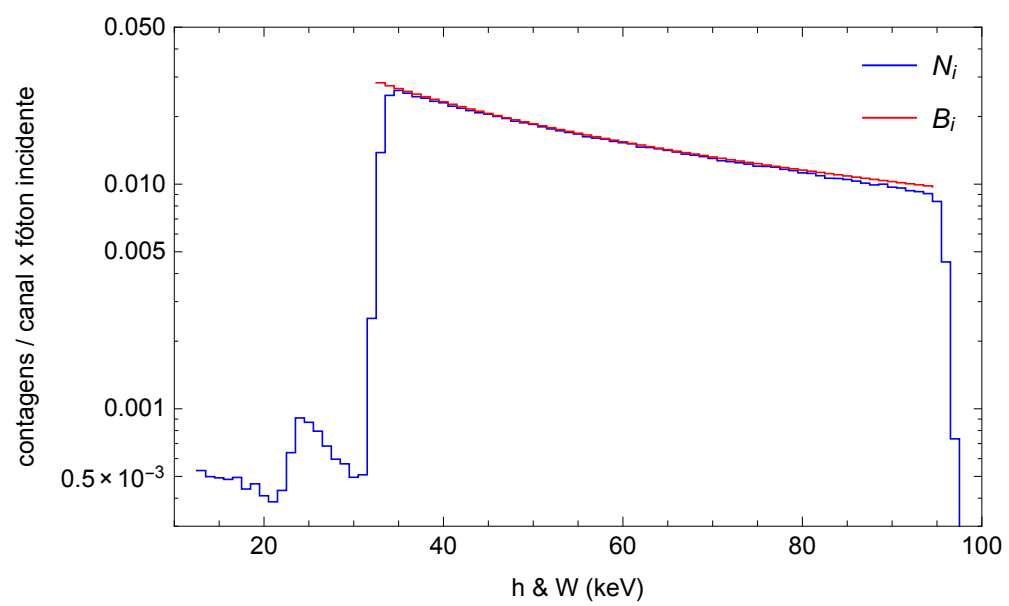

FiguRA 4.24: Espectro de fótons incidentes e distribuição de altura de pulso simulados com parâmetros de entrada $W_{\min }=32 \mathrm{keV}$ e $W_{\max }=95 \mathrm{keV}$.
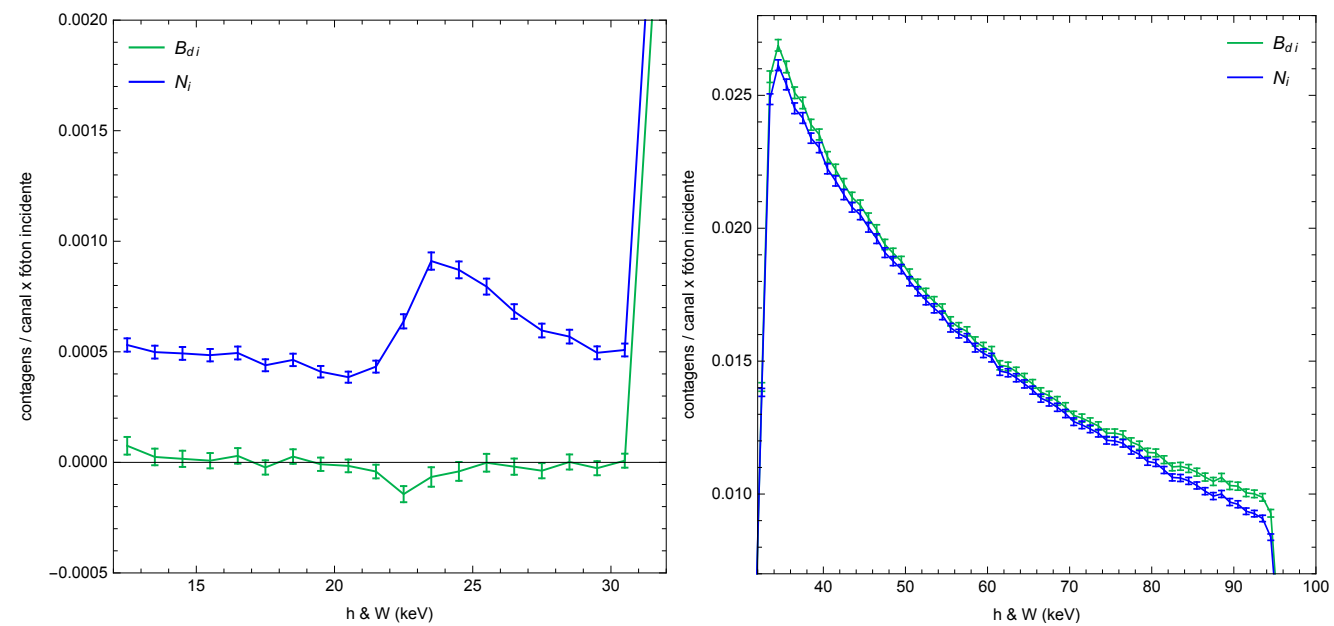

Figura 4.25: Comparação entre o EAP e o espectro deconvoluído.

risco. Como já foi descrito, em um espectro real $W_{\min }=0$, e no limite inferior que será utilizado na deconvolução $(h \sim 12 \mathrm{keV})$, não existiram descontinuidades como a do exemplo anterior. A Fig. 4.27 exibe uma outra situação em que é deconvoluído um espectro incidente com parâmetros $W_{\min }=10 \mathrm{keV}$ e $W_{\max }=95 \mathrm{keV}$ entre $h=12 \mathrm{keV}$ e $h=100 \mathrm{keV}$. Segundo o esperado o limite inferior não apresenta um comportamento anômalo. Finalmente o ponto do tip, que nos dois exemplos o resultado está abaixo do valor verdadeiro de dois a três desvios padrões, será obtido por outra metodologia que inclui a determinação da energia do feixe de elétrons (ver secção 7.2). 

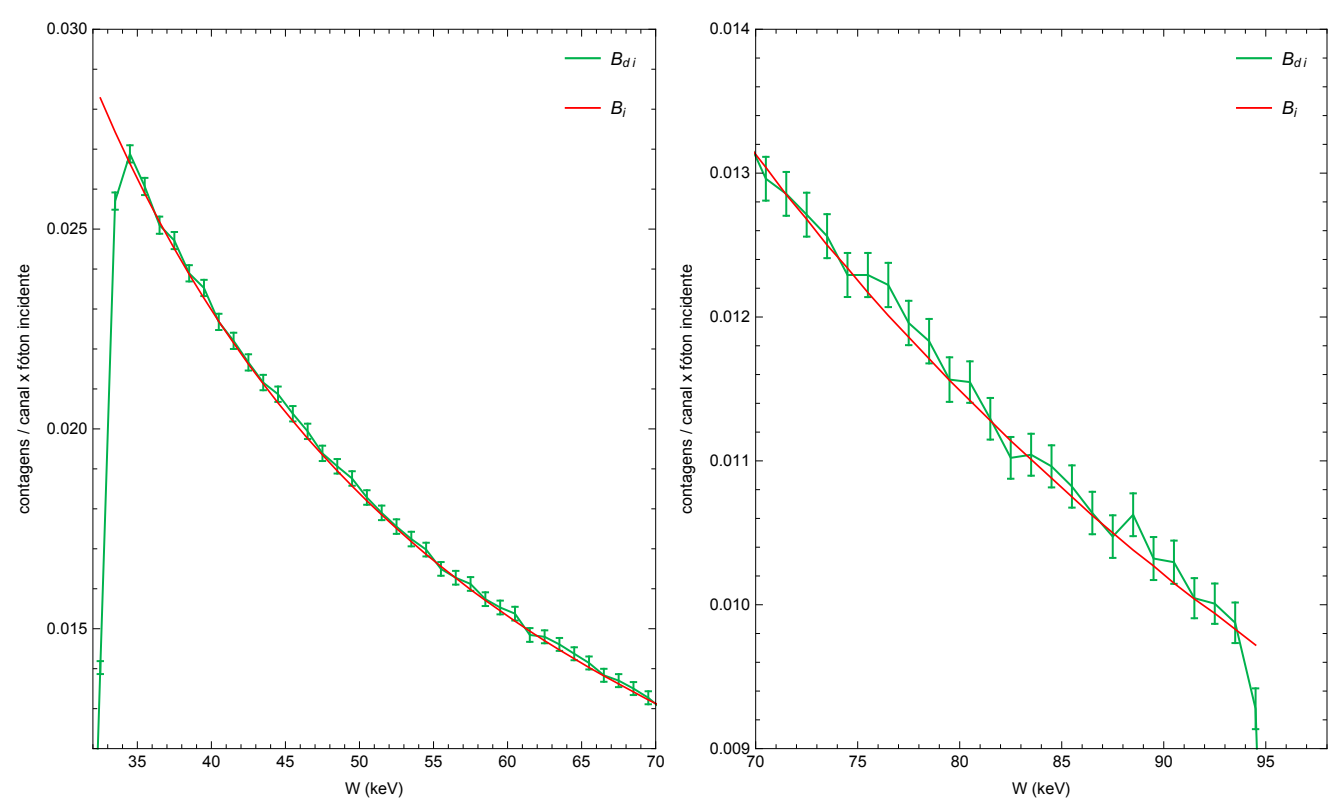

Figura 4.26: Comparação entre o espectro deconvoluído com a matriz $\mathbf{R}$ calculada segundo o modelo analítico e o espectro verdadeiro utilizado na simulação MC. Os valores limites de $\mathbf{B}$ são $W_{\min }=32 \mathrm{keV}$ e $W_{\max }=95 \mathrm{keV}$.
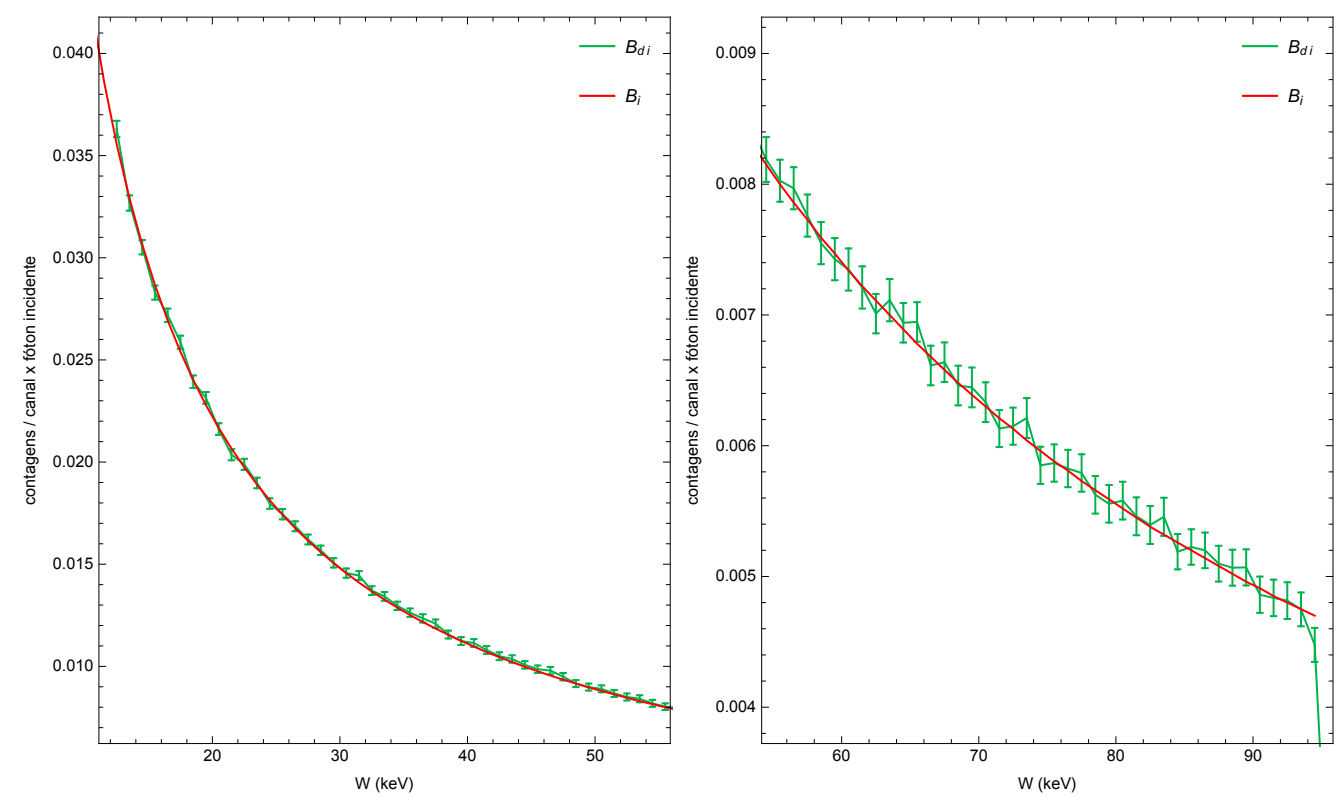

FigurA 4.27: Comparação entre o espectro deconvoluído com a matriz $\mathbf{R}$ calculada segundo o modelo analítico e o espectro verdadeiro utilizado na simulação MC. Os valores limites de B são $W_{\min }=10 \mathrm{keV}$ e $W_{\max }=95 \mathrm{keV}$. 


\section{Capítulo 5}

\section{Influência da geometria na função resposta: teste experimental}

Neste capítulo são descritas as experiências realizadas para testar a FR de um dos detetores utilizados nas medidas de bremsstrahlung. Os espectros coletados de uma fonte ${ }^{241}$ Am são comparados com o modelo analítico desenvolvido a fim de validar suas componentes. Os experimentos mostram a influência no EAP do colimador e da fonte. É exposta a metodologia adotada para a avaliação desses efeitos. Finalmente são quantificadas ambas as contribuições.

\subsection{Função resposta: experimento de teste}

No capítulo anterior os testes realizados utilizaram a simulação MC com o código PENELOPE para a comparação e validação do modelo analítico de FR. Os experimentos propostos a seguir tem o objetivo de avaliar a FR com um EAP real, e examinar a influência que introduzem os elementos do arranjo.

A Fig. 5.1 apresenta o esquema da montagem experimental onde foram coletados espectros de uma fonte radioativa de ${ }^{241} \mathrm{Am}$ sob condições semelhantes às assumidas no modelo de FR. A fim de reduzir o efeito da radiação de fundo, o detetor planar de Ge tomado como referência nas simulações MC da seção 4.4 se colocou no interior de uma blindagem de $\mathrm{Pb}$ de geometria paralelepipedal, com dimensões internas de $400 \times 400 \times 200 \mathrm{~mm}^{3}$ e paredes de espessura $100 \mathrm{~mm}$. Um dos colimadores de $\mathrm{Cu}$ utilizado nas medidas de bremsstrahlung (comprimento $100 \mathrm{~mm}$ e diâmetro $10 \mathrm{~mm}$ ) se posicionou na frente do detetor para garantir a incidência dos fótons em torno ao eixo de simetria do cristal de $\mathrm{Ge}$. $\mathrm{O} \mathrm{Cu}$ foi escolhido como material do colimador fundamentalmente para evitar a 
influência dos raios-x característicos na faixa de deconvolução dos espectros de bremsstrahlung (a energia da transição $\mathrm{K} \beta_{1}$ é 8905,413(38) eV [83]). O detetor também foi blindado por uma caixa de $\mathrm{Cu}$ (na região do volume ativo) para impedir a detecção de raios-x do $\mathrm{Pb}$.
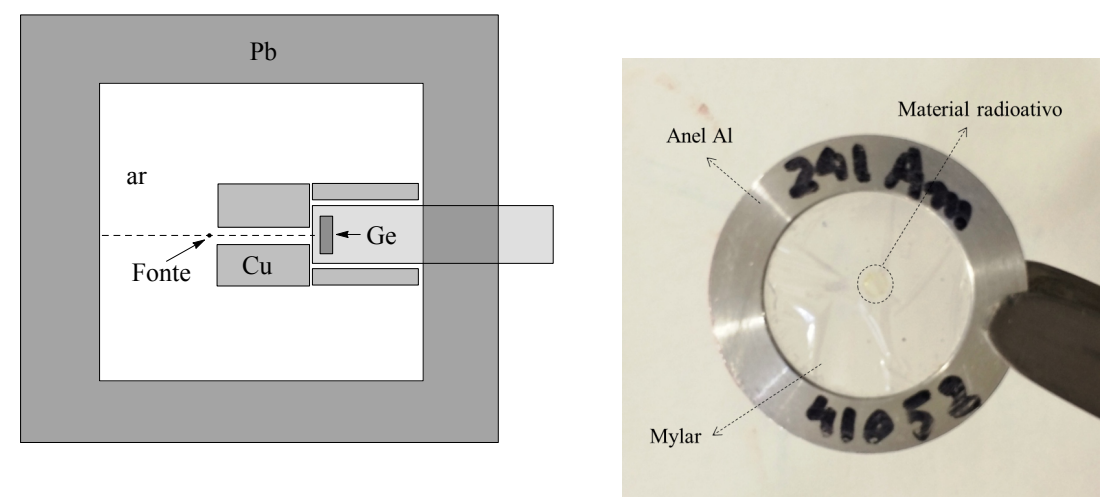

FiguRA 5.1: Esquema bidimensional do arranjo experimental e fotografia da fonte de ${ }^{241}$ Am utilizada nas medidas de teste da FR.

A fonte de ${ }^{241} \mathrm{Am}$ foi construída no Laboratório de Metrologia do Instituto de Pesquisas Energéticas e Nucleares, a partir da deposição de uma gota de solução radioativa sobre um filme de mylar ${ }^{1}$. Depois de seca, uma outra folha desse material colocou-se por cima da primeira para garantir o selado. Os dois filmes se fixaram por meio de anéis de $\mathrm{Al}$ (Fig. 5.1). O procedimento permite diminuir os efeitos de espalhamento inelástico na fonte $[97,98]$. A área que cobre o material radioativo apresenta um contorno aproximadamente circular com raio médio de $2 \mathrm{~mm}$. Nos experimentos, essa região da fonte foi centrada em relação ao orifício do colimador e a separação entre as superfícies de ambos elementos foi $d_{\mathrm{f}}=3 \mathrm{~mm}$. A escolha do ${ }^{241} \mathrm{Am}$ resulta especialmente atrativa para a comparação do modelo de FR pois a máxima energia dos fótons emitidos é $59,54 \mathrm{keV}$. Essa característica garante um estudo livre de contínuos de Compton produzidos por energias superiores à faixa estudada, como é o caso de outros radionuclídeos que com frequência se utilizam nos laboratórios ( $p$. ex. ${ }^{133} \mathrm{Ba},{ }^{137} \mathrm{Cs}$ ou $\left.{ }^{152} \mathrm{Eu}\right)$.

A Fig. 5.2 apresenta os espectros coletados da fonte de ${ }^{241} \mathrm{Am}$, do fundo radioativo característico do lugar da instalação experimental (normalizado ao tempo da medida anterior) e o espectro líquido calculado como a diferença entre os dois. Não foram feitas correções de empilhamento devido à baixa atividade do radionuclídeo ( $\sim 40 \mathrm{kBq})$. Cada canal nos EAP da Fig. 5.2 possui uma largura $\delta=57,7$ eV. Podem se observar os picos de absorção total correspondentes às principais transições $\gamma$ com energias $E=59,54$ $\left(\gamma_{2,0}\right), 55,55\left(\gamma_{6,4}\right), 43,42\left(\gamma_{4,2}\right), 33,20\left(\gamma_{1,0}\right)$ e $26,34 \mathrm{keV}\left(\gamma_{2,1}\right)$, e dos raios-x L do Np, compreendidos na faixa $11-23 \mathrm{keV}$. Além disso, são visíveis os picos de escape dos

\footnotetext{
${ }^{1}$ Mylar: tereftalato de polietileno, fórmula molecular: $\left(\mathrm{C}_{10} \mathrm{H}_{8} \mathrm{O}_{4}\right)_{n}$.
} 
raios-x K do Ge produzidos pelos raios-x L do Np e pelos fótons de 59,54 keV. O pico alargado à direita deste último, corresponde a espalhamentos Compton em torno de $\theta=90^{\circ}$, também de fótons de $59,54 \mathrm{keV}$, nas folhas de mylar da fonte.

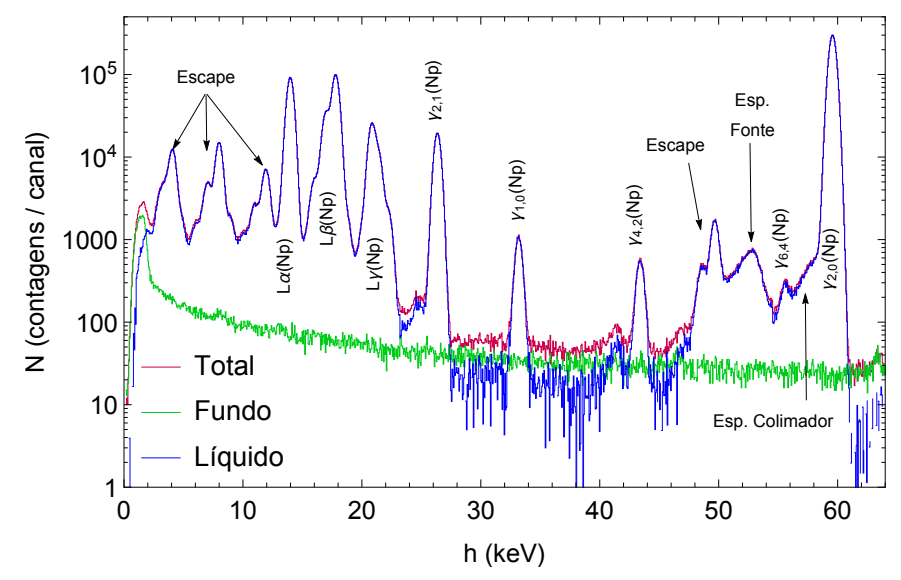

FigurA 5.2: Espectro de ${ }^{241} \mathrm{Am}$ coletado durante $t_{\mathrm{v}}=446400 \mathrm{~s}$ e do fundo radioativo normalizado ao mesmo tempo.

Os fótons de 59,54 keV tem a maior probabilidade de emissão no decaimento do ${ }^{241} \mathrm{Am}$ $(0,3578(9)$ [99]). Porém, a parte contínua do espectro provocada por essa energia incidente (escape de fótons do volume ativo de Ge após experimentar colisões inelásticas) não pode ser observada diretamente pela influência dos raios-x L do Np. Com o objetivo de estudar o efeito e comparar a componente $P_{\text {inc }}$ do modelo analítico de FR, Eq. (4.33), um outro experimento foi realizado. Entre a fonte de ${ }^{241} \mathrm{Am}$ e o colimador foi colocada uma lâmina de $\mathrm{Fe}$ (espessura $t_{\mathrm{Fe}}=0,4 \mathrm{~mm}$ ) e a continuação uma outra de $\mathrm{Al}$ (espessura $t_{\mathrm{Al}}=0,52 \mathrm{~mm}$ ). Com a primeiro material a intensidade de fótons de $21 \mathrm{keV}$ (cota superior para os raios-x L do Np) se reduz três ordens de grandeza, enquanto os fótons de $59,54 \mathrm{keV}$ se atenuam em $30 \%$. Com a folha de $\mathrm{Al}$, a fração transmitida de raios-x $\mathrm{K}$ do $\mathrm{Fe}\left(\mathrm{K} \beta_{1}=7058,175(16) \mathrm{eV}[83]\right)$ na direção do detetor é inferior a $9 \times 10^{-4}$.

A Fig. 5.3 mostra a comparação dos espectros coletados nas duas experiências. Notase a definição do contínuo de Compton que se procurava com o segundo experimento. Sobre essa estrutura também se observam raios-x $\mathrm{K}$ característicos de $\mathrm{Cu}$ produto da fluorescência do colimador. Uma outra diferença resulta o significativo incremento das contagens no intervalo compreendido entre o fotopico de $59,54 \mathrm{keV}$ e os picos de escape de raios-x K do Ge. Esse aumento está associado a espalhamentos inelásticos nas lâminas atenuadoras. 


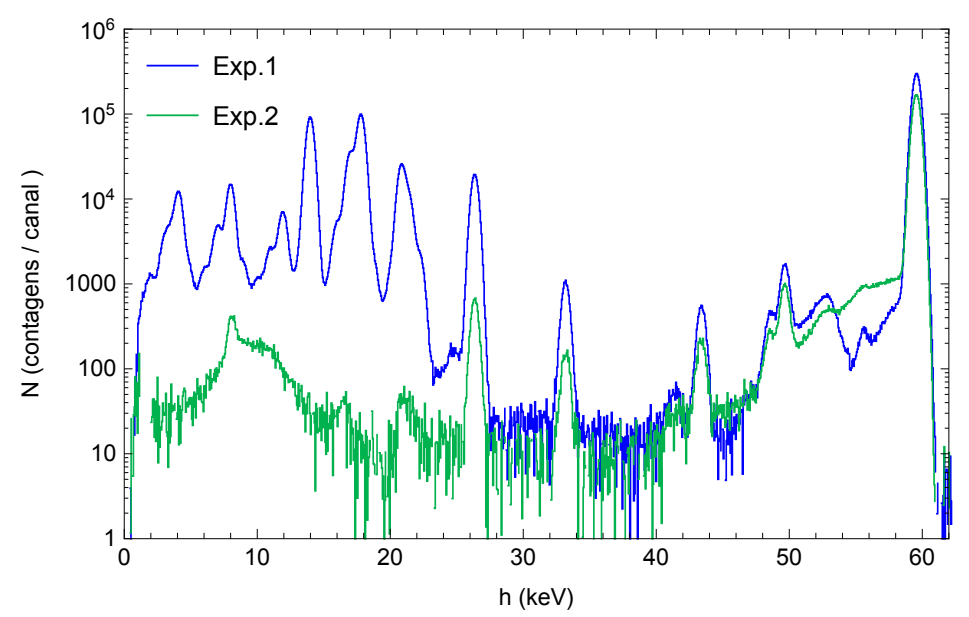

Figura 5.3: Espectros netos de ${ }^{241} \mathrm{Am}$ coletados na primeira experiencia (Exp. 1) e na segunda (Exp. 2) incluindo as lâminas atenuadoras entre a fonte e o colimador. $\mathrm{O}$ tempo de medida no segundo experimento foi $t_{\mathrm{v}}=345600 \mathrm{~s}$.

\subsubsection{Comparação do modelo de FR com o experimento}

O espectro de energia $F(E)$ que emite uma fonte radioativa e atinge o detetor pode se escrever como

$$
F(E)=\mathcal{A} t_{\mathrm{v}} \frac{\Omega}{4 \pi} \sum_{j} I_{j} \delta\left(E-E_{j}\right),
$$

onde $\mathcal{A}$ é a atividade do radionuclídeo na data da medida, $t_{\mathrm{v}}$ é o tempo vivo de adquisição do EAP, $\Omega$ é o ângulo sólido subtendido pelo detetor em relação à fonte, $I_{j}$ representa a probabilidade de emissão dos fótons de energia $E_{j}$ e $\delta\left(E-E_{j}\right)$ a função delta de Dirac. De acordo com a Eq. (4.4), o número de contagens $N_{i}$ coletados no canal $i$, correspondente às energias entre $h_{i}-\delta / 2$ e $h_{i}+\delta / 2$, foi calculado segundo a expressão

$$
N_{i}=\mathcal{A} t_{\mathrm{v}} \frac{\Omega}{4 \pi} \delta \sum_{j} I_{j} R\left(h_{i}, E_{j}\right),
$$

onde se utilizou na Eq. (4.3) a aproximação

$$
r\left(h_{i}, E_{j}\right) \approx \delta R\left(h_{i}, E_{j}\right)
$$

levando em conta a ordem da largura $\delta$ de cada canal. Para avaliar a Eq. (5.2) se utilizaram as intensidades das transições $\gamma$ do ${ }^{241} \mathrm{Am}$ e dos raios-x L fornecidas na referência [99] e no trabalho de Lépy e colaboradores [100], respectivamente.

A modo didático, na Fig. 5.4 é apresentada uma comparação entre os espectros medido (Exp. 1) e calculado incluindo sucessivamente: (A) a energia de 59,54 keV, (B) as outras transições $\gamma$ e finalmente $(\mathrm{C})$ os raios-x L do Np. Os gráficos (A) e (B) permitem verificar a concordância de ambos espectros nos picos de absorção total a diferentes 
energias. Além disso, conclui-se que o espalhamento na janela de Be não é a única causa do contínuo à esquerda do foto-pico de 59,54 keV. Essa contribuição está vinculada a eventos inelásticos no colimador e será abordada e quantificada na seção 5.2. Constata-se que os picos que surgem devido ao escape dos raios-x K do Ge para as energias incidentes dos raios-x L do Np, tem como base o contínuo de Compton provocado pelos fótons de $59,54 \mathrm{keV}$. Por outro lado, na faixa das transições $\mathrm{Np} \mathrm{L} \beta$, o espectro é composto pela convolução dos fotopicos dessas energias e os picos de escape associados aos fótons de 26,34 keV. As principais diferenças entre o cálculo e o experimento na Fig. 5.4 (C) $(h<11 \mathrm{keV})$ são devidas a efeitos não incluídos no modelo como: $i)$ fótons de autofluorescência do Am [100], ii) espalhamento nas folhas de mylar da fonte, iii) raios-x K de Ge que são produzidos na camada morta e atingem o volume ativo, e $i v$ ) raios-x K de $\mathrm{Cu}$ do colimador.

Na Fig. 5.5 se faz uma comparação similar à anterior no caso da segunda experiência. Além das diferenças já discutidas à esquerda do foto-pico de $59,54 \mathrm{keV}$, observa-se na região do contínuo de Compton a excelente concordância entre o espectro experimental e o calculado sob a AIR. Torna-se visível no gráfico B a forma distorcida que segue o EAP analítico em relação aos dados coletados se a FR fosse computada seguindo a aproximação de KN. O resultado ressalta a importância de considerar o alargamento Doppler e conclui de maneira exitosa a validação das componentes intrínsecas do modelo de FR. No entanto, deixa aberta a avaliação da influência introduzida pelo arranjo experimental na coleção dos EAP. As seguintes seções modelam e quantificam os efeitos do colimador e da fonte. 

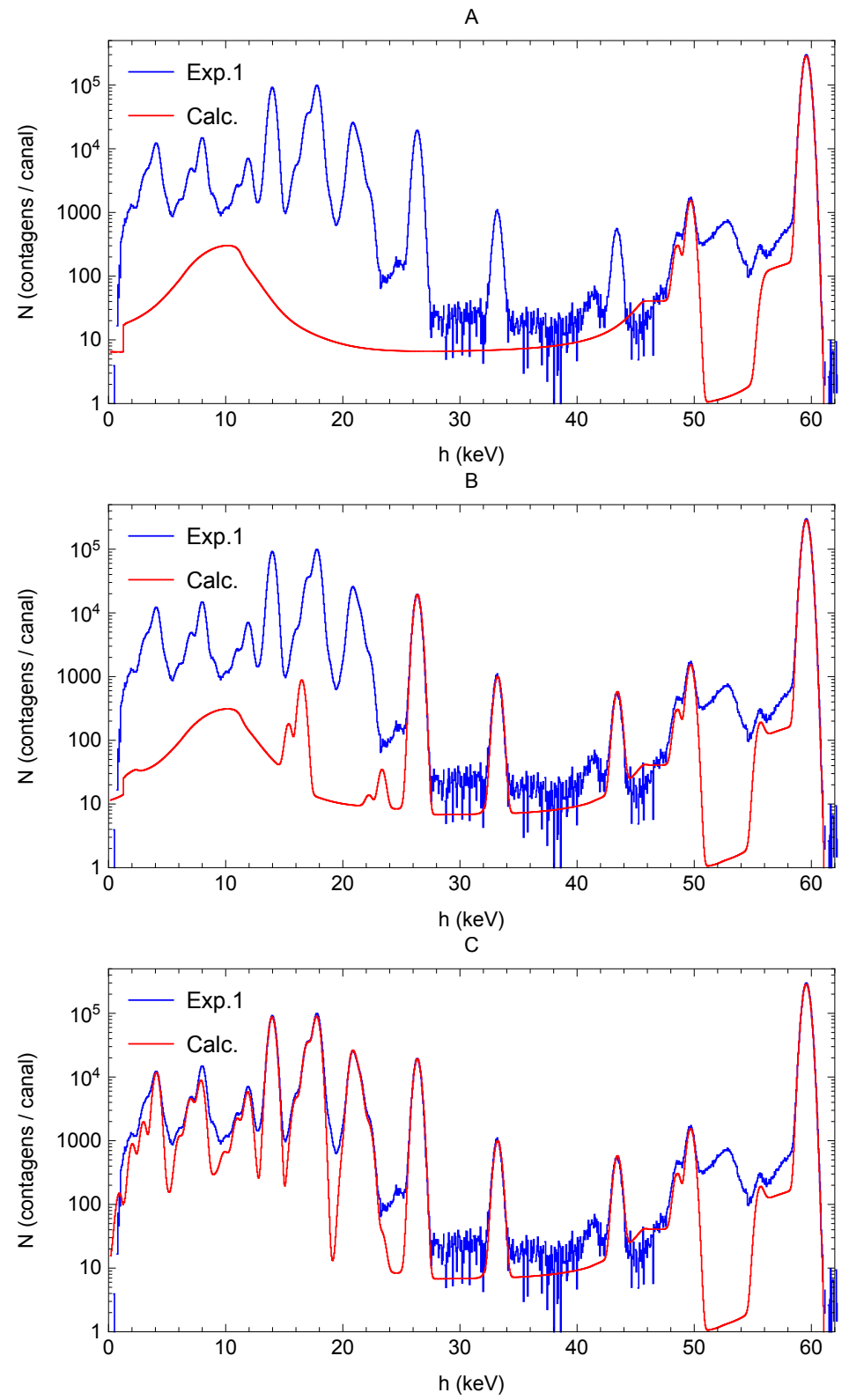

Figura 5.4: Espectros da fonte de ${ }^{241}$ Am medido e calculado segundo o modelo de FR na primeira experiência (Exp. 1). O gráfico A mostra o cálculo da resposta incluindo somente fótons incidentes de 59,54 keV. O gráfico B inclui todas as transições $\gamma$ do ${ }^{241} \mathrm{Am}$ no intervalo estudado. O gráfico $\mathrm{C}$, além das anteriores, inclui no cálculo os raios-x L do Np. 

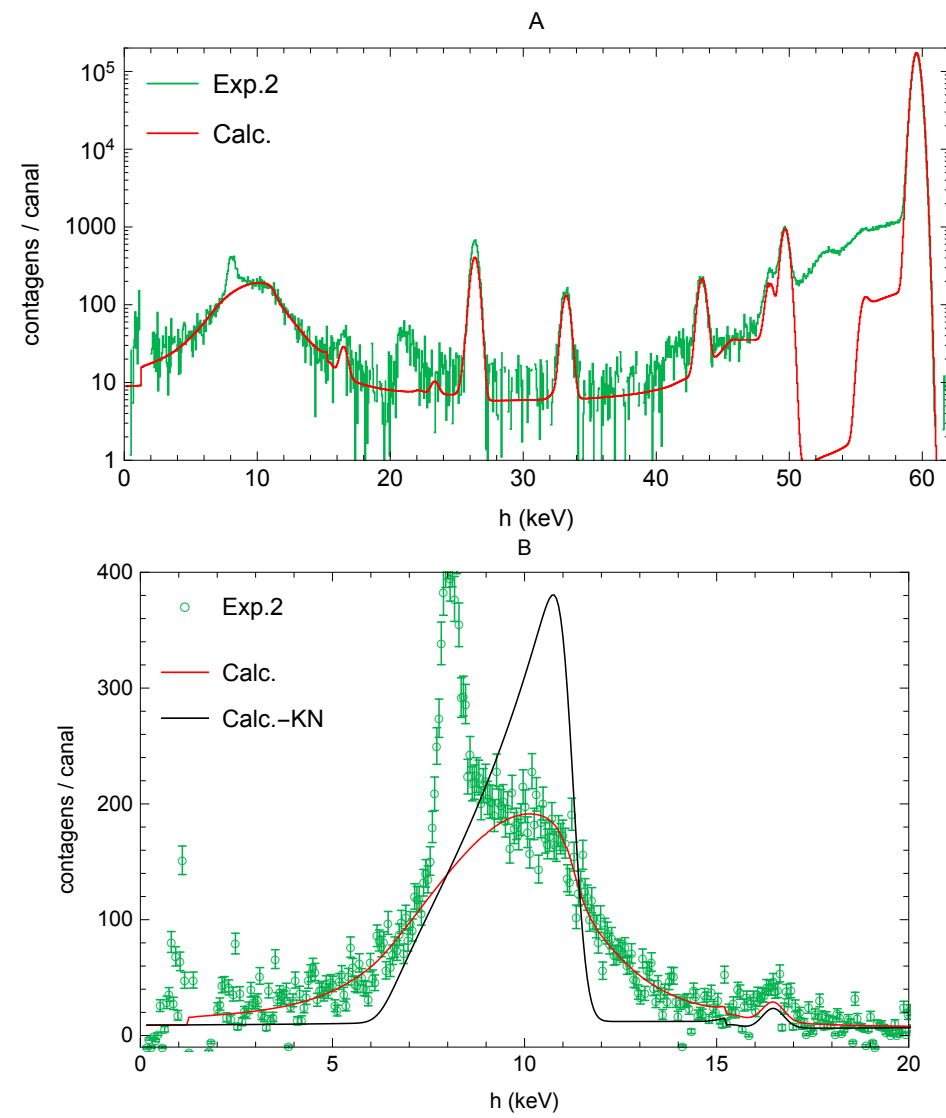

Figura 5.5: Espectros da fonte de ${ }^{241} \mathrm{Am}$ medido na segunda experiência (Exp. 2) e calculado segundo o modelo de FR. O gráfico B apresenta a região do contínuo de Compton em escala linear, incluindo também a resposta calculada considerando a SCD de $\mathrm{KN}$ (Calc.-KN).

\subsection{Influência do colimador na função resposta}

Um colimador ideal tem a função de deixar passar a radiação exclusivamente através de seu orifício central. Dependendo do material e da energia dos fótons o dispositivo será mais ou menos efetivo. Levando em conta as evidências observadas nas experiências relatadas na seção anterior, a continuação é analisada a influência do colimador na FR e modelada a sua contribuição. Como foi descrito na seção 3.1, entre as janelas da câmara de irradiação e os detetores se colocaram colimadores de $\mathrm{Cu}$. Logo, todas as seções choque, coeficientes de atenuação, etc. são referidas a esse elemento.

Considera-se um colimador de comprimento $d_{\mathrm{c}}$, com raio da abertura cilíndrica $r_{\mathrm{c}}$, e um ponto de emissão isotrópica de fótons de energia $E$, posicionado no eixo de simetria $z$ à distância $d_{\mathrm{f}}$ da origem de coordenadas (Fig. 5.6). A separação entre o colimador e o cristal do detetor é $d_{\mathrm{cc}}$.

São estudados dois tipos de eventos: $i$ ) fótons que atravessam o colimador sem interagir e atingem o volume ativo (trajetória da direita na Fig. 5.6), e ii) interações Compton 
correspondentes às trajetórias onde o fóton espalhado com energia $E^{\prime}$ atinge o cristal do detetor saindo pelo orifício do colimador (esquema da esquerda na Fig. 5.6).

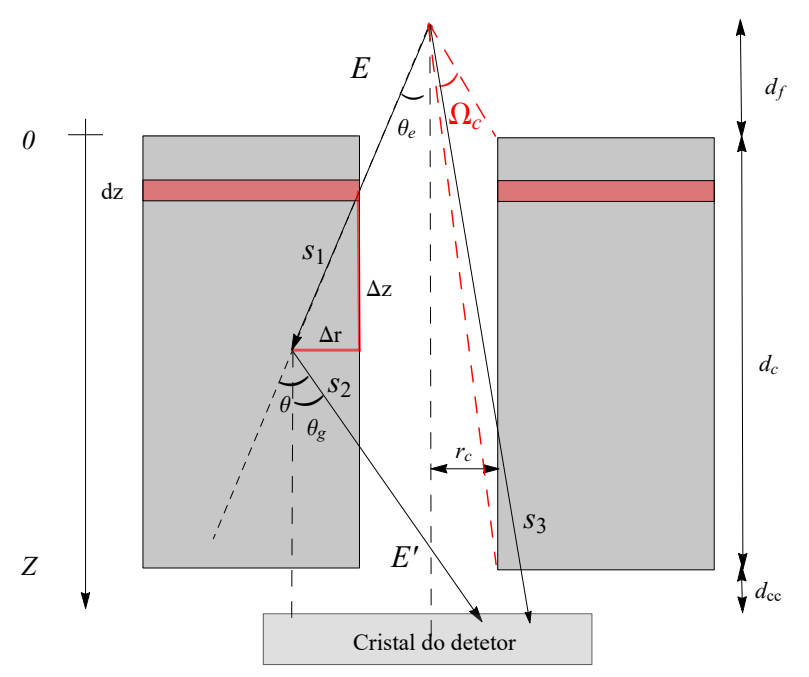

Figura 5.6: Esquema bidimensional da geometria do colimador, onde se representam as possíveis trajetórias dos fótons.

A função densidade de probabilidade que descreve o número de fótons $\mathrm{d} N$ emitidos na direção $\theta_{\mathrm{e}}$ em torno do ângulo sólido $\mathrm{d} \Omega=\sin \theta_{\mathrm{e}} \mathrm{d} \theta_{\mathrm{e}} \mathrm{d} \phi_{\mathrm{e}}$ pode se escrever como

$$
\mathrm{d} N=\frac{1}{4 \pi} \mathrm{d} \Omega
$$

onde $\theta_{\mathrm{e}}$ e $\phi_{\mathrm{e}}$ são os ângulos polar e azimutal respetivamente. Levando em conta a simetria cilíndrica do problema, a Eq. (5.4) se transforma segundo

$$
\mathrm{d} N=\frac{1}{2} \mathrm{~d}\left(\cos \theta_{\mathrm{e}}\right)
$$

que representa o número de fótons emitidos no anel de raio unitário e espessura $\mathrm{d} \theta_{\mathrm{e}}$. Por outro lado, $\cos \theta_{\mathrm{e}}$ se relaciona com a coordenada $z$ do colimador por meio de

$$
\cos \theta_{\mathrm{e}}=\frac{z+d_{\mathrm{f}}}{\sqrt{r_{\mathrm{c}}^{2}+\left(z+d_{\mathrm{f}}\right)^{2}}} .
$$

Logo, realizando uma transformação de variáveis é possível calcular o número de fótons que atingem o colimador no anel definido pelo intervalo $[z, z+\mathrm{d} z]$ (faixa vermelha na Fig. 5.6):

$$
\mathrm{d} N(z)=\frac{1}{2 r_{\mathrm{c}}}\left[1+\left(\frac{z+d_{\mathrm{f}}}{r_{\mathrm{c}}}\right)^{2}\right]^{-3 / 2} \mathrm{~d} z
$$


A função densidade de probabilidade anterior permite extrair algumas conclusões previas analisando a iluminação recebida pelas paredes internas do colimador $\left(r_{\mathrm{c}}=5 \mathrm{~mm}, d_{\mathrm{c}}=\right.$ $100 \mathrm{~mm}$ ) nos dois casos típicos de medidas. Em experimentos para testar a FR, como os descritos na seç̧ão 5.1, a fonte se colocou à distância $d_{\mathrm{f}}=3 \mathrm{~mm}$. Porém, para as medidas de bremsstrahlung de elétrons, o colimador se posiciona fora da câmara de irradiação e $d_{\mathrm{f}} \approx 300 \mathrm{~mm}$ (ver seç̧ão 3.1, Tabela 3.1). A aplicação da Eq. (5.7) a ambas situações é ilustrada na Fig. 5.7. Observa-se que quando a fonte é colocada perto, a quantidade de partículas que atingem os primeiros milímetros do colimador é varias ordens de grandeza maior do que o número que chega na parte final. Portanto, espera-se que os efeitos provocados por espalhamentos incoerentes do tipo (ii) sejam dominados pela porção superior do dispositivo. Contrariamente, o afastamento do ponto de emissão propicia uma iluminação mais uniforme, e por conseguinte, toda a superfície interna do orifício deve contribuir ao espectro de fótons espalhados.

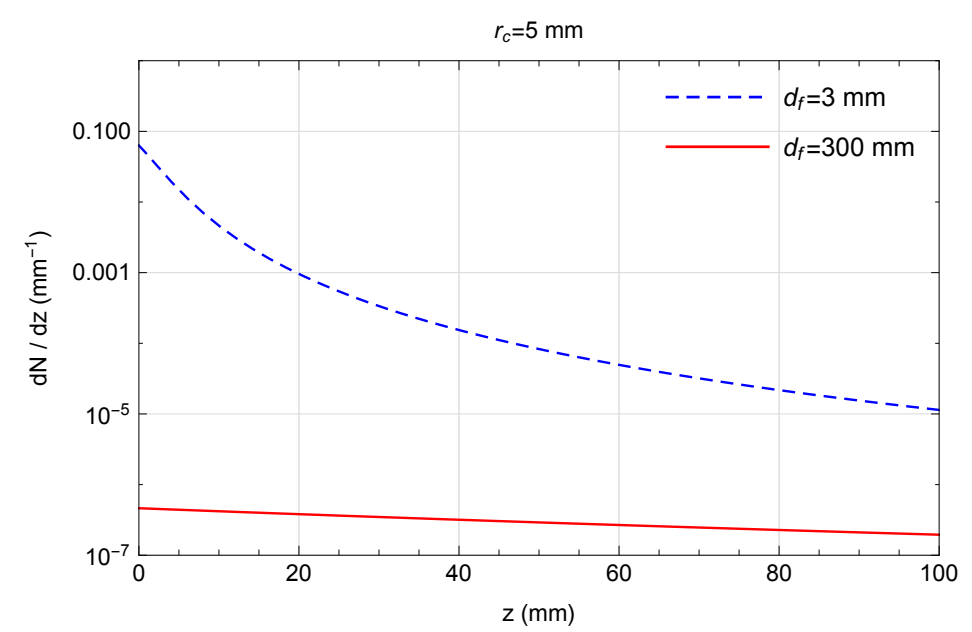

Figura 5.7: Iluminação das paredes do colimador quando o parâmetro $d_{\mathrm{f}}=3 \mathrm{ou}$ $300 \mathrm{~mm}$.

Uma outra ideia que ilustra quantitativamente a diferença da influência do colimador na FR, extrai-se do cálculo do número de fótons que passam pelo orifício central ou que atingem as paredes internas nas duas configurações experimentais. A partir das Eqs. (5.4) e (5.7) se define o ângulo sólido subtendido pelas paredes internas do colimador $\Omega_{\mathrm{c}}$ em relação ao ponto de emissão como

$$
\Omega_{\mathrm{c}}=4 \pi \int_{0}^{d_{\mathrm{c}}} \mathrm{d} N(z)
$$

e ângulo sólido geométrico $\Omega_{\mathrm{g}}$ subtendido pelo detetor em relação ao mencionado ponto

$$
\Omega_{\mathrm{g}}=4 \pi \int_{d_{\mathrm{c}}}^{\infty} \mathrm{d} N(z) .
$$


Quando o parâmetro $d_{\mathrm{f}}=3 \mathrm{~mm}$, se obtém $\Omega_{\mathrm{c}}=2,63$ sr e $\Omega_{\mathrm{g}}=0,42 \mathrm{sr}$, ou seja o número de fótons sobre as paredes é 6 vezes maior. No outro caso, com $d_{\mathrm{f}}=300 \mathrm{~mm}$, $\Omega_{\mathrm{c}}=5,54 \times 10^{-5}$ sr e $\Omega_{\mathrm{g}}=8,17 \times 10^{-4}$ sr e a situação se torna contrária, o orifício do colimador permite o passo de uma quantidade de fótons superior em mais de uma ordem aos fótons que atingem as paredes internas do dispositivo.

\subsubsection{Transparência do colimador}

A comparação anterior deixa um marco propício para estudar agora o que chamaremos transparência do colimador. Essa grandeza pode se definir como o incremento efetivo no ângulo sólido geométrico causado pelos fótons que atravessam sem interagir o colimador e saem pela superfície posterior (trajetória $s_{3}$ na Fig. 5.6). Sob esse enfoque, a transparência em termos matemáticos se expressa como

$$
\Omega_{\mathrm{ef}}\left(E ; r_{\mathrm{c}}, d_{\mathrm{c}}, d_{\mathrm{f}}\right)=4 \pi \int_{0}^{d_{\mathrm{c}}} \mathrm{d} N(z) \exp \left[-\mu_{\mathrm{Cu}}(E) s(z)\right]
$$

com

$$
s(z)=\frac{d_{\mathrm{c}}-z}{z+d_{\mathrm{f}}} \sqrt{r_{\mathrm{c}}^{2}+\left(z+d_{\mathrm{f}}\right)^{2}} .
$$

Na Eq. (5.10), o primeiro fator do integrando representa a densidade de probabilidade de que um fóton atinja a parede interior do colimador, a uma profundidade entre $(z, z+\mathrm{d} z)$, e o segundo fator, a probabilidade de sair pela superfície posterior sem interagir depois de percorrer o caminho $s(z)$. A Fig. 5.8 mostra a fração porcentual $\Delta \Omega$ que representa $\Omega_{\mathrm{ef}}$ em relação ao ângulo sólido geométrico $\Omega_{\mathrm{g}}$, em função da energia incidente.

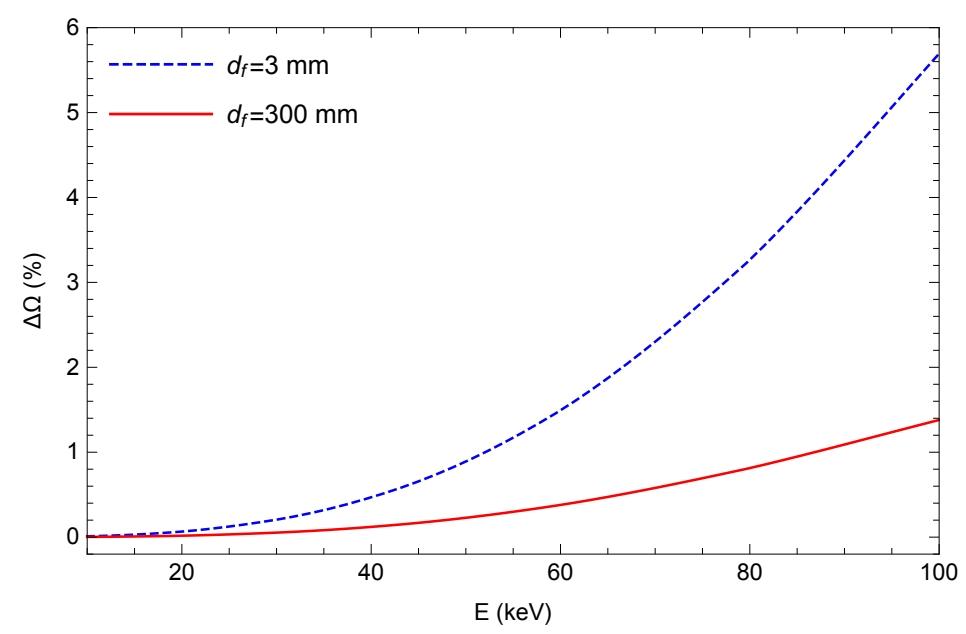

Figura 5.8: Relação $\Omega_{\mathrm{ef}} / \Omega_{\mathrm{g}}$ em porcento, para parâmetros $d_{\mathrm{f}}=3$ ou $300 \mathrm{~mm}$.

Percebe-se que a transparência do colimador de $\mathrm{Cu}$ avaliado depende em grande medida do parâmetro $d_{\mathrm{f}}$. Na configuração de irradiação, as correções que precisam ser feitas ao 
ângulo sólido na determinação da SCDD, Eq. (2.77), são menores que 0,5\% para fótons até $65 \mathrm{keV}$, enquanto a $100 \mathrm{keV}$ o incremento chega ser de 1,4\%.

\subsubsection{Contribuição do espalhamento Compton no colimador}

Como foi observado nos experimentos o outro efeito que o colimador introduz no EAP deve-se a fótons espalhados incoerentemente (trajetórias $s_{1}$ e $s_{2}$ na Fig. 5.2). Os cálculos apresentados nesta secção quantificam esse fenômeno.

Seguindo o formalismo desenvolvido no capítulo anterior, o espectro produzido pelos fótons que atingem a porção do colimador situada à profundidade $z$ com espessura $\mathrm{d} z$ pode se deduzir a partir do produto de probabilidades

$$
\begin{aligned}
\mathrm{d}^{4} P_{\mathrm{col}}\left(E, E^{\prime}, z, s_{1}, \cos \theta, \phi\right)= & \mathrm{d} N(z) \mu_{\mathrm{Cu}}^{\mathrm{inc}}(E) \exp \left[-\mu_{\mathrm{Cu}}(E) s_{1}\right] \mathrm{d} s_{1} \\
& \times \frac{1}{\sigma_{\mathrm{Cu}}^{\mathrm{inc}}(E)} \frac{\mathrm{d}^{2} \sigma_{\mathrm{AIR}}\left(E, E^{\prime}, \cos \theta\right)}{\mathrm{d} E^{\prime} \mathrm{d} \Omega^{\prime}} \mathrm{d}(\cos \theta) \mathrm{d} \phi \\
& \times \exp \left[-\mu_{\mathrm{Cu}}\left(E^{\prime}\right) s_{2}\right],
\end{aligned}
$$

onde o grupo de fatores da primeira linha do membro direito representa a probabilidade de que os fótons que atingem o colimador no ponto $z$ experimentem um espalhamento inelástico ao longo da trajetória $s_{1}$, o grupo da segunda linha fornece a distribuição de energias $E^{\prime}$ e ângulos $(\theta, \phi)$ segundo a AIR, e o último fator estabelece a probabilidade de escape após percorrer um caminho $s_{2}$ no colimador (Fig. 5.9).

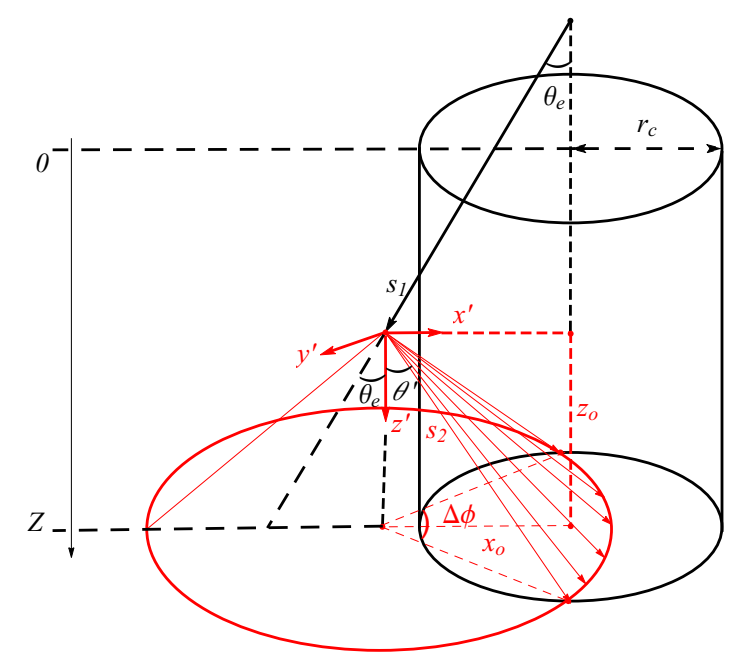

Figura 5.9: Esquema tridimensional da geometria do espalhamento incoerente de fótons no colimador. O cilindro representa o orifício do colimador. 
Na Eq. (5.12) a dependência com o ângulo $\phi$ está contida em $s_{2}$. Para descrever essa relação foi escolhido o sistema de coordenadas $\left(x^{\prime}, y^{\prime}, z^{\prime}\right)$ com origem no ponto de interação do fóton e direção $z^{\prime}$ paralela ao eixo do colimador (Fig. 5.9). Nesse referencial, a simetria do problema limita a saída dos fótons através do primeiro e do quarto octantes, mas basta calcular as trajetórias $s_{2}$ em um deles e multiplicar por dois para levar em conta a intensidade total do processo de escape. No primeiro octante, o ângulo de espalhamento pode ser expresso como $\theta=\theta_{\mathrm{e}}+\theta^{\prime}$, onde $\theta^{\prime}$ é medido em relação ao eixo $z^{\prime}$, e $\phi^{\prime}$ (medido respeito ao eixo $x^{\prime}$ ) varia no intervalo $\left[0, \phi_{\mathrm{m}}\right]$. Por outro lado, os caminhos $s_{2}$ se inscrevem sobre a superfície do cone

$$
x^{\prime 2}+y^{\prime 2}=z^{\prime 2} \tan ^{2} \theta^{\prime}
$$

e vão desde a origem até a intersecção com o cilíndrico

$$
\left(x^{\prime}-x_{\mathrm{o}}\right)^{2}+y^{\prime 2}=r_{\mathrm{c}}^{2}
$$

definido pela abertura do colimador. O valor $\phi_{\mathrm{m}}$ se obtém igualando ambas equações no plano $z=z_{\mathrm{o}}$, delimitado pela superfície final do colimador:

$$
\phi_{\mathrm{m}}=\arctan \left(y_{\mathrm{m}} / x_{\mathrm{m}}\right),
$$

sendo

$$
x_{\mathrm{m}}=\frac{1}{2 x_{\mathrm{o}}}\left(z_{\mathrm{o}}^{2} \tan ^{2} \theta^{\prime}+x_{\mathrm{o}}^{2}-r_{\mathrm{c}}^{2}\right) \quad \text { e } \quad y_{\mathrm{m}}=\sqrt{r_{\mathrm{c}}^{2}-x_{\mathrm{m}}^{2}}
$$

As distâncias $z_{\mathrm{o}}$ e $x_{\mathrm{o}}$ são funções das variáveis $z$ e $s_{1}$ (ver Figs. 5.6 e 5.9):

$$
z_{\mathrm{o}}=d_{\mathrm{c}}-z-s_{1} \cos \theta_{\mathrm{e}} \quad \text { e } \quad x_{\mathrm{o}}=s_{1} \sin \theta_{\mathrm{e}}+r_{\mathrm{c}}
$$

Dessa forma, os limites de integração de $\phi=\phi^{\prime}$ na Eq. (5.12) estão totalmente definidos para valores fixos de $z$ e $s_{1}$. O passo seguinte consiste em determinar os caminhos $s_{2}$ em função dos ângulos $\theta^{\prime}$ e $\phi^{\prime}$. Neste caso, as variáveis $x^{\prime}, y^{\prime}$ estão definidas pela quádrica do cilindro, Eq. (5.14), e $z^{\prime}$ se relaciona com $x^{\prime}$ e $y^{\prime}$ por médio da Eq. (5.13). Sob essas condições se obtém

$$
s_{2}\left(z, s_{1}, \theta^{\prime}, \phi^{\prime}\right)=\sqrt{x^{\prime 2}+y^{\prime 2}+z^{\prime 2}}=\sqrt{\rho_{\text {cil }}^{2}+z_{\text {con }}^{2}}
$$

com

$$
\rho_{\text {cil }}=x_{\mathrm{o}} \cos \phi^{\prime}-\sqrt{r_{\mathrm{c}}^{2}-\left(x_{\mathrm{o}} \sin \phi^{\prime}\right)^{2}},
$$

e

$$
z_{\text {con }}=\cot \theta^{\prime} \sqrt{2 x_{\mathrm{o}} \rho_{\text {cil }} \cos \phi^{\prime}-\left(x_{\mathrm{o}}^{2}-r_{\mathrm{c}}^{2}\right)} .
$$


Logo, com a relação anterior e fixando as variáveis $z, s_{1}$ e $\theta^{\prime}$, é possível fazer a primeira integração na Eq. (5.12) para levar em conta a contribuição do ângulo azimutal $\phi^{\prime}$

$$
\begin{aligned}
\mathrm{d}^{3} P_{\text {col }}\left(E, E^{\prime}, z, s_{1}, \cos \theta\right)= & \mathcal{N}_{\mathrm{Cu}} \mathrm{d} N(z) \exp \left[-\mu_{\mathrm{Cu}}(E) s_{1}\right] \mathrm{d} s_{1} \\
& \times \frac{\mathrm{d}^{2} \sigma_{\mathrm{AIR}}\left(E, E^{\prime}, \cos \theta\right)}{\mathrm{d} E^{\prime} \mathrm{d} \Omega^{\prime}} I_{3}\left(E^{\prime}, z, s_{1}, \cos \theta\right) \mathrm{d}(\cos \theta),
\end{aligned}
$$

onde

$$
I_{3}\left(E^{\prime}, z, s_{1}, \cos \theta\right)=2 \int_{0}^{\phi_{\mathrm{m}}} \exp \left[-\mu_{\mathrm{Cu}}\left(E^{\prime}\right) s_{2}\right] \mathrm{d} \phi^{\prime}
$$

$\mathcal{N}_{\mathrm{Cu}}$ é o número de átomos por unidade de volume do $\mathrm{Cu}$. De maneira similar, para valores fixos de $z$ e $s_{1}$ é realizada a segunda integração que considera todos os possíveis ângulos de espalhamento $\theta$ :

$$
\mathrm{d}^{2} P_{\mathrm{col}}\left(E, E^{\prime}, z, s_{1}\right)=\mathcal{N}_{\mathrm{Cu}} \mathrm{d} N(z) \exp \left[-\mu_{\mathrm{Cu}}(E) s_{1}\right] I_{2}\left(E^{\prime}, z, s_{1}\right) \mathrm{d} s_{1},
$$

com

$$
I_{2}\left(E^{\prime}, z, s_{1}\right)=\int_{\cos \theta_{\max }}^{\cos \theta_{\min }} \frac{\mathrm{d}^{2} \sigma_{\mathrm{AIR}}\left(E, E^{\prime}, \cos \theta\right)}{\mathrm{d} E^{\prime} \mathrm{d} \Omega^{\prime}} I_{3}\left(E^{\prime}, z, s_{1}, \cos \theta\right) \mathrm{d}(\cos \theta) .
$$

Os limites de integração vêm dados por

$$
\cos \theta_{\max }=\cos \theta_{\text {gmax }} \cos \theta_{\mathrm{e}}-\sin \theta_{\text {gmax }} \sin \theta_{\mathrm{e}}
$$

com

$$
\theta_{\operatorname{gmax}}=\arctan \left(\frac{x_{\mathrm{o}}+r_{\mathrm{c}}}{z_{\mathrm{o}}}\right)
$$

$\mathrm{e}$

$$
\cos \theta_{\text {min }}=\cos \theta_{\text {gmin }} \cos \theta_{\mathrm{e}}-\sin \theta_{\text {gmin }} \sin \theta_{\mathrm{e}},
$$

com

$$
\theta_{\text {gmin }}=\arctan \left(\frac{x_{\mathrm{o}}-r_{\mathrm{c}}}{z_{\mathrm{o}}}\right)
$$

A contribuição do anel de espessura $\mathrm{d} z$ situado à profundidade $z$ é calculado agora integrando a variável $s_{1}$

$$
\mathrm{d} P_{\operatorname{col}}\left(E, E^{\prime}, z\right)=\mathcal{N}_{\mathrm{Cu}} I_{1}\left(E, E^{\prime}, z\right) \mathrm{d} N(z),
$$

sendo

$$
I_{1}\left(E, E^{\prime}, z\right)=\int_{0}^{3 \lambda_{\mathrm{Cu}}(E)} \exp \left[-\mu_{\mathrm{Cu}}(E) s_{1}\right] I_{2}\left(E^{\prime}, z, s_{1}\right) \mathrm{d} s_{1} .
$$


No limite superior, $\lambda_{\mathrm{Cu}}(E)=\mu_{\mathrm{Cu}}^{-1}(E)$ representa o caminho livre médio no $\mathrm{Cu}$ de um fóton de energia $E$. Finalmente, a probabilidade de que um fóton atinja o cristal de Ge depois ser espalhado no colimador e sair pelo orifício central, se obtém como

$$
P_{\text {col }}\left(E, E^{\prime}\right)=\mathcal{N}_{\mathrm{Cu}} \int_{0}^{d_{\mathrm{c}}-5 \lambda_{\mathrm{Cu}}(E)} I_{1}\left(E, E^{\prime}, z\right) \mathrm{d} N(z) .
$$

Nesse caso, a integração é realizada até o valor $d_{\mathrm{c}}-5 \lambda_{\mathrm{Cu}}(E)$ para garantir caminhos de entrada $s_{1}$ entre 0 e $3 \lambda_{\mathrm{Cu}}(E)$, e caminhos de saída $s_{2}$ através do orifício central como considerado no modelo.

Para calcular o EAP gerado pelo espalhamento no colimador se assume que a energia $E^{\prime}$ é depositada no cristal do detetor com uma probabilidade dada por $\varepsilon_{\text {int }}\left(E^{\prime}\right)$.

\subsection{Influência da fonte na função resposta}

O efeito que introduz no EAP a massa que rodeia o material radioativo de uma fonte é conhecido e tem sido estudado para diferentes geometrias [97, 98]. Nesta seção será analisado esse fenômeno na configuração do experimento descrito anteriormente para o teste da FR. Considera-se que a deposição do material radioativo de ${ }^{241} \mathrm{Am}$ é um ponto, posicionado entre duas folhas de mylar de espessuras $l_{1}$ e $l_{2}$ (Fig. 5.10). Toma-se um sistema de referência com origem nesse ponto, de maneira que o eixo $z$ coincide com o eixo do colimador. Além disso, assume-se que a direção dos fótons espalhados é paralela a esse eixo. A condição anterior estabelece a ligação $\theta_{\mathrm{e}}=\theta$, ou seja, o ângulo de emissão medido em relação ao eixo $z$ resulta o mesmo que o ângulo do espalhamento Compton.

Sob as simplificações adotadas, e seguindo a notação utilizada nas seções anteriores, a função densidade de probabilidade que descreve o espectro de espalhamento na fonte, emitido na direção do detetor, pode se calcular a partir de

$$
\begin{aligned}
\mathrm{d}^{2} P_{\text {fon }}\left(E, E^{\prime}, x_{\mathrm{e}}, s_{1} ; l_{1}, l_{2}, r_{\mathrm{c}}\right)= & \mathrm{d} N\left(x_{\mathrm{e}}\right) \mu_{\mathrm{p}}^{\mathrm{inc}}(E) \exp \left[-\mu_{\mathrm{p}}(E) s_{1}\right] \mathrm{d} s_{1} \\
& \times \frac{2 \pi}{\sigma_{\mathrm{p}}^{\text {inc }}(E)} \frac{\mathrm{d}^{2} \sigma_{\mathrm{AIR}}\left(E, E^{\prime}, x_{\mathrm{e}}\right)}{\mathrm{d} E^{\prime} \mathrm{d} \Omega^{\prime}} \\
& \times \exp \left[-\mu_{\mathrm{p}}\left(E^{\prime}\right) s_{2}\right],
\end{aligned}
$$

onde $\cos \theta_{\mathrm{e}}=x_{\mathrm{e}}$, e o subíndice "p" nos coeficientes de atenuação e na seção de choque incoerente total se refere ao plástico das folhas que encapsulam a fonte. O fator $\mathrm{d} N\left(x_{\mathrm{e}}\right)$ vem dado pela Eq. (5.5), e como os outros termos do membro esquerdo da Eq. (5.32), conservam o mesmo significado físico descrito ao longo deste trabalho. Note-se que a SCDD segundo a AIR está avaliada diretamente no valor dado por $x_{\mathrm{e}}$ toda vez que 


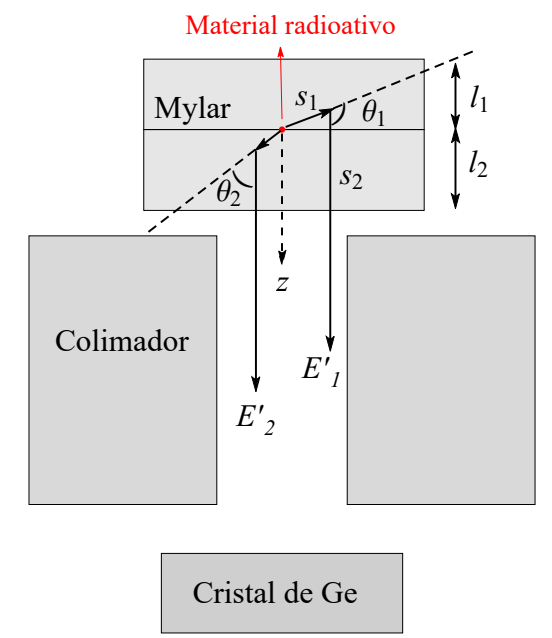

Figura 5.10: Esquema do espalhamento Compton no material que compõe a fonte. Desenho fora de escala.

$\theta_{\mathrm{e}}=\theta$. Por outro lado, de acordo com a simetria do problema, o caminho $s_{2}$ que o fóton espalhado percorre antes de sair da fonte pode ser diretamente relacionado com o trajeto $s_{1}$ do fóton emitido dentro desta:

$$
s_{2}=l_{2}-s_{1} x_{\mathrm{e}}
$$

Assim, calcula-se o fator geométrico da fonte que leva em conta todos os caminhos possíveis $s_{1}$ em função de $x_{\mathrm{e}}$

$$
\begin{aligned}
G_{\text {fon }}\left(E, E^{\prime}, x_{\mathrm{e}}\right)= & \exp \left[-\mu_{\mathrm{p}}\left(E^{\prime}\right) l_{2}\right] \int_{0}^{s_{1 l}} \exp \left[-\mu_{\mathrm{p}}(E) s_{1}\right] \exp \left[\mu_{\mathrm{p}}\left(E^{\prime}\right) s_{1} x_{\mathrm{e}}\right] \mathrm{d} s_{1} \\
& =\exp \left[-\mu_{\mathrm{p}}\left(E^{\prime}\right) l_{2}\right] \xi^{-1}\left[1-\exp \left(-\xi s_{1 l}\right)\right]
\end{aligned}
$$

onde $\xi=\mu_{\mathrm{p}}(E)-\mu_{\mathrm{p}}\left(E^{\prime}\right) x_{\mathrm{e}}$. O limite superior da integral $s_{1 l}$ depende da região da fonte onde o espalhamento tem lugar:

1. Se $x_{\mathrm{e}} \in\left[l_{2} / \sqrt{l_{2}^{2}+r_{\mathrm{c}}^{2}}, 1\right]$ então $s_{1 l}=l_{2} / x_{\mathrm{e}}$.

2. Se $x_{\mathrm{e}} \in\left[0, l_{2} / \sqrt{l_{2}^{2}+r_{\mathrm{c}}^{2}}\right]$ então $s_{1 l}=r_{\mathrm{c}} / \sqrt{1-x_{\mathrm{e}}^{2}}$.

3. Se $x_{\mathrm{e}} \in\left[-l_{1} / \sqrt{l_{1}^{2}+r_{\mathrm{c}}^{2}}, 0\right]$ então $s_{1 l}=r_{\mathrm{c}} / \sqrt{1-x_{\mathrm{e}}^{2}}$.

4. Se $x_{\mathrm{e}} \in\left[-1,-l_{1} / \sqrt{l_{1}^{2}+r_{\mathrm{c}}^{2}}\right]$ então $s_{1 l}=l_{1} / x_{\mathrm{e}}$.

Nas situações 2 e 3 se considerou que o raio do colimador $r_{\mathrm{c}}$ limita geometricamente a região de interação. Logo, o espectro $P_{\text {fon }}\left(E, E^{\prime}\right)$ se obtém integrando sobre todos os 
valores de $x_{\mathrm{e}}$ :

$$
P_{\text {fon }}\left(E, E^{\prime}\right)=\pi \mathcal{N}_{\mathrm{p}} \int_{-1}^{1} \frac{\mathrm{d}^{2} \sigma_{\mathrm{AIR}}\left(E, E^{\prime}, x_{\mathrm{e}}\right)}{\mathrm{d} E^{\prime} \mathrm{d} \Omega^{\prime}} G_{\text {fon }}\left(E, E^{\prime}, x_{\mathrm{e}}\right) \mathrm{d} x_{\mathrm{e}},
$$

sendo $\mathcal{N}_{\mathrm{p}}$ o número de átomos por unidade de volume do material da fonte. Como nos casos da janela de Be e do colimador, para calcular o EAP gerado pela fonte, é assumido que a energia $E^{\prime}$ se deposita no cristal do detetor com uma probabilidade dada por $\varepsilon_{\text {int }}\left(E^{\prime}\right)$.

\subsection{Quantificação dos efeitos da geometria}

As expressões analíticas desenvolvidas para o espalhamento Compton no colimador e na fonte, permitem agora fazer uma comparação com o EAP coletado na primeira experiência relatada na seção 5.1, especificamente, no intervalo compreendido entre $h=45 \mathrm{keV}$ e o fotopico de $59,54 \mathrm{keV}$. Nessa região se evidenciaram os efeitos desses elementos do arranjo.

Para a determinação das contagens produzidas pelo espalhamento Compton na fonte, utilizaram-se espessuras $l_{1}=0,5 \mathrm{~mm}$ e $l_{2}=0,1 \mathrm{~mm}$. Adotou-se o valor $\rho_{\mathrm{p}}=1,4 \mathrm{~g} / \mathrm{cm}^{3}$ como densidade do mylar [84]. As outras grandezas envolvidas nos cálculos onde aparece este composto, como a SCDD segundo a AIR e o número de massa efetivo, foram derivadas levando em conta a quantidade de átomos por elemento segundo a sua fórmula molecular: $\left(\mathrm{C}_{10} \mathrm{H}_{8} \mathrm{O}_{4}\right)_{n}$. Os coeficientes de atenuação de todos os materiais se tomaram do programa XCOM [84].

A Fig. 5.11 apresenta os resultados da comparação. São plotadas de maneira separada as contribuições da FR do detetor para fótons incidentes de $59,54 \mathrm{keV}$ e $55,55 \mathrm{keV}$, bem como as componentes contínuas de espalhamento Compton no colimador e na fonte de ${ }^{241} \mathrm{Am}$. Observa-se que sob as aproximações consideradas, a forma do EAP experimental é bem reproduzida.

O colimador gera um espectro que decresce rapidamente com a diminuição da energia a esquerda do pico de absorção total de 59,54 keV. O resultado indica que os espalhamentos acontecem a baixo ângulo e muito perto da superfície das paredes do orifício. No intervalo $56 \mathrm{keV} \leqslant h \leqslant 58 \mathrm{keV}$ o modelo subestima o experimento. Espalhamentos múltiplos a baixo ângulo, ou trajetórias em que os fótons saem pela superfície final do colimador poderiam explicar esse fato. Nenhum dos dois casos são modelados e ambos favoreceriam um aumento das contagens nessa faixa do EAP. 


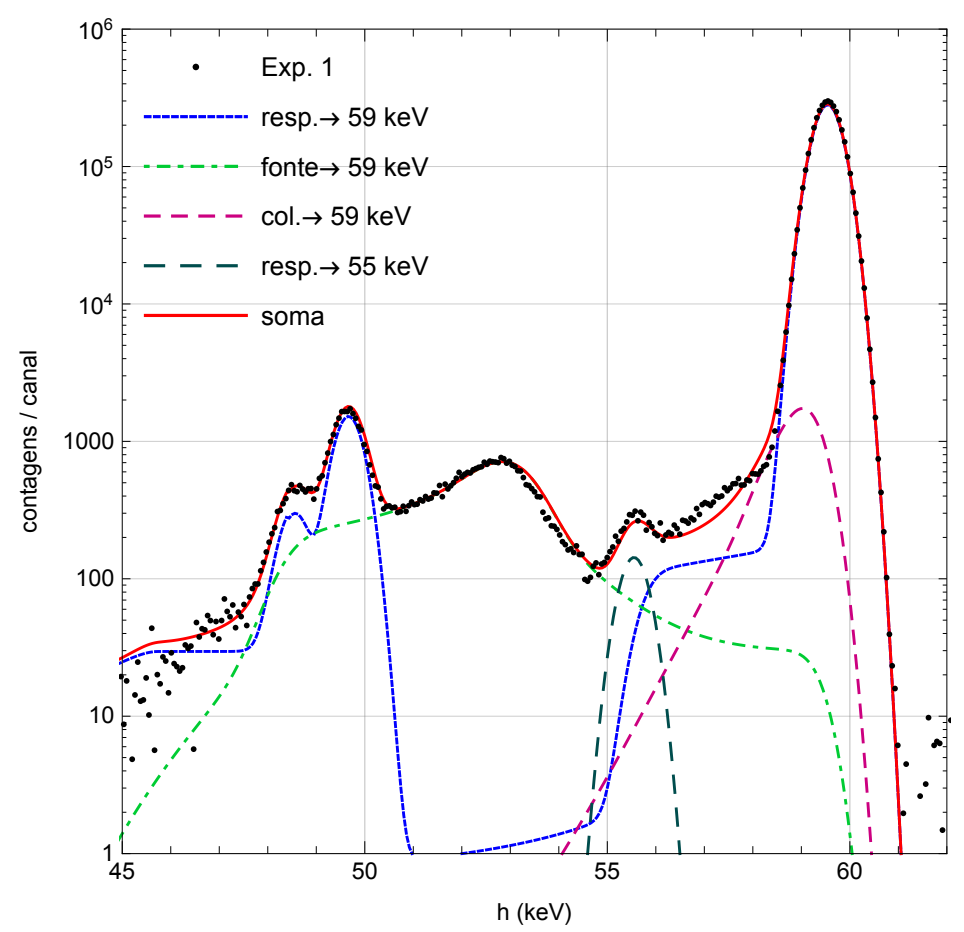

Figura 5.11: Comparação entre o espectro experimental e os modelos analíticos da contribuição dos elementos do arranjo na função resposta.

A contribuição da fonte domina o EAP coletado no intervalo $51 \mathrm{keV} \leqslant h \leqslant 55 \mathrm{keV}$, apresentando um pico alargado com um máximo perto de $h=52,8 \mathrm{keV}$, energia que equivale a espalhamento sob o ângulo $\theta \sim 92^{\circ}$ com a maior probabilidade. $O$ fato de $l_{1}$ ser maior que $l_{2}$ explica essa observação, e além disso, a maior intensidade obtida do lado esquerdo da mencionada estrutura, que corresponde fundamentalmente a eventos de retroespalhamento. Note-se que a porção esquerda dessa componente da FR interfere com os picos de escape de raios-x de Ge impedindo a melhor separação $\mathrm{K} \alpha-\mathrm{K} \beta$. É importante destacar que somente utilizando a SCDD sob a AIR se consegue descrever a considerável faixa energética aportada pelo espalhamento Compton no mylar.

A Fig. 5.12 mostra a função de acumulação de probabilidade $\Gamma(h)$, Eq. (4.67), para cada uma das componentes consideradas no modelo. O gráfico permite quantificar com maior clareza a contribuição relativa dos efeitos da geometria. Desse modo se estima que o colimador e a fonte aportam 1\% e 1,2\% da área total observada no EAP respectivamente, quando incidem fótons de $59,54 \mathrm{keV}$.

Embora as estimativas anteriores sejam específicas para uma energia e configuração experimental, algumas conclusões gerais podem se extrair. Tomando como exemplo $E \approx 60 \mathrm{keV}$, e de acordo com a discussão realizada na seç̧ão 5.2, o efeito que o colimador introduz na FR do detetor tem que ser pelo menos uma ordem menor quando o ponto de emissão é afastado à distância $d_{\mathrm{f}}=300 \mathrm{~mm}$. Essa análise permite garantir que, na configuração de irradiação e para energias incidentes menores que $60 \mathrm{keV}$, a influência 


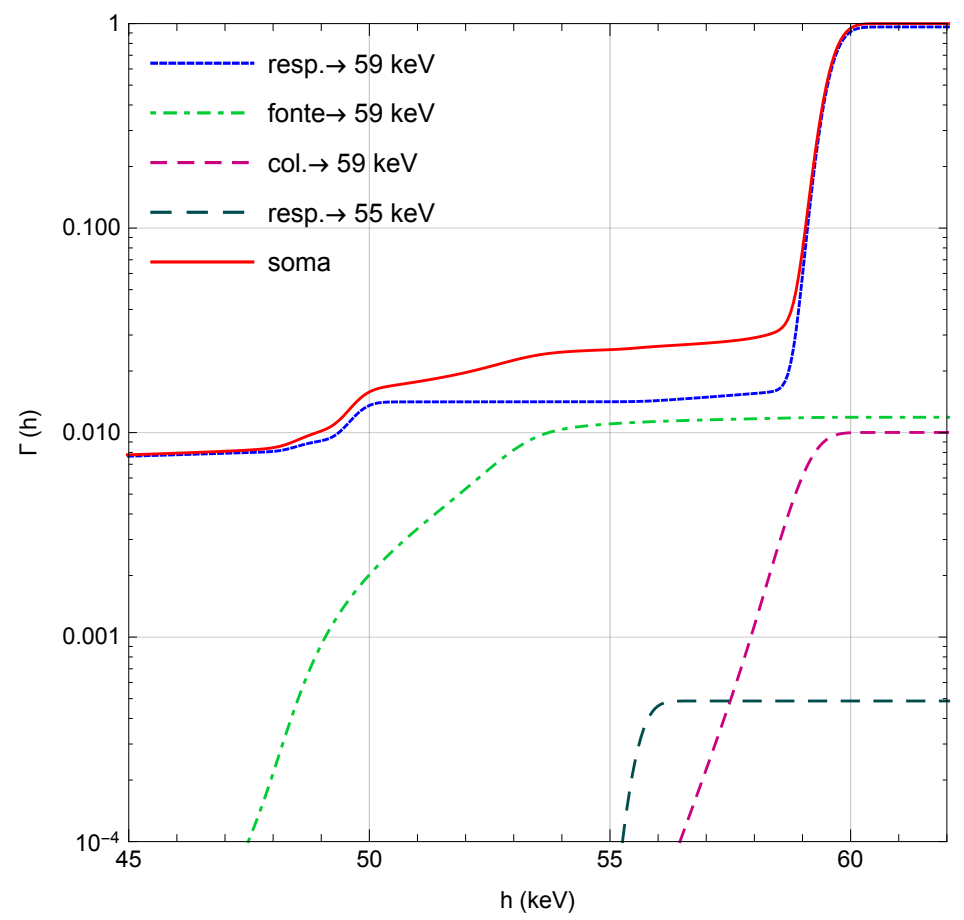

Figura 5.12: Função de acumulação de probabilidade de cada uma das contribuições analisadas no espectro medido da fonte de ${ }^{241} \mathrm{Am}$.

do colimador em termos de área aportada ao EAP será menor do que $0,1 \%$. Por outro lado, examinemos as seções de choque de espalhamento Compton $\sigma_{\mathrm{Cu}}^{\text {inc }}(E)$, e fotoefeito $\sigma_{\mathrm{Cu}}^{\mathrm{fe}}(E)$, do $\mathrm{Cu}$ na faixa $60 \mathrm{keV} \leqslant E \leqslant 100 \mathrm{keV}$. Enquanto $\sigma_{\mathrm{Cu}}^{\text {inc }}(E)$ se mantém praticamente constante, passando de 13,86 b/átomo a 13,32 b/átomo nos extremos do intervalo, $\sigma_{\mathrm{Cu}}^{\mathrm{fe}}(E)$ varia de $142,8 \mathrm{~b} /$ átomo para $60 \mathrm{keV}$ a 30,37 b/átomo para $100 \mathrm{keV}$ [84]. Esses números indicam que a probabilidade de espalhamento irá ser similar e o caminho livre médio dos fótons irá se acrescentar em mais de três vezes com o aumento da energia de 60 para $100 \mathrm{keV}$. Logo, para fótons incidentes superiores a $60 \mathrm{keV}$ a transparência do colimador será o efeito más importante.

Quando as medidas de bremsstrahlung são realizadas, os alvos tem um papel equivalente à fonte deste experimento. Porém, como poderá ser visto no Capítulo 6, a espessura deles está na ordem dos nm, de maneira que a massa na vizinhança do ponto de emissão se reduz em relação à fonte radioativa em mais de 4 ordens de grandeza. Além disso, a distribuição dos fótons gerados não é isotrópica. Pode se afirmar então que a contribuição do alvo irá ser muito menor que $1 \%$ na faixa energética de interesse neste trabalho. 


\section{Capítulo 6}

\section{Caracterização do arranjo experimental no Microtron e dos alvos}

Neste capítulo são relatados os testes e procedimentos de optimização do sistema de adquisição de dados que envolve a eletrônica associada aos espectrômetros. Além disso, são expostos os métodos experimentais de calibração da resolução e da eficiência dos detetores, a partir das quais são obtidas grandezas necessárias para a avaliação da FR e da SCDD. Relatam-se os procedimentos seguidos para determinar a espessura dos alvos a partir de medidas de retroespalhamento Rutherford de partículas ${ }^{4} \mathrm{He}^{2+}$. São discutidos alguns critérios quantitativos em relação à mudança da direção de incidência original, e às perdas de energia do elétron ao atravessar o alvo, que permitem avaliar as condições da emissão de bremsstrahlung.

\subsection{Otimização do sistema de aquisição}

A forma que apresentam as diferentes estruturas observadas num EAP depende dos parâmetros de amplificação escolhidos para o processamento do sinal proveniente do detetor. Nesta seção são estudados os tempos de conformação dos pulsos nos amplificadores da eletrônica de aquisição, sob as condições de irradiação dos alvos no acelerador. Procura-se a optimização do sistema a fim de obter os espectros de bremsstrahlung com a melhor resolução possível. Além disso, é verificado o modelo de tempo morto adotado pelo software de aquisição MAESTRO [52] para o cálculo do tempo vivo de coleção dos espectros. Esse último fator resulta de vital importância uma vez que a emissão do bremsstrahlung não é isotrópica, logo, a taxa de contagens a diferentes ângulos é 
distinta, e portanto o tempo morto irá ser diferente em cada detetor. Como resultado, a carga coletada pelo integrador deverá ser corrigida em função da fração que representa o tempo vivo do tempo real em cada caso.

\subsubsection{Conformação de pulsos}

Para o estudo da variação da resolução dos detetores se tomaram como referência os amplificadores digitais DSPEC e DSPEC-Jr 2.0 [51]. Nesses dispositivos um pulso de voltagem $h$ é conformado segundo a função trapezoidal

$$
h^{\prime}(t)= \begin{cases}h \frac{t}{t_{\mathrm{s}}} & 0 \leqslant t \leqslant t_{\mathrm{s}} \\ h & t_{\mathrm{s}}<t<t_{\mathrm{s}}+t_{\mathrm{p}} \\ h\left(1+\frac{t_{\mathrm{s}}+t_{\mathrm{p}}-t}{t_{\mathrm{d}}}\right) & t_{\mathrm{s}}+t_{\mathrm{p}} \leqslant t \leqslant t_{\mathrm{s}}+t_{\mathrm{p}}+t_{\mathrm{d}} \\ 0 & t \geqslant t_{\mathrm{s}}+t_{\mathrm{p}}+t_{\mathrm{d}},\end{cases}
$$

onde $h^{\prime}(t)$ é a altura do pulso em função do tempo $t, t_{\mathrm{s}}$ e $t_{\mathrm{d}}$ são os tempos de subida e descenso respectivamente, e $t_{\mathrm{p}}$ é o tempo em que o pulso permanece à voltagem máxima $h$. Entretanto, é conhecido que $t_{\mathrm{d}}=2 t_{\mathrm{s}}$ no DSPEC [51].

Em uma primeira etapa foram medidos espectros de bremsstrahlung correspondentes a um alvo de $\mathrm{Au}(\sim 90 \mathrm{~nm})$ para uma corrente fixa de elétrons, uma energia $E_{0} \approx 100 \mathrm{keV}$ e tempo vivo constante $t_{\mathrm{v}}=300 \mathrm{~s}$, variando $t_{\mathrm{s}}$ no intervalo $2-6 \mu \mathrm{s}$, com $t_{\mathrm{p}}=0,6 \mu \mathrm{s}$. A Fig. 6.1 mostra um exemplo da forma em que são conformados pulsos de altura unitária para diferentes valores de $t_{\mathrm{s}} \operatorname{com} t_{\mathrm{p}}$ fixo.

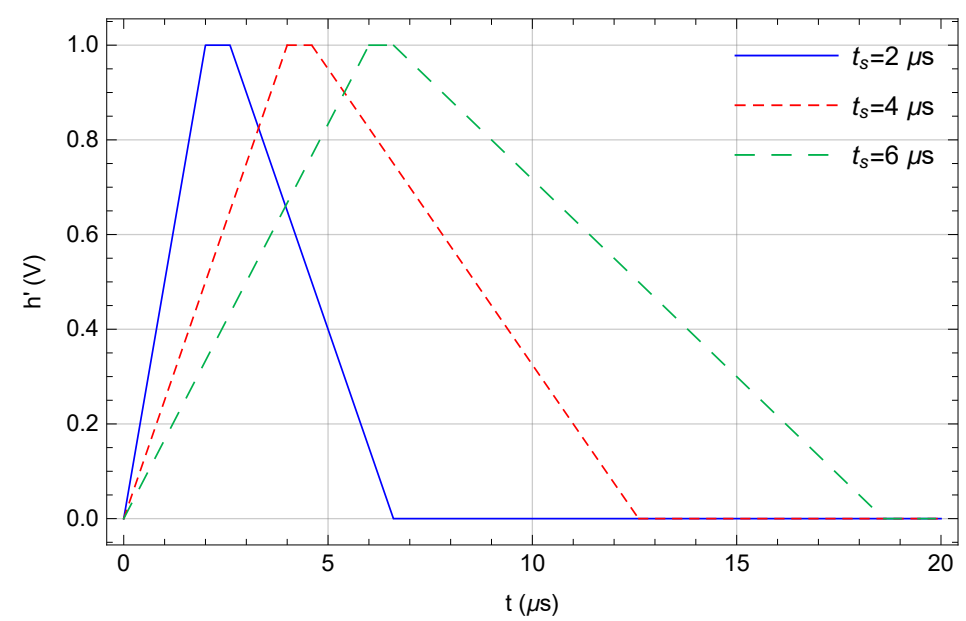

Figura 6.1: Forma dos pulsos amplificados no DSPEC para diferentes valores de $t_{\mathrm{s}}$ e $t_{\mathrm{p}}=0,6 \mu \mathrm{s}$.

Como medida da resolução foi quantificada a largura à meia altura do pico $\left(\mathrm{FWHM}^{1}\right)$

\footnotetext{
${ }^{1}$ FWHM: "Full Width at Half Maximum".
} 
correspondente à linha $\mathrm{L} \alpha$ do $\mathrm{Au}^{2}$. A Fig. 6.2 apresenta um exemplo da variação da largura do fotopico para tempos $t_{\mathrm{s}}=2,4$ e $6 \mu \mathrm{s}$, no detetor situado em $\theta=35^{\circ}$ (gráfico da esquerda), e a tendência observada no valor absoluto de FWHM (gráfico da direita) no intervalo de $t_{\mathrm{s}}$ estudado.
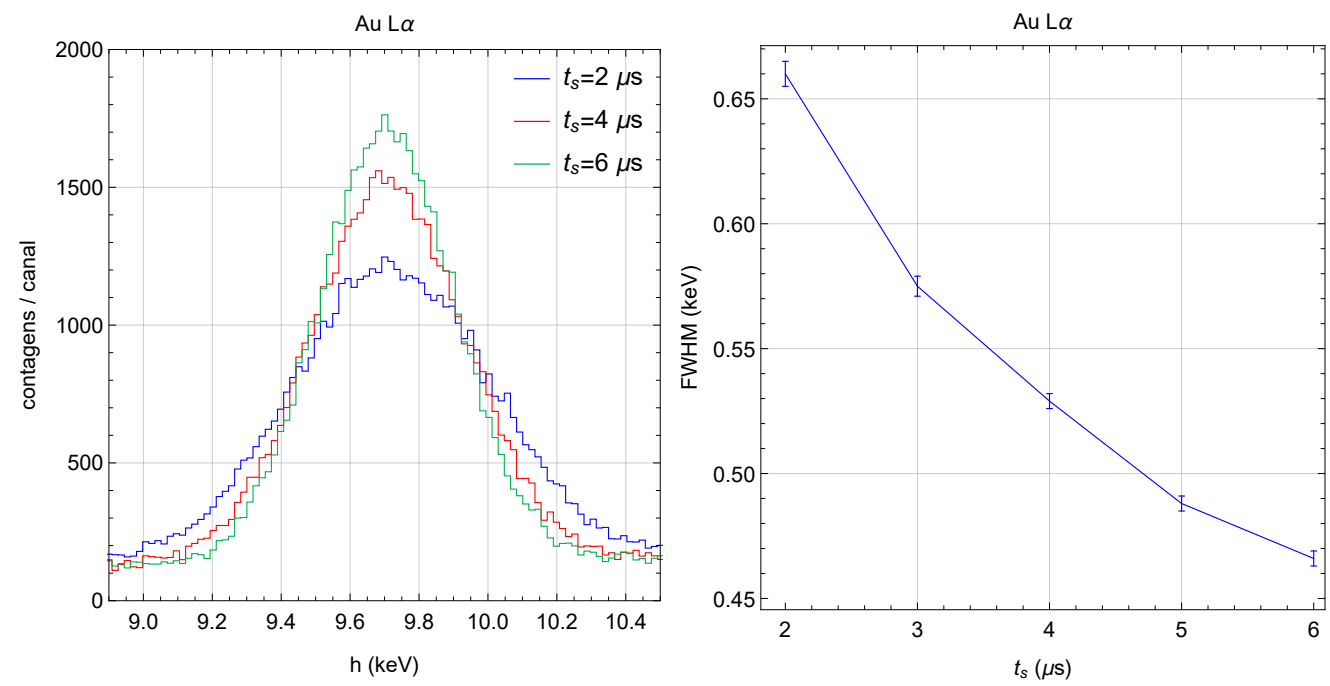

Figura 6.2: O gráfico da esquerda mostra a mudança na largura fotopico correspondente à linha $\mathrm{L} \alpha$ do $\mathrm{Au}$, para $t_{\mathrm{s}}=2,4$ e $6 \mu \mathrm{s}$, com $t_{\mathrm{p}}=0,6 \mu \mathrm{s}$. O gráfico da direita apresenta a variação de FWHM $(\mathrm{Au}-\mathrm{L} \alpha)$ em função de $t_{\mathrm{s}}$, com $t_{\mathrm{p}}=0,6 \mu \mathrm{s}$. Ambos exemplos são associados a medidas realizadas com o detetor situado a $\theta=35^{\circ}$, no arranjo experimental do Microtron.

Posteriormente foram obtidos espectros fixando o $t_{\mathrm{s}}$ em 4 e $6 \mu \mathrm{s}$ e variando $t_{\mathrm{p}}$ entre 0,2 e $0,8 \mu$ s. Este parâmetro não muda a largura do pico L $\alpha$ na faixa estudada (Fig. 6.3). A afirmação se sustenta no valor de $\chi^{2}$ obtido a partir do ajuste realizado segundo

$$
\operatorname{FWHM}\left(t_{\mathrm{p}}\right)=A\left(t_{\mathrm{p}}\right) \quad \text { com } \quad A\left(t_{\mathrm{p}}\right)=\text { cte. }
$$

Em ambos os casos a hipótese é aceita com um nível de significância de $0,05\left(\chi^{2}=5,5\right.$ com $\nu=5$ graus de liberdade para os dados coletados com $t_{\mathrm{s}}=4 \mu \mathrm{s}$, e $\chi^{2}=3,7$ para $\left.t_{\mathrm{s}}=6 \mu \mathrm{s}\right)$.

A partir dos resultados obtidos e considerando a distribuição angular do bremsstrahlung foram escolhidos para o detetor situado a $\theta=35^{\circ}$ os tempos de conformação $t_{\mathrm{s}}=4 \mu \mathrm{s}$ e $t_{\mathrm{p}}=0,4 \mu \mathrm{s}$. Nesse caso colocar um tempo de subida maior a fim de lograr melhor resolução aumentaria a fração de tempo morto do detetor em relação aos outros dois $\left(\theta=90^{\circ}\right.$ e $\left.131^{\circ}\right)$, devido a que a emissão de bremsstrahlung em ângulos dianteiros é dominante na faixa analisada de energia dos elétrons. Seguindo o mesmo critério, mas em sentido contrário, para o detetor colocado a $\theta=131^{\circ}$ se fixou o parâmetro $t_{\mathrm{s}}=6 \mu \mathrm{s}$ (o maior possível), mantendo $t_{\mathrm{p}}=0,4 \mu \mathrm{s}$, já que a taxa de emissão bremsstrahlung

\footnotetext{
${ }^{2}$ As energias correspondentes as transições $\mathrm{L}_{3} \mathrm{M}_{4}\left(\mathrm{~L} \alpha_{2}\right)$ e $\mathrm{L}_{3} \mathrm{M}_{5}\left(\mathrm{~L} \alpha_{1}\right)$ no $\mathrm{Au}$ são: 9628,05(33) e $9713,44(34) \mathrm{eV}$ respectivamente [83].
} 


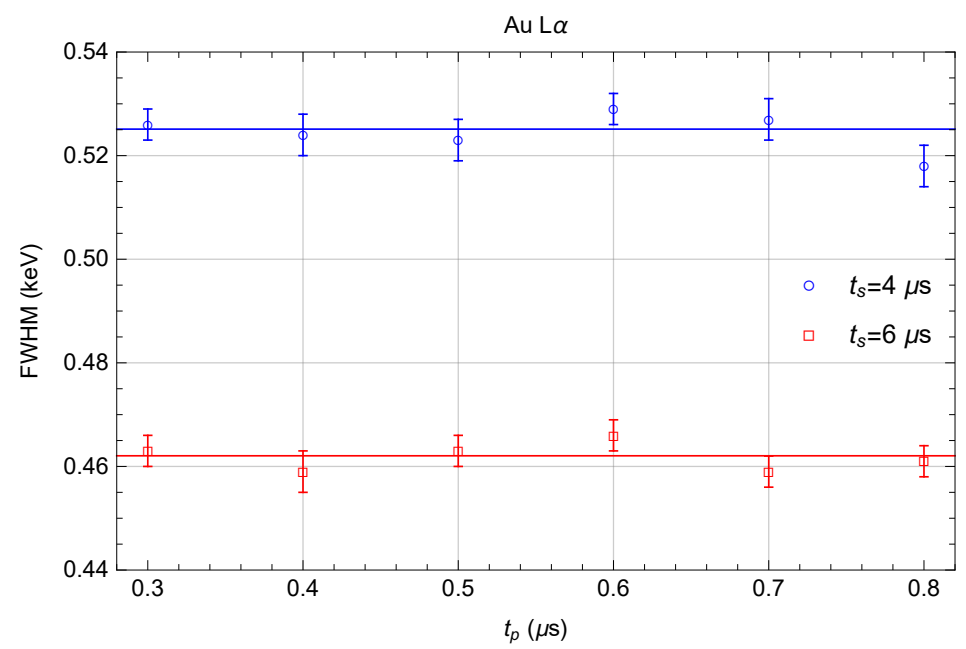

Figura 6.3: Variação da resolução da linha $\mathrm{L} \alpha$ do $\mathrm{Au}$, no detetor colocado em $\theta=35^{\circ}$, em função do $t_{\mathrm{p}} \operatorname{com} t_{\mathrm{s}}=4$ e $6 \mu \mathrm{s}$.

para esse ângulo é a menor das três posições estudadas. O detetor coaxial também foi colocado a $131^{\circ}$ conhecendo que apresenta a menor resolução, logo, ao receber menor taxa de contagens, permite trabalhar com $t_{\mathrm{p}}=6 \mu$ s conservando o equilíbrio do sistema de aquisição. Finalmente, para o detetor a $90^{\circ}$ acoplado a uma eletrônica analógica, em que os pulsos são amplificados de maneira Gaussiana, verificou-se que um tempo característico de conformação ( "shaping time" [81]) de $2 \mu$ s fornece resultados em quanto a resolução e tempo morto similares ao detetor situado a $35^{\circ}$.

\subsubsection{Estudo do tempo morto do sistema de aquisição}

Anteriormente foi mostrada a importância da correção por tempo morto $\tau$, na carga $\mathcal{Q}$ coletada durante o tempo real $t_{\mathrm{r}}$, em que são adquiridos os espectros de bremsstrahlung. A partir de $\mathcal{Q}$ se calcula o número de elétrons incidentes $N_{0}$ durante o tempo vivo $t_{\mathrm{v}}$ de medida dos EAP em cada detetor. A correção a $\mathcal{Q}$ se determina a partir da razão $t_{\mathrm{v}} / t_{\mathrm{r}}$, que depende exclusivamente de $\tau$, sendo $t_{\mathrm{v}}$ e $t_{\mathrm{r}}$ fornecidos pelo software de adquisição MAESTRO [52] durante a medida. O objetivo do estudo é comprovar a precisão e a faixa de taxas de contagens em que esses dados são confiáveis.

O experimento é similar ao descrito na subseção anterior, mas neste caso foram adquiridos os EAP de bremsstrahlung variando a corrente $I$ do feixe incidente sobre o alvo de Au entre 30 e $1000 \mathrm{nA}$, durante intervalos fixos de $t_{\mathrm{v}}=300 \mathrm{~s}$. A Fig. 6.4 apresenta três dos espectros coletados para diferentes correntes do feixe.

São conhecidos dois comportamentos ideais para a descrição do tempo morto $\tau$ de um sistema de detecção de radiação: o Modelo Paralisável (MP) e o Modelo Não Paralisável 


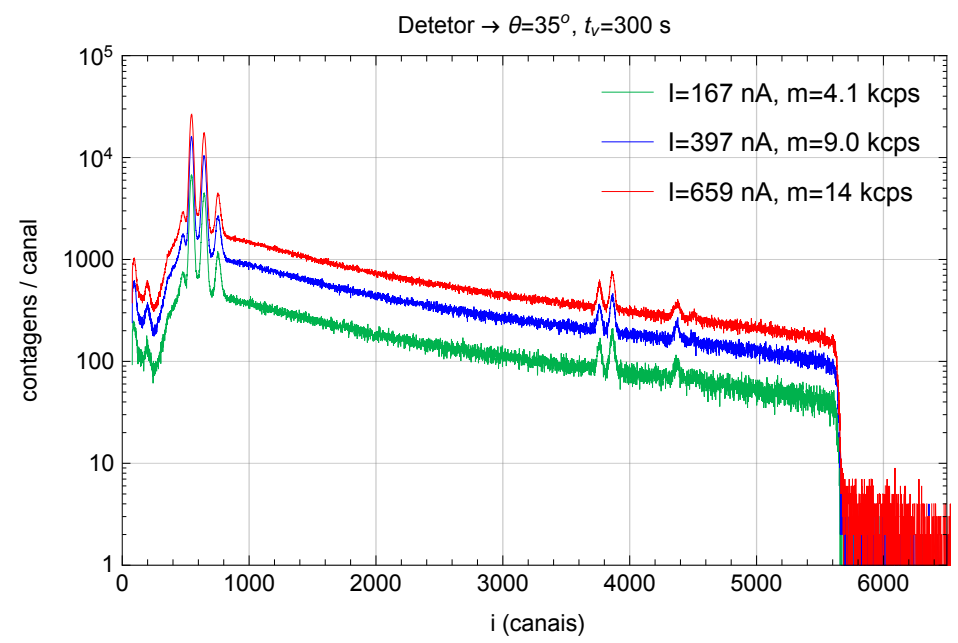

Figura 6.4: Exemplo dos EAP coletados para diferentes correntes $I$ do feixe de elétrons, que equivalem às taxas $m$ de contagens, no detetor colocado a $\theta=35^{\circ}$. Nas medidas se utilizou um alvo de Au.

(MNP) [81]. Seguindo notação do livro de Knoll [81], podem se adotar as seguintes definições:

- $n$ é taxa de eventos reais, ou o número de fótons por unidade de tempo que atingem o detetor.

- $m$ é a taxa de eventos coletados.

A expressão matemática que caracteriza o MNP é

$$
m=\frac{n}{1+n \tau}
$$

enquanto o MP é descrito por:

$$
m=n \mathrm{e}^{-n \tau}
$$

No presente experimento $m$ pode ser calculada em função de $t_{\mathrm{r}}$ segundo:

$$
m=t_{\mathrm{r}}^{-1} \sum_{i} N(i)
$$

onde $N(i)$ são as contagens no canal $i$ do histograma multicanal. A incerteza da taxa registrada se estimou como

$$
\sigma_{m}=t_{\mathrm{r}}^{-1} \sqrt{\sum_{i} N(i)} .
$$


Por outro lado, $n$ é proporcional à corrente média $I$ que incide sobre o alvo, determinada a partir da carga coletada $\mathcal{Q}$ durante $t_{\mathrm{r}}$ :

$$
n=\vartheta \frac{\mathcal{Q}}{t_{\mathrm{r}}}=\vartheta I
$$

sendo $\vartheta$ um parâmetro constante para a mesma energia $E_{0}$, que depende somente da seção de choque de interação dos elétrons e da geometria do experimento. Logo, substituindo $n$ nas Eqs. (6.3) e (6.4) obtemos respectivamente para o MNP e o MP

$$
m=\frac{\vartheta I}{1+\zeta I}
$$

e

$$
m=\vartheta I \mathrm{e}^{-\zeta I},
$$

onde $\zeta=\vartheta \tau$.

No experimento foram medidos os valores de $m$ para diferentes correntes $I$ (que são equivalentes a diferentes taxas $n$ ), assim foi possível ajustar os parâmetros $\vartheta$ e $\zeta$ para determinar $\tau=\zeta / \vartheta$, e testar tanto o MP quanto o MNP. O programa MAESTRO calcula a fração $t_{\mathrm{v}} / t_{\mathrm{r}}$ utilizando a Eq. (6.3) do MP, a partir da taxa de contagens coletada e o tempo morto do sistema de aquisição [52]. Segundo o manual do DSPEC [51], o tempo morto vem dado por:

$$
\tau=3 t_{\mathrm{s}}+t_{\mathrm{p}}
$$

Para o detetor colocado a $35^{\circ}$ a experiência foi realizada com $t_{\mathrm{s}}=4 \mu \mathrm{s}$ e $t_{\mathrm{p}}=0,6 \mu \mathrm{s}$, portanto a estimativa inicial é $\tau=12,6 \mu \mathrm{s}$.

As Eqs. (6.8) e (6.9) foram ajustadas aos dados medidos utilizando o algoritmo não linear NonlinearModelFit do software Mathematica [87] (Fig. 6.5). Os resíduos reduzidos são mostrados no gráfico inferior da Fig. 6.5 para cada modelo e na Tabela 6.1 se resumem os resultados obtidos. 

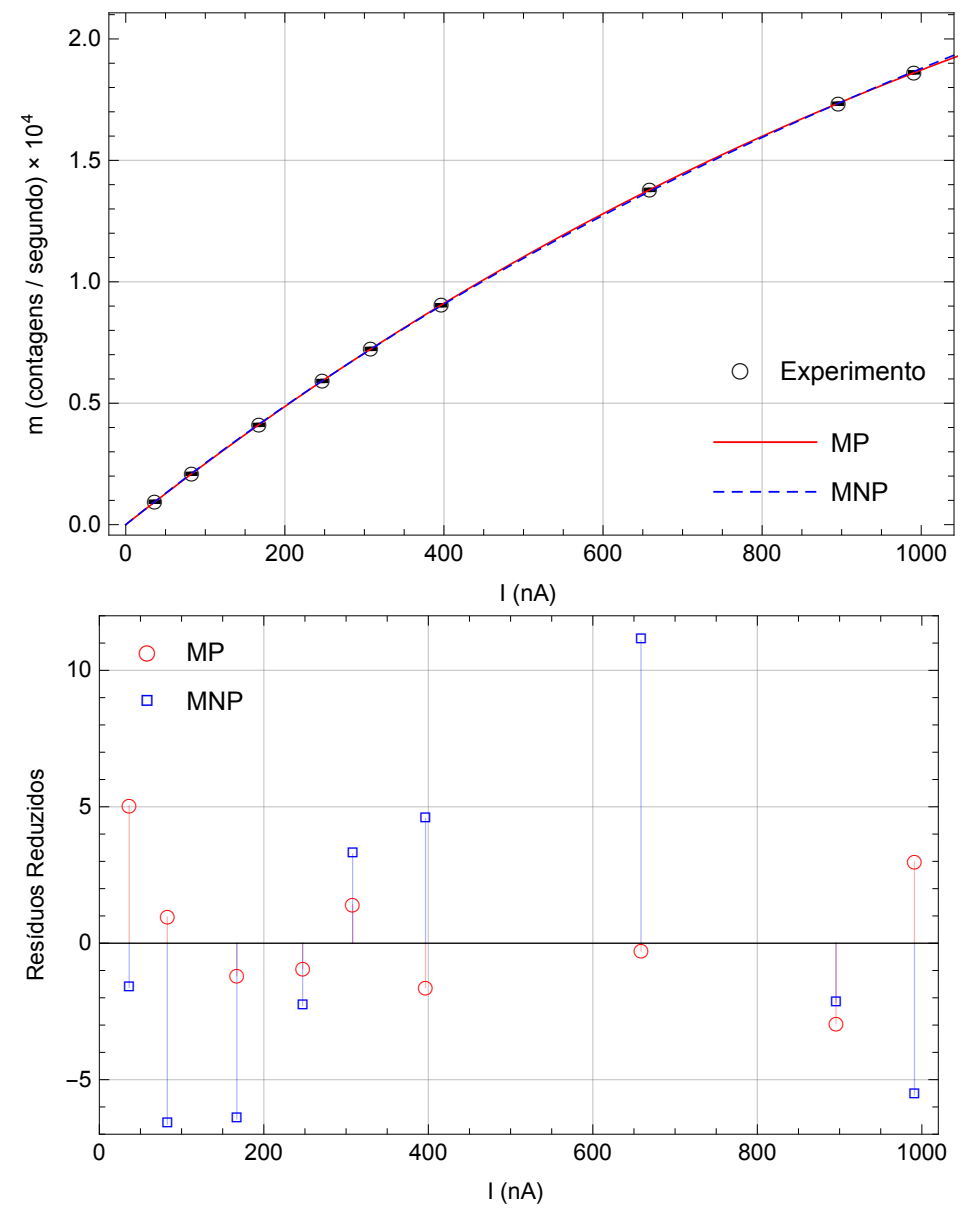

Figura 6.5: O gráfico superior apresenta os pontos experimentais e as curvas correspondentes a cada modelo com os parâmetros $\vartheta$ e $\zeta$ ajustados. Os resíduos reduzidos dos ajustes se mostram no gráfico inferior.

TABela 6.1: Parâmetros $\vartheta$ e $\zeta$ ajustados segundo o MP e o MNP, e tempo morto $\tau$ determinado em cada caso.

\begin{tabular}{c||cccc}
\hline \hline & $\vartheta(1 / \mathrm{nC})$ & $\zeta(\mu \mathrm{s} / \mathrm{nC})$ & $\tau(\mu \mathrm{s})$ & $\chi^{2}(\nu=7)$ \\
\hline MP & $25,968(12)$ & $327,2(6)$ & $12,60(15)$ & 51,1 \\
MNP & $26,366(13)$ & $403,6(9)$ & $15,31(20)$ & 284 \\
\hline \hline
\end{tabular}


De acordo com os elevados valores dos resíduos e de $\chi^{2}$, além da pouca concordância de $\tau$ com o valor esperado segundo a Eq. (6.10), pode se concluir o MNP não é adequado para descrever o comportamento medido. No caso do MP, o ajuste não passa o teste de $\chi^{2}$, mas o fato de ser compatível o tempo morto determinado com a estimava prevista indica que as incertezas $\sigma_{m}$ foram subestimadas. Esta última hipótese é razoável considerando que a fórmula (6.6) só leva em conta as flutuações estatística nas contagens registradas, porém não inclui possíveis erros associados à eletrônica do sistema de aquisição (o integrador de carga). Logo, o ajuste foi repetido utilizando incertezas nos pontos experimentais iguais a $2 \sigma_{m}$. A Fig. 6.6 mostra os resíduos para esta situação. Nesse caso se obtém $\chi^{2}=12,8$ com $\nu=7$ graus de liberdade, que passa no teste com um nível de significância de 0,05, enquanto $\tau=12,60(29)$.

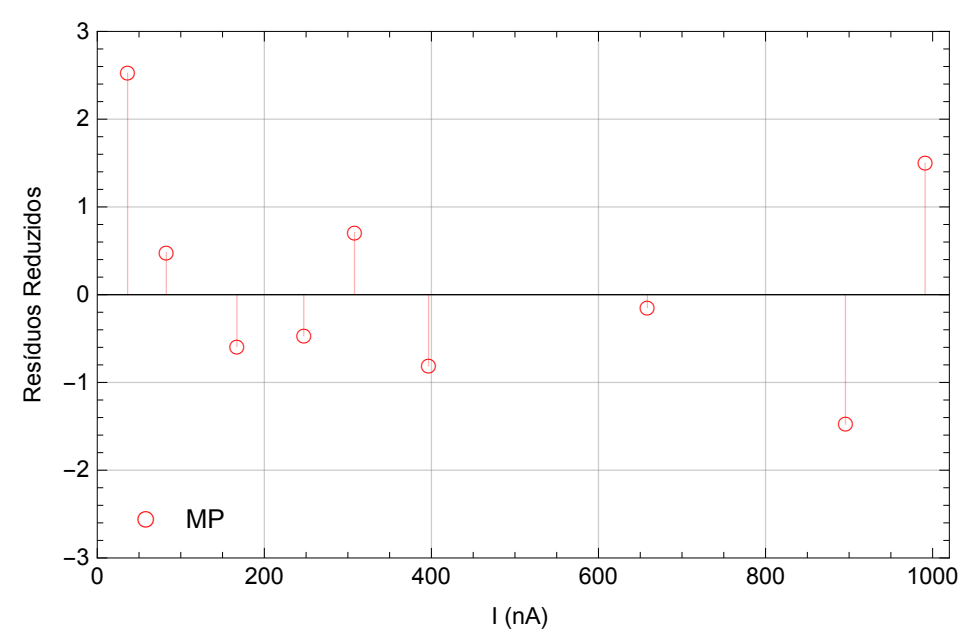

Figura 6.6: Resíduos reduzidos do ajuste segundo o MP considerando as incertezas em cada ponto $m$ como $2 \sigma_{m}$.

No intervalo de taxas estudado, a fração do tempo real em que os detetores não registraram contagens esteve entre $1 \%$ e $30 \%$. Os resultados anteriores permitem concluir que nessa faixa o sistema de adquisição corrige o tempo morto adequadamente. Dessa maneira, os espectros de bremsstrahlung para a determinação das SCDDs se coletaram escolhendo taxas de contagens com frações de tempo morto inferiores a $10 \%$.

\subsection{Caracterização experimental dos detetores}

A coleção de EAP produzidos pelos raios-x e $\gamma$ emitidos por radionuclídeos de fontes calibradas, permite a determinação experimental da resolução dos detetores e da eficiência absoluta de foto pico $\varepsilon_{\mathrm{A}}(E)$. Essas duas calibrações são essenciais, a partir delas se obtêm os parâmetros necessários para avaliar a função $\mathcal{G}(h, \epsilon ; \sigma)$ na Eq. (4.14), que carateriza a resposta dos detetores, e além disso, o ângulo sólido $\Omega$ subtendido pelo detetor 
em relação à posição do alvo, envolvido diretamente na Eq. (2.77) para a determinação das SCDD de emissão de bremsstrahlung.

\subsubsection{Calibração da resolução dos detetores: determinação dos parâmetros de dispersão Gaussiana da resposta}

Nas calibrações de resolução tipicamente é utilizada a FWHM como grandeza caraterística. A escolha se deve a que a largura à meia altura propicia, intuitivamente, mais clareza da capacidade que tem um detetor para definir um pico do que a variância $\sigma$ da Gaussiana $\mathcal{G}(h, \epsilon ; \sigma)$. Ambas quantidades estão relacionadas linearmente por FWHM = $2 \sqrt{2 \ln 2} \sigma \simeq 2,3548 \sigma$, logo, é equivalente fazer um ajuste dos parâmetros $a$ e $b$ da fórmula (4.64) por meio de

$$
\mathrm{FWHM}^{2}=a^{\prime}+b^{\prime} \epsilon
$$

onde $a^{\prime}=8 \ln 2 a, b^{\prime}=8 \ln 2 b$ e $\epsilon=E$, sendo $E$ a energia do centroide da Gaussiana que descreve o pico absorção total no EAP.

Para a calibração em FWHM dos detetores foram coletados espectros dos radionuclídeos ${ }^{133} \mathrm{Ba},{ }^{152} \mathrm{Eu} \mathrm{e}{ }^{241} \mathrm{Am}$. As fontes utilizadas foram preparadas no Laboratório de Metrologia Nuclear, do Instituto de Pesquisas Energéticas e Nucleares, tomando como base um suporte de carbono igual aos adotados na confecção dos alvos (ver seção 3.2.1). De maneira similar ao procedimento descrito no Capítulo 5, foi depositada uma gota de solução radioativa sobre um filme de kapton colocado na abertura central da moldura (Fig. 6.7). Uma vez seca a deposição, foi colocado por cima desta mais um filme de collodio para evitar o escape do material radioativo.

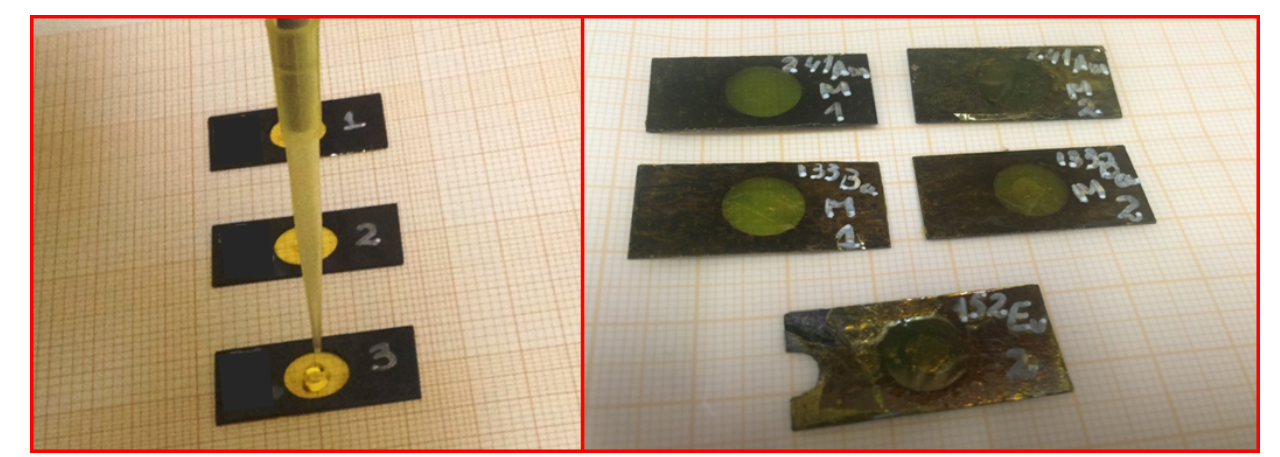

Figura 6.7: Na fotografia da esquerda se observa o momento da deposição da gota radioativa. Á direita são apresentadas as fontes no formato final.

As fontes foram posicionadas na torre de alvos, com uma inclinação de $\alpha=60^{\circ} \mathrm{em}$ relação a direção de incidência do feixe, a fim de realizar as calibrações com uma geometria igual à de irradiação. Para a análise dos EAP foi desenvolvido um programa em 
Mathematica [87] que permite o ajuste dos parâmetros de até oito funções Gaussianas ao mesmo tempo, bem como os coeficientes de polinômios de grau 0, 1 ou 2 que levam em conta as contagens na base dos picos. O programa fornece a energia do centroide, a FWHM e a área dos picos, empregando os algoritmos de ajuste LinearModelFit e NonLinearModelFit [87].

A Fig. 6.8 apresenta os resultados das calibrações em FWHM para os três detetores. Nesse caso somente foram utilizados os picos de absorção total correspondes as transições $\gamma$ nos EAP coletados de duas fontes de ${ }^{133} \mathrm{Ba}$, duas de ${ }^{241} \mathrm{Am}$ e uma de ${ }^{152} \mathrm{Eu}$. A Tabela 6.2 resume os coeficientes $a^{\prime}$ e $b^{\prime}$ obtidos a partir dos ajustes, os parâmetros $a$ e $b$ característicos da variância de $\mathcal{G}(h, \epsilon ; \sigma)$, o valor de $\chi_{\nu}^{2}$ com $\nu=9$ graus de liberdade, e a probabilidade $P\left(\chi_{\nu}^{2}\right)$ de exceder o valor de $\chi^{2}$ crítico para $\nu=9$ [53]. Observa-se que os ajustes realizados são adequados com um nível de significância de $5 \%$ no teste de $\chi^{2}$. Pode se visualizar a semelhança obtida $b^{\prime}$ nos três detetores, em acordo com as predições teóricas que estabelecem a dependência dessa constante só com as características do material de deteç̧ão: fator de Fano e energia para criar um par elétron-buraco [81].

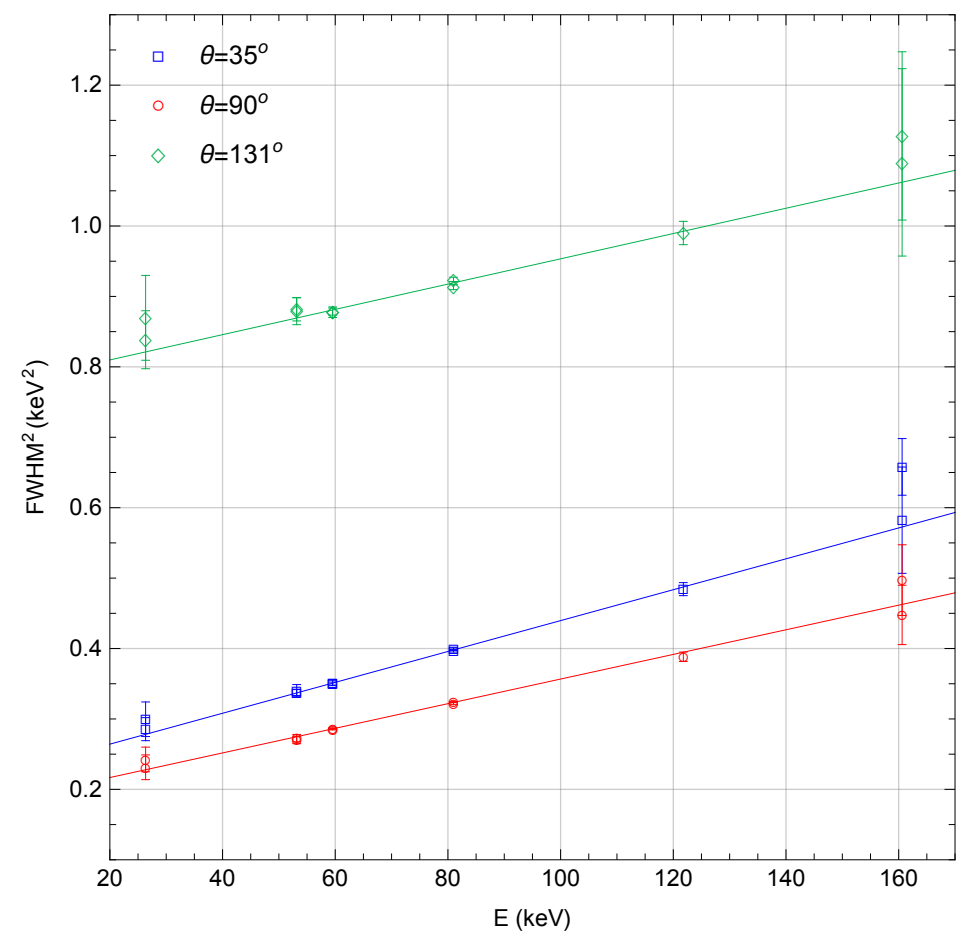

Figura 6.8: Calibração de resolução dos três detetores na faixa de energia 20-160 keV. As linhas contínuas representam os ajustes realizados em cada caso. Observe-se a semelhança nos coeficientes angulares das retas ajustadas. 
TABela 6.2: Resultados do ajuste dos coeficientes da largura à meia altura $\left(a^{\prime}\right.$ e $\left.b^{\prime}\right)$ e dos parâmetros de dispersão Gaussiana ( $a$ e $b)$.

\begin{tabular}{c||cccccc}
\hline \hline \multirow{2}{*}{ Detetor } & $a^{\prime}\left(\mathrm{eV}^{2}\right)$ & $b^{\prime}(\mathrm{eV})$ & $a\left(\mathrm{eV}^{2}\right)$ & $b(\mathrm{eV})$ & $\chi_{\nu}^{2}$ & $P\left(\chi_{\nu}^{2}\right)$ \\
\hline \hline \multirow{2}{*}{$\theta=35^{\circ}$} & $220(6)$ & $2,19(8)$ & $39,7(11)$ & $0,395(14)$ & 6,85 & 0,65 \\
$\theta=90^{\circ}$ & $182(3)$ & $1,75(4)$ & $32,8(4)$ & $0,315(6)$ & 6,44 & 0,70 \\
$\theta=131^{\circ}$ & $774(13)$ & $1,79(17)$ & $140(3)$ & $0,32(3)$ & 8,14 & 0,52 \\
\hline \hline
\end{tabular}

\subsubsection{Calibração de eficiência: determinação do ângulo sólido}

Para a calibração de eficiência se utilizaram os mesmos EAP coletados das fontes de ${ }^{133} \mathrm{Ba}$ (duas), ${ }^{152} \mathrm{Eu}$ (uma) e ${ }^{241} \mathrm{Am}$ (duas). A eficiência absoluta de fotopico se determinou segundo

$$
\varepsilon_{\mathrm{A}, i}=\frac{a_{i}}{\mathcal{A}_{s} I_{i} t_{\mathrm{v}}},
$$

onde $a_{i}$ é a área do pico correspondente à linha $i$ analisada, $I_{i}$ a sua probabilidade de emissão por decaimento, $\mathcal{A}_{s}$ a atividade da fonte $s$ no momento da medida, e $t_{\mathrm{v}} \mathrm{o}$ tempo vivo de adquisição dos espectros. Os dados de vida média dos radionuclídeos e do rendimento das transições avaliadas foram tomados da referência [99], exceto as probabilidades de emissão dos raios-x L do ${ }^{241} \mathrm{Am}$, para as quais se adotaram os valores fornecidos por Lépy et al. [100]. As áreas $a_{i}$ foram determinadas a partir do ajuste de funções Gaussianas com o mesmo programa descrito na subseção anterior.

Por outra parte, neste trabalho foi seguido um procedimento similar ao desenvolvido por Maidana e colaboradores [95], para ajustar o ângulo sólido $\Omega$ subtendido pelo detetor em relação à fonte (ou ao alvo) e a espessura $d$ da camada morta de Ge, a partir das medidas de eficiência $\left(E_{i}, \varepsilon_{\mathrm{A}, i}\right)$. Para a geometria de feixes bem colimados e para a emissão de fótons por uma fonte isotrópica, $\varepsilon_{\mathrm{A}}$ pode se expressar em termos de $\varepsilon_{\mathrm{int}}(E ; l, r)$, Eq. (4.50), que descreve a probabilidade de absorção total no volume ativo de um detetor descoberto, de acordo com

$$
\varepsilon_{\mathrm{A}}(E ; \Omega, d)=\frac{\Omega}{4 \pi} \exp \left[-\mu_{\mathrm{Ge}}(E) d\right] T_{0}(E) \varepsilon_{\mathrm{int}}(E ; l, r),
$$

onde $\exp \left[-\mu_{\mathrm{Ge}}(E) d\right]$ é o fator que descreve a atenuação de fótons na camada morta (de espessura $d)$, e $T_{0}(E)$ a fração de fótons transmitidos através das diferentes camadas de materiais com espessuras conhecidas na frente do volume ativo. Note-se que a equação anterior é linear no parâmetro $\Omega$ e não linear em $d$, logo, para ajustar ambas grandezas 
foi minimizada a função de mérito $Q(\Omega, d)[53,95]$ dada por

$$
Q(\Omega, d)=\mathbf{q}^{\mathrm{t}} \mathbf{V} \mathbf{q}
$$

onde as componentes do vetor dos resíduos $\mathbf{q}$ e as da matriz de covariância $\mathbf{V}$ são respectivamente

$$
q_{i}=\varepsilon_{\mathrm{A}, i}-\varepsilon_{\mathrm{A}}\left(E_{i} ; \Omega, d\right)
$$

e

$$
V_{i, j}=\varepsilon_{\mathrm{A}, i} \varepsilon_{\mathrm{A}, j}\left\{\left[\frac{\operatorname{var}\left(a_{i}\right)}{\left(a_{i}^{2}\right)}+\frac{\operatorname{var}\left(I_{i}\right)}{\left(I_{i}^{2}\right)}\right] \delta_{i, j}+\frac{\operatorname{var}\left(A_{s}\right)}{A_{s}^{2}} \delta_{s_{i}, s_{j}}\right\} .
$$

O uso de mais de uma linha da mesma fonte introduz covariâncias entre os valores experimentais $\varepsilon_{\mathrm{A}, i}$. A fórmula (6.16) considera esse fato, onde $\operatorname{var}(x)$ representa a variância da grandeza $x$ e $\delta_{i, j}$ são deltas de Kronecker.

A minimização de $Q(\Omega, d)$ foi realizada fornecendo valores ao parâmetro não linear $d$ e calculando a estimativa $\Omega_{d}$ de variância mínima, por meio da expressão

$$
\Omega_{d}=\left(\mathbf{Y}^{\mathrm{t}} \mathbf{V}^{-1} \mathbf{Y}\right)^{-1} \mathbf{Y}^{\mathrm{t}} \mathbf{V}^{-1} \mathbf{E}_{\mathrm{f}}
$$

sendo Y a matriz de desenho do Método dos Mínimos Quadrados [53], quando somente é ajustado o ângulo sólido $\Omega$ :

$$
Y_{i}=\frac{\partial \varepsilon_{\mathrm{A}}\left(E_{i} ; \Omega, d\right)}{\partial \Omega},
$$

enquanto $\mathbf{E}_{\mathrm{f}}$ é o vetor das eficiências experimentais com componentes dadas pela Eq. (6.12). Foi escolhida como a melhor estimativa dos parâmetros ajustados o par $\left(\Omega_{a}, d_{a}\right)$ que corresponde ao mínimo observado no gráfico de $Q(\Omega, d)$ vs $d$. Finalmente, a matriz de variância dos parâmetros ajustados $\mathbf{V}_{\Omega, d}$ [53], foi calculada a partir da matriz de desenho $\mathbf{X}$, com componentes

$$
X_{i, 1}=\left.\frac{\partial \varepsilon_{\mathrm{A}}\left(E_{i} ; \Omega, d\right)}{\partial \Omega}\right|_{d_{a}}, \quad X_{i, 2}=\left.\frac{\partial \varepsilon_{\mathrm{A}}\left(E_{i} ; \Omega, d\right)}{\partial d}\right|_{\Omega_{a}, d_{a}},
$$

e a matriz de variância $\mathbf{V}$ como

$$
\mathbf{V}_{\Omega, d}=\left(\mathbf{X}^{\mathrm{t}} \mathbf{V}^{-1} \mathbf{X}\right)^{-1}
$$

Em acordo com a analise da subseção 5.2.1 o ângulo sólido $\Omega$ depende da energia do fóton incidente por causa da transparência do colimador. Logo, dois procedimentos de ajuste foram realizados:

M-1) Não foi levada em conta a dependência energética de $\Omega$, portanto se estabeleceu diretamente $\Omega=\Omega_{\mathrm{g}}=$ cte. 
M-2) Foi considerado

$$
\Omega(E)=\Omega_{\mathrm{g}}+\Omega_{\mathrm{ef}}\left(E ; r_{\mathrm{c}}, d_{\mathrm{c}}, d_{\mathrm{f}}\right),
$$

onde $\Omega_{\mathrm{ef}}\left(E ; r_{\mathrm{c}}, d_{\mathrm{c}}, d_{\mathrm{f}}\right)$ se calculou segundo a Eq. (5.10) utilizando para cada detetor os dados de $r_{\mathrm{c}}, d_{\mathrm{c}}$ e $d_{\mathrm{f}}=R+d_{1}$ especificados na Tabela 3.1 .

Em ambas situações foi minimizada a função $Q$ e ajustados os parâmetros $\omega_{\mathrm{g}}$ e $d$. Os gráficos da Fig. 6.9 mostram os resultados deste procedimento para os três detetores, enquanto os pontos experimentais, bem como as curvas de eficiência são apresentados na Fig. 6.10. A Tabela 6.3 resume os parâmetros desses ajustes e os testes estatísticos correspondentes.

Embora que os dois modelos sejam adequados segundo o teste de $\chi^{2}$, os valores de $Q_{\text {mín }}$ alcançados segundo M-2 se aproximam mais ao número de graus de liberdade do ajuste em cada caso. Observa-se que as espessuras de camadas mortas de Ge obtidas por ambos modelos são compatíveis dentro de um desvio padrão, e sistemicamente M-1 fornece resultados maiores que M-2. Além disso, note-se que as eficiências geométricas $\omega_{\mathrm{g}}=$ $\Omega_{\mathrm{g}} / 4 \pi$ também são compatíveis entre elas e com os valores calculados (última coluna da Tabela 3.1) a partir das distâncias medidas que caracterizam a posição dos colimadores em relação à câmara de irradiação. Este resultado demonstra que o alinhamento dos componentes do arranjo experimental (detetores-colimadores-alvo) é correto. Para a avaliação da FR utilizada na deconvolução dos espectros de bremsstrahlung se tomaram os dados obtidos pelo método M-2. Por outro lado, estabelece-se a relação (6.21) para o cálculo do ângulo sólido $\Omega(E)$ na determinação da SCDD segundo a Eq. (2.77), cuja parte constante é conhecida com uma incerteza relativa inferior a $1 \%$. 

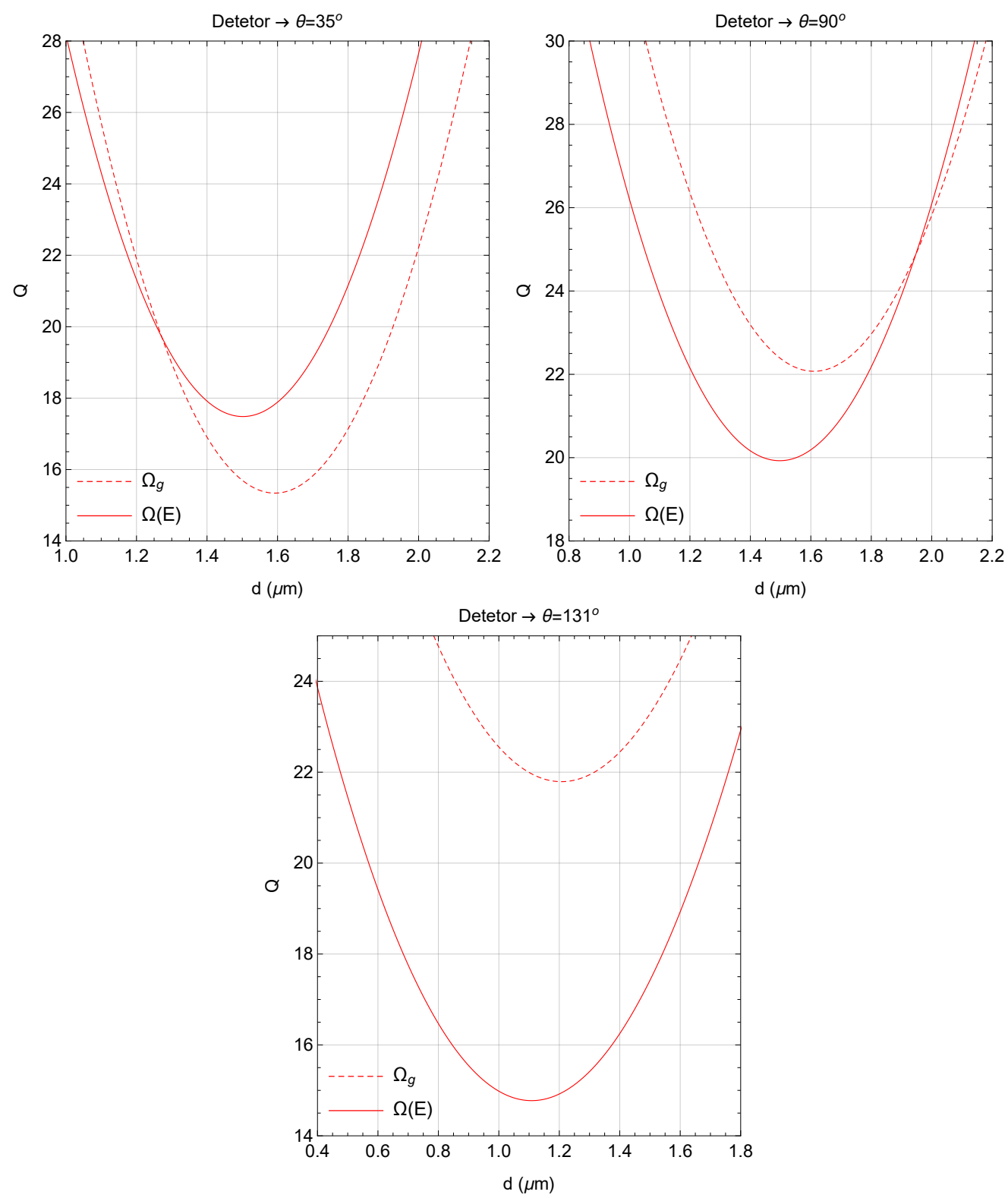

Figura 6.9: Minimização da função de mérito $Q(\Omega, d)$ considerando os modelos de ajuste M-1 e M-2, nos três detetores. 

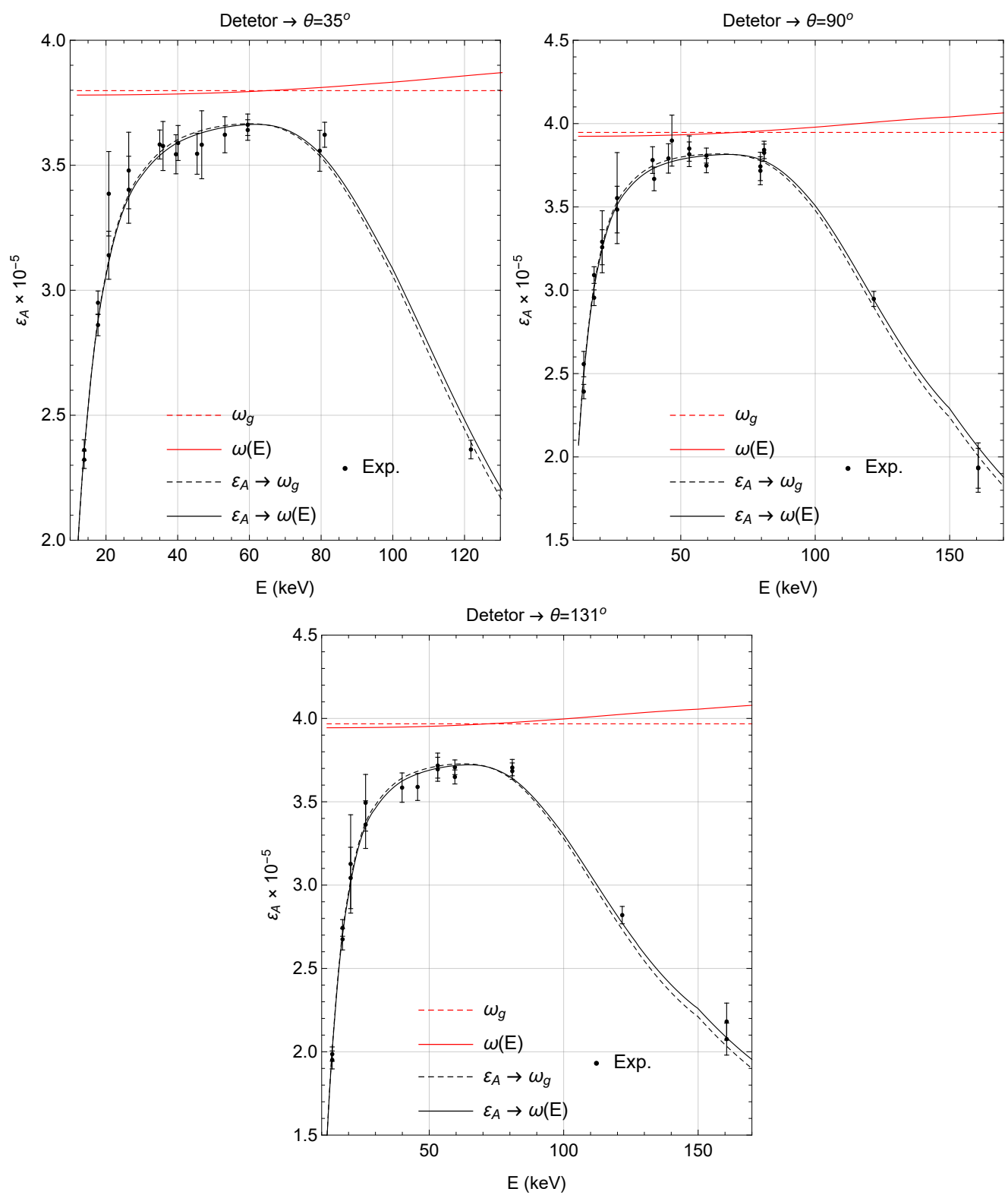

FiguRA 6.10: Eficiências experimentais e curvas ajustadas para os três detetores. São plotadas as eficiências geométricas $\omega_{\mathrm{g}}$ e $\omega(E)$. 
TABela 6.3: Mínimos $Q_{\text {mín }}$ da função de mérito $Q$, probabilidades $P\left(Q_{\text {mín }}\right)$ do valor de $\chi_{\nu}^{2}=Q_{\text {mín }}$ exceder o valor crítico e resultados dos parâmetros $d$ e $\omega_{\mathrm{g}}$, ajustados segundo os modelos 1 (M-1) e 2 (M-2). Os graus de liberdade são $\nu=18$, 21 e 16 para os detetores colocados a $\theta=35^{\circ}, 90^{\circ}$ e $131^{\circ}$ respectivamente.

\begin{tabular}{c||cc|cc|cc|cc}
\hline \hline \multirow{2}{*}{ Detetor } & \multicolumn{2}{c|}{$Q_{\text {mín }}$} & \multicolumn{2}{c|}{$P\left(Q_{\text {mín }}\right)$} & \multicolumn{2}{c|}{$d(\mu \mathrm{m})$} & \multicolumn{2}{c}{$\omega_{\mathrm{g}} \times 10^{-5}$} \\
\cline { 2 - 8 } & $\mathrm{M}-1$ & $\mathrm{M}-2$ & $\mathrm{M}-1$ & $\mathrm{M}-2$ & $\mathrm{M}-1$ & $\mathrm{M}-2$ & $\mathrm{M}-1$ & $\mathrm{M}-2$ \\
\hline$\theta=35^{\circ}$ & 15,3 & 17,5 & 0,64 & 0,49 & $1,59(15)$ & $1,50(15)$ & $3,798(22)$ & $3,781(22)$ \\
$\theta=90^{\circ}$ & 22,1 & 19,9 & 0,39 & 0,52 & $1,61(20)$ & $1,50(20)$ & $3,945(21)$ & $3,924(21)$ \\
$\theta=131^{\circ}$ & 21,8 & 14,8 & 0,19 & 0,61 & $1,21(24)$ & $1,11(24)$ & $3,968(22)$ & $3,944(22)$ \\
\hline \hline
\end{tabular}




\subsection{Determinação da espessura dos alvo}

Pela sua precisão, a técnica RBS (Rutherford Backscattering Spectrometry) é uma das mais usadas para analisar filmes finos. No método, feixes monoenergéticos de íons ${ }^{1} \mathrm{H}^{+}$, ${ }^{4} \mathrm{He}^{+}$ou ${ }^{4} \mathrm{He}^{2+}$, com correntes da ordem dos $\mathrm{nA}$, incidem sobre as amostras pesquisadas, e são medidas as partículas retro-espalhadas nos átomos do alvo. As perdas de energia da partícula dentro do material são proporcionais à distancia percorrida nele, logo, é possível estabelecer uma escala de profundidade a partir do EAP coletado dos íons espalhados elasticamente.

A densidade superficial dos alvos foi determinada no Laboratório de Análise de Materiais por Feixes Iônicos (LAMFI) do IFUSP. Nas medidas usou-se o feixe de ${ }^{4} \mathrm{He}^{2+}$ de energia $E=2200 \mathrm{keV}$ produzido no acelerador tandem van de Graaff, que incidiu com um ângulo $\alpha=7^{\circ}$ em relação ao alvo (Fig. 6.11), e dois detetores de Si de barreira de superfície, posicionados a $\theta=120^{\circ}$ e $\theta=170^{\circ}$ em relação à direção das partículas incidentes, em uma câmara cilíndrica (diâmetro interno $43 \mathrm{~cm}$, altura $15 \mathrm{~cm}$ ) ao vácuo $\left(2 \times 10^{-5}\right.$ torr). Os filmes foram colocados em uma porta amostras que permite fazer medidas de três alvos sem precisar fazer a quebra do vácuo da câmara de irradiação. A descrição detalhada das instalações experimentais está disponível no sítio web do LAMFI [101]. Os conceitos físicos da técnica RBS podem ser encontrados no livro clássico de Chu, Mayer e Nicolet [102].

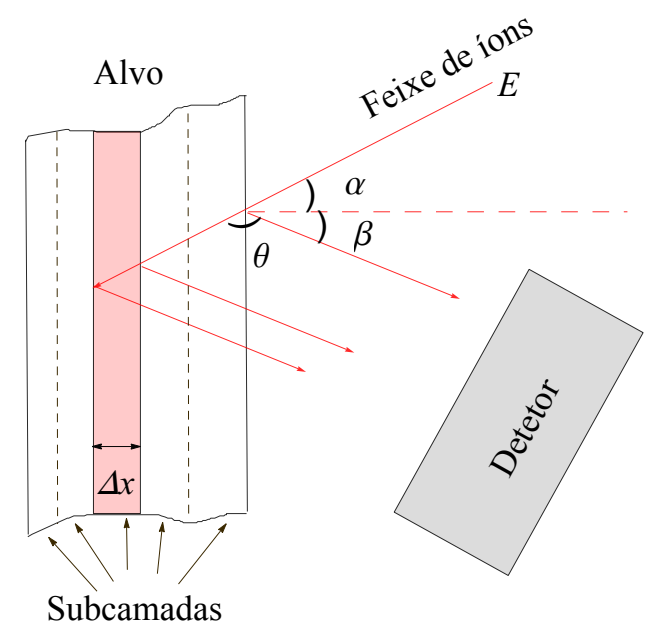

FIGURA 6.11: Representação do retroespalhamento das partículas no alvo.

Os softwares SIMNRA [103] e MultiSIMNRA [104] foram utilizados para a análise dos resultados. O SIMNRA permite simulação de espectros RBS a partir de parâmetros de entrada relacionados com i) o arranjo experimental: tipo de partículas do feixe, 
energia incidente, ângulos $\alpha$ e $\theta$, tipo de detetores e resolução dos mesmos, etc., e ii) a composição do alvo: camadas de elementos, substrato, rugosidade, entre outros. Com base nos espectros coletados para um alvo, o programa possibilita o ajuste de parâmetros de interesse na sua caracterização: 1) composição e espessura das camadas, 2) rugosidade, 3) calibração de energia (ganho e coeficiente linear) e 4) o produto do número de partículas incidentes e o ângulo sólido subtendido pelo detetor em relação ao alvo $\left(N_{0} \Omega\right)$. A metodologia baseia-se na minimização do desvio quadrático

$$
\chi^{2}=\sum_{i} \frac{\left[N_{\exp }(i)-N_{\operatorname{sim}}(i)\right]^{2}}{\sigma_{i}^{2}},
$$

a partir da simulação das contagens $N_{\text {sim }}(i)$ de cada canal $i$, variando os parâmetros de entrada. $N_{\exp }(i)$ e $\sigma_{i}^{2}$ representam as contagens observadas no experimento e a incerteza estatística $^{3}$ associada.

Para a simulação, cada camada ${ }^{4}$ do alvo é subdivida em subcamadas (Fig. 6.11), de maneira que o espectro é composto como a soma das contribuições de cada isótopo em cada subcamada. Quando as partículas incidentes penetram uma subcamada perdem energia ao interagir com os elétrons e núcleos do meio. Como consequência das flutuações que caracterizam essas perdas se produz um alargamento energético do feixe inicial ("energy straggling"). O SIMNRA calcula a energia das partículas retroespalhadas na superfície frontal e posterior de cada subcamada, e a energia das mesmas ao atingir o detetor depois de atravessar o alvo no seu caminho de volta (Fig. 6.11). O espectro de energia da contribuição de cada subcamada ao espectro total é convoluído com uma função Gaussiana cuja variância vem dada por $\sigma^{2}=\sigma_{\mathrm{S}}^{2}+\sigma_{\mathrm{D}}^{2}$, a fim de levar em conta o straggling ( $\sigma_{\mathrm{S}}^{2}$ é a variância da distribuição de energia das partículas retroespalhadas) e a resolução do detetor $\left(\sigma_{\mathrm{D}}^{2}\right)$.

No SIMNRA o número $Q$ de íons retroespalhados numa subcamada de espessura $\Delta x$ (faixa vermelha na Fig. 6.11) é calculado como

$$
\begin{aligned}
Q & =\frac{N_{0} \Omega}{\cos \alpha} \int_{x_{0}}^{x_{1}} \frac{\mathrm{d} \sigma^{\mathrm{e}}}{\mathrm{d} \Omega}(E(x), \theta) \mathrm{d} x \\
& =\frac{N_{0} \Omega}{\cos \alpha} \int_{E_{0}}^{E_{1}} \frac{\mathrm{d} \sigma^{\mathrm{e}}}{\mathrm{d} \Omega}(E, \theta) \frac{\mathrm{d} x}{\mathrm{~d} E} \mathrm{~d} E,
\end{aligned}
$$

onde $x_{0}$ e $x_{1}$ são as profundidades anterior e posterior da subcamada, e $E_{0}=E\left(x_{0}\right)$, $E_{1}=E\left(x_{1}\right)$ as correspondentes energias, $\mathrm{d} \sigma^{\mathrm{e}} / \mathrm{d} \Omega$ é a SCD de espalhamento elástico da partícula incidente no elemento da camada [102, 103]. Considerando que o poder de

\footnotetext{
${ }^{3}$ O SIMNRA utiliza $\sigma_{i}=\sqrt{N_{\exp }(i)}$ para $N_{\exp }>4$, e $\sigma_{i}=2$ para $N_{\exp } \leqslant 4$.

${ }^{4}$ Entenda-se por camada uma região do alvo composta por um ou vários elementos em determinada proporção.
} 
freamento $S=\mathrm{d} E / \mathrm{d} x(\bar{E})$ se mantém constante ao longo da subcamada ( $\bar{E}$ é a energia média do intervalo $\Delta x$ ), a relação entre o caminho percorrido dentro do alvo e a energia do íon incidente vem dada por $E(x)=E_{0}-S x$, logo

$$
Q=\frac{N_{0} \Omega}{\cos \alpha} \frac{1}{S} \int_{E_{0}}^{E_{1}} \frac{\mathrm{d} \sigma^{\mathrm{e}}}{\mathrm{d} \Omega}(E, \theta) \mathrm{d} E
$$

Duas grandezas precisam ser bem conhecidas para a avaliação da Eq. (6.24): d $\sigma^{\mathrm{e}} / \mathrm{d} \Omega$ e o poder de freamento $S$, esta última em duas partes da expressão, no cálculo do valor médio de $S$ na subcamada, e na determinação da energia $E_{0}$ com que as partículas incidentes atingem o ponto $x_{0}$ no intervalo $\Delta x$ considerado.

O SIMNRA avalia a SCD de espalhamento elástico (no sistema referencial de laboratório, e para experimentos com ${ }^{4} \mathrm{He}^{2+}$ a $2200 \mathrm{keV}$ ) segundo a expressão:

$$
\frac{\mathrm{d} \sigma^{\mathrm{e}}}{\mathrm{d} \Omega}(E, \theta)=F_{\mathrm{A}}(E, \theta) \frac{\mathrm{d} \sigma^{\mathrm{R}}}{\mathrm{d} \Omega}(E, \theta)
$$

sendo $F_{\mathrm{A}}$ o fator de correção de Andersen [105] por efeitos da blindagem dos elétrons atômicos à carga nuclear na SCD elástica de Rutherford:

$$
\begin{aligned}
\frac{\mathrm{d} \sigma^{\mathrm{R}}}{\mathrm{d} \Omega}(E, \theta)= & 5,1837436 \times 10^{6}\left(\frac{Z_{1} Z_{2}}{E}\right)^{2} \\
& \times \frac{\left[\left(M_{2}^{2}-M_{1}^{2} \sin ^{2} \theta\right)^{1 / 2}+M_{2} \cos \theta\right]^{2}}{M_{2} \sin ^{4} \theta\left(M_{2}^{2}-M_{1}^{2} \sin ^{2} \theta\right)^{1 / 2}} .
\end{aligned}
$$

As unidades da equação anterior são mb/sr quando a energia incidente é dada em keV, enquanto $Z_{1}$ e $M_{1}$ representam a carga nuclear e o número de massa do projétil, e $Z_{2}$ e $M_{2}$ tem o mesmo significado no elemento do alvo. $F_{\mathrm{A}}$ pode se expressar de acordo com $[105]$

$$
F_{\mathrm{A}}(E, \theta)=\left(1+\frac{1}{2} \frac{V_{1}}{E_{\text {c.m. }}}\right)^{2}\left\{1+\frac{V_{1}}{E_{\text {c.m. }}}+\left[\frac{1}{2} \frac{V_{1}}{E_{\text {c.m. }}}\left(\frac{1}{\sin \left(\theta_{\text {c.m. }} / 2\right)}\right)\right]^{2}\right\}^{-2}
$$

onde $V_{1}$ equivale ao incremento efetivo na energia cinética, experimentada neste caso, pelo núcleo ${ }^{4} \mathrm{He}$, a causa do campo elétrico dos elétrons (ou de maneira análoga à menor desaceleração em relação a um núcleo sem a nuvem de elétrons), e vem dado por

$$
V_{1}=0,04873 Z_{1} Z_{2}\left(Z_{1}^{2 / 3}+Z_{2}^{2 / 3}\right)^{1 / 2}
$$

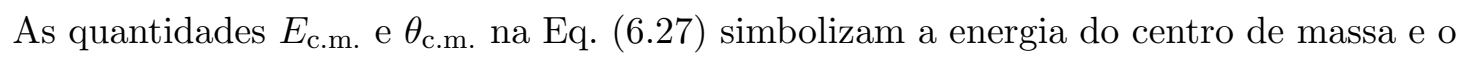
ângulo de espalhamento nesse referencial. Na Eq. (6.28), $V_{1}$ possui unidades energéticas 
de keV. A Fig. 6.12 mostra comportamento do fator $F_{\mathrm{A}}$ para a incidência de íons ${ }^{4} \mathrm{He}$ sobre átomos de C, Ag e Au.

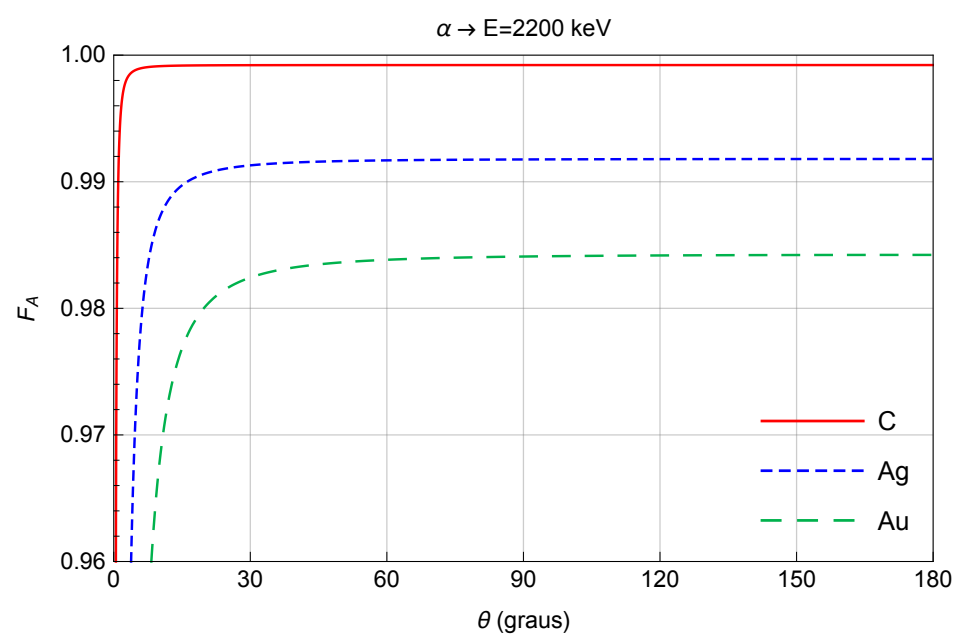

Figura 6.12: Fator de Andersen para a blindagem da carga nuclear em C, Ag e Au quando incidem partículas $\alpha$ de $2200 \mathrm{keV}$.

A Fig. 6.13 apresenta uma comparação entre as SCDs calculadas por meio da Eq. (6.25) para a reação $\alpha(\mathrm{C}, \mathrm{C}) \alpha$, e as SCDs non-Rutherford tomadas da base de dados IBANDL $^{5}[106$, 107], que contém os resultados experimentais mais relevantes para a análise por técnicas de feixes iônicos, considerada o estado da arte nesse tema. Na energia de irradiação (2200 keV, indicada com a linha vertical tracejada) as discrepâncias observadas são menores que $1 \%$ para $\theta=120^{\circ}$, porém, para $\theta=170^{\circ}$ a diferença é maior que $7 \%$. Logo, na análise da espessura deste elemento, utilizaram-se os espectros coletados a $120^{\circ}$. Para os outros alvos, o cálculo das SCDs de espalhamento é preciso segundo a Eq. (6.25).

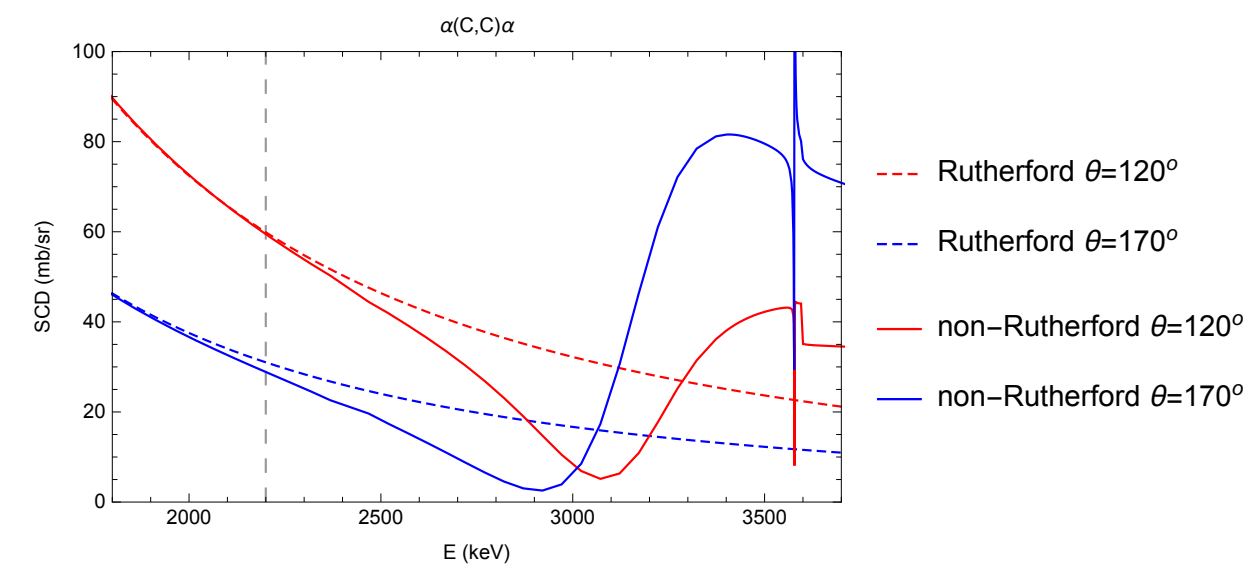

FigurA 6.13: Comparação entre as SCD Rutherford e non-Rutherford para a reação $\alpha(\mathrm{C}, \mathrm{C}) \alpha$. A linha tracejada vertical indica a energia $E=2200 \mathrm{keV}$.

\footnotetext{
${ }^{5}$ IBANDL: Ion Beam Analysis Nuclear Data Library.
} 
Por outro lado, a energia $E$ de uma partícula após passar uma camada de espessura $x$ está dada pela equação integral

$$
E(x)=E_{0}-\int_{0}^{x} \frac{\mathrm{d} E}{\mathrm{~d} x^{\prime}}\left(E, x^{\prime}\right) \mathrm{d} x^{\prime},
$$

onde se assume que a energia inicial do íon é $E_{0}$ na superfície $x=0$. Diferenciando a relação anterior se obtém

$$
\frac{\mathrm{d} E}{\mathrm{~d} x}=-\epsilon(E)
$$

que se conhece como a seção de choque de freamento dependente da energia, e representa uma equação diferencial de primeira ordem, com condições de contorno $E(x=0)=E_{0} \mathrm{e}$ $\mathrm{d} E / \mathrm{d} x(x=0)=-\epsilon\left(E_{0}\right)$. O SIMNRA utiliza um método Runge-Kutta de quinta ordem para resolver a Eq. (6.30). Os erros estimados deste cálculo numérico são menores que $20 \mathrm{eV}$ para perdas de energias de $1 \mathrm{MeV}$ [103]. Finalmente, nas analises dos alvos deste trabalho, foram selecionados os dados de poder de freamento eletrônico de Ziegler, Biersack e Littmark [108], recomendados pelos autores do SIMNRA.

Para a obtenção dos parâmetros que caracterizam aos alvos, os espectros coletados foram ajustados com o programa MultiSIMNRA [104]. O software se baseia nos cálculos SIMNRA segundo a metodologia até agora descrita, e faz uma análise auto consistente impondo restrições entre parâmetros ajustáveis que possuem ligações físicas ( $p$. ex. a calibração de energia dos espectros coletados por um mesmo detetor).

A Fig. 6.14 apresenta os espectros coletados para os alvos de Te e Au, assim como as respectivas simulações MultiSIMNRA de acordo com o procedimento de minimização segundo a Eq. (6.22). Os picos observados no extremo direito de ambos gráficos, centrados em 1983 e $2065 \mathrm{keV}$ para Te e Au em cada caso, correspondem ao retroespalhamento nas camadas desses elementos. Enquanto os picos mais alargados e com menos estatística em torno de $715 \mathrm{keV}$ são devidos ao mesmo efeito no substrato de C. À direita dessas estruturas, e com menor intensidade ainda, aparecem as contribuições associadas à oxidação dos alvos. A concentração de $\mathrm{O}$ achada em todas as análises é menor que a concentração de $\mathrm{C}$ pelo menos duas ordens de grandeza.

A Tabela 6.4 resume os resultados do ajuste da densidade superficial de átomos $t$ (e da espessura equivalente $d$ ) dos alvos utilizados nas medidas de bremsstrahlung. As densidades mássicas utilizadas para o cálculo de $d$ foram obtidas da referência [109]. A incerteza final leva em conta os erros relacionados com $i$ ) o procedimento numérico de ajuste (fornecidos pelo MultiSIMNRA), ii) o posicionamento dos alvos e iii) a estatística de contagem. Os modelos físicos utilizados para a simulação MultiSIMNRA-SIMNRA, como seções de choque e poderes de freamento, não foram considerados na propagação de incertezas. 

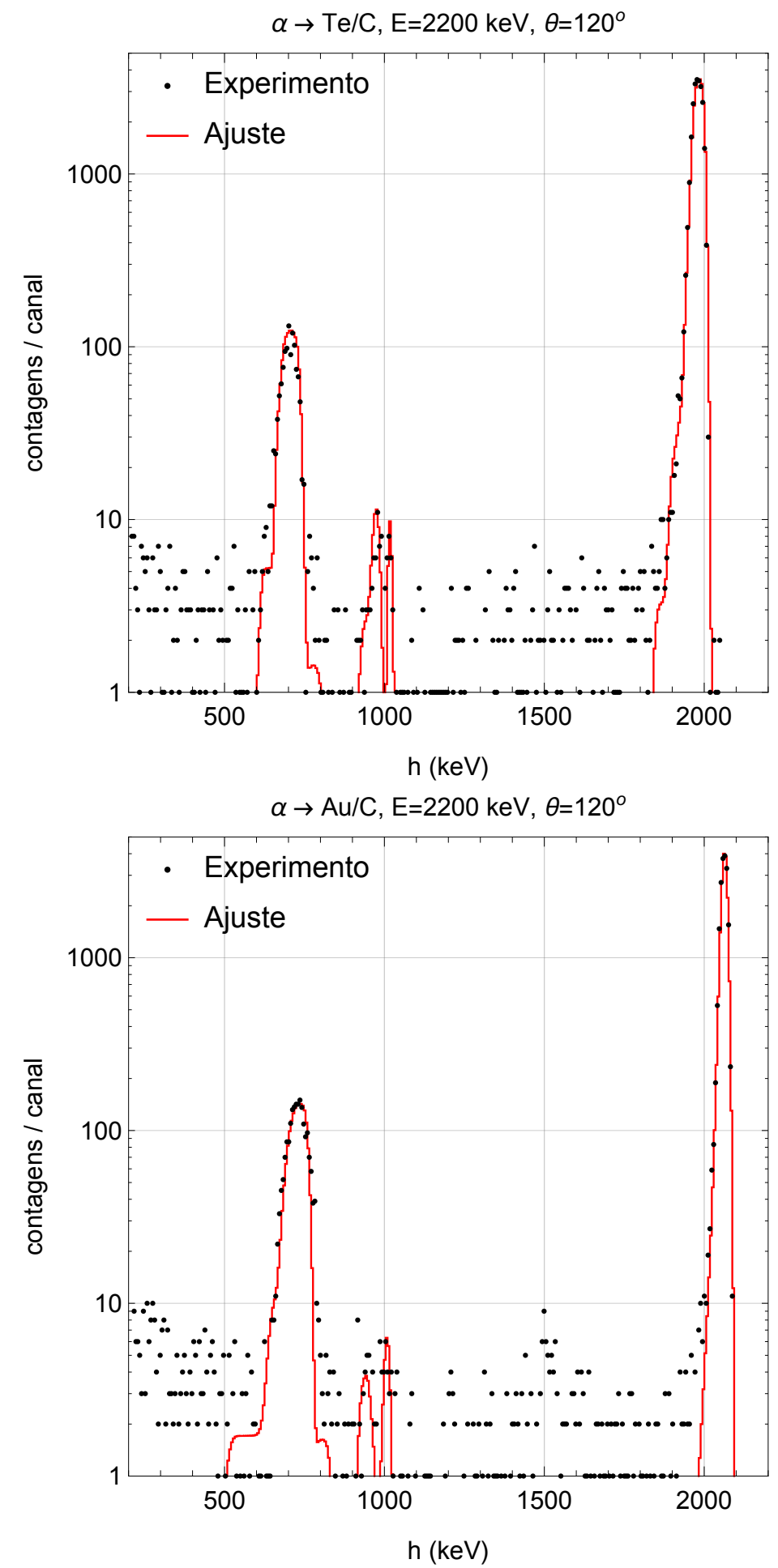

Figura 6.14: Espectros RBS experimentais e simulados com o programa MultiSIMNRA para o ajuste dos parâmetros dos alvos de Te e Au. A energia incidente do feixe de ${ }^{4} \mathrm{He}^{2+}$ foi $E=2200 \mathrm{keV}$ e o ângulo de espalhamento $\theta=120^{\circ}$. 
TABELA 6.4: Resultados do ajuste da densidade superficial de átomos $t$, equivalente à espessura $d$, das camadas do elemento e do substrato (C) que conformam cada alvo.

\begin{tabular}{c||cc||cc||cc}
\hline \hline \multirow{2}{*}{ Alvo } & \multicolumn{2}{c||}{$t\left(\mathrm{at} / \mathrm{cm}^{2} \times 10^{15}\right)$} & \multicolumn{2}{c||}{$d(\mathrm{~nm})$} & \multicolumn{2}{c}{ Incerteza Relativa (\%) } \\
\cline { 2 - 7 } & Elemento & Substrato & Elemento & Substrato & Elemento & Substrato \\
\hline $\mathrm{C}$ & $450(11)$ & - & $39,6(10)$ & - & 2,4 & - \\
$\mathrm{Al}$ & $100(4)$ & $562(20)$ & $16,6(7)$ & $49,4(18)$ & 4,0 & 3,6 \\
$\mathrm{Ag}$ & $124(3)$ & $350(9)$ & $21,2(5)$ & $30,8(8)$ & 2,4 & 2,6 \\
$\mathrm{Te}$ & $110(3)$ & $525(10)$ & $37,4(10)$ & $46,2(9)$ & 2,7 & 1,9 \\
$\mathrm{Ta}$ & $60,6(24)$ & $461(10)$ & $11,2(4)$ & $40,6(9)$ & 3,9 & 2,2 \\
$\mathrm{Au}$ & $29,9(6)$ & $610(20)$ & $5,07(10)$ & $53,7(18)$ & 2,0 & 3,3 \\
\hline \hline
\end{tabular}

\subsection{Alguns critérios quantitativos em relação aos alvos}

Uma vez determinadas as espessuras podem se realizar algumas avaliações em relação ao carácter "fino" dos alvos. A intenção é conhecer quão perto das condições iniciais será emitido o bremsstrahlung quando o elétron atravessa o filme. Entenda-se neste caso, os possíveis desvios da direção e da energia original com que o feixe atinge a superfície do alvo.

Um dos procedimentos usualmente seguidos é calcular o incremento médio $\langle\Delta d\rangle$ na trajetória do elétron ao atravessar um filme, em relação a sua espessura $d$. Motz e Placious [6] por exemplo, adotaram que um alvo é fino se o cociente $\langle\Delta d\rangle / d$ é inferior a $2 \%$. Com base na teoria de espalhamento múltiplo e para elétrons entre 10 e 130 keV, Hubbell e Birkhoff [110] mostraram que o incremento médio $\langle\Delta d\rangle$ na trajetória do elétron, depois de passar uma espessura $d$, pode ser calculado como

$$
\begin{aligned}
\langle\Delta d\rangle= & 2 \pi r_{\mathrm{e}}^{2} N_{\mathrm{A}} \frac{Z(Z+1) \rho d^{2}}{A} \frac{1-\beta^{2}}{\beta^{4}} \\
& \times\left\{\ln \left[\frac{\beta}{\alpha Z^{1 / 3}\left(1-\beta^{2}\right)^{1 / 2}}\right]+\ln 1,76-\left(1-\beta^{2} / 4\right)\right\},
\end{aligned}
$$

onde $N_{\mathrm{A}}$ é a constante de Avogadro, $Z$ e $A$ o número e peso atômico, e $\rho$ a densidade mássica do filme. A expressão é válida para valores de $d$ muito menores do que o alcance do elétron no material.

A Fig. 6.15 apresenta a relação $\langle\Delta d\rangle / d$ para cada um dos alvos utilizados nas medidas de bremsstrahlung em função da energia do elétron incidente $E_{0}$. No cálculo foi considerada a inclinação $\alpha=60^{\circ}$ da torre de alvos que aumenta a espessura $d$ num fator multiplicativo $\left(\sin 60^{\circ}\right)^{-1}=1,155$ (ver Eq. (2.78)). A estimativa fornece um critério quantitativo que ilustra a respeito das deflexões angulares experimentadas pelo elétron dentro do filme. Observa-se que em todos os casos, e para energias menores que $50 \mathrm{keV}$, o incremento porcentual na espessura $d$ é inferior a $2 \%$. Já para elétrons de $20 \mathrm{keV}$ 
a fração $\langle\Delta d\rangle / d$ alcança valores em torno de $3 \%$ para o alvo de $\mathrm{Au}$, e entre $5 \%$ e $6 \%$ para Ag, Te e Ta. O resultado indica que a $20 \mathrm{keV}$ o espalhamento elástico dos elétrons começa a ter maior importância e a emissão de bremsstrahlung não poderá ser associada unicamente a direção de incidência do feixe.
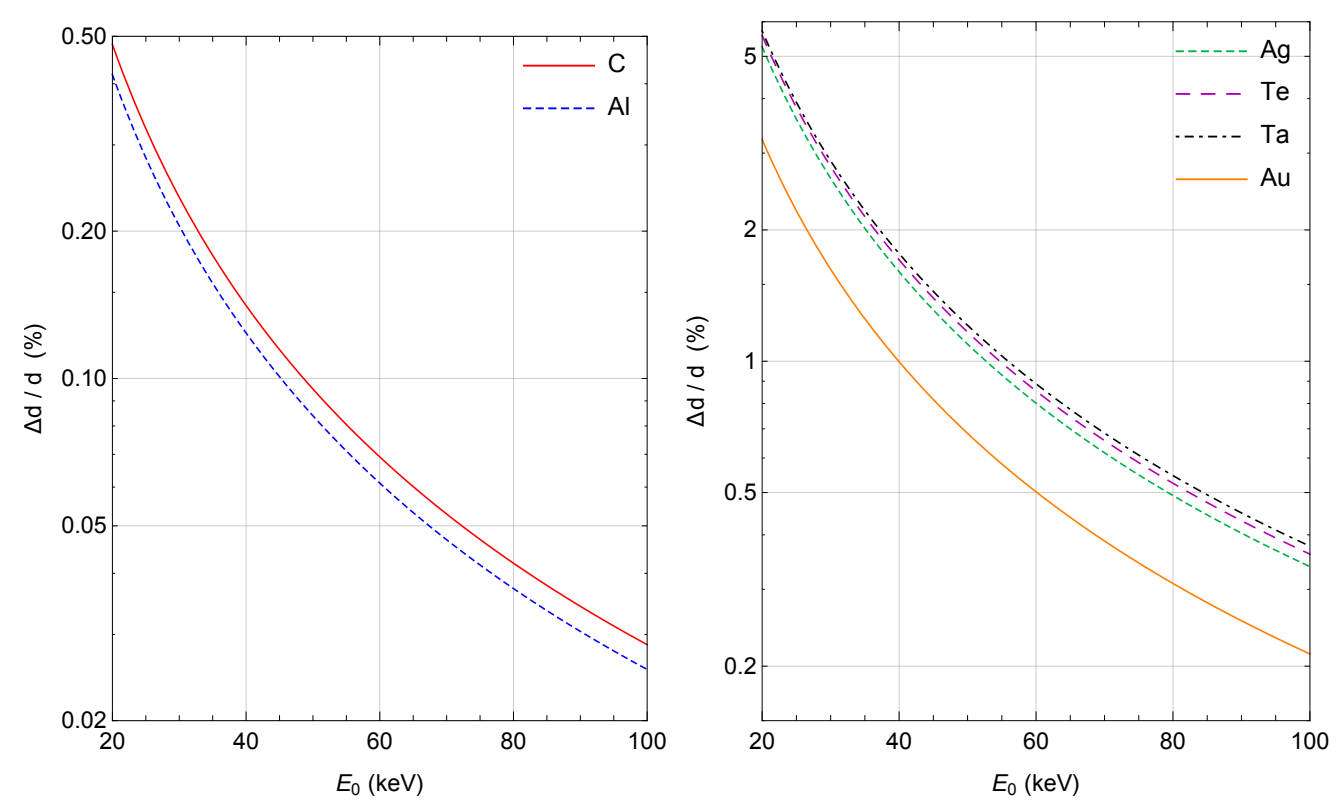

FigurA 6.15: Relação $\langle\Delta d\rangle / d$ em função da energia do elétron incidente para cada um dos alvos utilizados nas medidas de bremsstrahlung.

Por outro lado na Fig. 6.16 são mostradas as perdas de energia $\Delta E_{0}$ (em keV e a fração porcentual) que experimenta um elétron ao atravessar os filmes deste trabalho. Os poderes de freamento utilizados para esse cálculo foram adquiridos da base de dados ESTAR [111]. De acordo com os valores obtidos em todo o intervalo de $E_{0}$ considerado, a relação $\Delta E_{0} / E_{0}$ é inferior a $1 \%$, portanto este parâmetro resulta menos crítico que os desvios da direção de incidência estudados anteriormente. 

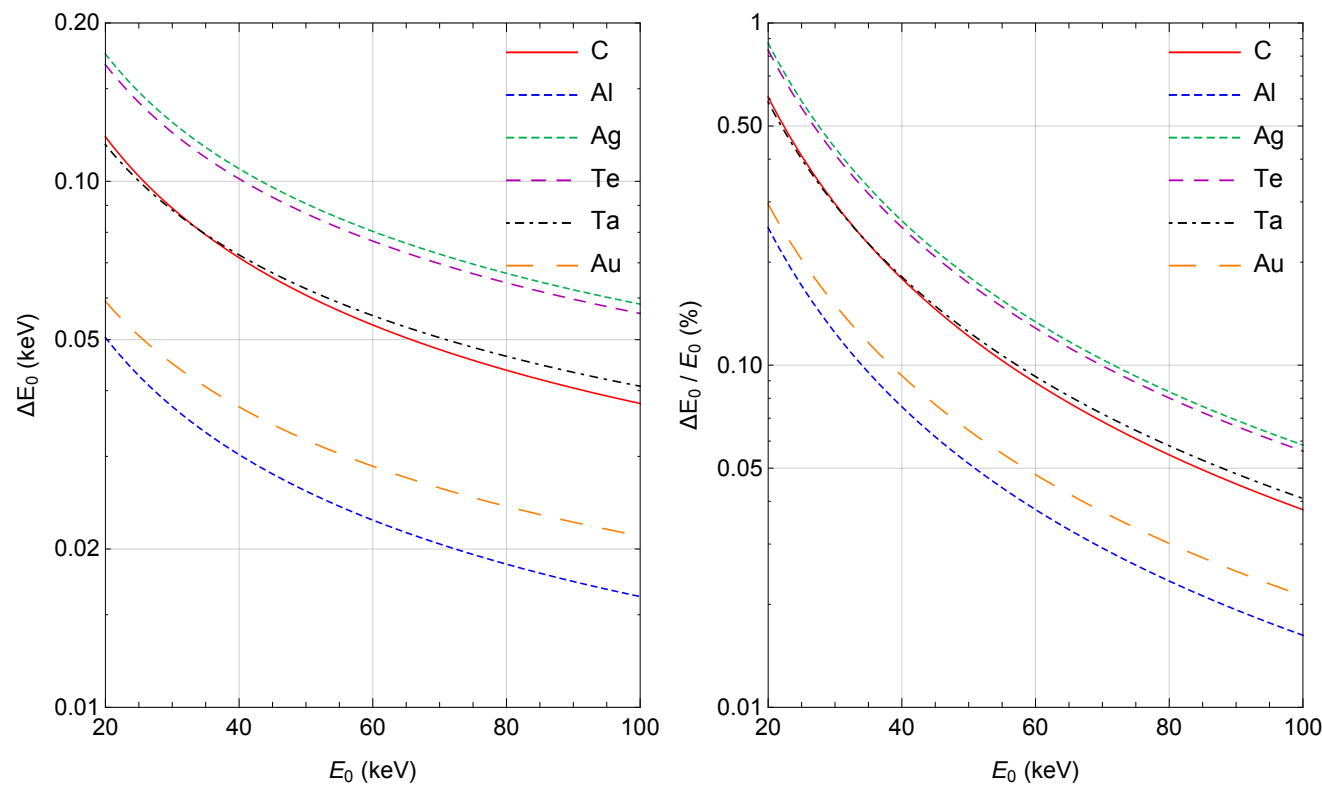

Figura 6.16: Perdas de energia total $\left(\Delta E_{0}\right)$ e relativa $\left(\Delta E_{0} / E_{0}\right)$ experimentada pelos elétrons ao atravessar os filmes utilizados nas medidas de emissão de bremsstrahlung. 



\section{Capítulo 7}

\section{Medidas das SCDDs de bremsstrahlung: tratamento de dados}

Neste capítulo se detalham as correções realizadas os espectros coletados pelos efeitos de fundo radioativo, empilhamento de pulsos e pelo bremsstrahlung gerado no substrato de C. É descrita a metodologia desenvolvida para o ajuste da energia e a dispersão do feixe de elétrons, bem como para a obtenção do intensidade do espectro de bremsstrahlung no ponto de máxima transferência de energia. Finalmente são apresentados exemplos do procedimento de deconvolução dos espectros entre os $12 \mathrm{keV}$ e a região do tip.

\subsection{Correções aos espectros de bremsstrahlung coletados}

As medidas se realizaram em duas campanhas de irradiação. Na primeira delas se estudaram os elementos $\mathrm{C}$, Te e Ta, posteriormente se tomaram espectros de C, Al, $\mathrm{Ag}$ e Au.

Antes de realizar o procedimento de deconvolução, os EAP coletados precisam ser corrigidos devido a contribuições externas à interação dos elétrons no filme do elemento estudado. A primeira delas se deve aos raios- $\gamma$ das desintegrações de radionuclídeos naturais, bem como a radiação cósmica, que de conjunto produzem o conhecido fenômeno de fundo radioativo. Durante um período de aproximadamente 15 dias foram medidos os espectros de fundo característicos de cada detetor. Uma vez normalizadas, subtraíramse as contagens relacionadas com esse efeito em todos EAP de bremsstrahlung obtidos (ver Fig. 7.1). 
Outra contribuição a considerar é o empilhamento de pulsos (EP) associado á eletrônica dos espectrômetros. Nesse caso se produz um espectro soma como consequência dos eventos que se registram num intervalo menor que o tempo de resolução $T$ do sistema de aquisição [81]. A metodologia descrita a seguir modela o espectro gerado por esse processo a partir das contagens coletadas em cada canal.

Dada a natureza aleatória da ocorrência temporal dos eventos registrados na energia $h_{i}$, correspondente ao canal $i$, o tempo total que demora o sistema em processar pulsos deste tipo pode ser calculado como $T_{i}=N\left(h_{i}\right) T$. Se durante $T_{i}$ pulsos de energia $h_{j}$ são registrados, se obterão contagens em $N\left(h_{i}+h_{j}\right)$ que podem ser determinadas segundo

$$
N\left(h_{i}+h_{j}\right)=N\left(h_{i}\right) T \frac{N\left(h_{j}\right)}{t_{\mathrm{v}}}
$$

onde $N\left(h_{j}\right) / t_{\mathrm{v}}$ representa a probabilidade por unidade de tempo de ocorrência de eventos de energia $h_{j}$, sendo $t_{\mathrm{v}}$ o tempo vivo da medida. A analise leva em conta situações em que está sendo processado um pulso de altura $h_{i}$ e é registrado um outro de altura $h_{j}$. Porém, pode acontecer o efeito contrário produzindo uma contagem na mesma energia $h_{j}+h_{i}$, portanto é necessário multiplicar por 2 o resultado anterior. Assim, as contagens de $\operatorname{EP} N_{\mathrm{m}}^{\mathrm{e}}\left(h_{k}\right)$ observadas na energia $h_{k}$, sendo $k=i+j$, vêm dadas por

$$
N_{\mathrm{m}}^{\mathrm{e}}\left(h_{k}\right)=2 \frac{T}{t_{\mathrm{v}}} N\left(h_{i}\right) N\left(h_{j}\right)
$$

Para um espectro contínuo o número de contagens em cada canal $k$ produto do EP é

$$
N_{\mathrm{m}}^{\mathrm{e}}\left(h_{k}\right)=2 \frac{T}{t_{\mathrm{v}}} \sum_{i=1}^{k-1} N\left(h_{i}\right) N\left(h_{k-i}\right), \quad \text { com } \quad N_{\mathrm{m}}^{\mathrm{e}}\left(h_{1}\right)=0, \quad k=2, \ldots, M
$$

Como o fator $2 T / t_{\mathrm{v}}$ é constante, a forma do espectro de EP só depende do somatório, logo, a Eq. (7.4) pode ser calculada fixando um valor de $2 T / t_{\mathrm{v}}$ a conveniência ( $p$. ex. $\left.10^{-9}\right)$. Por outro lado, as contagens observadas para energias superiores a $E_{0}$ no EAP de bremsstrahlung (ver região do gráfico da Fig. 7.1 acima do tip) se devem exclusivamente ao fenômeno de EP, uma vez corrigido o fundo radioativo. A área nesta faixa foi calculada no espectro experimental $\left(A_{\mathrm{exp}}^{\mathrm{e}}\right)$ e no espectro modelado $\left(A_{\mathrm{m}}^{\mathrm{e}}\right)$, de maneira que o espectro final de EP foi determinado de acordo com:

$$
N^{\mathrm{e}}\left(h_{k}\right)=\frac{A_{\mathrm{exp}}^{e}}{A_{\mathrm{m}}^{e}} N_{\mathrm{m}}^{\mathrm{e}}\left(h_{k}\right)
$$


A Fig. 7.1 apresenta um exemplo do EAP coletado no detetor colocado a $90^{\circ}$, para o alvo de Te e elétrons incidentes de $E_{0}=51,19 \mathrm{keV}$, bem como os respectivos espectros de fundo radioativo e de EP. Pode se observar o espectro de bremsstrahlung típico de alvo fino, com o tip bem definido, além dos raios-x K característicos do Te. Note-se como são modeladas de forma satisfatória as contagens acima do tip pelo espectro calculado de EP e a contribuição de fundo.

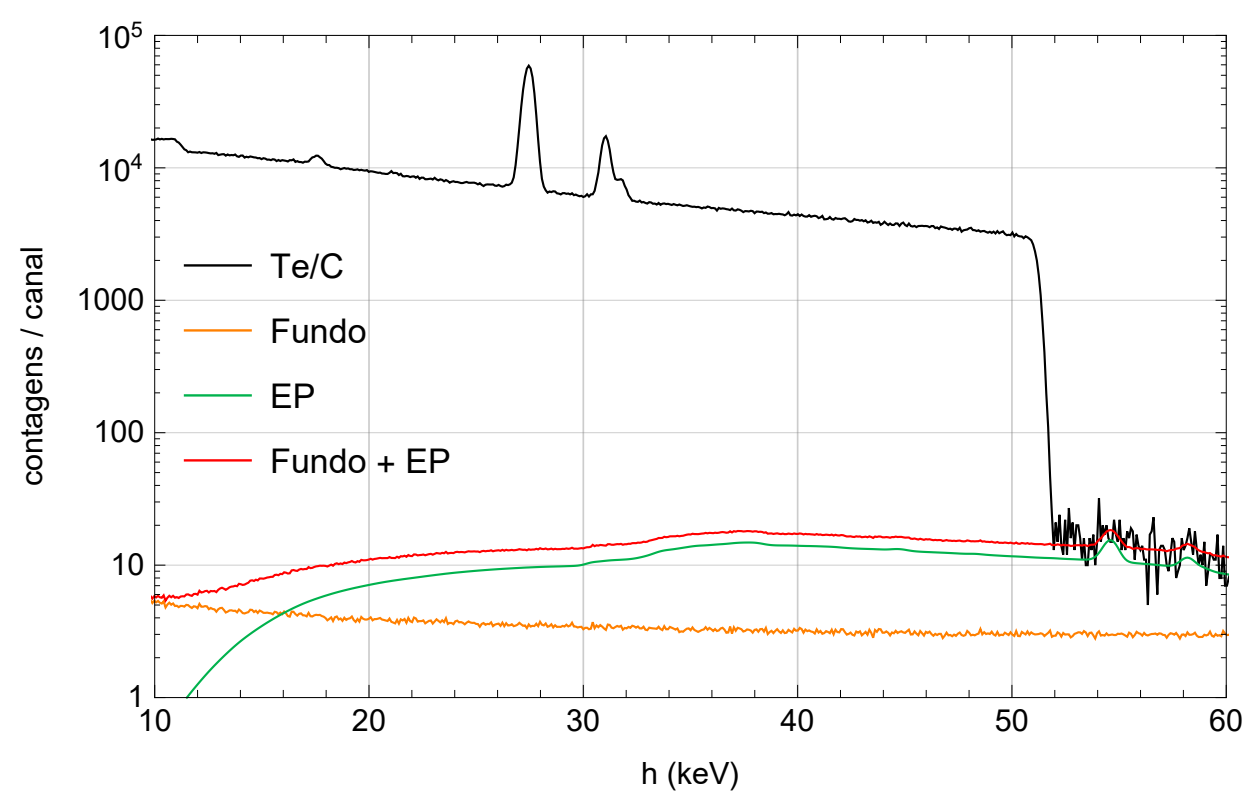

Figura 7.1: Espectro coletado para o alvo de Te, com $E_{0}=51,19 \mathrm{keV}$ a $90^{\circ}$, e correções devidas à radiação de fundo (Fun.) e ao empilhamento de pulsos (EP).

No gráfico da Fig. 7.2 se mostram os EAP medido e corrigido correspondentes ao alvo de Te do exemplo anterior, na faixa energética 50-60 keV. A área $A_{\text {exp }}^{\mathrm{e}}$ é obtida com as contagens observadas no EAP medido acima de $52 \mathrm{keV}$. Os dois pequenos picos que se apreciam no espectro de EP em 54,6 e 58,2 keV coincidem com a soma dos raios$\mathrm{x} \mathrm{K} \alpha+\mathrm{K} \alpha$ e $\mathrm{K} \alpha+\mathrm{K} \beta$ do $\mathrm{Te}^{1}$. Em geral a importância das correções por EP e fundo dependem do tempo de coleção dos espectros, o elemento, ângulo e energia analisados.

A última das correções, por serem os alvos depositados sobre C, consiste em subtrair as contagens da radiação gerada no substrato. Para esse fim são medidos sob as mesmas condições de irradiação o EAP do alvo $\left[N\left(h_{i}\right)\right]_{\mathrm{X} / \mathrm{C}}$, onde o subíndice $\mathrm{X} / \mathrm{C}$ indica que o espectro é composto pelo elemento $\mathrm{X}$ estudado e o substrato de $\mathrm{C}$, e posteriormente o alvo que só contém o $\mathrm{C}\left[N\left(h_{i}\right)\right]_{\mathrm{C}}$. Para isolar as contagens $\left[N\left(h_{i}\right)\right]_{\mathrm{X}}$ produzidas no material X é realizada canal a canal $i$ a correção

$$
\left[N\left(h_{i}\right)\right]_{\mathrm{X}}=\left[N\left(h_{i}\right)\right]_{\mathrm{X} / \mathrm{C}}-\left[\frac{N_{0}(\mathrm{X} / \mathrm{C})}{N_{0}(\mathrm{C})}\right]\left[\frac{t(\mathrm{X} / \mathrm{C})}{t(\mathrm{C})}\right]\left[N\left(h_{i}\right)\right]_{\mathrm{C}}
$$

\footnotetext{
${ }^{1}$ As energias das transições $\mathrm{KL}_{2}\left(\mathrm{~K} \alpha_{2}\right), \mathrm{KL}_{3}\left(\mathrm{~K} \alpha_{1}\right), \mathrm{KM}_{2}\left(\mathrm{~K} \beta_{3}\right), \mathrm{KM}_{3}\left(\mathrm{~K} \beta_{1}\right)$ no Te são 27201,99(26), $27472,57(27), 30944,60(46)$ e 30995,97(34) eV respectivamente [83].
} 


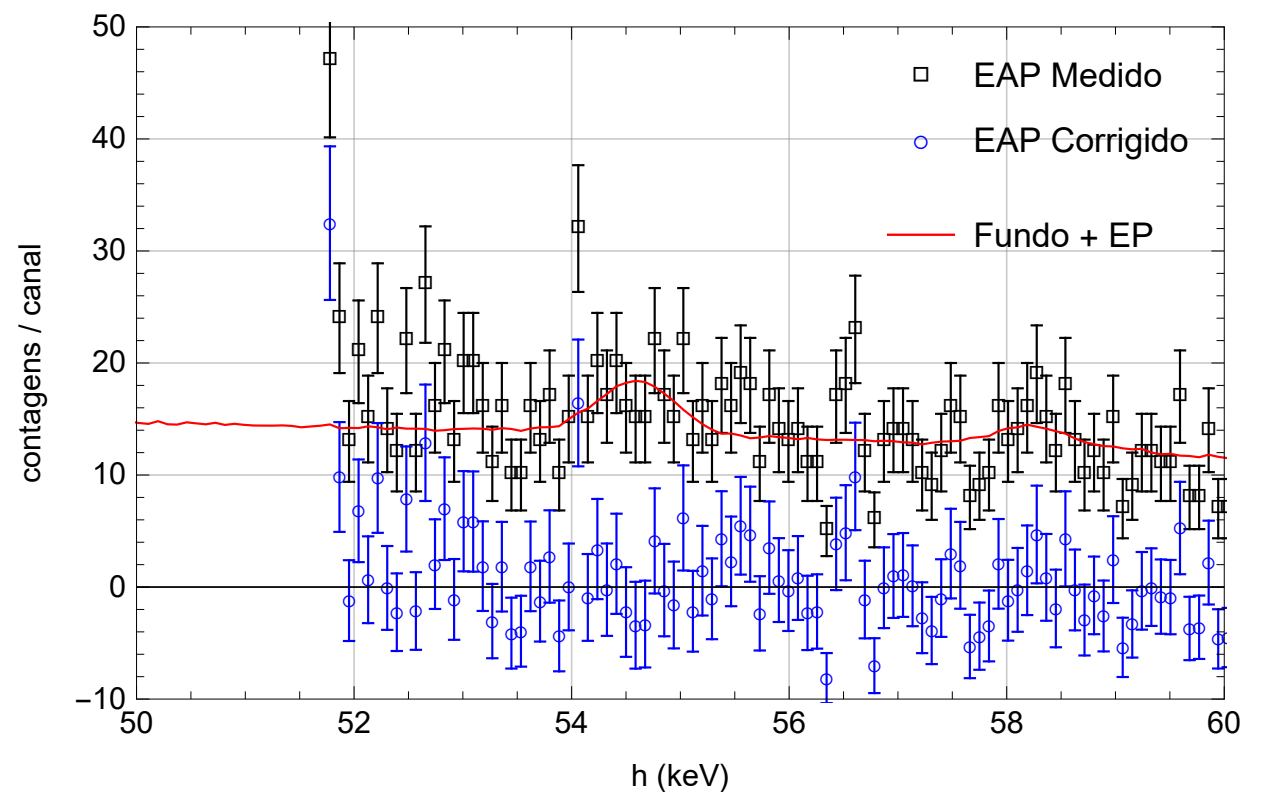

Figura 7.2: Faixa do espectro de Te em que se observam as contagens por EP. Os pontos azuis oscilando em torno de zero mostram o EAP corrigido.

onde as frações $N_{0}(\mathrm{X} / \mathrm{C}) / N_{0}(\mathrm{C})$ e $t(\mathrm{X} / \mathrm{C}) / t(\mathrm{C})$ são fatores de normalização pelo número de elétrons incidentes em cada medida, e pela espessura de $\mathrm{C}$ no substrato do elemento $\mathrm{X}(t(\mathrm{X} / \mathrm{C}))$ em relação ao alvo de $\mathrm{C}(t(\mathrm{C}))$ medido (ver Tabela 6.4).

No gráfico superior da Fig. 7.3 são mostrados os EAP de bremsstrahlung do alvo de Te do exemplo citado acima, do alvo de $\mathrm{C}$ correspondente as mesmas condições de irradiação, normalizado à primeira medida, e o espectro resultante da correção. O gráfico inferior apresenta a porcentagem que constituem as contagens devidas à interação radiativa dos elétrons no substrato do alvo de Te, em função de $h$. Observa-se o comportamento decrescente da curva atingindo um mínimo no tip em torno de 3,5\%. Esse resultado indica a maior intensidade de emissão de fótons nessa região para o Te em relação ao $\mathrm{C}$. Além disso, está em acordo qualitativo com a Eq. (2.44), que descreve a SCDD sob a aproximação de SM para o limite não relativístico, na região de máxima transferência de energia, e estabelece uma proporcionalidade com o número atômico do elemento avaliado segundo $Z^{3}$.

A modo ilustrativo nas Figs. 7.4-7.6 são apresentados os espectros corrigidos pelos efeitos discutidos para $\mathrm{Al}, \mathrm{Ag}$ e $\mathrm{Au}$, energias $E_{0}$ em torno de 20, 50 e $100 \mathrm{keV}$ e os três ângulos de detecção. As contagens foram normalizadas pelo número de elétrons incidentes e a largura do canal em cada caso. Ainda sem deconvouir, algumas conclusões qualitativas podem ser derivadas desses gráficos. A primeira delas se relaciona com a distribuição angular, nota-se como para $E_{0} \approx 20 \mathrm{keV}$ a intensidade dos fótons emitidos a $35^{\circ} \mathrm{e}$ $90^{\circ}$ é similar para $\mathrm{Al}$ e $\mathrm{Ag}$, sendo superior a emissão lateral em Au. Ao aumentar a energia para $E_{0} \approx 50$ e $100 \mathrm{keV}$ se observa como a distribuição angular é dominada pela 

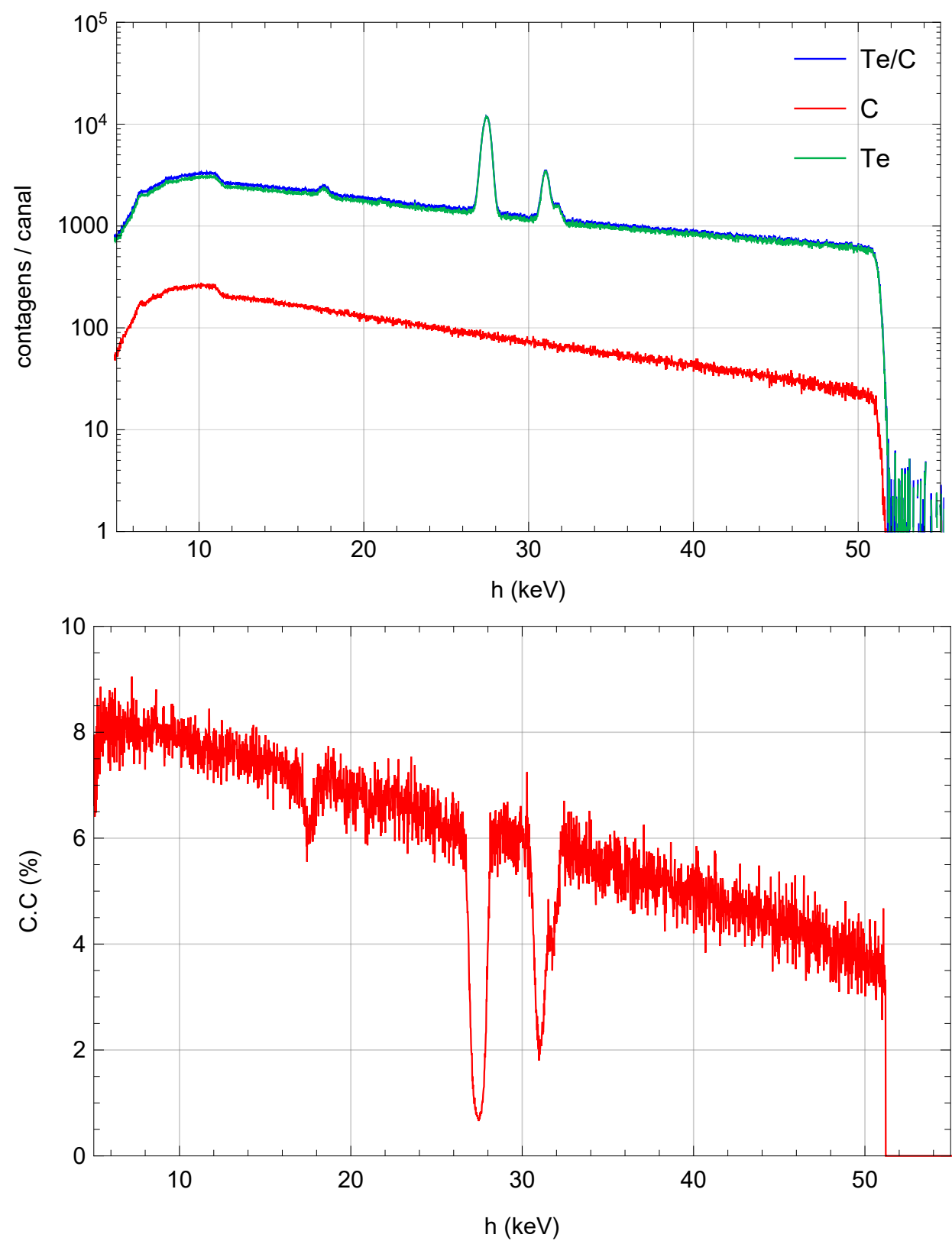

Figura 7.3: Correção do espectro de bremsstrahlung do alvo de Te, coletado a $90^{\circ}$, para elétrons de $51,19 \mathrm{keV}$, pela radiação gerada no substrato de $\mathrm{C}$ (gráfico superior).

Porcentagem da correção (gráfico inferior).

emissão frontal de fótons em todos os casos. Em geral a intensidade do tip experimenta um decrescimento com o aumento de $E_{0}$, perceba-se em particular como desaparece a queda brusca do espectro de bremsstrahlung no caso do Al para elétrons de $\sim 100 \mathrm{keV}$ e $\theta=131^{\circ}$. Uma outra característica notável é a forma diferente que apresentam os espectros coletados com o detetor coaxial $\left(\theta=131^{\circ}\right)$ abaixo de $10 \mathrm{keV}$. A janela de $\mathrm{C}$ de $0,9 \mathrm{~mm}$ absorbe uma maior quantidade de fótons em relação as janelas mais finas de Be $(\sim 0,25 \mathrm{~mm})$ que possuem os outros dois detetores planares.

Uma menção aparte merece o claro efeito de detecção, mais visíveis nos espectros de $\mathrm{Al}$, 
$\mathrm{Al}, E_{0}=20.36 \mathrm{keV}$

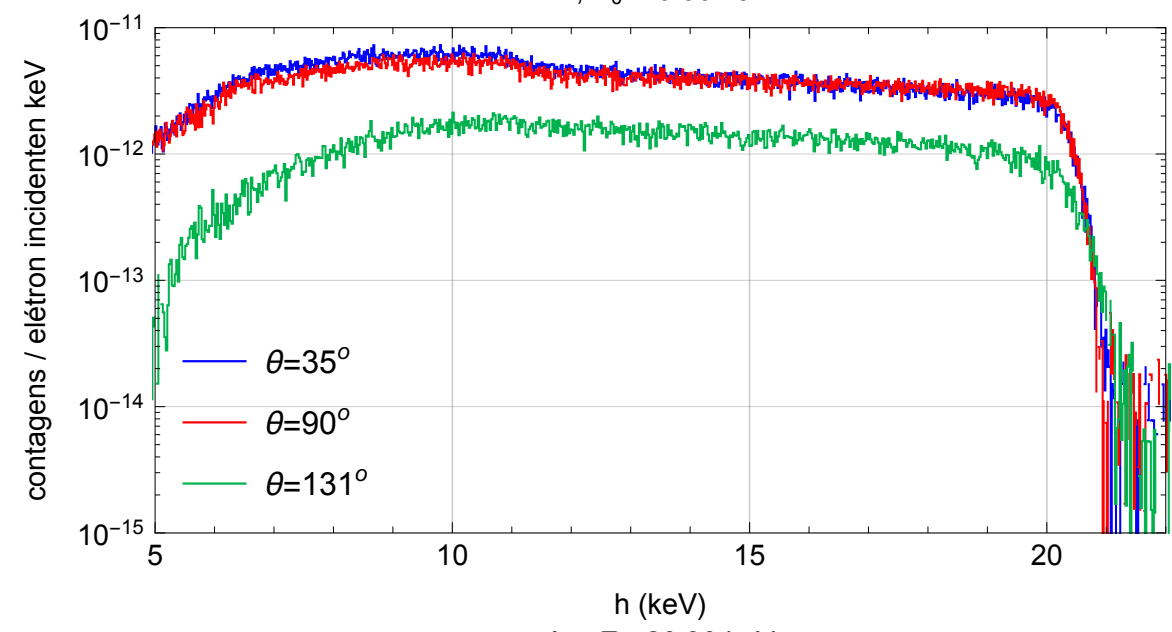

Ag, $E_{0}=20.30 \mathrm{keV}$

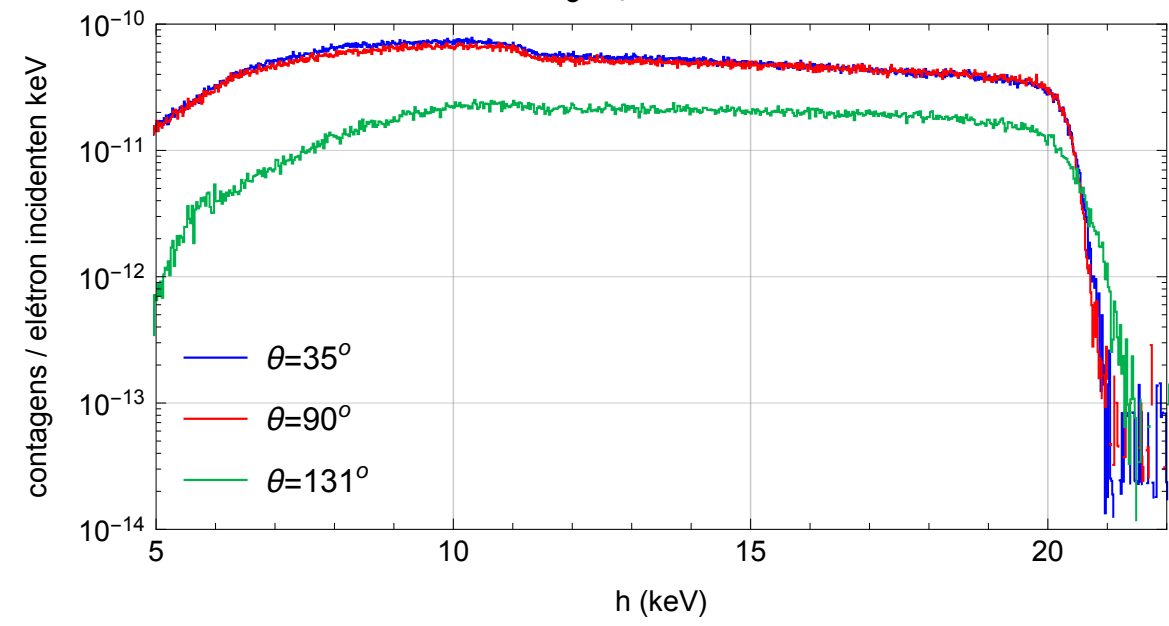

$\mathrm{Au}, E_{0}=20.38 \mathrm{keV}$

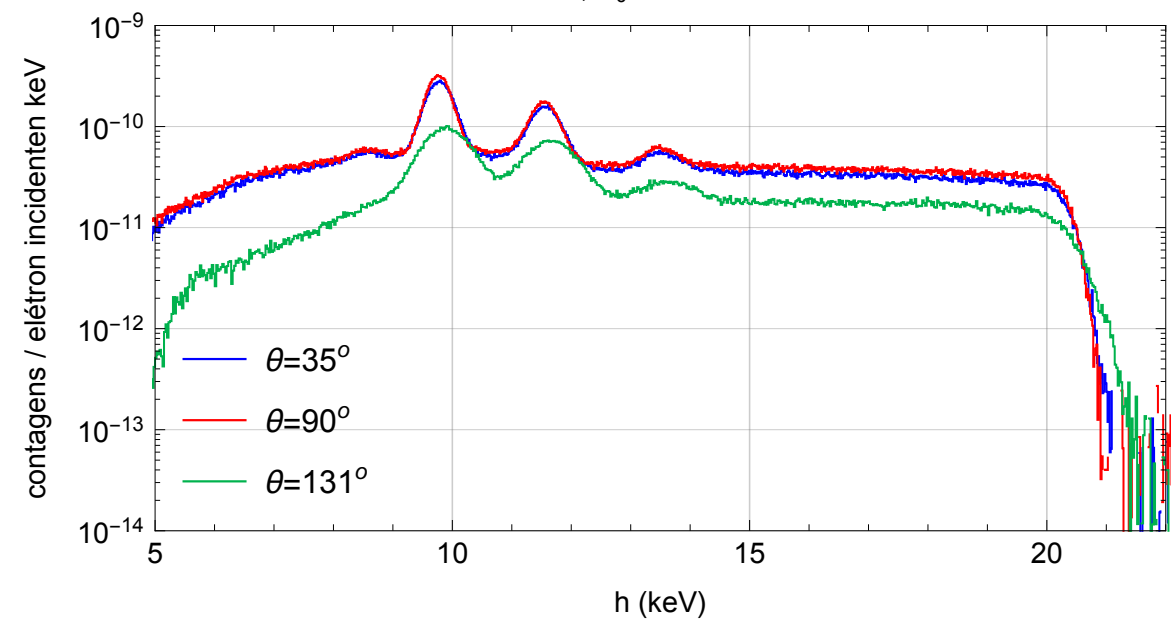

FiguRA 7.4: Espectros de bremsstrahlung corrigidos por fundo radioativo, empilhamento de pulsos e radiação gerada no substrato, para $E_{0}$ em torno de $20 \mathrm{keV}$, nos três ângulos de coleção e os elementos $\mathrm{Al}, \mathrm{Ag}$ e $\mathrm{Au}$. 
$\mathrm{Al}, E_{0}=50.96 \mathrm{keV}$

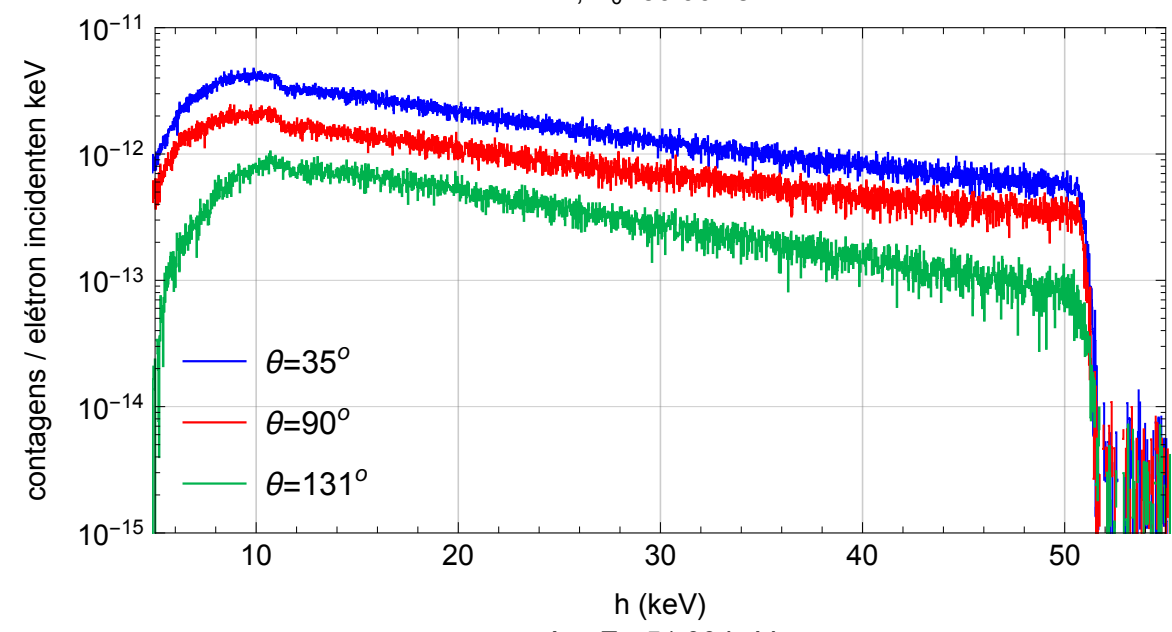

$\mathrm{Ag}, E_{0}=51.09 \mathrm{keV}$

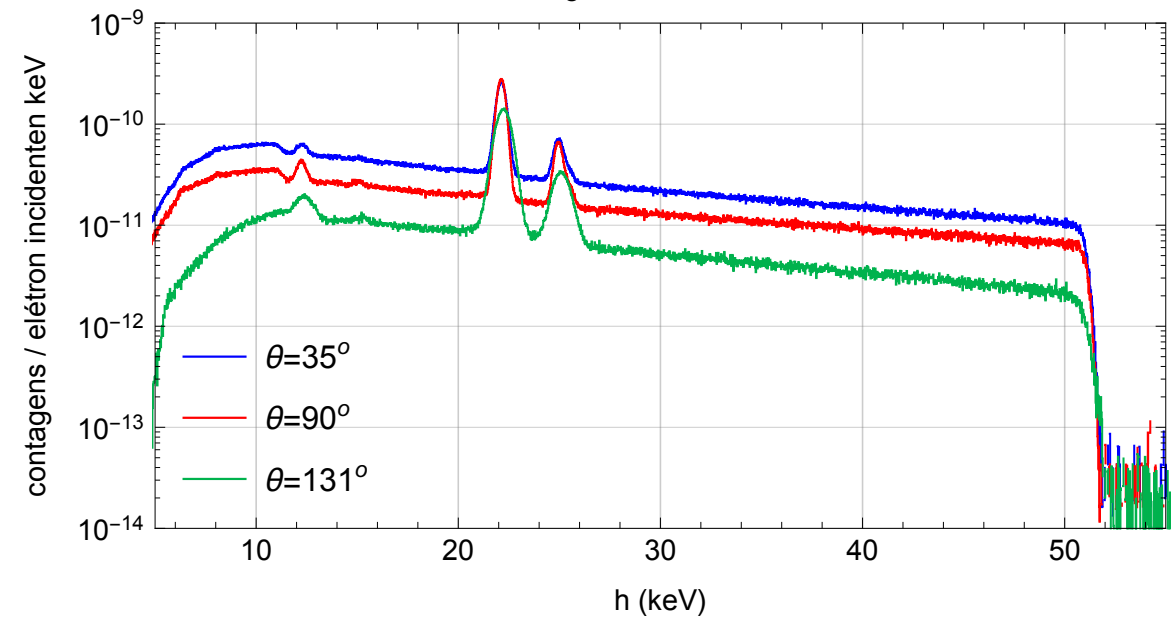

$\mathrm{Au}, E_{0}=51.09 \mathrm{keV}$

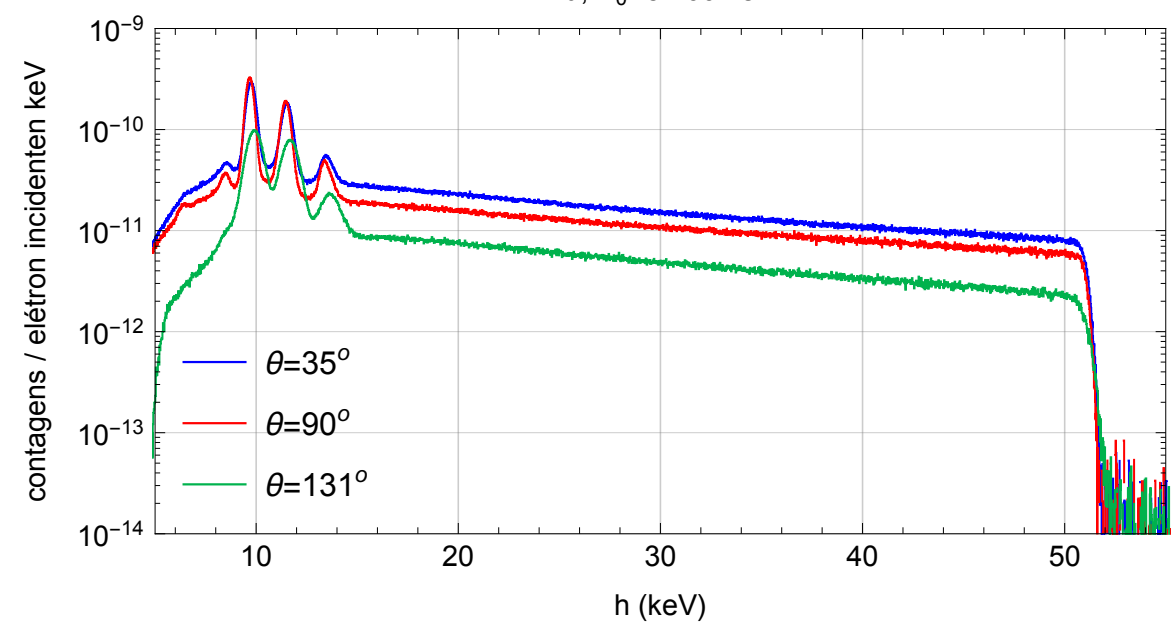

FiguRA 7.5: Espectros de bremsstrahlung corrigidos por fundo radioativo, empilhamento de pulsos e radiação gerada no substrato, para $E_{0}$ em torno de $50 \mathrm{keV}$, nos três ângulos de coleção e os elementos $\mathrm{Al}, \mathrm{Ag}$ e $\mathrm{Au}$. 


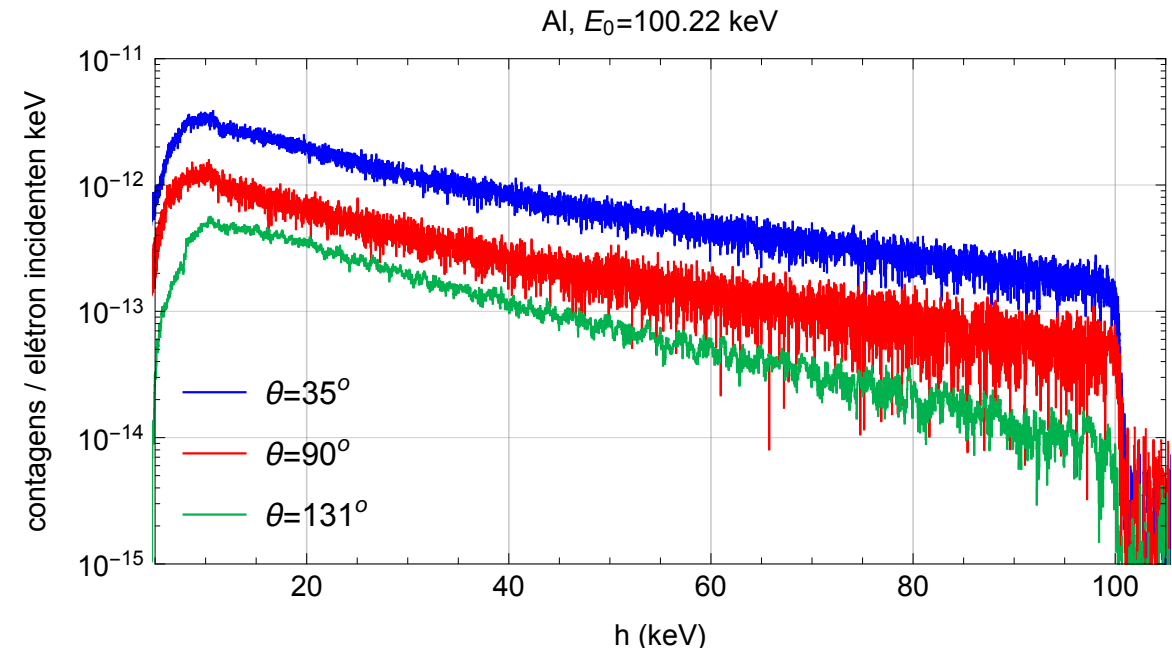

$\mathrm{Ag}, E_{0}=100.44 \mathrm{keV}$

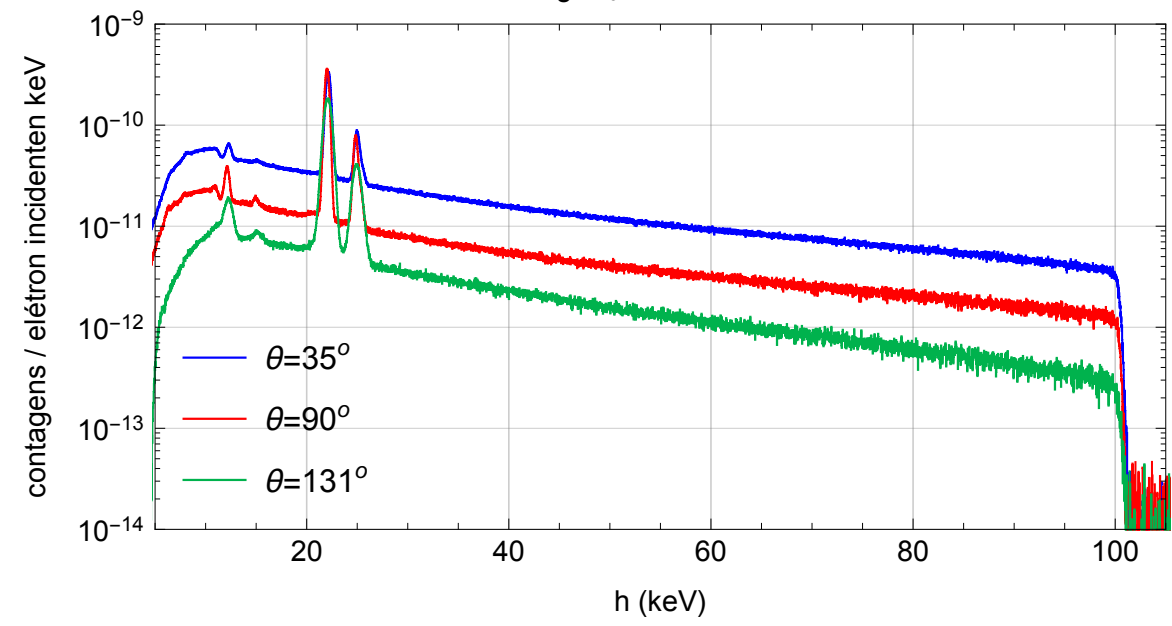

$\mathrm{Au}, E_{0}=100.48 \mathrm{keV}$

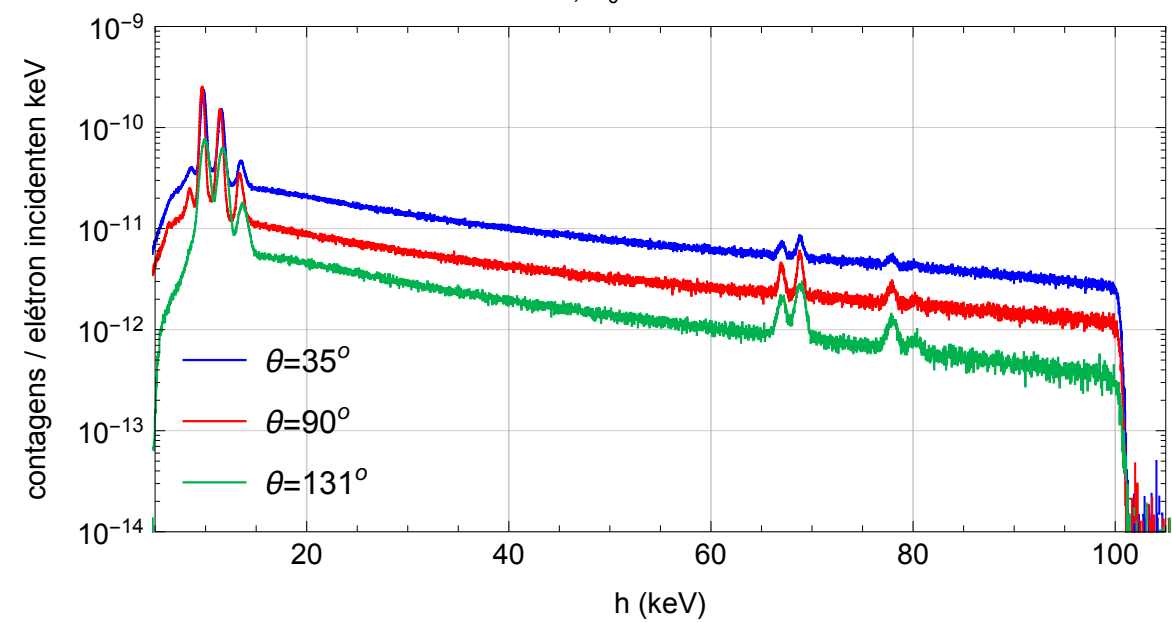

FiguRA 7.6: Espectros de bremsstrahlung corrigidos por fundo radioativo, empilhamento de pulsos e radiação gerada no substrato, para $E_{0}$ em torno de $100 \mathrm{keV}$, nos três ângulos de coleção e os elementos $\mathrm{Al}, \mathrm{Ag}$ e $\mathrm{Au}$. 
em torno da borda $\mathrm{K}$ de absorção do $\mathrm{Ge}\left(U_{\mathrm{K}}=11103,76(74) \mathrm{eV}\right.$ [83]). Ao contrário do que a intuição sugere, abaixo de $U_{\mathrm{K}}$ onde a seção de foto efeito cai de $2,374 \times$ $10^{4}$ barn/átomo para $3,206 \times 10^{3}$ barn/átomo [84], as contagens observadas no espectro aumentam (ver Fig. 7.7). O fenômeno pode ser explicado considerando a eficiência intrínseca de um detetor de Ge dada pela Eq. (4.50). Quando os fótons incidentes são menores que $U_{\mathrm{K}}$, não existe mais absorção de radiação pelos elétrons da camada $\mathrm{K}$ do átomo, e portanto, não há emissão de raios-x $\mathrm{K}$ característicos. Logo, os termos $P_{\mathrm{K} \alpha} \mathrm{e}$ $P_{\mathrm{K} \beta}$ são iguais a zero, o que faz que a probabilidade de absorção total aumente ainda quando a seção de choque diminui quase uma ordem de grandeza.

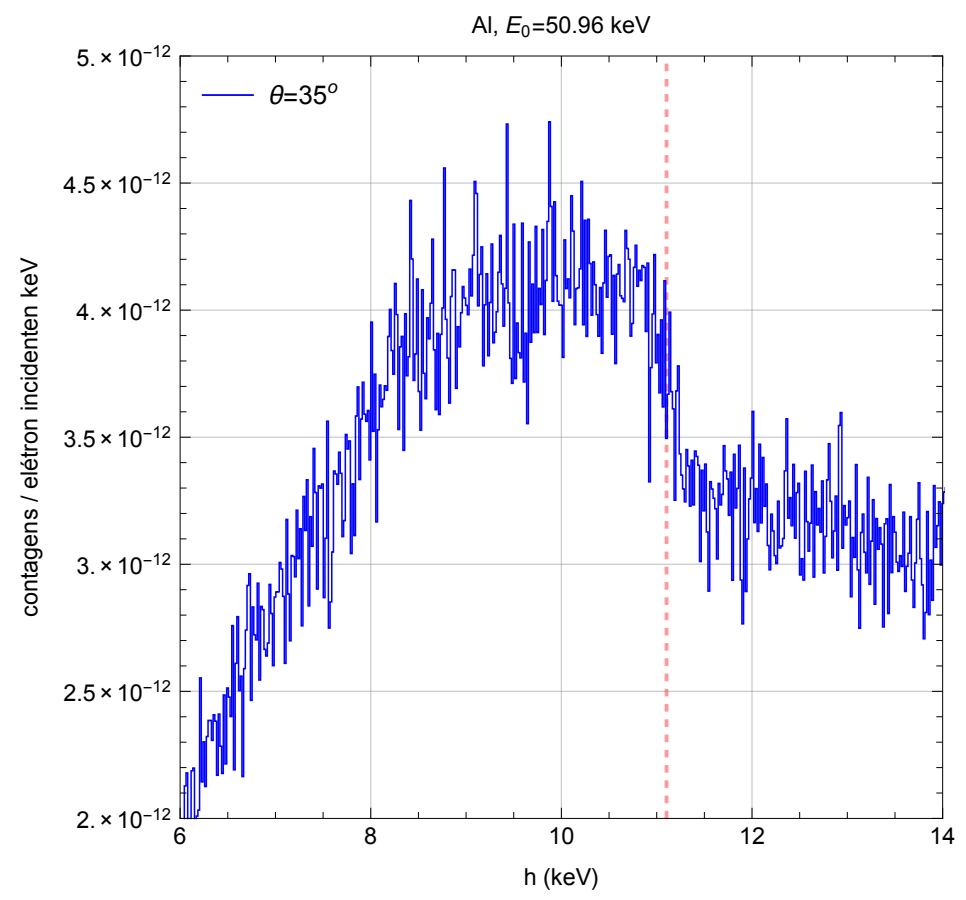

FigurA 7.7: Espectro de bremsstrahlung do alvo Al, coletado para a energia incidente $E_{0}=50,96 \mathrm{keV}$ no ângulo $\theta=35^{\circ}$ na faixa de $h$ entre 6 e $14 \mathrm{keV}$. A linha vermelha tracejada está situada sobre a energia da borda K do Ge.

\subsection{Determinação da energia do feixe de elétrons e da in- tensidade do espectro de bremsstrahlung no ponto de máxima transferência de energia}

O feixe de elétrons do acelerador que incide sobre o alvo a estudar não é exatamente monocromático. Para descrever a sua dispersão em energia generalmente é proposta uma distribuição Gaussiana com média $E_{0}$ e desvio padrão $\sigma_{0}$. Neste trabalho é utilizada e testada essa hipótese por meio do Método dos Mínimos Quadrados, para o ajuste de uma função que modela o espectro coletado na região do tip. O procedimento permite a 
determinação dos parâmetros $E_{0}$ e $\sigma_{0}$, e além disso, obter o número de fótons emitidos em cada ângulo, no ponto do espectro de máxima transferência energia $\kappa=W / E_{0}=1$.

Quando elétrons de energia $E_{e}$ incidem sobre um alvo o número de fótons $B\left(E_{e} ; W\right)^{2}$, gerados entre $W$ e $W+\mathrm{d} W$, que atingem o detetor situado no ângulo $\theta$, pode ser calculado como

$$
B\left(E_{e} ; W\right)=N_{0} t \Omega \frac{\mathrm{d}^{2} \sigma}{\mathrm{d} W \mathrm{~d} \Omega}=N_{0} t \Omega \frac{Z^{2}}{\beta^{2}} \frac{1}{W} \chi\left(Z, E_{e}, W\right) S\left(Z, E_{e}, W, \theta\right)
$$

onde $N_{0}, t, \Omega, Z$ e $\beta$ tem os significados habituais adotados neste trabalho, e a SCDD foi escrita em função da seção de choque reduzida $\chi\left(Z, E_{e}, W\right)$ [3] e a distribuição angular $S\left(Z, E_{e}, W, \theta\right)[5]$. Para valores fixos de $Z, E_{e}$ e $\theta$ a dependência de $B\left(E_{e} ; W\right)$ com $W$ tem a forma

$$
B\left(E_{e} ; W\right)=\frac{C}{W} f(W)
$$

sendo $C$ uma constante e $f(W)=\chi(W) S(W)$. Note-se que $C$ e $f(W)$ possuem unidades recíprocas: $\left(\mathrm{sr} / \mathrm{cm}^{2}\right)$ e $\left(\mathrm{cm}^{2} / \mathrm{sr}\right)$ respectivamente. Para modelar o tip de bremsstrahlung é utilizada a seguinte estimativa

$$
f(W) \approx\langle\chi(W) S(W)\rangle_{\Delta W}=\bar{f}
$$

equivalendo $\bar{f}$ ao valor médio de $f(W)$ no intervalo $\Delta W$ onde é avaliado o espectro de fótons. A Eq. (7.8) tem maior validade quanto menor for $\Delta W$. Sob esses pressupostos o tip de bremsstrahlung pode se aproximar a

$$
B\left(E_{e} ; W\right)=\frac{A}{W} \Theta\left(E_{e}-W\right)
$$

sendo $A=C \bar{f}$ um fator adimensional de amplitude que depende das condições experimentais. Além disso, na Eq. (7.9) tem sido considerado o princípio de conservação da energia $\left(W \leq E_{e}\right)$ por meio da função degrau de Heaviside.

Incorporando a hipótese inicial e seguindo o modelo adotado, o espectro de fótons gerado por um feixe com uma distribuição de energia Gaussiana de parâmetros $E_{0}$ e $\sigma_{0}$ pode ser calculado como

\footnotetext{
${ }^{2}$ Note-se que $B\left(E_{e} ; W\right)$ é uma função densidade de probabilidade.
} 


$$
\begin{aligned}
B\left(E_{0}, \sigma_{0} ; W\right) & =\int_{0}^{\infty} B\left(E_{e} ; W\right) \frac{1}{\sqrt{2 \pi} \sigma_{0}} \exp \left[-\frac{\left(E_{e}-E_{0}\right)^{2}}{2 \sigma_{0}^{2}}\right] \mathrm{d} E_{e} \\
& =\frac{A}{2 W}\left[1+\operatorname{erf}\left(\frac{E_{0}-W}{\sqrt{2} \sigma_{0}}\right)\right]=A b\left(E_{0}, \sigma_{0} ; W\right)
\end{aligned}
$$

onde $\operatorname{erf}(x)$ representa a função erro. Igualmente se aceitou que a variação de $A$ durante a integração é desprezível sendo tomado como um fator constante.

A convolução da FR dos detetores $R(h, W)$ com o espectro incidente $B\left(E_{0}, \sigma_{0} ; W\right)$ permite modelar as contagens registradas no tip. Logo, substituindo ambas funções na Eq. (4.1) se obtém a densidade de probabilidade

$$
N(h)=A \int_{0}^{\infty} R(h, W) b\left(E_{0}, \sigma_{0} ; W\right) \mathrm{d} W=A F\left(E_{0}, \sigma_{0} ; h\right),
$$

que descreve o espectro coletado no intervalo $[h, h+\mathrm{d} h]$. A Eq. (7.11) depende linearmente do parâmetro $A$ e de maneira não linear de $E_{0}$ e $\sigma_{0} . F\left(E_{0}, \sigma_{0} ; h\right)$ se calculou numericamente com o software Mathematica 10.0.2 [87] utilizando o Método de Quadraturas Gaussianas de ordem 12.

A Fig. 7.8 apresenta os modelos dos espectros $B\left(E_{0}, \sigma_{0} ; W\right)$ e $F\left(E_{0}, \sigma_{0} ; h\right)$, para o caso de $E_{0}=100 \mathrm{keV}$ com desvios $\sigma_{0}=30$ e $150 \mathrm{eV}$. Nesse intervalo de $\sigma_{0}$ é esperado que o acelerador opere. A função $F\left(E_{0}, \sigma_{0} ; h\right)$ foi calculada utilizando $\mathrm{FR}$ do detetor planar colocado a $35^{\circ}$. Observa-se que ainda quando existe uma marcada diferença entre os comportamentos de $B\left(E_{0}, \sigma_{0} ; W\right)$ para os valores de $\sigma_{0}$ estudados, o resultado da convolução com a resposta $R(h, W)$ em ambos casos é muito próximo (maior discrepância entorno de $3 \%$ ), demostrando que sob essas condições o efeito de deformação no tip estará dominado pela Gaussiana $\mathcal{G}(h, W)$ que carateriza a resolução do detetor.

A energia do feixe e sua dispersão é ajustada minimizando simultaneamente a função de mérito

$$
Q\left(E_{0}, \sigma_{0}\right)=\sum_{j, i}\left[N_{j, i}-N_{j, i}^{\bmod }\right]^{2} / \sigma_{j, i}^{2},
$$

a partir das contagens $N_{j, i}$ coletadas no experimento e modeladas $N_{j, i}^{\text {mod }}$, onde o subíndice $j$ simboliza o espectro medido por cada detetor, $\sigma_{j, i}$ é o desvio padrão das contagens, e o somatório se realiza sobre os canais $i$ considerados na avaliação de $Q\left(E_{0}, \sigma_{0}\right)$. O modelo de ajuste utiliza a Eq. (4.2) para discretizar da densidade de probabilidade (7.11) segundo a aproximação 

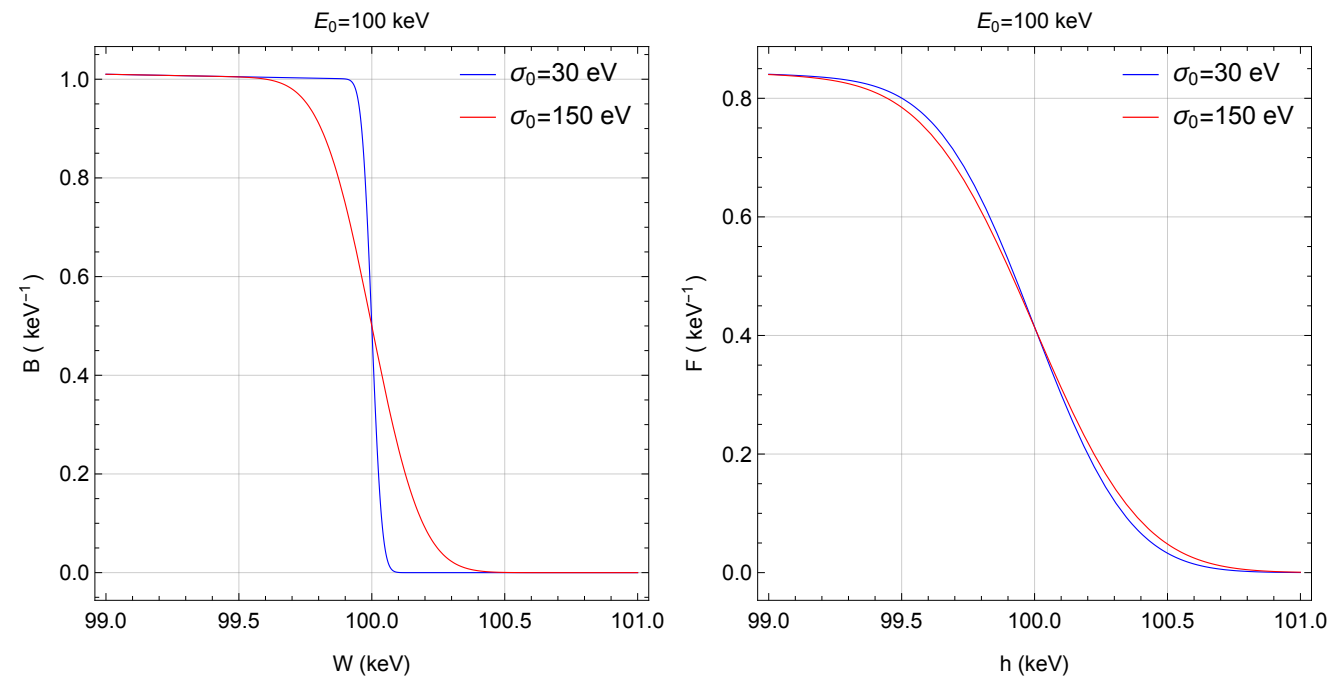

Figura 7.8: Funções densidade de probabilidade para os espectros de fótons gerado no alvo $(B)$ e medido no detector $(F)$ quando $E_{0}=100 \mathrm{keV}$, com larguras de $30 \mathrm{e}$ $150 \mathrm{eV}$. O fator de escala utilizado foi $A=10^{2}$.

$$
N_{j, i}^{\mathrm{mod}}=\int_{h_{j, i}-\delta_{j} / 2}^{h_{j, i}+\delta_{j} / 2} N(h) \mathrm{d} h \approx \delta_{j} N\left(h_{j, i}\right)=\delta_{j} \widehat{A}_{j} F_{j}\left(E_{0}, \sigma_{0} ; h_{j, i}\right),
$$

sendo $\delta_{j}$ a largura de cada canal no detetor $j$. Para um dado par coordenado $\left(E_{0}, \sigma_{0}\right)$, $\widehat{A}_{j}$ é a estimativa de menor variância do parâmetro $A$, determinada a priori pelo Método dos Mínimos Quadrados como:

$$
\widehat{A}_{j}=\frac{\sum_{i} \delta_{j} F_{j}\left(E_{0}, \sigma_{0} ; h_{j, i}\right) N_{j, i} / \sigma_{j, i}^{2}}{\sum_{i}\left[\delta_{j} F_{j}\left(E_{0}, \sigma_{0} ; h_{j, i}\right)\right]^{2} / \sigma_{j, i}^{2}}
$$

Finalmente, de acordo com as Eqs. (7.6) e (7.9) a seção de choque no ponto de máxima transferência de energia pode se determinar a partir das grandezas ajustadas por meio de:

$$
\frac{\mathrm{d}^{2} \sigma}{\mathrm{d} W \mathrm{~d} \Omega}=\frac{\widehat{A}_{j} / E_{0}}{N_{0, j} t \Omega_{j}} .
$$

O cociente $\widehat{A}_{j} / E_{0}$ representa o número de fótons com $W=E_{0}$ emitidos no alvo durante a incidência de $N_{0, j}$ elétrons.

Para realizar o procedimento de ajuste e garantir a maior precisão possível, as calibrações em energia obtidas de maneira preliminar com as medidas de espectros de fontes radioativas, são refeitas para cada espectro analisado a partir da posição dos picos de raios-x característicos do elemento. No caso do $\mathrm{C}$ e do Al, foram tomadas as calibrações derivadas dos alvos irradiados imediatamente antes o depois. O procedimento permite levar 
em conta as variações que pode experimentar o sistema de aquisição pela influência de fenômenos externos temporais ( $p$. ex. mudanças na linha base dos amplificadores pelo ruído eletrônico).

A Fig. 7.9 apresentam as curvas de nível de $\chi^{2}$ da minimização da função de mérito (7.12) para quatro dos espectros coletados de Ag. No exemplo as energias ajustadas $E_{0}=$ 26,97, 51,09, 70,63 e 100,44 keV, para valores de largura do feixe de 0,11, 0,09, 0,08 e $0,10 \mathrm{keV}$ respectivamente. As superfícies fechadas representam o valor mais provável $Q_{\min }\left(E_{0}, \sigma_{0}\right)=\chi^{2}$ aumentado em uma unidade quadrática $\left(\chi^{2}+i^{2}, \operatorname{com} i=1,2, \ldots, n\right)$. Os resultados dos ajustes são mostrados nas Figs. 7.10-7.11, observa-se que quanto maior é a estatística de contagens no tip maior é número de curvas de nível fechadas nos gráficos da Fig. 7.9. Os canais dos espectros das Figs. 7.10-7.11 possuem uma largura $\delta_{j}=17,78,17,57$ e $25,14 \mathrm{eV}$ para os detetores situados a $35^{\circ}, 90^{\circ}$ e $131^{\circ}$ respectivamente.
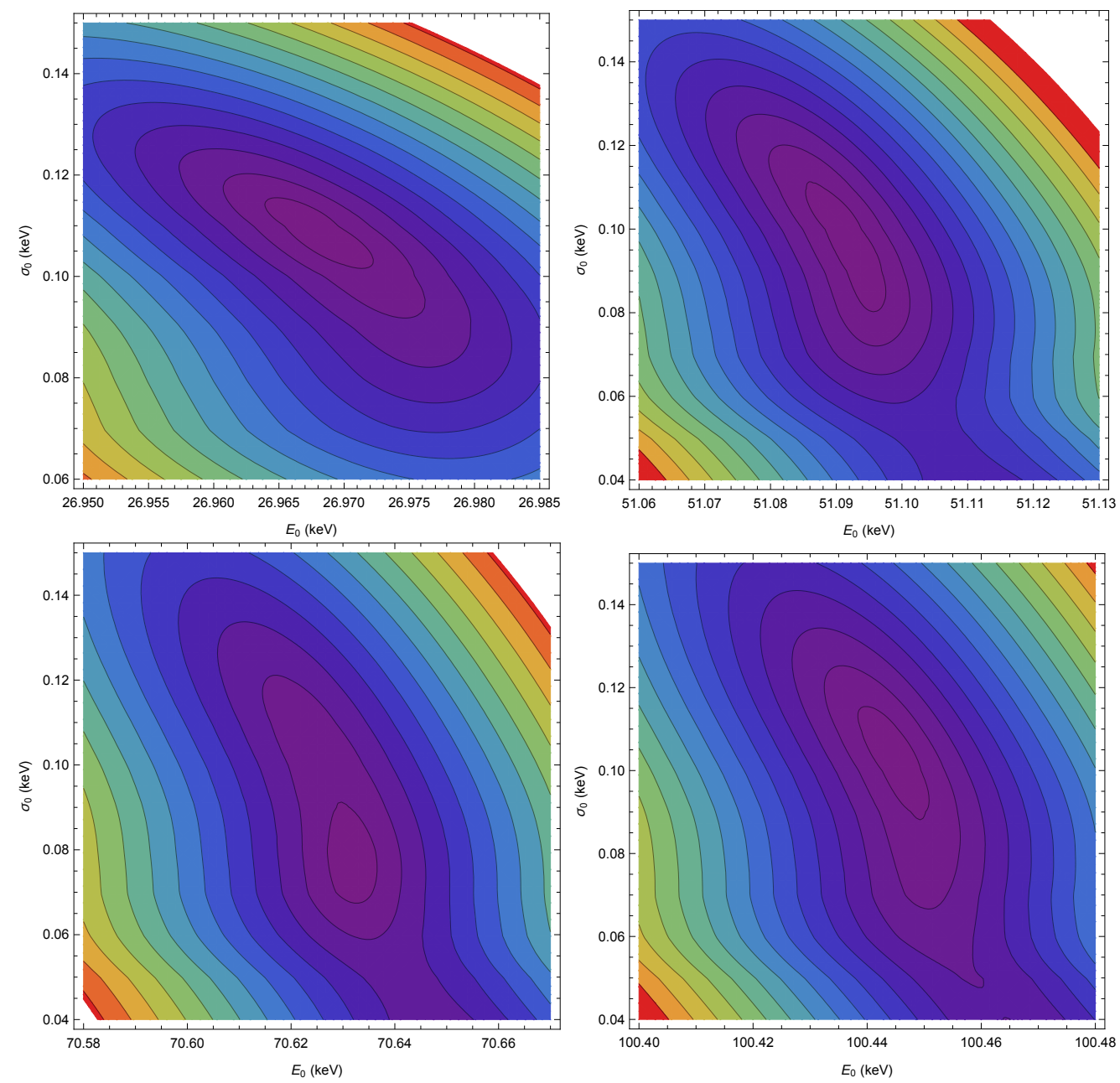

Figura 7.9: Curvas de nível de $\chi^{2}$ do ajuste dos parâmetros $E_{0}$ e $\sigma_{0}$, para diferentes energias do feixe de elétrons e o alvo de Ag. 

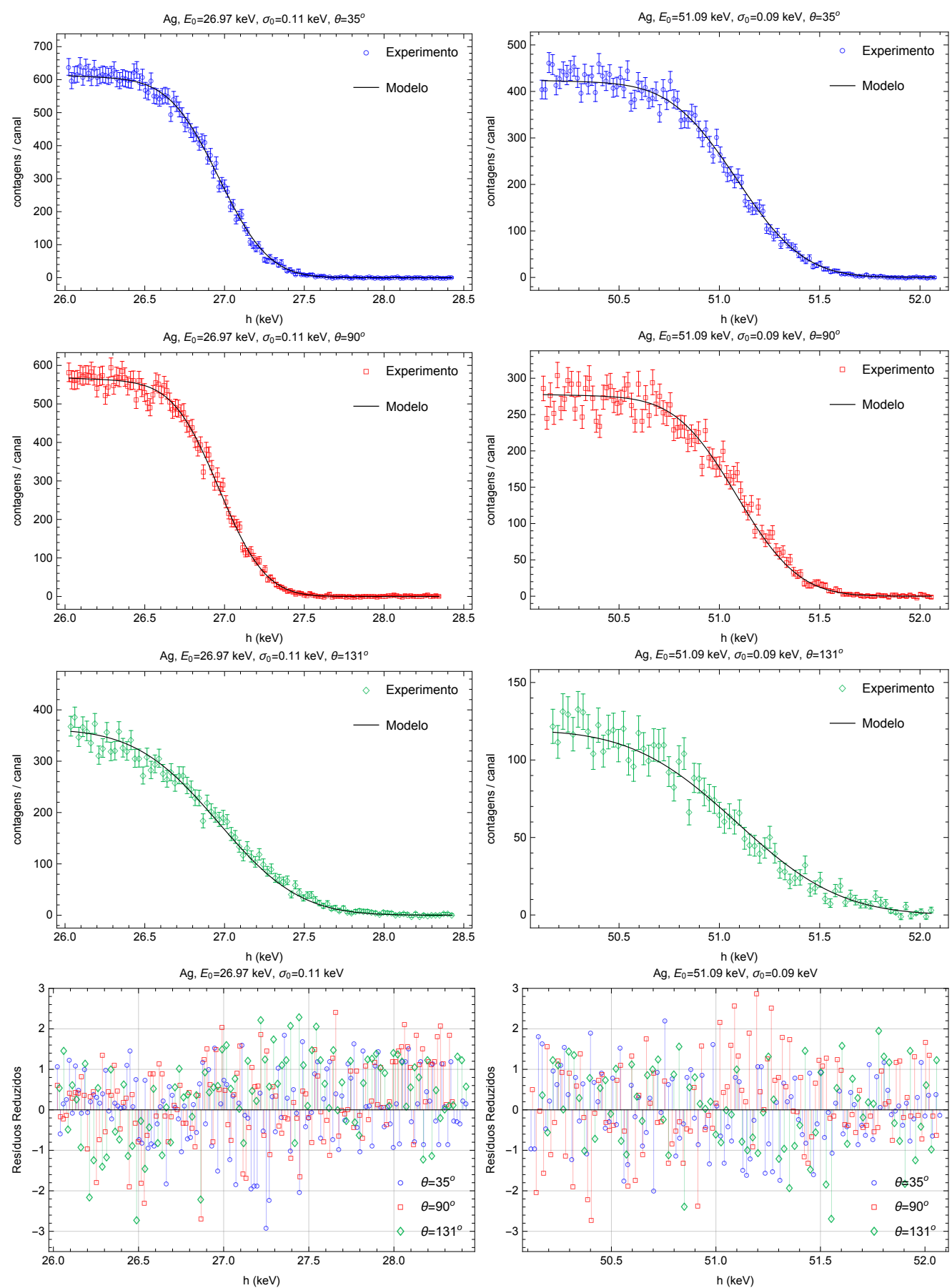

Figura 7.10: Ajuste do modelo proposto às contagens coletadas no tip, para o alvo de $\mathrm{Ag}$ e os três detetores. Os parâmetros obtidos são $E_{0}=26,97 \mathrm{keV}$ e $\sigma_{0}=0,11 \mathrm{keV}$ (coluna esquerda) e $E_{0}=51,09 \mathrm{keV}$ e $\sigma_{0}=0,09 \mathrm{keV}$ (coluna direita). 

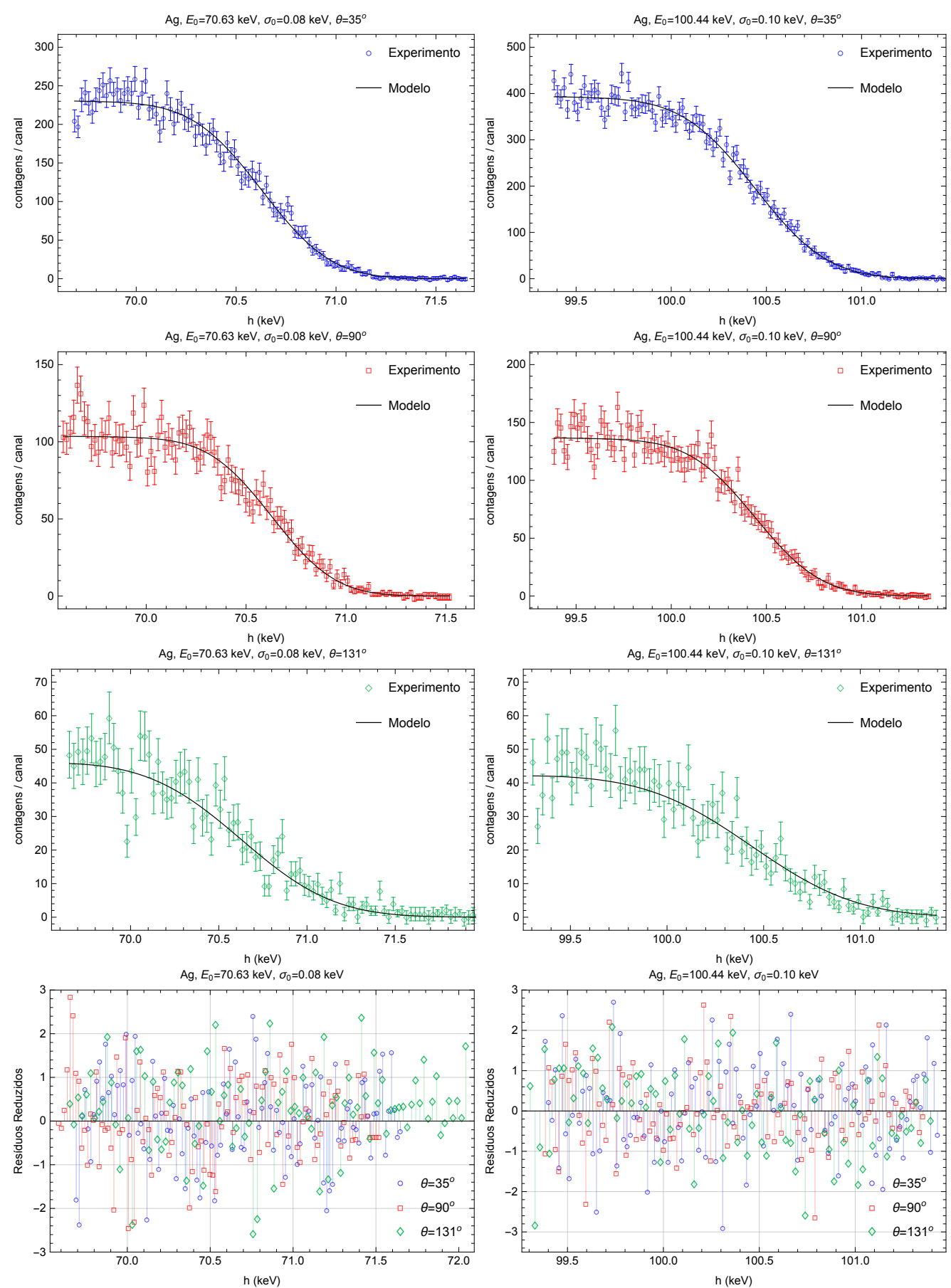

Figura 7.11: Ajuste do modelo proposto às contagens coletadas no tip, para o alvo de $\mathrm{Ag}$ e os três detetores. Os parâmetros obtidos são $E_{0}=70,63 \mathrm{keV}$ e $\sigma_{0}=0,08 \mathrm{keV}$ (coluna esquerda) e $E_{0}=100,44 \mathrm{keV}$ e $\sigma_{0}=0,10 \mathrm{keV}$ (coluna direita). 


\subsection{Deconvolução dos espectros de bremsstrahlung}

A deconvolução dos espectros foi realizada entre os $12 \mathrm{keV}$ (acima da borda $\mathrm{K}$ do Ge) e o tip de bremsstrahlung. Para compor o vetor $\mathbf{N}$ da Eq. (4.13), foram somadas e agrupadas em 1 canal as contagens correspondentes a 57 canais dos EAP coletados originalmente pelos detetores colocados em $\theta=35^{\circ}$ e $90^{\circ}$, e 40 canais no caso do terceiro detetor $\left(\theta=131^{\circ}\right)$. Desta maneira, o EAP $N(h)$ apresenta uma resolução $\delta=1 \mathrm{keV}$, que equivale a $\Delta=1 \mathrm{keV}$ no espectro bremsstrahlung $B(W)$. Essa largura evita posteriores procedimentos de regularização em $B(W)$ [54]. Consequentemente, a matriz $\mathbf{R}$ foi calculada integrando numericamente a FR analítica $R(h, W)^{3}$ na Eq. (4.3) sobre intervalos de largura $\delta=1 \mathrm{keV}$.

O gráfico da Fig. 7.12 representa as matriz $\mathbf{R}$ correspondente ao detetor colocado em $\theta=35^{\circ}$. Cada quadrado simboliza a FR integrada sobre um canal de $1 \mathrm{keV}$, cuja intensidade é dada pela cor. As colunas indicam para um valor dado de energia incidente $W_{j}$ os possíveis valores de energia registrada $h_{i}$. O primeiro elemento da matriz designa o valor $W_{j}=h_{i} \simeq 12 \mathrm{keV}$. A partir desse ponto à direita aumenta $W_{j}$, enquanto da mesma referência para abaixo aumenta $h_{i}$. A diagonal, onde $W_{j}=h_{i}$, contêm os picos de absorção total. Aproximadamente 10 unidades acima desses pontos, define-se uma linha paralela que demarca a posição dos picos de escape de raios-x K do Ge. Observa-se como essa estrutura é mais intensa quanto menor é a energia $W_{j}$. Os tons amarelos na região superior direita representam o escape Compton para os maiores valores $W_{j}$. A faixa $h_{i}>W_{j}=0$, salvo um quadro à esquerda do fotopico que considera os efeitos Gaussianos de resolução, revelando o carácter quase triangular da matriz $\mathbf{R}$.

Na Fig. 7.13 se representa a matriz inversa $\mathbf{R}^{-1}$ do mesmo detetor. Nota-se como a região à direita da diagonal toma agora valores negativos, que indicam o número de contagens registradas em $h_{i}$ que são incorporadas ao espectro incidente $\mathbf{B}$ na energia $W_{j}$, com $j>i$, quando se realiza o produto $\mathbf{R}^{-1} \mathbf{N}$.

Por outro lado, na Fig. 7.14 são mostrados dois exemplos dos espectros de Ta medidos com o detetor situado em $\theta=35^{\circ}$, para energias $E_{0}=51,19$ e 100,80 keV e largura dos canais $\Delta=1 \mathrm{keV}$, o os espectros obtidos a partir do processo de deconvolução. Observase, como era de esperar para o intervalo de energias analisado, que o espectro $B(W)$ é sempre maior que o EAP $N(h)$. Finalmente, a Fig. 7.15 apresenta para os mesmos exemplos uma comparação entre $B(W)$, e o espectro $B^{\prime}(W)$, determinado segundo

$$
B^{\prime}(W)=\left.\frac{N(h)}{T(W) \varepsilon_{\text {int }}(W)}\right|_{h=W},
$$

\footnotetext{
${ }^{3} \operatorname{Em} R(h, W)$ foram incluídos os efeitos do colimador.
} 


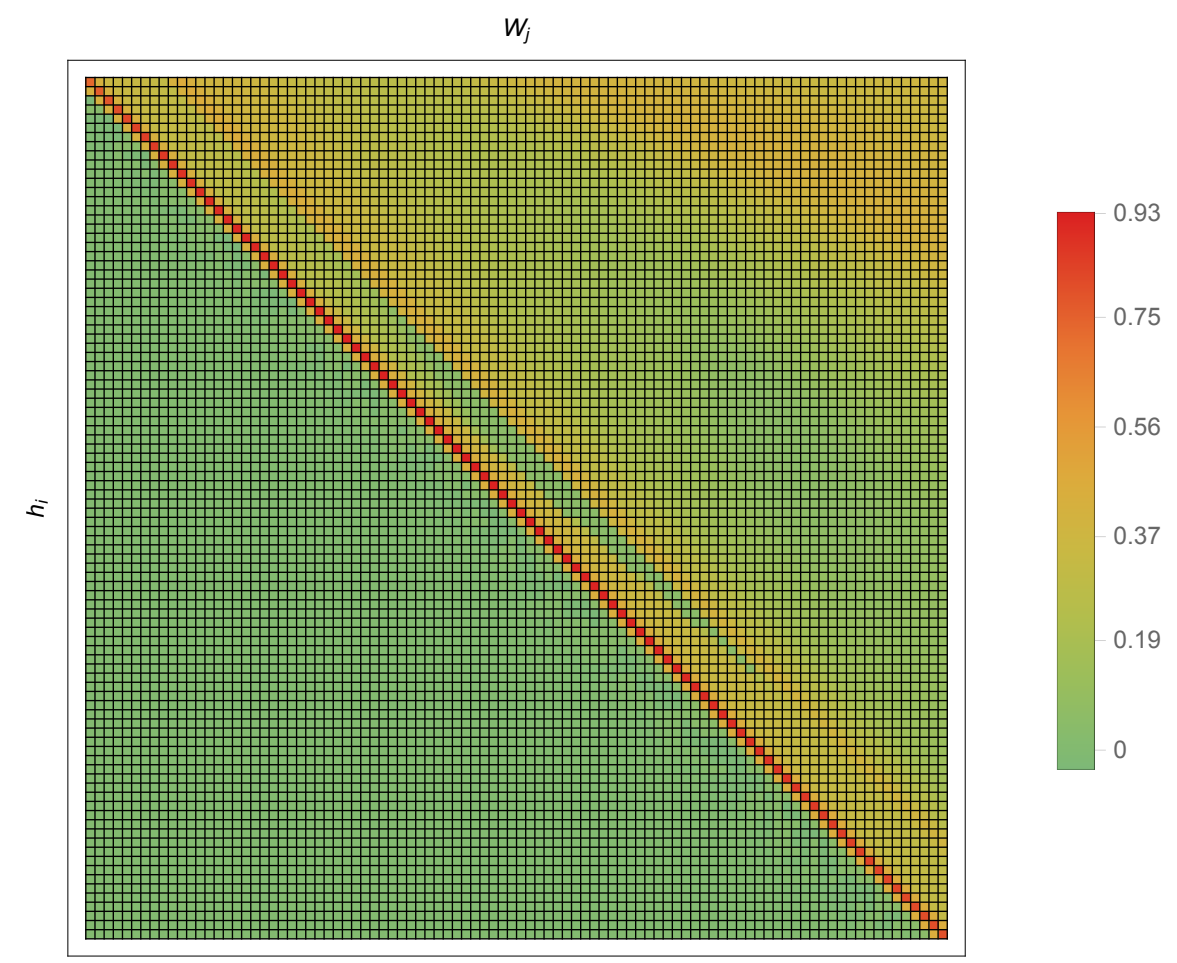

Figura 7.12: Representação da matriz resposta $\mathbf{R}$ do detetor colocado a $35^{\circ}$.

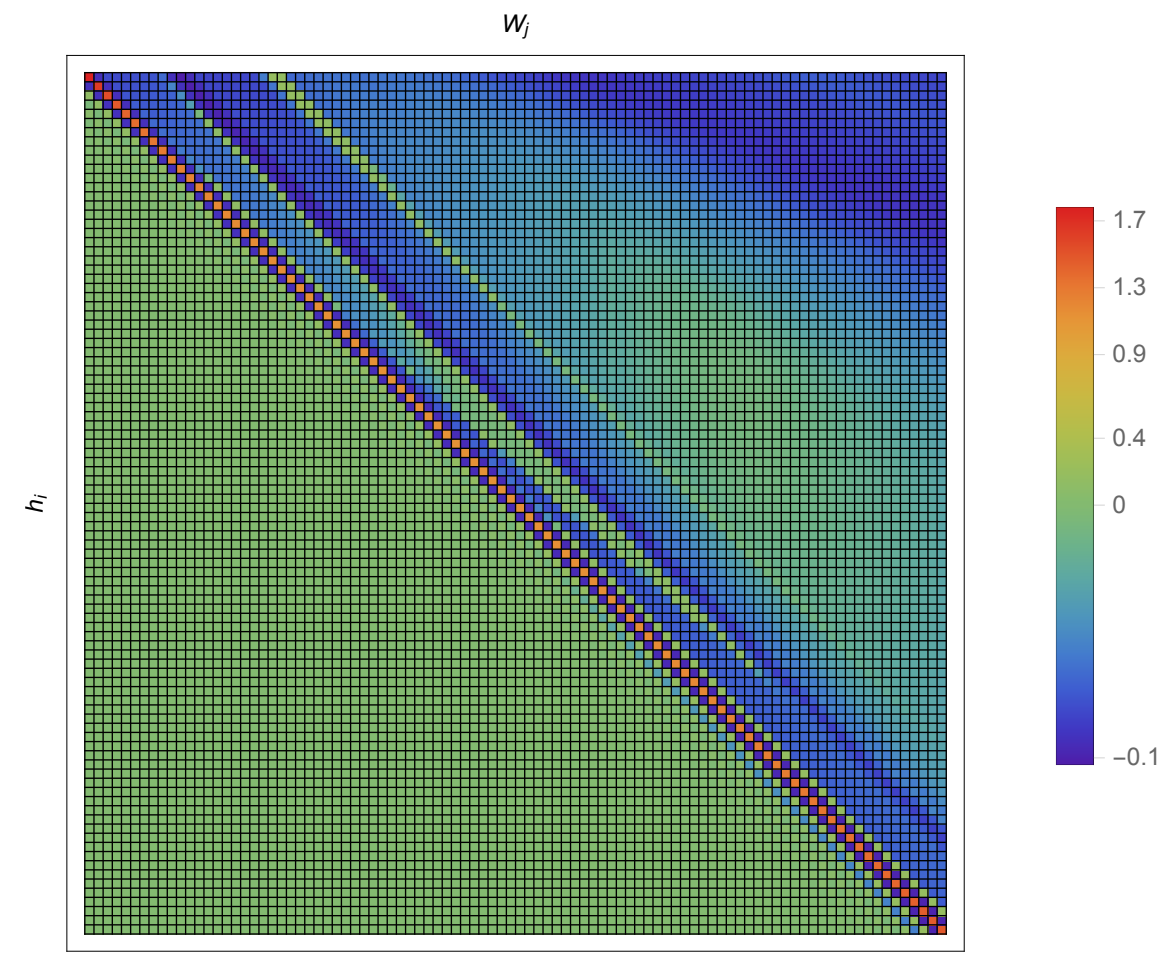

Figura 7.13: Representação da inversa da matriz resposta $\mathbf{R}^{-1}$ do detetor colocado a $35^{\circ}$. 
onde $T(W)$ é dado pela Eq. (4.15) e representa a fração de fótons transmitidos através dos materiais na frente do volume ativo do detector, e $\varepsilon_{\text {int }}(W)$ é a eficiência intrínseca de fotopico. Do ponto de vista matemático a Eq. (7.16) é equivalente a realizar a deconvolução considerando exclusivamente os elementos diagonais da matriz resposta do detetor. Nos gráficos inferiores da figura tem sido calculada a fração porcentual 1 $B^{\prime}(W) / B(W)$ que ilustra com maior clareza a diferença entre $B(W)$ e $B^{\prime}(W)$. Na região do tip $B^{\prime}(W)$ subestima o espectro real por não considerar as contagens registradas fora do fotopico. Quando diminui a energia acontece o efeito contrário respondendo ao fato de associar os pulsos de altura $h$ à energia $W=h$.
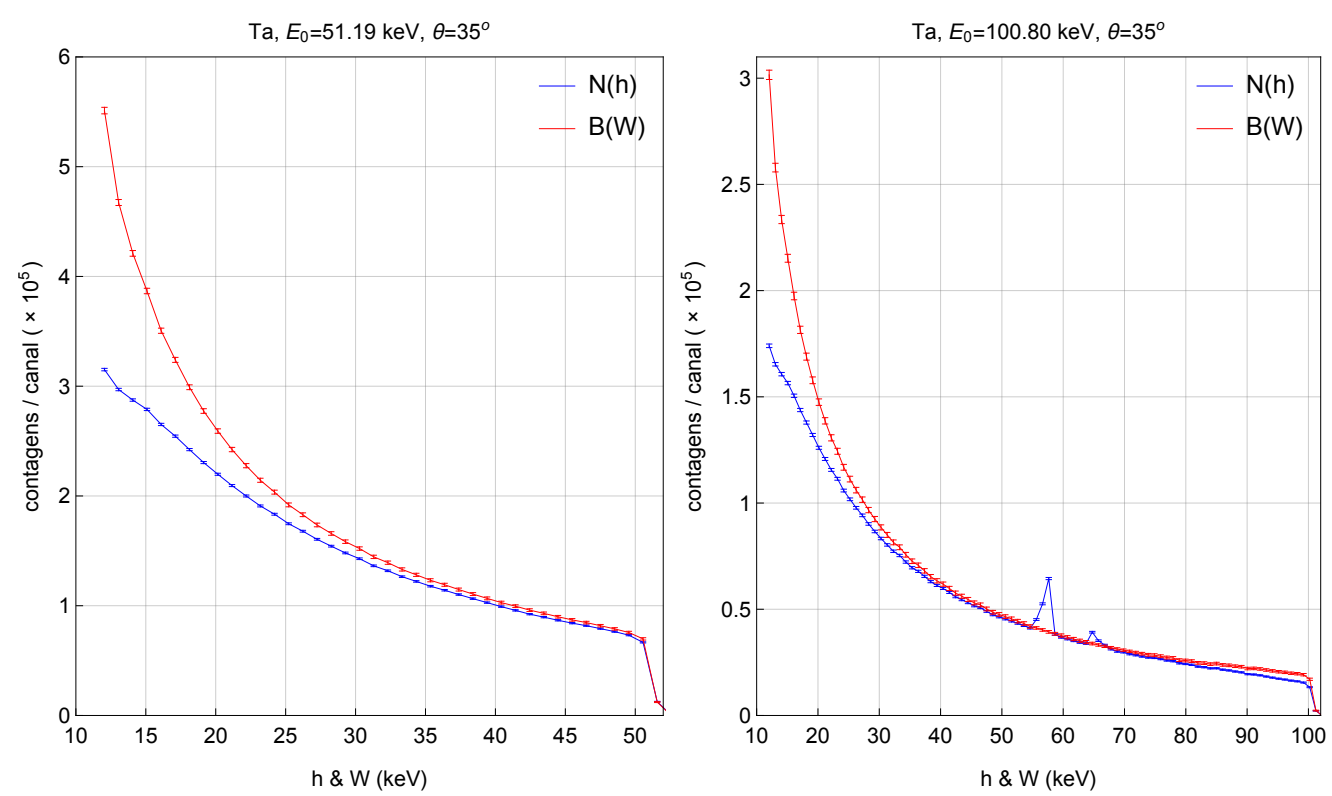

FigurA 7.14: Espectro medido $N(h)$ e deconvoluído $B(W)$, para o alvo de Ta a $35^{\circ}$ e as energias incidentes $E_{0}=51,19$ e $100,80 \mathrm{keV}$.

\subsection{Verificação cruzada: Ionização da camada K da Ag por impacto de elétrons}

A seção de choque de ionização da camada K (SCIK) por impacto de elétrons tem sido uma grandeza amplamente estudada nos últimos 80 anos [112]. Um dos elementos com mais experimentos encontrados na literatura é a Ag [113-121]. Alguns desses resultados são compatíveis entre eles e também, com a estimativa teórica da SCIK dada pela Aproximação de Born de Ondas Distorcidas (ABOD) [122]. Porem, como se indica no recente artigo de Vanin et al. [121], a grande maioria dos trabalhos experimentais não descrevem adequadamente os métodos aplicados e as SCIK apresentam grandes incertezas. Vanin e colaboradores mediram a SCIK da Ag no acelerador Microtron de São Paulo, para elétrons com energias incidentes entre 50 e $100 \mathrm{keV}$. Os autores utilizaram um método 

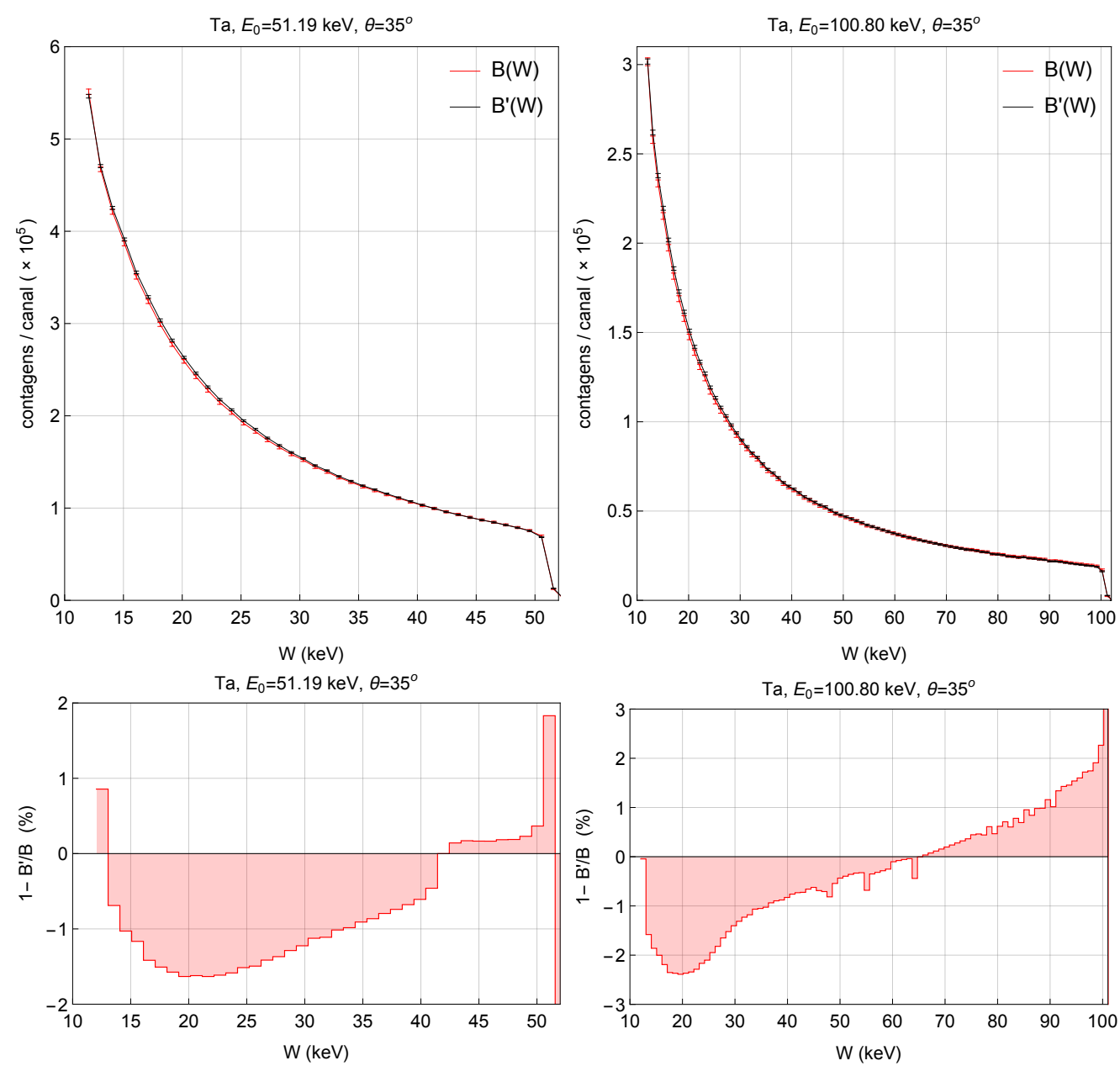

FiguRA 7.15: Comparação entre os espectros deconvoluído com a matriz resposta $\mathbf{R}$ $(B(W))$ e calculado com a eficiência intrínseca $\varepsilon_{\mathrm{i}}(W)\left(B^{\prime}(W)\right)$, para o mesmo alvo de

Ta da Fig. 7.14. Os gráficos inferiores mostram a diferença porcentual entre ambos.

relativo, ajustando a espessura do alvo a partir do tip dos espectros coletados, a FR do detetor usado e a SCDD teórica de emissão de bremsstrahlung [121]. As incertezas obtidas nesse experimento estão em torno de $12 \%$ e são devidas fundamentalmente ao cálculo da SCDD segundo as tabelas de Seltzer e Berger [3], e Kissel et al. [5]. Além disso, no artigo se expressa a necessidade de melhorar a precisão desses dados por meio de experimentos em que todas as grandezas envolvidas na SCIK sejam determinadas de maneira absoluta.

No contexto anterior, e considerando que a partir dos espectros de bremsstrahlung coletados para o alvo de Ag podem ser obtida as SCIKs para diferentes energias dos elétrons incidentes, ver Figs. 7.5-7.6, no presente trabalho são medidos esses dados. O objetivo fundamental é realizar uma verificação cruzada que permita avaliar a instalação experimental uma vez que os processos físicos de ionização e de emissão de bremsstrahlung são independentes, e que varias das grandezas envolvidas na determinação das SCIKs e SCDDs são comuns a ambos: a densidade superficial de átomos do alvo $t$, ângulo sólido 
$\Omega$ subtendido por cada detetor em relação ao alvo, e o número de elétrons incidentes $N_{0}$.

A seção de choque de produção de raios-x $\mathrm{K}_{i}, \sigma_{\mathrm{K} i}^{\mathrm{x}}\left(E_{0}\right)$, para a energia dos elétrons $E_{0}$ se determina segundo

$$
\sigma_{\mathrm{K} i}^{\mathrm{x}}\left(E_{0}\right)=\frac{N_{\mathrm{K} i}}{N_{0} t \varepsilon_{\mathrm{A}}\left(E_{\mathrm{K} i} ; \Omega\right)},
$$

onde $N_{\mathrm{K} i}$ é o número de contagens observadas no EAP devido às transições radiativas $\mathrm{K} i$ no elemento analisado, e $\varepsilon_{\mathrm{A}}\left(E_{\mathrm{x}} ; \Omega\right)$ é a eficiência absoluta de fotopico, dada pela Eq. (6.13), avaliada na energia $E_{\mathrm{K} i}$ da transição. Note-se que $\sigma_{\mathrm{K} i}^{\mathrm{x}}\left(E_{0}\right)$ foi escrita de maneira compacta na Eq. (7.17), já que o fator $\Omega / 4 \pi$, que representa a fração de raios-x $\mathrm{K}$ emitidos na direção do detetor, está intrinsecamente contido em $\varepsilon_{\mathrm{A}}\left(E_{\mathrm{x}} ; \Omega\right)$, visto que a distribuição angular desse fenômeno é isotrópica. A SICK, $\sigma_{\mathrm{K}}$, relaciona-se com $\sigma_{\mathrm{K} i}^{\mathrm{x}}$ por meio de

$$
\sigma_{\mathrm{K}}\left(E_{0}\right)=\left(\frac{\Gamma_{\mathrm{K} i}}{\Gamma_{\mathrm{K}, \mathrm{tot}}} \omega_{\mathrm{K}}\right)^{-1} \sigma_{\mathrm{K} i}^{\mathrm{x}}\left(E_{0}\right)
$$

sendo $\omega_{\mathrm{K}}$ o rendimento fluorescente, e $\Gamma_{\mathrm{K} i} / \Gamma_{\mathrm{K}, \text { tot }}$ a razão entre a taxa de emissão de raios-x $\mathrm{K}_{i}$ e a taxa de emissão total.

Devido a sua maior intensidade a linha $\mathrm{K} \alpha$ da $\mathrm{Ag}$ foi selecionada para determinar $\sigma_{\mathrm{K}}$. Os métodos para a obtenção das grandezas presentes no denominador da Eq. (7.17) tem sido discutidos ao longo deste trabalho. Por outra parte, a área $N_{\mathrm{K} \alpha}$ do pico $\mathrm{K} \alpha$ foi ajustada considerando um modelo composto por duas funções Gaussianas, para descrever as contribuições das transições $\mathrm{K} \alpha_{1}$ e $\mathrm{K} \alpha_{2}$, e uma função $Y(h)$ que considera o contínuo de bremsstrahlung observado no EAP segundo:

$$
Y(h)=\frac{1}{h}(a+b h) .
$$

O algoritmo NonLinearModelFit do software Mathematica [87] foi utilizado para ajustar os parâmetros de amplitude, os desvios padrões e um dos centroides das funções Gaussianas (o outro foi fixado pela diferença energética $\mathrm{K} \alpha_{1}-\mathrm{K} \alpha_{2}$ ), bem como $a$ e $b$ em $Y(h)$. A forma funcional proposta para $Y(h)$ constitui uma aproximação simples baseada na Eq. (2.76) que caracteriza a SCDD de emissão de bremsstrahlung. Os dados de rendimento fluorescente e as probabilidades de emissão $\Gamma_{\mathrm{K} \alpha}$ e $\Gamma_{\mathrm{K}, \text { tot }}$, foram tomados 
dos trabalhos de Krause [123] e Scofield [124], respectivamente. Como no artigo de Vanin e colaboradores [121], assumiram-se incertezas relativas de $2 \%$ para $\omega_{\mathrm{K}}$ e a razão $\Gamma_{\mathrm{K} \alpha} / \Gamma_{\mathrm{K}, \mathrm{tot}}$. 



\section{Capítulo 8}

\section{Resultados das SCDDs de emissão de bremsstrahlung de elétrons}

Neste capítulo se apresentam os resultados das medidas de SCDDs para todos os elementos estudados, energias e ângulos, e se realiza uma comparação com os cálculos teóricos de maior precisão. Discutem-se as tendências observadas para cada elemento em quanto a valor absoluto e forma funcional das SCDDs experimentais em relação à teoria. De maneira complementar também são mostrados os resultados obtidos para a SICK da Ag, correspondentes ao experimento cruzado.

\subsection{Estrutura de apresentação dos resultados}

Com a deconvolução dos espectros de bremsstrahlung, foram determinadas todas as grandezas necessárias para avaliar absolutamente a SCDD segundo a Eq. (2.77). A continuação são apresentadas as SCDDs obtidas para cada elemento, acompanhados da comparação com os correspondentes resultados teóricos. Para esse fim, foram interpolados os dados de seção de choque reduzida $\chi\left(Z, E_{0}, \kappa\right)$ e de distribuição angular $S\left(Z, E_{0}, \kappa, \theta\right)$, tabulados por Seltzer e Berger [3] e Kissel et al. [5], respectivamente (ver Apêndice B), e calculada a SCDD teórica conforme a Eq. (2.76). De acordo com os autores espera-se que a precisão desse procedimento seja em torno de $11 \%$.

As incertezas relativas nas SCDDs medidas neste trabalho estão entre 3\% e 5\%, com a seguinte contribuição: a) $1 \%$ devido à estatística de contagem e ao procedimento de deconvolução do espectro $B(W), b) 1 \%$ associado à coleção da carga, a partir da qual 
se cacula $\left.N_{0}, c\right)<1 \%$ devido à determinação do ângulo sólido $\Omega$, e $d$ ) entre $2 \%$ e $4 \%$ na determinação da densidade superficial de átomos dos alvos.

\subsection{Resultados da SCDD de emissão de bremsstrahlung no ponto de máxima transferência de energia e da SCIK da Ag, discussão}

As Tabelas 8.1 e 8.2 resumem os valores de energia $E_{0}$ e dispersão do feixe $\sigma_{0}$ ajustados a partir do tip de bremsstrahlung, em cada irradiação, para cada elemento, bem como as SCDDs no ponto $\kappa=1$ e nos três ângulos de medida. Além disso, são calculadas as SCDDs teóricas no valor $E_{0}$. Este primeiro estudo permite examinar a variação da SCDD em função de $E_{0}$, quando são fixados o ângulo e a energia de emissão dos fótons. No caso dos elementos $\mathrm{Ag}$ e $\mathrm{Au}$, o número de medidas realizadas foi maior, tomando 10 y 11 pontos entre 20 e $100 \mathrm{keV}$, respectivamente.

Os valores de largura energética $\sigma_{0}$ do feixe de elétrons representam uma fração menor que $1 \%$ em relação à média ajustada $E_{0}$. A variação experimentada pela SCDD teórica por uma mudança dessa ordem em $E_{0}$ é muito menor que a incerteza obtida nos dados experimentais da mesma grandeza. As incertezas associadas ao procedimento de ajuste dos parâmetros $E_{0}$ e $\sigma_{0}$ estão no intervalo compreendido entre $0,04 \mathrm{e} 0,07 \mathrm{keV}$.

O gráficos das Figs. 8.1-8.3 apresentam o comportamento dos resultados experimentais e teóricos, incluindo nestes últimos a faixa de $\pm 11 \%$ atribuída ao procedimento de interpolação das tabelas de Seltzer e Berger [3] e Kissel et al. [5]. Observa-se que no caso do $\mathrm{Al}$ e $\mathrm{Au}$, elementos para os quais os cálculos de ondas parciais foram desenvolvidos, a maioria dos pontos experimentais contêm o valor teórico dentro de um desvio padrão. As SCDDs do C encontram-se dentro do intervalo de $\pm 11 \%$ previsto pela teoria, mas sistematicamente acima, enquanto as SCDDs do Te e do Ta possuem um comportamento similar, nesse caso sistematicamente abaixo da teoria. Para estes três últimos elementos as SCDDs teóricas são totalmente interpoladas em número atômico $Z$, além das outras variáveis: $E_{0}, \kappa$ e $\theta$.

Uma analise separada é necessária para as SCDDs da Ag, percebe-se que se encontram sistematicamente abaixo da teoria para todos os ângulos e energias, em um fator que oscila entre 20 e $30 \%$ (ver Tabela 8.2). Assim como para o Al e o Au, os cálculos de ondas parciais de SCDDs foram também realizados para Ag [3]. Logo, resulta chamativo a grande discrepância obtida entre os resultados experimentais e teóricos, especialmente quando as SCDDs dos outros elementos medidos estão dentro da margem esperada. Uma primeira explicação baseia-se na hipótese de haver cometido um erro sistemático 
(superestimação) na determinação da densidade superficial de átomos $t$, que neste caso, sendo uma característica intrínseca do alvo, explicaria as discrepâncias observadas para todos os valores de $E_{0}$ estudados.

A fim de discutir com mais argumentos o comportamento das SCDDs de emissão de bremsstrahlung em Ag e $\kappa=1$, na Tabela 8.3 são apresentados os resultados das medidas da SCIK. Esses dados foram obtidos a partir dos mesmos EAP utilizados para determinar as SCDDs da Tabela 8.2. Além das SCIKs derivadas das medidas com cada detetor, é calculada a média, a estimativa fornecida pela ABOD [122], e a razão R experimento/teoria. Nas Figs. 8.4-8.5 são comparados os resultados do presente experimento com as SCIKs apresentadas por Vanin e colaboradores [121] e com todos os dados encontrados na literatura para a faixa de energia estudada [113-121].

Constata-se que para todas os valores de $E_{0}$ os resultados das SCIKs deduzidas dos espectros coletados nos três ângulos são totalmente compatíveis entre eles. O fato de obter, para energias entre 70 e $100 \mathrm{keV}$, dados também compatíveis com os resultados anteriores de Vanin et al. [121] e com o cálculo da ABOD [122], confirma que a densidade superficial do alvo foi corretamente determinada. Por outra parte, os pontos em 51,09 e $60,86 \mathrm{keV}$, seguem a tendência observada por Vanin e colaboradores, mostrando que a SCIK experimental é menor que a estimativa fornecida pela ABOD nessa região. Entre o limiar da ionização e os $\sim 40 \mathrm{keV}$, observa-se um comportamento similar ao mostrado por outros experimentos em que os resultados estão abaixo da curva da ABOD.

Os resultados deste experimento cruzado permitem descartar a hipótese de superestimação da espessura do alvo de Ag, e ao mesmo tempo, validam os procedimentos experimentais seguidos como: a coleção da carga, a determinação do ângulo sólido e eficiência de cada detetor, etc. Na literatura não foram encontradas medidas de SCDDs explicitamente para $\kappa=1$ e nenhum dos elementos analisados neste trabalho. 


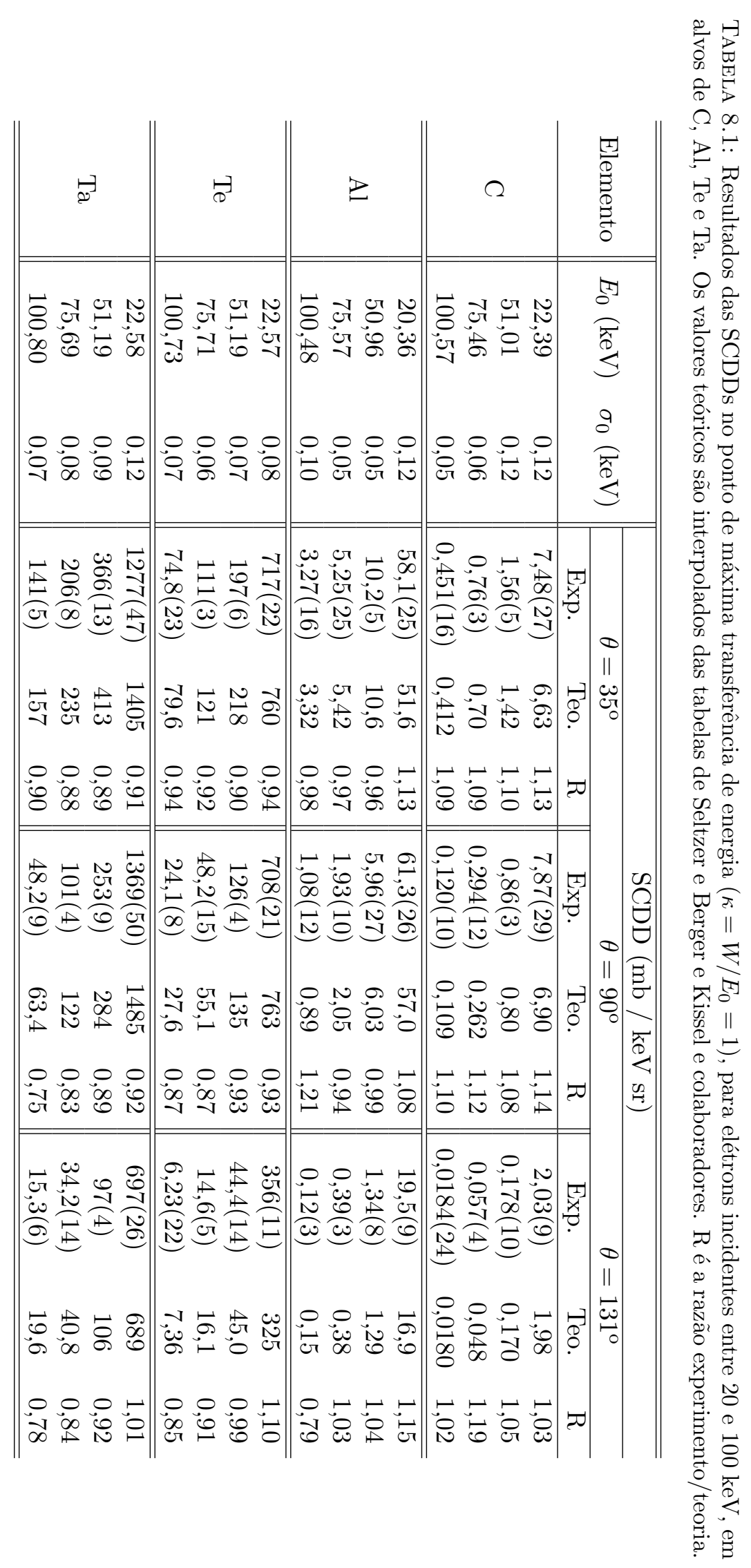




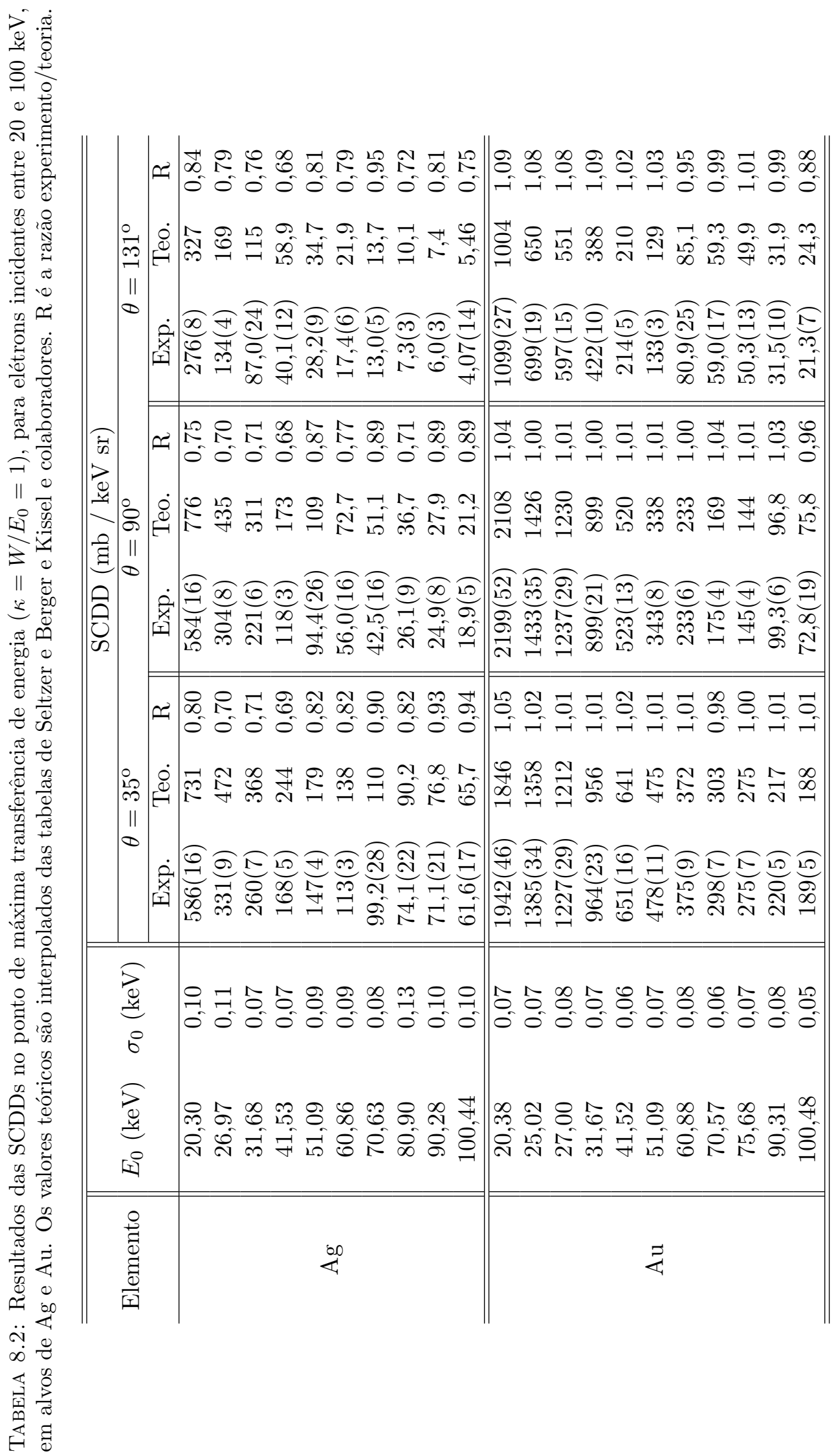



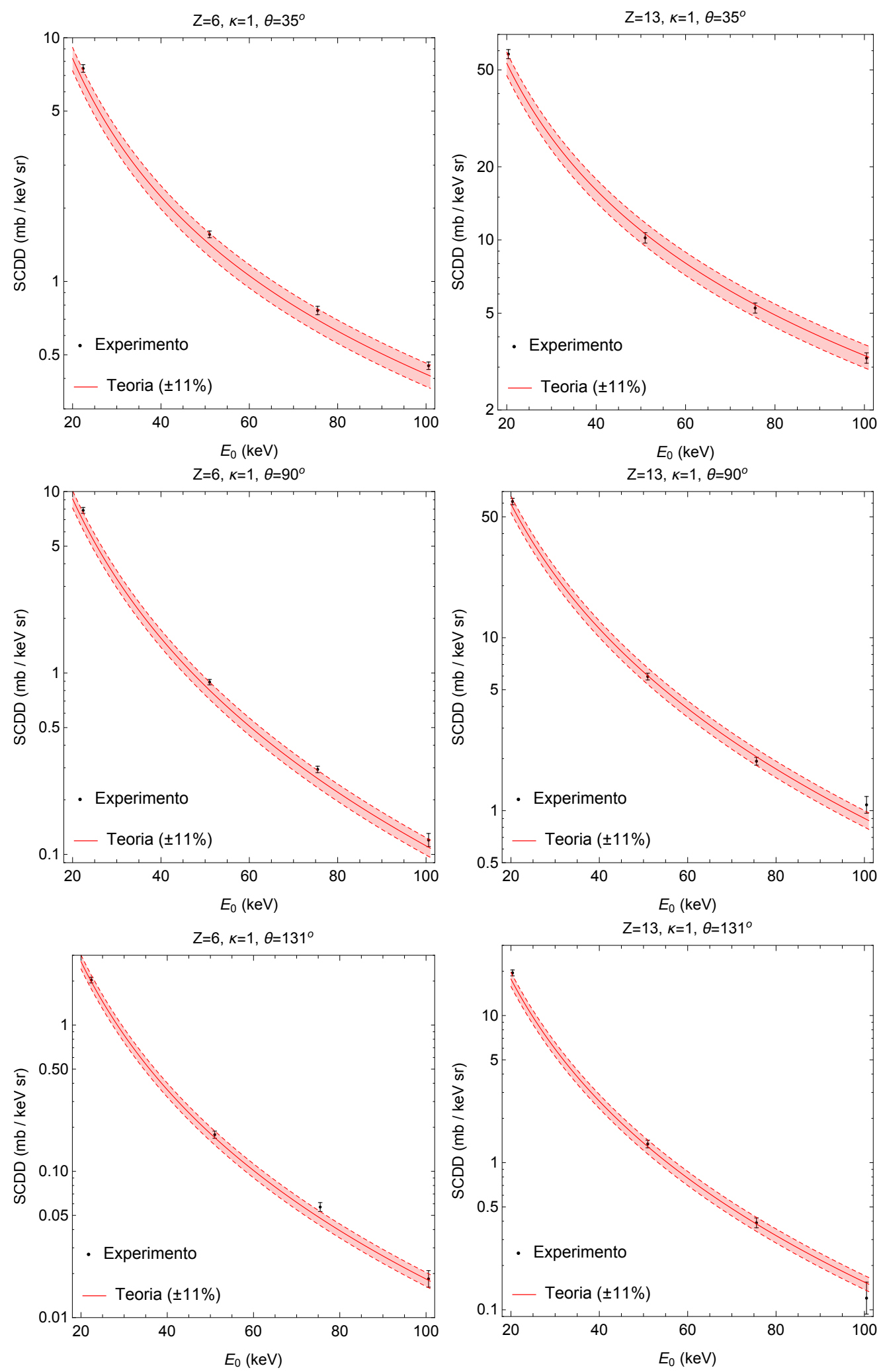

Figura 8.1: Comparação entre a SCDD experimental e teórica no ponto $\kappa=1$, para os elementos $\mathrm{C}$ (coluna esquerda) e $\mathrm{Al}$ (coluna direita). 

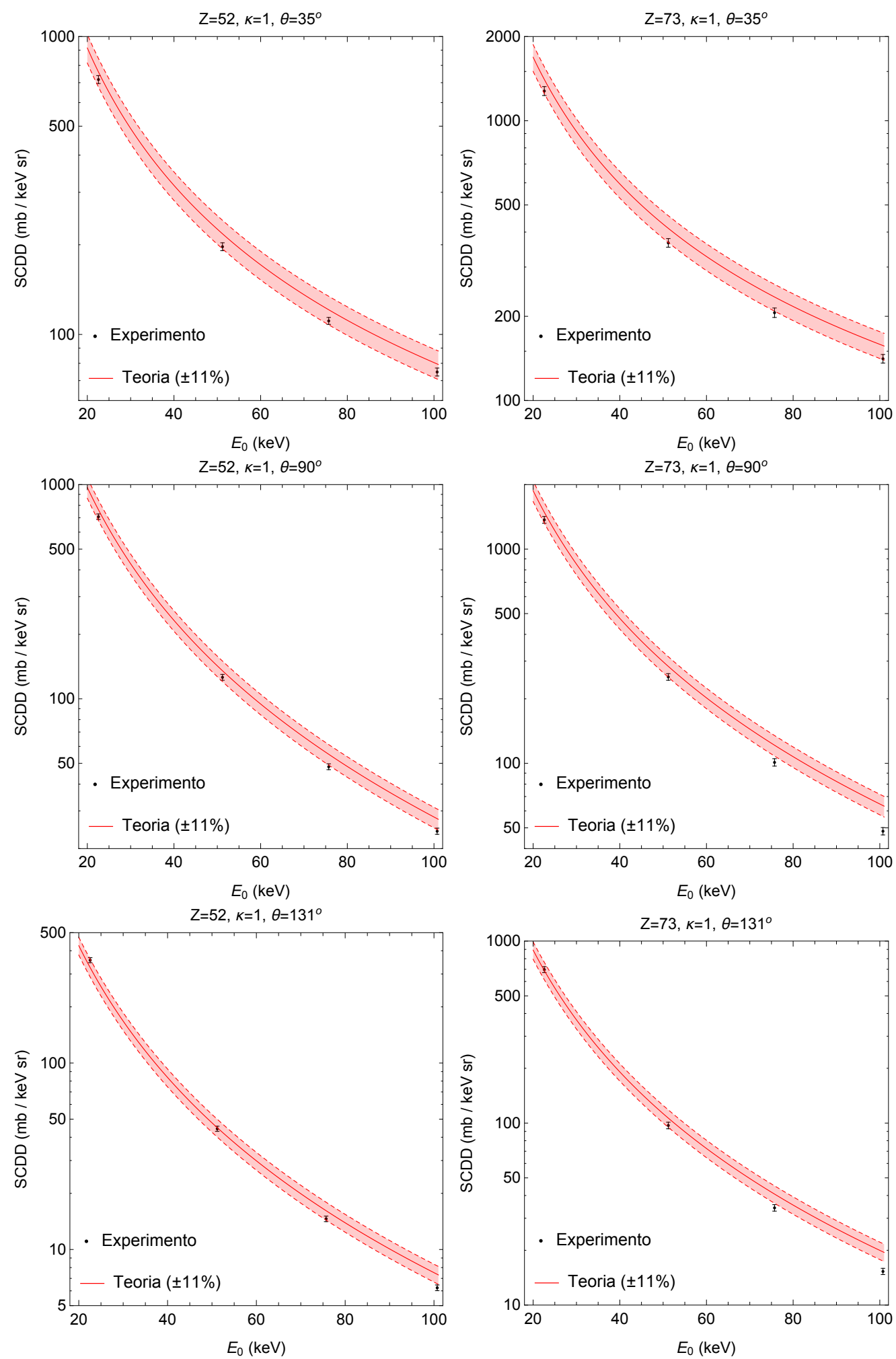

Figura 8.2: Comparação entre a SCDD experimental e teórica no ponto $\kappa=1$, para os elementos Te (coluna esquerda) e Ta (coluna direita). 

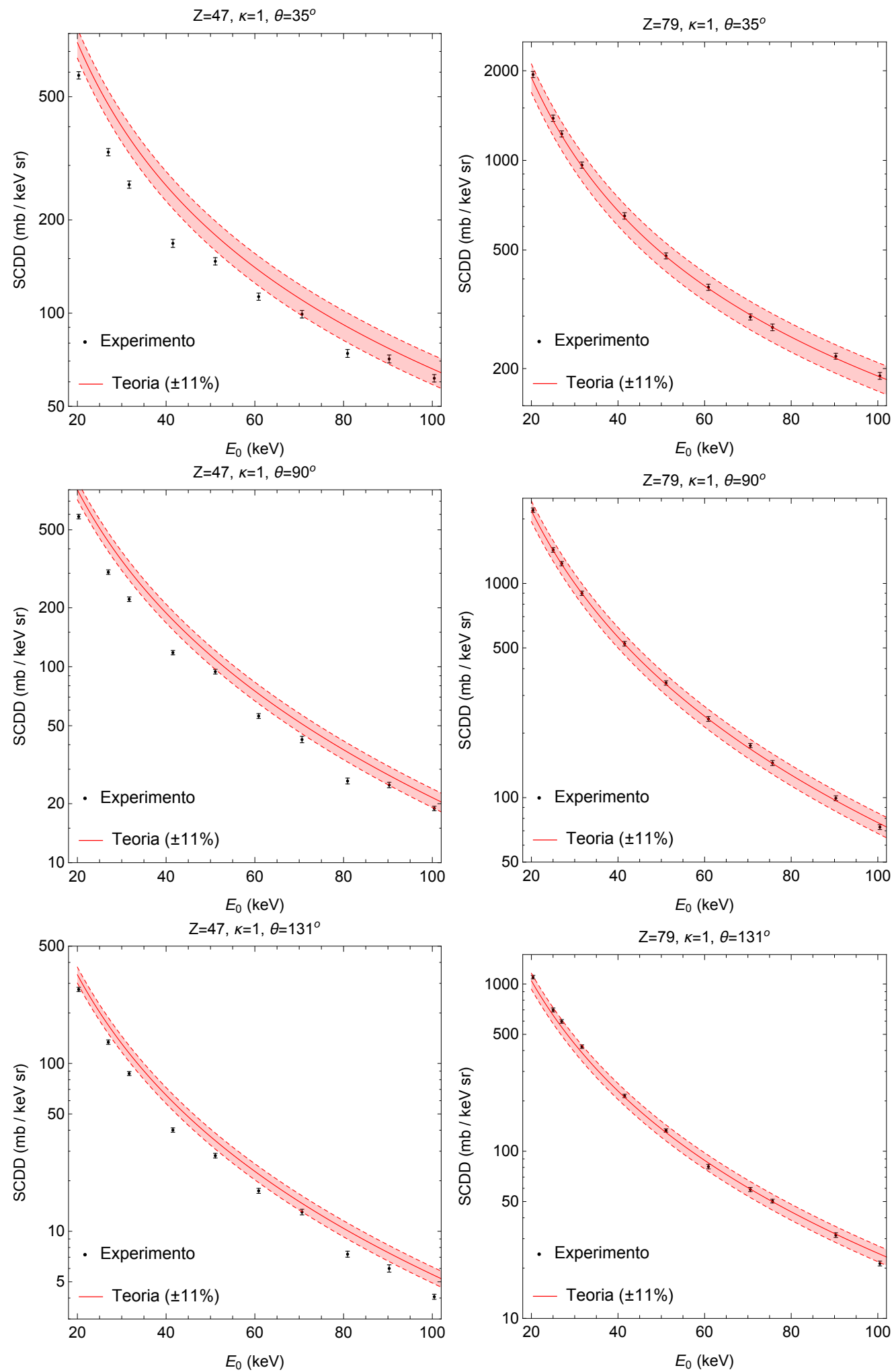

Figura 8.3: Comparação entre a SCDD experimental e teórica no ponto $\kappa=1$, para os elementos Ag (coluna esquerda) e Au (coluna direita). 
TABELA 8.3: Resultados das SCIKs da Ag obtidos em cada ângulo $\theta$ de medida, valor médio experimental e estimativa teórica segundo a ABOD. $\mathrm{R}$ é a razão entre a média experimental e a SCIK teórica.

\begin{tabular}{c||cccccc}
\hline \hline \multirow{2}{*}{$E_{0}(\mathrm{keV})$} & \multicolumn{5}{c}{$\sigma_{\mathrm{K}}(\mathrm{b})$} \\
\cline { 2 - 7 } & $\theta=35^{\mathrm{O}}$ & $\theta=90^{\circ}$ & $\theta=131^{\circ}$ & Média & ABOD & $\mathrm{R}$ \\
\hline 26,97 & $5,28(21)$ & $5,18(23)$ & $5,32(26)$ & $5,26(16)$ & 6,45 & 0,82 \\
31,68 & $18,0(7)$ & $17,7(8)$ & $18,2(7)$ & $18,0(5)$ & 22,2 & 0,81 \\
41,53 & $29,9(12)$ & $29,5(13)$ & $29,7(12)$ & $29,7(10)$ & 40,2 & 0,74 \\
51,09 & $44,5(18)$ & $44,2(20)$ & $44,1(18)$ & $44,3(14)$ & 48,8 & 0,91 \\
60,86 & $48,5(19)$ & $48,0(21)$ & $47,7(19)$ & $48,0(17)$ & 53,4 & 0,90 \\
70,63 & $56,9(23)$ & $56,0(25)$ & $55,7(22)$ & $56,2(21)$ & 55,9 & 1,01 \\
80,96 & $58,4(23)$ & $57,7(28)$ & $57,2(23)$ & $57,8(21)$ & 57,2 & 1,01 \\
90,28 & $59,4(24)$ & $58,4(26)$ & $58,5(23)$ & $58,8(21)$ & 57,7 & 1,02 \\
100,44 & $60,1(24)$ & $59,3(26)$ & $59,0(24)$ & $59,4(21)$ & 57,9 & 1,03 \\
\hline \hline
\end{tabular}

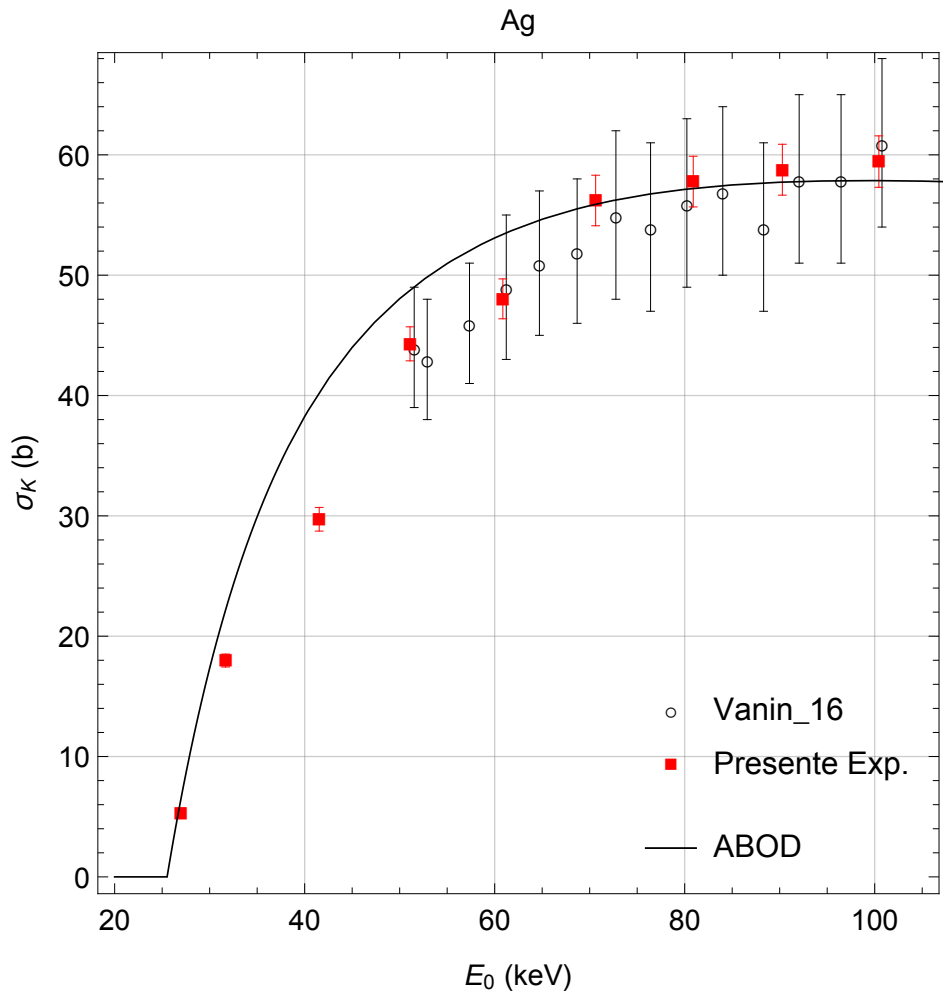

Figura 8.4: Comparação entre as SCIKs em Ag obtidas no presente experimento, os resultados de Vanin e colaboradores e o cálculo segundo a ABOD. 


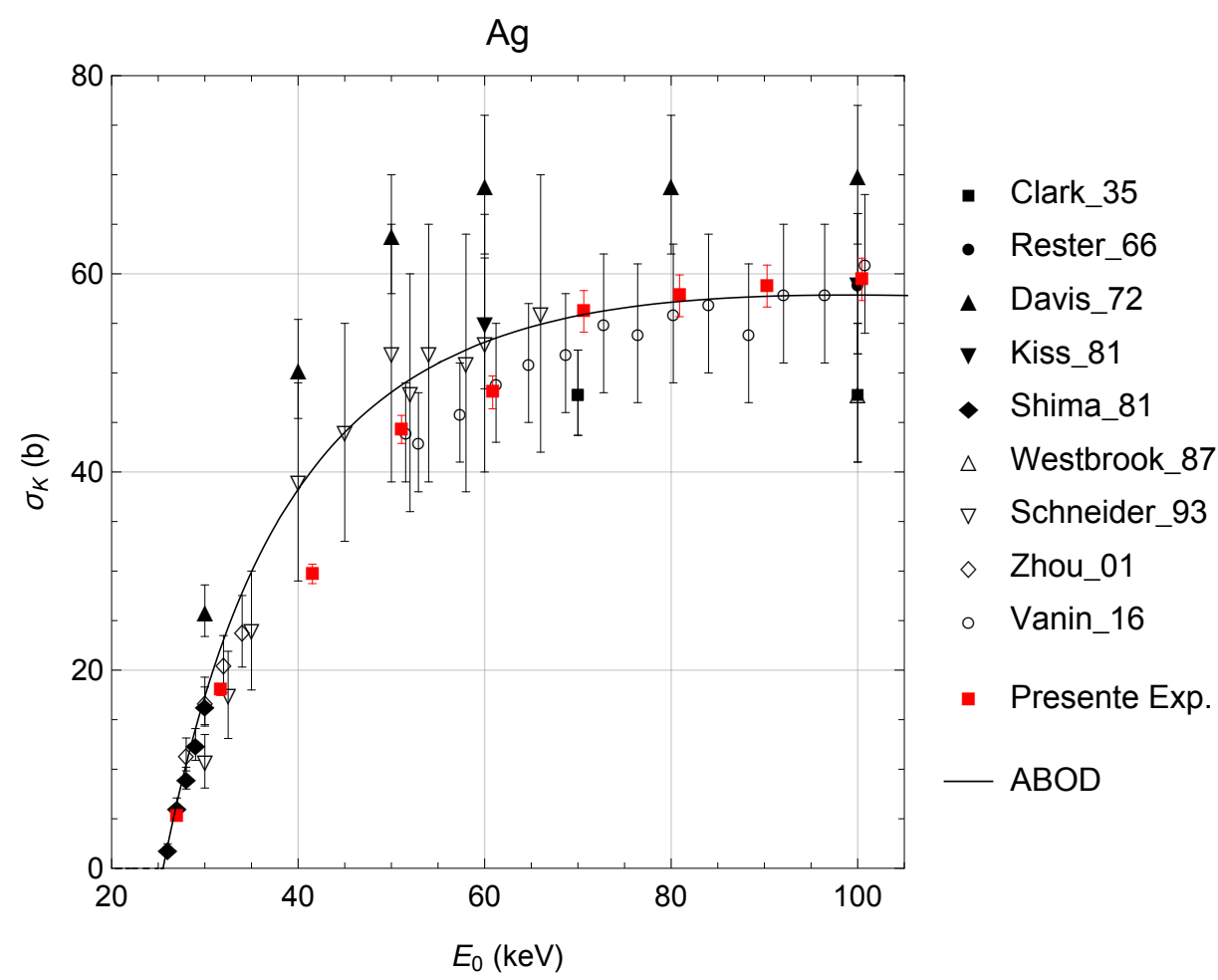

Figura 8.5: Comparação entre as SCIKs em Ag obtidas no presente experimento, os dados experimentais encontrados na literatura e o cálculo segundo a ABOD.

\subsection{Resultados da SCDD de emissão de bremsstrahlung para fótons de diferentes energias, discussão}

A continuação são apresentados para cada elemento, ângulo e energia dos elétrons incidentes o comportamento das SCDD de emissão de bremsstrahlung, em função da energia reduzida $\kappa$ do fóton. Cada gráfico está acompanhado pela razão entre as SCDDs experimental e teórica.

\subsubsection{Carbono $Z=6$}

Os gráficos das Figs. 8.6-8.10 mostram os resultados obtidos para o C. Observa-se que para as quatro energias dos elétrons incidentes, e os três ângulos, as SCDDs estão acima dos valores teóricos em torno de $20 \%$, e para $\kappa \leqslant 0,4$, a diferença ascende até $40 \%$. Só para $E_{0}=100,57 \mathrm{keV}$ e $\theta=90^{\circ}$ e $131^{\circ}$ o comportamento registrado é diferente, onde a relação experimento/teoria passa de maneira monótona, de 1,1 em $\kappa=1 \mathrm{a} \sim 1,6 \mathrm{em}$ $\kappa \approx 0,12$. Em geral, na região do tip se alcança a melhor concordância em todos os casos. A razão experimento/teoria aproximadamente constante evidencia que a forma funcional das SCDDs é similar para a maioria dos valores de $\kappa$. 

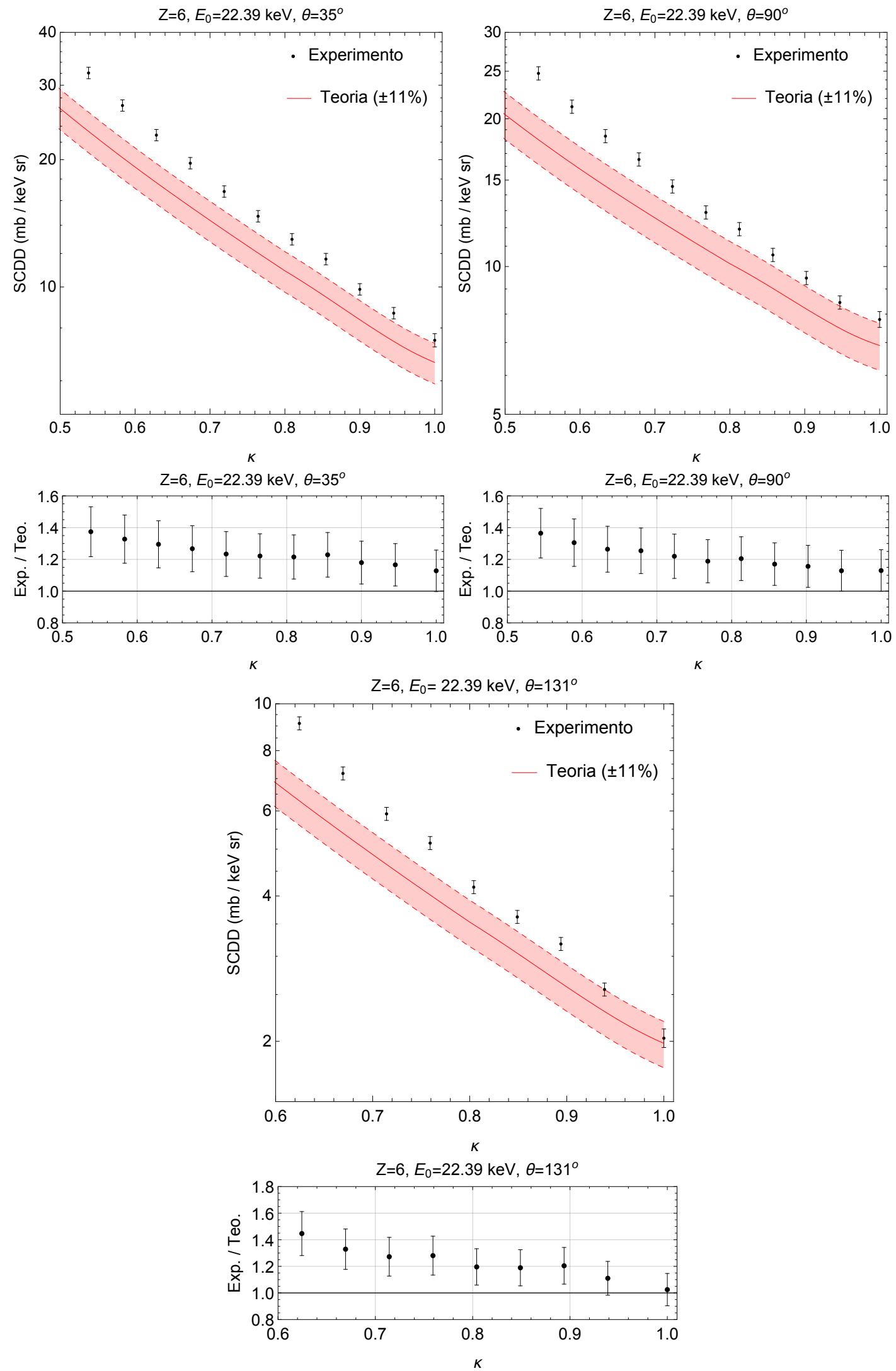

Figura 8.6: Resultados das SCDDs de emissão de bremsstrahlung em C para elétrons incidentes de $22,39 \mathrm{keV}$. 

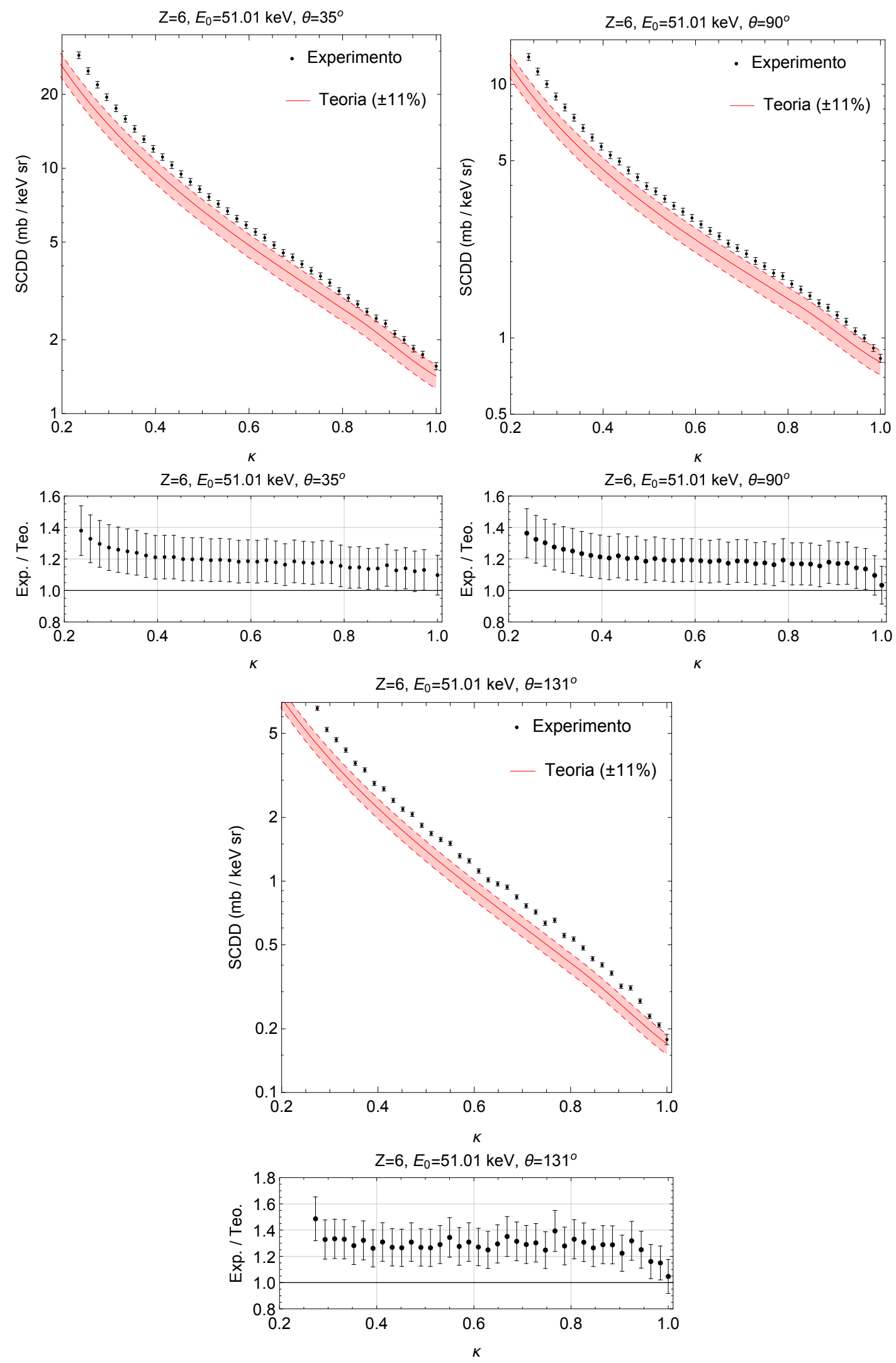

Figura 8.7: Resultados das SCDDs de emissão de bremsstrahlung em C para elétrons incidentes de $51,01 \mathrm{keV}$. 

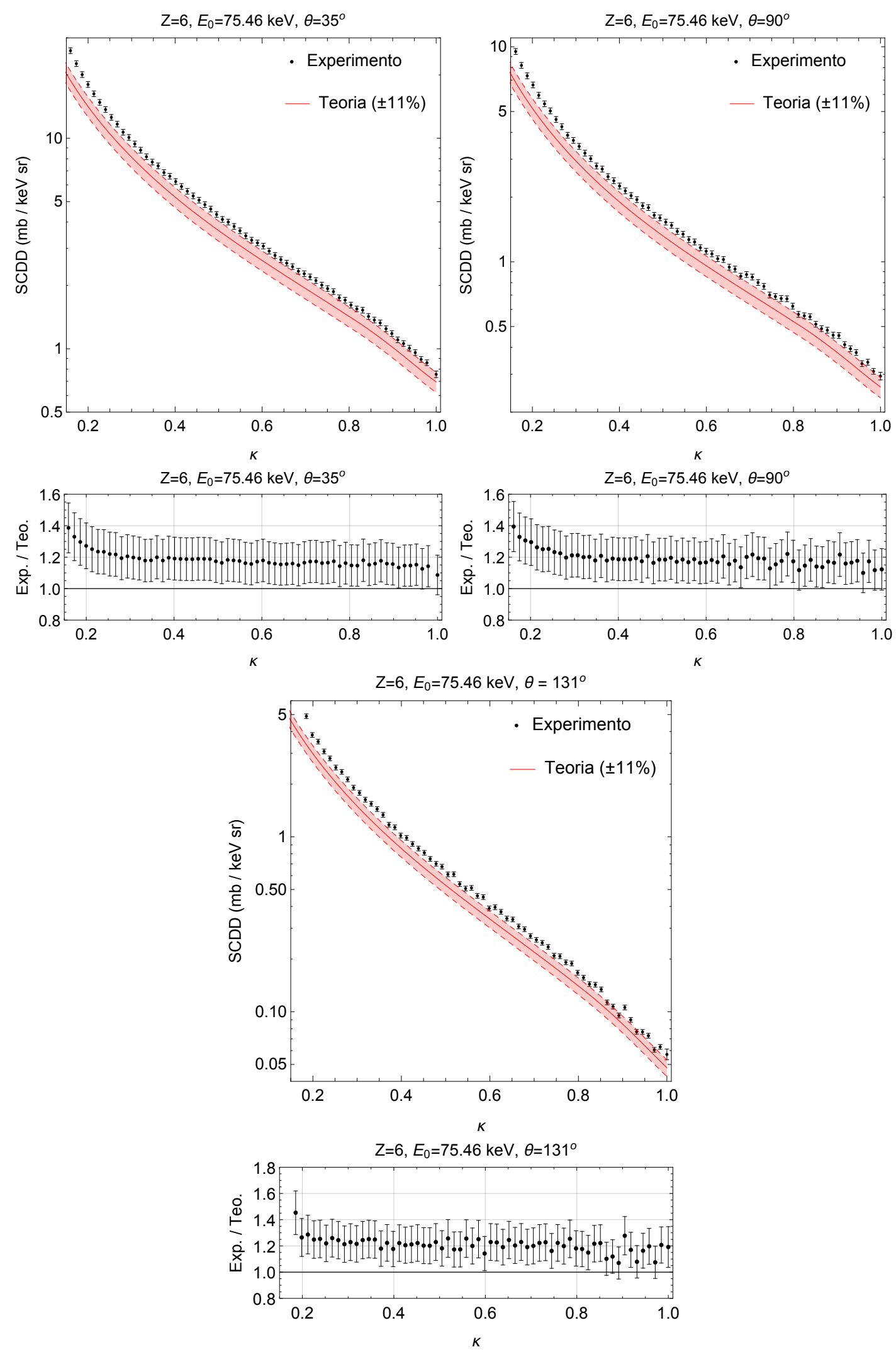

Figura 8.8: Resultados das SCDDs de emissão de bremsstrahlung em C para elétrons incidentes de $75,46 \mathrm{keV}$. 

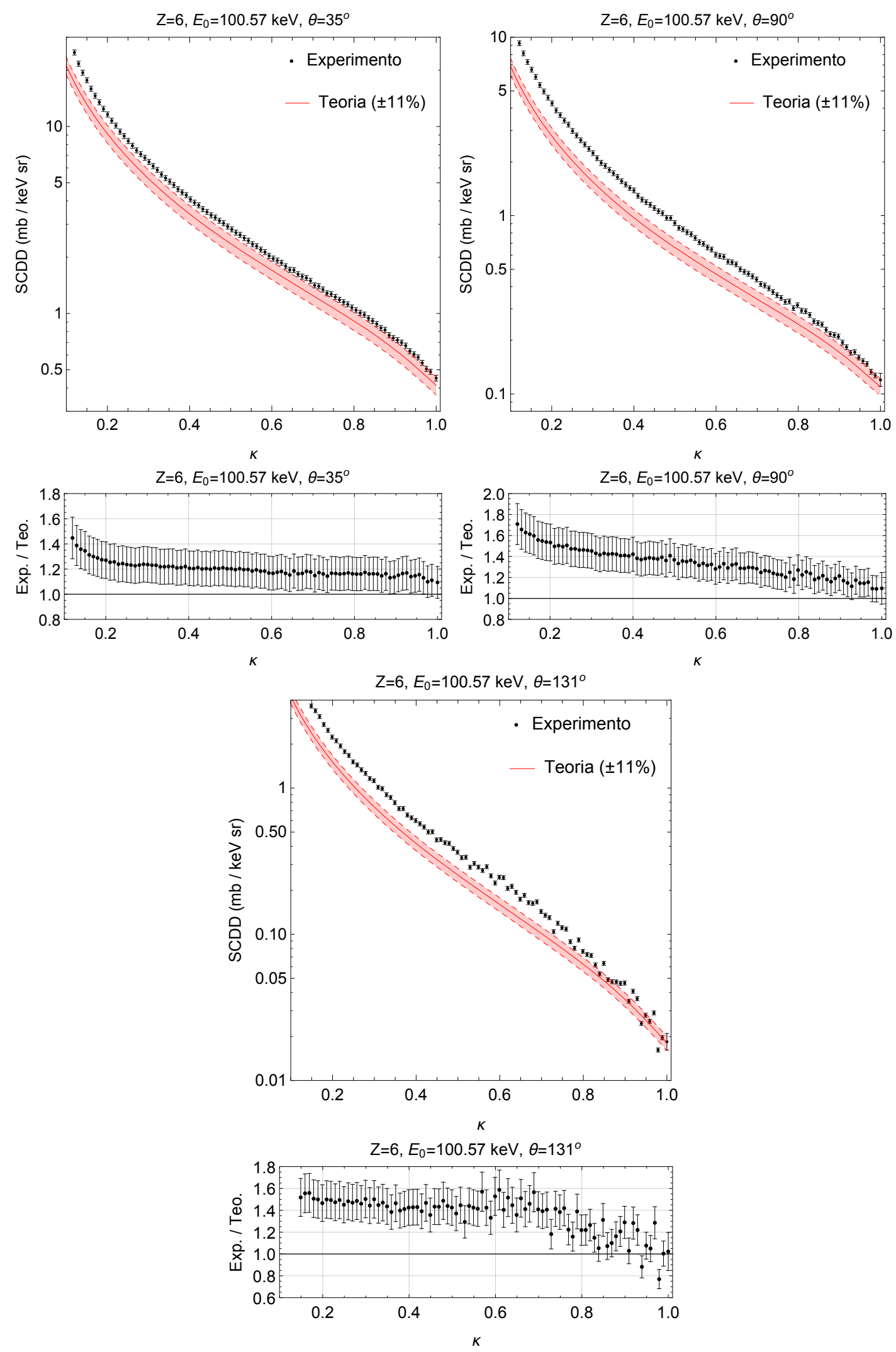

Figura 8.9: Resultados das SCDDs de emissão de bremsstrahlung em C para elétrons incidentes de 100,57 keV. 

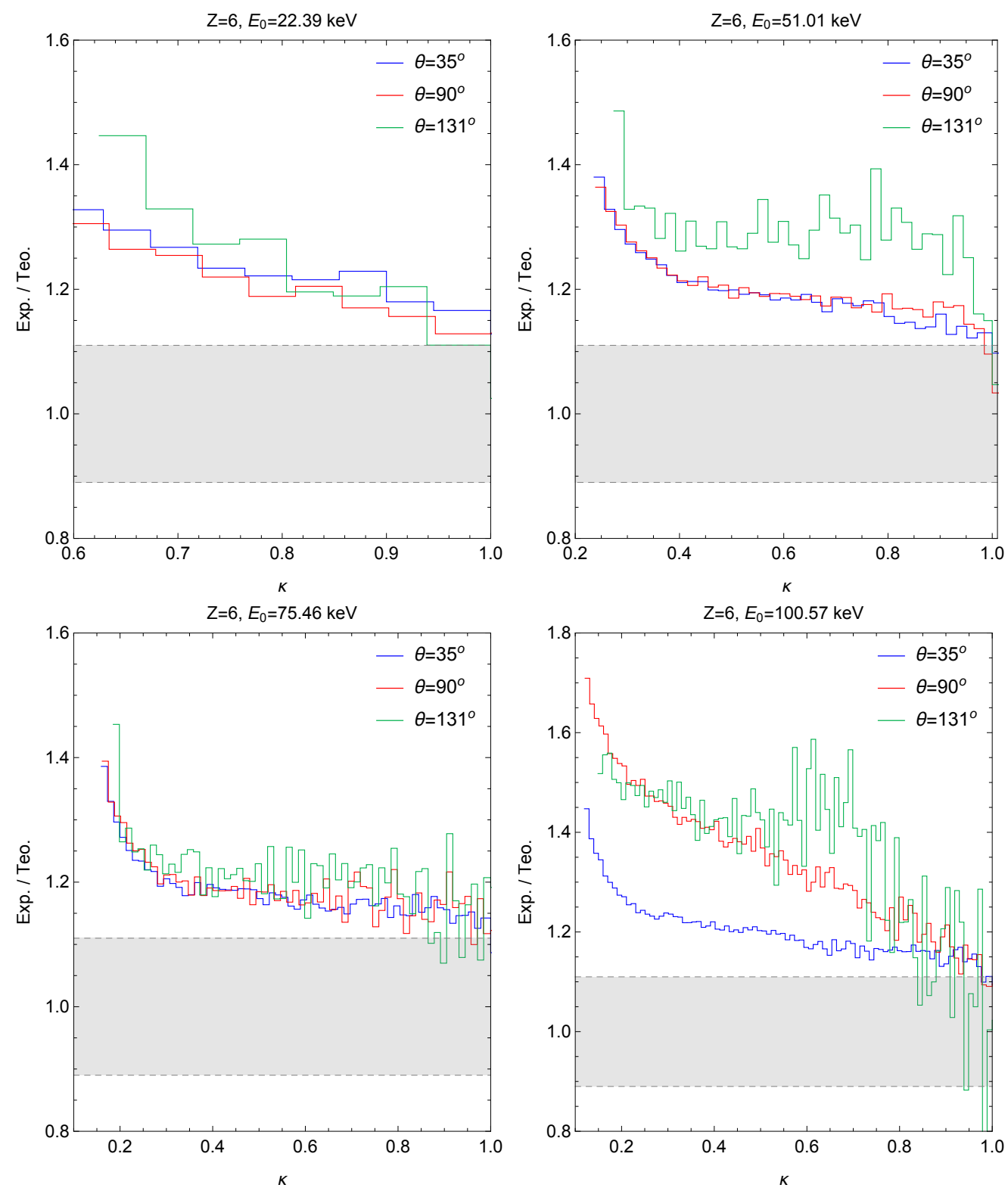

FIgURA 8.10: Razão experimento/teoria entre as SCDDs obtidas para o C, considerando todas energias e ângulos medidos. A faixa cinza indica o intervalo de $\pm 11 \%$. 


\subsubsection{Alumínio $Z=13$}

As Figs. 8.11-8.15 mostram os resultados do Al. Para $E_{0}=20,36 \mathrm{keV}$ e os três ângulos, as SCDDs medidas estão acima dos valores teóricos aproximadamente um 20\%, sendo menor a diferença no tip.

Por outro lado, ao aumentar a energia incidente a $E_{0}=50,96 \mathrm{keV}$, as SCDDs experimentais apresentam um comportamento oscilatório em torno da teoria; todos os valores de $\kappa$ estudados estão dentro da faixa de $\pm 11 \%$, e para $\theta=90^{\circ}$ os desvios são menores de $5 \%$. Para $E_{0}=75,57$ e 100,48 $\mathrm{keV}$ se obteve uma forma espectral similar. As variações observadas nas SCDDs para essas duas energias em $\theta=131^{\circ}$ e $\kappa \gtrsim 0,6$, devem-se à menor estatística de contagem nos espectros coletados, em relação a $\theta=35^{\circ}$ ou $90^{\circ}$ (ver Fig. 7.6).

De igual maneira que no caso do $\mathrm{C}$, a razão experimento/teoria aproximadamente constante evidencia que a forma funcional das SCDDs é similar para a maioria dos valores de $\kappa$. 

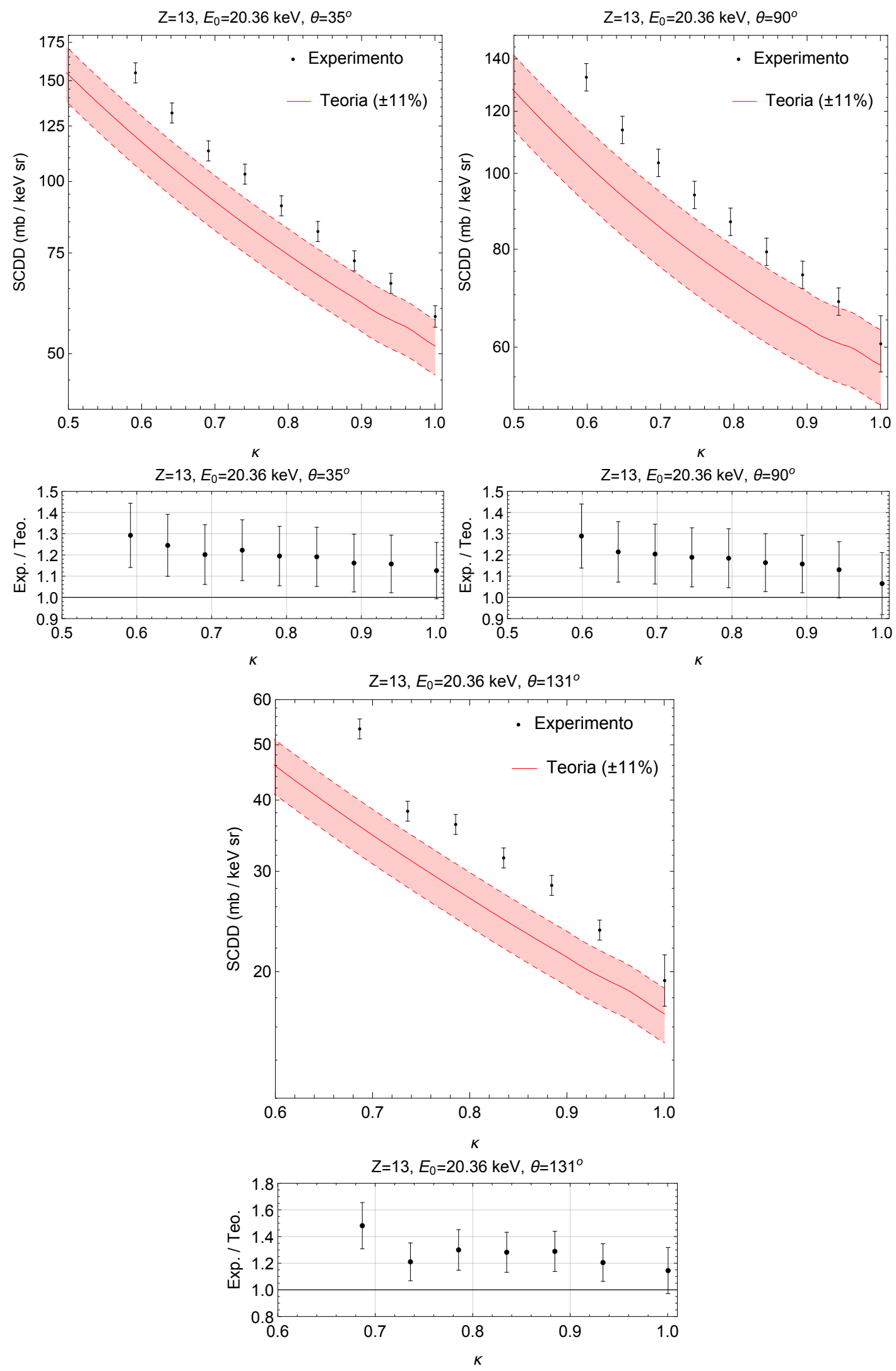

Figura 8.11: Resultados das SCDDs de emissão de bremsstrahlung em Al para elétrons incidentes de $20,36 \mathrm{keV}$. 

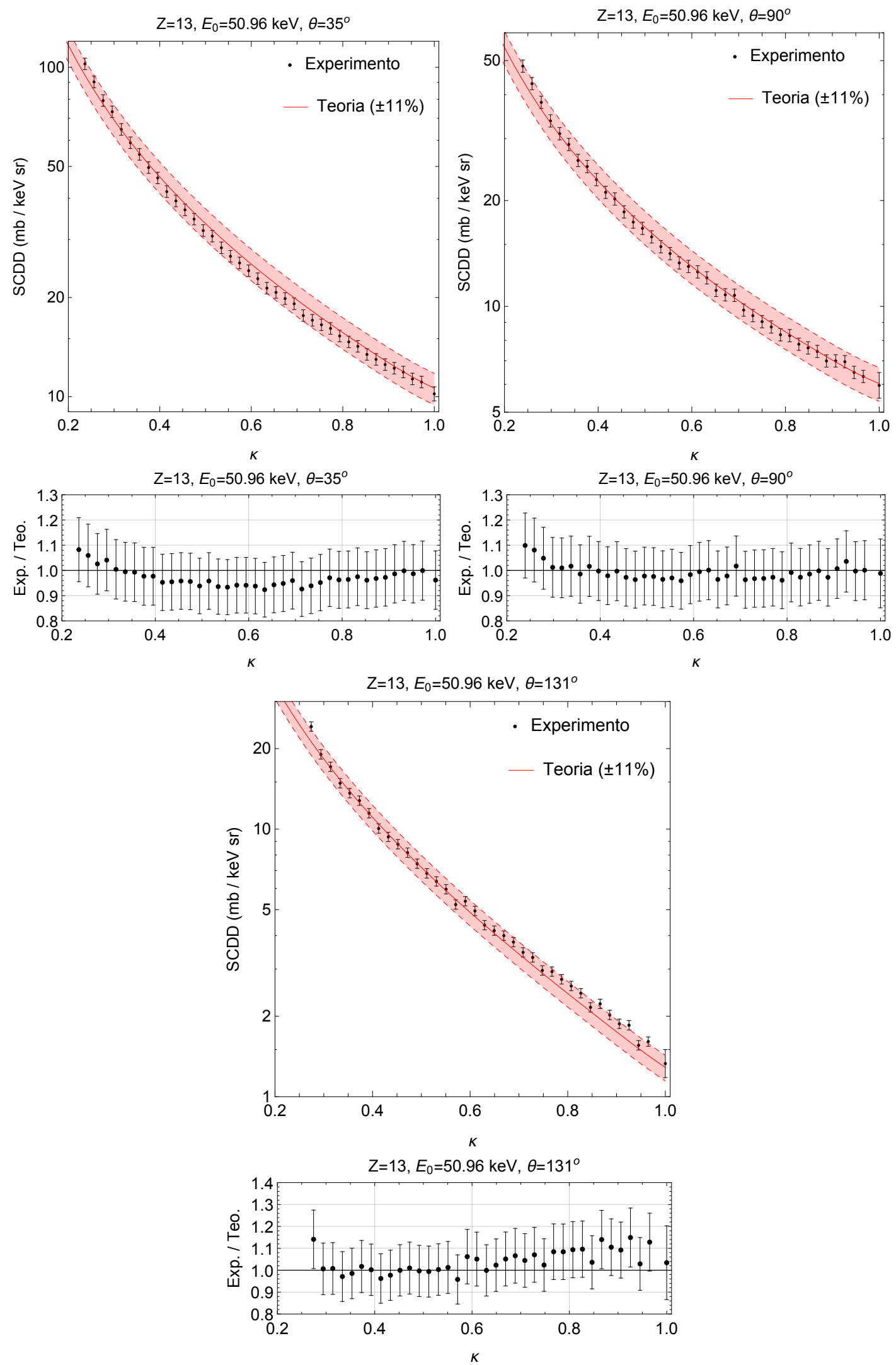

Figura 8.12: Resultados das SCDDs de emissão de bremsstrahlung em Al para elétrons incidentes de 50,96 keV. 

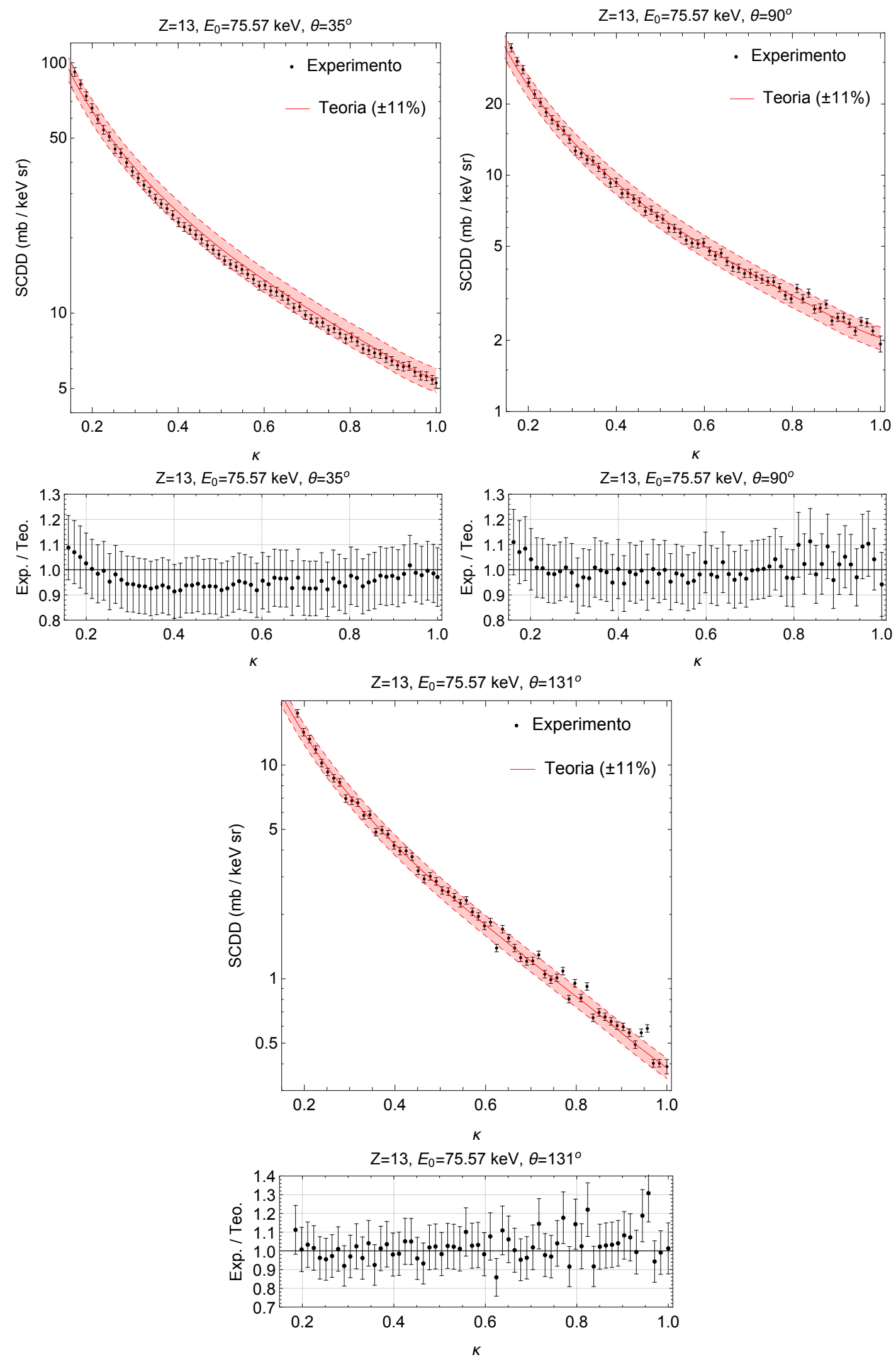

Figura 8.13: Resultados das SCDDs de emissão de bremsstrahlung em Al para elétrons incidentes de $75,57 \mathrm{keV}$. 

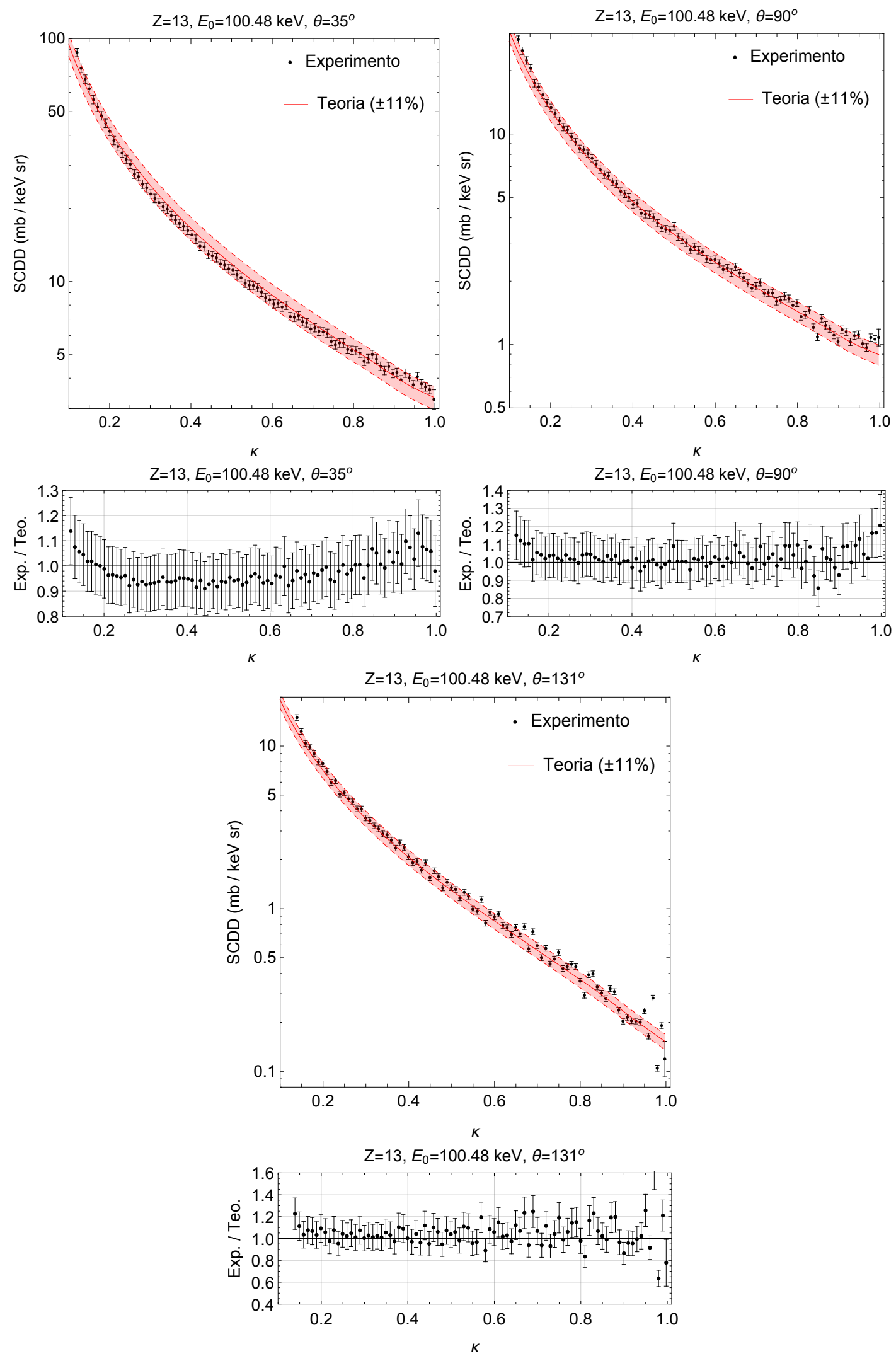

Figura 8.14: Resultados das SCDDs de emissão de bremsstrahlung em Al para elétrons incidentes de 100,48 keV. 

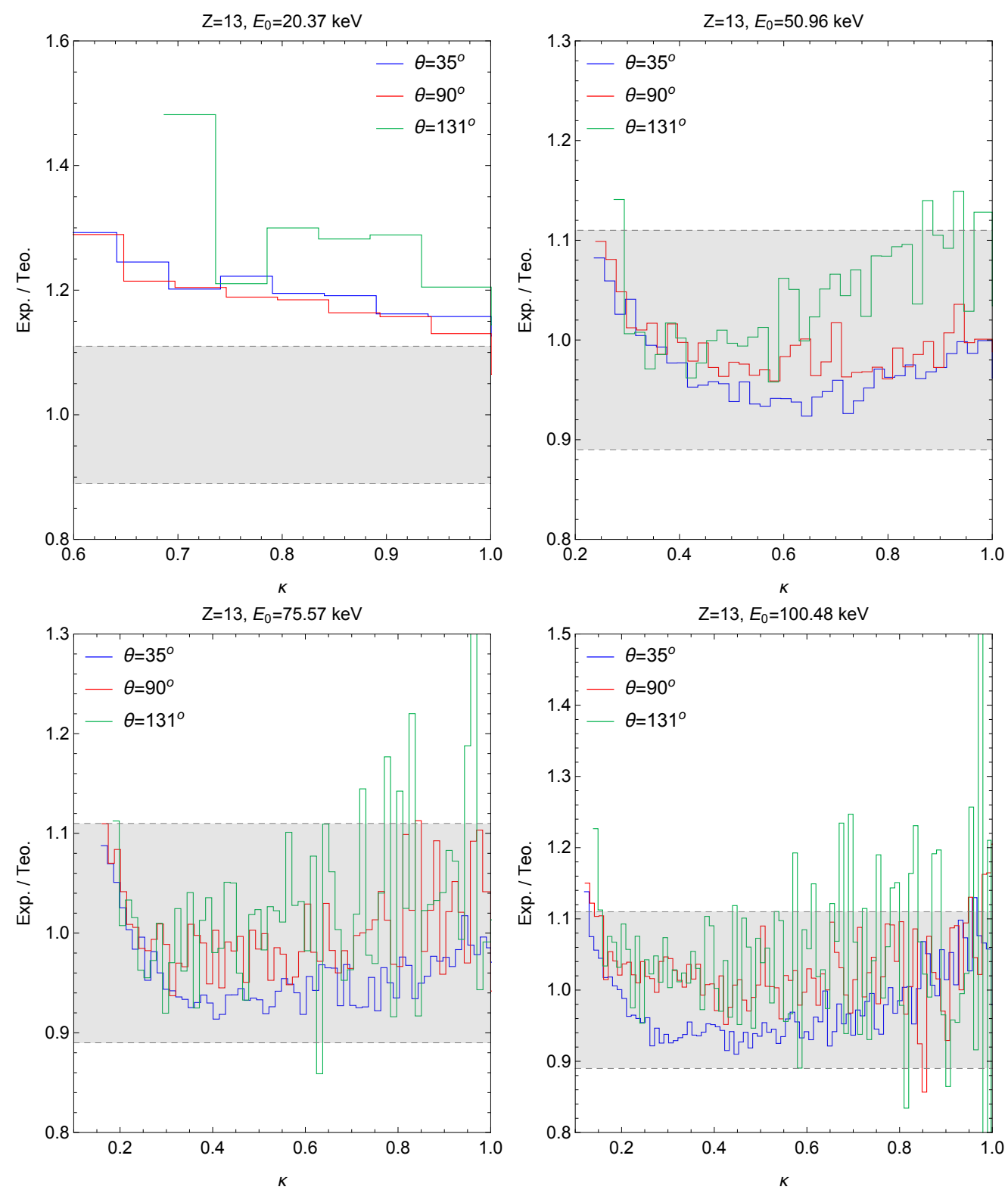

FIGURA 8.15: Razão experimento/teoria entre as SCDDs obtidas para o Al, considerando todas energias e ângulos medidos. A faixa cinza indica o intervalo de $\pm 11 \%$. 


\subsubsection{Prata $Z=47$}

Nas Figs. 8.16-8.20 são mostrados os resultados da Ag. Para $E_{0}=20,30 \mathrm{keV}$ e a maioria dos valores de $\kappa$, a SCDD experimental obtida é sistematicamente menor que a estimativa teórica, observando-se no tip a maior diferença. Só no ângulo $\theta=131^{\circ}$ experimento e teoria são compatíveis.

Para $E_{0}=51,09 \mathrm{keV}$ os resultados apresentam comportamentos diferentes em função do ângulo. $\operatorname{Em} \theta=35^{\circ}$ o experimento está sistematicamente abaixo da teoria em torno de $18 \%$, salvo para $\kappa \leqslant 0,3$ onde ambos valores são mais próximos ( $<10 \%$ de diferença). $\operatorname{Em} \theta=90^{\circ}$, exceto na região do tip $(\kappa>0,9)$, os dados experimentais e teóricos são compatíveis, sendo os primeiros menores que os segundos entre $0,5 \leqslant \kappa \leqslant 0,9$, e maiores entre $0,25 \leqslant \kappa<0,5$. $\operatorname{Em} \theta=131^{\circ}$ as SCDDs experimentais são menores que as teóricas em aproximadamente $5 \%$.

Para $E_{0}=70,63$ e 100,44 keV são observadas as mesmas tendências. Em $\theta=35^{\circ}$ os dados experimentais são inferiores à teoria entre 5 e $10 \%$. Para $\theta=90^{\circ}$ os pontos experimentais tendem a ser um $5 \%$ superiores à curva teórica, enquanto para $\theta=131^{\circ}$ a razão experimento/teoria oscila em torno de 1,0.

Em geral, segundo os resultados experimentais, a intensidade de emissão de fótons a $35^{\circ}$ é menor em relação à teoria, a $90^{\circ}$ é maior, e a $131^{\circ}$ é similar, exceto na região do tip onde o experimento é sempre inferior. 

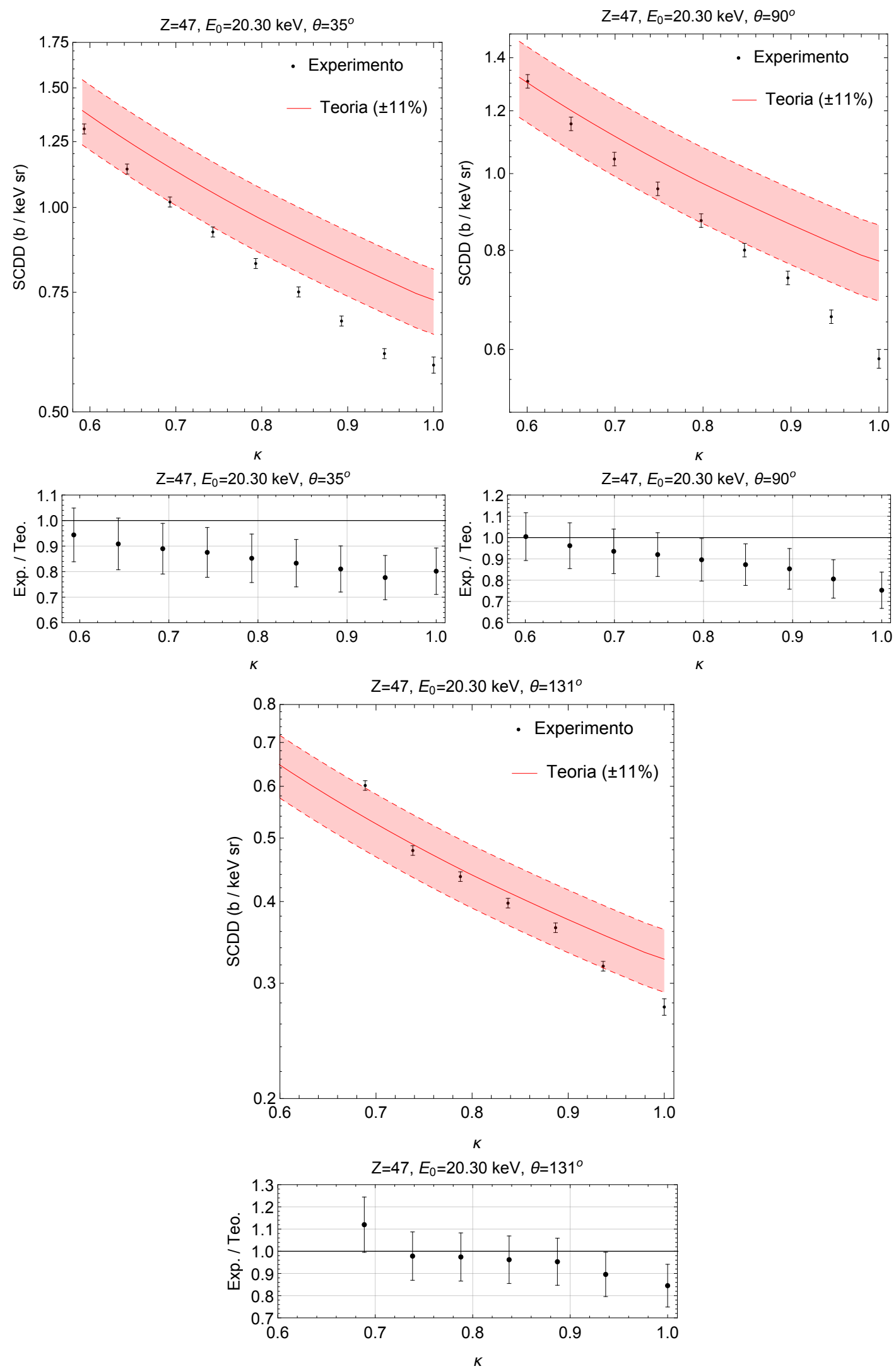

Figura 8.16: Resultados das SCDDs de emissão de bremsstrahlung em Ag para elétrons incidentes de 20,30 keV. 

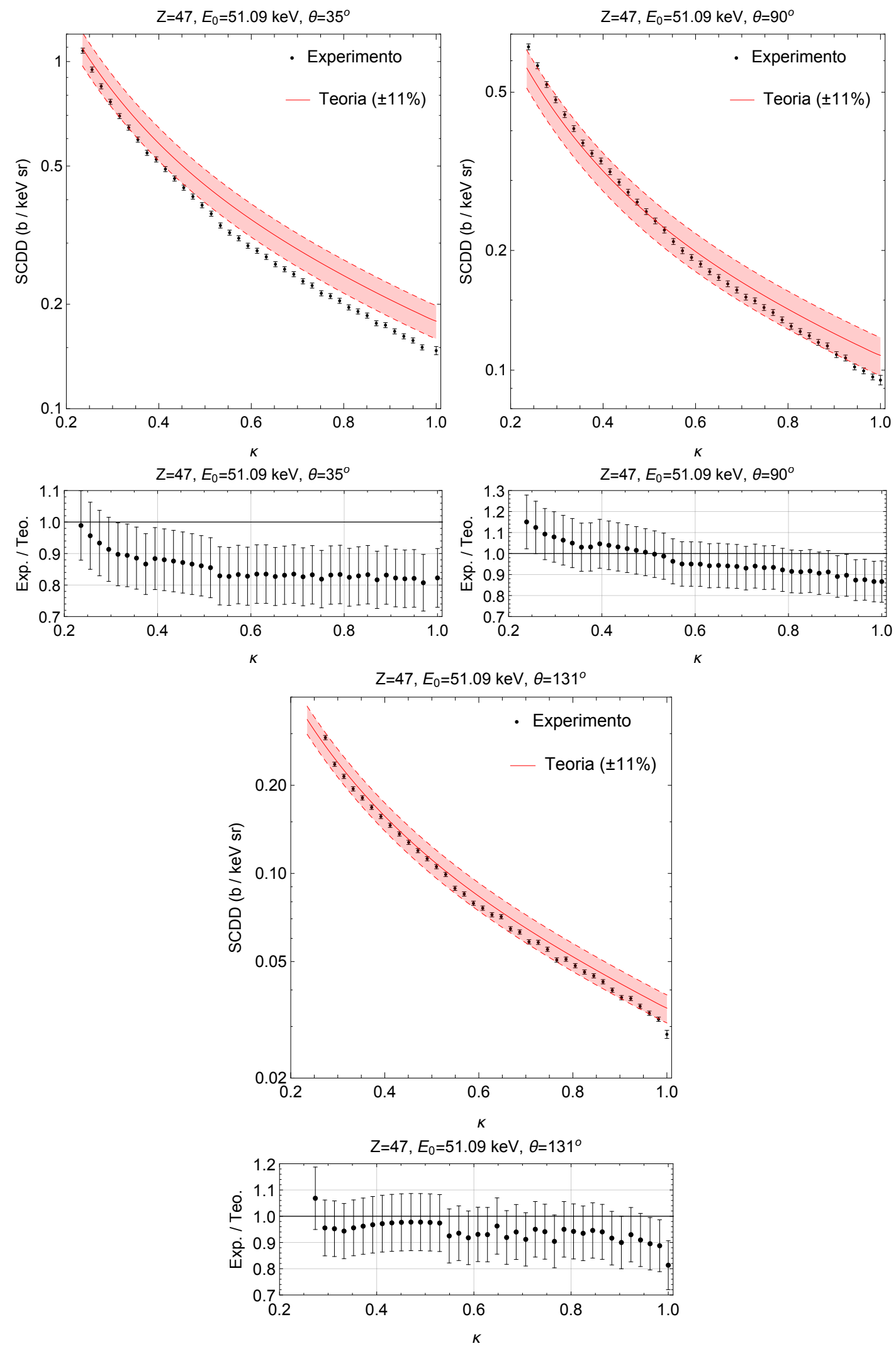

Figura 8.17: Resultados das SCDDs de emissão de bremsstrahlung em Ag para elétrons incidentes de 51,09 keV. 

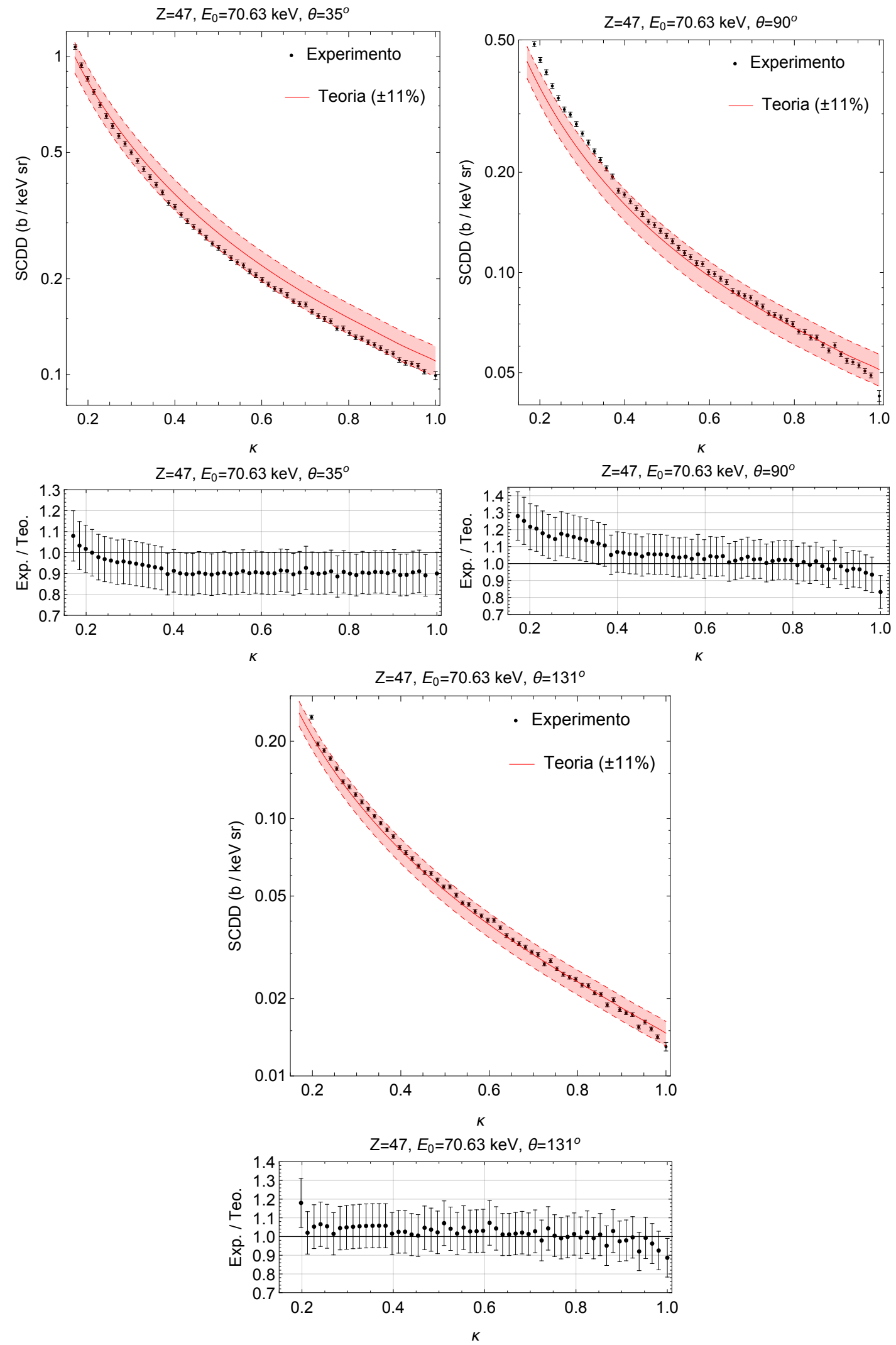

Figura 8.18: Resultados das SCDDs de emissão de bremsstrahlung em Ag para elétrons incidentes de $70,63 \mathrm{keV}$. 

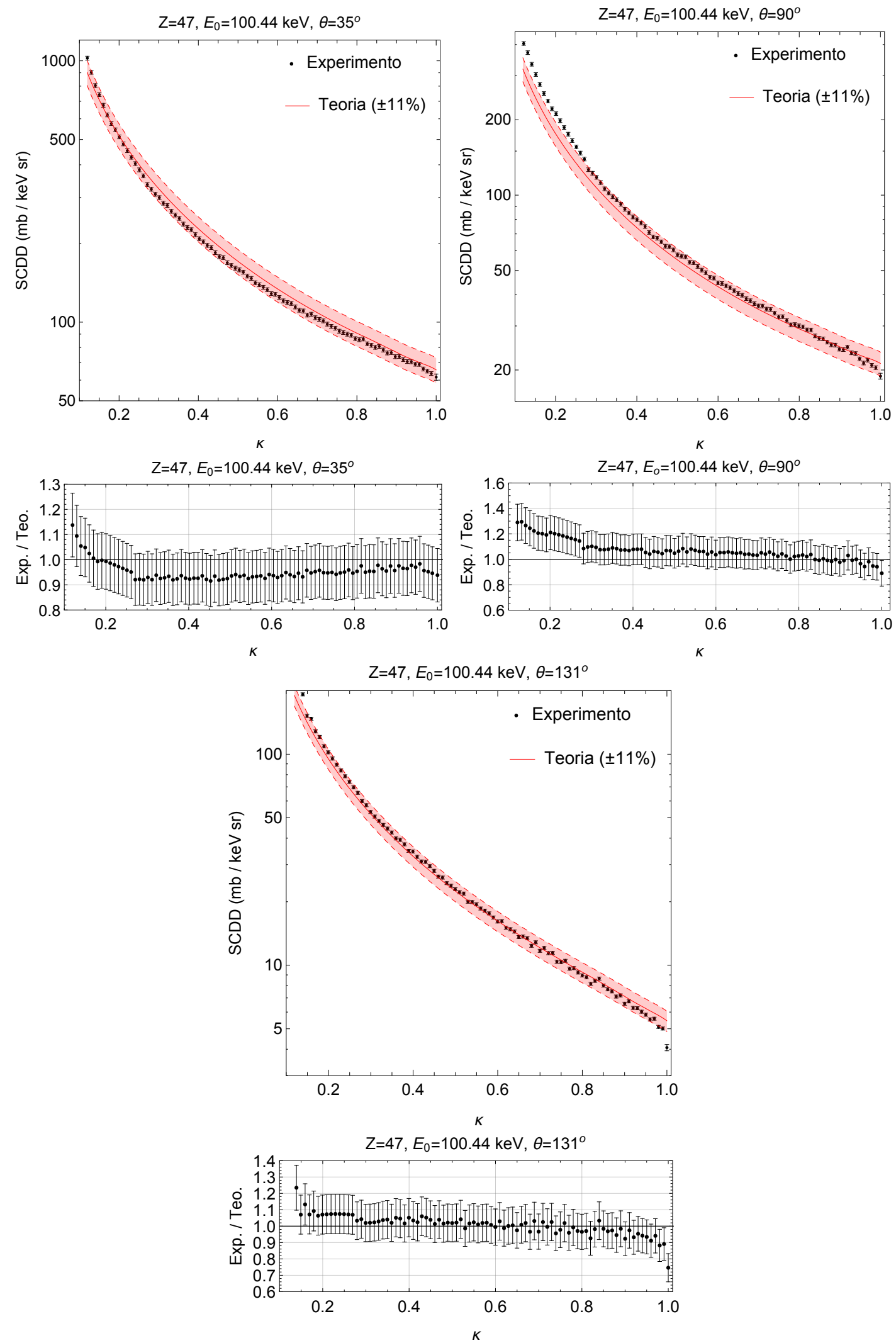

Figura 8.19: Resultados das SCDDs de emissão de bremsstrahlung em Ag para elétrons incidentes de 100,44 keV. 

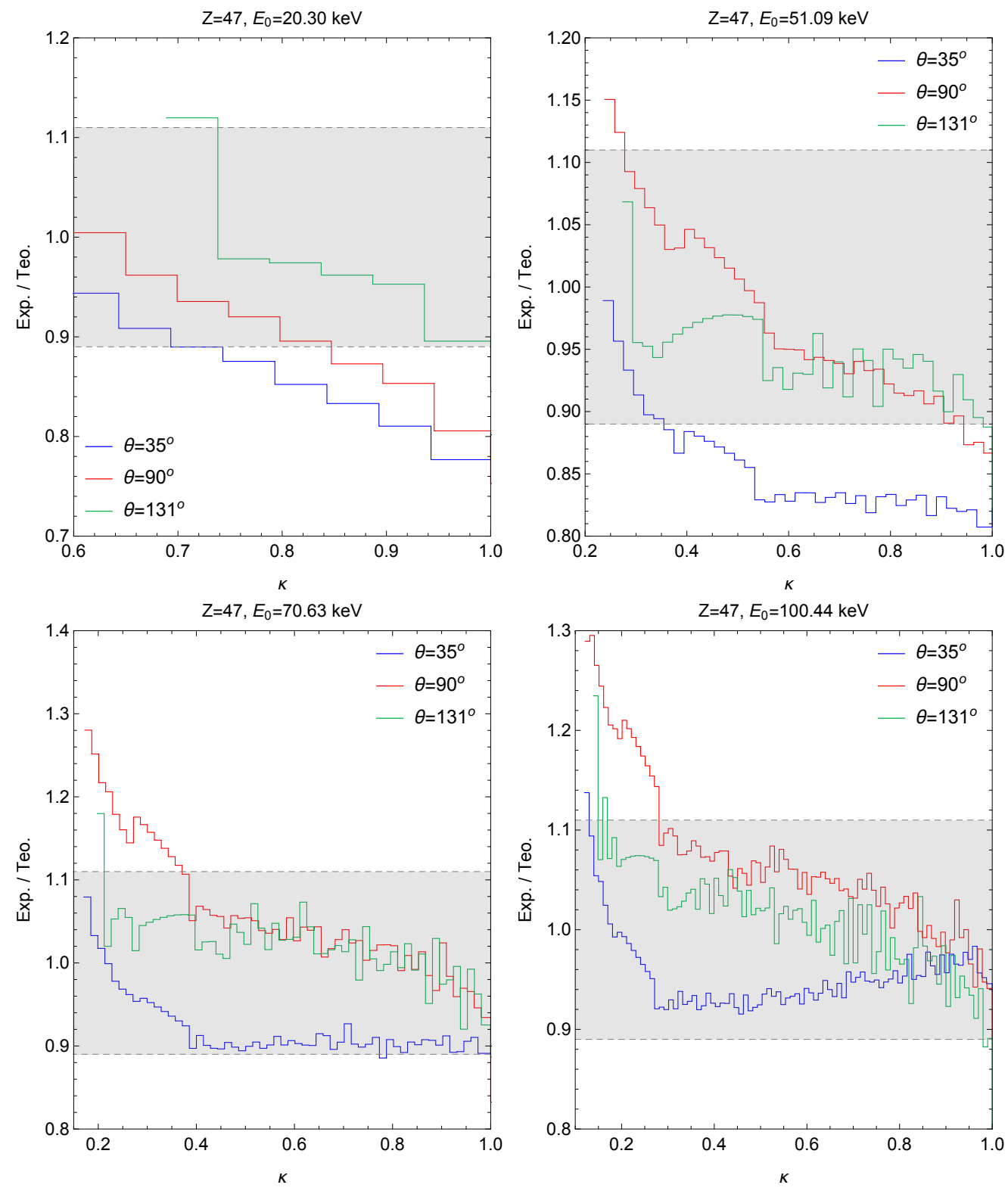

FiguRA 8.20: Razão experimento/teoria entre as SCDDs obtidas para o Ag, considerando todas energias e ângulos medidos. A faixa cinza indica o intervalo de $\pm 11 \%$. 


\subsubsection{Telúrio $Z=52$}

As Figs. 8.21-8.25 apresentam os resultados do Te. Para a energia dos elétrons de $22,57 \mathrm{keV}$, os resultados experimentais são compatíveis com a teoria somente na região do tip $(\kappa>0,9)$ nos três ângulos de medida. Nos restantes valores de $\kappa$ o experimento é superior à estimativa teórica com diferenças entre $10 \%$ e $30 \%$.

Para $E_{0}=51,19 \mathrm{keV}$, o comportamento associado às medidas em $\theta=35^{\circ}$ e $90^{\circ}$ é similar. Na região $\kappa>0,85$ o experimento é inferior à teoria (menos de $10 \%$ ), entre $0,6 \leqslant \kappa \leqslant 0,85$ a razão experimento/teoria é aproximadamente 1,0 , e para $\kappa<0,6$ a relação anterior aumenta até alcançar o valor de $1,25 \mathrm{em} \kappa \approx 0,25$. No ângulo $\theta=131^{\circ}$, o experimento é superior à estimativa teórica em torno de um $10 \%$.

Para as energias $E_{0}=75,71$ e $100,73 \mathrm{keV}$ se observam tendências semelhantes em função do ângulo. $\operatorname{Em} \theta=35^{\circ}$ a relação experimento/teoria é 1,0 entre $0,6 \leqslant \kappa \leqslant 1$, mostrando logo um comportamento crescente com a diminuição de $\kappa$, atingido valores entre 1,4 e 1,5 para $\kappa \approx 0,15$. Na posição $\theta=90^{\circ}$, os valores experimentais e teóricos seguem a mesma conduta entre a região do tip e $\kappa \approx 0,6$, entre $0,15 \leqslant \kappa<0,6$ os pontos experimentais se afastam da teoria no sentido contrário ao observado em $\theta=35^{\circ}$. Em ambos ângulos a forma funcional da SCDD experimental é diferente à curva teórica. Finalmente, em $\theta=131^{\circ}$ os resultados experimentais oscilam dentro de $\pm 5 \%$ com respeito à teoria. 

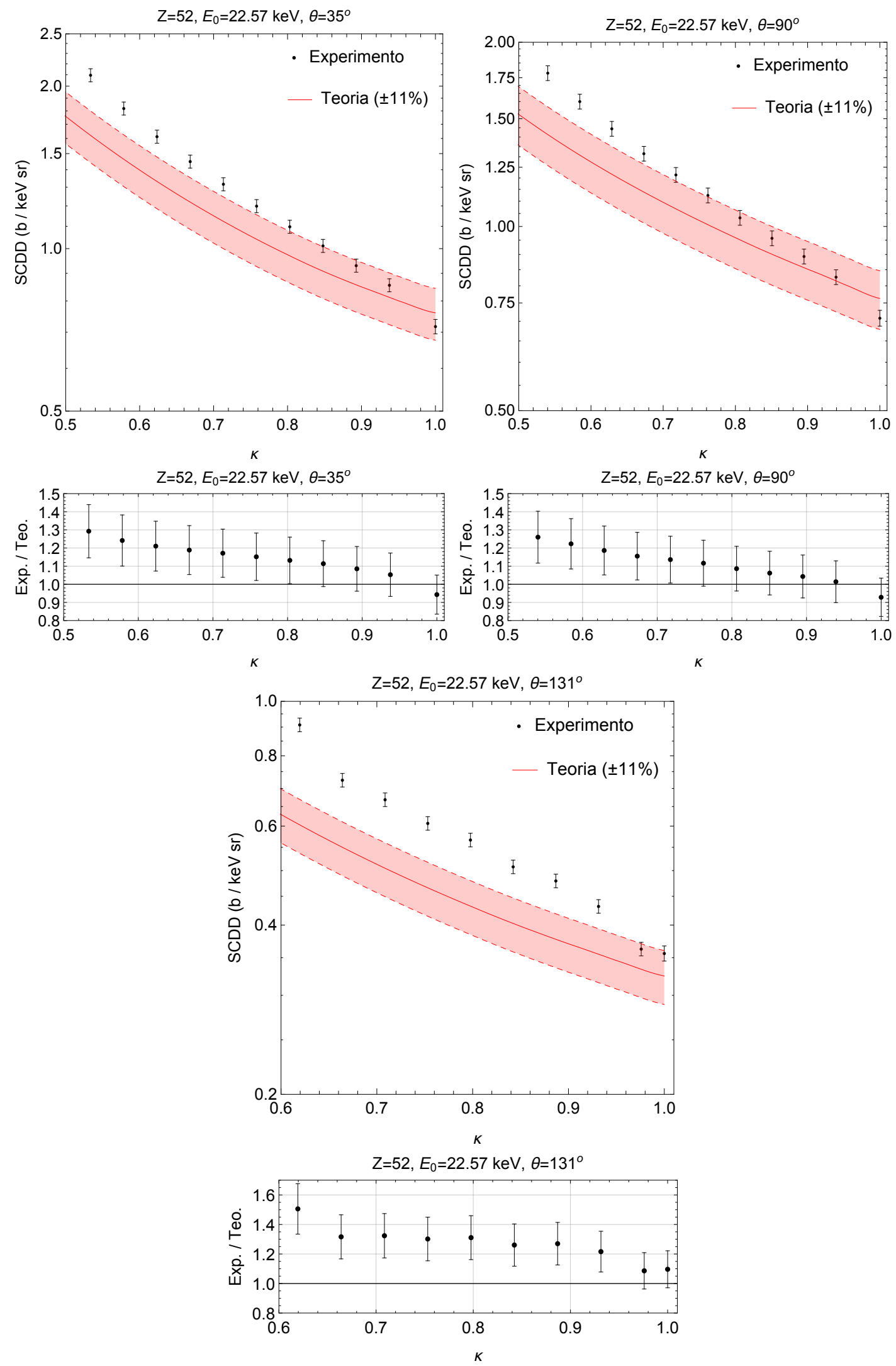

Figura 8.21: Resultados das SCDDs de emissão de bremsstrahlung em Te para elétrons incidentes de 22,57 keV. 

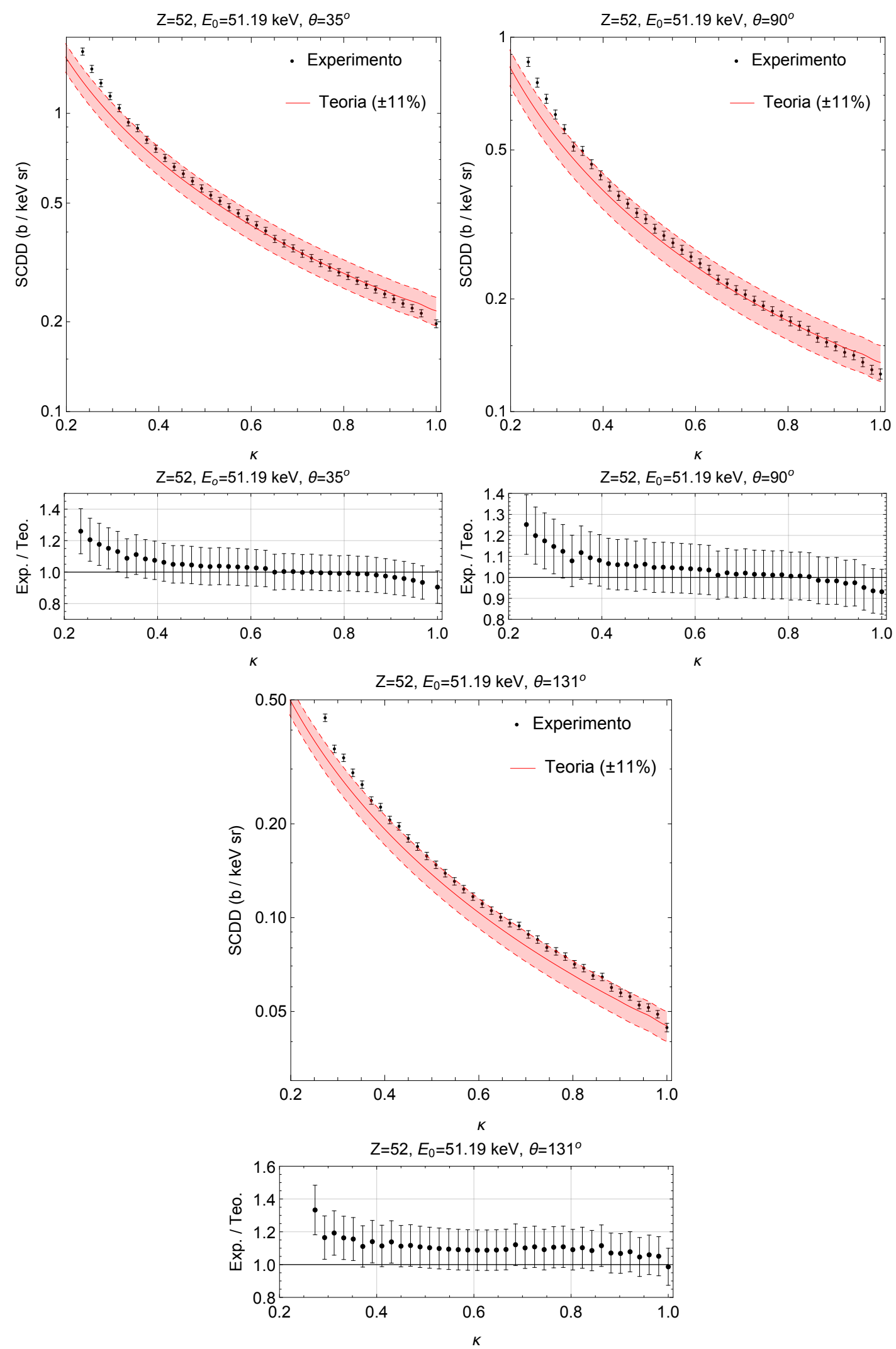

Figura 8.22: Resultados das SCDDs de emissão de bremsstrahlung em Te para elétrons incidentes de 51,19 keV. 

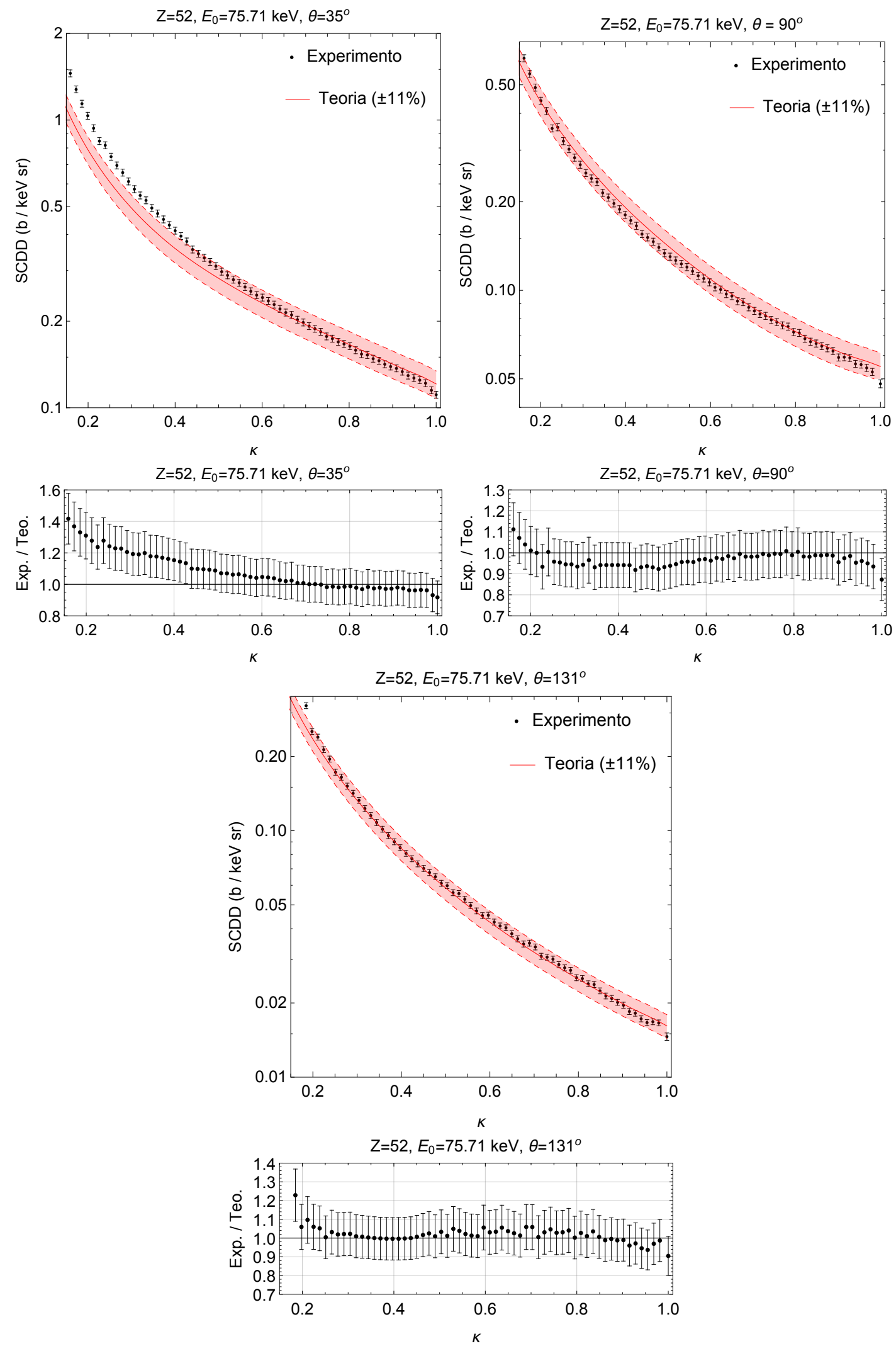

Figura 8.23: Resultados das SCDDs de emissão de bremsstrahlung em Te para elétrons incidentes de $75,71 \mathrm{keV}$. 

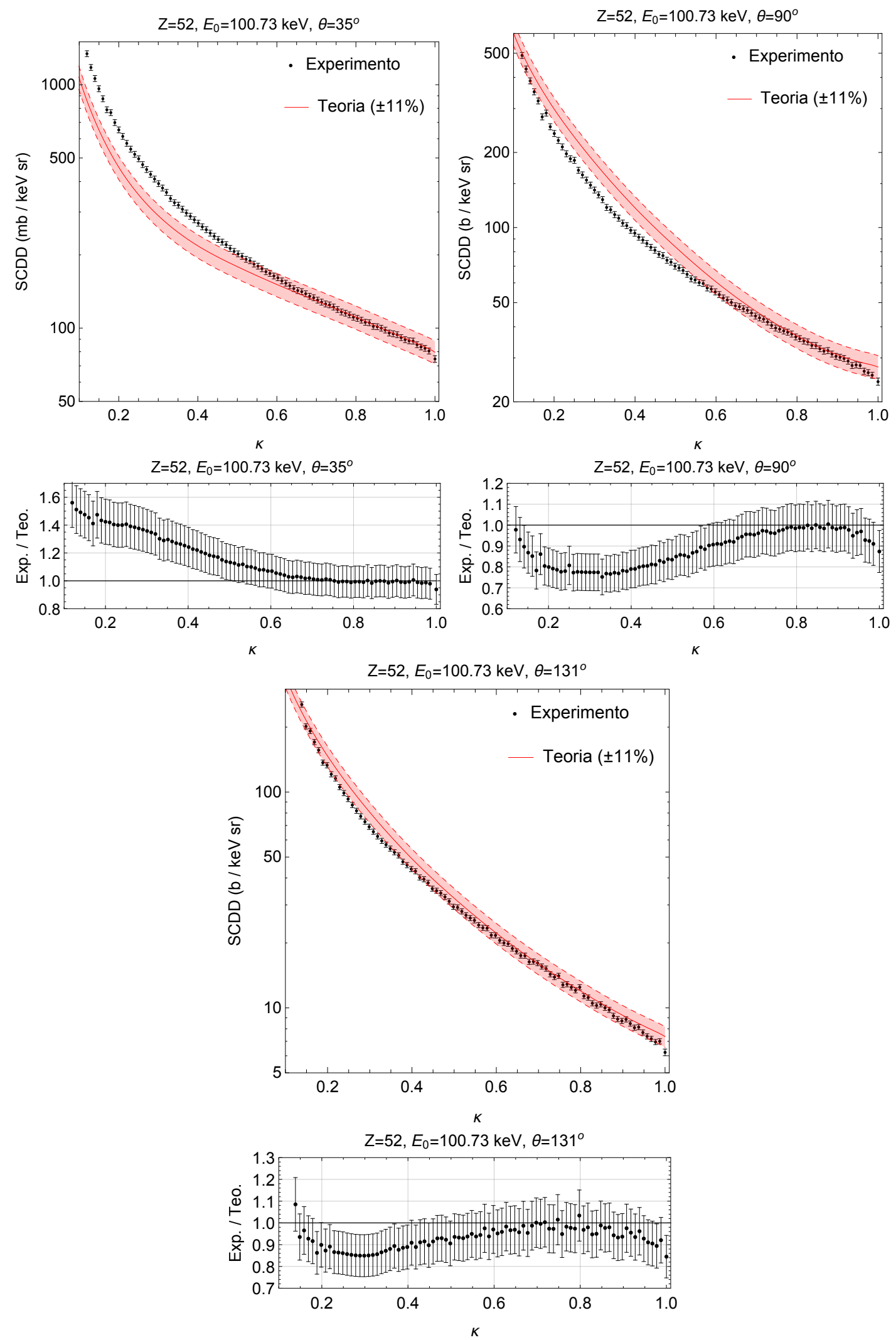

Figura 8.24: Resultados das SCDDs de emissão de bremsstrahlung em Te para elétrons incidentes de 100,73 keV. 

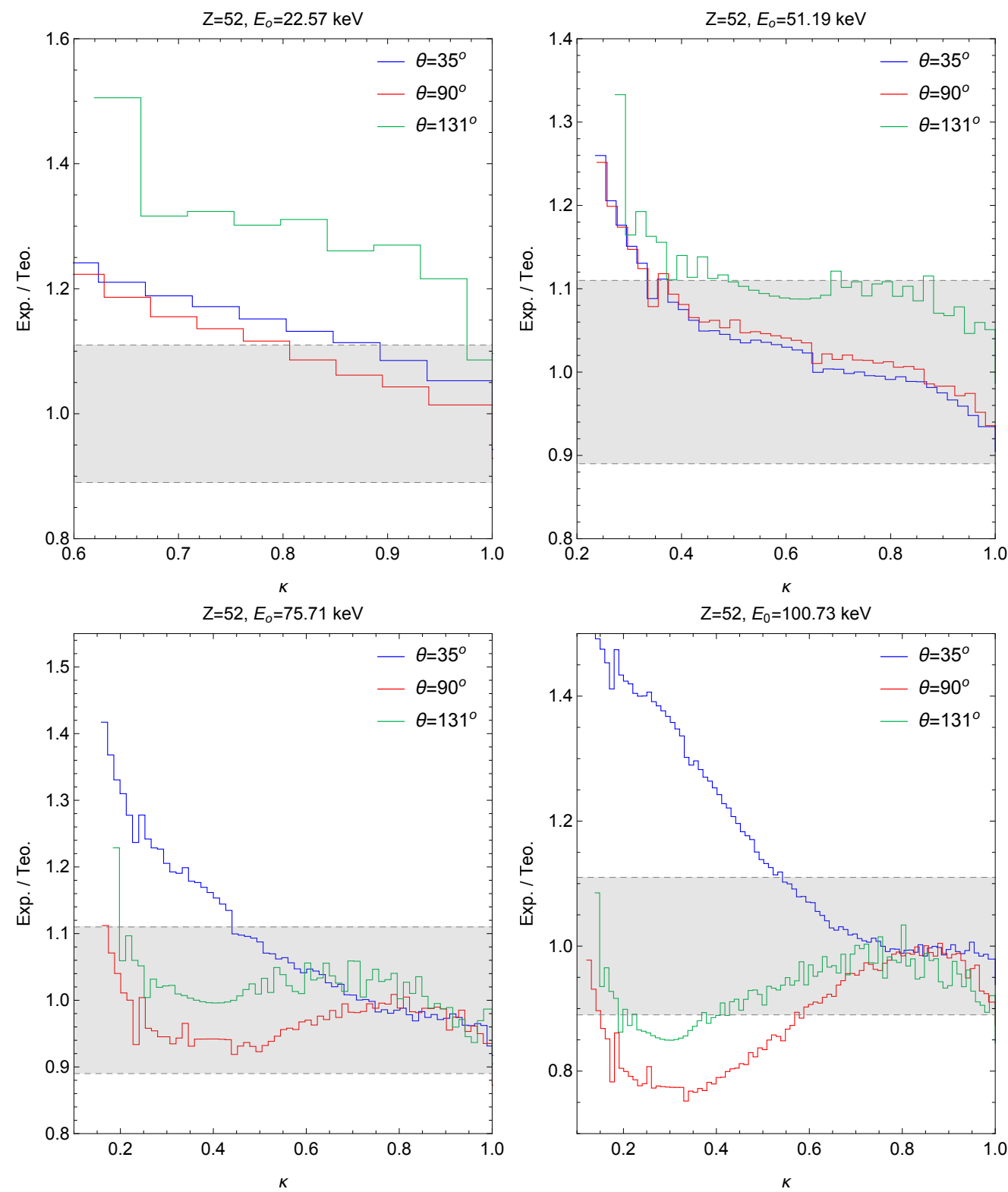

FiguRA 8.25: Razão experimento/teoria entre as SCDDs obtidas para o Te, considerando todas energias e ângulos medidos. A faixa cinza indica o intervalo de $\pm 11 \%$. 


\subsubsection{Tântalo $Z=73$}

Os resultados do Ta são mostrados nas Figs. 8.26-8.30. Observa-se um comportamento similar ao descrito para o Te. Para $E_{0}=22,58 \mathrm{keV}$ os pontos experimentais estão acima da teoria em até $20 \%$ para os três ângulo de medida, excepto na região do tip, em que ambos dados são compatíveis.

Para $E_{0}=51,19 \mathrm{keV}$ a faixa teórica de $\pm 11 \%$ contém a maioria das SCDDs experimentais. Nos gráficos correspondentes aos ângulos $\theta=35^{\circ}$ e $90^{\circ}$, o ponto $\kappa \approx 0,6$ define dois comportamentos: à direita as SCDDs experimentais são menores que a teoria, enquanto à esquerda acontece o contrário. Para $\theta=131^{\circ}$ o experimento é superior à curva teórica entre $5 \%$ e $15 \%$.

Para $E_{0}=75,69$ e 100,80 keV se observam desvios entre experimento e teoria, a partir de $\kappa \approx 0,6$, com a mesma forma funcional descrita para o Te nas correspondentes energias de incidência dos elétrons. 

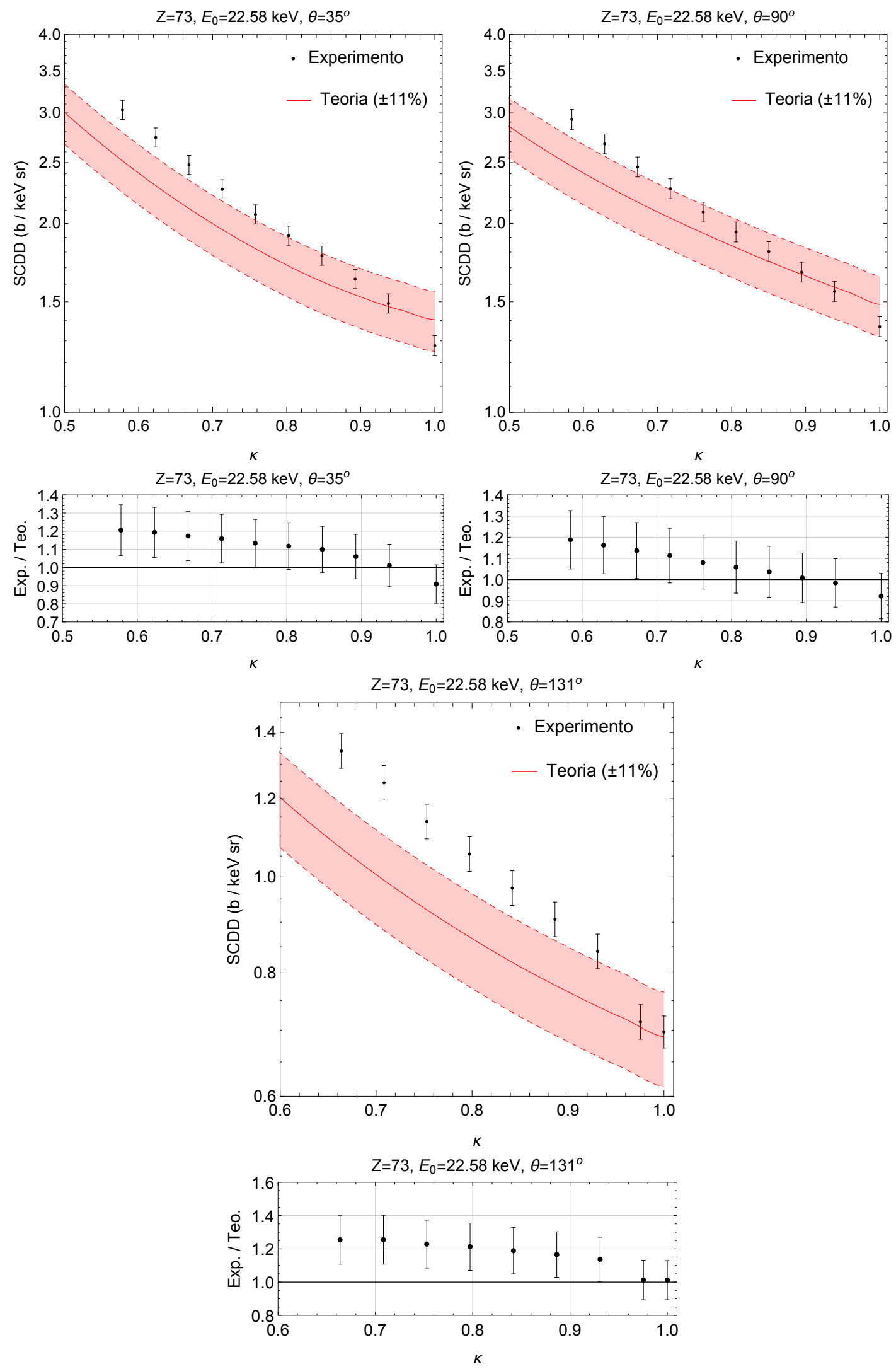

Figura 8.26: Resultados das SCDDs de emissão de bremsstrahlung em Ta para elétrons incidentes de $22,58 \mathrm{keV}$. 

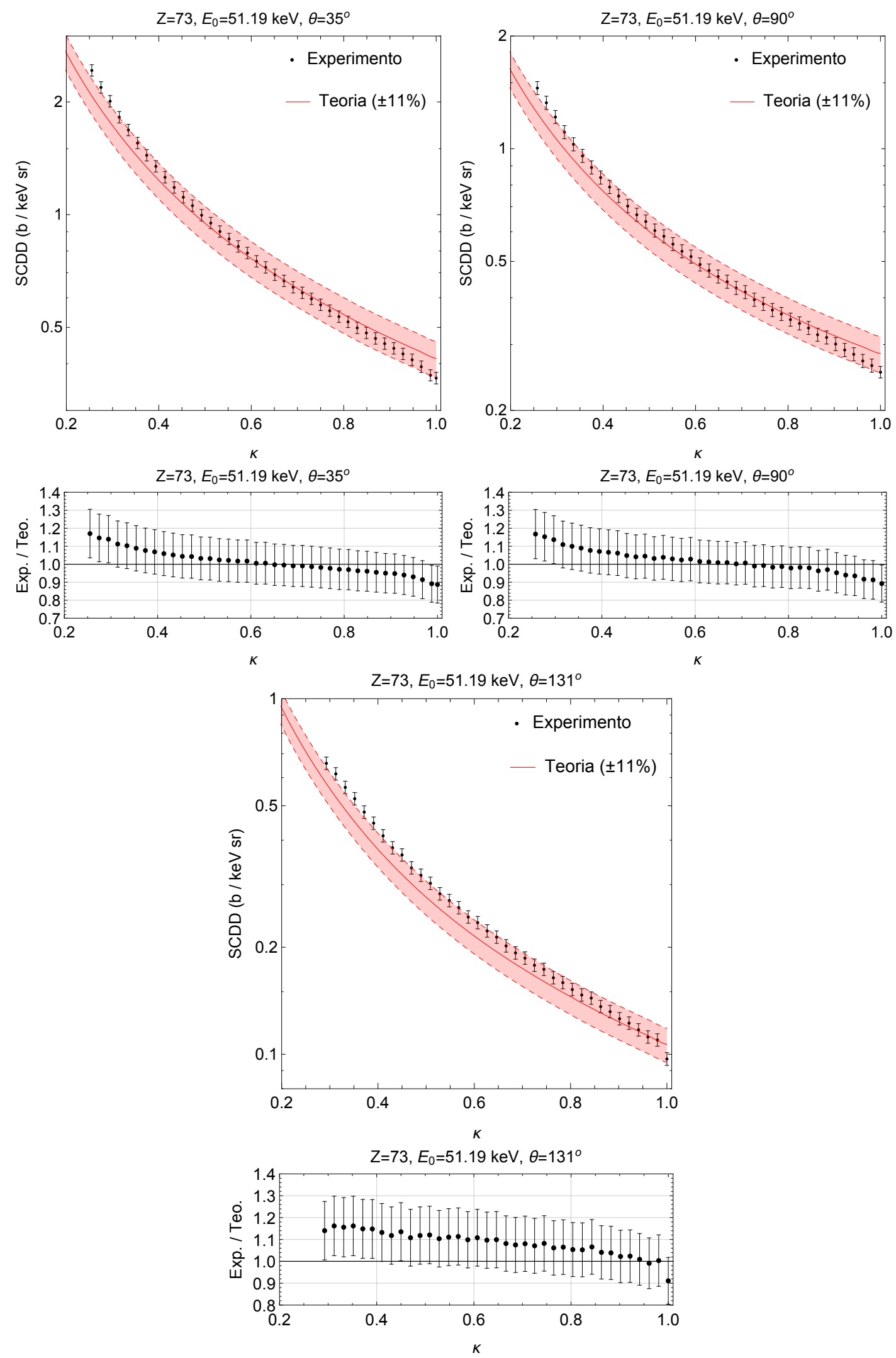

Figura 8.27: Resultados das SCDDs de emissão de bremsstrahlung em Ta para elétrons incidentes de $51,19 \mathrm{keV}$. 

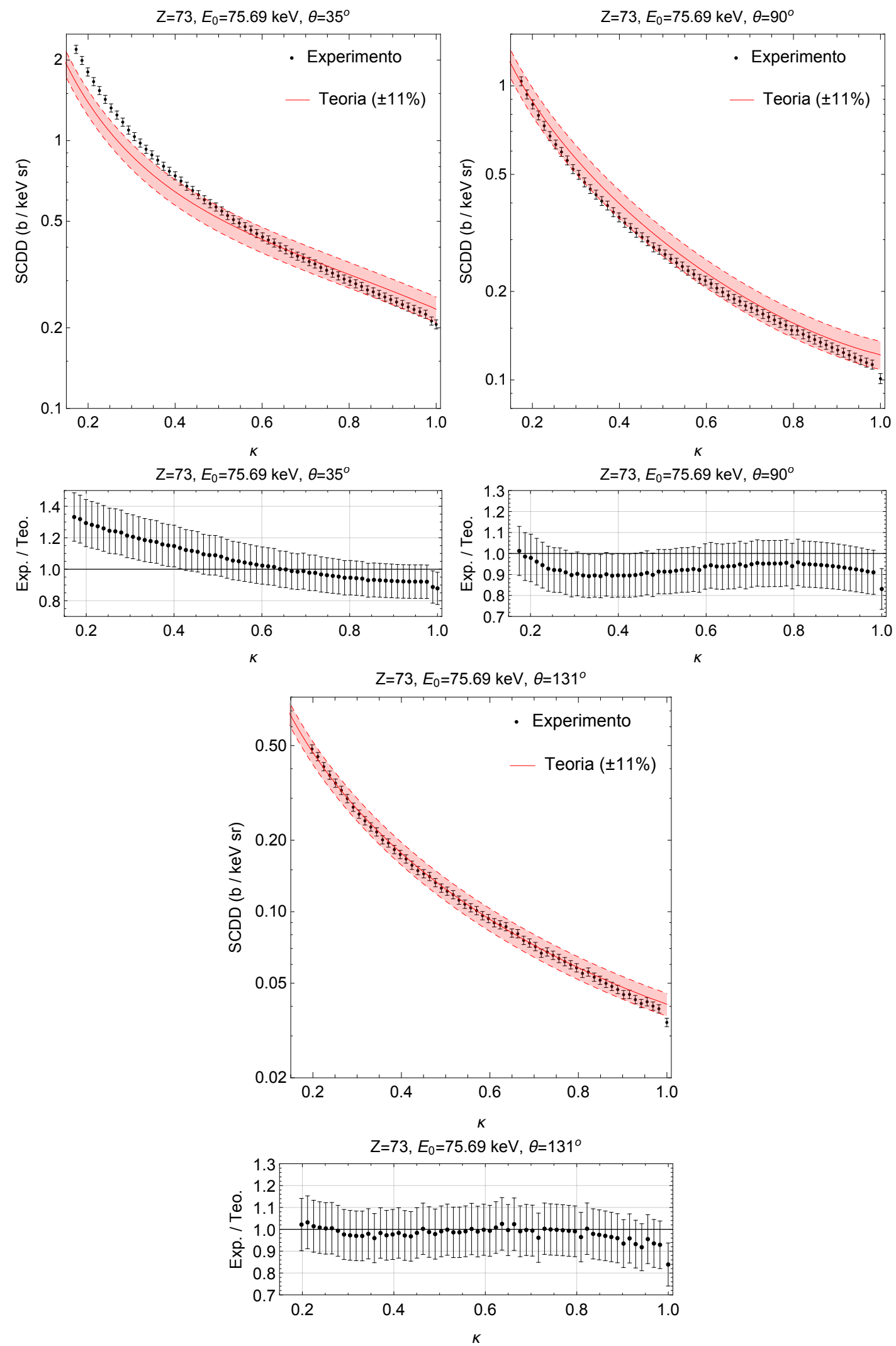

Figura 8.28: Resultados das SCDDs de emissão de bremsstrahlung em Ta para elétrons incidentes de $75,69 \mathrm{keV}$. 

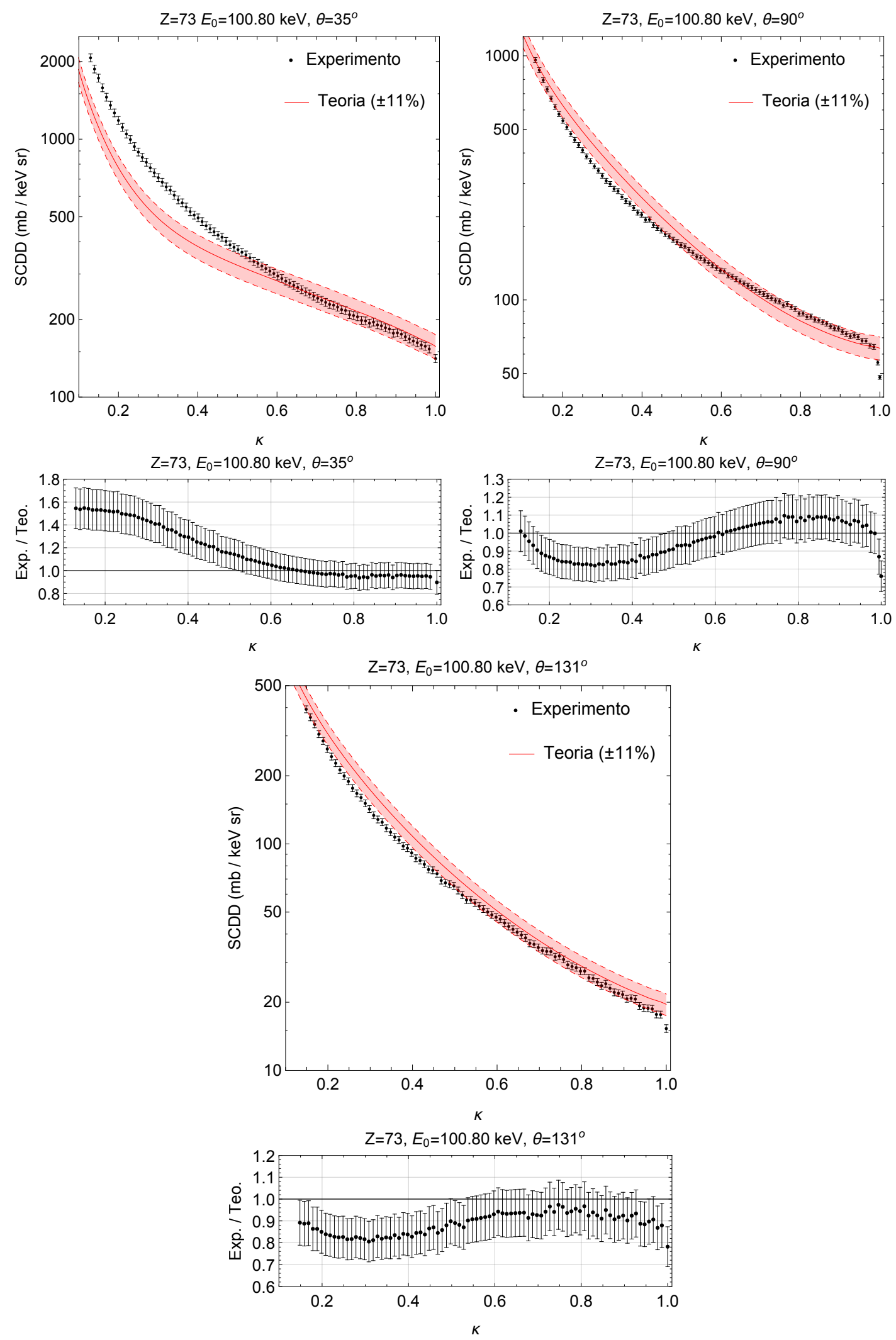

Figura 8.29: Resultados das SCDDs de emissão de bremsstrahlung em Ta para elétrons incidentes de 100,80 keV. 

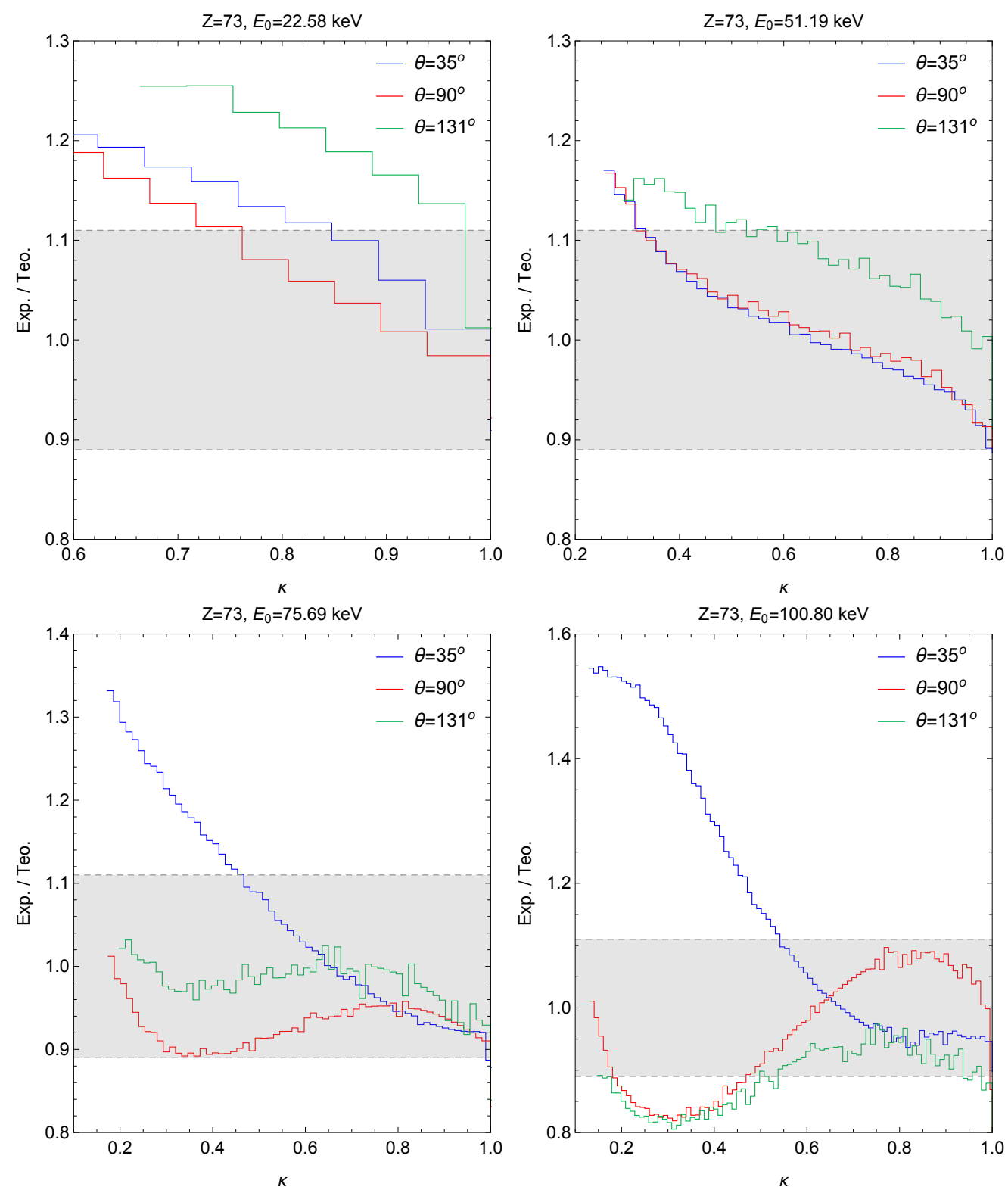

Figura 8.30: Razão experimento/teoria entre as SCDDs obtidas para o Ta, considerando todas energias e ângulos medidos. A faixa cinza indica o intervalo de $\pm 11 \%$. 


\subsubsection{Ouro $Z=79$}

Nas Figs. 8.31-8.35 se apresentam os resultados do Au. No caso de $E_{0}=20,38 \mathrm{keV}$, observa-se o mesmo comportamento obtido para energias de incidência similares nos outros elementos estudados: os pontos experimentais estão acima da curva teórica aproximadamente um $20 \%$.

Na energia de incidência $E_{0}=51,09 \mathrm{keV}$, as SCDDs experimentais passam de ter um total acordo com a teoria em $\kappa=1$, a superestimar esta curva em um fator entre $20 \%$ e $25 \%$ em $\kappa \approx 0,3$. O melhor acordo se alcança nas medidas realizadas a $35^{\circ}$.

Para os três ângulos e as energias $E_{0}=75,68$ e 100,48 keV, a relação experimento/teoria é praticamente constante com um valor de 1,1, excepto no tip, que como já foi mostrado, o acordo é melhor. A forma funcional seguida pelas SCDDs experimentais e teórica é semelhante e não se observam as oscilações obtidas nos elementos Te e Ta. 

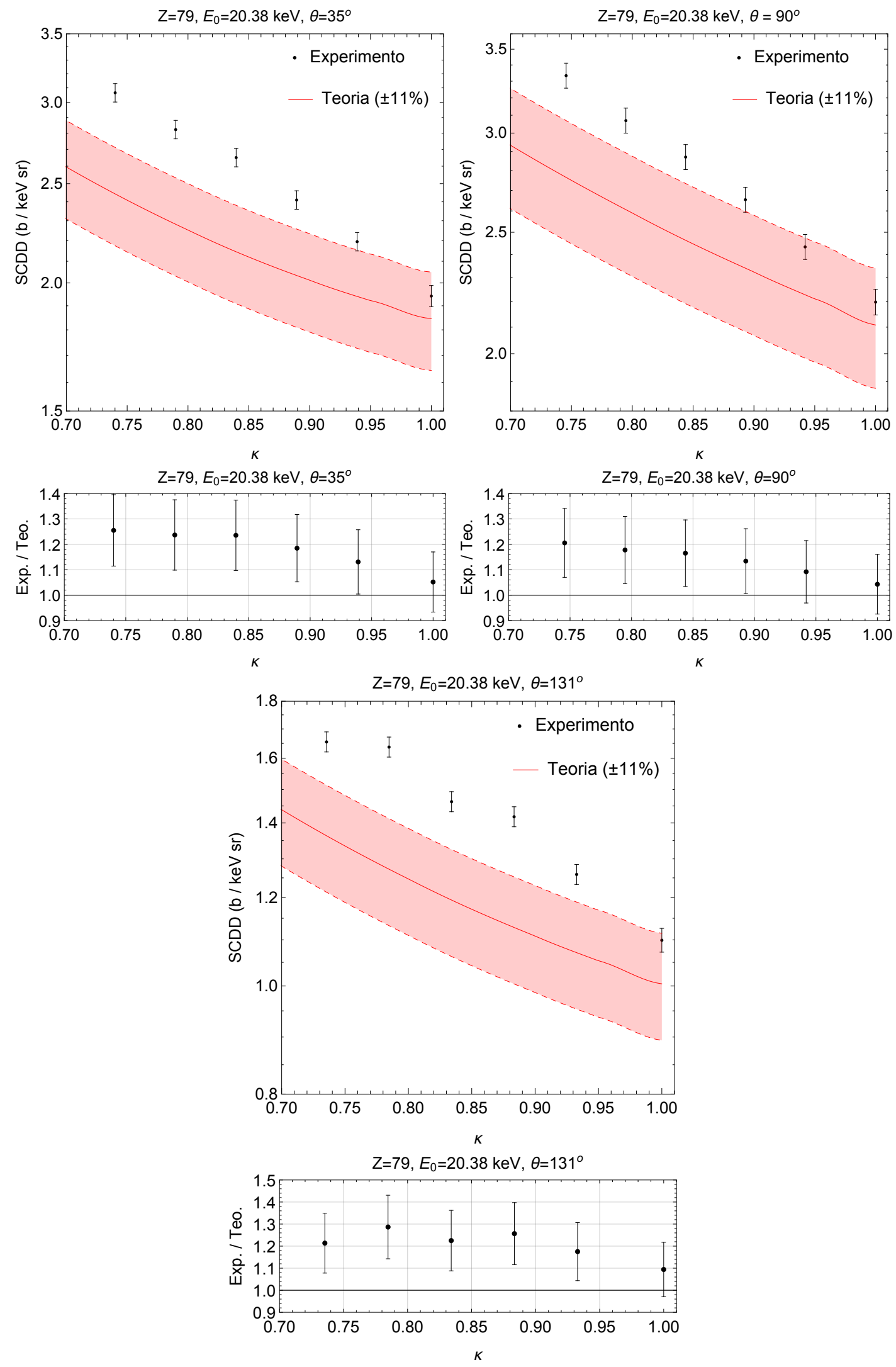

Figura 8.31: Resultados das SCDDs de emissão de bremsstrahlung em Au para elétrons incidentes de $20,38 \mathrm{keV}$. 

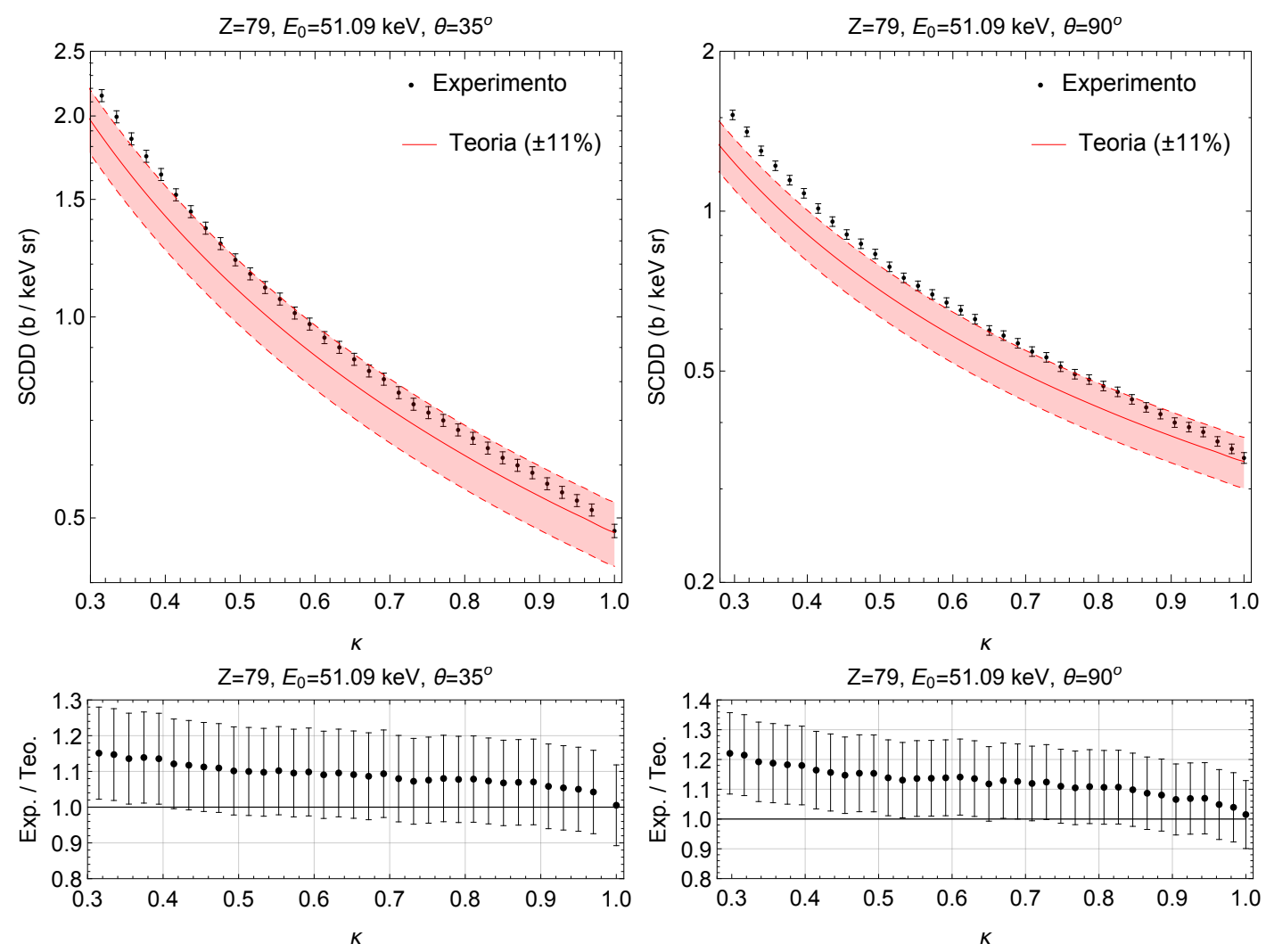

$\mathrm{Z}=79, E_{0}=51.09 \mathrm{keV}, \theta=131^{\circ}$
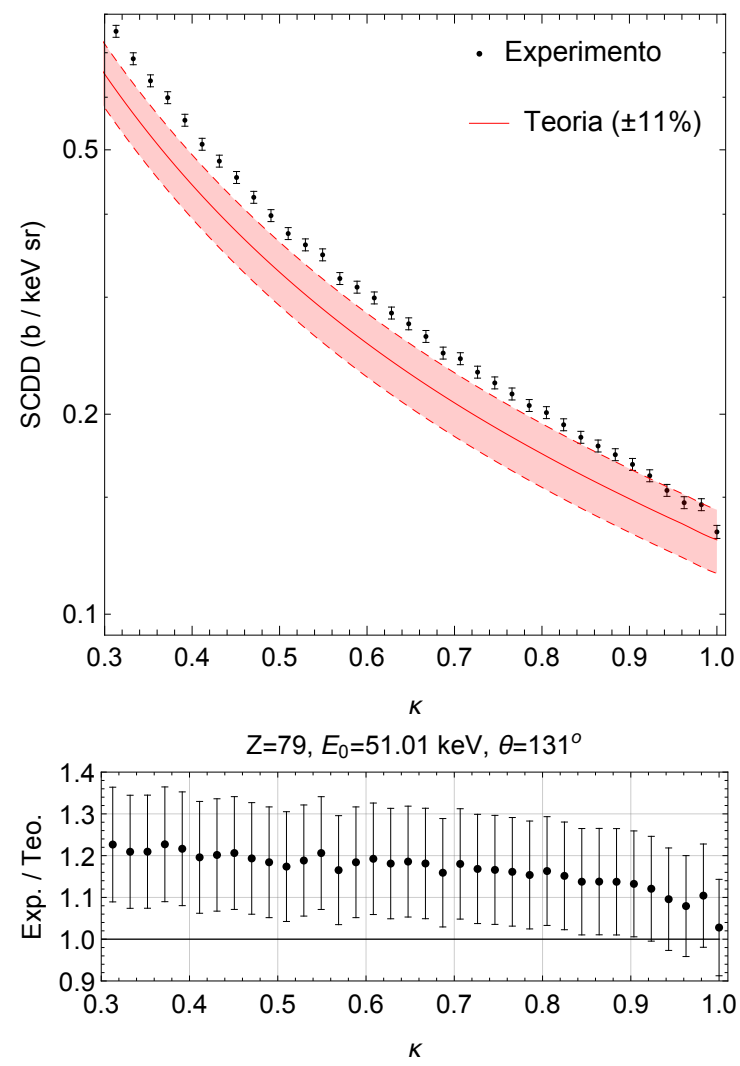

Figura 8.32: Resultados das SCDDs de emissão de bremsstrahlung em Au para elétrons incidentes de $51,09 \mathrm{keV}$. 

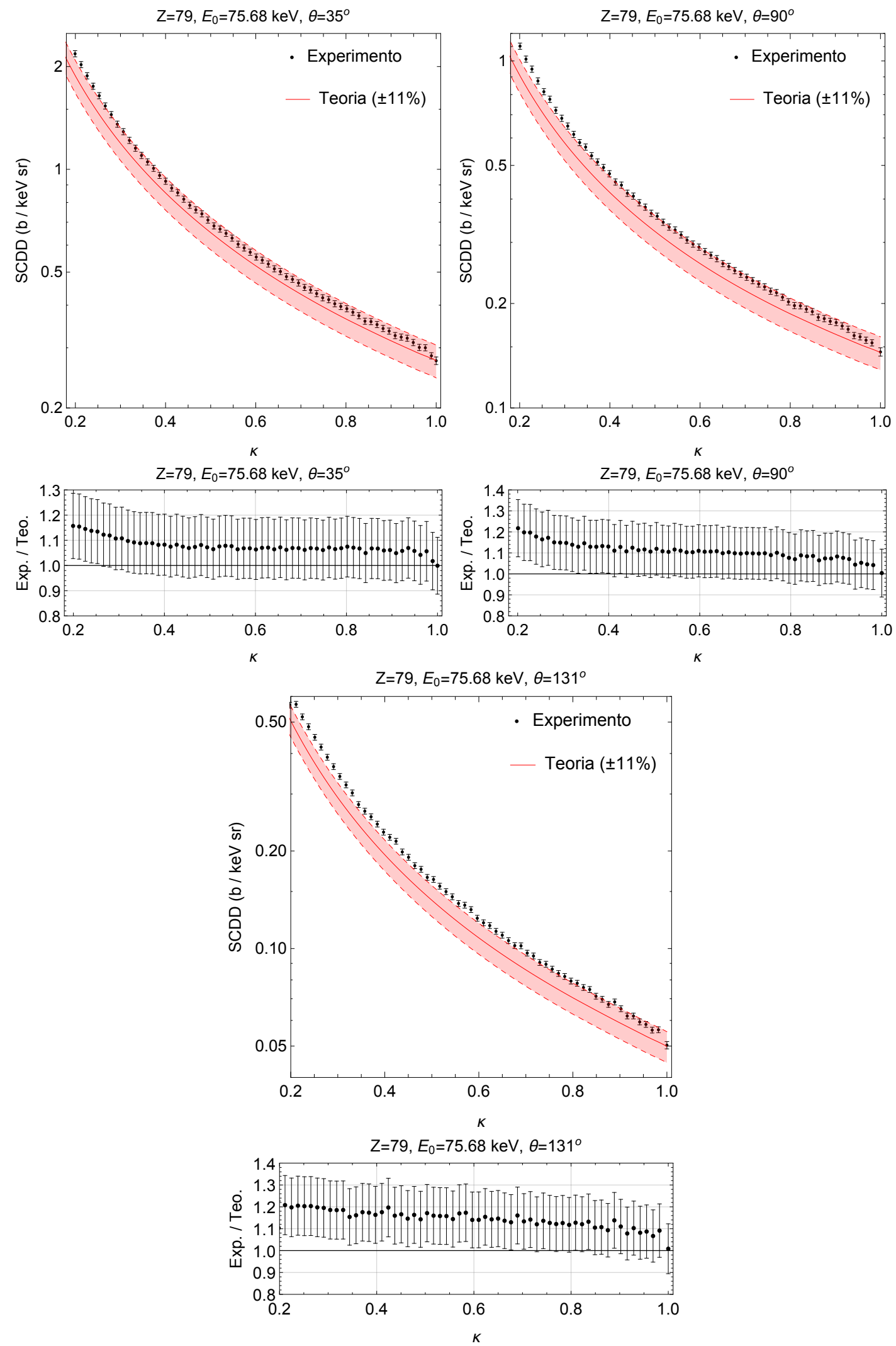

Figura 8.33: Resultados das SCDDs de emissão de bremsstrahlung em Au para elétrons incidentes de $75,68 \mathrm{keV}$. 

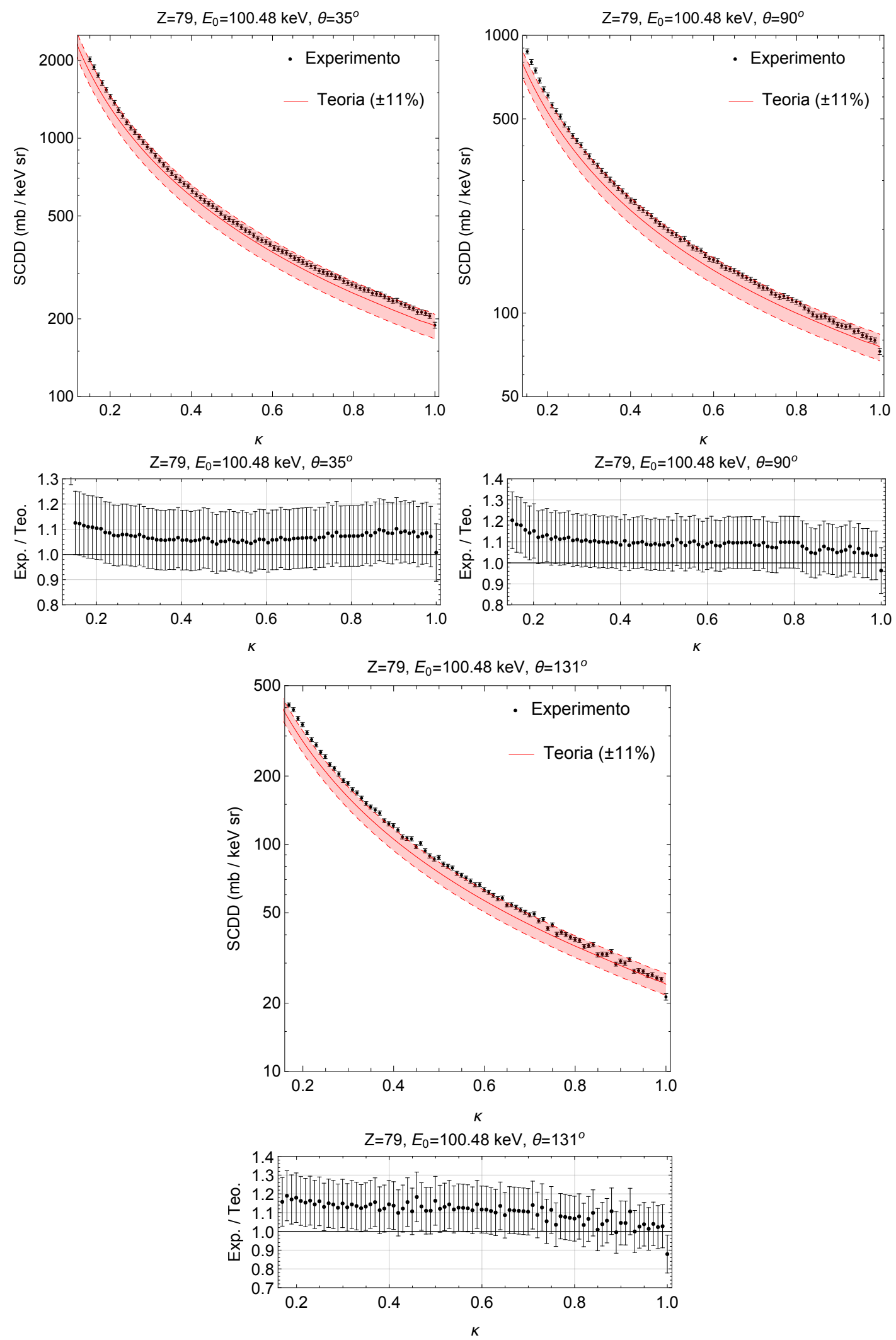

Figura 8.34: Resultados das SCDDs de emissão de bremsstrahlung em Au para elétrons incidentes de 100,48 keV. 

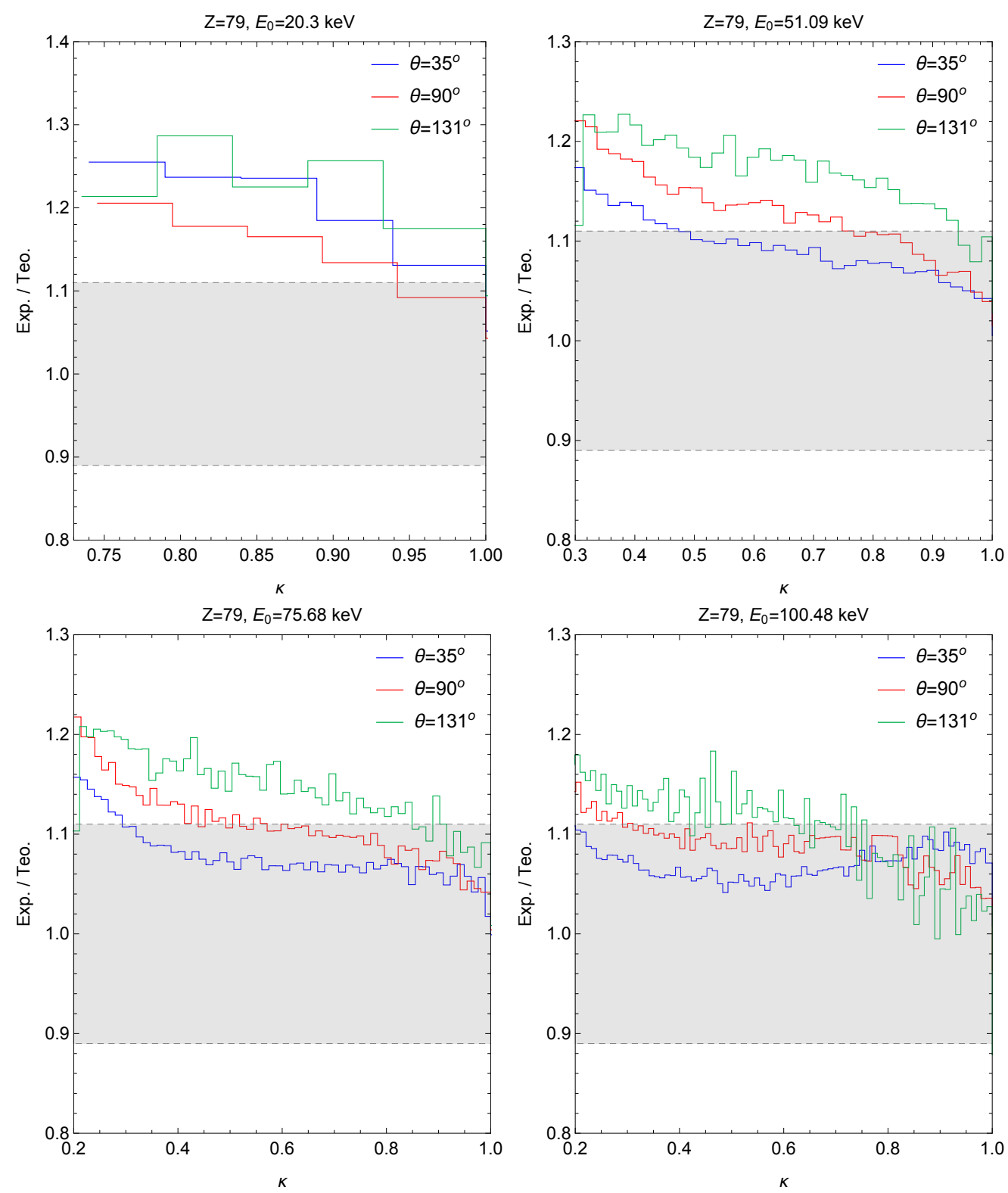

Figura 8.35: Razão experimento/teoria entre as SCDDs obtidas para o Au, considerando todas energias e ângulos medidos. 


\subsubsection{Comparação com os resultados publicados na literatura}

Os resultados das SCDDs obtidos para elétrons incidentes em torno de $50 \mathrm{keV}$ e $\theta=90^{\circ}$, foram comparados com os dados de Motz e Placious [6] para os elementos $\mathrm{Al}$ e $\mathrm{Au}$, e com os dados de Ambrose e colaboradores [9] para Al, Ag, Te e Au (Fig. 8.36). Nas duas publicações se encontraram as únicas medidas compatíveis com o conjunto experiências realizadas neste trabalho. Resulta importante lembrar que Motz e Placious desenvolveram os primeiros experimentos absolutos de SCDDs, em 1958, com incertezas relativas de 15\%. Por outro lado, no estudo de Ambrose et al. [9] as SCDDs se determinaram com uma incerteza relativa de $22 \%$, mas não podem ser consideradas exatamente medidas absolutas devido a que os autores utilizaram a SCDD teórica e espectros de bremsstrahlung coletados a $100 \mathrm{keV}$, para calibrar a eficiência de fotopico do detetor (ver Eq. (2.81)). A linha contínua em cada gráfico da Fig. 8.36 representa a seção de choque teórica calculada a partir das tabulações de Seltzer e Berger [3] e Kissel et al. [5].

Em geral, devido à grande incerteza das SCDDs de Motz e Placious [6] e de Ambrose et al. [9], os três conjuntos comparados são compatíveis dentro de um desvio padrão na maioria do intervalo de $\kappa$ estudado. No caso do Al, observa-se a melhor concordância dos resultados, tanto entre os experimentos, quanto com a teoria. Para o Au, as SCDDs de Motz e Placious são mais próximas às SCDDs deste trabalho, e estão sistematicamente acima da teoria, enquanto o experimento de Ambrose e colaboradores apresenta uma tendência contrária: sistematicamente abaixo da teoria. Nos elementos Ag e Te, as SCDDs de Ambrose et al. [9] estão sistematicamente abaixo das medidas deste trabalho, alcançando melhor acordo no Te. 

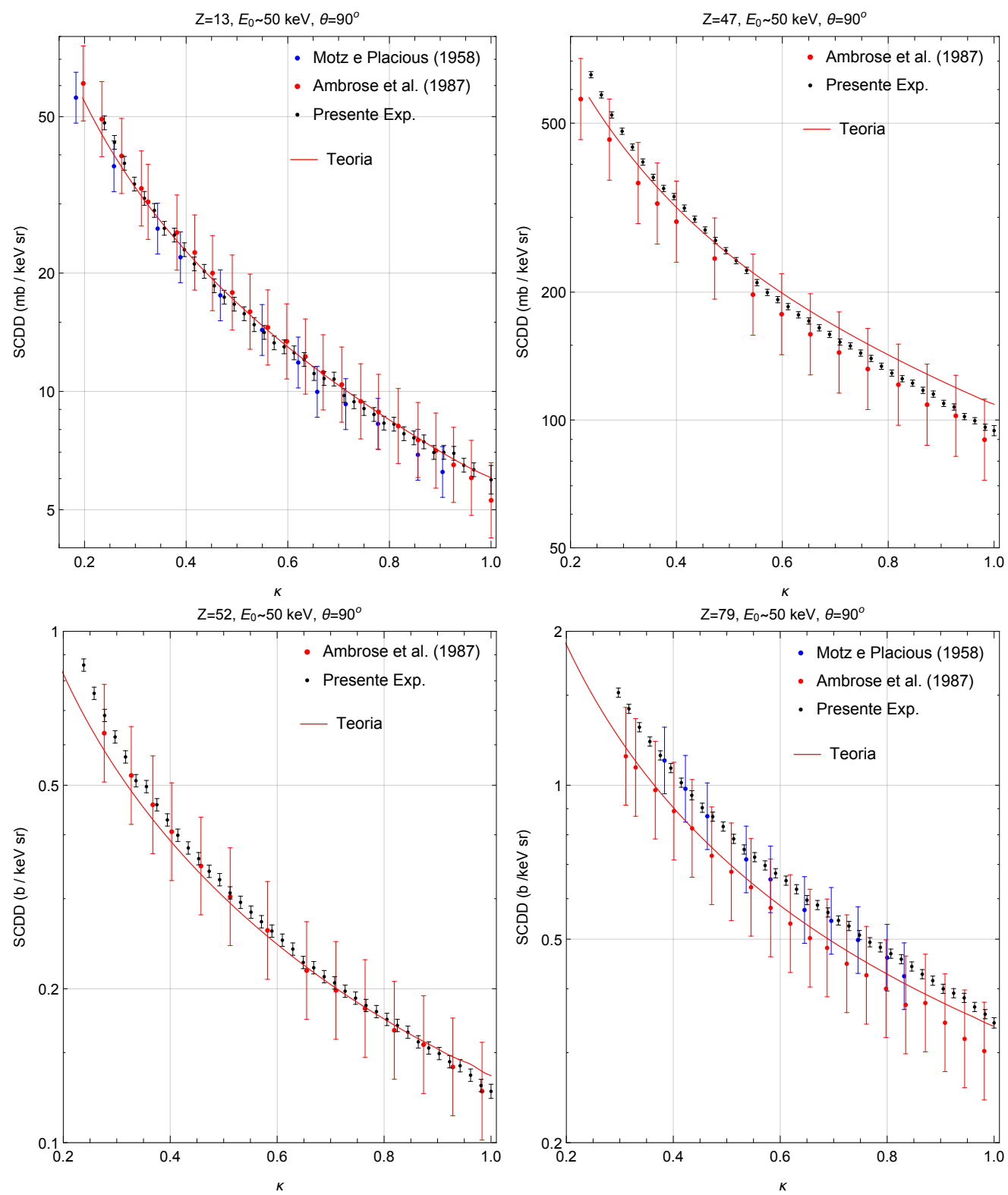

Figura 8.36: Comparação entre os resultados obtidos neste trabalho e os dados encontrados na literatura, para $E_{0} \sim 50 \mathrm{keV}, \theta=90^{\circ}$ e os elementos Al, Ag, Te e Au. 


\subsection{Discussão das tendências gerais observadas nas SCDDs experimentais}

A comparação das medidas realizadas com as SCDDs teóricas deixa em evidência alguns comportamentos gerais. Entre estes, destaca a sistemática observada para energias incidentes em torno dos $20 \mathrm{keV}$. Em todos os elementos estudados, salvo na Ag, observase que o experimento é superior as estimativas teóricas independentemente do ângulo de emissão dos fótons. Como foi discutido na seção 6.4, nos filmes de Ag, Te e Ta, os elétrons com valores $E_{0} \approx 20 \mathrm{keV}$ percorrem em média dentro do alvo, produto do espalhamento elástico, uma distância aproximadamente $5 \%$ maior que a espessura $d$ dos mesmos. No alvo de $\mathrm{Au}$, a porcentagem é de $\sim 3 \%$, enquanto nos alvos de $\mathrm{C}$ e $\mathrm{Al}$, esse aumento é menor que 0,5\%. Em nenhum caso esse fenômeno explicaria os resultados obtidos, em que a SCDD experimental é superior $20 \%$ em média à teoria.

Outro fato derivado da comparação indica que as medidas realizadas no entorno dos pontos da grade de interpolação teórica (2.69), entenda-se $Z=13,47$ e 79 , e $E_{0} \approx 50$ e $100 \mathrm{keV}$, apresentam a maior concordância com os dados deduzidos das tabulações de Seltzer e Berger [3] e Kissel et al. [5]. Perceba-se que a relação entre as SCDDs experimental e teórica conserva um comportamento aproximadamente constante, o com pequenas e monótonas variações da derivada, para a maioria dos valores de $\kappa$ estudados. Já nos elementos C, Te e Ta, as variações da referida razão são mais abruptas, e se observam (acima de tudo em Te e Ta) prominentes diferenças na forma espectral das SCDDs, que mudam em função do ângulo $\theta$ e da energia $E_{0}$. O fenômeno pode estar associado ao procedimento de cálculo da distribuição angular, em específico dos coeficiente $B_{n}\left(Z, E_{0}, \kappa\right)$ que multiplicam os polinômios de Legendre na Eq. (2.75) (ver Apêndice B). O resultado indica que a sensibilidade da metodologia proposta por Kissel e colaboradores [5] para a avaliação de $S\left(Z, E_{0}, \kappa, \theta\right)$ a partir da interpolação de $B_{n}\left(Z, E_{0}, \kappa\right)$ em $Z$, $E_{0}$ e $\kappa$, parece diminuir com o aumento tanto do número atômico $Z$ quanto da energia incidente $E_{0}$ dos elétrons.

No que se refere à distribuição angular, observou-se que para as energias $E_{0} \approx 20 \mathrm{e}$ $50 \mathrm{keV}$, os resultados da razão experimento/teoria correspondentes a $\theta=131^{\circ}$, em proporção com similar análise para os outros dois ângulos $\theta=35^{\circ}$ e $90^{\circ}$, situam-se sistematicamente acima.

Por outro lado, constata-se que de maneira geral no intervalo $0,8 \leqslant \kappa \leqslant 1$ se alcança o melhor acordo entre as SCDDs experimentais e as estimativas teóricas, independentemente do elemento analisado, o ângulo de emissão dos fótons e da energia de incidência dos elétrons. Nota-se que um grande número de resultados experimentais estão dentro da margem de erro teórica de $\pm 11 \%$ (faixa cinza nos gráficos das Figs. 8.10, 8.15, 8.20, 
8.25, 8.30, 8.35). Em trabalhos anteriores desenvolvidos no Laboratório do Acelerador Linear do IFUSP, para o estudo de seções de choque de ionização das camadas K e L por impacto de elétrons [121, 125, 126], a espessura dos alvos foi ajustada aplicando um método relativo que utiliza a SCDD teórica, e a região do tip do espectro de bremsstrahlung medido. As evidências encontradas na presente investigação em relação a essa faixa do espectro corroboram a validade do tratamento adotado pelos autores [121, 125, 126]. 



\section{Capítulo 9}

\section{Conclusões}

Em conformidade com o objetivo principal deste trabalho, foram determinadas de maneira absoluta SCDDs de produção de bremsstrahlung, para elétrons com energias cinéticas incidentes entre 20 e $100 \mathrm{keV}$, nos elementos C, Al, Ag, Te, Ta e Au, e os ângulos de emissão de fótons $35^{\circ}, 90^{\circ}$ e $131^{\circ}$. A incerteza relativa dos resultados finais esteve entre $3 \%$ e $5 \%$. Estas medidas superam consideravelmente a precisão dos poucos dados encontrados na literatura, e ao mesmo tempo, fornecem um amplo conjunto de SCDDs medidas pela primeira vez.

Nos diferentes capítulos foram expostos os procedimentos seguidos para determinar cada uma das grandezas envolvidas na SCDD.

Assim, foi desenvolvido um modelo analítico de função resposta de detetores planares de Ge, para fótons incidentes entre 10 e $100 \mathrm{keV}$, a partir de primeiros princípios. A FR analítica depende unicamente dos parâmetros geométricos do detetor. A AIR foi utilizada para calcular o espectro produzido pelo escape Compton dos fótons, conseguindo uma excelente concordância entre os espectros simulados com o código Penelope [43] e o modelo adotado. Neste sentido, o EAP pode ser descrito considerando somente uma interação inelástica no cristal de Ge. Os resultados desse modelo se publicaram na revista Radiation Physics and Chemistry [55]. Igualmente, a FR analítica foi estendida para se aplicar a detetores coaxiais. Por outro lado, estudou-se a influência na FR dos elementos do arranjo experimental, com maior ênfase nos efeitos introduzidos pelo colimador. As expressões obtidas na modelação, tanto para a FR intrínseca do detetor, quanto para a contribuição do arranjo, descreveram com sucesso os EAP medidos.

A calibração de largura dos detetores usados nas medidas de bremsstrahlung permitiu obter os parâmetros de dispersão Gaussiana associados à FR. Além disso, a partir do ajuste da componente de absorção total do modelo de FR aos pontos experimentais de 
eficiência de fotopico, foram determinados os ângulos sólidos subtendidos pelos detetores em relação ao alvo, considerando a transparência do colimador, com uma incerteza relativa inferior a $1 \%$. Os resultados desse procedimento possibilitaram também estimar a camada morta de Ge dos três detetores.

As medidas de retroespalhamento de partículas ${ }^{4} \mathrm{He}^{+2}$, realizadas sobre os alvos finos no acelerador tandem van de Graaff do LAMFI, e a posterior simulação dos espectros coletados com os programas SIMNRA [103] e MultiSIMNRA [104], permitiram ajustar a espessura das diferentes camadas dos mencionados alvos. A incerteza relativa relacionada às medidas de RBS esteve entre $2 \%$ e $4 \%$.

Foram identificadas e aplicadas correções aos espectros de bremsstrahlung coletados para subtrair as contagens devidas ao fundo radioativo, ao empilhamento de pulsos e à radiação produzida no substrato de C. A energia do feixe e sua dispersão se determinou por meio do ajuste dos parâmetros de uma função, proposta para descrever a região do tip, aos pontos experimentais medidos nessa faixa. Demostrou-se estatisticamente que a aplicação do modelo é apropriada para esse fim, e foi possível obter a energia média $E_{0}$ dos elétrons incidentes com uma precisão em torno dos $50 \mathrm{eV}$. Paralelamente se derivou a intensidade de fótons emitidos à máxima energia transferida na interação elétron-átomo. A deconvolução dos espectros se realizou entre esse último ponto $(\kappa=1)$ e aproximadamente os $12 \mathrm{keV}$ (acima da borda $\mathrm{K}$ do Ge, $\kappa=12 \mathrm{keV} / E_{0}$ ), com uma resolução energética de $1 \mathrm{keV}$, utilizando a $\mathrm{FR}$ analítica.

No caso das medidas realizadas para $\mathrm{Al}$ e $\mathrm{Au}$, com $E_{0} \approx 50 \mathrm{keV}$ e $\theta=90^{\circ}$, a comparação com os resultados experimentais publicados na literatura demonstrou compatibilidade com os dados de Motz e Placious [6], em vários pontos, dentro mesmo do desvio padrão das SCDDs do presente trabalho.

Sob outra perspectiva, a comparação com as estimativas teóricas evidenciou que a forma das SCDDs experimentais em função de $\kappa$, foi melhor descrita em aqueles elementos que constituem nodos: Al, Ag e Au, do que em aqueles calculados a partir da interpolação dos resultados dos primeiros, onde apareceram maiores discrepâncias nesse sentido.

Foi realizado um experimento cruzado onde se determinaram as SCIKs da Ag, para energias dos elétrons incidentes entre o limiar de ionização e $100 \mathrm{keV}$. Os valores obtidos mostraram total compatibilidade entre as medidas correspondentes aos três ângulos dentro de um desvio padrão, e também com resultados de experimentos anteriores como os de Vanin a colaboradores [121]. Em relação a esse último trabalho, os presentes resultados compreendem um intervalo de energia maior e superam a precisão precedente. 


\section{Apêndice A}

\section{Perfis de Compton do átomo de Ge}

As Figs. A.1 e A.2 apresentam uma comparação entre os PCs analíticos, utilizados no cálculo da SCDD de espalhamento Compton segundo a aproximação de Brusa e colaboradores [80], Eq. (4.37), e os PCs numéricos obtidos das tabulações de Biggs et al. [85]. As grandezas $J\left(p_{z}\right)$ e $p_{z}$ estão expressas em unidades atômicas (u.a).
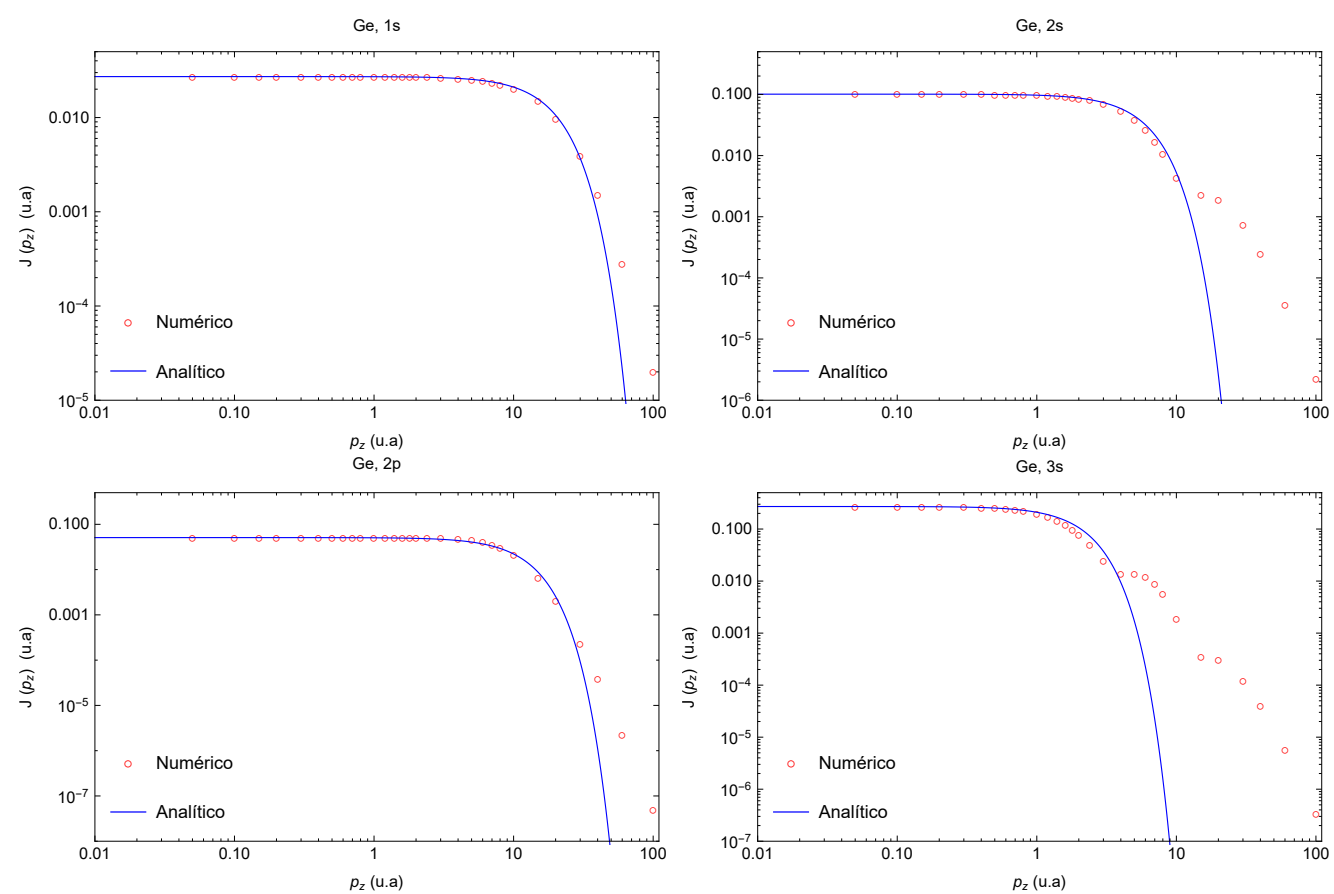

Figura A.1: Perfis de Compton para os orbitais 1s, 2s, 2p e 3s do átomo de Ge.

Comparação entre os valores tabulados por Biggs et al. e a aproximação analítica utilizada neste trabalho. 

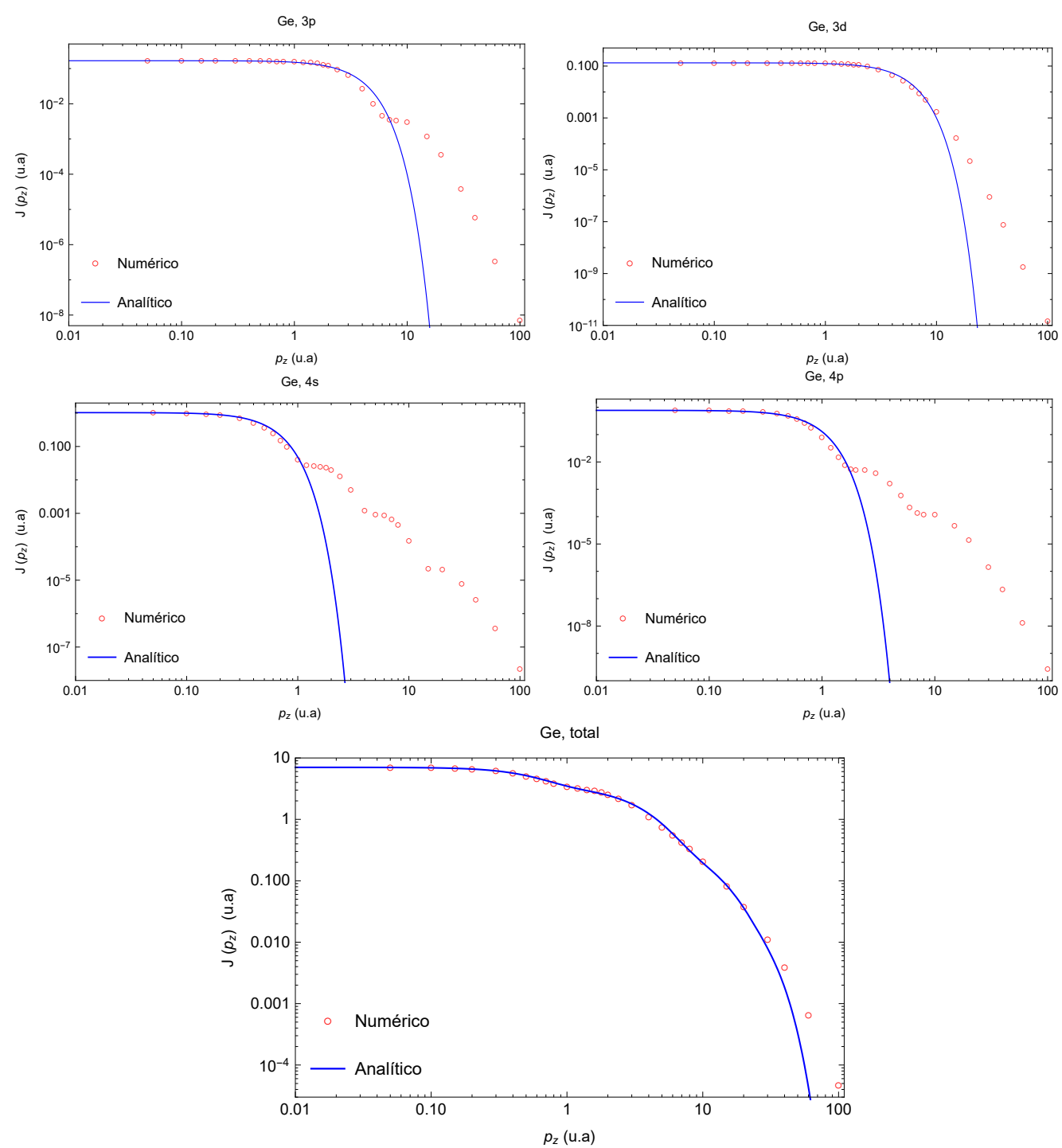

Figura A.2: Perfis de Compton para os orbitais 3p, 3d, 4s, 4p e contribuição de todos os orbitais (total) do átomo de Ge. Comparação entre os valores tabulados por Biggs et al. e a aproximação analítica utilizada neste trabalho. 


\section{Apêndice B}

\section{Coeficientes $B_{n}$ para o cálculo da distribuição angular de bremsstrahlung}

As Tabelas B.1, B.2 e B.3 apresentam os coeficientes $B_{n}$ calculados por Kissel e colaboradores [5] para a avaliação da distribuição angular de emissão de bremsstrahlung. No presente trabalho, esses pontos foram interpolados nas variáveis $E_{0}, Z$ e $\kappa$, a fim de computar a função $S\left(Z, E_{0}, \kappa, \theta\right)$ nos valores correspondentes aos experimentos. O software Mathematica [87] foi utilizado para realizar o mencionado procedimento por meio da rotina Interpolation, que ajusta polinômios de ordem 3 aos dados. Os parâmetros $E_{0}$ e $Z$ foram interpolados em escala logarítmica: $\log E_{0}$ e $\log Z$. 
TABela B.1: Coeficientes $B_{n}$ tabulados por Kissel e colaboradores para o cálculo da distribuição angular de bremsstrahlung (Parte 1).

\begin{tabular}{|c|c|c|c|c|c|c|c|}
\hline $\bar{E}_{E_{0}(\mathrm{keV})}$ & 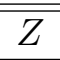 & $\kappa$ & 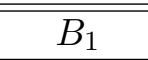 & $\overline{\bar{B} B_{2}}$ & $\overline{\bar{B} B_{3}}$ & $\overline{\bar{B} B_{4}}$ & 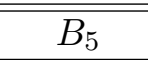 \\
\hline 1 & 2 & 0,00 & $-0,21151$ & 0,17523 & $-0,01017$ & 0,00003 & 0,00000 \\
\hline 1 & 2 & 0,60 & $-0,08591$ & $-0,59025$ & 0,01747 & 0,00062 & 0,00017 \\
\hline 1 & 2 & 0,80 & $-0,05333$ & $-0,80182$ & 0,02101 & 0,00069 & 0,00024 \\
\hline 1 & 2 & 0,95 & $-0,03139$ & $-0,95045$ & 0,02314 & 0,00041 & 0,00031 \\
\hline 1 & 8 & 0,00 & $-0,20609$ & 0,02951 & $-0,00243$ & 0,00001 & 0,00000 \\
\hline 1 & 8 & 0,60 & $-0,10855$ & $-0,56474$ & 0,02936 & 0,00252 & $-0,00014$ \\
\hline 1 & 8 & 0,80 & $-0,08863$ & $-0,69444$ & 0,03411 & 0,00325 & $-0,00017$ \\
\hline 1 & 8 & 0,95 & $-0,07663$ & $-0,77359$ & 0,03705 & 0,00253 & $-0,00010$ \\
\hline 1 & 13 & 0,00 & $-0,20121$ & $-0,10160$ & 0,00943 & $-0,00025$ & 0,00000 \\
\hline 1 & 13 & 0,60 & $-0,12071$ & $-0,59570$ & 0,04347 & 0,00141 & $-0,00014$ \\
\hline 1 & 13 & 0,80 & $-0,10563$ & $-0,68214$ & 0,04725 & $-0,00020$ & 0,00005 \\
\hline 1 & 13 & 0,95 & $-0,09638$ & $-0,73304$ & 0,04887 & 0,00008 & 0,00047 \\
\hline 1 & 47 & 0,00 & $-0,18676$ & $-0,49059$ & 0,07122 & $-0,00460$ & 0,00020 \\
\hline 1 & 47 & 0,60 & $-0,14121$ & $-0,71105$ & 0,09273 & $-0,00664$ & $-0,00058$ \\
\hline 1 & 47 & 0,80 & $-0,14223$ & $-0,71696$ & 0,09391 & $-0,00475$ & $-0,00025$ \\
\hline 1 & 47 & 0,95 & $-0,14796$ & $-0,72188$ & 0,09995 & $-0,00618$ & 0,00028 \\
\hline 1 & 79 & 0,00 & $-0,18581$ & $-0,51588$ & 0,06850 & $-0,00373$ & 0,00013 \\
\hline 1 & 79 & 0,60 & $-0,16373$ & $-0,58320$ & 0,07512 & $-0,00358$ & $-0,00215$ \\
\hline 1 & 79 & 0,80 & $-0,16957$ & $-0,55865$ & 0,07451 & $-0,00534$ & 0,00065 \\
\hline 1 & 79 & 0,95 & $-0,16884$ & $-0,55066$ & 0,07219 & $-0,00379$ & 0,00025 \\
\hline 1 & 92 & 0,00 & $-0,18850$ & $-0,44352$ & 0,05487 & $-0,00263$ & 0,00008 \\
\hline 1 & 92 & 0,60 & $-0,16409$ & $-0,52443$ & 0,06063 & $-0,00345$ & $-0,00254$ \\
\hline 1 & 92 & 0,80 & $-0,17369$ & $-0,50279$ & 0,06351 & $-0,00329$ & $-0,00128$ \\
\hline 1 & 92 & 0,95 & $-0,18934$ & $-0,49102$ & 0,06918 & $-0,00553$ & 0,00288 \\
\hline 5 & 2 & 0,00 & $-0,46909$ & 0,28693 & $-0,02403$ & $-0,00016$ & 0,00000 \\
\hline 5 & 2 & 0,60 & $-0,17091$ & $-0,56724$ & 0,02152 & 0,00117 & 0,00198 \\
\hline 5 & 2 & 0,80 & $-0,10490$ & $-0,78669$ & 0,02935 & 0,00171 & 0,00281 \\
\hline 5 & 2 & 0,95 & $-0,05779$ & $-0,94635$ & 0,03549 & 0,00082 & 0,00374 \\
\hline 5 & 8 & 0,00 & $-0,46403$ & 0,22465 & $-0,02137$ & 0,00006 & 0,00001 \\
\hline 5 & 8 & 0,60 & $-0,19769$ & $-0,54140$ & 0,03240 & 0,00123 & 0,00151 \\
\hline 5 & 8 & 0,80 & $-0,14082$ & $-0,72554$ & 0,04108 & 0,00142 & 0,00211 \\
\hline 5 & 8 & 0,95 & $-0,10116$ & $-0,85693$ & 0,04673 & 0,00064 & 0,00257 \\
\hline 5 & 13 & 0,00 & $-0,45993$ & 0,17414 & $-0,01814$ & 0,00016 & 0,00001 \\
\hline 5 & 13 & 0,60 & $-0,21740$ & $-0,52585$ & 0,04164 & 0,00112 & 0,00118 \\
\hline 5 & 13 & 0,80 & $-0,16772$ & $-0,68286$ & 0,05087 & 0,00106 & 0,00162 \\
\hline 5 & 13 & 0,95 & $-0,13338$ & $-0,79346$ & 0,05679 & 0,00048 & 0,00195 \\
\hline 5 & 47 & 0,00 & $-0,43671$ & $-0,11460$ & 0,03807 & $-0,00357$ & 0,00014 \\
\hline 5 & 47 & 0,60 & $-0,26991$ & $-0,59377$ & 0,11035 & $-0,00634$ & 0,00093 \\
\hline 5 & 47 & 0,80 & $-0,25158$ & $-0,65149$ & 0,11837 & $-0,00694$ & 0,00109 \\
\hline 5 & 47 & 0,95 & $-0,24229$ & $-0,68255$ & 0,12294 & $-0,00740$ & 0,00090 \\
\hline 5 & 79 & 0,00 & $-0,42259$ & $-0,29149$ & 0,09680 & $-0,01278$ & 0,00103 \\
\hline 5 & 79 & 0,60 & $-0,29272$ & $-0,64623$ & 0,16387 & $-0,01775$ & 0,00130 \\
\hline 5 & 79 & 0,80 & $-0,28554$ & $-0,67436$ & 0,17043 & $-0,01879$ & 0,00143 \\
\hline 5 & 79 & 0,95 & $-0,28354$ & $-0,68701$ & 0,17387 & $-0,01951$ & 0,00153 \\
\hline 5 & 92 & 0,00 & $-0,42096$ & $-0,31221$ & 0,10657 & $-0,01485$ & 0,00134 \\
\hline 5 & 92 & 0,60 & $-0,30060$ & $-0,64235$ & 0,16990 & $-0,01979$ & 0,00203 \\
\hline 5 & 92 & 0,80 & $-0,29657$ & $-0,65999$ & 0,17602 & $-0,02126$ & 0,00218 \\
\hline 5 & 92 & 0,95 & $-0,29615$ & $-0,66850$ & 0,17964 & $-0,02207$ & 0,00230 \\
\hline
\end{tabular}


TABEla B.2: Coeficientes $B_{n}$ tabulados por Kissel e colaboradores para o cálculo da distribuição angular de bremsstrahlung (Parte 2).

\begin{tabular}{|c|c|c|c|c|c|c|c|}
\hline $\bar{E}_{E_{0}(\mathrm{keV})}$ & 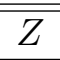 & $\kappa$ & 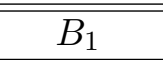 & $\overline{\bar{B} B_{2}}$ & $\overline{\bar{B} B_{3}}$ & $\overline{\bar{B} B_{4}}$ & 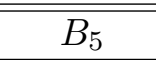 \\
\hline 10 & 2 & 0,00 & $-0,64745$ & 0,33754 & $-0,03338$ & $-0,00039$ & $-0,00001$ \\
\hline 10 & 2 & 0,60 & $-0,23918$ & $-0,54496$ & 0,02827 & 0,00177 & 0,00082 \\
\hline 10 & 2 & 0,80 & $-0,14794$ & $-0,77383$ & 0,04150 & 0,00243 & 0,00076 \\
\hline 10 & 2 & 0,95 & $-0,08019$ & $-0,94598$ & 0,05382 & 0,00254 & 0,00025 \\
\hline 10 & 8 & 0,00 & $-0,64274$ & 0,29526 & $-0,03257$ & $-0,00011$ & 0,00001 \\
\hline 10 & 8 & 0,60 & $-0,25757$ & $-0,53299$ & 0,03462 & 0,00283 & 0,00108 \\
\hline 10 & 8 & 0,80 & $-0,16976$ & $-0,74547$ & 0,04741 & 0,00312 & 0,00114 \\
\hline 10 & 8 & 0,95 & $-0,10898$ & $-0,90126$ & 0,06083 & 0,00291 & 0,00059 \\
\hline 10 & 13 & 0,00 & $-0,63911$ & 0,26258 & $-0,03085$ & 0,00007 & 0,00001 \\
\hline 10 & 13 & 0,60 & $-0,27257$ & $-0,52485$ & 0,04107 & 0,00319 & 0,00124 \\
\hline 10 & 13 & 0,80 & $-0,20420$ & $-0,70347$ & 0,06209 & 0,00180 & 0,00018 \\
\hline 10 & 13 & 0,95 & $-0,13446$ & $-0,86309$ & 0,06727 & 0,00288 & 0,00079 \\
\hline 10 & 47 & 0,00 & $-0,61683$ & 0,05977 & 0,00831 & $-0,00169$ & 0,00006 \\
\hline 10 & 47 & 0,60 & $-0,35398$ & $-0,51928$ & 0,10586 & $-0,00542$ & 0,00167 \\
\hline 10 & 47 & 0,80 & $-0,31981$ & $-0,60136$ & 0,11696 & $-0,00581$ & 0,00198 \\
\hline 10 & 47 & 0,95 & $-0,30162$ & $-0,64874$ & 0,12461 & $-0,00584$ & 0,00117 \\
\hline 10 & 79 & 0,00 & $-0,59910$ & $-0,10419$ & 0,07132 & $-0,01219$ & 0,00099 \\
\hline 10 & 79 & 0,60 & $-0,37796$ & $-0,59419$ & 0,17769 & $-0,02076$ & 0,00271 \\
\hline 10 & 79 & 0,80 & $-0,36008$ & $-0,64482$ & 0,18972 & $-0,02218$ & 0,00259 \\
\hline 10 & 79 & 0,95 & $-0,35977$ & $-0,64143$ & 0,19061 & $-0,02317$ & 0,00249 \\
\hline 10 & 92 & 0,00 & $-0,59531$ & $-0,13971$ & 0,09036 & $-0,01712$ & 0,00174 \\
\hline 10 & 92 & 0,60 & $-0,38559$ & $-0,60691$ & 0,19926 & $-0,02760$ & 0,00313 \\
\hline 10 & 92 & 0,80 & $-0,37116$ & $-0,64978$ & 0,21147 & $-0,02968$ & 0,00321 \\
\hline 10 & 92 & 0,95 & $-0,36553$ & $-0,67114$ & 0,21869 & $-0,03129$ & 0,00327 \\
\hline 50 & 2 & 0,00 & $-1,20197$ & 0,51537 & $-0,06593$ & $-0,00184$ & $-0,00019$ \\
\hline 50 & 2 & 0,60 & $-0,54605$ & $-0,37404$ & 0,04287 & 0,01007 & $-0,00421$ \\
\hline 50 & 2 & 0,80 & $-0,34511$ & $-0,66855$ & 0,07319 & 0,01287 & $-0,00304$ \\
\hline 50 & 2 & 0,95 & $-0,17483$ & $-0,91768$ & 0,09855 & 0,01435 & $-0,00168$ \\
\hline 50 & 8 & 0,00 & $-1,19966$ & 0,50321 & $-0,06800$ & $-0,00148$ & $-0,00011$ \\
\hline 50 & 8 & 0,60 & $-0,54835$ & $-0,38190$ & 0,04475 & 0,00560 & 0,00040 \\
\hline 50 & 8 & 0,80 & $-0,33237$ & $-0,69222$ & 0,07723 & 0,00615 & 0,00169 \\
\hline 50 & 8 & 0,95 & $-0,15975$ & $-0,94230$ & 0,10352 & 0,00689 & 0,00223 \\
\hline 50 & 13 & 0,00 & $-1,19819$ & 0,49529 & $-0,06898$ & $-0,00117$ & $-0,00006$ \\
\hline 50 & 13 & 0,60 & $-0,55550$ & $-0,38128$ & 0,04675 & 0,00324 & 0,00297 \\
\hline 50 & 13 & 0,80 & $-0,33571$ & $-0,69409$ & 0,08026 & 0,00267 & 0,00421 \\
\hline 50 & 13 & 0,95 & $-0,16599$ & $-0,93876$ & 0,10672 & 0,00302 & 0,00427 \\
\hline 50 & 47 & 0,00 & $-1,18844$ & 0,44269 & $-0,06298$ & 0,00036 & 0,00007 \\
\hline 50 & 47 & 0,60 & $-0,67464$ & $-0,29153$ & 0,08325 & 0,00302 & 0,00029 \\
\hline 50 & 47 & 0,80 & $-0,56025$ & $-0,46312$ & 0,11229 & 0,00550 & $-0,00040$ \\
\hline 50 & 47 & 0,95 & $-0,47901$ & $-0,58510$ & 0,13124 & 0,00781 & $-0,00057$ \\
\hline 50 & 79 & 0,00 & $-1,17382$ & 0,36052 & $-0,02617$ & $-0,00431$ & 0,00023 \\
\hline 50 & 79 & 0,60 & $-0,73369$ & $-0,31543$ & 0,16859 & $-0,01936$ & 0,00107 \\
\hline 50 & 79 & 0,80 & $-0,67500$ & $-0,41587$ & 0,19740 & $-0,01995$ & 0,00090 \\
\hline 50 & 79 & 0,95 & $-0,63897$ & $-0,47771$ & 0,21339 & $-0,01803$ & $-0,00003$ \\
\hline 50 & 92 & 0,00 & $-1,16731$ & 0,32281 & 0,00062 & $-0,01121$ & 0,00085 \\
\hline 50 & 92 & 0,60 & $-0,74292$ & $-0,35116$ & 0,21105 & $-0,03366$ & 0,00341 \\
\hline 50 & 92 & 0,80 & $-0,68958$ & $-0,44524$ & 0,24131 & $-0,03735$ & 0,00400 \\
\hline 50 & 92 & 0,95 & $-0,65976$ & $-0,49891$ & 0,25747 & $-0,03628$ & 0,00244 \\
\hline
\end{tabular}


TABEla B.3: Coeficientes $B_{n}$ tabulados por Kissel e colaboradores para o cálculo da distribuição angular de bremsstrahlung (Parte 3).

\begin{tabular}{|c|c|c|c|c|c|c|c|}
\hline$E_{0}(\mathrm{keV})$ & $\bar{Z}$ & $\kappa$ & $\overline{B_{1}}$ & $\overline{B_{2}}$ & $\overline{B_{3}}$ & $\overline{B_{4}}$ & $\overline{B_{5}}$ \\
\hline 100 & 2 & 0,00 & $-1,42451$ & 0,61636 & $-0,08210$ & $-0,00300$ & $-0,00046$ \\
\hline 100 & 2 & 0,60 & $-0,77465$ & $-0,19315$ & 0,03815 & 0,00553 & 0,00042 \\
\hline 100 & 2 & 0,80 & $-0,50828$ & $-0,53707$ & 0,08307 & 0,00163 & 0,00649 \\
\hline 100 & 2 & 0,95 & $-0,24203$ & $-0,88026$ & 0,11905 & 0,00809 & 0,00818 \\
\hline 100 & 8 & 0,00 & $-1,42436$ & 0,61392 & $-0,08472$ & $-0,00270$ & $-0,00036$ \\
\hline 100 & 8 & 0,60 & $-0,77861$ & $-0,19025$ & 0,03828 & 0,00668 & 0,00020 \\
\hline 100 & 8 & 0,80 & $-0,51479$ & $-0,53058$ & 0,08051 & 0,00816 & 0,00321 \\
\hline 100 & 8 & 0,95 & $-0,25359$ & $-0,86753$ & 0,11950 & 0,00833 & 0,00777 \\
\hline 100 & 13 & 0,00 & $-1,42425$ & 0,61225 & $-0,08631$ & $-0,00243$ & $-0,00029$ \\
\hline 100 & 13 & 0,60 & $-0,78591$ & $-0,18410$ & 0,03835 & 0,00764 & 0,00001 \\
\hline 100 & 13 & 0,80 & $-0,52902$ & $-0,51607$ & 0,07881 & 0,01228 & 0,00112 \\
\hline 100 & 13 & 0,95 & $-0,27653$ & $-0,84268$ & 0,11936 & 0,00906 & 0,00716 \\
\hline 100 & 47 & 0,00 & $-1,42277$ & 0,59773 & $-0,09033$ & $-0,00011$ & $-0,00004$ \\
\hline 100 & 47 & 0,60 & $-0,89289$ & $-0,10235$ & 0,05388 & 0,00923 & $-0,00125$ \\
\hline 100 & 47 & 0,80 & $-0,74775$ & $-0,30489$ & 0,09096 & 0,01277 & $-0,00165$ \\
\hline 100 & 47 & 0,95 & $-0,61492$ & $-0,49051$ & 0,12546 & 0,01565 & $-0,00017$ \\
\hline 100 & 79 & 0,00 & $-1,41839$ & 0,56396 & $-0,07703$ & 0,00021 & $-0,00025$ \\
\hline 100 & 79 & 0,60 & $-0,96388$ & $-0,09604$ & 0,11911 & $-0,00560$ & $-0,00195$ \\
\hline 100 & 79 & 0,80 & $-0,87542$ & $-0,23057$ & 0,15553 & $-0,00498$ & $-0,00218$ \\
\hline 100 & 79 & 0,95 & $-0,80929$ & $-0,33104$ & 0,18196 & $-0,00168$ & $-0,00309$ \\
\hline 100 & 92 & 0,00 & $-1,41536$ & 0,54243 & $-0,06101$ & $-0,00304$ & $-0,00026$ \\
\hline 100 & 92 & 0,60 & $-0,98320$ & 0,10932 & 0,15389 & $-0,01429$ & $-0,00214$ \\
\hline 100 & 92 & 0,80 & $-0,90427$ & $-0,23127$ & 0,18990 & $-0,01361$ & $-0,00282$ \\
\hline 100 & 92 & 0,95 & $-0,85250$ & $-0,31090$ & 0,21377 & $-0,01318$ & $-0,00332$ \\
\hline 500 & 2 & 0,00 & $-1,65989$ & 0,76417 & $-0,09205$ & $-0,00614$ & $-0,00302$ \\
\hline 500 & 2 & 0,60 & $-1,40681$ & 0,44048 & $-0,04181$ & 0,01998 & $-0,00530$ \\
\hline 500 & 2 & 0,80 & $-1,19734$ & 0,17722 & $-0,00426$ & 0,03628 & $-0,00574$ \\
\hline 500 & 2 & 0,95 & $-0,69097$ & $-0,48385$ & 0,11209 & 0,07157 & $-0,00526$ \\
\hline 500 & 8 & 0,00 & $-1,66151$ & 0,76688 & $-0,09311$ & $-0,00646$ & $-0,00288$ \\
\hline 500 & 8 & 0,60 & $-1,40952$ & 0,44396 & $-0,04223$ & 0,01858 & $-0,00451$ \\
\hline 500 & 8 & 0,80 & $-1,20107$ & 0,17796 & 0,00424 & 0,02720 & $-0,00227$ \\
\hline 500 & 8 & 0,95 & $-0,70839$ & $-0,47208$ & 0,14536 & 0,02431 & 0,01415 \\
\hline 500 & 13 & 0,00 & $-1,66258$ & 0,76870 & $-0,09384$ & $-0,00668$ & $-0,00276$ \\
\hline 500 & 13 & 0,60 & $-1,41210$ & 0,44717 & $-0,04285$ & 0,01781 & $-0,00396$ \\
\hline 500 & 13 & 0,80 & $-1,20612$ & 0,18161 & 0,00879 & 0,02174 & $-0,00008$ \\
\hline 500 & 13 & 0,95 & $-0,73114$ & $-0,45009$ & 0,16207 & $-0,00246$ & 0,02485 \\
\hline 500 & 47 & 0,00 & $-1,67232$ & 0,78575 & $-0,10192$ & $-0,00728$ & $-0,00188$ \\
\hline 500 & 47 & 0,60 & $-1,44395$ & 0,48743 & $-0,05817$ & 0,02331 & $-0,00405$ \\
\hline 500 & 47 & 0,80 & $-1,28622$ & 0,27868 & $-0,01495$ & 0,02729 & $-0,00036$ \\
\hline 500 & 47 & 0,95 & $-1,02201$ & $-0,08890$ & 0,07938 & 0,02868 & 0,00611 \\
\hline 500 & 79 & 0,00 & $-1,69196$ & 0,82154 & $-0,12197$ & $-0,00442$ & $-0,00139$ \\
\hline 500 & 79 & 0,60 & $-1,50242$ & 0,56789 & $-0,09399$ & 0,04172 & $-0,01043$ \\
\hline 500 & 79 & 0,80 & $-1,40535$ & 0,44032 & $-0,07802$ & 0,05738 & $-0,01164$ \\
\hline 500 & 79 & 0,95 & $-1,29395$ & 0,28137 & $-0,04155$ & 0,07042 & $-0,01385$ \\
\hline 500 & 92 & 0,00 & $-1,70461$ & 0,84508 & $-0,13614$ & 0,00111 & $-0,00165$ \\
\hline 500 & 92 & 0,60 & $-1,53042$ & 0,60714 & $-0,11171$ & 0,05089 & $-0,01391$ \\
\hline 500 & 92 & 0,80 & $-1,45859$ & 0,51386 & $-0,10730$ & 0,07119 & $-0,01719$ \\
\hline 500 & 92 & 0,95 & $-1,39449$ & 0,42246 & $-0,08480$ & 0,07464 & $-0,01593$ \\
\hline
\end{tabular}




\section{Referências Bibliográficas}

[1] E. Haug and W. Nakel. The Elementary Process of Bremsstrahlung. World Scientific Lectures Notes in Physics, World Scientific Publishing Co. Pte. Ltd, Singapore, 73,2004 .

[2] R. H. Pratt, H. K. Tseng, C. M. Lee, L. Kissel, C. MacCallum, and M. Riley. Bremsstrahlung energy spectra from electrons of kinetic energy $1 \mathrm{keV} \leqslant T_{1} \leqslant 2000$ $\mathrm{keV}$ incident on neutral atoms $2 \leqslant Z \leqslant 92$. Atomic Data and Nuclear Data Tables, 20(2):175-209, 1977.

[3] S. M. Seltzer and M. J. Berger. Bremsstrahlung energy spectra from electrons with kinetic energy $1 \mathrm{keV}-10 \mathrm{GeV}$ incident on screened and orbital electrons of neutral atoms with $Z=1-100$. Atomic Data and Nuclear Data Tables, 35(3): 345-418, 1986.

[4] H. K. Tseng, R. H. Pratt, and C. M. Lee. Electron bremsstrahlung angular distribution in the 1-500 keV energy range. Physical Review A, 19(1):187-195, 1979.

[5] L. Kissel, C. A. Quarles, and R. H. Pratt. Shape functions for atomic field bremsstrahlung from electrons of kinetic energy $1-500 \mathrm{keV}$ on selected neutral atoms $2 \leqslant Z \leqslant 92$. Atomic Data and Nuclear Data Tables, 28(3):381-460, 1983.

[6] J. W. Motz and R. C. Placious. Bremsstrahlung cross-section measurements for $50 \mathrm{keV}$ electrons. Physical Review, 109(2):235-242, 1958.

[7] D. H. Rester, N. Edmonson, and Q. Peasley. Bremsstrahlung cross sections for $\mathrm{Al}$ and $\mathrm{Au}$ for incident electron energies of 0.05 and $0.20 \mathrm{MeV}$. Physical Review A, 2 (6):2190-2196, 1970.

[8] C. A. Quarles and D. B. Heroy. Atomic field bremsstrahlung from 50-140-keV electrons. Physical Review A, 24(1):48-54, 1981.

[9] R. Ambrose, J. C. Altman, and C. A. Quarles. Atomic field bremsstrahlung cross section for incident electron energy from $50-150 \mathrm{keV}$ for atomic numbers from 6 to 92. Physical Review A, 35(2):529-539, 1987. 
[10] N. L. Pino. Bremsstrahlung de elétrons no microtron do IFUSP: medidas, simulação e proposta de aplicação ao estudo da ressonância fluorescente nuclear. Tese de Doutorado, Instituto de Física, Universidade de São Paulo, 2011.

[11] V. Jahnke. Medidas das seções de choque de ionização da camada K de Au e Bi por impacto de elétrons. Dissertação de Mestrado, Instituto de Física, Universidade de São Paulo, 2012.

[12] S. F. de Barros. Ionização atômica da camada L de Au e Ta por impacto de elétrons com o acelerador Microton de São Paulo. Dissertação de Mestrado, Instituto de Física, Universidade de São Paulo, 2014.

[13] O. C. B. dos Santos. Medida da seção de choque de ionização atômica da camada K de Ta e Te por impacto de elétrons com energias do limiar até $100 \mathrm{keV}$. Dissertação de Mestrado, Instituto de Física, Universidade de São Paulo, 2016.

[14] J. J. Sakurai and J. J. Napolitano. Modern Quantum Mechanics. Addison-Wesley 1301 Sansome Street, San Francisco, CA 94111, 2014.

[15] W. Nakel. The elementary process of bremsstrahlung. Physics Reports, 243:317353, 1994.

[16] S. M. Seltzer and M. J. Berger. Bremsstrahlung spectra from electron interactions with screened atomic nuclei and orbital electrons. Nuclear Instruments and Methods B, 12:95-134, 1985.

[17] H. K. Tseng. Nonrelativistic calculation of an elementary process of electron bremsstrahlung from atoms. Physical Review A, 40(12):6826-6830, 1989.

[18] E. Haug. Bremsstrahlung cross-section with screening and Coulomb corrections at high energies. Radiation Physics and Chemistry, 77:207-214, 2008.

[19] E. Haug. Cross section of electron-nucleus bremsstrahlung near the shortwavelength limit. The European Physical Journal D, 58:297-310, 2010.

[20] F. Salvat, J. D. Martínez, R. Mayol, and J. Parellada. Analytical Dirac-HartreeFock-Slater screening function for atoms $(Z=1-92)$. Physical Review A, 36(2): 467-474, 1987.

[21] M. Abramowitz and I. A. Stegun. Handbook of Mathematical Functions: with Formulas, Graphs and Mathematical Tables. National Bureau of Standards, Dover Publications, Inc., New York, 2012.

[22] H. K. Tseng. Energy-angle distributions of bremsstrahlung from intermediateenergy electrons on atoms. Physical Review A, 56(4):2868-2871, 1997. 
[23] H. K. Tseng. Relativistic calculation of an elementary process of electron bremsstrahlung from atoms. Journal of Physics B, 30:317-321, 1997.

[24] H. W. Koch and J. W. Motz. Bremsstrahlung cross section formulas and related data. Reviews of Modern Physics, 31(4):920-956, 1959.

[25] G. Elwert and E. Haug. Calculation of bremsstrahlung cross sections with Sommerfeld-Maue eigenfunctions. Physical Review, 183(1):90-105, 1969.

[26] H. K. Tseng and R. H. Pratt. Exact screened calculation of atomic field bremsstrahlung. Physical Review A, 3(1):100-115, 1971.

[27] B. H. Bransden and C. J. Joachain. Physics of Atoms and Molecules. Pearson Education Limited, Malaysia, 2nd Edition, 2003.

[28] P. Csavinszky. Approximate variational solution of the Thomas-Fermi equation for atoms. Physical Review, 166(1):53-57, 1968.

[29] D. A. Liberman, D. T. Cromer, and J. T. Waber. Relativistic self-consistent field Program for atoms and ions. Computer Physics Communications, 2:107-113, 1971.

[30] C. M. Lee, L. Kissell, R. H. Pratt, and H. K. Tseng. Electron bremsstrahlung spectrum, 1-500 keV. Physical Review A, 13(5):1714-1727, 1976.

[31] E. Haug. Bremsstrahlung and pair production in the field of free-electrons. Zeitschrift für Naturforschung A, 30(9):1099-1113, 1975.

[32] J. N. Chapman, C. C. Gray, B. W. Robertson, and W. A. P. Nicholson. X-Ray production in thin films by electrons with energies between 40 and $100 \mathrm{keV}$. X-Ray Spectrometry, 12(4):153-162, 1983.

[33] R. Ambrose, D. L. Kahler, H. E. Lehtihet, and C. A. Quarles. Atomic field bremsstrahlung at zero degrees. Nuclear Instruments and Methods B, 57:330-333, 1991.

[34] V. Ambrose, R. Ambrose, and C. A. Quarles. Thin target bremsstrahlung at $0^{\circ}$ from $50 \mathrm{keV}$ electrons. Nuclear Instruments and Methods B, 124:457-463, 1997.

[35] C. A. Quarles. Bremsstrahlung: an experimentalist's personal perspective on the postmodern era. Radiation Physics and Chemistry, 59:159-169, 2000.

[36] R. Shanker. Experimental results on 2-30 keV bremsstrahlung from thick and thin targets. Radiation Physics and Chemistry, 75:1176-1186, 2006.

[37] S. Williams and Q. A. Quarles. Absolute bremsstrahlung yields at $135^{\circ}$ from 53-keV electrons on gold film targets. Physical Review A, 78:062704 (9 pp), 2008. 
[38] W. J. Gallagher and S. J. Cipolla. A model-based eficiency calibration of a $\mathrm{Si}(\mathrm{Li})$ detector in the energy region from 3 to $140 \mathrm{keV}$. Nuclear Instruments and Methods, 122:405-414, 1974.

[39] J. C. Altman, R. Ambrose, C. A. Quarles, and G. L. Westbrook. Measurement of the efficiency of solid state detectors using atomic-field bremsstrahlung. Nuclear Instruments and Methods B, 24/25:1028-1030, 1987.

[40] N. F. Mott and H. S. W. Massey. The Theory of Atomic Collisions. Oxford University Press, London, 3rd Edition, 1965.

[41] L. Tain, J. Zhu, M. Liu, and Z. An. Bremsstrahlung spectra produced by kilovolt electron impact on thick targets. Nuclear Instruments and Methods B, 267: 3495-3499, 2009.

[42] S. Czarnecki, D. Gonzales, and S. Williams. Comparison of the bremsstrahlung absolute probability densities produced by experiment and PENELOPE for lowenergy (keV) electrons incident on solid silver. X-Ray Spectrometry, 45:100-102, 2016.

[43] F. Salvat, J. M. Fernández-Varea, and J. Sempau. PENELOPE, a Code System for Monte Carlo Simulation of Electron and Photon Transport. NEA/OECD, Issy-les-Moulineaux, 2011.

[44] A. S. Dhaliwal. Bremsstrahlung spectra produced by ${ }^{89} \mathrm{Sr}$ beta particles in thick targets. X-Ray Spectrometry, 32:367-372, 2003.

[45] T. Singh, K. S. Kahlon, and S. Dhaliwal. Thick target bremsstrahlung spectra produced by ${ }^{204} \mathrm{Tl}$ beta particles in the photon energy region of $10-30 \mathrm{keV}$. Journal of Physics B, 41:235001 (6pp), 2008.

[46] T. Singh and K. S. Kahlon andA. S. Dhaliwal. Total bremsstrahlung spectra in targets of $\mathrm{Al}, \mathrm{Ti}, \mathrm{Sn}$ and $\mathrm{Pb}$ produced by complete absorption of ${ }^{90} \mathrm{Sr} \beta$ particles. X-Ray Spectrometry, 39:291-295, 2010.

[47] W. R. Leo. Techniques for Nuclear and Particle Physics Experiments: a how-to approach. Springer-Verlag, New York, 2nd Edition, 1994.

[48] N. Ueta, W. G. P. Engel, and J. J. G. Leandro. Confecção de Alvos Nuclares. Instituto de Física da Universidade de São Paulo, 2007.

[49] RBS. Chemical Products R. Borghgraef S.A. aceso em 30 de junho de 2016. URL http://www.rbs-cp.be/.

[50] J. E. Mahan. Physical Vapor Deposition of Thin Films. Wiley, New York, 2000. 
[51] ORTEC. ORTEC Advance Measurement Technology, Oak Ridge, USA. aceso em 30 de abril de 2016. URL http://www.ortec-online.com/.

[52] MAESTRO. A65-B32-MAESTRO-32-MCA. Ortec Catalogues. Aceso em 30 de abril de 2016. URL http://www.ortec-online.com/.

[53] O. A. M. Helene and V. R. Vanin. Tratamento Estatístico de Dados em Física Experimental. Edgard Blucher, São Paulo, 1991.

[54] O. Helene, V. R. Vanin, Z. O. Guimarães-Filho, and C. Takiya. Variances, covariances and artifacts in image deconvolution. Nuclear Instruments and Methods A, 580:1466-1473, 2007.

[55] J. A. García-Alvarez, N. L. Maidana, V. R. Vanin, and J. M. Fernández-Varea. Analytical response function for planar Ge detectors. Radiation Physics and Chemistry, 121:23-34, 2016.

[56] S. M. Seltzer. Calculated response function of intrinsic germanium detectors to narrow beams of photons with energies up to $\sim 300 \mathrm{keV}$. Nuclear Instruments and Methods, 188:133-151, 1981.

[57] R. P. Gardner and J. M. Doster. Treatment of the $\mathrm{Si}(\mathrm{Li})$ detector response as a probability density function. Nuclear Instruments and Methods, 198:381-390, 1982.

[58] A. M. Yacout, R. P. Gardner, and K. Verghese. A semi-empirical model for the X-ray $\mathrm{Si}(\mathrm{Li})$ detector response function. Nuclear Instruments and Methods A, 243: 121-130, 1986.

[59] Y. Inagaki, K. Shima, and H. Maezawa. Response function of a $\mathrm{Si}(\mathrm{Li})$ detector for 1.3-4.0 keV monochromatic photons. Nuclear Instruments and Methods B, 27: 353-359, 1987.

[60] Y. Jin, R. P. Gardner, and K. Verghese. A semi-empirical model for the gammaray response function of germanium detectors based on fundamental interaction mechanism. Nuclear Instruments and Methods A, 242:416-426, 1986.

[61] M. C. Lee, K. Verghese, and R. P. Gardner. Extension of the semiempirical germanium detector response function to low energy gamma rays. Nuclear Instruments and Methods A, 262:430-438, 1987.

[62] J. Y. Zevallos-Chávez, M. T. F. da Cruz, M. N. Martins, V. P. Likhachev, Z. B. Zamboni, S. P. Camargo, F. A. Genezini, J. A. G. Medeiros, and M. M. Indi. Response function of a germanium detector to photon energies between 6 and 120 keV. Nuclear Instruments and Methods A, 457:212-219, 2001. 
[63] N. V. de Castro and R. J. A. Lévesque. Photopeak and double escape peak efficiencies of germanium lithium drift detectors. Nuclear Instruments and Methods, 46:325-332, 1967.

[64] J. X. Wang. Monte Carlo simulation of the response function of $\mathrm{Si}(\mathrm{Li})$ detectors to photon monoenergetic X-ray. Nuclear Instruments and Methods B, 54:499-506, 1991.

[65] J. L. Campbell, L. McDonald, T. Hopman, and T. Papp. Simulations of Si(Li) x-ray detector response. X-Ray Spectrometry, 30:230-241, 2001.

[66] J. M. O'Meara and J. L. Campbell. Corrections to the conventional approach to $\mathrm{Si}(\mathrm{Li})$ detector efficiency. X-Ray Spectrometry, 33:146-157, 2004.

[67] J. E. Fernandez and V. Scot. Simulation of the detector response function with the code MCSHAPE. Radiation Physics and Chemistry, 78:882-887, 2009.

[68] S. Hurtado, M. García-León, and R. García-Tenorio. Monte Carlo simulation of the response of a germanium detector for low-level spectrometry measurements using GEANT4. Applied Radiation and Isotopes, 61:139-143, 2004.

[69] C. M. Salgado, C. C. Conti, and P. H. B. Becker. Determination of HPGe detector response using MCNP5 for 20-150 keV X-rays. Applied Radiation and Isotopes, 64:700-705, 2006.

[70] A. Tomal, D. M. Cunha, M. Antoniassi, and M. E. Poletti. Response functions of $\mathrm{Si}(\mathrm{Li}), \mathrm{SDD}$ and CdTe detectors for mammographic x-ray spectroscopy. Applied Radiation and Isotopes, 70:1355-1359, 2012.

[71] A. Tomal, J. C. Santos, P. R. Costa, A. H. Lopez Gonzales, and M. E. Poletti. Monte Carlo simulation of the response functions of CdTe detectors to be applied in x-ray spectroscopy. Applied Radiation and Isotopes, 100:32-37, 2015.

[72] S. Goto. Response function of a $\mathrm{Si}(\mathrm{Li})$ detector for photon energies from 1 to 10 keV. Nuclear Instruments and Methods A, 333:452-457, 1993.

[73] B. G. Lowe. An analytical description of low-energy X-ray spectra in $\mathrm{Si}(\mathrm{Li})$ and HPGe detectors. Nuclear Instruments and Methods A, 439:247-261, 2000.

[74] F. Scholze and M. Procop. Measurement of detection efficiency and response functions for an $\mathrm{Si}(\mathrm{Li})$ x-ray spectrometer in the range 0.1-5 keV. X-Ray Spectrometry, 30:69-76, 2001.

[75] F. Scholze and M. Procop. Modelling the response function of energy dispersive X-ray spectrometers with silicon detectors. X-Ray Spectrometry, 38:312-321, 2009. 
[76] X-5 Monte Carlo Team. MCNP-A General Monte Carlo N-Particle Transport Code, Version 5, volume I: Overview and Theory, LA-UR-03-1987. Los Alamos National Laboratory, 2003.

[77] S. Agostinelli et al. Geant4-a simulation toolkit. Nuclear Instruments and Methods A, 506:250-303, 2003.

[78] O. Klein and Y. Nishina. About the scattering of radiation by free electrons after the new relativistic quantum dynamics of Dirac. Zeitschrift für Physik, 52:853-868, 1929.

[79] R. Ribberfors and K. F. Berggren. Incoherent x-ray scattering functions and cross sections by means of a pocket calculator. Physical Review A, 26:3325-3333, 1982.

[80] D. Brusa, G. Stutz, J. A. Riveros, J. M. Fernández-Varea, and F. Salvat. Fast sampling algorithm for the simulation of photon Compton scattering. Nuclear Instruments and Methods A, 379:167-175, 1996.

[81] G. F. Knoll. Radiation Detection and Measurement. 3rd edition, Wiley and Sons, 2000 .

[82] J. M. Sampaio, T. I. Madeira, J. P. Marques, F. Parente, A. M. Costa, P. Indelicato, J. P. Santos, M. C. Lépy, and Y. Ménesguen. Approaches for theoretical and experimental determinations of K-shell decay rates and fluorescence yields in Ge. Physical Review A, 89:012512 (8 pp), 2014.

[83] R. D. Deslattes, E. G. Kessler Jr, P. Indelicato, L. de Billy, E. Lindroth, and J. Anton. X-ray transition energies: new approach to a comprehensive evaluation. Reviews of Modern Physics, 75:35-99, 2003.

[84] M. J. Berger, J. H. Hubbell, S. M. Seltzer, J. Chang, J. S. Coursey, R. Sukumar, D. S. Zucker, and K. Olsen. XCOM: Photon Cross Section Database (version 1.5). National Institute of Standards and Technology, Gaithersburg, MD., 2010. URL http://physics.nist.gov/xcom.

[85] F. Biggs, L. B. Mendelsohn, and J. B. Mann. Hartree-Fock Compton profiles for the elements. Atomic Data and Nuclear Data Tables, 16:201-309, 1975.

[86] L. B. Mendelsohn and B. J. Bloch. Atomic $L$-shell Compton profiles: Calculations for atoms and comparison with experiment. Physical Review A, 12:551-555, 1975.

[87] Mathematica 10.0.1. 2014. URL http://www.wolfram.com/mathematica.

[88] J. W. Cooper and S. T. Manson. Photo ionization in the soft X-ray range: angular distribution of photo-electrons and interpretation in terms of subshell structure. Physical Review, 177:157-164, 1969. 
[89] A. Jablonski. Angular distribution of photoelectrons emitted by the laboratory soft and hard X-ray radiation sources. Journal of Electron Spectroscopy and Related Phenomena, 189:81-95, 2013.

[90] L. Sabbatucci and F. Salvat. Theory and calculation of the atomic photoeffect. Radiation Physics and Chemistry, 121:122-140, 2016.

[91] F. Sauter. The atomical photo effect in the K-shell according to the relativistic wave mechanics of Dirac. Annalen der Physik, 11:454-488, 1931.

[92] J. M. Fernández-Varea, P. Andreo, and T. Tabata. Detuour factors in water and plastic phantoms and their use for range and depth scaling in electron-beam dosimetry. Physics in Medicine and Biology, 41:1119-1139, 1996.

[93] J. H. Hubbell, W. J. Veigele, E. A. Briggs, R. T. Brown, D. T. Cromer, and R. J. Howerton. Atomic form factors, incoherent scattering functions, and photon scattering cross sections. Journal of Physical and Chemical Reference Data, 4: 471-538, 1975.

[94] J. Sempau, A. Badal, and L. Brualla. A PENELOPE-based system for the automated Monte Carlo simulation of clinacs and voxelized geometries - application to far-from-axis fields. Medical Physics, 38:5887-5895, 2011.

[95] N. L. Maidana, V. R. Vanin, V. Jahnke, J. M. Fernández-Varea, M. N. Martins, and L. Brualla. Efficiency calibration of x-ray HPGe detectors for photons with energies above the Ge K binding energy. Nuclear Instruments and Methods A, 729:371-380, 2013.

[96] F. Gao, L. W. Campbell, R. Devanathan, Y. L. Xie, Y. Zhang, A. J. Peurrung, and W. J. Weber. Gamma-ray interaction in Ge: A Monte Carlo simulation. Nuclear Instruments and Methods B, 255:286-290, 2007.

[97] M. Majer, M. Budanec, G. Jerbić-Zorc, S. Pašić, M. Uroić, B. Vuković, and K. Ilakovac. Effects of near-source photon scattering at the energy of $60 \mathrm{keV}$. Nuclear Instruments and Methods A, 524:227 - 235, 2004.

[98] M. Majer, M. Uroić, T. Bokulić, S. Pašić, B. Vuković, and K. Ilakovac. Effects of near-source Compton scattering in low-energy $\gamma$-ray spectra. Nuclear Instruments and Methods A, 555:243 - 250, 2005.

[99] M. M. Bé, V. Chisté, C. Dulieu, E. Browne, V. Chechev, N. Kusmenko, R. Helmer, A. Nichols, E. Schonfeld, and R. Dersch. Table of Radionuclides. Bureau International des Poids et Mesures, Pavillon de Breteuil, F-92310, Sèvres, France, 2004. 
[100] M. C. Lépy, J. Plagnard, and L. Ferreux. Measurement of ${ }^{241}$ Am L X-ray emission probabilities. Applied Radiation and Isotopes, 66:715-721, 2008.

[101] LAMFI. Instituto de Física da Universidade de São Paulo. Aceso em 29 de abril de 2016. URL http://www2.if.usp.br/ lamfi/.

[102] W. K. Chu, J. W. Mayer, and M. A. Nicolet. Backscattering Spectrometry. Academic Press, New York, 1978.

[103] M. Mayer. SIMNRA User's Guide. Max-Planck-Institut für Plasmaphysik, Aceso em 29 de abril de 2016. URL http://home.rzg.mpg.de/ mam/.

[104] T. F. Silva, C. L. Rodrigues, M. Mayer, M. V. Moro, G. F. Trindade, F. R. Aguirre, N. Added, M. A. Rizzutto, and M. H. Tabacniks. MultiSIMNRA: A computational tool for self-consistent ion beam analysis using SIMNRA. Nuclear Instruments and Methods B, 371:86 - 89, 2016.

[105] H. H. Andersen, F. Besenbacher, P. Loftager, and W. Moller. Large-angle scattering of light ions in the weakly screened Rutherford region. Physical Review A, 21 (6):1891-1901, 1980.

[106] A. F. Gurbich. Evaluation of non-Rutherford cross sections for IBA: Theory and results. Nuclear Instruments and Methods B, 261:401-404, 2007.

[107] IAEA. Ion Beam Analysis Nuclear Data Library. Data Compilation: Valentina Semkova, NDS, International Atomic Energy Agency, 2014, Aceso em 7 de julho de 2016. URL https://www-nds.iaea.org/exfor/ibandl.htm.

[108] J. F. Ziegler, J. P. Biersack, and U. Littmark. The Stopping and Range of Ions in Solids, vol. 1 of The Stopping and Ranges of Ions in Matter. Pergamon Press, New York, 1985.

[109] NIST. X-Ray Mass Attenuation Coefficients. Aceso em 4 de abril de 2016. URL http://physics.nist.gov/PhysRefData/XrayMassCoef/tab1.html.

[110] H. H. Hubbell and R. D. Birkhoff. Calorimetric measurement of electron stopping power of aluminium and copper between 11 and $127 \mathrm{keV}$. Physical Review A, 26 (5):2460-2467, 1982.

[111] NIST. ESTAR: Stopping Powers and Ranges for Electrons. Aceso em 4 de abril de 2016. URL http://physics.nist.gov/PhysRefData/Star/Text/method.html.

[112] X. Llovet, C. J. Powell, F. Salvat, and A. Jablonski. Cross Sections for Inner-Shell Ionization by Electron Impact. Journal of Physical and Chemical Reference Data, 43:013102 (105 pp), 2014. 
[113] J. C. Clark. A measurement of the absolute probability of K-electron ionization of silver by cathode rays. Physical Review, 48:30-42, 1935.

[114] D. H. Rester and W. E. Dance. K-shell ionization of Ag, Sn, and Au from electron bombardment. Physical Review, 152:1-3, 1966.

[115] D. V. Davis, V. D. Mistry, and C. A. Quarles. Inner shell ionization of copper, silver and gold by electron bombardment. Physics Letters, 38A:169-170, 1972.

[116] K. Kiss, Gy. Kálmán, J. Pálinkás, and B. Schlenk. Investigation of inner shell ionization by electron impact in the $60-600 \mathrm{keV}$ energy region. Acta Physica Academiae Scientiarum Hungaricae, 50:97-102, 1981.

[117] K. Shima, T. Nakagawa, K. Umetani, and T. Mikuno. Threshold behavior of Cu-, Ge-, Ag-K, and Au-L3-shell ionization cross sections by electron impact. Physical Review A, 24:72-74, 1981.

[118] G. L. Westbrook and C. A. Quarles. Total cross sections for ionization of the K-shell by electron bombardment. Nuclear Instruments and Methods B, 24/25: 196-198, 1987.

[119] H. Schneider, I. Tobehn, F. Ebel, and R. Hippler. Absolute cross section for inner shell ionization by lepton impact. Physical Review Letters, 71:2707-2709, 1993.

[120] C.-G. Zhou, Y.-C. Fu, Z. An, C.-H. Tang, and Z.-M. Lou. Measurement and multiple scattering correction of $\mathrm{K}$-shell ionization cross sections of silver by electron impact. Chinese Physical Letters, 18:531-532, 2001.

[121] V. R. Vanin, M. V. Manso Guevara, N. L. Maidana, M. N. Martins, and J. M. Fernández-Varea. Ag K-shell ionization by electron impact: New cross-section measurements between 50 and $100 \mathrm{keV}$ and review of previous experimental data. Radiation Physics and Chemistry, 119:14-23, 2016.

[122] D. Bote and F. Salvat. Calculations of inner-shell ionization by electron impact with the distorted-wave and plane-wave Born approximations. Physical Review A, 77:042701 (24pp), 2008.

[123] M. O. Krause. Atomic radiative and radiationless yields for K and L shells. Journal of Physical and Chemical Reference Data, 8:307-327, 1979.

[124] J. H. Scofield. Relativistic Hartree-Slater values for K and L X-ray emission rates. Atomic Data and Nuclear Data Tables, 14:121-137, 1974.

[125] J. M. Fernández-Varea, V. Jahnke, N. L. Maidana, A. A. Malafronte, and V. R. Vanin. Cross sections of K-shell ionization by electron impact, measured from threshold to $100 \mathrm{keV}$, for Au and Bi. Journal of Physics B, 47:155201 (9pp), 2014. 
[126] S. F. Barros, V. R. Vanin, N. L. Maidana, and J. M. Fernández-Varea. Ionization cross sections of the $\mathrm{L}$ subshells of Au by 50 to $100 \mathrm{keV}$ electron impact. Journal of Physics B, 48:175201 (12pp), 2015. 